\title{
NREL Photovoltaic Program FY 1994 Annual Report
}

K.A. Summers, Coordinator

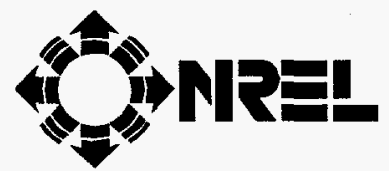

National Renewable Energy Laboratory 1617 Cole Boulevard Golden, Colorado 80401-3393

A national laboratory of the U.S. Department of Energy Managed by Midwest Research Institute for the U.S. Department of Energy under contract No. DE-AC36-83CH10093

Prepared under Task No. PV510101 June 1995 


\section{NOTICE}

This report was prepared as an account of work sponsored by an agency of the United States government. Neither the United States government nor any agency thereof, nor any of their employees, makes any warranty, express or implied, or assumes any legal liability or responsibility for the accuracy, completeness, or usefulness of any information, apparatus, product, or process disclosed, or represents that its use would not infringe privately owned rights. Reference herein to any specific commercial product, process, or service by trade name, trademark, manufacturer, or otherwise does not necessarily constitute or imply its endorsement, recommendation, or favoring by the United States government or any agency thereof. The views and opinions of authors expressed herein do not necessarily state or reflect those of the United States government or any agency thereof.

Available to DOE and DOE contractors from:

Office of Scientific and Technical Information (OSTI)

P.O. Box 62

Oak Ridge, TN 37831

Prices available by calling (615) $576-8401$

Available to the public from:

National Technical Information Service (NTIS)

U.S. Department of Commerce

5285 Port Royal Road

Springfield, VA 22161

(703) $487-4650$ 


\section{DISCLAIMER}

Portions of this document may be illegible electronic image products. Images are produced from the best available original document. 


\section{PREFACE}

This report summarizes the in-house and subcontracted research and development (R\&D) activities under the National Renewable Energy Laboratory (NREL) Photovoltaics (PV) Program from October 1, 1993, through September 30, 1994 (fiscal year [FY] 1994). The NREL PV Program is part of the U.S. Department of Energy's (DOE's) National Photovoltaics Program, as described in the DOE Photovoltaics Program Plan, FY 1991 - FY 1995. The mission of the DOE National Photovoltaics Program is to work in partnership with U.S. industry to develop and deploy photovoltaic technology for the generation of economically competitive electric power, making photovoltaics an important contributor to the nation's and the world's energy use and environmental improvement. The two primary goals of the national program are to maintain the U.S. PV industry's world leadership in research and technology development and to help the U.S. industry remain a major, profitable force in the world market. The NREL PV Program provides leadership and support to the national program toward achieving its mission and goals.

The FY 1994 budget authority (BA) for carrying out the NREL PV Program was $\$ 50.29$ million in operating funds and $\$ 3.323$ million in capital equipment funds. Subcontract activities represent a major part of the NREL PV Program, with more than $\$ 31.9$ million (nearly $63.5 \%$ ) of the FY 1994 operating funds going to 105 subcontractors. Cost sharing by industry added approximately $\$ 16.1$ million to the subcontract R\&D activities with industry.

The NREL PV Program activities involve conducting basic, applied, and engineering research; managing subcontracted R\&D projects; performing research complementary to subcontracted work; developing and maintaining state-of-the-art measurement and device capabilities; supporting PV manufacturing technology and module development; transferring results to industry; and evolving viable partnerships for PV systems and market development. The program is carried out under six project areas: PV Program Management, Crystalline Silicon and Advanced Devices, Thin-Film PV Technologies, Photovoltaic Manufacturing Technology, PV Module and System Performance and Engineering, and PV Applications and Markets Development.

\section{Approved for the NATIONAL RENEWABLE ENERGY LABORATORY}

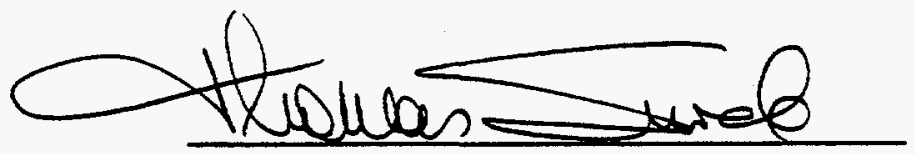

Thomas Surek, Manager

Photovoltaics Program
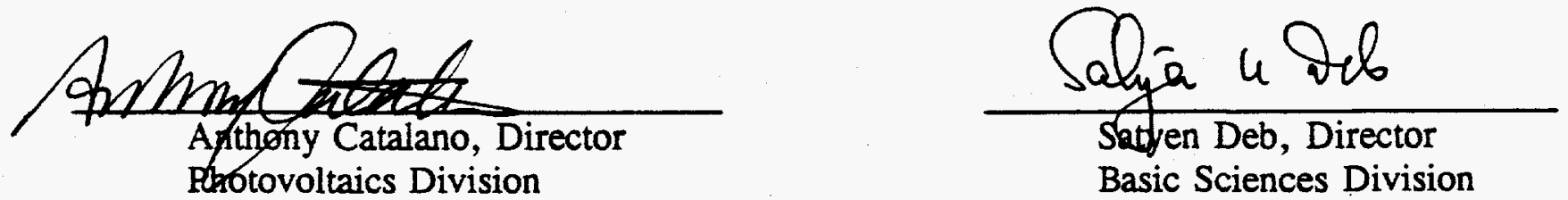

Notice: This publication was reproduced from camera-ready copy submitted by the individual subcontractors. The efficiency values reported by the subcontractors may not have been independently confirmed by NREL or Sandia National Laboratories. 
$1.0 \quad$ Introduction $\ldots \ldots \ldots \ldots \ldots \ldots \ldots \ldots \ldots \ldots \ldots \ldots \ldots \ldots \ldots$

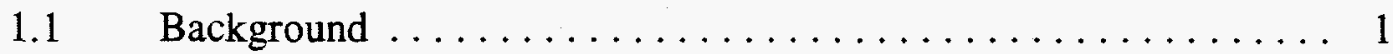

$1.2 \quad$ Key FY 1994 Accomplishments $\ldots \ldots \ldots \ldots \ldots \ldots \ldots \ldots 6$

1.3 Technology Transfer ...................... 14

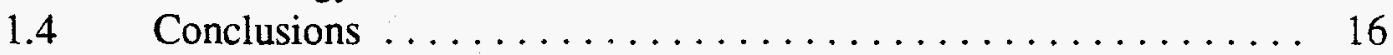

2.0 Crystalline Silicon and Advanced Devices Project $\ldots \ldots \ldots \ldots \ldots, 17$

Crystalline Silicon and Advanced Devices In-House Research

Crystalline Silicon Research;

NREL In-House Crystalline Silicon and Advanced Devices Research . . . 43

Material and Interface Characterization of PV Devices;

NREL In-House Crystalline Silicon and Advanced Devices Research . . . 47

Electro-Optical Characterization;

NREL In-House Crystalline Silicon and Advanced Devices Research . . . 51

GaInP/GaAs Tandem Solar Cells;

NREL In-House Crystalline Silicon and Advanced Devices Research . . . $\quad 55$

Solid State Theory;

NREL In-House Crystalline Silicon and Advanced Devices Research . . . 58

\section{Crystalline Silicon and Advanced Devices Subcontracts}

Influence of Self-Interstitials Injected by Phosphorus Diffusion on

Defect Structures and Electronic Properties in Crystalline Silicon;

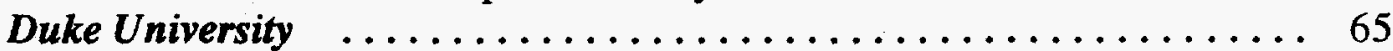

Fundamental Research on Post-Growth Quality Enhancement Techniques;

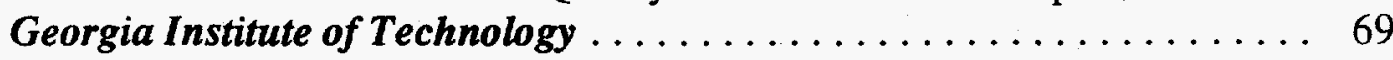

Solar Energy Conversion at Dye Sensitized Nanostructured Electrodes

Fabricated by Sol-Gel Processing; The Johns Hopkins University . . . . . 73 


\section{TABLE OF CONTENTS (continued)}

The Role of Point Defects and Impurities in the Processing Performance of Crystalline Silicon Solar Cells;

Back Surface Fields for n/p and p/n GaInP Solar Cells;

North Carolina State University $\ldots \ldots \ldots \ldots \ldots \ldots \ldots \ldots \ldots, 81$

Porous Silicon Solar Cell Development; Spire Corporation ............. 85

Thin-Film Silicon by Constitutional Supercooling;

State University of New York at Buffalo $\ldots \ldots \ldots \ldots \ldots \ldots \ldots 89$

Theoretical Analysis and Hydrogen-Vacancy Complex Formation and

Diffusion in Silicon; Texas Tech University ................. 93

New Plasma Source of Hydrides for Epitaxial Growth;

University of Florida $\ldots \ldots \ldots \ldots \ldots \ldots \ldots \ldots \ldots \ldots \ldots \ldots \ldots$

Fundamental Studies of the Effect of Crystal Defects on CuInSe $/ 2 \mathrm{CdS}$

Heterojunction Behavior; University of Illinois . ........... 100

Optimization of Gettering Processes for Photovoltaic Silicon;

University of South Florida . . . . . . . . . . . . . . . . . . . . 105

Advanced Processing Technology for High Efficiency Thin-Film CuInSe

and CdTe Solar Cells; Heterojunction Development and Optimization in

Thin-Film Compound Semiconductor Solar Cells;

University of South Florida .......................... 109

Electronic Processes in Thin-Film PV Materials;

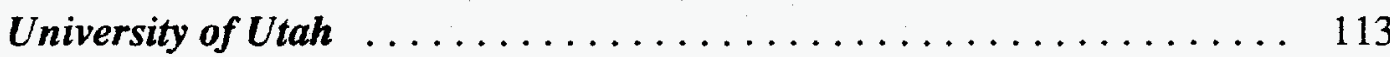

3.0 Thin-Film Technologies Project $\ldots \ldots \ldots \ldots \ldots \ldots \ldots \ldots \ldots, 117$

\section{Thin-Film Technologies In-House Research}

Development of Polycrystalline Thin-Film CdTe Solar Cells Deposited by Close-Spaced Sublimation; NREL In-House CdTe Research ........... 123

Development of Polycrystalline $\mathrm{Cu}(\mathrm{In}, \mathrm{Ga}) \mathrm{Se}_{2}$ Thin Films and Devices;

NREL In-House CIS Research ..................... 127 


\section{TABLE OF CONTENTS (continued)}

Connection Between Dangling Bond Relaxation and Metastability in Hydrogenated Amorphous Silicon; NREL In-House Amorphous Silicon Research . . . 131

Studies of H Diffusion and H Bonding in Device Quality a-Si:H; NREL In-House Amorphous Silicon Research ............. 135

Amorphous Silicon Solar Cells Incorporating Hot-Wire Deposited Intrinsic Layers; NREL In-House Amorphous Silicon Research . . . . . . . . . . . 136

\section{Thin-Film Technologies Subcontracts}

Development of Large Area, Monolithically Integrated Silicon-Film ${ }^{\mathrm{TM}}$ Photovoltaic Modules; AstroPower, Inc.

Polycrystalline Thin-Film Cadmium Telluride Solar Cells Fabricated by Electrodeposition; Colorado School of Mines . ............ 143

Microstructure of Amorphous-Silicon-Based Solar Cell Materials by Small-Angle X-Ray Scattering; Colorado School of Mines ........ 147

Device Physics of Thin-Film Polycrystalline Cells and Modules; Colorado State University

Development of High, Stable-Efficiency Triple-Junction a-Si Alloy Solar Cells; Energy Conversion Devices, Inc.

Non- $\mathrm{H}_{2} \mathrm{Se}$, Ultra-Thin CIS Devices;

Energy Photovoltaics

Polycrystalline CuInSe $\mathrm{C}_{2}$ and CdTe PV Solar Cells;

Florida Solar Energy Center ........................ 163

Development of High-Efficiency CdTe Solar Cells;

Georgia Institute of Technology ....................... 167

Optimization of Transparent and Reflecting Electrodes for Amorphous

Silicon Solar Cells; Harvard University

Processing and Modeling Issues for Thin-Film Solar Cell Devices;

University of Delaware, Institute of Energy Conversion 


\section{TABLE OF CONTENTS (continued)}

Novel Two-Stage Selenization Methods for Fabrication of Thin-Film CIS Cells and Sub-Modules; International Solar Electric Technology . . . . . . . 179

Research on Stability and Electronic Properties of a-Si:H and a-(Si,Ge):H Devices; Iowa State University . . . . . . . . . . . . . . . . . . . . . . . 183

Atomic Scale Characterization of Hydrogenated Amorphous Silicon Films and Devices; National Institute of Standards and Technology . . ......

Wide Bandgap Solar Cells with High Stabilized Performance;

The Pennsylvania State University . . . . . . . . . . . . . . .

Development and Application of a Computer Model for CdTe- and CuInSe $\mathrm{C}_{2}$-Based Solar Cells; Purdue University . . . . . . . . . . . . . . . . .

Research on High-Efficiency, Large-Area CuInSe ${ }_{2}$-Based Thin-Film Modules;

Siemens Solar Industries ......................... 199

Fabrication of Stable Large-Area Thin-Film Cadmium Telluride Photovoltaic Modules; Solar Cells, Inc. . . . . . . . . . . . . . . . . . . 203

Research on Stable, High-Efficiency Amorphous Silicon Multijunction Modules;

United Solar Systems Corporation . . . . . . . . . . . . . . . . 207

Photocharge Transport and Recombination Measurements in Amorphous Silicon Films and Solar Cells by Photoconductive Frequency Mixing;

University of California at Los Angeles . . . . . . . . . . . 210

Novel Thin-Film CuInSe ${ }_{2}$ Fabrication;

University of Colorado, Boulder . . . . . . . . . . . . . . . 214

Experimental Study of Photodegradation in a-Si:H Solar Cells;

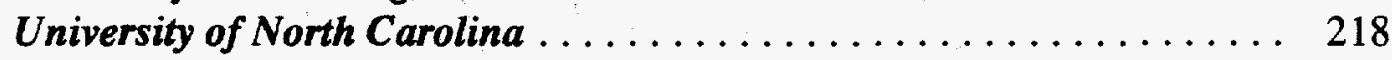

Microscopic Origins of Metastable Effects in a-Si:H and Deep Defect

Characterization in a-Si,Ge:H Alloys; University of Oregon ............ 222

Advanced Processing Technology for High-Efficiency Thin-Film CuInSe $\mathrm{C}_{2}$ and CdTe Solar Cells; Heterojunction Development and Optimization in Thin-Film

Compound Semiconductor Solar Cells; University of South Florida _.. 226 


\section{TABLE OF CONTENTS (continued)}

Research on Wide Bandgap, Amorphous-Silicon-Based Solar Cells;

Syracuse University

High-Efficiency Thin-Film Cadmium Telluride Photovoltaic Cells;

The University of Toledo

Investigation of Polycrystalline Thin-Film CuInSe ${ }_{2}$ Solar Cells Based on

ZnSe Windows; Washington State University

4.0 Photovoltaic Manufacturing Technology (PVMaT) Project

\section{PVMaT Subcontracts}

Amorphous Silicon Photovoltaic Manufacturing Technology—Phase II;

Advanced Photovoltaic Systems . . . . . . . . . . . . . . . . . . . . 261

Silicon-Film ${ }^{\mathrm{TM}}$ Photovoltaic Manufacturing Technology;

AstroPower, Inc.

Continuous Roll-to-Roll a-Si Photovoltaic Manufacturing Technology;

Energy Conversion Devices, Inc.

Photovoltaic Manufacturing Technology (PVMaT) Improvements for

ENTECH's Concentrator Module; ENTECH, Inc.

Commercial Production of Thin-Film CdTe Photovoltaic Modules;

Golden Photon, Inc.

PV Cz Silicon Manufacturing Technology Improvements;

Siemens Solar Industries

Development of CdTe Module Manufacturing;

Solar Cells, Inc.

Large-Area, Multijunction, a-Si Alloy Production Scale-Up;

Solarex Corporation .............................. 289

Cast Polycrystalline Silicon Photovoltaic Module Manufacturing

Technology Improvements; Solarex Corporation

Automated Solar Cell Assembly Teamed Process Research;

Spire Corporation 
Advanced Development of PV Encapsulants;

Springborn Laboratories, Inc.

Amorphous Silicon Photovoltaic Manufacturing Technology;

Utility Power Group . . . . . . . . . . . . . . . . . . . . . . 305

5.0 PV Module and System Performance and Engineering Project ... . . 309

PV Module and System Performance and Engineering In-House Research

PV Efficiency Measurements - Standard Reporting Conditions;

NREL In-House PV Module and System Performance and

Engineering Project ........................... 315

PV Module Materials and Encapsulation Research;

NREL In-House PV Module and System Performance and

Engineering Project ........................... 319

PV Module Qualification Testing and Test Method Development;

NREL In-House PV Module and System Performance and

Engineering Project ........................... 323

Photovoltaic Module and System Technology Validation;

NREL In-House PV Module and System Performance and

Engineering Project ........................ 327

Photovoltaic Component Diagnostics and Failure Analysis;

NREL In-House PV Module and System Performance and

Engineering Project $\ldots \ldots \ldots \ldots \ldots \ldots \ldots \ldots \ldots \ldots \ldots \ldots \ldots \ldots \ldots$

Photovoltaic Solar Radiometric Measurements and Evaluation;

NREL In-House PV Module and System Performance and

Engineering Project ......................... 335

PV Standards and Codes;

NREL In-House PV Module and System Performance and

Engineering Project ............................. 339

PV Module and System Performance and Engineering Subcontracts

PV Module Encapsulant Formulations;

University of Colorado at Boulder 


\section{TABLE OF CONTENTS (continued)}

PV Module Degradation-EVA Pore Volume;

Pennsylvania State University ..................... 348

Long-Term Effects on Roof-Mounted Photovoltaic Modules;

Southwest Technology Development Institute . . . . . . . . . . . . . . 349

NREL Module Energy Rating Methodology Project;

Endecon Engineering . . . . . . . . . . . . . . . . 353

PV Performance Modeling and Support;

Sunset Technology Co ...................... 355

Support for the National Electrical Code;

Solar Energy Industries Association $\ldots \ldots \ldots \ldots \ldots 7$

Management and Administration of IEC/TEC-82 Secretariat;

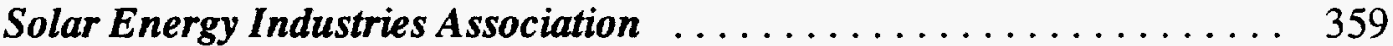

PV Utility/Industry Engineering Projects;

Advanced Photovoltaic Systems

Energy Conversion Devices

Energy Photovoltaics

Integrated Power Corporation

Solarex Corporation

Utility Power Group . . . . . . . . . . . . . . . . . . . . . . . 361

6.0 PV Applications and Market Development Project .......... 365

Design for Photovoltaics: Curriculum Development for North American

Architecture School Faculty; AIA/ACSA Council . . . . . . . . . . 373

Joint U.S./Brazilian Rural Electrification Pilot Project;

Centro de Pesquisas de Energia Elétrica (CEPEL) . . . . . . . . . 377

Solar Resource Utility Load Matching Assessment;

The University at Albany, New York,

Atmospheric Sciences Research Center . . . . . . . . . . . . . . 378

Evaluation of DSM Incentive Opportunities for Photovoltaics;

University of Delaware, Center for Energy and Environmental Policy . . 382

Rooftop PV System;

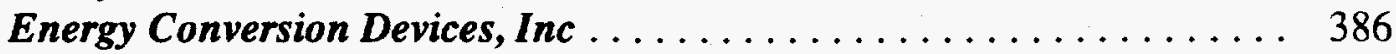




\section{TABLE OF CONTENTS (continued)}

PV Program Support to Electric Utilities;

NEOS Corporation

7.0 List of Active Subcontracts .

8.0 NREL Photovoltaic Program FY 1994 Bibliography 405 


\subsection{INTRODUCTION}

This report summarizes the in-house and subcontracted research and development (R\&D) activities under the NREL PV Program from October 1, 1993, through September 30, 1994 (FY 1994). The NREL PV Program is part of DOE's National Photovoltaics Program, as described in the DOE Photovoltaics Program Plan, FY 1991-FY 1995. The FY 1994 budget authority for carrying out the NREL PV Program was $\$ 50.29$ million in operating funds and $\$ 3.323$ million in capital equipment funds. The operating funds included \$0.375 million of FY 1993 funds and $\$ 0.775$ million of non-PV Program funds from DOE. Subcontract activities represent a major part of the NREL PV Program, with more than $\$ 31.9$ million (nearly 63.5\%) of the FY 1994 operating funds going to 105 subcontractors. Cost sharing by industry added about $\$ 16.1$ million to the subcontract R\&D activities with industry.

\subsection{Background}

The NREL PV Program is part of the DOE National Photovoltaics Program managed by the Photovoltaics Technology Division in the Office of Solar Energy Conversion (OSEC). OSEC is under the Office of Utility Technologies in DOE's Office of Energy Efficiency and Renewable Energy. Major program thrusts in FY 1994 continued to be implemented based on DOE's Photovoltaics Program Plan FY 1991-FY 1995. The program mission is to:

Work in partnership with U.S. industry to develop and deploy photovoltaic technology for generating economically competitive electric power, making photovoltaics an important contributor to the nation's and the world's energy use and environmental improvement.

The two primary goals of the national program are to (1) maintain the U.S. PV industry's world leadership in research and technology development, and (2) help the U.S. industry remain a major, profitable force in the world market. The NREL PV Program provides leadership and support to the national program toward achieving its mission and goals.

The DOE strategy over the next few years will be to lay the groundwork for a growing U.S. PV technology and industrial base, with increased emphasis on market and project development activities with industry. To accomplish this, the national program embraces three relatively equal priority activities: (1) technology development and validation, (2) market conditioning, and (3) project venturing. The new strategy continues a strong technology development program, but emphasizes R\&D for the technologies and companies that are positioned to substantially penetrate the market, reduce prices, and scale up manufacturing. Program activities include continuing efforts to form partnerships with manufacturers and utilities (the ultimate benefactors and users), with universities, and with federal and state agencies.

Under the DOE National Photovoltaics Program, the NREL PV Program supports fundamental and applied R\&D, manufacturing development, and systems and market development in PV energy technology. The project also provides services to industry, electric utilities, and other users, and it provides overall programmatic support for the National Photovoltaics Program. The 
NREL subcontract program is responsible for most of the R\&D, manufacturing technology development, and some of the systems and market development task areas under the National Photovoltaics Program. It is implemented via competitive public solicitations. One of the most important subcontracting mechanisms is government/industry partnerships, with industry sharing the cost of research with DOE/NREL.

Closer work with industry, utilities, and other end users on PV manufacturing technology, systems, and market needs is being increasingly emphasized. Approaches for this emphasis include mitigating barriers to PV adoption in the utility and international marketplaces, and project venturing with decision makers and organizations representing domestic and international market sectors for PV. As appropriate for the system and market development areas, NREL is supporting activities such as education; technical assistance and training; market, economic, and financial analysis; technology characterizations; regulatory, rate, and value analysis; codes and standards assessment and development; working with customers in project development activities; and co-financing demonstration projects. Under project venturing, DOE is placing particular emphasis on supporting and strengthening programs already in place, such as PV:BONUS (Building Opportunities in the U.S. for Photovoltaics), the Americas' 21st Century Project, and the Utility PhotoVoltaics Group (UPVG).

NREL's PV activities include laboratory research and subcontract project management. We also provide technical support to efforts contracted from the DOE Golden Field Office (GO), such as PV:BONUS and UPVG. The primary research activities are conducted in advanced PV material technologies, including amorphous silicon (a-Si) thin-film materials; polycrystalline thin films, such as copper indium diselenide (CIS), cadmium telluride (CdTe), and their alloys; and highefficiency crystalline cells, including silicon ( $\mathrm{Si}$ ) and gallium arsenide and their alloys. These activities are conducted in-house and through subcontracts with industry (mostly cost shared) and universities. The research activities are closely coordinated through several NREL/industry/university "team" efforts. Improving PV device manufacturing is vital. We are pursuing two complementary approaches:

- Government/industry partnerships, such as the PVMaT project (which focuses on improving manufacturing processes and products, accelerating manufacturing cost reduction, and laying the foundation for increased production capacity)

- Module development research to evaluate modules (and module performance) and suggest solutions to manufacturers' module problems.

System and market development rounds out the balanced approach pursued. The objective is to create an environment in which system technology, user acceptance, and the PV industry can accommodate the continued expansion of PV into larger applications and markets. NREL subcontracts also support the continued influx of new ideas and highly qualified university research teams to expand the current limits of PV technology. Transferring research results into commercial products and applications in a timely and effective manner is another major activity of NREL's PV Program. 
Subcontracted R\&D is a significant part of the NREL PV Program, with nearly two-thirds of the program's budget allocated yearly to subcontracts. From FY 1978 through FY 1994, we awarded more than 1100 subcontracts totalling nearly $\$ 285$ million out of a total operating budget of $\$ 445$ million. In FY 1994, we awarded 105 subcontracts with a total funding of $\$ 31.9$ million. Cost sharing by industry subcontractors added about $\$ 16.1$ million to the $\$ 24.35$ million in NREL funding of 41 industry subcontracts. Fifty-five NREL subcontracts were with universities, with a total funding of $\$ 6.82$ million. Table 1.1-1 shows the distribution of in-house and subcontract budgets. Figure 1.1-1 shows the distribution of subcontract funds by business category. Table 1.1-2 shows the contacts for the various NREL PV Program areas. 
Table 1.1-1. Budget History of the NREL Photovoltaics Program

\begin{tabular}{|c|c|c|c|c|c|}
\hline & \multicolumn{5}{|c|}{ Fiscal Year } \\
\hline & $\begin{array}{l}\text { 1978-1990 } \\
(\$ M)\end{array}$ & $\begin{array}{l}1991 \\
(\$ M)\end{array}$ & $\begin{array}{l}1992 \\
(\$ M)\end{array}$ & $\begin{array}{l}1993 \\
(\$ M)\end{array}$ & $\begin{array}{l}1994 \\
(\$ M)\end{array}$ \\
\hline \multicolumn{6}{|l|}{ TASK AREA } \\
\hline In-House R\&D & 84.3 & 12.2 & 14.0 & 14.3 & 16.4 \\
\hline Capital Equipment & 15.3 & 0.6 & 1.3 & 5.7 & 3.3 \\
\hline Subtotal (in-house) & 99.6 & 12.8 & 15.3 & 20.0 & 19.7 \\
\hline \multicolumn{6}{|l|}{ Subcontracted $R \& D^{1}$} \\
\hline Amorphous Silicon Thin Films & 72.9 & 3.3 & 3.9 & 3.4 & 4.3 \\
\hline Polycrystalline Thin Films & 45.4 & 4.5 & 4.8 & 5.8 & 6.7 \\
\hline High-Efficiency Concepts & 34.5 & 1.3 & 0.9 & 1.0 & 0.6 \\
\hline Crystalline Silicon & 23.2 & 0.9 & 0.9 & 0.9 & 1.2 \\
\hline New Ideas & $18.4^{2}$ & 0.4 & 0.1 & 0.3 & 0.2 \\
\hline University Participation & 6.1 & 0.8 & 0.7 & 0.8 & 0.8 \\
\hline Subtotal (R\&D subcontracts) & 200.5 & 11.2 & 11.3 & 12.2 & 13.8 \\
\hline \multicolumn{6}{|c|}{$\begin{array}{l}\text { Manufacturing Technology Development and System and Market Development } \\
\text { Subcontracts }{ }^{1}\end{array}$} \\
\hline PVMaT Project & 1.7 & 6.7 & 10.2 & 10.6 & 16.6 \\
\hline $\begin{array}{l}\text { Module and System Performance and } \\
\text { Engineering Project }{ }^{3}\end{array}$ & N/A & 2.0 & 0.1 & 0.5 & 0.6 \\
\hline $\begin{array}{l}\text { PV Analysis and Applications } \\
\text { Development Project }^{4}\end{array}$ & N/A & N/A & 0.7 & 2.5 & 2.9 \\
\hline Subtotal (other subcontracts) & 1.7 & 8.7 & 11.0 & 13.6 & 20.1 \\
\hline Subtotal (subcontracts) ${ }^{1}$ & 202.2 & 19.9 & 22.3 & 25.8 & 33.9 \\
\hline $\begin{array}{l}\text { TOTAL NREL PV } \\
\text { PROGRAM }\end{array}$ & 301.8 & 32.7 & 37.6 & 45.8 & 53.6 \\
\hline
\end{tabular}

' Includes about 15\%-20\% for technical program management, fees, etc.

${ }^{2}$ Includes $\$ 9$ million for photoelectrochemical cell research

${ }^{3}$ Significant subcontracted effort initiated in FY 1991

4 Significant subcontracted effort initiated in FY 1992 
Figure 1.1-1. Business category distribution of FY 1994 subcontract funds

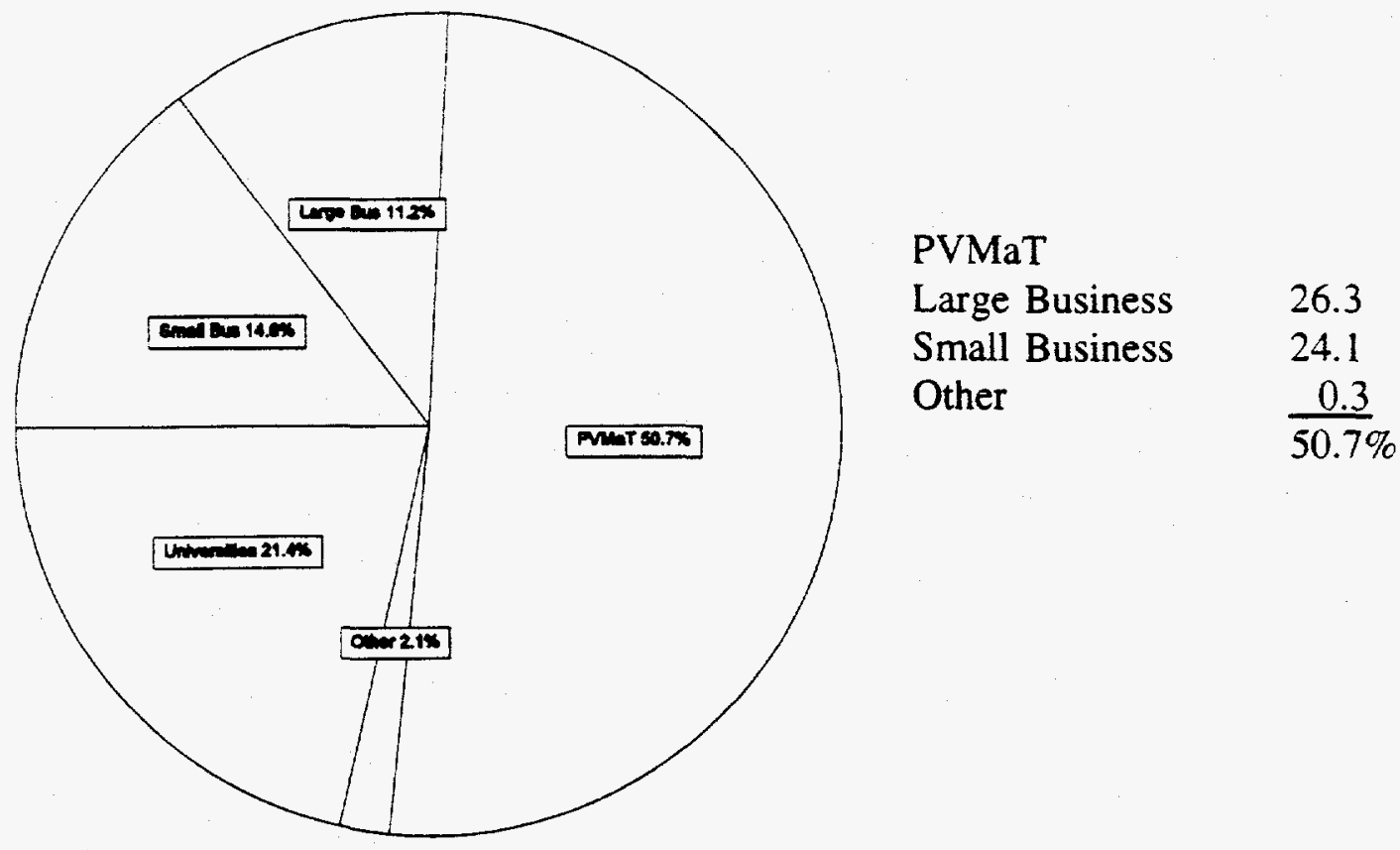

Total Subcontracts $=\$ 31.9$ million

Cost-share by Industry $=\$ 16.1$ million

\begin{tabular}{|l|l|l|}
\hline \multicolumn{2}{|c|}{ Table 1.1-2. NREL Photovoltaics Program Contacts by Project Area } \\
\hline \multicolumn{1}{|c|}{ Project Area } & \multicolumn{1}{|c|}{ Contact Name } & Telephone \\
\hline PV Program & $\begin{array}{l}\text { Thomas Surek, Manager } \\
\text { Kathy Summers }\end{array}$ & $\begin{array}{l}\text { (303) 384-6471 } \\
(303) 384-6595\end{array}$ \\
\hline $\begin{array}{l}\text { Crystalline Silicon and } \\
\text { Advanced Devices Project }\end{array}$ & John Benner, Manager & $(303) 384-6496$ \\
\hline $\begin{array}{l}\text { Thin-Film Photovoitaics } \\
\text { Technologies Project }\end{array}$ & Kenneth Zweibel, Manager & (303) 384-6441 \\
\hline PVMaT Project & Ed Witt. Manager & (303) 384-6402 \\
\hline $\begin{array}{l}\text { PV Module and System } \\
\text { Performance and Engineering }\end{array}$ & $\begin{array}{l}\text { Richard DeBlasio, } \\
\text { Manager }\end{array}$ & (303) 275-3786 \\
\hline $\begin{array}{l}\text { PV Applications and Market } \\
\text { Development Project }\end{array}$ & Roger Taylor, Manager & (303) 384-6432 \\
\hline
\end{tabular}




\subsection{Key FY 1994 Accomplishments}

Table 1.2-1 describes some key achievements in the NREL PV Program during FY 1994. The accomplishments are grouped by the six project areas of the program. Sections 2.0 through 6.0 of this report provide further details of the in-house and subcontracted research performed under these projects.

\begin{tabular}{|c|c|}
\hline Research Group & Achievement \\
\hline \multicolumn{2}{|c|}{ PV PROGRAM MANAGEMENT } \\
\hline NREL & $\begin{array}{l}\text { The new Solar Energy Research Facility (SERF) was } \\
\text { opened. We moved } 37 \text { laboratories and } 170 \text { staff } \\
\text { members into the facility with minimal impact on the } \\
\text { research activities. }\end{array}$ \\
\hline NREL & $\begin{array}{l}\text { We developed an innovative program aimed at } \\
\text { attracting minority science and engineering students } \\
\text { from Historically Black Colleges and Universities into } \\
\text { PV. }\end{array}$ \\
\hline NREL & $\begin{array}{l}\text { We organized a review of the DOE PV Program by } \\
\text { DOE's Office of Program Analysis. The reviews were } \\
\text { conducted during late FY } 1994 \text { and early FY } 1995 \text {. }\end{array}$ \\
\hline \multicolumn{2}{|c|}{ CRYSTALLINE SILICON AND ADVANCED DEVICES PROJECT } \\
\hline NREL & $\begin{array}{l}\text { Achieved } 30.2 \% \text { efficiency in a monolithic, multiple- } \\
\text { junction concentrator cell }\left(\mathrm{GaInP}_{2} / \mathrm{GaAs}\right) \text { at } 150 \text { suns } \\
\text { illumination. }\end{array}$ \\
\hline $\begin{array}{l}\text { NREL, North Carolina } \\
\text { State University } \\
\text { (NCSU), MEMC, } \\
\text { Siemens Solar } \\
\text { Industries (SSI) }\end{array}$ & $\begin{array}{l}\text { Demonstrated that minority carrier lifetimes in single- } \\
\text { crystal Si can be increased by post-growth processing } \\
\text { using a combination of phosphorus diffusion with } \\
\text { rapid thermal anneal followed by hydrogen } \\
\text { passivation. }\end{array}$ \\
\hline NREL, Labsphere & $\begin{array}{l}\text { Agreed to license and produce the Scanning Defect } \\
\text { Mapping System developed at NREL. The } \\
\text { commercial system will rapidly analyze crystal defects } \\
\text { in Si wafers, providing industry with a tool to monitor } \\
\text { processes and process development. }\end{array}$ \\
\hline
\end{tabular}




\begin{tabular}{|c|c|}
\hline Research Group & Achievement \\
\hline $\begin{array}{l}\text { NREL, Texas Tech } \\
\text { University, Georgia } \\
\text { Institute of Technology } \\
\text { (GIT) }\end{array}$ & $\begin{array}{l}\text { Established a theoretical and experimental foundation } \\
\text { to explain mechanisms involved in hydrogenating } \mathrm{Si} \text {, } \\
\text { an essential step in developing advanced processes. } \\
\text { Hydrogen has been observed to diffuse at rates that } \\
\text { are orders of magnitude greater than conventionally } \\
\text { expected values. }\end{array}$ \\
\hline $\begin{array}{l}\text { NREL, Texas } \\
\text { Instruments (TI) }\end{array}$ & $\begin{array}{l}\text { As a result of research covered under a cooperative } \\
\text { research and development agreement (CRADA), TI } \\
\text { will install a minority carrier lifetime measurement } \\
\text { system (designed at NREL) into its line for process } \\
\text { monitoring. }\end{array}$ \\
\hline NREL & $\begin{array}{l}\text { Organized the First NREL Conference on } \\
\text { Thermophotovoltaic Generation of Electricity to } \\
\text { provide a catalyst for forming a national R\&D } \\
\text { program in thermophotovoltaic technology. }\end{array}$ \\
\hline NREL & $\begin{array}{l}\text { Selected and awarded subcontracts for research in } \\
\text { high-efficiency PV technologies. }\end{array}$ \\
\hline \multicolumn{2}{|c|}{ THIN-FILM PV TECHNOLOGIES PROJECT } \\
\hline NREL & $\begin{array}{l}\text { Increased the world record for all thin films from } \\
\text { previous best (15.5\%) to } 16.8 \% \text { on a copper indium } \\
\text { gallium diselenide (CIGS) thin-film cell. Shared } \\
\text { technology with U.S. CIGS industry through extended } \\
\text { on-site visits; for example, improved Solarex CIGS } \\
\text { from } 11 \% \text { to } 14.5 \% \text { through hands-on technology } \\
\text { transfer. }\end{array}$ \\
\hline $\begin{array}{l}\text { United Solar Systems } \\
\text { Corporation (USSC) }\end{array}$ & $\begin{array}{l}\text { Produced the first }>10 \% \text {-efficient, stabilized a-Si } \\
\text { prototype module (1- } \mathrm{ft}^{2} \text { area). }\end{array}$ \\
\hline Solar Cells, Inc. (SCI) & $\begin{array}{l}\text { Rapidly improved the efficiency/output of its large- } \\
\text { area }\left(7200-\mathrm{cm}^{2} \text { or } 8-\mathrm{ft}^{2}\right) \text { CdTe modules to } 8.4 \%(60 \\
\text { watts). Delivered } 1 \mathrm{~kW} \text { of modules to NREL for } \\
\text { testing. }\end{array}$ \\
\hline $\begin{array}{l}\text { University of South } \\
\text { Florida (USF) }\end{array}$ & $\begin{array}{l}\text { Produced best CdTe solar cell on low-cost sodalime } \\
\text { glass ( } 13.5 \% \text { efficient). }\end{array}$ \\
\hline NREL & $\begin{array}{l}\text { Demonstrated a "baseline" capability of } 10 \% \text {-efficient } \\
\text { CdTe solar cells ( } 12 \% \text { best). }\end{array}$ \\
\hline
\end{tabular}




\begin{tabular}{|c|c|}
\hline Research Group & Achievement \\
\hline NREL & $\begin{array}{l}\text { Initial encouraging stability results on hot-wire a-Si:H } \\
\text { (no degradation observed in first set of cells). }\end{array}$ \\
\hline $\begin{array}{l}\text { Siemens Solar } \\
\text { Industries }\end{array}$ & $\begin{array}{l}\text { Achieved } 10.2 \% \text {-efficient, encapsulated CIGS power } \\
\text { module (4-ft } \mathrm{ft}^{2} \text { area). Delivered world's first } 1-\mathrm{kW} \text { CIS } \\
\text { array to NREL for testing. }\end{array}$ \\
\hline Golden Photon, Inc. & $\begin{array}{l}\text { Introduced first commercial U.S. CdTe products } \\
\text { (demonstration scale). Built 2-MW production plant } \\
\text { (first CdTe plant in the world). }\end{array}$ \\
\hline $\begin{array}{l}\text { Energy Photovoltaics, } \\
\text { Inc. }\end{array}$ & $\begin{array}{l}\text { Achieved } 9 \% \text { efficiency on } 200-\mathrm{cm}^{2} \text { CIGS submodule; } \\
\text { built equipment for depositing } 7-\mathrm{ft}^{2} \text { modules. }\end{array}$ \\
\hline NREL & $\begin{array}{l}\text { Co-hosted, with Brookhaven National Laboratory, an } \\
\text { international workshop on the toxicology of CIS-based } \\
\text { and CdTe PV compounds. }\end{array}$ \\
\hline NREL & $\begin{array}{l}\text { Developed Thin-Film PV Partnership Program and } \\
\text { carried out solicitation on schedule. Formed } \\
\text { Partnership's CIS, CdTe, and Environmental Safety } \\
\text { and Health (ES\&H) R\&D teams (with NREL, } \\
\text { industry, and university participation) to add to } \\
\text { existing a-Si teams. }\end{array}$ \\
\hline \multicolumn{2}{|c|}{$\begin{array}{l}\text { PHOTOVOLTAIC MANUFACTURING TECHNOLOGY } \\
\text { (PVMaT) PROJECT }\end{array}$} \\
\hline NREL & $\begin{array}{l}\text { Working with the PVMaT Implementation Group, } \\
\text { NREL developed a solicitation for the next phase of } \\
\text { the program, PVMaT Phase } 4 a-\text { Product-Driven } \\
\text { Manufacturing. The solicitation was issued and } \\
\text { proposals were selected for award in FY } 1995 \text {. }\end{array}$ \\
\hline AstroPower, Inc. (API) & $\begin{array}{l}\text { API's accomplishments included fabricating a } 15-\mathrm{cm} \mathrm{x} \\
45-\mathrm{cm} \text {, Si-Film }{ }^{\mathrm{TM}} \text { cell, the largest Si solar cell ever } \\
\text { produced. API also increased its wafer production } \\
\text { capacity to } 4.4 \mathrm{MW} / \mathrm{yr} \text { and reduced the cost of } \\
\text { producing the wafers by } 53 \% \text {. All together, API } \\
\text { reduced module fabrication costs by } 42 \% \text {. }\end{array}$ \\
\hline
\end{tabular}




\begin{tabular}{|c|c|}
\hline Research Group & Achievement \\
\hline $\begin{array}{l}\text { Energy Conversion } \\
\text { Devices (ECD), Inc. }\end{array}$ & $\begin{array}{l}\text { ECD demonstrated full-scale production runs of rolls } \\
\text { of a-Si film with a total length of } 762 \mathrm{~m}(2506 \mathrm{ft}) \text {. } \\
\text { The film had excellent uniformity with subcell yields } \\
\text { of } 99.7 \% \text { (uniformity is a critical property of thin } \\
\text { films). ECD also produced a triple-junction module, } \\
0.37 \mathrm{~m}^{2}\left(4 \mathrm{ft}^{2}\right) \text { in size, with a stabilized efficiency of } \\
8.0 \% \text {. Overall, ECD reduced material costs by } 56 \% \text {. }\end{array}$ \\
\hline ENTECH, Inc. & $\begin{array}{l}\text { ENTECH developed several improved production } \\
\text { processes to reduce material and labor costs. Working } \\
\text { with the } 3 \mathrm{M} \text { Company, ENTECH developed an } \\
\text { improved prismatic cell cover, reducing the material } \\
\text { and labor costs of that step by } 90 \% \text {. ENTECH also } \\
\text { identified a team that can produce all parts of its } \\
\text { concentrator modules at a production capacity of more } \\
\text { than } 10 \mathrm{MW} / \mathrm{yr} \text {. As part of this team, three companies } \\
\text { produce PV cells compatible with ENTECH's module, } \\
\text { with efficiencies greater than } 19 \% \text {. }\end{array}$ \\
\hline $\begin{array}{l}\text { Mobil Solar Energy } \\
\text { Corporation (MSEC) }\end{array}$ & $\begin{array}{l}\text { MSEC produced thin }(250-\mu \mathrm{m}) \text { Si wafers from unique, } \\
\text { octagon-shaped tubes, resulting in cells with an } \\
\text { efficiency of } 13.8 \% \text {. In addition, MSEC developed a } \\
\text { workstation on the production line for cutting wafers } \\
\text { with a laser beam to increase throughput on the line } \\
\text { by a factor of two. Overall, MSEC reduced its } \\
\text { module production costs by } 15 \% \text {. MSEC's PVMaT } \\
\text { project ended early in FY 1994; the company was } \\
\text { purchased by ASE Americas, Inc. }\end{array}$ \\
\hline $\begin{array}{l}\text { Siemens Solar } \\
\text { Industries }\end{array}$ & $\begin{array}{l}\text { SSI improved the design of its crystal growers, } \\
\text { reducing by } 30 \% \text { the cost of producing crystalline } \\
\text { ingots and saving } \$ 300,000 / y r \text {. In addition, SSI } \\
\text { concentrated the waste streams, reducing waste } \\
\text { volume by } 10 \% \text { and waste-handling costs by } 20 \% \text {. }\end{array}$ \\
\hline Solarex Corporation & $\begin{array}{l}\text { Solarex developed a monolithic (made in a single } \\
\text { piece), multi-junction, a-Si module, } 3700 \mathrm{~cm}^{2}\left(4 \mathrm{ft}^{2}\right) \text { in } \\
\text { size, with an initial efficiency of } 8.9 \% \text {. Solarex } \\
\text { started commercial production of these modules and } \\
\text { plans to increase production to } 10 \mathrm{MW} / \mathrm{yr} \text {. }\end{array}$ \\
\hline
\end{tabular}




\begin{tabular}{|c|c|}
\hline Research Group & Achievement \\
\hline $\begin{array}{l}\text { Utility Power Group } \\
\text { (UPG) with lower-tier } \\
\text { subcontractor, } \\
\text { Advanced Photovoltaic } \\
\text { Systems, Inc. (APS) }\end{array}$ & $\begin{array}{l}\text { UPG substantially streamlined its module-production } \\
\text { processes. For example, UPG eliminated } 30 \text { steps } \\
\text { from the process used to encapsulate and connect the } \\
\text { electrical leads of the module, reducing the cost of this } \\
\text { production step by } 81 \% \text {. Overall, UPG reduced } \\
\text { module production costs by } 28 \% \text { and increased its } \\
\text { manufacturing capacity by a factor of five. APS } \\
\text { developed prototypes of new modules and reduced } \\
\text { processing time for producing modules, significantly } \\
\text { reducing costs. APS completed startup of its } \\
\text { production line in Fairfield, CA. }\end{array}$ \\
\hline Golden Photon, Inc. & $\begin{array}{l}\text { Golden Photon completed design of its manufacturing } \\
\text { line with a production capacity of } 2 \mathrm{MW} / \mathrm{yr} \text {. }\end{array}$ \\
\hline Solar Cells, Inc. & $\begin{array}{l}\text { SCI improved the mechanism for conveying raw } \\
\text { materials and for controlling pressure and temperature } \\
\text { during processing. Overall, SCI increased production } \\
\text { capacity by a factor of } 100 \text {. }\end{array}$ \\
\hline Solarex Corporation & $\begin{array}{l}\text { Solarex modified the design of one casting station, } \\
\text { resulting in a } 73 \% \text { increase in capacity, and began } \\
\text { using a new wire saw for cutting wafers from cast } \\
\text { ingots. }\end{array}$ \\
\hline Texas Instruments & $\begin{array}{l}\text { TI developed equipment that can produce several } \\
\text { MW/yr, demonstrated cell yields as high as } 90 \% \text { on a } \\
\text { pilot production line, and produced a } 4000-\mathrm{cm}^{2}, 8.3 \% \text { - } \\
\text { efficient pilot PV module. (TI closed its PV } \\
\text { operations January } 26,1995 \text {.) }\end{array}$ \\
\hline Spire Corporation & $\begin{array}{l}\text { Spire developed automated equipment that assembles } \\
\text { and solders Si solar cells into cell strings. Spire } \\
\text { developed and conducted demonstration tests of a } \\
\text { prototype machine using Si cells from several } \\
\text { manufacturers and will test the machine in a } \\
\text { production setting in FY } 1995 \text {. }\end{array}$ \\
\hline $\begin{array}{l}\text { Springborn } \\
\text { Laboratories, Inc. }\end{array}$ & $\begin{array}{l}\text { Springborn discovered that some glass products used } \\
\text { by PV module manufacturers transmit significantly } \\
\text { less ultraviolet (UV) radiation than normal glass. } \\
\text { Through accelerated UV testing, Springborn } \\
\text { determined that the new glass significantly reduces } \\
\text { premature aging of the encapsulants. }\end{array}$ \\
\hline
\end{tabular}




\begin{tabular}{|c|l|}
\hline \multicolumn{2}{|c|}{ Table 1.2-1. Key Achievements } \\
\hline Research Group & \multicolumn{1}{|c|}{ Achievement } \\
\hline PV MODULE AND SYSTEM PERFORMANCE \\
AND ENGINEERING PROJECT
\end{tabular}




\begin{tabular}{|c|c|}
\hline Research Group & Achievement \\
\hline NREL with PV Industry & $\begin{array}{l}\text { Designed, installed, and operated technology validation } \\
\text { systems at the OTF site for USSC (1.8-kW a-Si } \\
\text { roofing modules), SSI (1.0-kW CIS modules), and SCI } \\
\text { ( } 0.4-\mathrm{kW} \text { CdTe modules). Monitored the SERF ( } 12- \\
\mathrm{kW} \text { crystalline-Si modules) for performance and } \\
\text { reliability. Test reports provided to individual } \\
\text { manufacturers. }\end{array}$ \\
\hline NREL with Industry & $\begin{array}{l}\text { Established a performance energy rating testing } \\
\text { (PERT) system at the NREL OTF and initiated testing } \\
\text { commercially available and advanced modules for } \\
\text { long-term performance to support establishing a data } \\
\text { base for developing and validating a module energy } \\
\text { rating methodology. Established industry technical } \\
\text { review committee to develop criteria for module } \\
\text { energy rating. }\end{array}$ \\
\hline $\begin{array}{l}\text { Arizona State } \\
\text { University }\end{array}$ & $\begin{array}{l}\text { Completed the first draft of a module certification and } \\
\text { laboratory accreditation criteria document. Criteria } \\
\text { were developed by industry representatives and are } \\
\text { currently under review. }\end{array}$ \\
\hline NREL & $\begin{array}{l}\text { Organized and conducted workshops: the } 7 \text { th PV } \\
\text { Performance and Reliability Workshop ( } 130 \text { attendees) } \\
\text { and a PV Standards and Codes Forum ( } 60 \text { attendees). }\end{array}$ \\
\hline NREL & $\begin{array}{l}\text { Provided leadership for several standards activities: } \\
\text { IEC TC- } 82 \text { Secretariat and National Electric } \\
\text { Code-Article } 690 \text { (subcontracts to Solar Energy } \\
\text { Industries Association); chaired Institute of Electrical } \\
\text { and Electronics Engineers (IEEE) SCC21 and } \\
\text { American Society for Testing and Materials (ASTM) } \\
\text { E44.09 committees. }\end{array}$ \\
\hline NREL with Industry & $\begin{array}{l}\text { Conducted cell and module intercomparisons (ASTM) } \\
\text { with Solarex, USSC, Spire, MSEC, SSI, SOLTECH, } \\
\text { and Sandia. Coordinated international comparisons } \\
\text { with Japan, Germany, and other countries. }\end{array}$ \\
\hline
\end{tabular}




\begin{tabular}{|c|c|}
\hline Research Group & Achievement \\
\hline NREL & $\begin{array}{l}\text { Coordinated the development of the } 7 \text { th draft of the } \\
\text { IEEE PAR } 1262 \text { module qualification test document, } \\
\text { which includes thin- and thick-film module } \\
\text { technologies. }\end{array}$ \\
\hline \multicolumn{2}{|c|}{ PV APPLICATIONS AND MARKET DEVELOPMENT PROJECT } \\
\hline NREL & $\begin{array}{l}\text { Installed Phase } 1 \text { systems in the Brazil rural } \\
\text { electrification program ( } 750 \text { homes and } 14 \text { schools in } \\
\text { two states). Extended the project to six other states in } \\
\text { Phase } 2 \text { (to be installed during FY 1995). Received } \\
\text { Award of Merit for Technology Transfer from the } \\
\text { Federal Laboratory Consortium. }\end{array}$ \\
\hline $\begin{array}{l}\text { NREL with DOE/GO } \\
\text { and Industry } \\
\text { Contractors }\end{array}$ & $\begin{array}{l}\text { Provided technical support to DOE/GO contracts } \\
\text { aimed at developing four prototype products for PV- } \\
\text { integrated buildings under the PV:BONUS project: } \\
\text { PV roofing, PV modular homes, alternating current } \\
\text { (ac) modules for buildings, and utility-dispatchable PV } \\
\text { systems. Each product is being developed by a team } \\
\text { of firms with a range of buildings- and PV-related } \\
\text { expertise. In addition, distributed a newly developed } \\
\text { curriculum to architectural schools dealing with PV- } \\
\text { integrated buildings. }\end{array}$ \\
\hline NREL & $\begin{array}{l}\text { Developed a draft bilateral agreement for cooperation } \\
\text { in renewable energy between the U.S. and India. } \\
\text { Organized industry visits and a Presidential Mission to } \\
\text { India led by U.S. Energy Secretary Hazel R. O'Leary. }\end{array}$ \\
\hline NREL & $\begin{array}{l}\text { Developed interactions for potential rural } \\
\text { electrification projects in China, Indonesia, and Egypt. }\end{array}$ \\
\hline $\begin{array}{l}\text { NREL, University of } \\
\text { Delaware, SUNY at } \\
\text { Albany, U.S. } \\
\text { Environmental } \\
\text { Protection Agency } \\
\text { (EPA) }\end{array}$ & $\begin{array}{l}\text { Continued to analyze and verify the value of PV for } \\
\text { demand-side management (DSM) applications. } \\
\text { Collected data from the SERF PV systems and the } \\
\text { systems installed by EPA on buildings owned by } 20 \\
\text { utilities. University of Delaware developed a } \\
\text { spreadsheet that building owners and utilities can use } \\
\text { to evaluate economic performance of PV DSM in } \\
\text { buildings. SUNY at Albany extended its correlations } \\
\text { of utility load profiles and solar availability to } \\
\text { additional utilities. }\end{array}$ \\
\hline
\end{tabular}




\begin{tabular}{|l|l|}
\hline \multicolumn{2}{|c|}{ Table 1.2-1. Key Achievements } \\
\hline Research Group & \multicolumn{1}{c|}{ Achievement } \\
\hline NREL & $\begin{array}{l}\text { Conducted a competition to design and build solar cars } \\
\text { for Sunrayce '95. The race will be held in June 1995 } \\
\text { and will run from Indianapolis, Indiana, to Golden, } \\
\text { Colorado. }\end{array}$ \\
\hline
\end{tabular}

\subsection{Technology Transfer}

Consistent with DOE policy, technology transfer within NREL's PV Program is defined as collaborative R\&D with industry to help industry commercialize products or services. An underlying theme of NREL technology transfer activities is the joint work accomplished by industry and NREL researchers focused on a common R\&D objective. Among government laboratories, seven principal tools effect technology transfer:

- $\quad$ Subcontracted R\&D

- $\quad$ Cooperative R\&D

- Industry-sponsored R\&D

- User facilities (at NREL)

- Technology licenses

- Researcher exchanges

- Information dissemination.

NREL's PV Program conducts its technology transfer primarily through subcontracts, cooperative $R \& D$, and information dissemination.

\section{Subcontracts with Industry}

Nearly $40 \%$ of the 105 subcontracts placed in FY 1994 were with the U.S. PV industry. The NREL funding of $\$ 24.35$ million to industry was supplemented by an additional $\$ 16.1$ million (estimated) of cost sharing by the industry partners. Most industry funding (and cost sharing) was in the PVMaT project (see Figure 1.1-1). Technically knowledgeable NREL research managers participate in defining, evaluating, awarding, and negotiating statements of work submitted by industry researchers in competitive solicitations. Following subcontract awards, NREL subcontract managers direct and evaluate research progress by visiting subcontractor sites and evaluating subcontractor deliverables in the NREL laboratories. 


\section{Cooperative R\&D}

NREL in-house researchers frequently perform informal cooperative R\&D with their industrial counterparts working under NREL subcontracts. These interactions have been ongoing since PV research started in 1977 at the Solar Energy Research Institute (now NREL). Most involve performance measurements and materials analyses performed with NREL's large and unique set of capabilities for PV efficiency and materials analysis. Informal cooperative $R \& D$, as distinguished from formal cooperative research and development agreements (CRADAs), is a natural complement to NREL's subcontracted PV program. Informal cooperative R\&D during FY 1994 included more than 15,000 measurements on PV materials, devices, and modules on properties ranging from composition and microstructure to cell and module performance. More than 150 organizations from the worldwide PV community worked with NREL researchers in this fashion.

CRADAs, as authorized by the National Competitiveness Technology Transfer Act of 1989, are formal agreements signed by the NREL Director and his industrial counterparts for conducting joint research projects that involve NREL and industrial researchers. We performed research under four CRADAs during FY 1994: API (thin-film Si characterization and process diagnostics), TI (spheral Si characterization), Martin Marietta (CIS deposition technology), and SCI (in-situ diagnostics and back contacts for CdTe). These CRADAs are intended to accelerate PV development by transferring NREL technology and by addressing industry's specific problems.

\section{Information Dissemination}

Effective traditional ways of transferring technology are to report $R \& D$ results to the technical community by publishing in scientific journals and by presenting at technical conferences and meetings. Section 8.0 contains a bibliography of FY 1994 publications, including subcontractor reports. During FY 1994, PV program staff helped organize several conferences and workshops, including the 12th NREL PV Program Review Meeting, the First World Conference on Photovoltaic Energy Conversion (held in December 1994), SOLTECH '94, and the American Solar Energy Society Conference. Other workshops included the Photovoltaic Performance and Reliability Workshop, the PV Standards and Codes Coordination Forum, and the Workshop on the Role of Point Defects and Impurities in Silicon. These gatherings provide important opportunities for industry researchers to exchange technical information with NREL and university researchers. 


\subsection{Conclusions}

This report reviews the in-house and subcontracted R\&D activities under the NREL PV Program during FY 1994. Major PV program thrusts during FY 1994 continued to be implemented based on DOE's Photovoltaics Program Plan, FY 1991-FY 1995.

In summary, the activities of the PV Program are to:

- Conduct basic, applied, and engineering research

- Manage subcontracted $R \& D$ projects

- Perform research complementary to subcontracted work

- Develop and maintain state-of-the-art measurement and device capabilities

- Develop PV manufacturing technology and modules

- Transfer results to industry

- Evolve viable partnerships for PV systems and market development.

This report describes the in-house and subcontracted R\&D activities, many of which encompass close collaborations between NREL and outside researchers.

The research activities under the program are summarized under the five project areas in the following sections: Crystalline Silicon and Advanced Devices Project, Thin-Film PV Technologies Project, PVMaT Project, PV Module and System Performance and Engineering Project, and PV Applications and Market Development Project. The sections include a brief overview that includes the objectives, approaches, and some key developments. Following that are technical summaries of the in-house and subcontract activities. The subcontract sections were, for the most part, provided by the subcontractors themselves. Some were compiled by NREL project managers from various reports submitted by the subcontractors. Section 7.0 provides a list of FY 1994 subcontracts, and Section 8.0 lists major research publications and subcontractor reports. 


\title{
2.0 CRYSTALLINE SILICON AND ADVANCED DEVICES PROJECT
}

\author{
John Benner, Manager
}

The goal of this project is to support the time-phased development of advanced technologies and analytical capabilities needed to help the PV industry develop high-efficiency products for evolving markets. During the past year, we addressed this goal by pursuing three objectives:

- $\quad$ Help the PV industry resolve current production problems by analyzing and optimizing $\mathrm{Si}$ crystal growth and post-growth cell fabrication processes, and continued technology transfer of the NREL-developed GaInP solar cell

- Investigate processes with potential for cost-effectively producing advanced thin-crystalline films of $\mathrm{Si}$, gallium arsenide (GaAs), and other semiconductors for ultra-high-efficiency flat-plate modules and concentrator cells

- Support fundamental and exploratory research to ensure the continued source of new ideas and techniques.

This project consists of five tasks:

1. Crystalline Silicon

2. High Efficiency

3. Advanced Measurement Techniques

4. Exploratory Materials and Devices

5. Photovoltaic CRADAs

The detailed project reports that follow describe research conducted in NREL's laboratories and in universities (predominantly) that build the technology base. This base underpins other elements of the National Photovoltaic Program that support development and manufacturing projects.

\section{Task 1. Crystalline Silicon}

In this task we seek to create viable and competitive materials growth, advanced processes for fabricating solar cells, and technological understanding that will help the manufacturers of crystalline and polycrystalline Si PV compete successfully in near- and long-term evolving PV markets, both domestically and internationally. In FY 1994, activities supporting this goal included: 
Helping the Si PV industry resolve current Si materials problems by growing materials with selected defects or impurities for controlled studies, characterizing materials, and analyzing Si crystal growth and post-growth processes

- Managing subcontracted research in experimental and theoretical analysis of Si and pointdefect processes to integrate these with industrial and in-house activities

- Initiating the development of new materials growth and processing strategies directed at high-performance and cost-effective future crystalline Si PV products

- Maintaining the infrastructure (Si growth apparatus, diagnostic device capability, and specialized characterization tools) needed for effective and timely progress in these tasks. This item demanded an inordinate amount of the project effort during FY 1994 because of moving laboratories and offices to the new DOE/NREL SERF building.

Because the crystalline Si PV industry is the most commercially advanced PV technology approach, the technical support role provided by DOE/NREL must be more diverse than for some PV technologies. One role is to provide expert consulting and analytical service to help companies optimize products. A number of established Si materials approaches are in various stages of commercial readiness. Each has a potential niche (to be confirmed by marketplace realities) in a cost-versus-performance matrix. The goal is not simply to achieve a certain PV efficiency, but to do so in the framework of an approach that has been heavily invested in (in some cases) and has a perceived cost advantage. Several advocates of these approaches express confidence that crystalline Si PV module prices can be significantly less than $\$ 2 / \mathrm{W}$ during the next few years. The approaches vary considerably, and each has a particular set of technical issues. NREL can serve the industries developing the various approaches by characterizing and analyzing growth, materials, and processes.

Investment in Si PV has been substantial, but the relative maturity of the technology would have been impossible without the multi-billion-dollar infrastructure in microelectronics. This supplements the availability of skilled personnel, applicable machinery, usable materials, and physical understanding needed in manufacturing. An important, but low-cost, activity of NREL's project is to nurture the linkages between these two Si technologies. We sponsored the fourth in a series of workshops under the title "Role of Point Defects and Defect Complexes in Silicon Device Processing" at Beaver Creek, Colorado, June 27-29, 1994. This meeting was unique in that it brought together researchers and manufacturers from the microelectronics and PV communities to exchange ideas and experiences in processing $\mathrm{Si}$. The meeting has stimulated independent investigations in adapting technologies and collaborations between communities to test new concepts.

For example, a team including North Carolina State University (NCSU), NREL, Siemens Solar Industries (SSI), and MEMC (a major supplier of Si wafers for microelectronics) participated in an experiment that involved gettering impurities and passivating Czochralski-grown Si wafers. Wafers from SSI and MEMC received different gettering processes done at MEMC, passivation by hydrogenation was done at NREL, and rapid thermal annealing (RTA) and minority carrier lifetime was measured at NCSU. The gettering processes included combinations of phosphorus 
diffusion from $\mathrm{POCl}_{3}, \mathrm{RTA}$, and polysilicon depositions. The results of these experiments on Siemens' material were somewhat surprising. Table 2-1 shows the results of this experiment:

\begin{tabular}{|c|c|c|c|}
\hline \multicolumn{3}{|c|}{ Table 2-1. Measured Values of Bulk Minority Carrier Lifetime (in usec) } \\
\hline Sample & Before Hydrogenation & $\begin{array}{c}\text { After Hydrogenation } \\
\text { (front) }\end{array}$ & $\begin{array}{c}\text { After Hydrogenation } \\
\text { (back) }\end{array}$ \\
\hline RR & $3.24-3.59$ & 7.42 & -- \\
\hline R1P & $2.73-4.15$ & 7.26 & 6.97 \\
\hline IP & $3.77-4.57$ & 5.14 & 4.73 \\
\hline RP & $8.38-11.51$ & 29.91 & $36.77-49.00$ \\
\hline P & $6.67-12.99$ & $15.73-22.19$ & $17.53-21.76$ \\
\hline
\end{tabular}

Note: $R R=$ reference

RIP $=$ RTA+lum poly

$1 \mathrm{P}=1 \mu \mathrm{m}$ poly

$\mathrm{RP}=\mathrm{RTA}+\mathrm{POCl}_{3}$

$\mathrm{P} \quad=\mathrm{POCl}_{3}$

This first experiment clearly demonstrated that hydrogen passivation can effectively increase the minority carrier lifetime if hydrogenation is carried out after a phosphorus diffusion and RTA process. Ongoing experiments with a new lot of samples will expand the number of elements in the process matrix.

An important outcome of the workshop was a consensus regarding the research technologies that need to be addressed to meet the $18 \%$ low-cost-cell efficiency goal. These findings are a first step in defining a road map for Si PV development. The results are grouped in three main categories, each containing a number of issues. Each item was designated as either a long-term or an immediate issue and was ranked from 1 (lowest) through 5 (highest) in terms of its priority of needing attention. Table 2.2 summarizes the results.

Research examining the effects of impurities and defects in $\mathrm{Si}$, evaluated by controlled introduction during crystal growth, was extended to evaluate the role of nitrogen. In a dislocationfree, float-zoned ingot, minority charge carrier lifetime $(\tau)$ was 6000-3000 $\mu$ s for the portion grown in $100 \% \mathrm{Ar}$ and only slightly lower $(\sim 2500 \mu \mathrm{s})$ for the portion grown in $5 \% \mathrm{~N}_{2} / 95 \% \mathrm{Ar}$. The lifetime dropped more significantly, to $\sim 400 \mu \mathrm{s}$, about $8 \mathrm{~min}$ after switching to a $100 \% \mathrm{~N}_{2}$ purge gas. Microdefects in the ingots were examined by copper $(\mathrm{Cu})$ decoration and Secco etching, and Fourier-transform infrared (FTIR) spectroscopy. In the dislocation-free segment grown in $100 \% \mathrm{~N}_{2}$, deep 4-fold-symmetry etch pits were observed after copper decoration and defect etching, and they may indicate nitride precipitate formation preceding the eventual 
nucleation of dislocations. However, in a multicrystalline ingot, $\tau$ was $40-60 \mu$ s over most of the ingot length and was not significantly different whether growth was conducted in $100 \% \mathrm{Ar}$, $5 \% \mathrm{~N}_{2} / 95 \% \mathrm{Ar}$, or $100 \% \mathrm{~N}_{2}$. Thus, it should be possible to conduct $\mathrm{Si}$ growth in a partial $\mathrm{N}_{2}$ ambient to take advantage of mechanical strengthening effects, lower purge gas costs, and other process advantages without large reductions in minority charge carrier lifetime.

Classical gettering approaches in poly-Si, using chlorine or phosphorus, improve the diffusion length in regions with relatively large minority carrier diffusion length (L) values, where recombination is controlled by dissolved heavy metals. They were less effective in wafer regions with short $\mathrm{L}$ values. Unfortunately, solar cell performance can be dominated by these poorquality regions that are difficult to upgrade. To improve $\mathrm{L}$ in these regions, alternative gettering approaches, such as Al gettering with RTA or furnace annealing, or a newer ultrasonic treatment (UST) developed at the University of South Florida (USF), must be used. USF has demonstrated that the RTA or furnace Al gettering improved L values (up to 3.5 times) especially in the lowdiffusion-length regions. Georgia Institute of Technology (GIT) has further shown that the large improvement in solar cell efficiency (roughly $10 \%$ relative improvement) results from the combined effects of forming a back surface field, textured back surface reflector, and improving the minority carrier diffusion length by gettering. Initial results with UST gettering improved parameters of commercial solar cells measured at 0.1 sun, but this effect vanished at 1-sun illumination. More research is needed to understand physical mechanisms involved. This could optimize the UST parameters to achieve the effect of ultrasound-stimulated improvement of solar cells at the arbitrary light intensities. This would provide an exciting new opportunity to improve the material and device parameters of finished poly-Si solar cells at low temperatures $\left(<1000^{\circ} \mathrm{C}\right)$.

Duke University developed a diffusion-segregation equation based on thermodynamic considerations used to simulate gettering. This model was applied to investigate gettering of fastdiffusing impurities such as $\mathrm{Ni}, \mathrm{Cu}, \mathrm{Fe}$, and $\mathrm{Au}$, by phosphorus diffusion and $\mathrm{Al}$ annealing. Duke's calculations show that a combined $\mathrm{P}$ and $\mathrm{Al}$ gettering can have a synergistic effect. This result agrees with the experimental data, probably because the diffusion-segregation equation also includes an impurity stabilization effect (related to a capture-and-release phenomenon), compared to the impurity diffusion alone.

The facilities at NREL, and all of the subcontracted universities, are tapped to help analyze commercial Si materials. Spatially resolved analysis of defects, dislocations, and dissolved and precipitated impurities are correlated with electronic characteristics to better control the initial crystal growth and optimized post-growth processes.

Although $\mathrm{Si}$ is the source of today's PV modules, to exclude Si from the realm of advanced products would be a serious mistake. Crystalline Si PV technology has shown laboratory-scale promise for considerably higher performance capability (23\% 1-sun total-area cell efficiency and $27 \%$ concentrator efficiency) than currently achieved by the industry approaches just discussed. In time, cost-effective variants of these laboratory achievements will likely be realized. Another role of the project is to provide industry with the leadership and advanced technology needed to exploit high- efficiency device structures. Here we seek both simplification of fabrication processes needed for high-efficiency and crystal-growth research to develop cost-effective and 
commercially realistic Si growth processes to produce Si material quality necessary to take advantage of the high-efficiency processes.

Thin crystalline Si solar cells made directly of deposited material on low-cost substrates is an attractive concept. More than $85 \%$ of the usable AM1.5 radiation can be absorbed by a $30-\mu \mathrm{m}$ thin layer even without any light trapping. However, growing device-quality thin-layer crystalline Si has not been easy. Liquid phase epitaxy (LPE) holds promise for this task because both highrate and low-temperature growth may be used. We have successfully grown smooth Si layers on multicrystalline Si substrates. To understand the growth mechanisms of the macroscopically smooth layers from $\mathrm{Cu} / \mathrm{Al}$ as opposed to the faceted, rough layers from an In solvent, thermodynamic calculations and atomic force microscopy (AFM) measurements were carried out. The thermodynamic calculations show a strong tendency for Al to replace $\mathrm{Si}$ in a silicon-oxygen bond. Thus, good wetting, and subsequently, dense nucleation sites, can be achieved because native Si oxides are removed from the substrate surface. AFM observations reveal drastically different surface roughnesses between layers grown from $\mathrm{Cu} / \mathrm{Al}$ and $\mathrm{In}$ solvents with an average roughness of $78 \AA$ and $5.2 \AA$ on (111) surfaces, respectively. In summary, Al-Cu appears to be an ideal solvent for Si LPE on multicrystalline substrates.

Solar cells in thin-layer $\mathrm{Si}$-in fact, all thin-films-benefit from light trapping. We have devised an interpolation model for our light-trapping calculations that predicts the degree of light trapping, optical absorption, transmission, and reflection using data from very few reflections within the cell. This improvement reduces the computational time from about 1 week to 2 hours without sacrificing the accuracy. This modification will be particularly useful for other groups at and outside NREL that plan to use our light-trapping model and software.

The benefits of hydrogen passivation for Si solar cell performance are clear, but the underlying processes are not defined. We are working to identify the state of passivating hydrogen in Si. Because of the low concentrations of hydrogen in Si and its low absorption coefficients, observing hydrogen absorption peaks by IR spectroscopy has been difficult. We have now successfully identified absorption bands caused by hydrogen in the bulk, associated with the surface damage caused by low-energy implantation, and the hydrogen in single and polycrystalline $\mathrm{Si}$. The peak shifts associated with substituting hydrogen with deuterium and the temperature dependencies at low temperatures (down to $10^{\circ} \mathrm{K}$ ) were measured. This is believed to be the first identification of the defect passivation-related hydrogen.

We have also developed an explanation for hydrogen's variability of diffusion in different types of Si. We postulated that diffusion was controlled by forming a complex of hydrogen with an Si vacancy. Calculations by Texas Tech showed that the vacancy is a strong trap for interstitial hydrogen and that, from molecular dynamic calculations, the vacancy and hydrogen will attract each other. Our experiments have shown that the diffusivity of deuterium (or hydrogen) is related to the material parameters that control the vacancy concentration in $\mathrm{Si}$, for example, growth speed, increasing carbon concentration, or decrease in oxygen concentration. Positron annihilation spectroscopy, performed by S.C. Sharma at the University of Texas at Arlington, showed a good correlation between increasing vacancy concentration and increasing hydrogen diffusivity. This finding is consistent with observations that hydrogen passivation greatly 
improves rapidly crystallized material such as ribbons, but has little benefit for wafers from cast ingots.

\begin{tabular}{|c|c|c|c|c|}
\hline \multicolumn{5}{|c|}{ WAFER } \\
\hline & & Long-Term & Immediate & Rank \\
\hline 1 & Larger Wafer & $\mathrm{X}$ & & 2 \\
\hline 2 & Improved Homogeneity & $\mathrm{X}$ & $\mathrm{X}$ & 5 \\
\hline 3 & Lower Cost Feedstock & & $X$ & 2 \\
\hline 4 & Reduced Cost Czochralski Crystal Growth & & $\mathrm{X}$ & 2 \\
\hline 5 & New Slicing Method & & $\mathrm{X}$ & 3 \\
\hline 6 & High T Czochralski Crystal Growth & $\mathrm{X}$ & & $3-4$ \\
\hline 7 & Non-Sliced Substrates & $\mathbf{X}$ & & $3-4$ \\
\hline 8 & New Growth Method; e.g. float zone, EMC & $\mathbf{X}$ & & 4 \\
\hline 9 & Thin-Film Si & $X$ & & 5 \\
\hline \multicolumn{5}{|c|}{ CELL PROCESSING } \\
\hline 1 & New Structures & $\mathrm{X}$ & & 4 \\
\hline 2 & Process Simplification & & & 3 \\
\hline 3 & Improved Screen Printing & & $\mathrm{X}$ & 1 \\
\hline 4 & Alternatives to Screen Printing & $\mathrm{X}$ & & 2 \\
\hline 5 & New Barriers (a-Si, SIPOS, etc.) & $\mathrm{X}$ & & 2 \\
\hline 6 & Gettering & $X$ & $X$ & 5 \\
\hline 7 & Surface/Defect Passivation & $X$ & $\mathrm{X}$ & 5 \\
\hline 8 & Hydrogenation & $X$ & $\mathbf{X}$ & 5 \\
\hline 9 & Handling Thin Wafers & & $X$ & 1 \\
\hline 10 & Optical Processing/RTP & $\mathbf{X}$ & $\mathrm{X}$ & 5 \\
\hline 11 & Reduced Chemical Usage & & $X$ & 1 \\
\hline
\end{tabular}




\begin{tabular}{|r|l|c|c|c|}
\hline \multicolumn{2}{|c|}{ LAMINATION } & $X$ & 3 \\
\hline 1 & New Encapsulants & & & 4 \\
\hline 2 & New Interconnect Schemes & $X$ & $X$ & 5 \\
\hline 3 & Assembly Automation & & & 2 \\
\hline 4 & Monolithic Module & $X$ & & \\
\hline
\end{tabular}

Task 2. High Efficiency

This task is the research within the National Photovoltaic Program expected to generate products that meet the high-efficiency, long-term targets of 25\%-efficient modules in flat plates and concentrators. These products may be essential to reducing system-level electricity prices to competitive levels, as increasing efficiency is the ultimate tool in avoiding area-related balanceof-systems costs. The efforts include:

- Exploring advanced concepts for low-cost fabrication of thin-crystalline semiconductor films

- Designing and evaluating concentrator solar cell architectures for increased efficiency and robust production tolerance

- $\quad$ Researching fundamental properties of high-efficiency semiconductors

- Maintaining and relocating the facilities needed to conduct research in high-efficiency solar cells.

We achieved a major milestone with the demonstration of a $30.2 \%$-efficient $\mathrm{GaInP}_{2} / \mathrm{GaAs}$ concentrator cell, on schedule this month. The III-V Materials and Devices Group received a test report from D. King, Sandia National Laboratories, on an NREL tandem solar cell. The 1-sun efficiency was measured as $26.4 \%$, in excellent agreement with the measurement by NREL's Device Characterization Group. (The performance is lower than the $29.5 \%$ efficiency of a device optimized for 1-sun performance because of changes in design needed for operation at high current.) The cell exceeded 30\% efficiency at concentrations from 115-260 suns, peaking at $30.2 \%$ in a range of roughly 140-180 suns. This is the first two-terminal solar cell to demonstrate a verified efficiency exceeding $30 \%$.

NREL initiated a follow-up study for the transfer of the GaInP/GaAs tandem-cell technology for space and terrestrial applications. Its objective is to demonstrate the feasibility of heteroepitaxial deposition of state-of-the-art tandem cells on low-cost germanium substrates. For space technology, this provides array-level compatibility with existing parts. Developing the space market, where the device has achieved $27.4 \%$ AM0, is seen as a path to move this technology along the manufacturing development curve to further reduce the cost of terrestrial systems. Relative to future applications as terrestrial products, the large low-cost germanium substrate cuts 
cell production costs in half to easily meet a $\$ 5 / \mathrm{cm}^{2}$ target. At this cost, the III-V-based solar cell is cheaper than $\mathrm{Si}$ and provides conversion efficiency that could reduce the systems price to levels compatible with $\$ 0.12 / \mathrm{kWh}$.

We initiated a study with a concentrator module manufacturer to address practical considerations in using the tandem cell structure in low-cost terrestrial products. A common question about tandem cells is how their performance changes with spectral change because of the atmosphere or chromatic aberration in the concentrating optics. The answer is that the performance changes very little. Previously, we reported that two-terminal, two-junction GaInP/GaAs cells introduced into the linear ENTECH concentrator module would suffer a power loss of $7 \%-10 \%$ as a result of two-terminal, instead of four-terminal, connection. We have now determined that this loss is a strong function of the module alignment and that the experimental module we purchased (with removable lens and missing endplate) was slightly out of alignment. If the alignment is corrected, the loss from two-terminal connection is reduced to about $4 \%$. The misalignment on our module is within what ENTECH considers to be normal tolerances for a module with Si cells. Therefore, replacing the $\mathrm{Si}$ cells with GaInP/GaAs cells would require a more careful alignment of the lens in the module. In this case, the $4 \%$ loss effectively reduces the efficiency of the GaInP/GaAs cells by about 1 percentage point, still leaving a significant difference between the efficiencies of the GaInP/GaAs cells (about 25\%) and the Si cells (18\%)-a commanding performance advantage.

These efficient cells rely on state-of-the-art materials technology that contribute to the advancement of many electronic and photonic technologies. The paper "Effect of Faceting on the Band Gap of Ordered GaInP" by D.J. Friedman et al., was submitted for publication in Applied Physics Letters. Under certain growth conditions, the pseudobinary semiconductor alloy GaInP shows cation site ordering into the $\mathrm{Cu}$ - $\mathrm{Pt}$ structure, and this ordering lowers the band gap, $E_{g}$, from that of the disordered alloy. The extent of the reduction in $E_{g}$ depends on growth conditions, including the substrate's orientation. This paper discusses the dependence of $E_{g}$ on epilayer thickness for GaInP grown by metal-organic vapor-phase epitaxy (MOVPE). For epilayers grown on precisely oriented (100) substrates under conditions typically used to produce ordered material, $E_{g}$ decreases dramatically with increasing epilayer thickness: $E_{g}$ for a 10 - $\mu \mathrm{m}$ thick epilayer is $\sim 40 \mathrm{meV}$ lower than for a 1-um-thick epilayer. This uncertainty dependence of $\mathrm{E}_{\mathrm{g}}$ on thickness can be understood in terms of the recently observed faceting of the GaInP growth surface.

S. Kurtz completed a study and presented a talk entitled "The Effect of Phosphine Pressure on the Band Gap of $\mathrm{Ga}_{0.5} \mathrm{In}_{0.5} \mathrm{P}^{\prime \prime}$ at the Materials Research Society meeting. The study yielded data implying that phosphine pressure plays a very important role in determining the band gap (and ordering) of $\mathrm{Ga}_{0.5} \mathrm{In}_{0.5} \mathrm{P}$ at high growth temperatures. The phosphine pressure is expected to affect the surface structure (reconstruction), and low phosphine pressures may be inadequate to maintain the structure which leads to ordering. Previously, for high growth temperatures, we had proposed that subsurface disordering was determining the band gap because the ordering increased at higher growth rates. In this study we raise the possibility that the changing growth rate actually affects the ordering by changing the effective phosphine pressure, casting doubt on the subsurface disordering model. At low growth temperatures, the data are consistent with models that predict the importance of surface diffusion. 
The subcontracted program that explores concepts for high-efficiency PV underwent a competitive procurement. In an environment of increasing pressure to commercialize existing technology, we worked with DOE to continue research and development (R\&D) in highefficiency PV. Initial guidance caused us to prepare an announcement of an indefinite postponement in the procurement issued in July. Before releasing that announcement, we delivered to DOE a number of reasons justifying the continued interest and need for research in the area. As SSI recently demonstrated, working with a technology that provides a sufficient high efficiency (such that some performance can be traded off for reduced production cost) presents a formidable tool to maintain competitive advantage. Although the U.S. retains leadership in high-efficiency PV, we identified a new and substantial foreign threat to the longterm intellectual property position of the United States. Ultimately, the action was deemed to have an unacceptable impact on the long-term position of the U.S. PV industry. Perhaps in response to the vacillating support, the R\&D community showed a new commitment to the field, with 12 organizations offering cost-sharing in new partnerships with NREL. In all, 38 proposals were received, with 26 rated above good. In next year's report, we will report progress from the three new subcontracts:

- California Institute of Technology (CalTech), which will investigate direct deposition of highly crystalline thin films of germanium onto glass to serve as a substrate for GaAs solar cells

- University of California at Los Angeles (UCLA), where E. Yablonovitch will use the access to both sides of the GaAs cell provided by lift-off technology, combined with innovative processing, to achieve surface texturing to test theoretical predictions of the efficiency improvements for optically confined, thin-film cells

- Research Triangle Institute (RTI), which will build on the "first-shot" record $15.8 \%$ efficiency achieved in thin-film polycrystalline GaAs solar cells to seek near-term cost goals for replacing the Si devices in linear concentrators, and fundamental understanding supporting longer-term goals of flat-plate viability.

\section{Task 3. Advanced Measurement Techniques}

This task supports the R\&D of new or upgraded techniques for analyzing materials and devices. NREL's history is filled with inventions of unique techniques for analyzing PV materials. These range from the apparent simplicity of creating a method to prepare peculiar samples for conventional analysis, such as Texas Instrument spheres, to the complexity of demonstrating entirely new analytical equipment suitable for R\&D 100 Awards. The strength of NREL's analytical support to the PV community does not rest on performing routine measurements that might have been obtained commercially at great expense; rather, it resides in the ability to create new analytical methods to extract needed data from unconventional materials never before synthesized or characterized.

In doped polar semiconductors, the macroscopic electric field associated with the longitudinal optical phonon allows for coupling with the plasmon in the material. Light scattering from these coupled phonon-plasmon modes is sensitive to the effective carrier concentration in the material. 
The position of the coupled phonon-plasmon modes scales with the concentration of carriers in the sample. We have examined several GaAs samples grown for different Se flow rates. From the position of the coupled plasmon modes for the different samples, we have determined the electron density in those samples. The values of the carrier concentration obtained from the Raman measurements were confirmed by independent capacitance-voltage (C-V) measurements on the samples. An excellent agreement is found between the two techniques. C-V measurements, however, require either a Schottky barrier or a $\mathrm{p} / \mathrm{n}$ junction to be fabricated. Hence, the new technique simplifies the measurements that enabled us to calibrate the MOVPE reactor for the number of carriers introduced into the GaAs samples as a function of Se flow rate.

To examine the feasibility of extending this technique to study GaAs tunnel junctions, we have studied a $\mathrm{n}-\mathrm{GaAs} / \mathrm{n}-\mathrm{GaAs} / \mathrm{GaInP}$ structure. From the Raman spectrum of this structure, we have been able to determine the carrier concentration in different heavily doped $\mathrm{GaAs}$ layers. These measurements have also shown the extreme sensitivity of the technique to study band bending at the $\mathrm{GaAs} / \mathrm{GaInP}$ interface.

Another important tool-measurement of minority carrier lifetime by photoconductivity decay sensed by if coupling - was developed through this task and the CRADA with Texas Instruments (TI).

\section{Task 4. Exploratory Materials and Devices}

This task covers the internal research in solid-state theory and the subcontracted projects in the University Participation and New Ideas Programs. We will finish developing theoretical methods that can predict the properties of mesoscopic-scale solids $\left(\geq 10^{3}\right.$ atoms/cell). These methods are needed to analyze inhomogeneous materials such as polycrystalline and nanostructured semiconductors. The new tools were applied to the analysis of porous Si to explain the unexpectedly strong photoluminescence and its possible uses in PV devices.

Nanostructure materials have been studied extensively during the past decade because of their potential in photoelectronic devices. The dielectric constants of these quantum nanostructures are important for modeling their electronic and optical properties. One example is quantum dots, with sizes around $40 \AA$. The magnitude of the dielectric constants of these quantum dots will determine whether the electron-hole exciton is free (as in the bulk) or confined by the quantum dot dimension. This, in turn, is important to determine which of the several disputed mechanisms of their photoluminescence is correct. The effective mass model is not sufficiently accurate for calculating the dielectric constant of $40 \AA$ for sized nanostructures because the parabolic approximation is no longer valid. Thus, atomic-scale mesoscopic calculations are necessary. We have used an empirical pseudopotential Hamiltonian and a plane wave basis set.

Because the conventional method of quantum mechanics cannot calculate such "large" (>1000 atom) systems, we have developed a generalized moments method to solve this problem. This new method uses statistical means (expansion in Chebychev polynomials) to calculate the optical absorption spectra, density of states, and dielectric constants. Using this method, the dielectric constants of different sized Si quantum dots were calculated. The calculated dielectric constant drops considerably from its bulk value; for example, for a sphere with diameter of $20 \AA$, it is $E_{r}=8$ 
instead of the bulk value of 11.4. However, it is still much larger than the value predicted by current models. We find that the exciton is confined by the quantum dot's physical dimension, not by the Coulomb attraction between the electron and the hole. A convenient parametrization of the dielectric constant as a function of the quantum dot's size is achieved from our calculations. The optical absorption spectra and density of states of different sized quantum dots were also calculated. We expect this new method to find many more applications in the quantum nanostructure optical property calculations.

The New Ideas Program periodically solicits proposals to investigate new technology applications for PV conversion. Two subcontracts selected from the last procurement seek to apply quantum size effects in new solar cells. Spire Corporation has demonstrated PV conversion in a solar cell made of porous $\mathrm{Si}$, which is created by etching crystalline $\mathrm{Si}$ under conditions that create fine "wires" on the surface. The size creates quantum wells, changing the optical properties of the material to a pseudo-direct bandgap such that the material emits light. The Spire program seeks to determine whether the direct absorption has potential for thin-film PV. Multiple quantum-well structures in organic semiconductors prepared at Princeton University may hold the key to exciton dissociation and charge transport issues that have limited the performance of all organicbased solar cells made to date.

The University Participation Program provides the research community with another forum to ensure that the National Photovoltaic Program is addressing key topics of relevance to its longterm well-being. Two key features of this program are essential for achieving this objective. First, it is the only PV Program area in which research is selected entirely by peer review. The scope of the solicitation is not specified. The proposal must justify the importance of the chosen topic. Reviewers from universities and industry evaluate these justifications and quality of the planned research. This selection process is designed to minimize the influence of "conventional wisdom" or program bias. The other key to success is the minimal management interference afforded the investigators after selection and award. University Participation subcontracts were the first in the NREL programs to provide 3-year, phased awards that match the time required for graduate students to complete their theses. Similar to a research grant, there may be no management performance milestones, and documentation is limited to annual and final reports. The program was reduced by about half in the most recent competition in 1993. It consists of five projects:

- "Fundamental Studies of the Effects of Crystal Defects on CuInSe $/ \mathrm{CdS}$ Heterojunction Behavior," University of Illinois

- $\quad$ "Electronic Processes in Thin-Film PV Materials," University of Utah

- $\quad$ "Heterojunction Development and Optimization in Thin-Film Compound Semiconductor Cells," University of South Florida

- $\quad$ "Solar Energy Conversion at Dye-Sensitized Nanostructured Electrodes Fabricated by SolGel Processing," Johns Hopkins University 


\section{Task 5. Cooperative Research and Development Agreements}

In the first PV Program CRADA (with TI), NREL helped characterize and understand the mechanisms underlying the upgrading process used to prepare spheral Si from metallurgical-grade starting material. Because of the heavy doping and very short lifetimes in spheral Si, extracting meaningful data from the TI spheres is quite difficult. The radio frequency coupled photoconductivity decay (RFPCD) systems available elsewhere generally do not have adequate sensitivity or fast enough response. Using our recently acquired yttrium aluminum garnet (YAG) laser and a new direct current (dc) pulse amplifier circuit, we succeeded in measuring the minority carrier lifetimes in samples ranging from a few hundred spheres down to a single sphere. TI and NREL performed a series of tests to determine whether the system provides highly reproducible results and the sample size needed to meaningfully evaluate the quality of a production lot. The tests indicate that a sample size of 1 gram or less may be adequate to characterize production. Based on these findings, TI duplicated the NREL system in its pilot facility for in-line process monitoring.

NREL's crystalline $\mathrm{Si}$ group supported AstroPower in evaluating and improving its thin-Si process. One purpose of this CRADA is to provide a mechanism to fully protect company proprietary data. Findings from the CRADA investigations are proprietary and not reported here.

New CRADAs were negotiated for work to continue into FY 1995. A "funds-in only" CRADA with UniSil Corporation on "Technology and Characterization Support for the High-Purity FloatZone Silicon Title III Project" helps UniSil in its efforts to establish a U.S.-based float-zoned Si manufacturing capability. NREL and UniSil are both interested in developing Si float-zoning technology for use in a variety of applications such as high-efficiency PV devices, high-power switching devices, and infrared detectors. The proposed CRADA would provide a highly interactive program that integrates Si float-zone materials and characterization expertise at NREL, with a fast-paced float-zone manufacturing initiation activity at UniSil. NREL/DOE expect to benefit from the proposed work because successfully implementing U.S. float-zoned $\mathrm{Si}$ commercial manufacturing would provide a source of material for U.S. companies engaged in high-efficiency Si PV module manufacturing. The higher performance capability of float-zoned $\mathrm{Si}$ is also expected to allow lower balance-of-system costs in utility applications.

NREL and Crystals Systems, Inc. (CSI) have agreed in principle to a CRADA intended to help develop technology to improve the performance and further reduce the cost of solar cells based on polycrystalline Si ingots. These goals will be met by investigating interactions of crucibles with the $\mathrm{Si}$ melt in the heat-exchanger method (HEM). CSI has developed a directional solidification process with HEM using silica crucibles. This process has been extended to $55-\mathrm{cm}$ $x$ 55-cm ingots. CSI has also demonstrated reusable crucible technology for ingots of $33 \mathrm{~cm} x$ $33 \mathrm{~cm}$ and $44 \mathrm{~cm} \times 44 \mathrm{~cm}$. CSI has designed and installed a totally automated furnace that requires labor for loading and unloading only. This furnace uses a custom-software-based control system, such that both the heat input and the heat extraction can be controlled during ingot growth. CSI sells the furnace for producing $55-\mathrm{cm} \times 55-\mathrm{cm}$ ingots. Other tasks of the CRADA will seek to improve on the already impressive $17.4 \%$ efficiency achieved in polycrystalline 
devices using CSI material by identifying mechanisms of impurity incorporation and defect generation and evaluating methods of gettering. 


\section{Crystalline Silicon and Advanced Devices In-House Research}




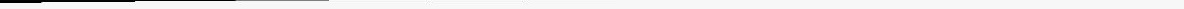




\section{CRYSTALLINE SILICON AND ADVANCED DEVICES PROJECT}

The goal of this project is to support the time-phased development of advanced technologies and analytical capabilities needed to assist the photovoltaics industry in developing high efficiency products for evolving markets. During the past year, we addressed this goal by pursuit of three objectives: (i) assist the photovoltaics industry on current production problems through analysis and optimization of silicon crystal growth and post-growth cell fabrication processes, as well as continuation of technology transfer of the NREL developed GaInP solar cell; (ii) investigate processes with potential for cost-effective production of advanced thin-crystalline-films of silicon, gallium arsenide $(\mathrm{GaAs})$ and other semiconductors for ultra-high-efficiency flat-plate modules and concentrator cells; and, (iii) support fundamental and exploratory research to assure the continued source of new ideas and techniques.

This project consists of five tasks: 1) Crystalline Silicon; 2) High Efficiency; 3) Advanced Measurement Techniques, 4) Exploratory Materials and Devices; and, 5) Photovoltaic Cooperative Research and Development Agreements (CRADAs). The detailed project reports that follow describe research carried out in NREL's laboratories and in universities (predominantly) that build the base of technology underpinning other elements of the National Photovoltaic Program that support development and manufacturing projects.

Crystalline Silicon:

In the first Task we seek too create viable and competitive materials growth, advanced processes for fabrication of solar cells, and technological understanding that will help the manufacturers of crystalline and polycrystalline silicon photovoltaics compete successfully in near-term as well as long range evolving PV markets, both domestically and internationally. In fiscal year 1994, activities supporting this goal included: (i) assisting the silicon PV industry with current silicon materials problems through growth of materials with selected defects or impurities for controlled studies, materials characterization, and analysis of silicon crystal growth and post growth processes; (ii) management of subcontracted research in experimental and theoretical analysis of silicon and point defect processes to integrate these with industrial and in-house activities; (iii) initiating the development of new materials growth and processing strategies directed at high-performance and cost-effective future crystalline silicon PV products; and (iv) maintaining the infra-structure (silicon growth apparatus, diagnostic device capability, and specialized characterization tools) needed for effective and timely progress in these tasks. Item (iv) demanded an inordinate amount of the project effort in fiscal year 1994 because of the moving laboratories and offices to the new DOE/NREL Solar Energy Research Facility (SERF) building.

Because the crystalline silicon PV industry is the most commercially advanced of the various PV technology approaches, the technical support role provided by DOENREL must be more diverse than for some of the other PV technologies. One role is providing expert consulting and analytical service to assist companies in product optimization. There are a number of established silicon materials approaches in various stages of commercial readiness. Each has a potential niche (to be confirmed by realities of the market place) in a cost versus performance matrix. The goal is not simply to achieve a certain PV efficiency, but to do so in the framework of an approach that has been heavily invested in (in some cases) and has a perceived cost advantage. A number of the advocates of these various approaches express confidence that crystalline silicon PV module prices can be significantly under $\$ 2 / \mathrm{W}$ in the next few years. The approaches vary considerably, and each has a particular set of technical issues. NREL can be of 
service to the industries that developed the various approaches by providing characterization and analysis assistance concerning these growth, materials, and process issues.

While the investment in silicon photovoltaics has been substantial, the relative maturity of the technology would not have been possible without reliance on the multi-billion dollar infrastructure in micro-electronics that supplements the availability of skilled personnel, applicable machinery, usable materials and physical understanding needed in manufacturing. An important, but low-cost, activity of NREL's project is to nurture the linkages between these two silicon applications. We sponsored the fourth in a series of workshops under the title "Role of Point Defects and Defect Complexes in Silicon Device Processing" at Beaver Creek, Colorado, June 27-29, 1994. This meeting in unique in that it draws together researchers and manufacturers from both the microelectronics and photovoltaics communities to exchange ideas and experiences in processing silicon. The meeting has stimulated both independent investigations in adapting technologies and collaborations between communities to test new concepts.

For example, a team including North Carolina State University, NREL, Siemens Solar Industries and MEMC (a major supplier of silicon wafers for microelectronics) participated in an experiment that involved gettering and passivation of Czochralski grown silicon wafers. The wafers from Siemens and MEMC received different gettering processes done at MEMC, passivation by hydrogenation was done at NREL, and rapid thermal annealing (RTA) and minority carrier lifetime was measured at NCSU. The gettering processes included combinations of phosphorus diffusion from $\mathrm{POCl}_{3}, \mathrm{RTA}$, and polysilicon depositions. The results of these experiments on Siemens' material were somewhat surprising. The following table shows the results of this experiment:

Table of Measured Values of Bulk Minority Carrier Lifetime

Sample before hydr. after hydr. (front) after hydr. (back)

$\begin{array}{llll}\text { RR } & 3.24-3.59 & 7.42 & - \\ \text { R1P } & 2.73-4.15 & 7.26 & 6.97 \\ \text { IP } & 3.77-4.57 & 5.14 & 4.73 \\ \text { RP } & 8.38-11.51 & 29.91 & 36.77-49.00 \\ \text { P } & 6.67-12.99 & 15.73-22.19 & 17.53-21.76\end{array}$

$\mathrm{RR}=$ reference, $\mathrm{R} 1 \mathrm{P}=\mathrm{RTA}+1 \mu \mathrm{m}$ poly, $1 \mathrm{P}=1 \mu \mathrm{m}$ poly, $\mathrm{RP}=\mathrm{RTA}+\mathrm{POCl}_{3}, \mathrm{P}=\mathrm{POCl}_{3}$

This first experiment clearly demonstrated that hydrogen passivation can be effective in increasing the minority carrier lifetime if hydrogenation is carried out after a phosphorus diffusion and RTA process. Ongoing experiments with a new lot of samples will expand the number of elements in the process matrix.

An important outcome of the workshop was a consensus of the research technologies that need to be addressed to meet the $18 \%$ low-cost cell efficiency goal. These findings are a first step in defining a roadmap for silicon photovoltaic development. The results are grouped in three main categories, each containing a number of issues. Each item was designated as either a long-term (Long) or an immediate (Int.) issue, and ranked (Rank) from 1 (lowest) through 5 (highest) in terms of its priority of needing attention. The following table summarizes the results: 
Long Int. Rank

1. Larger wafer

2. Improved homogeneity

3. Lower lost feedstock

4. Reduced cost $\mathrm{CZ}$

5. New slicing method

6. High t CZ

7. Non-sliced substrates

8. New growth method

(e.g., FZ, EMC)

9. Thin film Si
$\underline{X}$
$\underline{X}$
-
$\frac{\bar{X}}{\mathbf{X}}$
$\underline{X}$

$\underline{X}$ $\underline{2}$

$\underline{5}$

2

$\underline{2}$

$\underline{3}$

$\underline{3-4}$

$3-4$

$\underline{5}$
1. New structures

2. Process simplification

3. Improved screen printing

4. Alternatives to screen printing

5. New barriers ( a-Si, SIPOS, etc.)

6. Gettering

7. Surface/Defect passivation

8. Hydrogenation

9. Handling thin wafers

10. Optical processing/RTP

11. Reduced chemical usage
1. New encapsulants

2. New interconnect schemes

3. Assembly automation

4. Monolithic module

\section{Cell Processing}

$\begin{array}{lll}\text { Long } & \text { Int. } & \text { Rank } \\ \underline{X} & - & \frac{4}{3} \\ - & \underline{\mathbf{X}} & \frac{3}{1} \\ \overline{\bar{X}} & - & \frac{2}{2} \\ \underline{X} & \underline{\bar{X}} & \underline{\mathbf{5}} \\ \underline{\underline{X}} & \underline{\underline{X}} & \underline{5} \\ \underline{X} & \underline{X} & \underline{\mathbf{X}} \\ \underline{X} & \underline{X} & \underline{1} \\ - & \underline{X} & \underline{5}\end{array}$

Lamination

$\begin{array}{crc}\text { Long } & \text { Int. } & \text { Rank } \\ \overline{\bar{X}} & \underline{X} & \underline{3} \\ \overline{\bar{X}} & \underline{\mathbf{X}} & \frac{4}{5} \\ \underline{\mathbf{S}} & - & \underline{2}\end{array}$

Research examining the effects of impurities and defects in silicon, evaluated by controlled introduction during crystal growth, was extended to evaluate the role of nitrogen. In a dislocation-free float-zoned ingot, minority charge carrier lifetime, $\tau$, was $6,000-3,000 \mu$ s for the portion grown in $100 \%$ Ar and only slightly lower $(\sim 2,500 \mu \mathrm{s})$ for the portion grown in $5 \% \mathrm{~N}_{2} / 95 \%$ Ar. The lifetime dropped more significantly, to $-400 \mu$ s, about 8 minutes after switching to a $100 \% \mathrm{~N}_{2}$ purge gas. Microdefects in the ingots were examined by copper decoration and Secco etching, and Fourier-transform infrared spectroscopy (FTIR spectroscopy). In the dislocation-free segment grown in $100 \% \mathrm{~N}_{2}$, deep 4-fold-symmetry etch pits were observed after copper decoration and defect etching, and may be indicative of nitride precipitate formation 
preceding the eventual nucleation of dislocations. However, in a multicrystalline ingot, $\tau$ was $40-60 \mu \mathrm{s}$ over most of the ingot length and was not significantly different whether growth was conducted in $100 \%$ Ar, $5 \% \mathrm{~N}_{2} / 95 \% \mathrm{Ar}$, or $100 \% \mathrm{~N}_{2}$. Thus, it should be possible to conduct $\mathrm{Si}$ growth in a partial $\mathrm{N}_{2}$ ambient to take advantage of mechanical strengthening effects, lower purge gas costs, and other process advantages without large reductions in $\tau$.

Classical gettering approaches in poly-Si, using chlorine or phosphorus, provide an improvement of the diffusion length in regions with relatively large minority carrier diffusion length (L) values, where recombination is controlled by dissolved heavy metals. They were not as effective in wafer regions with short $L$ values. Unfortunately, solar cell performance can be dominated by these poor quality regions that are difficult to upgrade. To improve $\mathrm{L}$ in these regions, alternative gettering approaches, such $\mathrm{Al}$ gettering with RTA or furnace annealing or a newer ultrasonic treatment (UST) developed at the University of South Florida (USF), have to be used. USF has demonstrated that the RTA or furnace Al gettering improved L values (up to 3.5 times) especially in the low diffusion length regions. Georgia Institute of Technology (GIT) has further shown that that the large improvement in solar cell efficiency (roughly $10 \%$ relative improvement) it the result of the combined effects of forming a back surface field, textured back surface reflector and improving the minority carrier diffusion length by gettering. Initial results with UST gettering improved parameters of commercial solar cells measured at 0.1 sun, but this effect vanished at one sun illumination. Future research is needed to understand physical mechanism involved. This could result in optimization of the UST parameters to achieve the effect of ultrasound stimulated improvement of solar cells at the arbitrary light intensities. This would provide an exciting new opportunity to improve the material and device parameters of finished poly-Si solar cells at low temperatures $(<1000 \mathrm{C})$.

Duke University describes their work on the simulation of gettering, using diffusion-segregation equation developed by their group based on thermodynamic considerations. This model was applied to investigate gettering of fast diffusing impurities such as $\mathrm{Ni}, \mathrm{Cu}, \mathrm{Fe}$, and $\mathrm{Au}$, by phosphorous diffusion and $\mathrm{Al}$ annealing. Their calculations show that a combined $\mathbf{P}$ and $\mathrm{Al}$ gettering can have a beneficial synergistic effect. This result is in agreement with the experimental data. This agreement is believed to be due to the fact that their diffusion-segregation equation also includes an impurity stabilization effect, related to capture-and-release phenomenon, as compared to the impurity diffusion alone.

The facilities at NREL and all of the subcontracted universities are tapped to assist in analysis of commercial silicon materials. Spatially resolved analysis of defects, dislocations, dissolved and precipitated impurities are correlated with electronic characteristics to achieve better control of the initial crystal growth and optimized post-growth processes.

While silicon is the source of today's photovoltaic modules, to exclude silicon from the realm of advanced products would be a serious mistake. Crystalline silicon PV technology has shown laboratory-scale promise for considerably higher performance capability (23\% 1-sun total area cell efficiency, and $27 \%$ concentrator efficiency) than that currently achieved by the industry approaches just discussed. In time, it is likely that cost-effective variants of these lab achievements will become a reality. Another role of the project is to provide industry with the leadership and advanced technology needed to exploit high efficiency device structures. Here NREL can be of best service by not only assisting in the necessary simplification of the high-efficiency processes, but perhaps more importantly by developing and carrying out a strategy to assure that the prerequisite silicon material quality necessary to take advantage of the high efficiency processes can be achieved in a cost-effective and commercially realistic silicon growth process. 
Thin crystalline silicon solar cells made directly of deposited material on low-cost substrates is an attractive concept. Over $85 \%$ of the usable AM1.5 radiation can be absorbed by a $30 \mu \mathrm{m}$ thin layer even without any light trapping. However, growing device quality thin-layer crystalline silicon has not been easy. Liquid phase epitaxy holds promise for this task due to providing both high-rate and low-temperature growth. We have succeeded in growth of smooth silicon layers on multicrystalline silicon substrates. To understand the growth mechanisms of the macroscopically smooth layers from $\mathrm{Cu} / \mathrm{Al}$ as opposed to the faceted, rough layers from In solvent, thermodynamic calculations and Atomic Force Microscopy (AFM) were carried out. The thermodynamic calculations show a strong tendency for $\mathrm{Al}$ to replace $\mathrm{Si}$ in a silicon-oxygen bond. Thus, good wetting and subsequently, dense nucleation sites can be achieved because native silicon oxides are removed from the substrate surface. AFM observations reveal drastically different surface roughnesses between layers grown from $\mathrm{Cu} / \mathrm{Al}$ and In solvents with an average roughness of $78 \AA$ and $5.2 \AA$ on (111) surfaces, respectively. In summary, Al-Cu appears to be an ideal solvent for silicon LPE on multicrystalline substrates.

Solar cells in thin-layer silicon, and in fact all thin-films, benefit from light trapping. We have devised an interpolation model for our light trapping calculations that predicts the degree of light trapping, optical absorption, transmission, and reflection using data from very few reflections within the cell. This improvement reduces the computational time from about a week to two hours without sacrificing the accuracy. This modification will be particularly useful for other groups at and outside NREL who plan to use our light trapping model and software.

The benefits of hydrogen passivation for silicon solar cell performance are clear, but the underlying processes are not defined. We have been working on identifying the state of passivating-hydrogen in silicon. Due to the low concentrations of hydrogen in silicon, and due to its low absorption coefficients, it has been difficult in the past to observe hydrogen absorption peaks by IR spectroscopy. We have now successfully identified absorption bands due to hydrogen in the bulk, associated with the surface damage due to low-energy implantation, and the hydrogen in single and polycrystalline silicon. The peak shifts associated with the substitution of hydrogen by deuterium, and the temperature dependencies at low temperatures (down to $10^{\circ} \mathrm{K}$ ) were measured. This is believed to be the first identification of the defect passivation-related hydrogen.

We have also developed an explanation for the variability of diffusion of hydrogen in different types off silicon. We postulated that diffusion was controlled by formation of a complex of hydrogen with a silicon vacancy. Calculations by Texas Tech showed that the vacancy is a strong trap for interstitial hydrogen and that, from molecular dynamic calculations, the vacancy and hydrogen will attract each other. Our experiments have shown that the diffusivity of Deuterium (of $\mathrm{H}$ ) is related to the material parameters that control the vacancy concentration in silicon, e.g., growth speed, increasing carbon concentration, or decrease in oxygen concentration. Positron annihilation spectroscopy, performed by S. C. Sharma at the University of Texas at Arlington, showed a good correlation exists between increasing vacancy concentration and increasing $\mathrm{H}$ diffusivity. This finding is consistent with observations that hydrogen passivation greatly improves rapidly crystallized material, such as ribbons, but has little benefit for wafers from cast ingots.

\section{High Efficiency:}

Task 2 is the research within the National Photovoltaic Program that is expected to generate products meeting the high-efficiency, long-term targets of $25 \%$ efficient modules in both flat plates as well as concentrators. These products may be essential to reduce system level electricity prices to competitive levels, since increasing efficiency is the ultimate tool in avoiding area related balance-of-systems costs. 
The efforts include (i) exploration of advanced concepts for low-cost fabrication of thin-crystalli semiconductor films; (ii) design and evaluation of concentrator solar cell architectures for increase efficiency and robust production tolerance; (iii) research on fundamental properties of high efficienc semiconductors; and, (iv) maintenance and relocation of the facilities needed to carry-out research in hig efficiency solar cells.

We achieved a major milestone with the demonstration of a $30.2 \%$ efficient $\mathrm{GaInP}_{2} / \mathrm{GaAs}$ concentrato cell, on schedule this month. The III-V Materials and Devices Group received a test report from Dave King, Sandia National Laboratories, on one of the NREL tandem solar cells. The one-sun efficiency was measured at $26.4 \%$, in excellent agreement with the measurement by NREL's Device Characterization Group. (The performance is lower than the $29.5 \%$ efficiency of a device optimized for one sun performance due to changes in design needed for operation at high current.) The cell exceeded $30 \%$ efficiency at concentrations from 115 to 260 suns, peaking at $30.2 \%$ in a range of roughly 140 to 180 suns. This cell is the first two-terminal solar cell to demonstrate a verified efficiency exceeding $30 \%$.

NREL initiated a follow-up study for the transfer of the GaInP/GaAs tandem cell technology for space and terrestrial applications. The objective of the study is to demonstrate the feasibility of heteroepitaxial deposition of state-of-the-art tandem cells on low cost germanium substrates. For space technology, this provides array level compatibility with existing parts. Development of the space market, where the device has achieved $27.4 \%$ AM0, is seen as a path to move this technology along the manufacturing development curve so as to reduce further the cost of terrestrial systems. Relative to future applications as terrestrial product, the large low-cost germanium cuts cell production costs in half to easily meet a $\$ 5 \mathrm{~cm}^{-2}$ target. At this cost, the III-V based solar cell is cheaper than silicon and provides conversion efficiency that could reduce the systems price to levels compatible with $\$ 0.12 / \mathrm{kWh}$.

We initiated a study with a concentrator module manufacturer to address practical considerations of using the tandem cell structure in low-cost terrestrial products. A common question directed at tandem cells is how their performance changes with spectral change due to the atmosphere or chromatic aberration in the concentrating optics. The answer, fortunately, is very little loss of efficiency. Previously we reported that two-terminal, two-junction GalnP/GaAs cells introduced into the linear Entech concentrator module would suffer a power loss of $7-10 \%$ as a result of two-terminal, instead of four-terminal, connection. We have now determined that this loss is a strong function of the module alignment and that the experimental module we purchased (with removable lens and missing endplate) was slightly out of alignment. If the alignment is corrected we find that the loss from two-terminal connection is reduced to about $4 \%$. The misalignment that we note on our module is within what Entech considers to be normal tolerances for a module with silicon cells. Therefore, the replacement of the silicon cells with $\mathrm{GaInP} / \mathrm{GaAs}$ cells would require a more careful alignment of the lens in the module. In this case, the $4 \%$ loss effectively reduces the efficiency of the GaInP/GaAs cells by about a percentage point, still leaving a significant difference between the efficiencies of the GaInP/GaAs cells (about $25 \%$ ) and the Si cells (18\%).

These efficient cells rely on state-of-the-art materials technology that contribute to the advancement of many electronic and photonic technologies. The paper "Effect of faceting on the band gap of ordered GaInP" by D. J. Friedman et al., was submitted for publication in Applied Physics Letters. It is known that under certain growth conditions the pseudobinary semiconductor alloy GaInP shows cation site ordering into the $\mathrm{Cu}-\mathrm{Pt}$ structure, and that this ordering results in a lowering of the band gap, $\mathrm{E}_{\mathrm{g}}$, from that of the disordered alloy. The $\mathrm{E}_{\mathrm{g}}$ lowering is known to depend on growth conditions, including the orientation of the substrate. This paper discusses the dependence of $\mathrm{E}_{\mathbf{g}}$ on epilayer thickness for GalnP grown by metalorganic vapor-phase epitaxy. For epilayers grown on singular (100) substrates under growth conditions conventionally used to produce ordered material, $E_{\mathfrak{g}}$ decreases dramatically with increasing epilayer 
thickness: $E_{g}$ for a $10-\mu \mathrm{m}$-thick epilayer is $\sim 40 \mathrm{meV}$ lower than for a 1- $\mu \mathrm{m}$-thick epilayer. This dependence of $\mathrm{E}_{\mathrm{g}}$ on thickness can be understood in terms of the recently observed faceting of the GaInP growth surface.

A study was also completed and a talk entitled "The effect of phosphine pressure on the band gap of

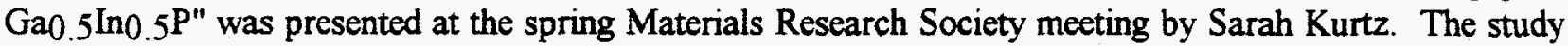
yielded data implying that phosphine pressure plays a very important role in determining the band gap (and ordering) of $\mathrm{Ga} 0.5 \mathrm{In} 0.5 \mathrm{P}$ at high growth temperatures. The phosphine pressure is expected to affect the surface structure (reconstruction) and low phosphine pressures may not be adequate to maintain the structure which leads to ordering. Previously, for high growth temperatures we had proposed that subsurface disordering was determining the band gap because the ordering increased at higher growth rates. In this study we raise the possibility that the changing growth rate actually affects the ordering by changing the effective phosphine pressure, casting doubt on the subsurface disordering model. At low growth temperatures, the data are consistent with models that predict the importance of surface diffusion.

The subcontracted program exploring concepts for high efficiency photovoltaics underwent a competitive procurement. In an environment of increasing pressure to commercialize existing technology we worked with DOE to carry-out the continuation of research and development in High Efficiency Photovoltaics. Initial guidance caused us to prepare an announcement of an indefinite postponement in the procurement issued in July. Prior to releasing that announcement we delivered to DOE a number of reasons justifying the continued interest and need for research in the area. As has recently been demonstrated by Siemens Solar, working with a technology that provides high enough efficiency such that some performance can be traded-off for reduced production cost presents a formidable tool to maintain competitive advantage. Although the U.S. retains leadership in high efficiency photovoltaics, we identified a new and substantial foreign threat to the long-term intellectual property position of the United States. Ultimately, the action was deemed to have an unacceptable impact on the long term position of the U.S. photovoltaic industry. Perhaps in response to the vacillating support, the $R \& D$ community showed a new commitment to the field with 12 organizations offering cost-sharing in new partnerships with NREL. In all, 38 proposals were received with 26 rated above good. While only about $10 \%$ of the requests can be funded, the high caliber of proposed research should assure protection of some advanced high efficiency technologies for future exploitation in flat-plate modules. In next years report, progress will be reported from the three new subcontracts, including: 1) California Institute of Technology that will investigate direct deposition of highly crystalline thin-films of germanium onto glass to serve as a substrate for GaAs solar cells; 2) University of California at Los Angeles where Eli Yablonovitch will use the access to both sides of the $\mathrm{GaAs}$ cell provided by lift-off technology combined with innovative processing to achieve surface texturing in order to test theoretical predictions of the efficiency improvements for optically confined, thin-film cells; and, 3) Research Triangle Institute that will build on the "first-shot" record $15.8 \%$ efficiency achieved in thin-film polycrystalline GaAs solar cells to seek near term cost goals for replacement of the silicon devices in linear concentrators and fundamental understanding supporting longer term goals of flat- plate viability.

\section{Advanced Measurement Techniques:}

The third task supports the research and development of new or upgraded techniques for materials and device analysis. NREL's history is filled with inventions of unique techniques for analysis of photovoltaic materials. These range from the apparent simplicity of creating a method to prepare peculiar samples for conventional analysis, such as Texas Instruments spheres, to the complexity of demonstrating whole new analytical equipment suitable for R\&D 100 Awards. The strength of NREL's analytical support to the PV community does not rest in the free performance of routine measurements that might have been obtained commercially at great expense. The strength resides in the ability to create new analytical methods to extract needed data from unconventional materials. 
In doped polar semiconductors the macroscopic electric field associated with the longitudinal optical phonon allows for the coupling with the plasmon in the material. Light scattering from these coupled phonon-plasmon modes is sensitive to the effective carrier concentration in the material. The position of the coupled phonon-plasmon modes scales with the amount of carriers in the sample. We have examined several $\mathrm{GaAs}$ samples grown for different Se flow rates. From the position of the coupled plasmon modes for the different samples we have determined the electron density in the samples. The values of the carrier concentration obtained from the Raman measurements were confirmed by independent $\mathrm{C}-\mathrm{V}$ measurements on the samples. An excellent agreement is found between the two techniques. Our measurements have enabled has to calibrate the OMVPE reactor for the amount of carriers introduced into the GaAs samples as a function of Se flow rate.

To examine the feasibility of extending this technique to study GaAs tunnel junctions, we have studied a n$\mathrm{GaAs} / \mathrm{n}-\mathrm{GaAs} / \mathrm{GaInP}$ structure. From the Raman spectra of this structure we have been able to determine the carrier concentration in different GaAs layers. These measurements have also shown the extreme sensitivity of the technique to study band bending at the GaAs/GaInP interface.

Another important tool, measurement of minority carrier lifetime by photoconductivity decay sensed by if coupling, was developed through this task and the CRADA with Texas Instruments.

\section{Exploratory Materials and Devices:}

Task four covers the internal research in solid state theory and the subcontracted projects in the University Participation Program and New Ideas Program. We will complete the development of theoretical methods capable of predicting the properties of mesoscopic-scale solids $\left(\geq 10^{3}\right.$ atoms/cell). These methods are needed to analyze inhomogenious materials such as polycrystalline and nanostructured semiconductors. The new tools were applied to the analysis of porous silicon to explain the strong photoluminescence and its possible uses in photovoltaic devices.

Nanostructure materials have been studied extensively in the last decade because of their potential in photo-electronic device. The dielectric constants of these quantum nanostructures are important for their electronic and optical properties. One example is the quantum dots with sizes around $40 \AA$. The magnitude of the dielectric constants of these quantum dots will determine whether the electron-hole exciton is free (as in the bulk ) or confined by the quantum dot dimension. This in turn is important to determine which of the several disputed mechanisms of their photoluminescence is correct. The effective mass model is not sufficiently accurate for the calculation of dielectric constant of $40 \AA$ for sized nanostructures because the parabolic approximation is no longer valid. Thus, atomic scale mesoscopic calculations are necessary. We have used an empirical pseudopotential Hamiltonian and a plane wave basis set. Since the conventional method of quantum mechanics can not calculate such 'large' (>1000 atom) systems, we have developed a generalized moments method to solve this problem. This new method uses statistical means (expansion in Chebychev polynomials) to calculate the optical absorption spectra, density of states and dielectric constants. Using this method, the dielectric constants of different sized Si quantum dots were calculated. The calculated dielectric constant drops considerably from its bulk value, e.g., for a sphere with diameter of $20 \AA$ it is $E=8$ instead of the bulk value of 11.4 . However, it is still much larger than the value predicted by current models. We find that the exciton is confined by the quantum dot's physical dimension, not by the Coulomb attraction between the electron and the hole. A convenient parametrization of the dielectric constant as a function of the quantum dot's size is achieved from our calculations. The optical absorption spectra and density of states of different sized quantum dots were also calculated. We expect this new method to find many more applications in the quantum nanostructure optical property calculations. 
The New Ideas program provides a periodic solicitation of proposals to investigate new application of technology for photovoltaic conversion. Two subcontracts selected from the last procurement seek to apply quantum size effects in new solar cells. Spire Corporation has demonstrated photovoltaic conversion in a solar cell made of porous silicon. Porous silicon is created by etching crystalline silicon under conditions that create fine "wires" on the surface. The size creates quantum wells changing the optical properties of the material to a pseudo-direct bandgap such that the material emits light. The Spire program seeks to determine if the direct absorption has potential for thin-film photovoltaics. Multiple quantum well structures in organic semiconductors prepared at Princeton University may hold the key to exciton dissociation and charge transport issues that have limited the performance of all organic-based solar cells made to date.

The University Participation Program provides the research community with another forum to ensure that the National Photovoltaic Program is addressing key topics its long-term well-being. Two key features of this program are essential for achieving this objective. First, it is the only PV Program area in which research is selected entirely by peer review. The scope of the solicitation is not specified. The proposal must justify the importance of the chosen topic. Reviewers from universities and industry evaluate these justifications and quality of the planned research. This selection process is designed to minimize the influence of "conventional wisdom" or program bias. The other key to success is the minimal management interference afforded the investigators after selection and award. University Participation subcontracts were the first in the NREL programs to provide three-year, phased-awards that match the time required for graduate students to complete their theses. Similar to a research grant, there may be no management performance milestones and documentation is limited to only Annual and Final Reports. The Program was reduced by about half in the most recent competition in 1993. It consists of five projects, including:

- "Fundamental Studies of the Effects of Crystal Defects on CuInSe2/CdS Heterojuntion Behavior" at the University of Illinois.

- "Electronic Processes in Thin-Film PV Materials", University of Utah

- "Heterojunction Development and Optimization in Thin-film Compound Semiconductor Cells", University of South Florida

- "Solar Energy Conversion at Dye-Sensiitized Nanostrucutred Electrodes Fabricated by Sol-Gel Processing", Johns Hopkins University

- "Back Surface Fields for n/pp and P/n GaInP Solar Cells" North Carolina State University.

Cooperative Research and Development Agreements:

In the first Photovoltaic Program CRADA, with Texas Instruments (TI), NREL assisted in helping to characterize and understand the mechanisms underlying the upgrading process they use to prepare spheral silicon from metallurgical grade starting material. Due to the heavy doping and very short lifetimes in Spheral Silicon it is quite difficult to extract meaningful data from the TI spheres. The radio frequency coupled photoconductivity decay (RFPCD) systems available elsewhere generally do not have adequate sensitivity nor fast enough response. Using our recently acquired YAG laser and a new DC pulse amplifier circuit we succeeded in measuring the minority carrier lifetimes in samples ranging from a few hundred spheres down to a single sphere. TI and NREL performed a series of tests to determine if the system provides highly reproducible results and to determine what sample size was needed to meaningfully evaluate the quality of a production lot. The result is a highlight for both the performance of the RFPCD system as well as TI's process uniformity. It indicates that a sample size of a gram or less may be adequate to characterize production. Based on these findings, TI duplicated the NREL system in their pilot facility for in line process monitoring. 
NREL's crystalline silicon group supported Astropower in evaluation and improvement of their thin-silicon process. One purpose of the CRADA is to provide a mechanism for full protection of company proprietary data. Findings from the CRADA investigations are not reported here because they are proprietary.

New CRADAs were negotiated for work to continue into fiscal year 1995. A "funds-in only" CRADA with UniSil Corporation on "Technology and Characterization Support for the High Purity Float Zone Silicon Title III Project" to assist UniSil in their efforts to set up a US-based float-zoned silicon manufacturing capability. NREL and UniSil are both interested in the development of silicon float zoning technology for use in a variety of applications such as high efficiency photovoltaic devices, high power switching devices, and infrared detectors. The proposed CRADA would provide a highly interactive program integrating silicon float-zone materials and characterization expertise at NREL, with a fast paced float-zone manufacturing initiation activity at UniSil. NREL/DOE expect to benefit from the proposed work because the successful implementation of US float zoned silicon commercial manufacturing would provide a source of material for those US companies engaged in high-efficiency silicon photovoltaic module manufacturing. The higher performance capability of float zoned silicon is also expected to allow lower balance of system costs in utility applications.

NREL and Crystals Systems, Inc. (CSI) have agreed in principle to a CRADA intended to assist in development of technology to further improve the performance and reduce the cost of polycrystalline silicon ingot based solar cells. These goals will be met by investigation of interactions of crucibles with the silicon melt in the Heat Exchanger Method (HEM). CSI has developed a directional solidification process with HEM using silica crucibles. This process has been extended to $55 \mathrm{~cm} \times 55 \mathrm{~cm}$ ingots. They have also demonstrated reusable crucible technology for ingots of $33 \mathrm{~cm} \times 33 \mathrm{~cm}$ and $44 \mathrm{~cm} \times 44 \mathrm{~cm}$. CSI has designed and installed a totally automated furnace that requires labor only for loading and unloading. This furnace uses a custom-software based control system such that both the heat input and the heat extraction can be controlled during ingot growth. They sell the furnace for production of $55 \mathrm{~cm} \times 55 \mathrm{~cm}$ ingots. Other tasks of the CRADA will seek to improve upon the already impressive $17.4 \%$ efficiency achieved in polycrystalline devices using CSI material by identifying mechanisms of impurity incorporation and defect generation and evaluating methods of gettering. 
Title:

Organization:

Contributors:
Crystalline Silicon Research

National Renewable Energy Laboratory

T. F. Ciszek, principal investigator; B. Sopori, S. Tsuo, R. Schwerdtfeger, R. Burrows, T. Wang, C. Marshall, M. Landry, M. Symko, M. Cudzinovic, and characterization staff

\section{Introduction}

This research addresses issues related to crystalline and multicrystalline silicon materials for PV use. A major effort was required this year to move the project's laboratory facilities from temporary buildings to a new lab building, the Solar Energy Research Facility or SERF. The technical activities were in the areas of Si materials research, Si devices, Si characterization, and Si industry interaction.

\section{SERF Move-In Activities}

These activities were the primary focus and time consumption of our work this year. The new facility houses laboratories for silicon crystal growth, wafering, polishing, electrical characterization, $x$-ray characterization, diagnostic solar cell fabrication, and processing studies. The move of silicon research equipment began in mid-January, 1994 and continued through the year. Modifications were made to existing equipment to reduce space requirements so that they would fit into the smaller SERF silicon labs. No new equipment was acquired. Most equipment is operational again, with the exception of $\mathrm{x}$-ray topography, hydrogen ion implantation, and the silicon diffusion furnace.

\section{Silicon Materials}

\section{Thin-Layer Silicon Growth}

We are investigating techniques for thin-layer Si growth on low-cost substrates. A new Cu-Al-based solvent has lead to successful liquid phase epitaxy (LPE) growth of smooth Si layers, $\sim 30 \mu \mathrm{m}$ thick, on multicrystalline metallurgical-grade (MG) Si substrates. Surface native oxides are removed via the energetically favorable reaction $4 \mathrm{Al}+3 \mathrm{SiO}_{2} \rightarrow 3 \mathrm{Si}+2 \mathrm{Al}_{2} \mathrm{O}_{3}$, and very good wetting between the melt and the substrate results. The high (>20\%) Si solute content (and thus higher growth rate) made possible by the $\mathrm{Cu}-\mathrm{Al}$ solvent eliminates faceted growth via a microscopically rough growth interface (about 80 Angstroms) compared to the microscopically smooth interface ( $\sim 5$ Angstroms) characteristic of more typical solvents providing low solute contents $(-5 \%)$. The surface of the layers grown from $\mathrm{Cu}-\mathrm{Al}$ is macroscopically smooth because of the isotropic growth resulting from the microscopic roughness and availability of lattice sites for new growth.

Impurity incorporation ( $\mathrm{Al}$ and $\mathrm{Cu}$ ) during liquid phase epitaxial growth of thin $\mathrm{Si}$ has been studied with secondary ion mass spectroscopy (SIMS) analysis on samples grown from different compositions of $\mathrm{Si}-\mathrm{Cu}-\mathrm{Al}$ solutions. Interactions between various solution components $\mathrm{Cu}$ and $\mathrm{Al}$, in this case) are seen to strongly affect their incorporation into the growing silicon matrix. To quantify these interactions, we determined incorporation factors $\alpha_{B}{ }^{A}$ for impurity B's contribution to incorporation of impurity $\mathbf{A}$ into the growing silicon, such that 
$n_{A}$ (solid) $=K_{A} n_{A}$ (iquid) $\left(n_{S i}+n_{B} \alpha_{B}^{A}+n_{C} \alpha_{C}{ }^{A}+\ldots\right.$,

where the n's are the mole fractions of the respective elements. $\alpha_{B}{ }^{A}>0$ means $A$ is expelled from solution into the growing $\mathrm{Si}$ by $\mathrm{B}$ and $\alpha_{B}{ }^{A}<0$ implies that $\mathrm{A}$ is attracted by $\mathrm{B}$ in the solution so that incorporation of $\mathrm{A}$ into the growing $\mathrm{Si}$ is retarded. The table lists the $\alpha_{C u}{ }^{A \prime}$ and $\alpha_{A l}{ }^{C u}$ values.

Table I Impurity incorporation factors for $\mathrm{Cu}$ and $\mathrm{Al}$ in $\mathrm{LPE}$-grown $\mathrm{Si}$ from $\mathrm{Cu} / \mathrm{AI}$ melt solutions

$\begin{array}{llllll}\text { Sample No. } & n_{S i}: n_{C u}: n_{A l} & n_{A l} \text { (solid } & & n_{C u} \text { (solid } \\ \text { w930520-1 } & .35: .65: 0 & & & 1.2 \times 10^{-7} & \mathrm{~K}_{\mathrm{Cu}=5.3 \times 10^{-7}} \\ \text { w930408-5 } & .32: 0: .68 & 2.4 \times 10^{-4} & \mathrm{~K}_{\mathrm{Al}}=1.11 \times 10^{-3} & & \\ \text { w931208-3 } & .34: .56: .10 & 0.1 \times 10^{-4} & \alpha_{C u}{ }^{A l}=-.44 & 1.6 \times 10^{-7} & \alpha_{A l}{ }^{C u}=1.01 \\ \text { w930127-1 } & .23: .49: .28 & 0.34 \times 10^{-4} & \alpha_{C u}{ }^{A l}=-.25 & \sim 5 \times 10^{-7} & \alpha_{A l}{ }^{C u}=1.70 \\ \text { w940208-1 } & .23: .57: .20 & 0.26 \times 10^{-4} & \alpha_{C u}{ }^{A l}=-.19 & -4.5 \times 10^{-7} & \alpha_{A l}{ }^{C u}=1.26\end{array}$

Thus, $\mathrm{Cu}$ partially restrains $\mathrm{Al}$ to remain in the $\mathrm{Si}-\mathrm{Cu}-\mathrm{Al}$ melt. Therefore, proper doping of the grown Si layer by $\mathrm{Al}$ can be realized through appropriate choice of solution composition. Previous NREL studies indicate that $\mathrm{Cu}$ retards the incorporation of many other impurities in $\mathrm{Si}$.

In summary, the $\mathrm{Cu} / \mathrm{Al}$ solvent system's advantages for thin $\mathrm{Si}$ growth on low-cost substrates are: high solvent power (with associated favorable growth kinetics and throughput economics), low $\mathrm{Cu}$ solubility in $\mathrm{Si}$, and a high capability for retaining impurities (including $\mathrm{Al}$ ) in solution rather than allowing them to incorporate into the growing layer. The $\mathrm{Al}$ component provides the capability to remove native oxides from the substrate surface, thus allowing uniform, dense, nucleation sites for growth. Together, $\mathrm{Al}$ and $\mathrm{Cu}$ show promise for rapid, uniform (microscopically rough and macroscopically smooth) growth on any random grain orientation in multicrystalline substrates without the need to partially dissolve or "melt back" the substrate, which would introduce unwanted impurities into the solution. The $\mathrm{Al} / \mathrm{Cu}$ solvent system thus is expected to be more effective than previously reported solvents such as In, $\mathrm{Ga}, \mathrm{Sn}$, or $\mathrm{Au}$.

\section{Controlled Defect and Impurity Studies}

We studied the effects of $\mathrm{Si}$ growth in atmospheres containing $\mathrm{N}_{2}$ on minority charge carrier lifetime $\tau$ using a high-purity, induction-heated, float-zone (FZ) crystal growth method and the ASTM F28-75 photoconductive decay (PCD) method. We found that Ga-doped multicrystalline silicon ingot growth in a partial or total $\mathrm{N}_{2}$ ambient has a minimal degradation effect on $\tau$, and no significant grain boundary passivation effect. $\tau$ is determined predominantly by the ingot grain size rather than the ambient composition. Multicrystalline growth in a $100 \% \mathrm{~N}_{2}$ ambient yields $\tau$ values around 40-100 $\mu$ s for the grain sizes obtained by the FZ method. However, nitride compounds will form on the melt surface and on the hot feed rod and crystal surfaces. The concentration of $\mathrm{N}$ in multicrystalline ingots grown in $\mathrm{N}_{2}$ approaches $1 \times 10^{16}$ atoms. $\mathrm{cm}^{-3}$.

For dislocation-free (DF) single-crystal growth, the effect of $\mathrm{N}_{2}$ on $\tau$ is minimal and can even be beneficial, provided that the amount of $\mathrm{N}_{2}$ in the purge gas is kept below the level at which nitride compounds form in the melt or on the rod surfaces and nucleate dislocations. DF FZ growth in a 
transient $25 \% \mathrm{~N}_{2} / 75 \% \mathrm{Ar}$ ambient yields $\tau$ values around 4,000 $\mu \mathrm{s}$. A beneficial effect of $\mathrm{N}_{2}$ is the elimination of swirl-type defects. The $\mathrm{N}$ concentrations in the crystals just prior to dislocation nucleation were measured to be about $8-10 \times 10^{15}$ atoms $\cdot \mathrm{cm}^{-3}$. We have not established the upper limit of $\mathrm{N}_{2}$ content in the purge gas for steady-state, "start-to-finish" DF growth, but it is < $20 \%$. It should be possible to conduct $\mathrm{Si}$ growth in a partial $\mathrm{N}_{2}$ ambient, within the limits discussed above, to take advantage of mechanical strengthening effects, lower purge gas costs, and other process advantages without significant reductions in $\tau$.

\section{Other Materials Studies}

- We demonstrated that 325 mesh, $99.999 \%$ pure Si can be controllably melted on various substrates using concentrated sunlight from NREL's high-flux solar furnace.

- We have succeeded in using NREL's high-flux solar furnace to convert hydrogenated amorphous $\mathrm{Si}$ into microcrystalline $\mathrm{Si}$ thin films by a solid-phase crystallization process.

- A simple chemical etching process was used to produce porous silicon on both sides of a MG Si wafer. This may be useful for gettering to upgrade MG Si for solar cell applications.

\section{Silicon Devices and Processes}

Our Si diagnostic device processing capability was shut down for moving in January, 1994 and will not be operational again until sometime in 1995. Several other device related activities were carried out:

- Optical Processing was applied to conventional finished Si cells without AR coating. Typically, a $0.2 \%$ absolute increase in efficiency, $1.6 \%$ absolute increase in fill factor, $5 \mathrm{mV}$ increase in $\mathrm{V}_{\mathrm{oc}}$, and $0.05 \mathrm{mAcm}^{-2}$ decrease in $\mathrm{J}_{\mathrm{x}}$ were seen.

- The feasibility of using sunlight as an energy source to fabricate silicon solar cells was demonstrated. It provides a cold wall process (reduced furnace contamination); temperature/time profiles can be precisely controlled; wavelength, intensity, and spatial distribution of the incident flux can be controlled and changed rapidly; a number of high-temperature processing steps can be performed simultaneously; quantum and thermal effects may benefit the cell performance; and the power source is clean, abundant, low-cost, and environmentally friendly.

- We have succeeded in fabricating triple-junction, silicon-based solar cells with $V_{\propto c}$ larger than 1.8 $\mathrm{V}$, as part of the Crystal Growth Research Group's collaborative efforts with NREL's Hydrogen Generation Program. These cells will be used for $\mathrm{H}$ generation by water splitting in a photoelectric conversion (PEC) process where a large $\mathrm{V}_{\alpha}$ is required.

- We have devised an interpolation model for our light trapping calculations that predicts the degree of light trapping, optical absorption, transmission, and reflection using data from very few reflections within the cell. This improvement reduces the computational time from about a week to two hours without sacrificing the accuracy.

\section{Silicon Characterization}

As part of a study to determine the synergistic effects of impurities, vacancies, and hydrogen and to develop a quantitative model of vacancy-hydrogen diffusion in $\mathrm{Si}$, $\mathrm{SIMS}$ depth profile analysis was used to follow the diffusion of $\mathrm{H}$ in various polycrystalline $\mathrm{Si}$ samples that had been hydrogenated by plasma, $\mathrm{Si}_{3} \mathrm{~N}_{4}$, and forming gas annealing processes. Diffusion profiles of deuterium in various silicon samples, believed to contain vacancy concentration in the following decreasing order: ribbon- 1 , ribbon- 
2 , cast poly-1, FZ, and CZ, were also determined by SIMS. The deuterium levels seen about $1 \mu \mathrm{m}$ below the surface of the various samples were in the same decreasing order, and ranged from $5 \times 10^{18}$ atomsocm ${ }^{-3}$ for ribbon-1 to $5 \times 10^{16}$ atomsocm $\mathrm{cm}^{-3}$ for the $\mathrm{CZ}$ wafer. These results agree with our previous vacancy model. Material with high carbon content passivates particularly well. We also showed that the hydrogen solubility at a Si surface depends on the nature of damage near that surface.

We can now routinely polish, defect etch, and defect map commercial size polycrystalline silicon wafers with the scanning defect mapping system (SDMS). The mapping time for 4-in $x$ 4-in samples is less than one hour. Design of the SDMS to incorporate improvements for cell characterization and automatic corrections for etch pit size for dislocation mapping was carried out, and we worked with Labsphere to incorporate the new design features into their commercial version of the SDMS. Scanning I-V system programs have also been written with the objective of implementing automatic probing of mesa diode arrays to evaluate material inhomogeneities and study influence of defect distributions on large-area cell performance.

\section{Silicon Industry Interaction}

\section{CRADAs}

A CRADA with AstroPower was completed this year, in which the focus was upon improvement of silicon film material quality. Late in the year, a CRADA was initiated with UniSil Corporation to assist them in FZ technology and characterization (lifetime, resistivity, and photoluminescence) of high purity $\mathrm{Si}$, as they establish a US-based manufacturing capability for FZ silicon. A third CRADA assisted Texas Instruments in characterization of spheral Si beads for a new PV cell structure.

\section{Other Interactions}

We continued to help Crystal Systems improve their casting using a reusable crucible. Diffusion length measurements and impurity analysis of many wafers was done. Several castings, grown under different process conditions, were analyzed. The objective of these experiments was to identify the growth conditions that will minimize the rejected top-portion of the casting. We have identified the cause of this degradation mechanism which in turm, has led to a significant reduction in the reject material. Interactions were also carried out with Siemens Solar, MEMC, NCSU, USF, ASE Americas, MRG, EBARA, and others.

\section{Publications}

- Eight papers from the Crystalline Silicon Project were published in the 23rd IEEE PVSC Proceedings (1993), pp. 65, 73, 97, 101, 127, 190, 281, and 287.

- T. F. Ciszek, "Electromagnetic and Float-Zone Methods for High-Purity Silicon Solidification," in: Containerless Processing: Techniques and Applications, William H. Hofmeister and Robert Schiffman eds., The Minerals, Metals \& Materials Society Publisher (1993) pp. 139-146.

- S. S. Ostapenko, L. Jastrzebski, J. Lagowski, and B. Sopori, "Increasing Short Minority Carrier Diffusion Lengths in Solar-grade Silicon by Ultrasound Treatment," Appl. Phys. Lett, 65, 1555 (1994)

- T.F. Ciszek, "Thin Silicon Layer Crystallization from High-Temperature Solutions of Si in Copper," U. S. Patent 5,314,571 (May 24, 1994). 
Organization: NREL

Contributors: Mowafak Al-Jassim (principal investigator), Sally Asher, Michael Bode, Amy Franz, Falah Hasoon, Kim Jones, Alice Mason, Rick Matson, David Niles, and Robert Reedy.

\section{Introduction}

The Materials Characterization task has provided extensive and crucial support for both our in-house and subcontract groups. A wide variety of techniques was utilized to characterize PV materials and devices. Compositional measurements are performed by electron probe microanalysis using either energy dispersive or wavelength dispersive X-ray analysis. A new, fully computerized microprobe was commissioned during this year. Structural and defect analyses are carried out by transmission electron microscopy (TEM). A project for the development of light element analysis on thin foils in the TEM was completed during this year enabling us to detect carbon and oxygen in various PV materials such as silicon. Additionally, scanning electron microscopy (SEM) in its various modes, such as secondary electron, CL and EBIC imaging, is providing a very wide support for measuring the topographical, luminescent and microelectrical properties of PV materials and devices.

In addition to these measurements, the Materials Characterization task was merged with the Surface and Interface Analysis Task during 1994 adding many powerful techniques. This includes scanning Auger microscopy (AES), X-ray photoelectron spectroscopy (XPS), ultra-violet photoelectron spectroscopy (UPS), secondary ion mass spectrometry (SIMS). A new field-emission AES instrument and a small-spot XPS instrument were commissioned during 1994.

Most of the research activities carried out by this task are planned through other in-house and subcontractors activities. The following is a brief description of some of the highlights of our work during 1994. These will be divided according to the material technology.

\section{$\underline{\text { CIS }}$}

The Materials Characterization task continued to support in-house as well as outside research and development on CIS with a large number of analytical studies on various material aspects. More than 400 analyses were performed using the electron microprobe facility, and over 200 devices and films were analyzed by SIMS and AES. In addition, a large number of selected devices were characterized by SEM and TEM. Many of these devices and films were of proprietary nature, showing the facility's value to in-house and industrial developers.

Aside from routine characterization of CIS technology related materials, much time was concentrated on more fundamental aspects of materials growth. John 
Scofield, together with Sally Asher took an in-depth look at the characteristics of the Molybdenum back contact. This contact provides electrical contact as well as a mechanical connection to the glass substrate. By using a two step approach to deposit the Mo, an improvement in both critical aspects was achieved.

In cooperation with John Tuttle, Michael Bode developed a sample preparation technique for TEM samples, which allows a much easier preparation of cross sectional TEM samples of CIS films on glass. This technique can be used to probe the composition of the film with an extremely high spatial resolution and to characterize individual grains within the material (Fig. 1). The technique was used for films in which an ordered phase of CIS was detected.

A mystery of CIS has always been the exact nature of the band alignments between the CIS and the CdS front contact. Investigations on PVD material always resulted in bandgaps that were too small to explain the contact characteristics. David Niles used target factor analysis techniques to analyze Auger electron spectroscopic data from real devices, and for the first time was able to show that the bandgap was $1.11 \mathrm{eV}$, sufficient to explain the contact's characteristics (Fig. 2).

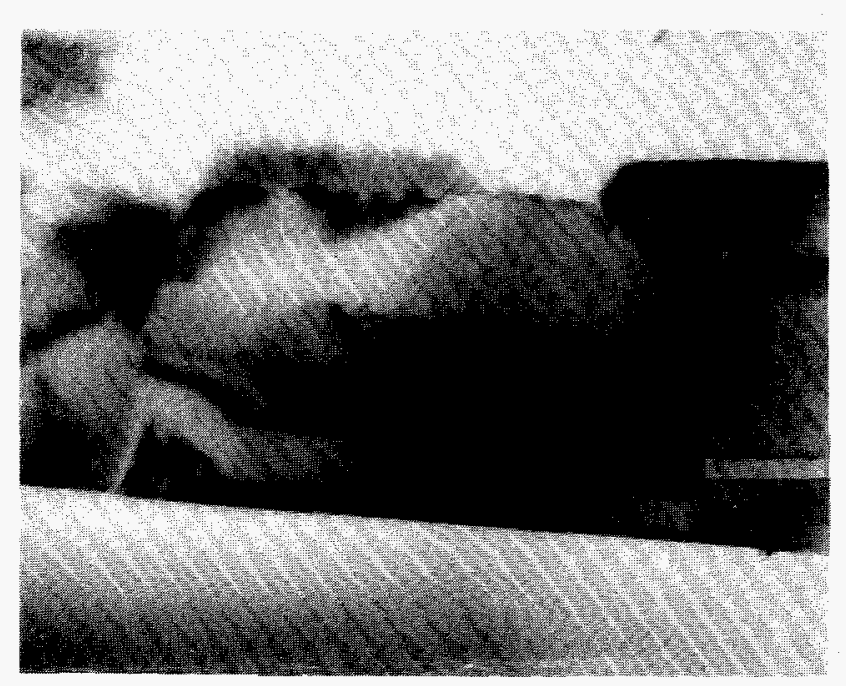

Fig. 1: TEM cross-sectional micrograph of a CIS film showing columnar grain structure and defects.

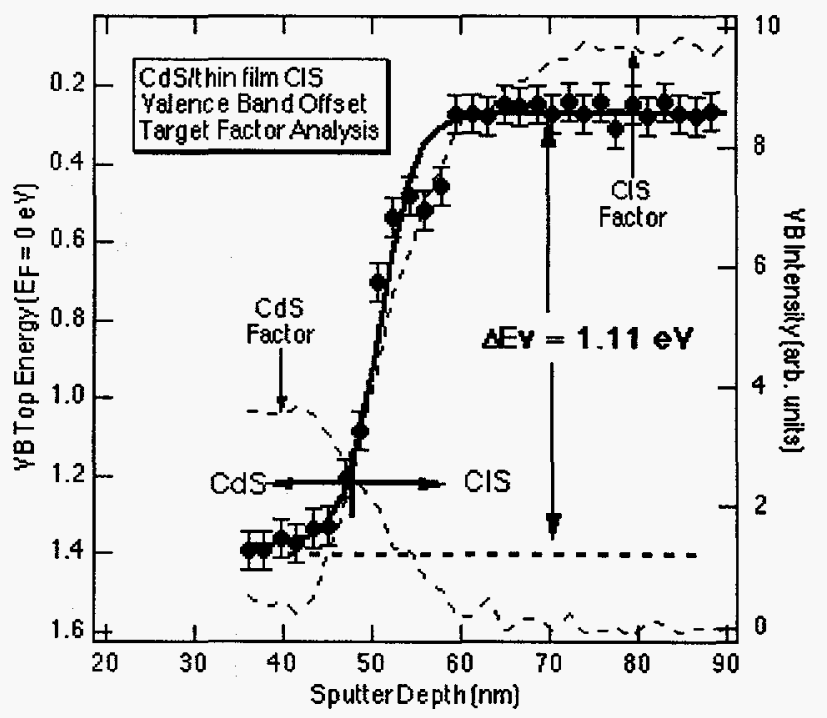

Fig. 2: Valence band structure of CdS/CIS interface, as determined by Auger Electron Spectroscopy.

\section{$\underline{\text { CdTe }}$}

Developing an electronic model for CdTe back contacts and understanding diffusion at the CdS/CdTe interface were two projects that dominated the efforts in the CdTe program of the Materials Characterization task. The fundamental issue in the formation of back contacts to CdTe pivots around the role of Te. Researchers in the CdTe program routinely treat the p-type CdTe thin-film in a concentrated etch of nitric and phosphoric acids before the application of the graphite back contact. Through a combination of XPS and AES analyses, we 
determined that the effect of the etch was to leave a Te layer on the p-type CdTe thin-film surface. Figure 3 contains XPS spectra that show the removal of Cd.

To understand why $\mathrm{Te}$ improves the performance of the back contact, we combined UPS and XPS to develop an electronic model for the back contact. In Figure 4, we show graphically how Au produces an inverted n-type region at the back contact whereas Te leaves a more favorable p-type region, thereby decreasing the series resistance of the back contact.
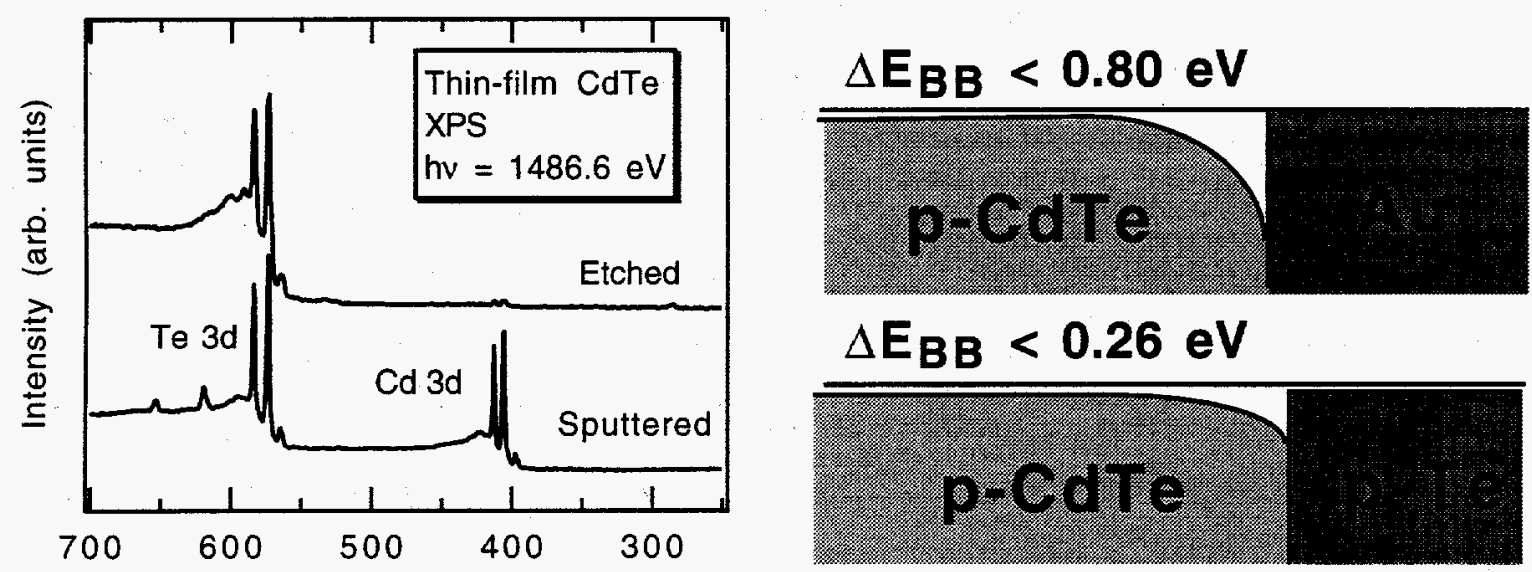

$\Delta \mathrm{E}_{\text {BB }}<0.26 \mathrm{eV}$

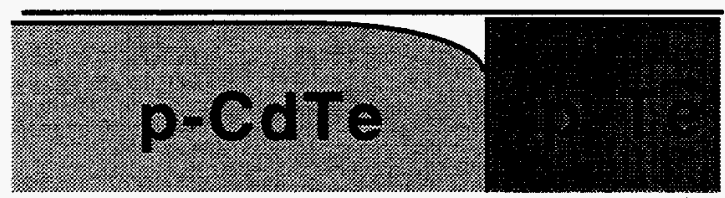

Binding Energy (eV)

Fig. 3: XPS spectra of acid-etched and Fig. 4: Models of back contacts to CdTe. sputtered CdTe surfaces.

Diffusion at the CdS/CdTe interface is critical to understanding the role of the $\mathrm{CdS}$ window layer. Figure 5 shows a secondary ion mass spectrometry depth profile of a $\mathrm{CdTe} / \mathrm{CdS}$ structure. The profile clearly shows an interface width of $-0.5 \mu \mathrm{m}$, not an atomically abrupt interface. This width leads to a region of mixed $\mathrm{CdS}$ and $\mathrm{CdTe}$ that changes the absorption of the device.

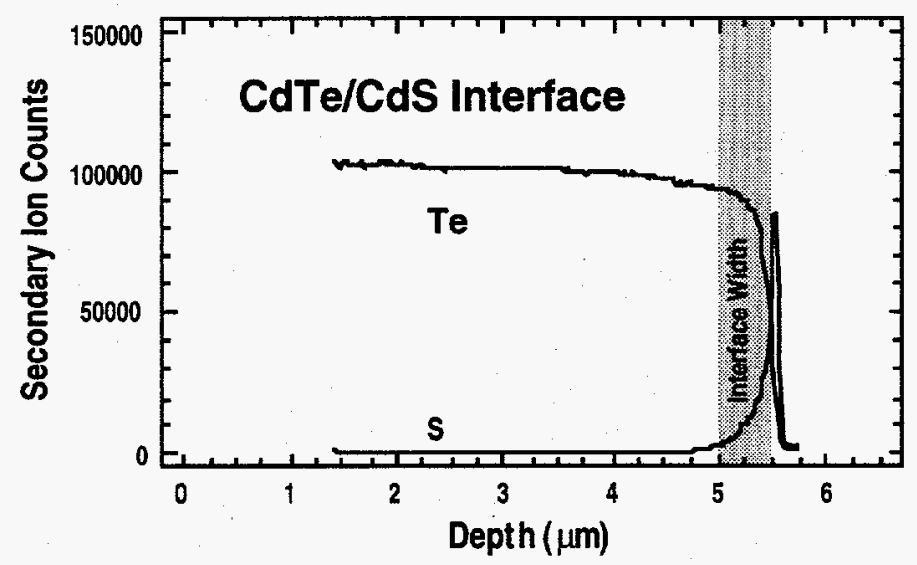

Fig. 5: SIMS depth profile through the $\mathrm{CdTe} / \mathrm{CdS}$ interface. 


\section{$\underline{\mathbf{S i}}$}

The Materials Characterization task supported in-house and subcontracted research, as well as CRADA work with industrial partners during 1994. In this period over 280 samples were analyzed by TEM, SIMS, SEM, EPMA, and laser scanning.

\section{LPE Grown Si}

SIMS was used to characterize impurity and residual solvent levels in LPE-grown thin film Si. Layers grown from various solvent melts were examined. Depth profile results were used to understand and control the growth kinetics and solvent atom diffusion. Silicon layers grown from the optimum melt composition show minority carrier lifetimes that are independent of the layer thickness.

\section{TI CRADA}

Spheral ${ }^{\mathrm{TM}}$ silicon was extensively studied to facilitate understanding of the upgrading and processing steps of metallurgical grade Si. Many spheres were removed after each step in the process. SIMS depth profile analysis was able to show the reduction of impurity levels after each step in the upgrading process. For example, Fe contaminant levels were found to decrease from $\sim 5 \times 10^{19} \mathrm{at} / \mathrm{cm}^{3}$ in first melt spheres to $<1 \times 10^{16} \mathrm{at} / \mathrm{cm}^{3}$ after four upgrading cycles, in fifth melt spheres.

Spheres from later processing steps were also studied, e.g. following the diffusion of phosphorus. Figure 6 shows a TEM cross-section of a post-diffusion sphere. The micrograph shows a layer close to the surface of the sphere containing a high density of dislocation loops. The thickness of this layer was consistent with depth of $\mathrm{P}$ diffusion observed by SIMS, and shown in Figure 7.

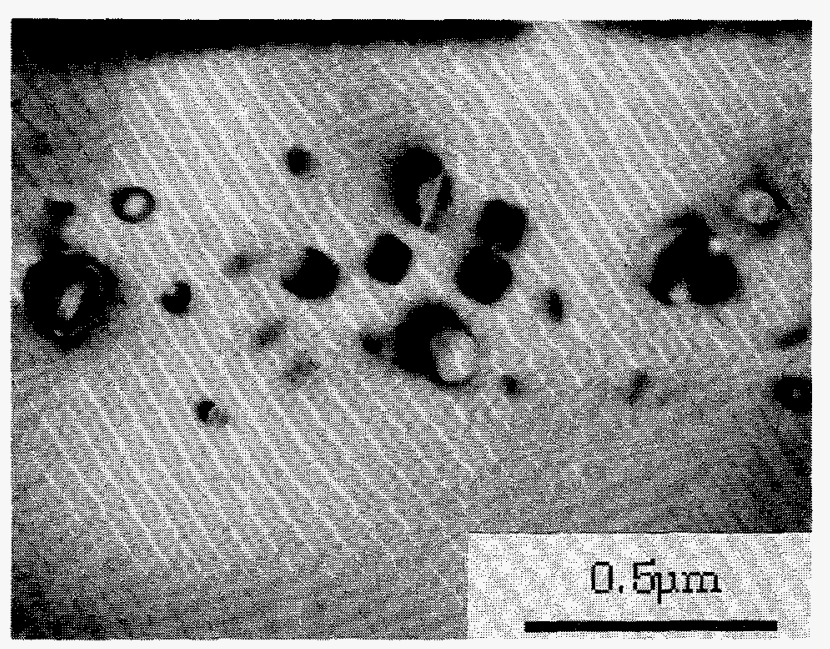

Fig. 6: TEM cross-section of a postdiffusion sphere revealing a high density of dislocation loops in a layer near the surface.

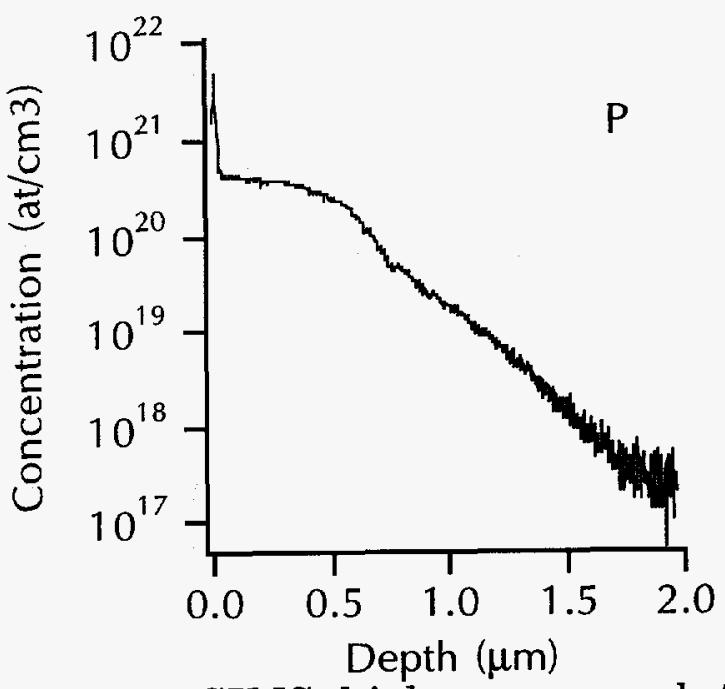

Fig. 7: SIMS high mass resolution depth profile of $\mathrm{P}$ in a post-diffusion $\mathrm{Si}$ sphere. 
Title:

Organization:

Contributors:

\section{Electro-Optical Characterization.}

National Renewable Energy Laboratory Golden Colorado 80401,

R. K. Ahrenkiel, B. M. Keyes, D. L. Levi, F. AbouElfotouh, A. Mascarenes, J. Webb

A great deal of progress was made in both relocating the laboratories and in research supporting the thin film polycrystalline and silicon programs. During the summer of 1994, the Electro-Optical Characterization Laboratories were relocated from Building 16 in Denver West Park to the Solar Energy Research Facility (SERF). All laboratories are now up and running in the SERF Facility. We began measuring samples on some of the facilities in early September.

A new mode-locked pumped dye laser was purchased as part of the SERF capital package. That system has been operational since the summer of 94 and has become the workhorse of the time-resolved photoluminescence. A new femtosecond titanium sapphire system was also purchased under the SERF capital budget. This will be used to extend the measurement capability in the time and frequency domain. This new facility will enable us to measure minority-carrier lifetime many materials that we cannot now measure. One of the first projects will be the measurement of CdS lifetime in CdS/absorber structures in thin film PV projects. We currently have no pulsed lasers capable of exciting CdS.

A new technique was developed during the course of the year for measuring minority carrier lifetime in non-light emitting materials such as silicon and germanium. The technique is called radio frequency photoconductive decay (RFPCD). This technique was also very useful for measuring lifetime in various InGaAs that are being developed for the thermophotovoltaic (TPV) program. A highlight of this work was to measure the lifetime in the small silicon spheres used in the Texas Instruments solar project[1,2]. A schematic of the RFPCD apparatus is shown in Figure 1.

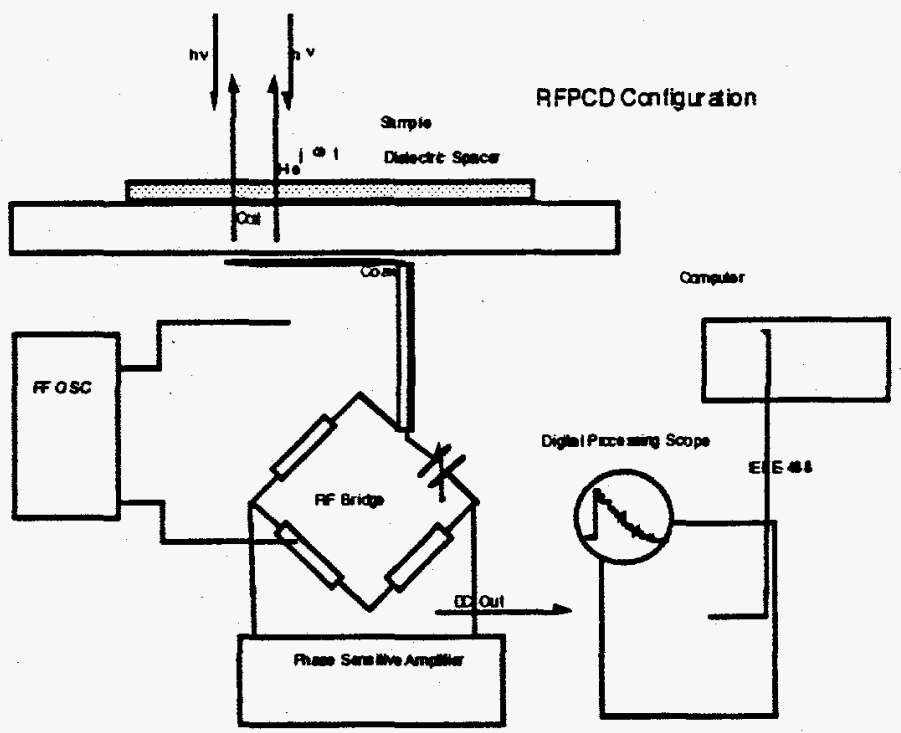

Figure 1. 
The system is a contactless technique based on a balanced VHF $(430 \mathrm{MHz})$ bridge. The bridge is unbalanced by excess carrier induced by a laser pulse. That excess conductivity is amplified and detected by a phase sensitive detector. That signal decays with the recombination or minority-carrier lifetime.

An example of measurements on $1 \mathrm{~mm}$ diameter silicon spheres are shown below in Figures 2 and 3. The data of Figure 1. comes from upgraded spheres with metallurgical grade silicon (MGS) as the starting material. The MGS materials is upgraded and purified by a series of cycles of melting and re-solidification.

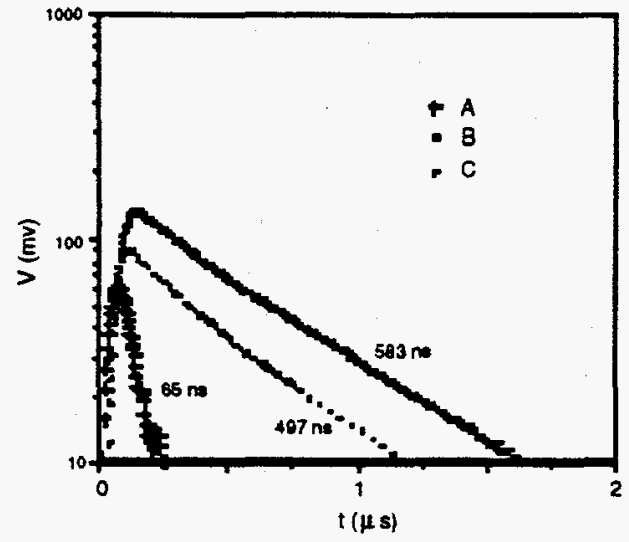

Fig. 2

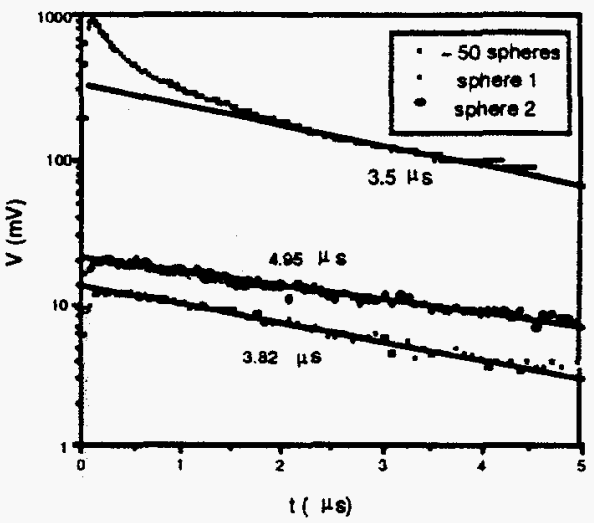

Fig. 3

Figure 1 data shows the RFPCD decay of a set of about 100 spheres from each set. Curve A shows the RFPCD decay of spheres that have undergone two melts followed by about 15 percent material removal after each melt. The lifetime here is $65 \mathrm{~ns}$ and solar cells fabricated from this material perform poorly. Curve B shows the RFPCD decay of the same material after five melts followed by 12 percent removal. There is a very striking lifetime improvement of about an order of magnitude. Finally, Curve $C$ shows the material after five melts followed by an oxygen denude process. There is again some improvement in lifetime and photoconductive response.

One of the changes in the Texas Instruments program was to switch to electronic grade silicon (EGS) which is much higher quality. Figure 3 shows some typical data from EGS grown spheres using two melting cycles. The sample size in Curve A is about 50 spheres and the injection level is quite low, about 10 to $20 \mathrm{~mJ}$ per pulse. The long term decay here is $3.5 \mu \mathrm{s}$ but the initial decay is about $1 \mu \mathrm{s}$. At the background doping is quite low here, the initial decay may be injection-Auger limited. This hypothesis is supported by a continuously varying lifetime rather than the two steep regimes seen in the trap controlled case. Decay times as long as $8.0 \mu \mathrm{s}$ are measured in some EGS samples. Because of the high sensitivity of this material, it was quite easy to measure the lifetime in single spheres. Data are shown in Curves B and C for two such spheres. The low injection lifetime of spheres $B$ and $C$ are 3.82 and $4.95 \mu \mathrm{s}$, respectively. This upgrade in materials pushed cell efficiency to above 11 percent AM1.5.

Detailed studies of recombination lifetimes in polycrystalline CdTe were also performed during the course of the year. These studies were performed on CdTe thin films at various stages of processing. This paper was presented at the first World Conference on Photovoltaic Energy Conversion[3] by Dean Levi. The paper was entitled: "Micro through Nanostructure Investigations of Polycrystalline CdTe: Correlation with Processing and Electronic Structures". The results are an extensive collaboration of in-house NREL researchers and outside subcontractors. The focus of this collaboration was to utilize NREL's characterization capabilities to delineate the evolution of the structural and electronic properties of CdTe as a function of processing steps. A key result of this research was the observation of a bandgap narrowing in the CdTe near the CdTe/CdS interface due to interdiffusion of $\mathrm{Te}$ and $\mathrm{S}$. This novel effect was observed using photoluminescence spectroscopy and confirmed using cross-sectional STM spectroscopy.

For the first time, TRPL data has been taken on polycrystalline CIGS samples 
supplied by in-house research. Although the luminescence was at the extreme limit of the detection equipment, it was possible to measure polycrystalline lifetimes that were above the system response of our detection system $(\sim 100 \mathrm{ps})$. The result indicates that useful information can be obtained on the CIGS composition thereby aiding development of the material.

Other highlights include time-resolve photoluminescence (TRPL) data was taken on MOCVD grown GaInP/GaAs double heterostructures grown at the Research Triangle Institute by R. Venkatasubramanian. This technique is providing direct feedback on the interface quality as a function of growth conditions. An extension of this work involved TRPL measurements of MOCVD grown GaAs. The sample structures were 6.5 $\mu \mathrm{m}$ double heterostructures (DHs) grown on Ge substrates of differing orientations and type. Initial results indicate that the crystallographic orientation of the Ge substrate can strongly influence the final DH material quality (lifetimes of $200 \mathrm{ps}$ vs. $7 \mathrm{~ns}$ ) [4].

We have successfully demonstrated the potential for determining the doping concentration in the heavily doped tunnel junction regions of GaInP solar cells using the technique of inelastic light scattering from plasmons. We have begun to examine the potential of using this technique to study the ability to passivate heavily doped GaAs using ultra thin ZnSe epilayers. For the purpose of this study we have established a collaboration with Henrik Temkin at Colorado State University who will provide us with samples of ultra thin $\mathrm{ZnSe}$ epilayersgrown on heavily doped GaAs substrates. The results are very exciting indicating that due to the large band gap difference between $\mathrm{ZnSe}$ and $\mathrm{GaAs}$ there is a region near the surface of $\mathrm{GaAs}$ that is depleted of carriers. This can be very easily seen in the light scattering spectrum which shows only bulk like LO and TO phonon modes from this depleted region but coupled LO phonon - plasmon modes from the undepleted region. Coordinated TRPL measurements studied the effects of passivation on minority carrier lifetime in the GaAs/ZnSe system.

One of the early applications of the RFPCD technique in the crystalline silicon program resulted from an interaction with Prof. A. Rohatagi's group at Georgia Tech. Recombination lifetime measurements, using the radio-frequency photoconductive decay technique (RFPCD), were performed on extensive series of EFG samples (ASE Americas) that were then processes by Georgia Tech Group. We are measuring the recombination lifetime as a function of the injection level in order to ascertain the concentrations of deep level impurities. These studies pointed toward efficient ways of gettering impurities from EFG materials and will be the subject of a future publication.

A CRADA was formulated by $T$. Ciszek involving high purity float zone silicon. Characterization contributions involved the analysis of trace impurites using both low temperature photoluminsecence and Fourier Transform infrared spectroscopy (FTIR). The resistivity of these samples ranged from 20,000 to $200,000 \Omega-\mathrm{cm}$ and the suspected impurities are $B$ and P. FTIR was able to detect the $316 \mathrm{~cm}^{-1} \mathrm{P}$ absorbance band in the $20,000 \Omega-\mathrm{cm}$ sample with a total concentration of $7 \times 10^{11} / \mathrm{cm}^{3} \mathrm{P}$ atoms. The theoretical resistivity of the sample should have been $7000 \Omega-\mathrm{cm}$, but the decrease in intensity of the $P$ absorbance band when the sample was analyzed in the dark suggests the presence of compensating impurities at lower concentrations. The $\mathrm{S} / \mathrm{N}$ was inadequate to detect these other impurities with the present apparatus. The near bandgap low temperature photoluminescence has recently produced spectra of bound excitons evolving form low densities of residual dopants. These two types of measurement will be the primary means of characterizing high purity materials.

Extensive lifetime measurements on InGaAs thin films were made for the first time. These were made possible because of the invention of the RFPCD technique. A paper was presented at the NREL sponsored Conference on Themophotovoltaic Technology which described these data. Lifetime measurements were made on both lattice-matched and lattice-mismatched InGaAs films grown on InP substrates. The latter were high dislocated despite grading layers to the substrate. The dislocation effects had a marked impact on the recombination lifetime. The recombination lifetimes of undoped lattice matched InGaAs/InP double heterostructures are shown in Fig. 4. The adverse effects of InP 


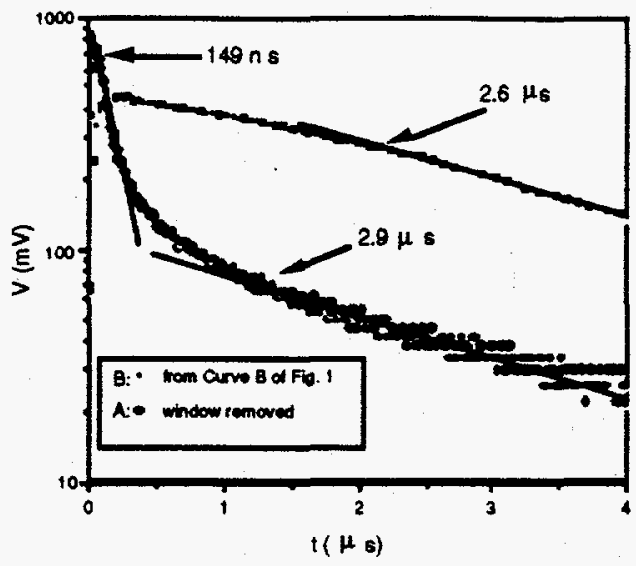

Fig. 4

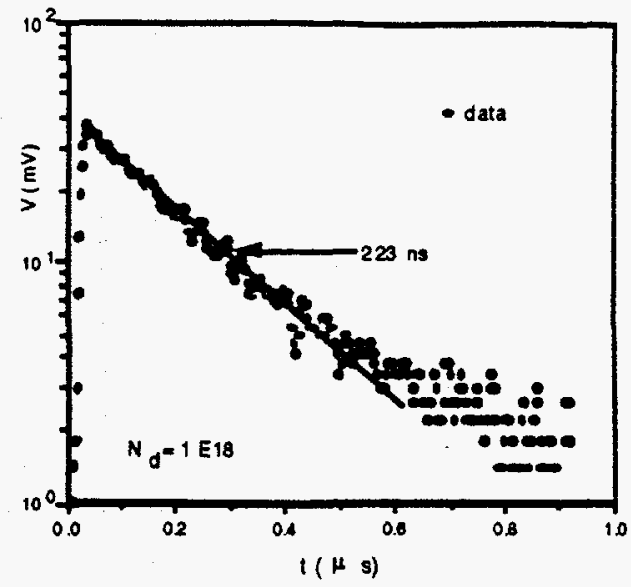

Fig. 5

To estimate the effects of dislocation activated recombination, an undoped film with $E_{g}=$ $0.5 \mathrm{eV}$ was grown on InP using a grading layer. These films had no confinement layers but the pulse energies were increased to values greater than those used in the data of Fig. 4. The RFPCD data from this film is shown in Fig. 5 and the signals are relatively weak. An initial lifetime of $52 \mathrm{~ns}$ is followed by a terminal lifetime somewhat longer. These data shown that dislocation reduction is one of the major goals of materials research for small bandgap InGaAs.

\section{Summary}

This was a productive year for the Electro-Optical Characterization Group despite moving the laboratories and changing programs direction. Beside a continuation of the more routine characterization work, a number of important innovations were implemented in the laboratory.

\section{References}

1. R. K. Ahrenkiel, D. Levi, and J. Arch, "Minority-carrier Lifetime Studies of Silicon Spheres", Proceedings of the WCPEC-1994 (in press).

2. J. K. Arch, J. S. Reynolds, M. D. Hammerbacher, R. K. Ahrenkiel, S. Asher, K. M, Jones, and M. Al-Jassim, "Characterization of Silicon Spheres for Spheral SolarTM Cells", Proceedings of the WCPEC-1994 (in press).

3. D. H. Levi, H. R. Moutinho, B. M. Keyes, R. K. Ahrenkiel, M. Al-Jassim, L. L. Kazmerski, and R. Birkmire, "Micro-Thorough Nanostructure Investigations of Polycrystalline CdTe: Correlations with Processing Electronic Structures", Proceedings of the WCPEC-1994 (in press).

4. R. Venkatasubramanian, D. P. Malta, M. L. Timmons, J. B. Posthill, J. A. Hutchby, R. K. Ahrenkiel , B. Keyes, and T. Wangensteen, "Material and Device Characterization Toward High-Efficiency GaAs Solar Cells on Optical-Grade Polycrystalline Ge Substrates", Proceedings of the WCPEC-1994 (inpress). 
Title:

Organization:

Contributors:

\section{GaInP/GaAs tandem solar cells}

\author{
National Renewable Energy Laboratory, Golden, CO
}

J. M. Olson, Principal Scientist

S. R. Kurtz, Senior Scientist

K. A. Bertness, Senior Scientist

D. J. Friedman, Staff Scientist

A. E. Kibbler, Staff Scientist

C. Kramer, Research Technician

J. Luo, Postdoctoral Scientist

D. J. Arent, Staff Scientist

\section{Objective}

Continue the development of the high-efficiency GaInP/GaAs multijunction cascade solar cell technology, for both 1-sun and concentrator illumination conditions.

\section{Approach}

We perform all aspects of the device development: design, growth, metallization, antireflection coating, and testing. The single-crystal devices are grown using organometallic vapor-phase epitaxy (OMVPE). Metallization is done using our own custom-designed grid patterns. Devices are tested in-house to extract the characteristics of the component cells of the tandem device, providing essential feedback to the iterative development process. ${ }^{1}$ Additional testing for verification of record efficiency results is done by independent laboratories at NREL and elsewhere.

The record-setting performance of the 1-sun device ${ }^{2}$ and its inherently low-current operation suggest that the GaInP/GaAs device should be readily adapted for high-efficiency operation under concentrated light. The results of preliminary work along these lines are discussed below.

\section{Results}

During the past year we completed the design/fabrication/testing cycle for a prototype concentrator device. ${ }^{3.4}$ This device demonstrated an efficiency exceeding $30 \%$ in the concentration range of 115 to 260 suns, making this the first two-terminal monolithic cell to exceed $30 \%$ efficiency.

Figure 1 shows a schematic cross-section of the device, as adapted from one-sun ${ }^{2}$ to concentrator operation. Devices are grown by atmospheric-pressure organometallic vapor-phase epitaxy. The front and back contacts are electroplated, unannealed gold. After the contacts are deposited, a mesa etch is used to define the device area. The contacting layer is then selectively etched away everywhere but under the contacts. After metallization, a $\mathrm{MgF}_{2} / \mathrm{ZnS}$ antireflectance (AR) coating is evaporated onto the devices.

Concentrator devices operate at high current densities; at 400 suns, even the inherently low-current $\mathrm{GaInP} / \mathrm{GaAs}$ tandem operates at about $5 \mathrm{amps} / \mathrm{cm}^{2}$. It is thus necessary in the design of the device to pay particular attention to the balance between resistive and grid shadowing losses. Contributions to the resistive loss which can be minimized by appropriate design of the front grids include top-cell emitter, grid fingers and bus bar, and grid contact resistances. The design of the front grids is discussed in more detail elsewhere. ${ }^{4}$ The prototype device, which is $3.4 \mathrm{~mm}$ square, has two bus bars, each $3.28 \mathrm{~mm}$ long with width tapered from $0.4 \mathrm{~mm}$ to $0 \mathrm{~mm}$ along the long 
dimension of the bus bar. The grid fingers are $3 \mu \mathrm{m}$ thick, $10 \mu \mathrm{m}$ wide, and are spaced $300 \mu \mathrm{m}$ apart, for a shadow loss of $3.2 \%$. The one-sun device, in comparison, has a grid finger spacing of $1320 \mu \mathrm{m}$. To further reduce the power loss due to lateral conduction in the top-cell emitter layer, this layer is made $0.2 \mu \mathrm{m}$ thick in the concentrator device, twice as thick as in the one-sun device, to reduce its sheet resistance. The prototype concentrator device has a top-cell emitter sheet resistance of $\sim 250 \Omega / \mathrm{sq}$. The grid fingers have a resistivity of $\sim 5 \times 10^{-6} \Omega-\mathrm{cm}$ and a front contact resistivity of $\sim 10^{-4} \Omega \mathrm{cm}^{2}$. With these parameters, we estimate that at 300 suns there is about a $3 \%$ power loss in the top-cell emitter, and a $2 \%$ power loss due to contact resistance and front metallization resistivity combined.

For the device structure of Fig. 1, the photocurrent generated by the tandem cell will be limited to the lesser of the photocurrents capable of being produced by the individual top and bottom subcells. Therefore, the thickness of the top cell is critical. Thinning the top cell decreases the amount of light it absorbs (and thus also increases that absorbed by the bottom cell), thereby decreasing the ratio of top to bottom cell photocurrents. The optimal top-cell thickness is the thickness at which the top and bottom cell photocurrents are equal. This thickness depends on the incident solar spectrum. Concentrators collect the direct normal spectrum, which lacks the blue-rich diffuse component of the global spectrum collected by one-sun devices. A typical tandem cell with a $0.7-\mu \mathrm{m}$-thick top cell, while current-matched under the standard AM1.5 global spectrum, is topcell-limited under the standard AM1.5 direct

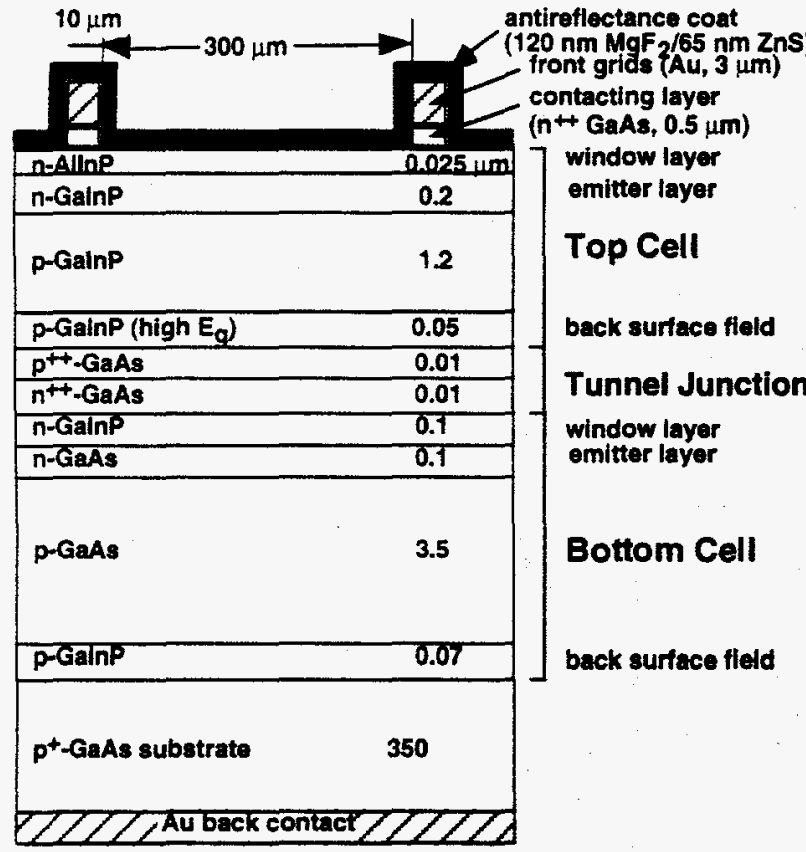

Figure 1. Simplified schematic cross-section of the NREL concentrator GaInP/GaAs tandem cell structure. The one-sun version of this device has a larger grid finger spacing and a thinner top-cell base and emitter layer, for reasons discussed in the paper. The schematic is not to scale. roughly 140-180 suns. The cell performance remains excellent at higher concentrations, showing an efficiency of $29.1 \%$ at a concentration of 425 suns. This cell is thus the first monolithic twoterminal device to demonstrate a verified efficiency exceeding $30 \% .^{5}$ The cell performance has room for further improvement and operation at higher concentration levels via a switch to evaporated front metallization, as well as fine-tuning of the emitter thickness and doping, AR coat, and grid-finger spacing. We anticipate that efficiencies greater than $31 \%$ at 1000 suns are achievable. which allows it to absorb more light at the expense of the bottom cell, current-matches the device for the direct spectrum.

A prototype device was designed and fabricated following the criteria just described. The device was measured under the ASTM standard AM1.5 direct spectrum. The one-sun performance was measured on a Spectrolab XT-10 solar simulator; appropriate spectral mismatch corrections were made using spectral response measurements for the individual cells in the tandem. The concentration-dependent measurements were done on a pulsed solar simulator with AM1.5 filter and additional spectral considerations. Figure 2 shows the dependence of open-circuit voltage $\left(V_{O C}\right)$, fill factor $(F F)$ and efficiency (Eff) on concentration. The concentration is determined by assuming linearity in $\mathrm{J}_{\mathrm{SC}}$. At one sun, the device has $\mathrm{V}_{\mathrm{OC}}=2.337 \mathrm{~V}$, short-circuit current $J_{S C}=13.0 \mathrm{~mA} / \mathrm{cm}^{2}$, and $F F=0.869$, for an efficiency of $26.4 \%$. The efficiency increases with concentration up to about 160 suns due to increases in $V_{O C}$ and FF. The device achieves over $30 \%$ efficiency at concentrations from 115 to 260 suns, peaking at $30.2 \%$ in a range of spectrum. Thickening the top cell to $1.5 \mu \mathrm{m}$, 


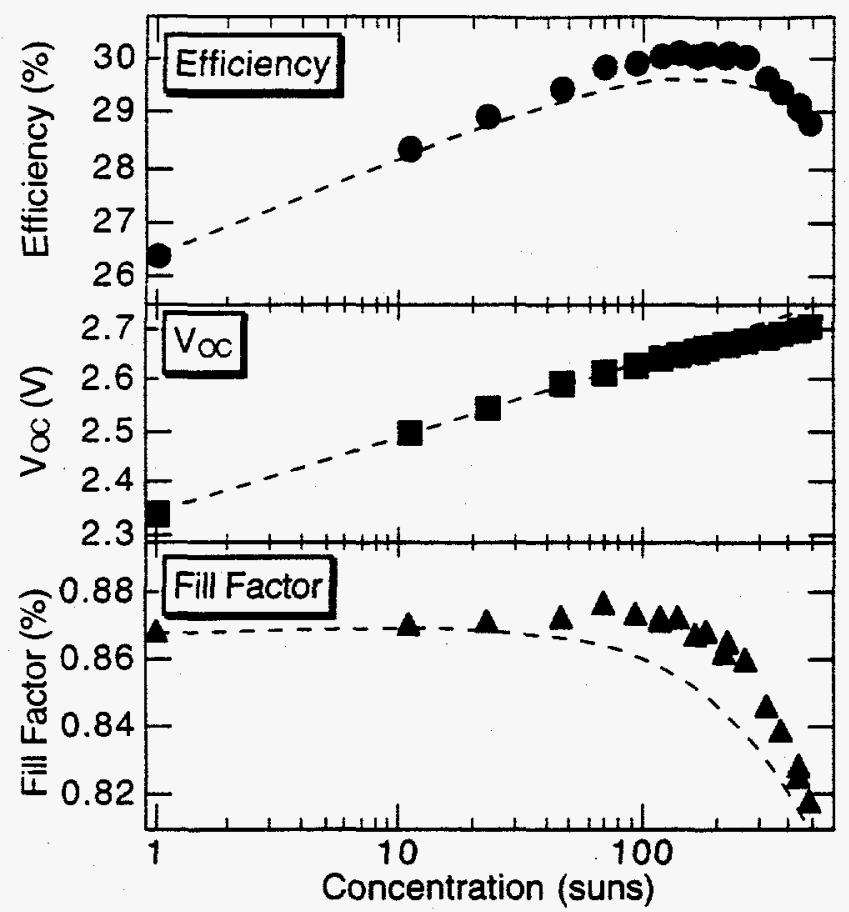

Cell performance as a function of concentration for the prototype concentrator device. The symbols are the measured data; the dashed curves are the modelled behavior. Measurements were made on a pulsed solar simulator at Sandia National Laboratories.
Over most of the concentration range, $V_{O C}$ shows the expected logarithmic dependence on $\mathrm{J}_{\mathrm{SC}}$, with an effective ideality factor of 2.5 . (For an idealized two-junction device the effective ideality factor would be 2.0.) At high concentrations, $V_{O C}$ starts to fall below the simple logarithmic prediction. There are a number of possible explanations for this deviation, including sample heating, Dember voltage, and bias voltage dependence of the dark current. The qualitative behavior of FF is also as expected: it initially rises with increasing concentration due to the exponential dependence of current on voltage, then starts to fall off as resistive losses in the grids and top-cell emitter layer become significant. Figure 2 compares the measured FF with a calculated FF extracted from I-V curves modelled by ideal diode curves for the top and bottom cells in series with an effective series resistance. The calculated FF curve is in qualitative agreement with the measurement, although not in quantitative agreement. The lack of precise agreement is attributed to the simplicity of the model.

\section{Other laboratory issues}

During the last year we moved our laboratory from its previous location in the Denver West site to its present location in the SERF. This tremendously time-consuming task, which is still ongoing, involves not only the moving of equipment but also a teardown and complete rebuild of our OMVPE reactor. This task has also involved much effort in debugging such services such as the SERF house hydrogen system.

\section{References}

1. S. R. Kurtz, K. Emery and J. M. Olson In $1^{s t}$ WCPEC; 1994; pp (in press).

2. K. A. Bertness, S. R. Kurtz, D. J. Friedman, A. E. Kibbler, C. Kramer and J. M. Olson, '29.5\%-efficient GaInP/GaAs tandem solar cells', Appl. Phys. Lett., 65, 989-991 (1994).

3. D. J. Friedman, S. R. Kurtz, K. A. Bertness, A. E. Kibbler, C. Kramer and J. M. Olson, ' $30.2 \%$ Efficient GaInP/GaAs Monolithic Two-Terminal Tandem Concentrator Cell', Progr. Photovolt., 3, 47-50 (1995).

4. D. J. Friedman, S. R. Kurtz, K. A. Bertness, A. E. Kibbler, C. Kramer, K. Emery, H. Field and J. M. Olson, 'Initial development of $\mathrm{GaInP}_{2} / \mathrm{GaAs}$ tandem concentrator cells', in AIP Conference Proceedings: Photovoltaic Advanced Research and Development Project, R. Noufi, Ed. (American Institute of Physics, New York, 1994) pp. 521-528.

5. M. A. Green and K. Emery, 'Solar cell efficiency tables (version 4)', Progr. Photovolt., 2, 231-234 (1994). 


\title{
Solid State Theory — In House
}

\author{
National Renewable Energy Laboratory \\ Golden Colorado
}

Alex Zunger (PI), S. H. Wei, S. Froyen

\section{Optical Properties of ordered $\mathbf{G a}_{x} \mathrm{In}_{1-x} \mathbf{P}$ Alloys [1-8] (S.-H. Wei and A. Zunger)}

The general features of ordering were recently reviewed in Refs. (1)-(2). Spontaneous ordering of $I I I-V$ alloys is known to cause a band gap reduction $\left|\Delta E_{g}\right|$ and a splitting $\Delta E_{12}$ of the valence band maximum. Strain also leads to a valence band splitting and a change in the band gap. We presented a general theory (Refs. 4-6) explaining how the strain produced by lattice mismatch with the substrate interacts with ordering effects. We find for (001) strain and (111) "CuPt" ordering that (i) atomic ordering removes the "cusp" in the band gap $v s$ strain curve of random alloys. (ii) Epitaxial strain always leads to an increase in the ordering-induced valence band splitting $\Delta E_{12}$ (iii) Atomic ordering reduces the slope of $\Delta E_{12} v s$ strain. (iv) While (a) strain, (b) ordering, and (c) clustering can all lead to a band gap reduction, we show here that the three effects can be partially distinguished on the basis of a $\Delta E_{12} v s\left|\Delta E_{g}\right|$ plot. (v) The wavefunctiontype at the valence band maximum (VBM) (and hence, the cause of the splitting) can be further determined by measuring the polarization dependence of the intensities of the transitions between the VBM split components and the conduction band minimum. We have derived a general formula (Ref. 7) relating the near band edge bulk reflectance difference spectroscopy (RDS) intensity to the degree of long range order. We used a full $6 \times 6$ Luttinger-type Hamiltonian which allows the coupling between ordering induced crystal field splitting and the spin-orbit splitting. We show that this coupling is important in determining the bulk RDS intensity near the band edge for $\mathrm{Ga}_{x} \mathrm{In}_{1-x} \mathrm{P}$ alloy due to its relatively small spin-orbit splitting and large crystal field splitting. We find that our calculated peak RDS intensity at $E=E_{g}$ agrees well with the experimental results of Luo et al. for $\mathrm{Ga}_{0.5} \mathrm{In}_{0.5} \mathrm{P}$. Our calculated peak bulk RDS intensity for $\mathrm{Ga}_{0.5} \mathrm{In}_{0.5}$ As is provided as prediction to be tested experimentally in the future. (vi) We predict that ordering can significantly enhance the degree of spin polarization of photoelectrons emitted from the VBM. Ordered III-V alloys can thus be used as a good polarized electron source. These general results open new avenues of band gap engineering by combining epitaxial strain with atomic ordering. We have also investigated theoretically the ordering-induced change of the $E_{1}, E_{2}$, and $E_{0}{ }^{\prime}$ transitions in $\mathrm{Ga}_{0.5} \mathrm{In}_{0.5} \mathrm{P}$ using symmetry arguments and first-principles band structure calculations (Ref. 8). We show that, as is the case in the fundamental band gap (the $E_{0}$ transition), (111) superlattice ordering causes significant splittings and shifts in the $E_{1}, E_{2}$, and $E_{0}{ }^{\prime}$ transitions. The new transition energies as function of ordering parameter $\eta$ are presented and compare well with recent experimental data. A microscopic explanation is given.

\section{Properties of Chalcopyrites and Related Compounds [3,9] (S.-H. Wei and A. Zunger)}

We have suggested different ways (e.g., alloying) to alter the material properties in chalcopyrite (and other) semiconductors in a review article (Ref. 3). Alloys of $\mathrm{CuInSe}_{2}$ (CIS) with either $\mathrm{CuGaSe}_{2}$ or with $\mathrm{CuInS}_{2}$ have been shown experimentally to be advantageous in increasing the efficiency of thin film CIS solar cells. However, while the properties of the end point materials are known, very little is known about the properties of their alloys, e.g. the amount by which the alloy gap differs from the average over the constituents ("bowing coefficients"). Our extensive 
literature search show that (i) optical bowing measurements are lacking for most Cu-III-VI alloys with $I I I=\mathrm{Al}, \mathrm{Ga}$, In and $V I=\mathrm{S}, \mathrm{Se}, \mathrm{Te}$. (ii) In those cases when data are available, the scatter between different measurements on the same alloy is very large, and (iii) the band offsets between the chalcopyrite constitutes (needed for device design) are not known. This lack of information can hinder systematic improvements of solar cell efficiency. Using first principles band structure theory we have calculated systematically (Ref. 9) the (i) alloy bowing coefficients, (ii) alloy mixing enthalpies and (iii) interfacial valence and conduction band offsets for

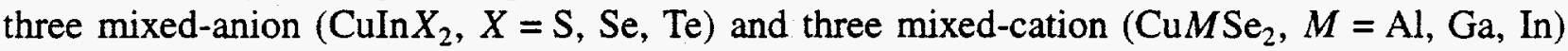
chalcopyrite systems. Our results are shown in Table I. The calculated bowing coefficients are in good agreement with the most reliable experimental data for stoichiometric alloys. Results for the mixing enthalpies and the band offsets are provided as predictions to be tested experimentally in the future. Comparing our calculated bowing and band offsets for the mixed-anion chalcopyrite alloys with those of the corresponding $\mathrm{Zn}$ chalcogenide alloys $(\mathrm{Zn} X, X=\mathrm{S}, \mathrm{Se}$, Te), we find that the larger $p-d$ coupling in chalcopyrite alloys reduces their band offsets and optical bowing. Bowing parameters for ordered, $\mathrm{Zn}$-based $I I-V I$ alloys in the $\mathrm{CuAu}, \mathrm{CuPt}$, and chalcopyrite structures are also presented: we find that ordered $\mathrm{Zn}_{2} \mathrm{SeTe}$ has a bowing coefficients of $1.44 \mathrm{eV}$ and $3.15 \mathrm{eV}$ in the $\mathrm{CuAu}$ and CuPt structures, while the random alloy $\mathrm{ZnSe}_{x} \mathrm{Te}_{1-x}$ has a bowing of $1.14 \mathrm{eV}$.

Table I. Calculated bowing parameters $b$, mixing enthalpies $\Delta H(x=1 / 2)$, valence band offset $\Delta E_{v}$ and conduction band offset $\Delta E_{C}$ for the six mixed-cation and mixed-anion chalcopyrites.

\begin{tabular}{lcccc}
\hline \hline \multicolumn{1}{c}{ Alloy } & $b(\mathrm{eV})$ & $\Delta H(\mathrm{meV} /$ atom $)$ & $\Delta E_{v}(\mathrm{eV})$ & $\Delta E_{c}(\mathrm{eV})$ \\
\hline $\mathrm{Cu}(\mathrm{Al}, \mathrm{Ga}) \mathrm{Se}_{2}$ & 0.39 & 0 & 0.22 & 0.77 \\
$\mathrm{Cu}(\mathrm{Al}, \mathrm{In}) \mathrm{Se}_{2}$ & 0.59 & 15 & 0.26 & 1.37 \\
$\mathrm{Cu}(\mathrm{Ga}, \mathrm{In}) \mathrm{Se}_{2}$ & 0.21 & 11 & 0.04 & 0.60 \\
$\mathrm{CuIn}(\mathrm{S}, \mathrm{Se})_{2}$ & 0.04 & 3 & 0.28 & 0.21 \\
$\mathrm{CuIn}(\mathrm{S}, \mathrm{Te})_{2}$ & 1.05 & 41 & 0.78 & 0.26 \\
$\mathrm{CuIn}(\mathrm{Se}, \mathrm{Te})_{2}$ & 0.44 & 13 & 0.50 & 0.47 \\
\hline \hline
\end{tabular}

\section{Surface structure of (001) GaAs [11,12] (S. Froyen and J. E. Northrup, Xerox PARC)}

Surface structure and surface reconstruction strongly influence the resulting material for $I I I-V$ semiconductors grown from gas phase constituents [usually on (001) surfaces]. The GaAs (001) surface is by far the most studied of the $I I I-V$ semiconductor surfaces, yet the detailed nature of its several surface reconstructions remain controversial. In collaboration with John E. Northrup at Xerox PARC, S. Froyen continued his studies of GaAs (001) surfaces. Based on (previous) total energy calculations, a simple electrostatic model was formulated that semi-quantitatively describes the energetics of many of the possible surface reconstructions. This electrostatic model lead Northrup and Froyen to reconsider a previously accepted reconstruction for As-rich surfaces (now found to be electrostatically unfavorable). Using first-principles pseudopotential calculations, a more stable surface reconstruction was subsequently demonstrated: the $\beta 2(2 \times 4)$ reconstruction, containing two As dimers in the top layer and a third As dimer in the third layer. This reconstruction is the analog of the Ga-terminated $\beta 2(4 \times 2)$ reconstruction but with the cations and anions swapped. Recent experiments by Hashizume et al. [Phys. Rev. Lett. 73, 2208 (1994)] appear to confirm the theoretical results. 
4. Surface-induced ordering and surface segregation of reconstructed GaxIn1-xP alloys [13] (S. Froyen and A. Zunger)

The structure of (001) III-V semiconductor surfaces is strongly dependent on the cation/anion ratio at the surface. The (001) GaAs surface, for instance, is observed to form a sequence of different reconstructions ranging from the $c(4 \times 4)$ and $(2 \times 4)$ As rich to the $(4 \times 2) / c(8 \times 2)$ Ga rich surface. (001) surfaces of other III-V materials are less well studied, but appear to show reconstructions similar to those of GaAs. For semiconductor alloy surfaces, in addition to the cation/anion ratio, the surface concentration of the alloying elements must be considered. The combination of surface reconstruction and surface alloying lead to two new features absent from the surfaces of binary zincblende semiconductors: surface segregation and atomic ordering.

By surface segregation of a given element, one means enrichment of that element at the surface with respect to the bulk composition. The effect has been extensively studied in metal alloys where thermodynamic equilibrium is easily attained and where the impact of segregation at grain boundaries on metal strength was recognized early. Over the past 10 years, surface segregation studies have also been performed for semiconductor alloys, and during the last few years the field has gained momentum because of its link to an inability to achieve abrupt interfaces in artificially grown $I I I-V$ superlattices and heterostructures. Specifically, when growing heterostructures containing two materials $A$ and $B$, the $A$-on- $B$ interface may be abrupt whereas the $B$-on- $A$ interface is diffuse. The non-abruptness of the second interface correlates with and is interpreted in terms of the surface segregation of material $A$. For technological reasons, most studies have focused on AlGaAs and GaInAs heterostructures using empirical thermodynamic models fit to experiment (formulated using a single exchange energy or coefficient).

Cation or anion sublattice CuPt-like order is observed in thick films for several pseudobinary III$V$ semiconductor alloys grown under certain conditions. The ordering process has been shown to occur at the surface as the material grows and can be observed directly using electron diffraction or indirectly through its effect on the band gap. Froyen and Zunger have previously studied the (001) gip alloy surfaces in the context of semiconductor ordering. They identified a $(2 \times 2)$ surface reconstruction where the cations would form a stable CuPt-like pattern, as a possible source for the ordering. The stability of this surface with respect to other types of surface reconstructions was not determined, however.

Here, Froyen and Zunger study the atomic structure and $T=0$ formation energies of the $\mathrm{Ga}_{x} \mathrm{In}_{1-x} \mathrm{P}(001)$ semiconductor alloy surface using the first principles pseudopotential method. They examine the $\beta 2(2 \times 4), \beta 2(4 \times 2), \beta(4 \times 2)$, and $(2 \times 2)$ reconstructions. These are stable or nearly stable surface structures for the GaAs (001) surface and will be used to derive candidate alloy surface structures by allowing the cations in the two uppermost layers to alloy. Froyen and Zunger find that size and chemical differences between $\mathrm{Ga}$ and In lead to dramatic differences in the properties of the alloy surface relative to the $\mathrm{GaAs}(001)$ surface and produces a surface stability diagram (constructed from the $T=0$ formation energy versus chemical potentials) that exhibit a strong asymmetry between $\mathrm{Ga}$ rich and In rich surfaces: Reconstructions that are unstable for GaAs surfaces such as the cation terminated $(2 \times 2)$ reconstruction, which promotes $\mathrm{CuPt}$ ordering in $\mathrm{Ga}_{x} \mathrm{In}_{1-x} \mathrm{P}$ alloys, are allowed for the alloy surface, and the partially cation terminated $\beta 2(4 \times 2)$ surface, which is stable for $\mathrm{GaAs}$ and $\mathrm{GaP}$, is unstable when the surface is In rich. 
Froyen has previously evaluated evaluated various methods for performing electronic structure calculations on large mesoscopic-sized semiconductor systems such as semiconductor heterostructures. Current first-principles methods typically use of the order of one hundred basis functions per atom and are therefore limited to systems containing of the order of one hundred atoms for a total of ten thousand basis functions. When used properly these very accurate methods can give us useful information about lattice properties and crystal stability in addition to the electronic structure. Much larger systems have traditionally been treated using approximate methods such as the k-dot-p based eight-band Luttinger model. This model relies on experiment to obtain the band structure (gap and and effective masses) of the constituents and makes assumptions/approximations (to some degree unverifyable) on the continuity of the basis functions and the envelope functions across material boundaries. Froyen has developed a first principles based method which can be seen as an extension of the Luttinger model and which removes any uncontrollable approximations. of the large system with little or no increase in the complexity of the calculation. The new method has the additional benefit of being able to handle smaller systems where the properties of the interfaces become important and where the traditional $\mathrm{k}$-dotp type models fail.

The new method is explicitly basis state based using states from both material $A$ and material $B$ (assuming here a two component heterostructure) including several bands and k-points, as necessary to obtain convergence. The Luttinger model uses a single set of basis states which are not specified explicitly, but are assumed to be eigenstates of the Hamiltonian both in region $A$ and $B$. The heterostructure Hamiltonian is constructed unit cell by unit cell using matrix elements calculated from small prototype systems. Initial tests of the method shows that it is accurate and fast once the matrix elements have been calculated.

The calculation of the literally millions of matrix elements currently form, however, a severe bottleneck to the usefulness of the method, and a matrix-element interpolation-scheme has been developed. In this scheme, a few high-symmetry-point matrix-elements and their derivatives up to second order are computed from small prototype systems. The derivatives are computed numerically using a wave-function phase locking scheme that was developed. The highsymmetry matrix-elements are then used to extrapolate to the matrix-elements needed for the much larger mesoscopic calculation. A problem with numerical errors (probably) caused by the over-completeness of the basis states remains. 
References:

(1) "Atomic Ordering and Phase-Separation in Epitaxial $I I I-V$ Alloys", A. Zunger and S. Mahajan, in Handbook of Semiconductors, Vol. 3, second edition, edited by S. Mahajan, (Elsevier, Amsterdam, 1994), pp. 1399-1513.

(2) "Structural Effects in Coherent Epitaxial Semiconductor films", A. Zunger, in Handbook of Crystal Growth, Vol. 3, edited by D. T. J. Hurle, (Elsevier, Amsterdam, 1994), p. 997.

(3) "Prediction of New Semiconductor and Transition Metal Structures and Their Properties”, A. Zunger, Jpn. J. Appl. Phys. Suppl. 32-33, 14 (1993).

(4) "Optical Properties of Zinc-Blende Semiconductor Alloys: effects of epitaxial strain and chemical ordering", S.-H. Wei and A. Zunger, Phys. Rev. B. 49, 14337 (1994).

(5) "Strain Effects on the Spectra of Spontaneously Ordered $\mathrm{Ga}_{x} \operatorname{In}_{1-x} \mathrm{P}$ ", S.-H. Wei and A. Zunger, Appl. Phys. Lett. 64, 757 (1994).

(6) "Optical Anisotropy and Spin Polarization in Ordered GaInP", S.-H. Wei and A. Zunger, Appl. Phys. Lett. 64, 1676 (1994).

(7) "Theory of Reflectance difference Spectroscopy in Ordered III-V Semiconductor Alloys", S.-H. Wei and A. Zunger, submitted to Phys. Rev. B.

(8) “ $E_{1}, E_{2}$, and $E_{0}$ ' Transitions in Ordered $\mathrm{Ga}_{0.5} \operatorname{In}_{0.5} \mathrm{P}$ ”, S.-H. Wei, A. Franceschetti, and A. Zunger, submitted to Phys. Rev. B.

(9) "Band Offset and Optical Bowing of Chalcopyrite and Zn-based II-VI Alloys", S.-H. Wei and A. Zunger, submitted to J. Appl. Phys.

(10) "Type-II $\rightarrow$ Type-I Transition in $(\mathrm{GaX})_{n}(\mathrm{InX})_{n}(001)$ Superlattices $(X=\mathrm{P}, \mathrm{Sb})$ as a Function of Period n", A. Franceschetti, S.-H. Wei, and A. Zunger, Phys. Rev. B (Rapid Communication) 50, 8094 (1994).

(11) "Structure of GaAs(100) surfaces: The role of electrostatic interactions," J. E. Northrup and S. Froyen, Phys. Rev. B 50, 2015 (1994).

(12) "Structure and thermodynamic stability of $\mathrm{GaAs}(100)$ surfaces," J. E. Northrup and S. Froyen, Materials Science and Engineering B (in press).

(13) "Surface segregation and ordering in GaInP," S. Froyen and A. Zunger, (in preparation). 


\section{Crystalline Silicon and Advanced Devices Subcontracts}





\title{
Title: Influence of Self-Interstitials Injected by Phosphorus Diffusion on Defect Structures and Electronic Properties in Crystalline Silicon
}

\author{
Organization: School of Engineering, Duke University, Durham, North Carolina \\ Contributors: T. Y. Tan and U. M. Göesele, principal investigators; \\ R. Gafiteanu and S. Joshi, graduate students
}

\section{Objective}

The overall objective is to identify means of effective gettering processes for improving crystalline Si minority carrier lifetimes so as to improve Si solar cell efficiencies. Presently the research focuses on two aspects. The first is to experimentally show the benefit of gettering of fast moving impurities in Si by a liquid Al-Si layer. Our experimental results demonstrated this benefit without the ambiguities involved in previous experiments for which many effects are present together. The second is to assess the effectiveness of a few different gettering methods and to understand the role of native point defect species in the gettering processes. To this end, gettering of the interstitialsubstitutional impurity Au by $\mathrm{P}$ indiffusion, by a liquid $\mathrm{Al}-\mathrm{Si}$ layer, and by the combination of the two methods, have been simulated. From the simulation results, the effectiveness of the different methods are ranked and the role of Si self-interstitials delineated.

\section{Gettering of Fast Moving Impurities in Si by Al}

The Si solar cell backside contact Al layer is known to be beneficial in improving solar cell efficiencies. The effects include a wafer backside field due to Al diffusion into Si to form a $\mathrm{p}^{+}-\mathrm{p}$ junction, formation of microscopic $\mathrm{p}^{+}-\mathrm{p}$ junctions around grain boundaries and dislocations by fast $\mathrm{Al}$ pipe diffusion, generation of atomic hydrogen at the Al-Si interface which passivates dislocations and grain boundaries, and gettering of metallic impurities [1]. These effects have not been individually delineated. We have conducted experiments [2] to unambiguously demonstrate the gettering effect of Al, which significantly improved the minority carrier diffusion lengths in Si crystals.

Dislocation-free, single crystal 4" (100) CZ Si wafers were used in our experiments. This ensures that passivation of dislocations or grain boundaries is not involved in our results. The Si wafer diffusion lengths were mapped using the ELYMAT technique before and after the Al treatment. The Al used was $99.9999 \%$ pure and deposited on only half of each wafer. The annealing temperature was $600-750^{\circ} \mathrm{C}$ for which a liquid $\mathrm{Al}-\mathrm{Si}$ layer forms. When annealed in nitrogen using a clean furnace, the diffusion lengths in the wafer halves covered by $\mathrm{Al}$ were substantially improved, while the diffusion lengths in the uncovered halves were also slightly improved. When annealed in a contaminated furnace, the Al covered wafer halves showed some diffusion length improvement, but the uncovered halves showed a significant diffusion length degradation. The ELYMAT measurement relies on the Schottky-like characteristics of the Si-HF electrolyte junction, and requires that the Si surface be p-type, not $\mathrm{p}^{+}$. After annealing, we have completely etched off the $\mathrm{p}^{+}$surface layer formed by Al indiffusion and have observed Si-electrolyte junction characteristics identical to those before Al deposition. This ensures that a wafer backsurface field did not exist. Clearly, the improvement in the diffusion lengths are due to gettering of metallic impurities from the bulk of the $\mathrm{Si}$ wafers to the Al layers during annealing, and the Al layer has also protected the wafers from furnace contamination. Since the gettering times are short $(30 \mathrm{~min})$ and temperatures are low, only fast-moving metals like $\mathrm{Fe}, \mathrm{Cu}$, Ni should have been gettered.

Following the first $\mathrm{Al}$ treatment, some wafers have received a second $\mathrm{Al}$ treatment at the same temperature. The second Al layer was also deposited over half the wafer, but with the wafer rotated by 90 degrees relative to the first $\mathrm{Al}$ deposition. This yields four wafer quadrants each of which received a different set of treatments. The quadrant received two anneals without Al showed the least 
diffusion length improvement, while the quadrant received two Al treatment showed the most improvement. The remaining two quadrants, each received one $\mathrm{Al}$ treatment and one anneal, showed intermediate improvement, see Fig. 1 for an example. This means that multiple Al gettering runs is more effective than a single gettering run with an Al volume equal to that of all the multiple gettering runs together, as has been suggested [3].

\section{$\mathrm{P}$ and $\mathrm{Al}$ Gettering in Si: Simulation and Optimization Considerations}

Compatible with the $\mathrm{Si}$ solar cell fabrication processes, wafer frontside $\mathrm{P}$ indiffusion and backside Al deposition can constitute as effective gettering methods for improving the cell efficiencies. These extrinsic gettering schemes are based on segregation-induced gettering or solution-stabilization mechanisms, for which the gettered impurity stabilization is due to the enhanced solubility of the impurity in the gettering regions. Up to now the gettering processes have not been satisfactorily explained on a quantitative basis. In previous simulations, only impurity diffusion have been treated quantitatively, but neither the process of impurity stabilization or removal at the gettering sites nor the role of the involved point defects has been accounted for. We have simulated the gettering processes by taking into account all these involved factors quantitatively.

Using our previously derived diffusion-segregation equation [3], we have simulated the $\mathrm{P}$ and $\mathrm{Al}$ gettering processes separately and simultaneously by considering both fast diffusers and substitutional-interstitial (s-i) impurities as the gettered species. Here we report that of the s-i species Au, because, for impurities and point defects, the processes of diffusion, segregation and stabilization, and mutual interactions are all involved. Au diffuses in Si via the migration of the interstitial species $\mathrm{Au}(\mathrm{i})$ and its changeover to become the substitutional species $\mathrm{Au}(\mathrm{s})$, but the Au(i) solubility is much lower than that of $\mathrm{Au}(\mathrm{s})$. During diffusion, a $\mathrm{Au}(\mathrm{i})$ atom can become a $\mathrm{Au}(\mathrm{s})$ atom by consuming a Si vacancy $V$ via the Frank-Turnbull (FT) mechanism, or by generating a Si self-interstitial $I$ via the kick-out (KO) mechanism. In the present study only the $\mathrm{KO}$ mechanism is assumed to be operative, because almost all Au experimental results are consistent with the $\mathrm{KO}$ mechanism, and because the presence of a high $I$ supersaturation induced by $\mathrm{P}$ indiffusion enhances the $\mathrm{KO}$ reaction but suppresses the FT reaction. The FT mechanism cannot be ruled out completely, it can be important close to the Si wafer surfaces, or when a large $I$ supersaturation does not exist in the $\mathrm{Si}$ wafer interior. Simulations with the FT mechanism fully considered are in progress.

The mathematical description of the Au gettering process by the different methods consists of a system of coupled nonlinear partial differential equations to account for the diffusion, segregation, and mutual interaction of all involved impurity and point defect species. This system of equations has been solved numerically using the software package ZOMBIE [4]. Details of the mathematical description, simulation conditions, and results have been reported [5].

Our simulation results showed that $P$ gettering possesses a greater gettering rate, because of the presence of an $I$ supersaturation induced by $\mathrm{P}$ indiffusion; while Al gettering possesses a greater gettering capacity and stability, because of the larger segregation coefficient of Au in the Al-Si-liquid/Si system, and because once gettered into the Al-Si liquid no Au atom will be further released as time progresses. The combined $\mathrm{P}$ and $\mathrm{Al}$ gettering has the advantages of both methods. For comparison purposes, we show in Fig. 2 the simulation results of the 2 hour gettering time cases for all three gettering schemes, as well as that for the Al gettering case with a gettering time of 7 hours. The $\mathrm{P}(2 \mathrm{~h})$ and the $\mathrm{Al}(2 \mathrm{~h})$ curves show that $\mathrm{P}$ gettering is faster. The $\mathrm{P}(2 \mathrm{~h})$ and the $\mathrm{Al}(7 \mathrm{~h})$ curves show that $\mathrm{Al}$ gettering is more stable and having a larger capacity, because, as time progresses, Al will getter more Au while P-gettering will loss Au due to the spreading of the P profile [5]. A comparison of the $\mathrm{P}(2 \mathrm{~h})$ and the $\mathrm{Al}(7 \mathrm{~h})$ curves with the $\mathrm{P}+\mathrm{Al}(2 \mathrm{~h})$ curve show that the gettering capacity of the combined $\mathrm{P}$ and $\mathrm{Al}$ gettering scheme is larger than that of the $\mathrm{P}$ indiffusion gettering scheme and that of the Al gettering scheme respectively by about two orders of magnitude and a few times. Clearly, the gettering rate and capacity of this scheme are the highest among all three schemes. Furthermore, the combined gettering scheme is as stable as Al gettering [5]. Thus, by all measures, the P+Al gettering scheme is the best one. Apparently, when the P indiffusion and 
Al gettering schemes are combined to work together, a synergistic effect exists for yielding the best result. This synergistic effect is on the one hand due to the supersaturation of $I$ produced by $P$ indiffusion which speeds up the gettering process by the Al layer, and on the other due to the very large and stable gettering capacity of the Al layer for $\mathrm{Au}$.

\section{Conclusions}

We have conducted experiments to show the benefit of gettering of fast moving impurities in Si by a liquid Al-Si layer. Our experimental results demonstrated this benefit without ambiguities involved in previous experiments for which many other effects existed simultaneous with the gettering effect. We have also simulated the process of gettering of the interstitial-substitutional impurity $\mathrm{Au}$ by $\mathrm{P}$ indiffusion, by a liquid $\mathrm{Al}-\mathrm{Si}$ layer, and by the combination of the two processes. From the simulation results, the effectiveness of the different methods are shown and the role of Si selfinterstitial delineated.

\section{References}

1. T. M. Bruton, A. Mitchell, L. Teale and J. Knobloch, "Maximising minority carrier lifetime in high efficiency screen printed silicon BSF cells" in Proceedings of the 10th European Photovoltaic Solar Energy Conference(Kluwer, Dordrecht, Netherlands, 1991) , p.667

2. S. M. Joshi, U. M. Gösele and T. Y. Tan, "Improvement of diffusion length in single crystal Si by Al gettering", accepted for J. Appl. Phy., tentative date 15 Apr 95

3. T. Y. Tan, R. Gafitsanu, and U. M. Gösele, "Diffusion-Segregation Equation and Simulation of the Diffusion-Segregation Phenomena" in Semiconductor Silicon 1994, eds. H. R. Huff, W. Bergholz, and K. Sumino (The Electrochem. Soc., Pennington, PA, 1994) p. 920 .

4. W. Jüngling, P. Pichler, S. Selberherr, E. Guerrero, and H. W. Pötzl, IEEE Trans. Electron. Devices ED-32, 156 (1985).

5. U. M. Gösele and T. Y. Tan, Second Annual Report, NREL Subcontract No. XD-2-110041 (1994).

\section{Figure Captions}

Fig. 1 Diffusion length map of Si wafer annealed twice at $600^{\circ} \mathrm{C}$ with an Al layer covering half the backside of the wafer each time. For the first anneal, the Al layer covered the lower half of the wafer, and for the second anneal, the Al layer covered the right half of the wafer. The lower right quadrant was $\mathrm{Al}$ treated twice and showed a net improvement in average diffusion length of $74.3 \mu \mathrm{m}$ over the original average of $151.3 \mu \mathrm{m}$. The upper left quadrant was annealed twice without $\mathrm{Al}$ and showed an improvement of $24.9 \mu \mathrm{m}$ from $132.3 \mu \mathrm{m}$. The upper right and lower left quadrants were Al treated once and annealed once, and showed improvements of $64.2 \mu \mathrm{m}$ and $50.7 \mu \mathrm{m}$ over the original 144.1 $\mu \mathrm{m}$ and $140.8 \mu \mathrm{m}$ respectively, most of which $(44.1 \mu \mathrm{m}$ and $41.6 \mu \mathrm{m}$ respectively) was obtained in the Al treatment step.

Fig. 2. Simulated $\mathrm{Au}$ concentrations after a gettering treatment by (i) $\mathbf{P}$ indiffusion gettering for 2 hours, the curve marked P (2h); (ii) Al gettering for 2 and 7 hours, respectively the curves marked $\mathrm{Al}(2 \mathrm{~h})$ and $\mathrm{Al}(7 \mathrm{~h})$; and (iii) combined $\mathrm{P}$ and $\mathrm{Al}$ gettering, the curve marked P+Al (2h). 

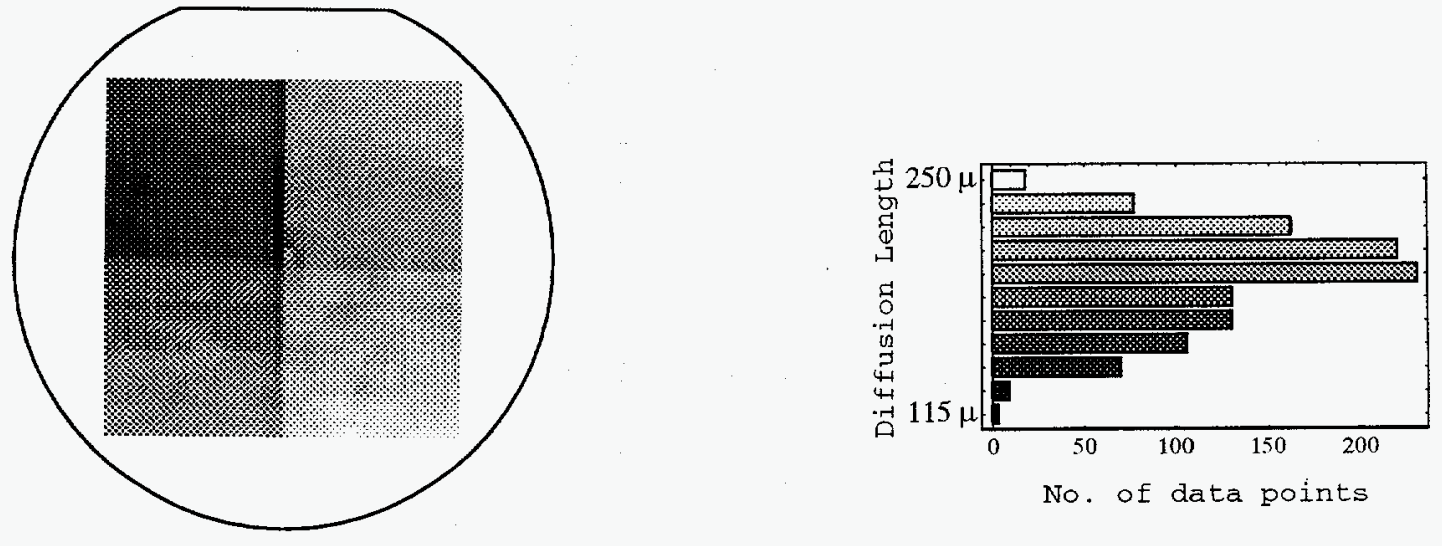

Figure 1

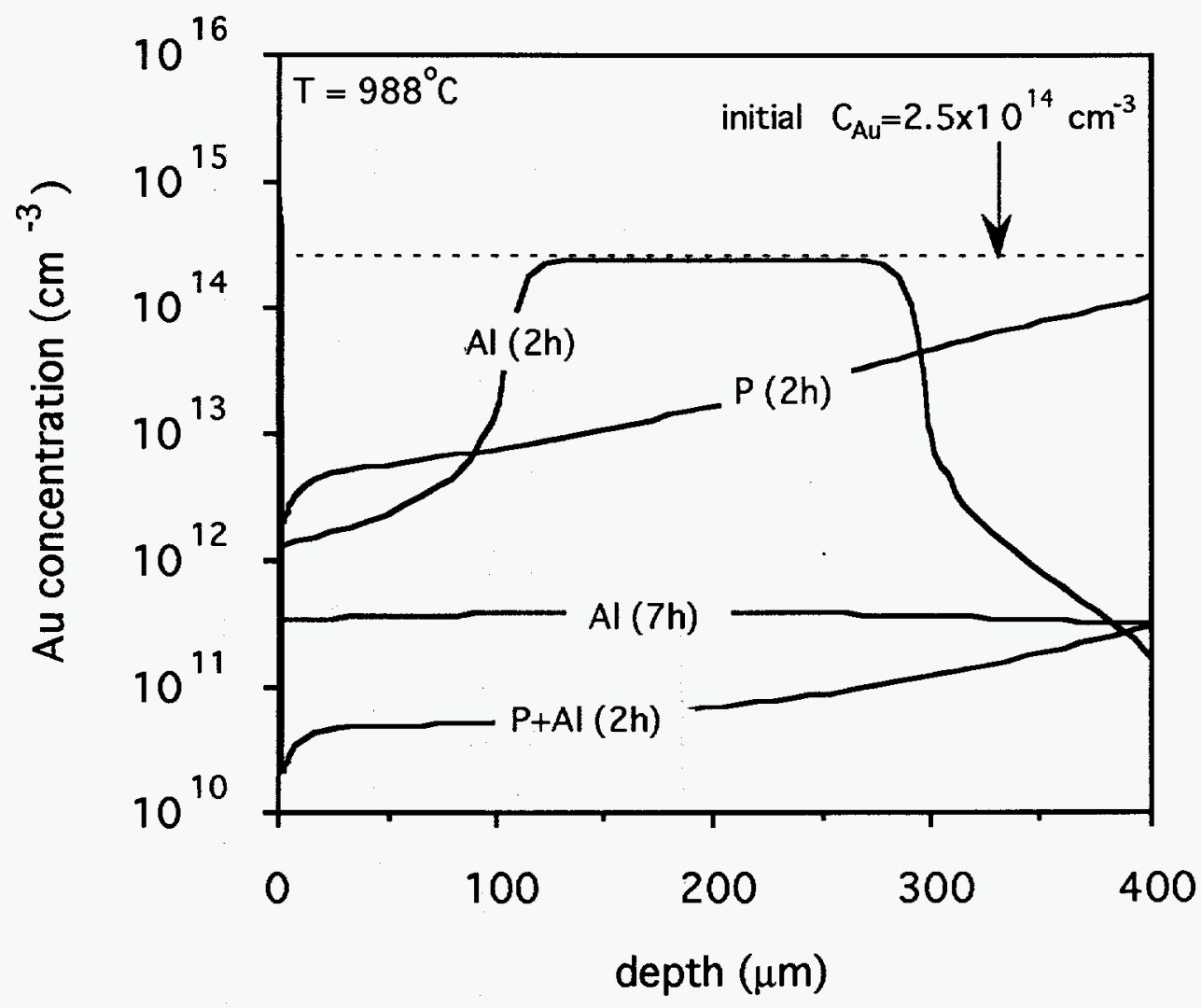

Figure 2 
Title:

Organization:

Contributors:
Fundamental Research on Post-Growth Quality Enhancement Techniques

School of Electrical and Computer Engineering Georgia Institute of Technology, Atlanta, Georgia

A. Rohatgi, director; P. Sana, graduate research assistant; and $Z$. Chen, research engineer.

\section{Objective}

The objective of this program is to investigate quality enhancement techniques, such as PECVD $\mathrm{SiO}_{2} / \mathrm{SiN}$ deposition and forming gas anneal (FGA) for defect passivation, and Al treatment for defect and impurity gettering.

Bulk and Surface Passivation of Multicrystalline Silicon Materials by $\mathrm{PECVD} \mathrm{SiO}_{2}$ and $\mathrm{SiN}$ Coatings

Three PECVD passivation schemes were investigated: (a) $100 \AA \mathrm{SiO}_{2}$; (b) $600 \AA \mathrm{SiN}$; and (c) $100 \AA \mathrm{SiO}_{2} / 600 \AA \mathrm{SiN}$. In order to determine the best passivation scheme, experiments were conducted on high resistivity $(-500 \mathrm{ohm}-\mathrm{cm}) \mathrm{FZ} \mathrm{Si}$ with very high bulk lifetime $\left(\tau_{\mathrm{b}}>15 \mathrm{~ms}\right)$. Photoconductive decay lifetime measurements were performed after the PECVD passivation for the three passivation schemes. It can be seen that the $100 \AA \mathrm{SiO}_{2} / 600 \AA \mathrm{SiN}$ double layer passivation gives the highest effective lifetime $(\sim 10 \mathrm{~ms})$ and lowest surface recombination velocity $(\mathrm{S}<1 \mathrm{~cm} / \mathrm{s})$. Such low $\mathrm{S}$ value results from the combination of excellent $\mathrm{SiO}_{2} / \mathrm{Si}$ interface and high positive charge density in SiN. (1)

\section{Effect of PECVD Passivation on Multicrystalline Silicon Cell Performance}

We demonstrated that PECVD films can passivate bulk defects and phosphorus diffused emitters in $\mathrm{n}^{+} \mathrm{pp}^{+}$multicrystalline solar cells. Internal quantum efficiency (IQE) measurements were performed on solar cells fabricated on different multicrystalline materials, including HEM, EFG, and Sitix wafers. Figs. 1 and 2 show the change in IQE of the EFG and HEM cells, before and after PECVD oxide/nitride deposition, with and without post-deposition photo-anneal. It was found that IQE decreases in the short wavelength range after PECVD the deposition, however, this damage was annealed out by a photo-assisted anneal in forming gas. The IQE in the short wavelength range is improved for both EFG and HEM cells after the $350^{\circ} \mathrm{C} / 20$ min, photo-anneal. However, IQE in the long wavelength range showed improvement only in the EFG cells. These results suggest that surface recombination is reduced by the PECVD deposition followed by photo-anneal in both the cells, but bulk defect passivation is observed only in the EFG cells.

\section{Effect of Al Treatment on LBIC Response of Multicrystalline Cells}

In order to investigate the effects of Al gettering, $\mathrm{n}^{+} \mathrm{p}-\mathrm{p}^{+}$solar cells were fabricated on $0.8 \Omega-\mathrm{cm}$ cast multicrystalline silicon from Sitix using neighboring wafers with similar microstructure. Light beam induced current (LBIC) measurements were performed on Sitix cells to investigate 
if any bulk diffusion length enhancement results from Al-gettering inside the grains or at the grain boundary. Samples covered with $1-\mu \mathrm{m}$ thick $\mathrm{Al}$ on back-side followed by $850^{\circ} \mathrm{C}$ drive-in were processed as solar cell. They were divided in four groups: no Al gettering, 1-min., 35min., and 120-min. Al drive-in, respectively. LBIC measurements were performed in a region with same microstructure on all four samples. The light spot size was $125 \mu \mathrm{m}$ for LBIC-map and $25 \mu \mathrm{m}$ for the scans over grain boundary to obtain a higher resolution. To generate carriers deeper in the bulk, light with wavelength of $975 \mathrm{~nm}$ was used. The LBIC maps for these four samples clearly showed that LBIC response is significantly enhanced by longer Al gettering time, with strong enhancement within the grains but not much change at grain boundaries.

\section{Effect of Al Gettering on Cell Performance}

In order to conduct a controlled investigation of $\mathrm{Al}$ gettering, cells were fabricated with one half of each wafer covered with Al on the back side (Al-diffused cells with BSF) while the other half was kept bare (Al-sintered cells with no BSF) during the $850^{\circ} \mathrm{C} / 35 \mathrm{~min}$. drive-in. All the cells were characterized by light and dark I-V and spectral response measurements. PC-ID device modeling was performed in selected instances to understand the multiple effects of Al treatment on cells. Table 1 shows a comparison of cell data for the Al-diffused and Al-sintered Sitix cast multicrystalline and EFG sheet Si cells. Cast multicrystalline cells showed an appreciable increase in the effective diffusion length from 195 to $261 \mu \mathrm{m}$ and a corresponding absolute cell efficiency improvement of $1.4 \%$ due to the Al process, Table 1 . Model calculations showed that in the $400 \mu \mathrm{m}$ thick Sitix polysilicon cells with $195 \mu \mathrm{m}$ diffusion length, Al BSF can only produce an efficiency improvement of $\sim 0.3 \%$. Model calculations also showed that the observed $1.4 \%$ increase in cell efficiency in this case results from the multiple effects of Al treatment. In addition to the BSF and gettering effects, Al treatment roughens the back surface which reduces the back surface reflectance and introduces some light trapping. Detailed cell analysis showed a reduction in BSR from $87 \%$ to $75 \%$ and path length enhancement by a factor of 1.4 due to back texturing in these cells. Model calculations revealed that Al treatment increased $\mathrm{J}_{\mathrm{sc}}$ by 0.5 $\mathrm{mA} / \mathrm{cm}^{2}$ due to $B S F$, decreased $J_{s c}$ by $0.1 \mathrm{~mA} / \mathrm{cm}^{2}$ due to reduced $B S R$, and increased $J_{s c}$ by 1.6 $\mathrm{mA} / \mathrm{cm}^{2}$ due to lifetime enhancement and light trapping, accounting for the observed increase of $2.1 \mathrm{~mA} / \mathrm{cm}^{2}$ in $\mathrm{J}_{\mathrm{sc}}$ in these cells.

\section{Defect Passivation by Forming Gas Anneal in Multicrystalline Si Cells}

Cell fabrication involved two forming gas treatments at $400^{\circ} \mathrm{C}$, one directly after the $850^{\circ} \mathrm{C} \mathrm{Al}$ drive-in step and the other after the Al back contact deposition. This was done intentionally to enhance the beneficial effect of FGA, if phosphorus diffused region, Al metal or $\mathrm{Al} / \mathrm{Si}$ alloy layers can generate atomic hydrogen by interacting with the $10 \%$ molecular $\mathrm{H}_{2}$ in the forming gas. Sintered Sitix multicrystalline cells showed no change in cell performance when both first and second forming gas anneals were replaced by nitrogen anneals. However, EFG cells showed a very significant effect of FGA. In order to investigate and decouple the effects of Al gettering and FGA passivation, we fabricated 4 kinds of cells on EFG materials, which is shown to respond quite favorably to hydrogen passivation. Fig. 3 shows that incorporation of $\mathrm{Al}$ gettering treatment and first and second FGA for defect passivation, resulted in efficiency of $13.8 \%$ to $14.1 \%$, which is comparable to good EFG cells made by intentional hydrogen ion implantation. If the $\mathrm{Al}$ diffusion is eliminated by fabricating $\mathrm{Al}$ sintered cells, then a significant drop in IQE 
and cell efficiency, from $13.8 \%$ to $12.4 \%$ is observed. This indicates that Al-induced gettering alone improves the EFG cell efficiency by $\sim 1.5 \%$, which is greater than what was observed in the case of cast material. This is not surprising since the EFG material has more grown-in defects and impurities. Fig. 4 shows that if the Al or alloyed Al layer interaction with forming gas is turned off by replacing the second FGA by nitrogen anneal in the case of sintered cells, then the IQE drops further and the EFG cell efficiency decreased from $12.4 \%$ to $11.2 \%$. This suggests that $\mathrm{Al}$ or $\mathrm{Al}$-diffused region/FGA interaction leads to some hydrogenation or defect passivation in the EFG material. Finally if both the $400^{\circ} \mathrm{C}$ FGA anneals are replaced by nitrogen anneals then the EFG cell efficiency drops significantly to $8.6 \%$, which indicates that the first FGA alone does significant defect passivation in the EFG material. Thus unlike the Sitix multicrystalline silicon, EFG material not only benefits from Al gettering but also from FGA-induced defect passivation (2). In order to prove that the FGA-induced improvement is related to hydrogenation, after the $400^{\circ} \mathrm{C} / 2 \mathrm{~h} \mathrm{FGA}$, the temperature was raised to $600^{\circ} \mathrm{C}$ and the ambient gas was switched to nitrogen to drive out the incorporated hydrogen. Indeed it was found that the cell efficiency decreased from $14 \%$ to $10 \%$, suggesting that hydrogen passivation is the most likely reason for FGA effect.

\section{$17.8 \%$ Efficient Solar Cells on Sitix Multicrystalline Si Material}

Oxide passivated solar cells were fabricated on Sitix cast multicrystalline silicon with $100 \AA$ thermal oxide passivation and $\mathrm{ZnS} / \mathrm{MgF}_{2}$ two layer $\mathrm{AR}$ coating. These cells went through the $\mathrm{Al}$ and phosphorus gettering as well as forming gas anneal but did not have PECVD coatings. We are conducting the study on the comparison between PECVD and thermal passivation, but not completed yet. The detailed process sequence has been published elsewhere (3). Table 2 and Fig. 6 show that the optimized process sequence resulted in a record high efficiency of $17.8 \%$ for multicrystalline cell (verified by Sandia) on $0.8 \Omega-\mathrm{cm}$ Sitix multicrystalline silicon with $\mathrm{V}_{\text {oc }}$ of $628 \mathrm{mV}$ and $J_{\mathrm{sc}}$ of $36.2 \mathrm{~mA} / \mathrm{cm}^{2}$. Dark I-V analysis gave a reserve saturation current density of $1.33 \times 10^{-12} \mathrm{~A} / \mathrm{cm}^{2}$ and a series resistance of $0.45 \Omega-\mathrm{cm}^{2}$. Open circuit voltage decay (OCVD) lifetime measurements gave effective carrier lifetime of $32 \mu \mathrm{sec}$ measured at $V_{o c}$, which is quite respectable for such low resistivity multicrystalline silicon. Fig. 4 also shows the reflectance and internal quantum efficiency of the $17.8 \%$ efficient cell.

\section{References}

1. Z. Chen, K, Yasutake, A. Doolittle, and A. Rohatgi, Appl. Phys. Lett., 63 (1993) 2117.

2. P. Sana, A. Rohatgi, J. P. Kalejs, and R.O. Bell, Appl. Phys. Lett., 1 (1994) 64.

3. P. Sana, J. Salami, and A. Rohatgi, IEEE Trans. Electron Devices, ED-40 (1993) 1461. 


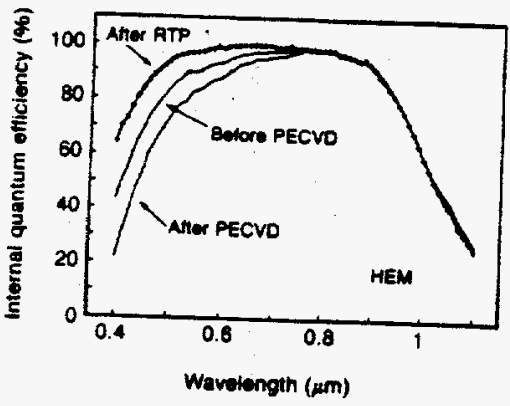

Fig. 1 The effect of the PECVD coating and photo-anneal in $\mathrm{N}_{2}$ and forming gas ambient on IQE solar celis fabricated on EFG sheet $\mathrm{Si}$

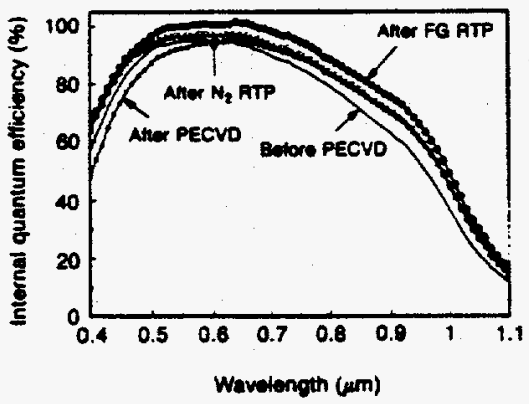

Fig. 2 The effect of the PECVD coating and post-deposition anneal on IQE of solar cells fabricaled on HEM Si material
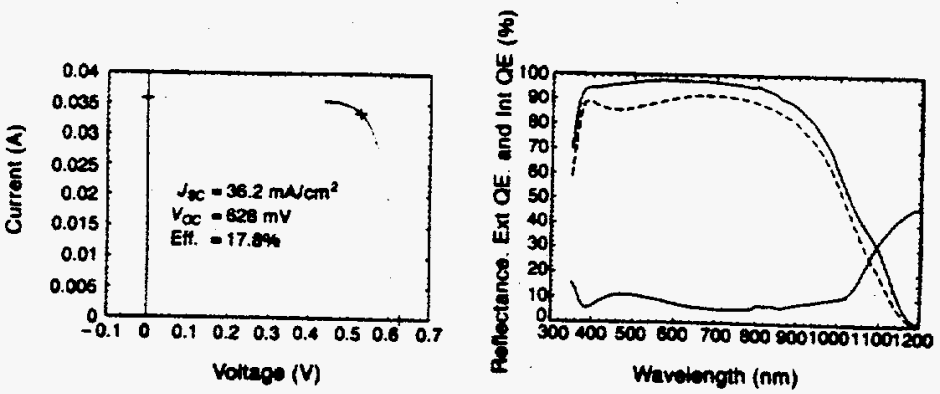

Fig. 4 The IQE and surface reflectance of record-high efficiency multicrystalline Sitix solar cell

\begin{tabular}{|c|c|c|c|c|c|c|c|c|}
\hline & \multicolumn{4}{|c|}{ Ahroinum diffused and gettered } & \multicolumn{4}{|c|}{ Auminam sintered whth no Al getseriag } \\
\hline & $\underset{(m V)}{V_{\omega}}$ & $\underset{\left(m \wedge / \mathrm{cm}^{2}\right)}{J_{x}}$ & $\begin{array}{c}\text { Efficiency } \\
(\%)\end{array}$ & $\begin{array}{l}\text { Diff. leogth } \\
(u \mathrm{~m})\end{array}$ & $(\mathrm{mV})$ & $\left(\mathrm{m} N / \mathrm{cm}^{2}\right)$ & $\begin{array}{c}\text { Bffeexy } \\
(\%)\end{array}$ & $\begin{array}{c}\text { Dif. lengt } \\
\text { (um) }\end{array}$ \\
\hline OTC & 628 & 36.2 & 17.8 & 261 & 618 & 34.1 & 16.4 & 195 \\
\hline EFG & 569 & 31.9 & 14.1 & 187 & 550 & 30.0 & 12.4 & 168 \\
\hline
\end{tabular}

Table 1. Effect of aluminum treatment on multicrystalline silicon cells

\begin{tabular}{cccccc}
\hline \hline Cell ID & $J_{m}\left(\mathrm{~mA} / \mathrm{cm}^{2}\right)$ & $V_{*}(\mathrm{mV})$ & FF & Enivieacy $(\%)$ & Measured at \\
\hline OPS-21 & 36.2 & 628 & 0.785 & 17.8 & Sandia \\
OPS-31 & 36.0 & 626 & 0.783 & 17.6 & Georgin Tech \\
OPS-13 & 35.7 & 626 & 0.783 & 17.5 & Sandia \\
OPS-12 & 35.6 & 625 & 0.782 & 17.4 & Georgin Tech \\
OPS-11 & 35.6 & 625 & 0.781 & 17.4 & Georgin Tech \\
OPS-22 & 35.4 & 621 & 0.781 & 17.2 & Georgin Tech \\
OPS-32 & 35.4 & 622 & 0.771 & 17.0 & Georgin Tech \\
OPS-33 & 35.4 & 621 & 0.768 & 16.9 & Georgia Tech \\
OPS-23 & 35.8 & 626 & 0.750 & 16.8 & Sandia \\
\hline \hline
\end{tabular}

Fig. 3 The effects of Al treatment and forming gas anneal on IQE of EFG solar cells

Table 2. Cell efficiency distribution on the wafer producing the $17.8 \%$ efficient cell 
Title: Solar Energy Conversion at Dye Sensitized Nanostructured

\section{Electrodes Fabricated by Sol-Gel Processing}

Organization: The Johns Hopkins University, Baltimore, MD 21218

Contributors: P. C. Searson and G. J. Meyer, Principle Investigators

F. Cao, T.A. Heimer, J.M. Stipkala, M. Bickson, L. Friedman, G. Oskam.

\section{Objectives}

In this program we are exploring the use of nanostructured materials as substrates for dyemediated light-to-electricity conversion in a photoelectrochemical cell. The objective of current work is to develop new materials, electrolytes, and molecular sensitizers which increase solar energy efficiency and stability. Materials processing and synthesis is carried out in conjunction with characterization of cell performance, detailed investigation of operating mechanisms, and modeling of charge transfer and transport in these systems.

\section{Results}

We have evaluated techniques for the fabrication and characterization of nanostructured films [1]. A colloidal suspension of nanometer size $\mathrm{TiO}_{2}$ particles is applied to a conducting glass substrate and subsequently sintered. We have evaluated $\mathrm{TiO}_{2}$ powders (anatase and rutile) from commercially available sources (Degussa, Johnson-Matthey, SCM) and the influence of sintering conditions. The position of the band edges and surface states, and the potential distribution at the interfaces are important in determining cell performance and efficiency. We have combined cyclic voltammetry, absorption measurements, EPR, and electrochemical impedance spectroscopy in order to analyze the potential distribution in porous nanostructured electrodes [2].

Nanostructured $\mathrm{TiO}_{2}$ films prepared from Degussa powders, are sufficiently transparent that optical measurements were performed in a transmission mode. The electronic properties of the films are dominated by a fundamental absorption in the ultra-violet region. The films are electrochromic and become uniformly black with negative applied bias. The transparency of the films can be restored by shifting the potential positive. The absorbance is related to the electrochemical process associated with the reversible trapping of an electron at Ti(IV) sites. Electron paramagnetic resonance measurements (EPR) on the electrochemically reduced films at $77 \mathrm{~K}$ reveal a sharp absorption with $\mathrm{g}=1.903$, consistent with literature values for $\mathrm{Ti}(\mathrm{III})$ surface sites. The spectroscopic and electrochemical data are more consistent with the presence of trapped electrons than free carriers.

We have prepared two families of sensitizers based on $\mathrm{Ru}(\mathrm{II})$ and $\mathrm{Os}(\mathrm{II})$ compounds for sensitization of the nanostructured $\mathrm{TiO}_{2}$ films [3,4]. The sensitizers utilize the chromophoric ligands 5,5'- $\left(\mathrm{CO}_{2} \mathrm{H}\right)_{2}-2,2^{\prime}$-bipyridine, abbreviated 5,5' $(\mathrm{LL})$ and 4,4' $-\left(\mathrm{CO}_{2} \mathrm{H}\right)_{2}-2,2^{\prime}-$ bipyridine, $4,4^{\prime}-(\mathrm{LL})$. The $\pi^{*}$ acceptor levels of the 5,5'-(LL) ligand are approximately 100 


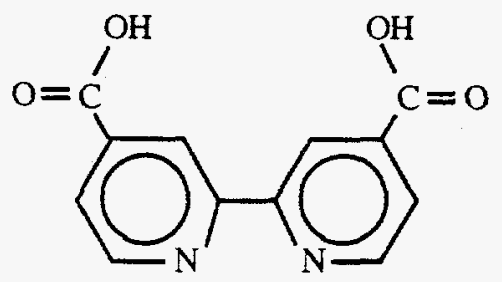

$4,4^{\prime}-(\mathrm{LL})$

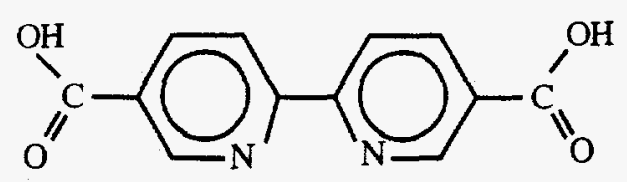

$5,5^{\prime}-(\mathrm{LL})$

$\mathrm{mV}$ below that of $4,4^{\prime}-(\mathrm{LL})$. Ruthenium polypyridyl compounds, cis-(LL) ${ }_{2} \mathrm{Ru}(\mathrm{X})_{2}$ where $\mathrm{X}$ $=\mathrm{Cl}^{-}, \mathrm{CN}^{-}$, and $\mathrm{SCN}^{-}$, have been prepared, spectroscopically characterized, and anchored to high surface area $\mathrm{TiO}_{2}$ electrodes described above. Vibrational studies reveal a surface ester linkage and indicate that the sensitizers bind to $\mathrm{TiO}_{2}$ through a distribution of interfacial interactions in a similar manner. When operating in a photoelectrochemical cell these materials convert visible photons into electrons. Figure 1 presents the incident-photon-to-current (IPCE) ratio for the sensitizers in $0.1 \mathrm{M} \mathrm{I}^{-}$/propylene carbonate electrolyte. Transition metal sensitizers based on [5,5'-(LL)] generally enhance photon-to-current efficiencies at low photon energies but at the expense of lower efficiencies at higher energies. Photoanodes based on cis-[(4,4'$\mathrm{LL})]_{2} \mathrm{Ru}(\mathrm{NCS})_{2}$ are the most efficient under simulated sunlight.

The observation of an electric field dependent luminescence from surface anchored sensitizers suggests that radiative and non-radiative recombination of the dye competes with electron injection into the conduction band in some cases. To gain insight into the rate of interfacial electron injection we have recently performed picosecond photoluminescence studies of cis- $\left[\left(4,4^{\prime}-\mathrm{LL}\right)\right]_{2} \mathrm{Ru}(\mathrm{NCS})_{2}$ and cis- $\left[\left(4,4^{\prime}-\mathrm{LL}\right)\right]_{2} \mathrm{Ru}(\mathrm{CN})_{2}$ anchored to $\mathrm{TiO}_{2}$ and $\mathrm{ZrO}_{2}$. The observed photoluminescence decays are kinetically complex. Modeling studies are consistent with the presence of an underlying skewed distribution of interfacial electron injection rates with maximum amplitude at $\sim 10^{8} \mathrm{~s}^{-1}[5]$.

In preliminary work we have fabricated photoelectrochemical cells using a polymer gel electrolyte based on polyacrylonitrile. Figure 2 a shows a schematic illustration of these cells. After fabrication of the porous $\mathrm{TiO}_{2}$ films and attachment of the sensitizer, the electrolyte is solution cast onto the film. Gelation is complete after 1 - 2 days. Figure $2 b$ shows a current voltage curve for a cell with a cis-Ru[(4,4'-LL) $]_{2}(\mathrm{CN})_{2}$ sensitizer. The fill factor for the cell shown in the figure was 0.61 . These results illustrate that this approach may be used to fabricate quasi-solid state devices.

We have used intensity modulated photocurrent spectroscopy (IMPS) to analyze the relaxation processes in these cells. Figure 3a shows an IMPS spectrum for a cell at a cell voltage of $0 \mathrm{~V}$. At high frequencies, above $100 \mathrm{~Hz}$, there is no response to the modulated perturbation. As the frequency is scanned in the negative direction, a loop is seen in the fourth quadrant. The low frequency intercept of the loop gives an efficiency of about $12 \%$ close to the value observed in the photocurrent spectrum shown in Figure $3 \mathrm{~b}$. The frequency at the 
maximum imaginary part of the IMPS spectrum is about $0.1 \mathrm{~Hz}$. corresponding to a time constant of about $2 \mathrm{~s}$. This spectrum shows that the response of the cell is very slow due to the filling of trap states in the film. The time constant for this process decreases with increasing light intensity since the rate of trap filling is faster at higher base light intensities.

\section{References}

1. Meyer G.J.; Searson. P.C. "Photelectrochemical Solar Energy Conversion at Nanostructured Materials" Interface 1993, 2, 23.

2. Cao, F.; Oskam, H.; Searson, P.C.; Stipkala, J.M.; Heimer, T.A.; Farzhad, F.; Meyer, G.J. " The Electronic and Optical Properties of Nanostructured $\mathrm{TiO}_{2}$ Films" submitted.

3. Heimer, T.A.; Meyer, G.J. "Molecular Level Photovoltaics: The Electro-Optical Properties of Metal Cyanide Complexes Ancored to Titanium Dioxide" J. Phys. Chem. 1993, 97, 11987.

4. Argazzi, R.A.; Bignozzi, C.A.; Heimer, T.A.; Castellano, P.N.; Meyer, G.J. "Enhanced Spectral Senitivity from Ru(II) Polypyridyl Photovoltaic Devices" Inorg. Chem. 1994, $\underline{33}, 5741$.

5. Heimer, T.A.; Meyer, G.J. "Distribution Analysis of Electron Injection Rates from Ruthenium(II) Sensitizers to Nanostructured Titanium Dioxide" submitted.

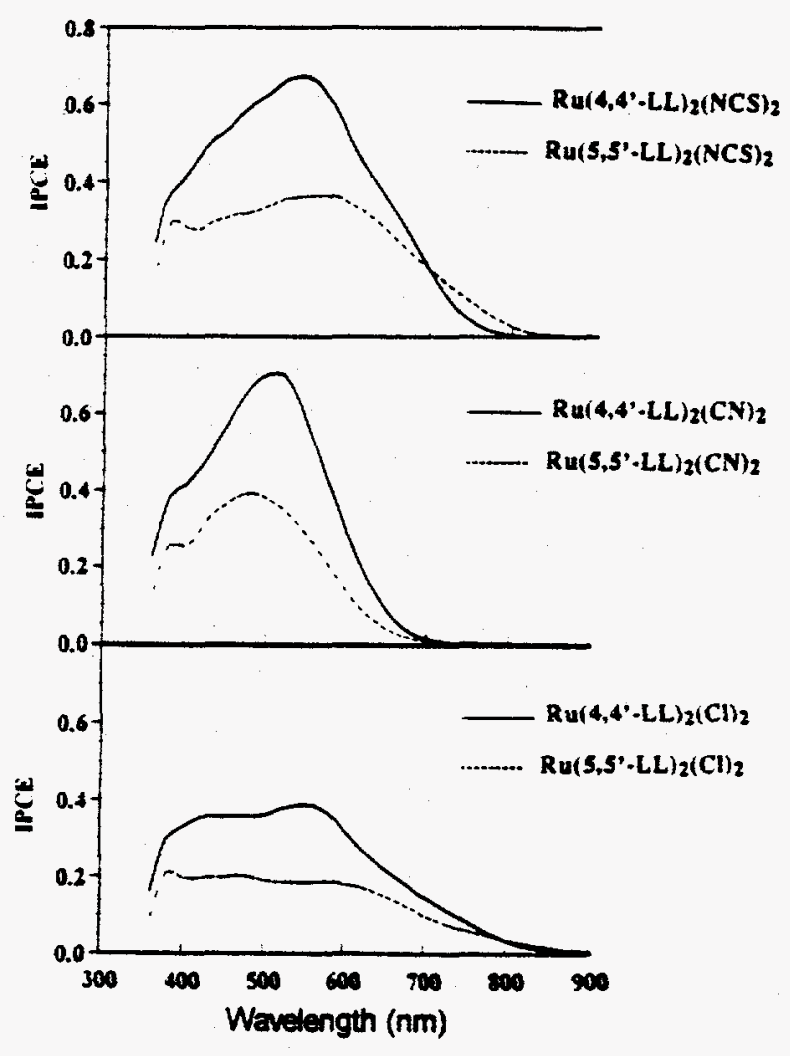

Figure 1. Photoaction spectra of the surface attached complexes $\left[4,4^{\prime}-(\mathrm{LL})\right]_{2} \mathrm{Ru}(\mathrm{X})_{2}$ and $\left[5,5^{\prime}-(\mathrm{LL})\right]_{2} \mathrm{Ru}(\mathrm{X})_{2}(-\cdots---)$ in propylene carbonate with $\mathrm{NaI} /{ }_{2}$ electrolyte, a) $\mathrm{X}=\mathrm{SCN}^{-}$, b) $\mathrm{X}=\mathrm{CN}^{-}$, and c) $\mathrm{X}=\mathrm{Cl}^{-}$. 

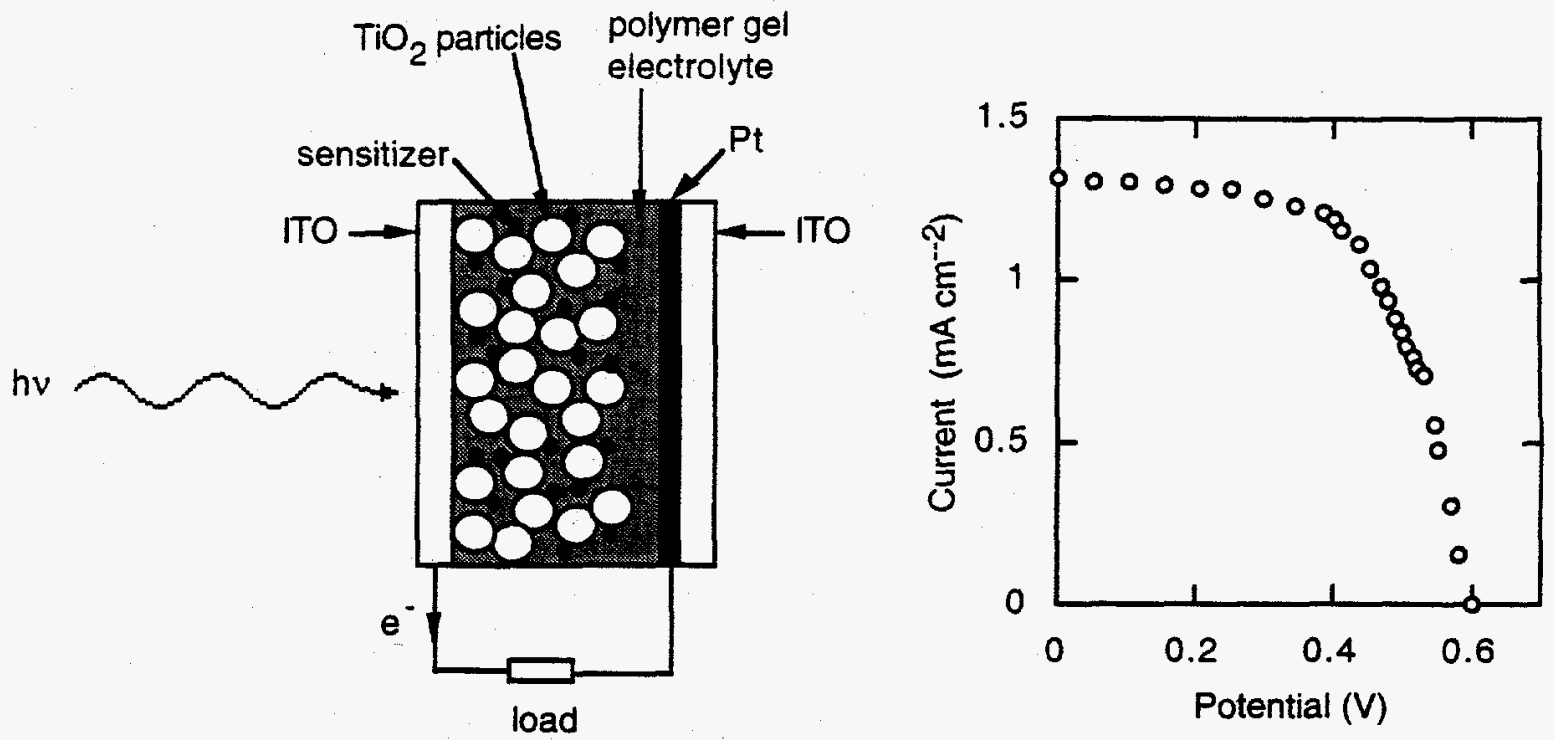

Figure 2. (a) Schematic ilustration of polymer gel photoelectrochemical cells. (b) Current voltage curve for gel electrolyte cell under white light illumination $\left(50 \mathrm{~mW} \mathrm{~cm}{ }^{-2}\right)$; sensitizer: cis- $\mathrm{Ru}\left[\left(4,4^{\prime}-\mathrm{LL}\right)\right]_{2}(\mathrm{CN})_{2}$.
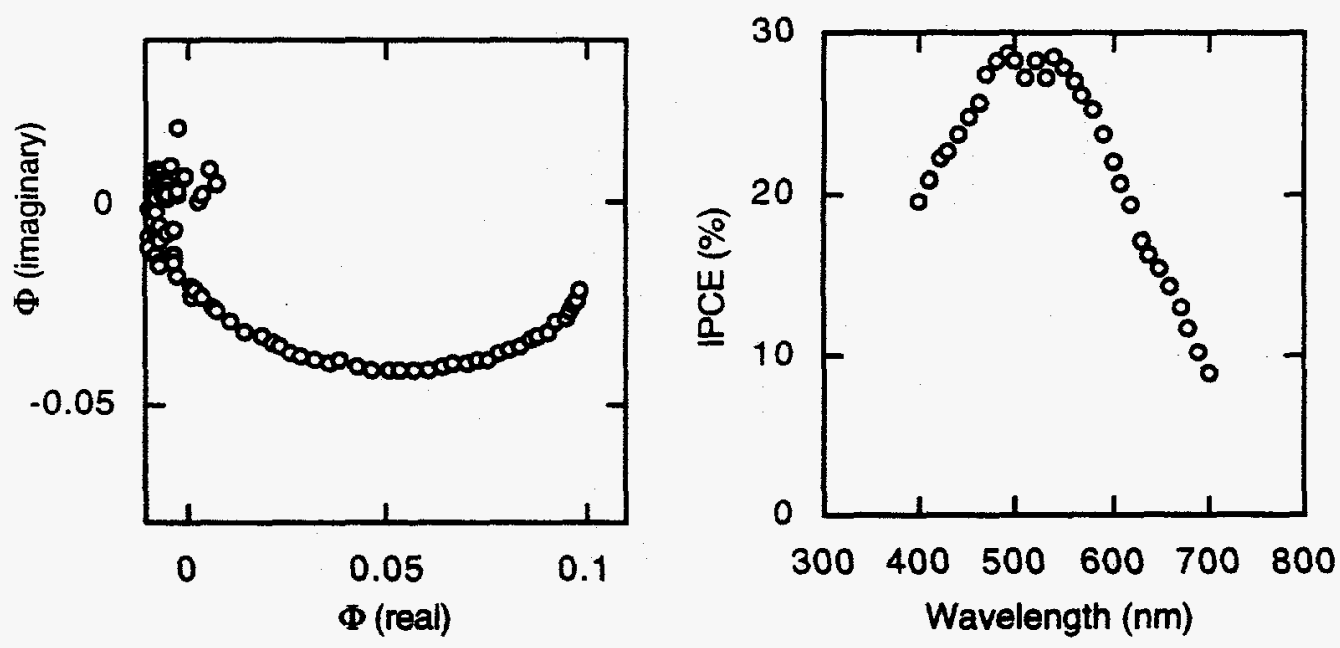

Figure 3. (a) IMPS spectrum for $\mathrm{TiO}_{2}$ film (Degussa) with cis-Ru[(4,4'-LL) $]_{2}(\mathrm{CN})_{2}$ sensitizer; electrolyte was $0.2 \mathrm{M} \mathrm{NaI}, 0.05 \mathrm{M} \mathrm{I}_{2}$ in PC; excitation at $595 \mathrm{~nm}$. (b) Incident photocurrent conversion efficiency at low light intensity. 
Title:

The Role of Point Defects and Impurities in the Processing Performance of Crystalline Silicon Solar Cells

Organization: Massachusetts Institute of Technology

Contributors: L. C. Kimerling, Principal Investigator; J. Michel, H. M'saad, L. Chalfoun, S. Zhao

\section{Introduction}

Our purpose is to develop and demonstrate processes that will enhance the yield and performance of crystalline silicon solar cells by the discovery of critical factors in defect control. We have refined Radio-Frequency Photoconductance Decay (RF-PCD) for use as a quantitative process contamination monitor; developed a thermodynamic model for metal contamination of silicon; continued atomistic simulations of iron in silicon; developed a generic model for interstitial point defect reactions in solar grade silicon; and defined the role of mechanical stress in the performance of Al-backside contacts.

\section{Experimental Results}

\section{Baseline Materials Handling and Defect Analysis}

The objective is evaluation of starting material and materials handling, and correlation with cell performance. The key technical issues are identification of persistent lifetime killers, and development of a methodology for monitor and control of materials quality during processing. The materials metric for cell performance is minority carrier lifetime. The philosophy is to monitor lifetime for control and to use spectroscopic diagnostics only when the process is out of control.

We designed and constructed a highly sensitive Radio Frequency Photoconductance Decay (RFPCD) measurement. It allows measurement of materials with a wide range of resistivity (large skin depth due to $50 \mathrm{MHz}$ carrier frequency) and in a variety of ambients (including wet chemical solutions). We demonstrated detection of surface contamination and roughening as an increase in surface recombination velocity. We detected bulk metal contamination in EFG (Mobil Solar, now ASE Americas) silicon, identified quantitative concentrations with DLTS and correlated, quantitatively, the measured concentrations with cell performance using PC-1D simulations. We defined a quality metric in TI's Spheral Solar ${ }^{\mathrm{TM}}$ silicon $\left(1 \mathrm{~mm}\right.$ diameter, $\left.\mathrm{p}=10^{17} \mathrm{~cm}^{-3}\right)$ by measuring trap tails in RF-PCD signals. This research provided the first in-situ monitor of surface cleanliness; a correlation of EFG cell yield with titanium contamination; and the first measurements and evaluations of Spheral Solar ${ }^{\mathrm{TM}}$ feedstock.

We employed iodine passivation of silicon surfaces to demonstrate the quantitative RF-PCD. Figure 1 shows the quantitative fit of iodine adsorption equilibria on silicon. A methanol solvent is used and the surface recombination velocity (SRV) is monitored as a function of solution composition. The coverage $\theta_{S}$ is determined by $\frac{N_{0}-N}{N_{0}}$, where $N_{o}$ and $N$ are the silicon surface state density in pure methanol and in the iodine:methanol solution. The line through the points is a plot of the classic Langmuir adsorption isotherm given by

$$
\frac{\theta_{\mathrm{s}}}{1-\theta_{\mathrm{s}}}=\text { const } \cdot[\text { iodine }]^{-1 / n}
$$

The excellent fit indicates the measurement is quantitative. The values of $0.85<\mathrm{n}<0.98$ for our sample reveal a first order atom-by-atom attachment mechanism.

The deposition of $\mathrm{Cu}$ on silicon from HF solution was detected at very low levels. Figure 2 shows the raw sensitivity of SRV to $\mathrm{Cu}$ contaminated HF. 


\section{Defect Reactions in Processing}

This project addresses stability of passivation and gettering during contact formation. RF-PCD served as the monitor. We established sensitivity to surface states at a record level of $10^{8} \mathrm{~cm}^{-2}$ $(100 \mathrm{ppb})$. Electronic passivation of the silicon surface by hydrogen and halogens was established. Reaction studies of surface deterioration in air were conducted using the same tool. Bulk lifetime changes due to $\mathrm{Al}$ alloy contact formation $(850 \mathrm{C}, 30 \mathrm{~min})$ were measured. Stress induced by the process was measured by laser deflection analysis of induced wafer curvature. The role of stress, stress relaxation, interface morphology, molten metal formation, and back surface field were correlated with bulk lifetime changes. The Al alloying step improved the bulk lifetime. Film stress is relieved with a time constant of days at room temperature.

Figure 3 shows a plot of film stress vs. time following an $850 \mathrm{C}, 30 \mathrm{~min}$ heat treatment. The 100 MPa level tensile stress persists for long times and is relieved only by loss of film continuity. Detailed studies of backside field and interfacial roughness reveal irregularities across the cell. Current research is focused on the root cause of the irregularities, the impact on cell performance, and the process window for optimized contact formation.

\section{Modeling and Simulation}

Models and process simulators were constructed from our experimental data to confirm, understand and guide cell fabrication. Multilevel models of metal contamination were constructed. Globally, a thermodynamic model of metal deposition on silicon surfaces from cleaning solutions was developed. Figure 4 shows a phase diagram for the stability of iron in silicon cleaning solutions. This "Pourbaix diagram" shows the field of stability of ions in solution (Fe $\mathrm{Fe}^{+++}$and $\mathrm{Fe}^{++}$) with respect to $\mathrm{E}(\mathrm{V})$, the oxidation potential of the solution and its $\mathrm{pH}$. This diagram can be constructed from the free energies of the reactions shown, and it provides an accurate guide for the design of silicon cleaning chemistry.

A model for metal solubility in silicon as a function of temperature and doping was developed to describe contamination during heat treatment. A local model of metal-acceptor pair determined the equilibrium defect species resulting at room temperature. Shockley-Read-Hall statistics were applied to the resulting species to yield the measured minority carrier lifetime. Figure 5 shows the variation in lifetime calculated for $\mathrm{Fe}$ contamination at different heat treatment temperatures. The variation reflects solubility changes with doping and temperature. These models allow evaluation of risk in process design, and specific applications to $\mathrm{Fe}$ in Si were correlated with experiment.

We have developed a global, time-dependent model for defect formation during processing. Figure 6 shows the evolution of interstitial defects as a function of boron content in silicon containing oxygen and carbon in the background. The coupled equations are fit to our experimental data. Similar data for oxygen and carbon variations are being generated.

\section{Conclusion}

This program addresses the limiting factors in cell performance with low cost manufacturing. Low cost silicon materials have high defect contents, wide initial state specifications and a large variation in resulting cell performance. Processes feature low control of purity and handling relative to integrated circuit fabrication. This project has created tools to monitor cleanliness; new process chemistries for handling; and fundamental formalisms to guide process development. The understanding and development of high tolerance processes are key to PV energy sources.

\section{References}

- Hichem M' saad, Jurgen Michel, J. J. Lappe, and L. C. Kimerling, "Electronic Passivation of Silicon Surfaces by Halogens," Journal of Electronic Materials, 23487 (1994).

- H. M'saad, G. J. Norga, J. Michel, and L. C. Kimerting, "Defect Monitoring and Control for Crystalline Silicon Processing," Proceedings of the Photovoltaic R\&D Meeting, Golden, CO, October 1993. 
- Hichem M'saad, Jurgen Michel, A. Reddy, and L. C. Kimerling, "Monitoring and Optimization of Silicon Surface Quality," J. Electrochem. Soc. 94-7, 505 (1994); presented at the Int'l Conference on Cleaning Technology in Semiconductor Devices, ECS, New Orleans, LA, October 10-15, 1993.

- Gerd J. Norga, K. Alexis Black, Hichem M'saad, Jurgen Michel, and Lionel C. Kimerling, "Metal Adsorption on Silicon Surfaces from Wet Wafer Cleaning Solutions." Proceedings of the Second International Symposium on Ultraclean Processing of Silicon Surfaces (UCPSS '94), (Acco, Leuven, 1994), p. 221.

- Gerd J. Norga and Lionel C. Kimerling, "Metal Removal from Silicon Surfaces in Wet Chemical Systems"; to be published in Journal of Electronic Materials.

- Gerd J. Norga, K. Alexis Black, Hichem M'saad, Jurgen Michel, and Lionel C. Kimerling, "Simulation and InSitu Monitoring of Metallic Contamination and Surface Roughening in Wet Wafer Cleaning Solutions," to be published in Materials Science and Technology.

- Hichem M'saad. J. T. Borenstein, and L. C. Kimerling, "Correspondence of Metallic Contamination of Silicon with Minority Carrier Lifetime: Mo and Ti," submitted to Joumal of Applied Physics.

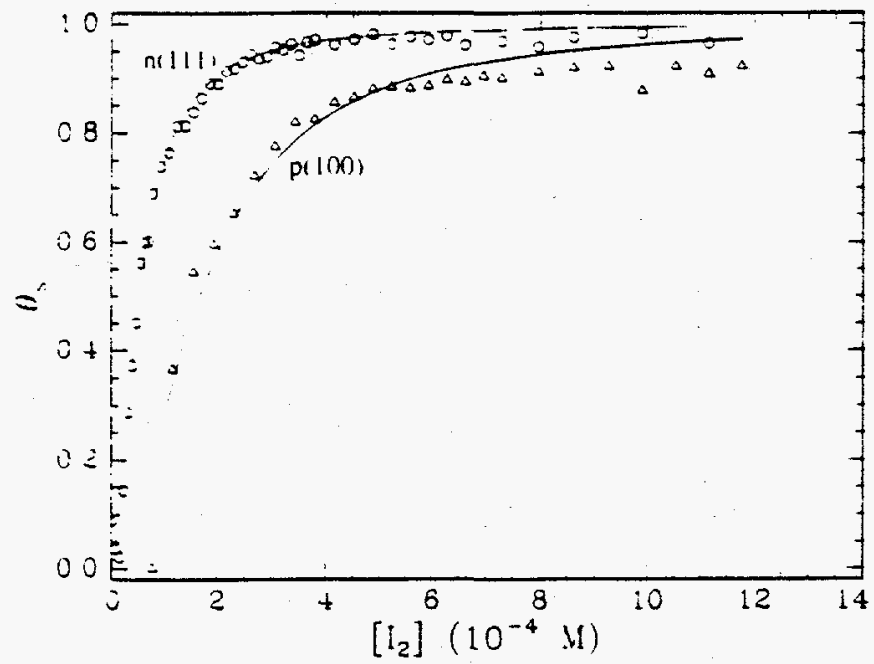

Figure 1: Adsorption isotherms for iodine on Si surface.

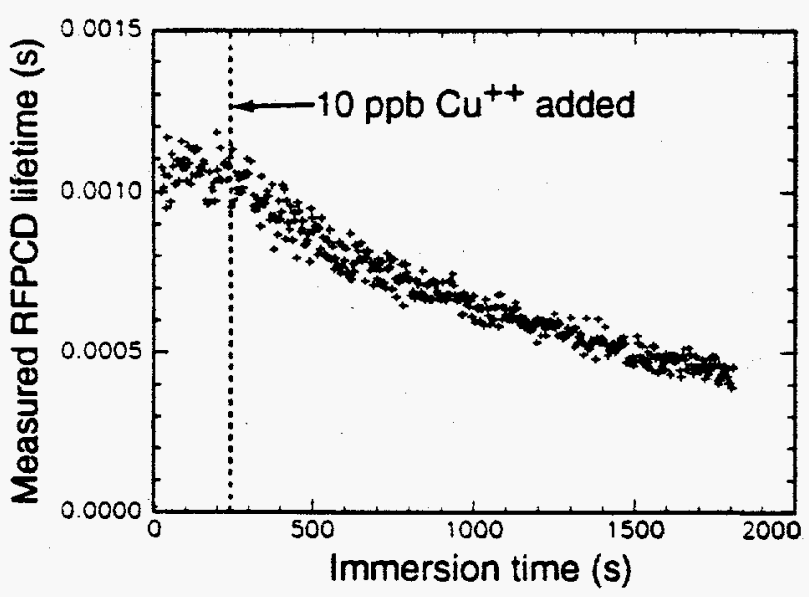

(a)

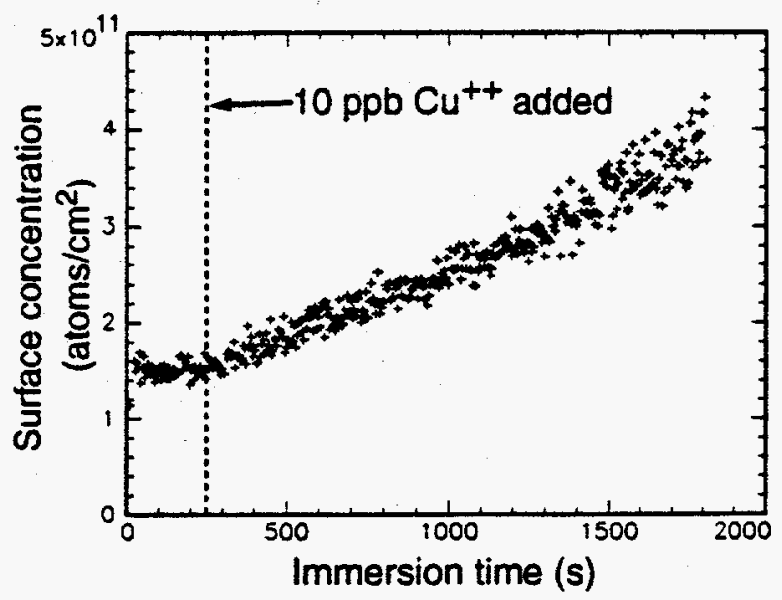

(b)

Figure 2: a) Detection of trace copper deposition on n-type Si from DHF solution using RF-PCD; b) Calculated surface concentration of $\mathrm{Cu}$, using a cross section $\sigma_{\mathrm{p}}(\mathrm{Cu})=2^{*} 10^{-17} \mathrm{~cm}^{2}$. 


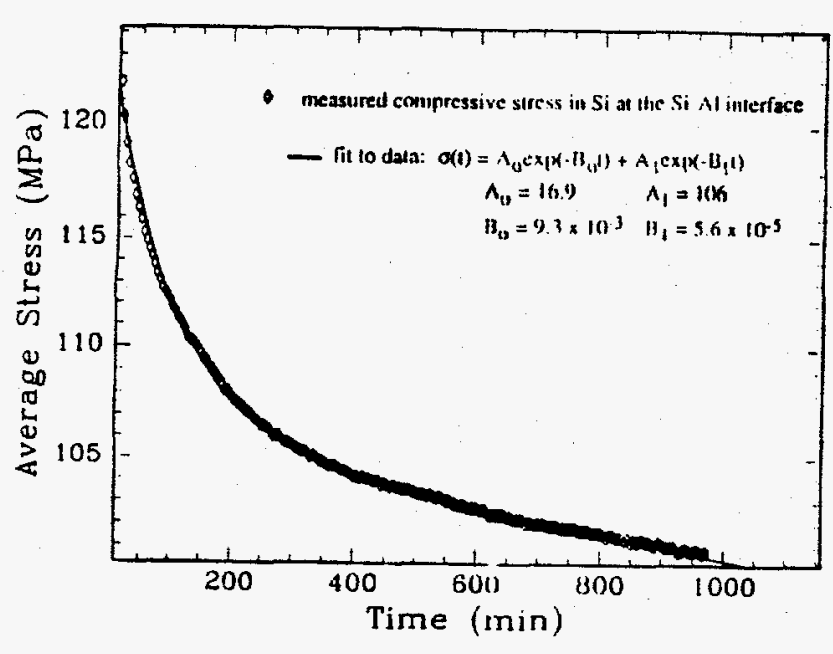

Figure 3: Decay of stress with time in a Si(100) wafer with Al metalization after $850 \mathrm{C}$ heat treatment.

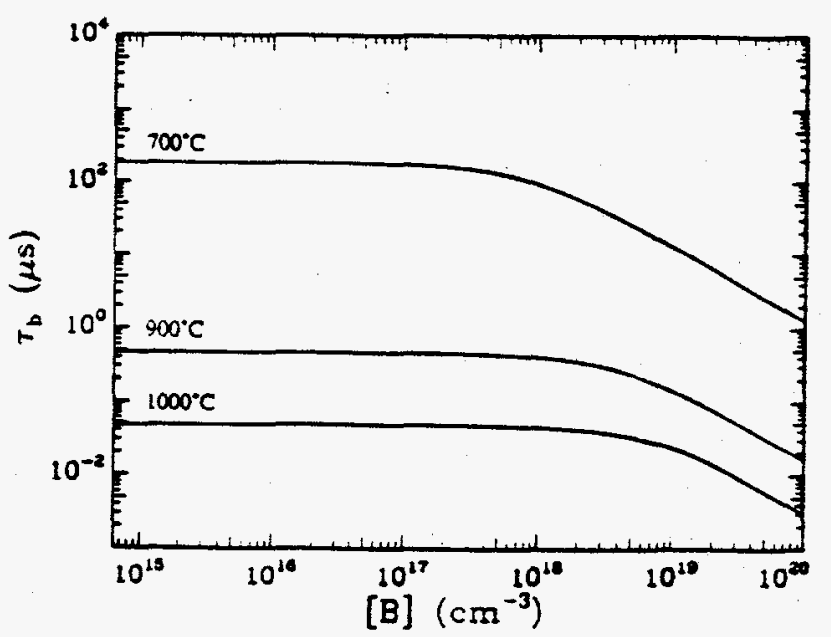

Figure 5: Bulk lifetime in Fecontaminated boron-doped $\mathrm{Si}$ following heat treatment at several temperatures.

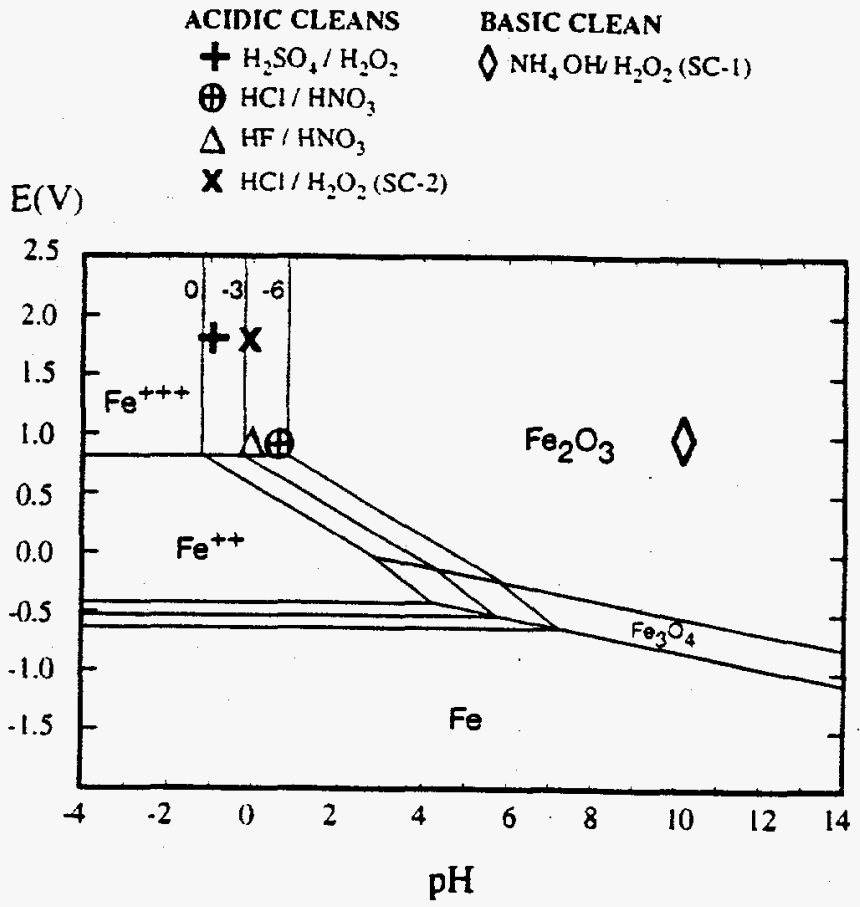

Figure 4: Modeling metals in cleaning solutions using Pourbaix diagrams.

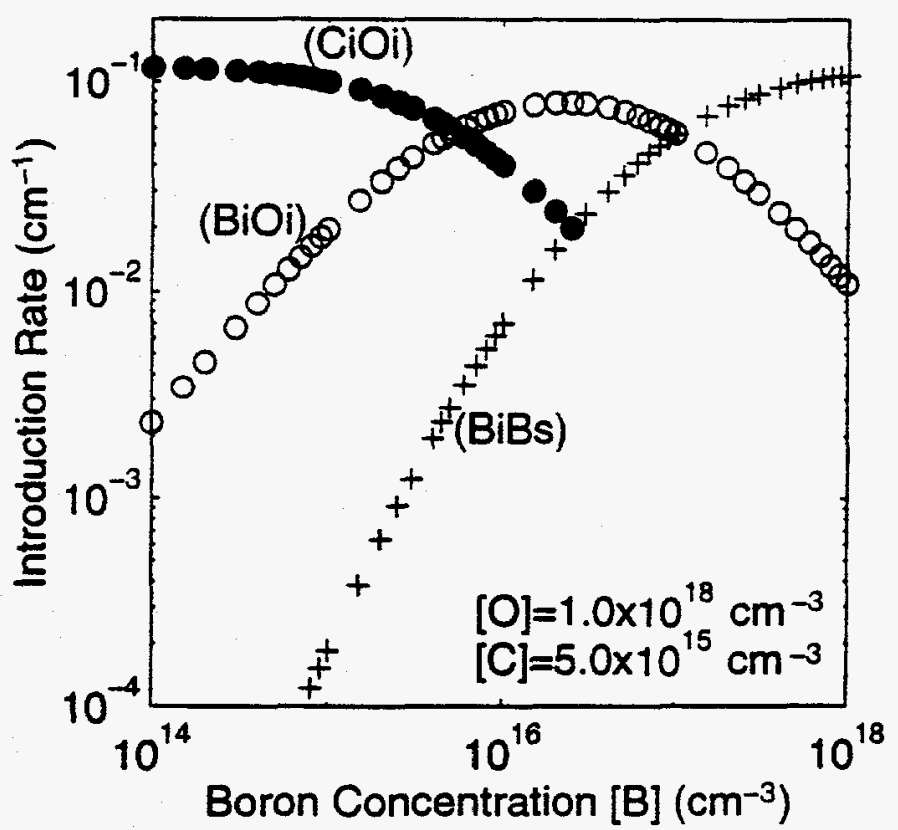

Figure 6: Introduction rate vs. boron concentration [B]. 
Title: Back Surface Fields for $\mathbf{n} / \mathbf{p}$ and $\mathbf{p} / \mathbf{n}$ GaInP Solar Cells

Organization: $\quad$ Electrical and Computer Engineering Department

North Carolina State University

Raleigh, North Carolina 27695 - 7911

Contributors: $\quad$ N. H. Rafat, M. L. Timmons, N.A. El-Masry and S. M. Bedair

\section{Objective}

The objective of this research is to study the effect of different types of back surface field (BSF) layers on $n / p$ and $p / n$ GaInP 2 cells. These cells are lattice matched to $\mathrm{GaAs}$ and to be used as top cells for multijunction tandem solar cells. The BSF layers studied are, abrupt strained $\mathrm{GaInP}_{2}$, graded strained GaInP $\mathrm{P}_{2}$, disordered $\mathrm{GaInP}_{2}$ and $\mathrm{AlGaAs}$ films. The measurements were done under 1-sun AM0 spectrum.

\section{Approach}

One of the most promising material for the top junction in multijunction solar cells, is $\mathrm{GaInP}_{2}$ lattice matched to GaAs. To achieve high-efficiency, the short-circuit current, the open-circuit voltage, and the fill factor must all be optimized. Reducing recombination at the back side, using BSF layer could increase the short circuit current, reduce the saturation current and hence increase the open circuit voltage and the efficiency. Fig. 1 shows the structure of the cells used in this work. We have either $n / p$ or $p / n \mathrm{GaInP}_{2}$ lattice matched to GaAs with energy gap around $1.87 \mathrm{eV}$. The thickness of this cell is around $0.55 \mu \mathrm{m}$ to ensure current matching with the bottom GaAs cell in the multijunction structure.

The GaInP $\mathrm{P}_{2}$ were grown using atmospheric pressure OMVPE growth. All the cells were grown at $650^{\circ} \mathrm{C}$ with growth rate of $0.04 \mu / \mathrm{min}$ for GaAs, $0.08 \mu / \mathrm{min}$ for GaInP 2 and $0.15 \mu / \mathrm{min}$ for AlGaAs. Trimethylgallium (TMGa), trimethylaluminum (TMAl), ethyldymethyle indium (EDMIn), arsine (As $\left.\mathrm{H}_{3}\right)$ and phosphine $\left(\mathrm{PH}_{3}\right)$ are the source materials for the growth. Diethylzinc (DEZn) and hydrogen selenide $\left(\mathrm{H}_{2} \mathrm{Se}\right)$ are used as the p-type and n-type dopants, respectively. Disordered $\mathrm{GaInP}_{2}$ was grown by doping the material heavily while using a low V/III ratio namely (50:1). Strained Ga.56In.44P BSF layer has unstrained band gap of about 1.95 $\mathrm{eV}$. Another strained Ga.63 In. $37 \mathrm{P}$ BSF layer has unstrained band gap of about $2.05 \mathrm{eV}$, was also tried. These strained layers are thin (about 150-200 A) to avoid exceeding the critical thicknesses of these alloys. A BSF layer graded from Ga.56In.44 $\mathrm{P}$ to $\mathrm{Ga} .{ }_{51} \mathrm{In} .49 \mathrm{P}$ over $150 \mathrm{~A}$ was also evaluated. The $\mathrm{Al}_{\mathrm{x}} \mathrm{Ga}_{1-\mathrm{x}} \mathrm{As}$ ( $\mathrm{x}$ about 0.8 ) BSF layer was not included in the $\mathrm{n} / \mathrm{p}$ device because most of the energy gap discontinuity between GaInP and AlGaAs is in the valence band only. The cells were processed as small-area devices using a concentrator grid metallization with a junction active-area of $0.141 \mathrm{~cm}^{2}$. The evaporated metallization was done using $\mathrm{Au}: \mathrm{Ge} / \mathrm{Ni} / \mathrm{Au}$ for the $\mathrm{n}$ type and using $\mathrm{T} \mathrm{i} / \mathrm{Au}$ for $\mathrm{p}$-type. In this study we have not used anti-reflecting coatings.

\section{Results and Discussions}

The measurements were done under 1-sun AM0 solar spectrum using a solar simulator. The results given in figures 2,3 , and 4 are for A) the "no BSF layer" , B) the disordered GaInP 2, C) the abrupt strained $\mathrm{GaIn} \mathrm{P}_{2}, \mathrm{D}$ ) the graded strained $\mathrm{GaIn} \mathrm{P}_{2}$, and $\mathrm{E}$ ) the AlGaAs. 
Fig. 2 shows the short circuit current under different types of BSF for both $n / p / p^{+}$and $p / n / n^{+}$ cells. The values of the short circuit current are comparable for the different types of BSF in the $\mathrm{n} / \mathrm{p} / \mathrm{p}^{+}$and $\mathrm{p} / \mathrm{n} / \mathrm{n}^{+}$structures. The short circuit currents for the abrupt and the graded strained layers $\mathrm{GaInP}_{2}$ (case $\mathrm{C}$ and $\mathrm{D}$ ) give the best results for both $\mathrm{n} / \mathrm{p} / \mathrm{p}^{+}$and $\mathrm{p} / \mathrm{n} / \mathrm{n}^{+}$. These values are: $\mathrm{J}_{\mathrm{sc}}=10 \mathrm{~mA} / \mathrm{cm}^{2}$ for $\mathrm{p} / \mathrm{n}^{+} \mathrm{n}^{+}$and $\mathrm{J}_{\mathrm{sc}}=5.6 \mathrm{~mA} / \mathrm{cm}^{2}$ for $\mathrm{n} / \mathrm{p} / \mathrm{p}^{+}$. In both structures, the short circuit currents for the disordered $\mathrm{GaInP}_{2}$ (case $\mathrm{B}$ ) give the worst results. It should also be noticed that the short circuit current for $p / n / n^{+}$is much higher than that of $n / p / p^{+}$. Since the abrupt strained BSF looks promising, the work has been extended to look for the effect of using a more strained $\mathrm{Ga}_{\mathrm{x}} \mathrm{In}_{1-\mathrm{x}} \mathrm{P}$ BSF layer $(\mathrm{x}=0.63)$ with a $\mathrm{p} / \mathrm{n}$ cell rather than an $\mathrm{n} / \mathrm{p}$ cell.

Fig. 3 shows the values of the fill factor under different types of BSF for both structures. The values for the $\mathrm{p} / \mathrm{n} / \mathrm{n}^{+}$structure still outperforms the $\mathrm{n} / \mathrm{p} / \mathrm{p}^{+}$structure. The AlGaAs BSF gives the worst results for $\mathrm{p} / \mathrm{n} / \mathrm{n}^{+}$. The abrupt strained BSF and the disordered BSF give the best results for the $\mathrm{p} / \mathrm{n} / \mathrm{n}^{+}$structure. For the $\mathrm{n} / \mathrm{p} / \mathrm{p}^{+}$structure the abrupt strained BSF outperforms the disordered BSF.

Fig. 4 shows the open circuit voltage for all BSF types. The AlGaAs is still the worst type of $\mathrm{BSF}$ for the $\mathrm{p} / \mathrm{n} / \mathrm{n}^{+}$cells. It can be noticed that there is no enhancement in the open circuit voltage for the $\mathrm{p} / \mathrm{n} / \mathrm{n}^{+}$structure which was not expected. For the $\mathrm{n} / \mathrm{p} / \mathrm{p}^{+}$structure, there is a reasonable enhancement ( $40 \mathrm{meV}$ ) in the open circuit voltage for the abrupt strained $\mathrm{GaInP}_{2}$ over the no BSF case. This may be due to that the $\mathrm{GaInP}_{2} \mathrm{BSF}$ layer is under tensile strain which will lead to a decrease in its energy gap than that of unstrained layer with the same composition. This decrease will lead to a lower band offset between the BSF layer and the base region which lies almost in the conduction band. Thus the barrier for electrons as minority carriers in the $\mathrm{p}$ - base region (and hence an enhancement in the cell parameters), is present where as there is no barrier for the holes in the n-base region.

To estimate the extent to which the Ga content of the BSF layer can be increased (to increase) without introducing a number of defects, a p/n/n ${ }^{+}$cell with a Ga.63In.37P BSF layer was studied and results are summarized in table $\mathrm{I}$. The performance due to this $\mathrm{Ga}$.63 In.37 $\mathrm{P}$ drops off dramatically for all parameters. As the mismatch between the base and the BSF layer increases beyond the critical thickness, misfit dislocations may be introduced leading to this poor performance.

From this study, the best parameters were obtained for the $\mathrm{p} / \mathrm{n} / \mathrm{n}^{+}$structure with a strained abrupt layer of Ga.56In.44P. The I-V characteristic of this cell is shown in fig. 5. With a proper optimization to the window layer and including anti-reflectings layers, more improvements can be expected.

\section{Conclusion}

In conclusion, the abrupt strained $\mathrm{GaInP}_{2} \mathrm{BSF}$ outperforms the other BSFs. Likely related to material quality, the AlGaAs layer clearly produce the least efficient BSF. Only limited amounts of strain in abrupt mismatched layers could be tolerated before device performance degraded severely.

\section{References}

1. N. H. Rafat, S. M. Bedair, P. R. Sharps, J. S. Hills, J. A. Hancock, and M. L. Timmons, First World Conference on Photovoltaic Energy Conversion, Hawaii, 1994. 


\begin{tabular}{|cccc|}
\hline por $n$ & GaAs & Cap & $0.2 \mu$ \\
\hline por $n$ & AllnP & Window & $250 \mathrm{~A}$ \\
\hline$p+$ or $n+$ & GalnP & Emitter & $0.2 \mu$ \\
\hline$n$ or $p$ & GainP & Base & $0.35 \mu$ \\
\hline$n+$ or $p+$ & BSF & Region & $150-200 \mathrm{~A}$ \\
\hline$n+$ or $P+$ & GaAs & Buffer & $0.2 \mu$ \\
\hline$n+$ or $p+$ & GaAs & Substrate \\
\hline
\end{tabular}

Fig. 1. The structure of the $\mathrm{GaInP}_{2}$ Cell. The cap layer is shown before etching. (not to scale).

Table [1] The short circuit current $I_{S c}$, the open circuit voltage $V_{o c}$ for the $p / n$ cell with no BSF layer and with abrupt strained $B S F$ layer of $\mathrm{Ga}_{x} \operatorname{In}_{1-x} P$ with $x=0.56$ and with $x=0.63$.

\begin{tabular}{|l|c|c|c|}
\hline & Voc volt & Isc $\mathrm{mA}$ & FF \\
\hline No BSF & 1.34 & 1.372 & 0.833 \\
\hline $\mathrm{x}(\mathrm{Ga})=0.56$ & 1.34 & 1.418 & 0.86 \\
& & & \\
\hline $\mathrm{x}(\mathrm{Ga})=0.63$ & 1.325 & 1.157 & 0.84 \\
\hline
\end{tabular}




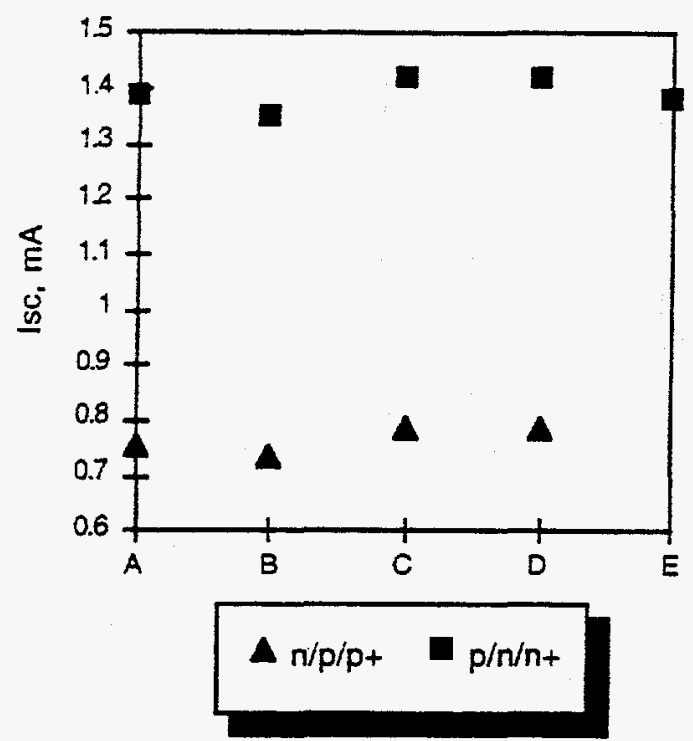

Fig. 2. The short circuit current in $m A$ over an active area $\left(0.141 \mathrm{~cm}^{2}\right)$ for different types of BSF for both $n / p / p^{+}$and $p / n / n^{+}$cells (no anti- reflecting coatings).

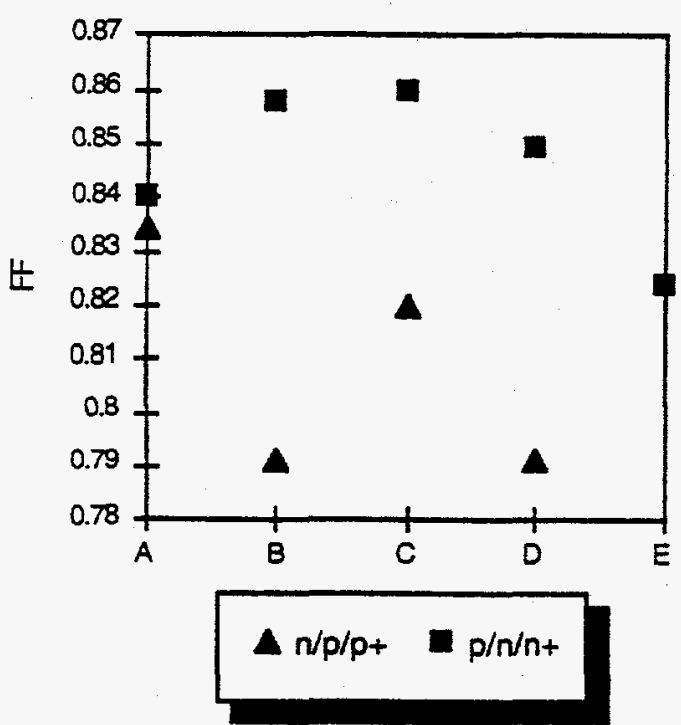

Fig. 3. The fill factor for different types of BSF for both $n / p / p^{+}$and $p / n / n^{+}$cells.

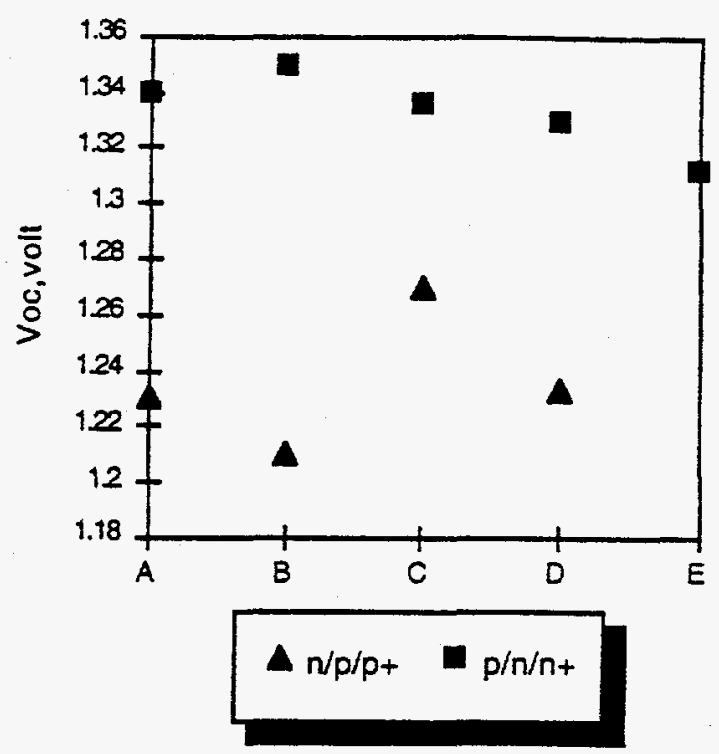

Fig. 4. The open circuit voltage for different types of BSF for both $\mathrm{n} / \mathrm{p} / \mathrm{p}^{+}$and $\mathrm{p} / \mathrm{n} / \mathrm{n}^{+}$cells.

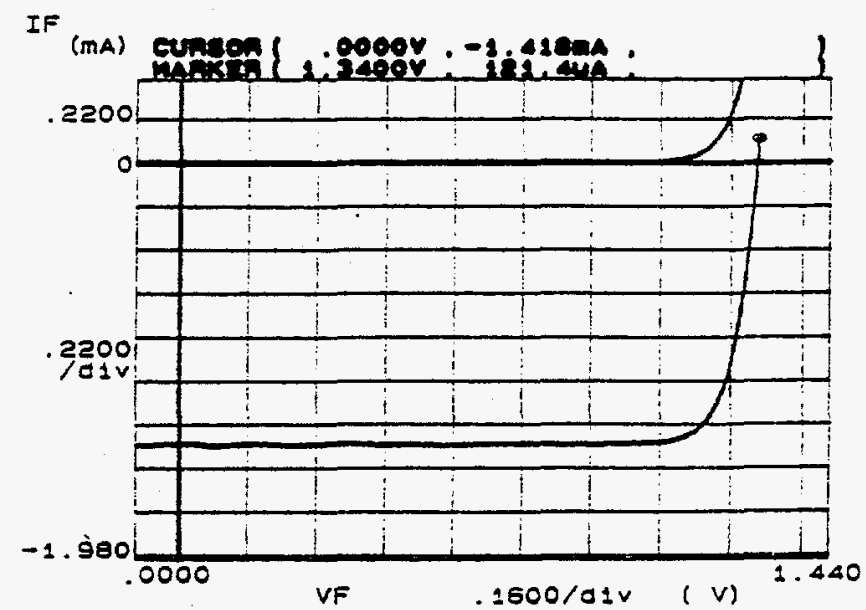

Fig. 5. The I-V characteristic of the best $\mathrm{p} / \mathrm{n} / \mathrm{n}+$ cell with an abrupt strained $\mathrm{Ga} .56$ In ${ }_{44} \mathrm{P}$ BSF. 
Title: Porous Silicon Solar Cell Development

Organization: Spire Corporation, Bedford, MA 01730-2396

Contributors: $\quad$ S.M. Vernon, Principal Investigator, N.M. Kalkhoran, Project Scientist

\section{Introduction}

The overall goal of this research is to establish technology to produce very-high-efficiency solar cells for terrestrial photovoltaic applications. Our approach involves the formation of a layer of porous $\mathrm{Si}$ on a conventional crystalline Si substrate. During this first year we have focussed on forming $\mathrm{p} / \mathrm{n}$ junctions in porous $\mathrm{Si}$, making ohmic contacts, and characterizing photovoltaic performance in this new material. We have demonstrated photovoltaic action in a porous Si $\mathrm{p} / \mathrm{n}$ junction for the first time; also, we have shown surface passivation and antireflection-coating properties of porous Si on crystalline Si solar cells.

\section{Porous Si p/n Junction Solar Cells}

We made porous Si solar cells by ion-implanting boron into $\mathrm{N}$-type crystalline Si wafers; junction depth is $=0.75 \mu \mathrm{m}$. Two conductivity levels of Si were used: N-type =three ohm-cm Si, and high-resistivity $\mathrm{n}^{-} \mathrm{Si},=5000 \mathrm{ohm}-\mathrm{cm}$. After annealing to remove implant damage, the Si was made porous, to a depth of several microns, by anodic etching under UV illumination. Next, top metallization was applied, using either shadow-mask dots or photolithographically defined evaporated grid lines Both front and back metallizations were $\mathrm{Ti} / \mathrm{Pd} / \mathrm{Au}$. Junction depth varies somewhat with Si n-type resistivity since the boron implant recipe was kept constant. Only the high-resistivity wafers were successful in allowing the formation of solar cells where the photoresist for narrow grid lines would flow properly to form a uniform pattern. The dot contacts were only done on the three-ohm-cm wafers. The structure is shown in Figure 1.

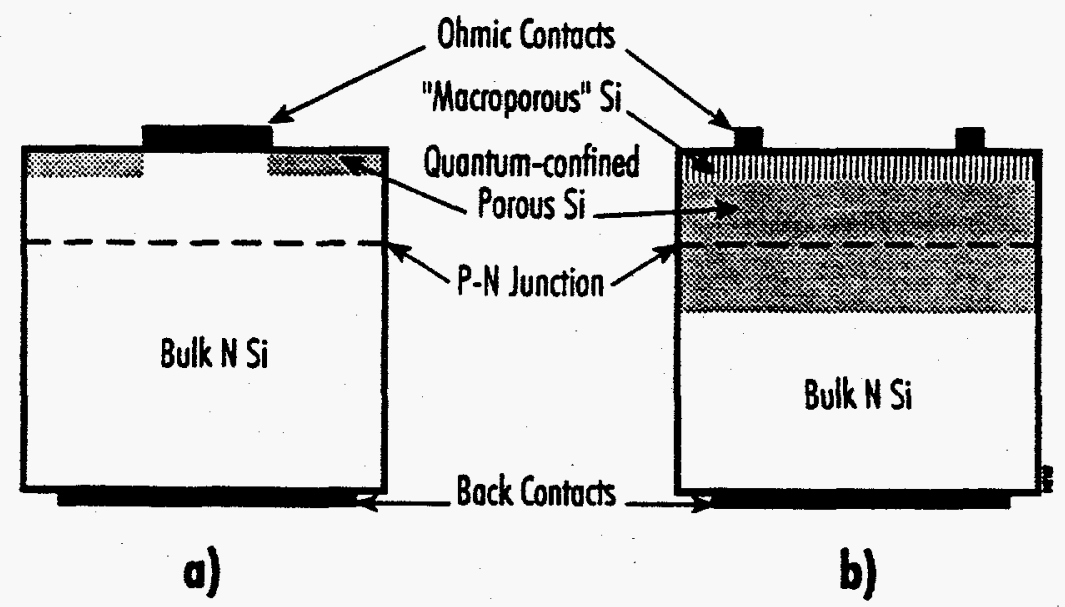

Figure 1 (a) Cell structure with shallow porous Si surface layer, metal grid contact is to the bulk $\mathrm{Si}$, which contains the junction; (b) cell structure with deep porous Si layer, containing the p-n junction, metal grid contact is to the porous Si. 


\section{Results- Junction Within the Deep Porous Si; Dot Contact to Porous Si on Top}

In these devices, we formed deep porous Si which penetrated the junction, before the application of the front ohmic contact. Characterization was performed with uncalibrated incandescent (microscope light) illumination. A photovoltaic effect was clearly observed. I-V characteristics are shown in Figure 2. In the dark, the device exhibits rectifying behavior, as can be expected from a $\mathrm{p} / \mathrm{n}$ junction. Dark current levels in reverse bias are below the instrument resolution limit on this scale. The photocurrent under white light illumination increases as intensity is raised. For the porous Si device shown, a photocurrent of about $2 \mu \mathrm{A}$ was generated. The largest $V_{\infty}$ measured was $0.11 \mathrm{~V}$. The low value of $\mathrm{V}_{\mathrm{oc}}$ is consistent with the small photocurrent.

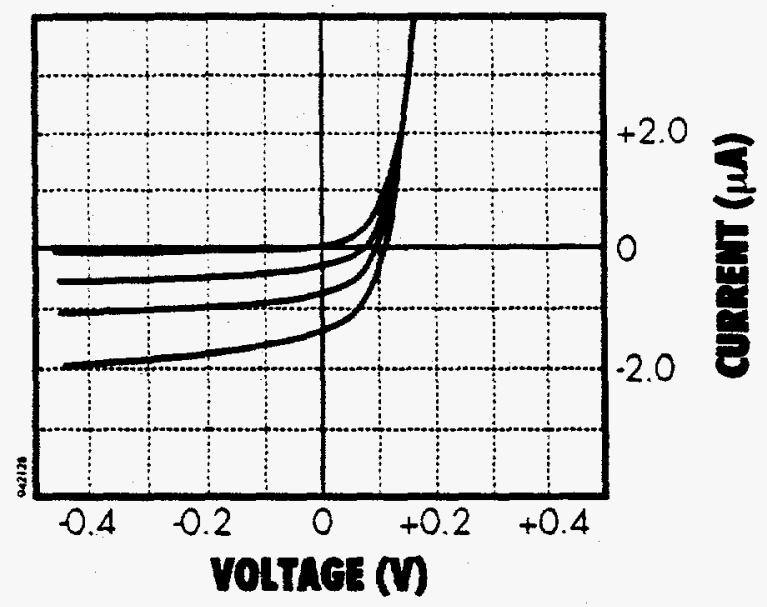

Figure 2 Current-voltage characteristics of porous $\mathrm{Si}$ diode exhibiting photovoltaic characteristics.

We explain the situation as follows. The metal contact is very thick, and cannot pass any light. The only light that is available for generating a photocurrent must be absorbed in the regions around the opaque contact. However, the surface $\mathrm{p}^{+}$region is composed of vertical wires, making horizontal carrier transport difficult. We suspect that the only photocurrent generated is by light scattered under the contact, and absorbed in material below the metal.

\section{Results - Junction Within the Deep Porous Si; Metal Grid Lines on Top}

Solar cells with photolithography-defined front contact fingers were attempted on both $3 \mathrm{ohm}-\mathrm{cm}$ and $5000 \mathrm{ohm}-\mathrm{cm} \mathrm{Si}$ substrates with porous Si formed several microns deep. The photoresist adhered well to the high-resistivity wafers, but was problematic on the higher doped ones. The cells on high-resistivity $\mathrm{Si}$ are essentially PIN diodes.

Illuminated porous Si cell data are shown in Figure $3 ; \mathrm{V}_{\mathrm{oc}}$ is about $0.3 \mathrm{~V}$. There is a significant increase in current with reverse bias, something often seen with amorphous Si PIN solar cells. Furthermore, the dark and the illuminated characteristics cross, again reminiscent of amorphous $\mathrm{Si}$. This observation indicates that superposition fails. That is, the illuminated I-V characteristic is not the difference between the photogenerated current and the dark current. Failure of the superposition principle in solar cells often indicates that the photogenerated current is voltage 


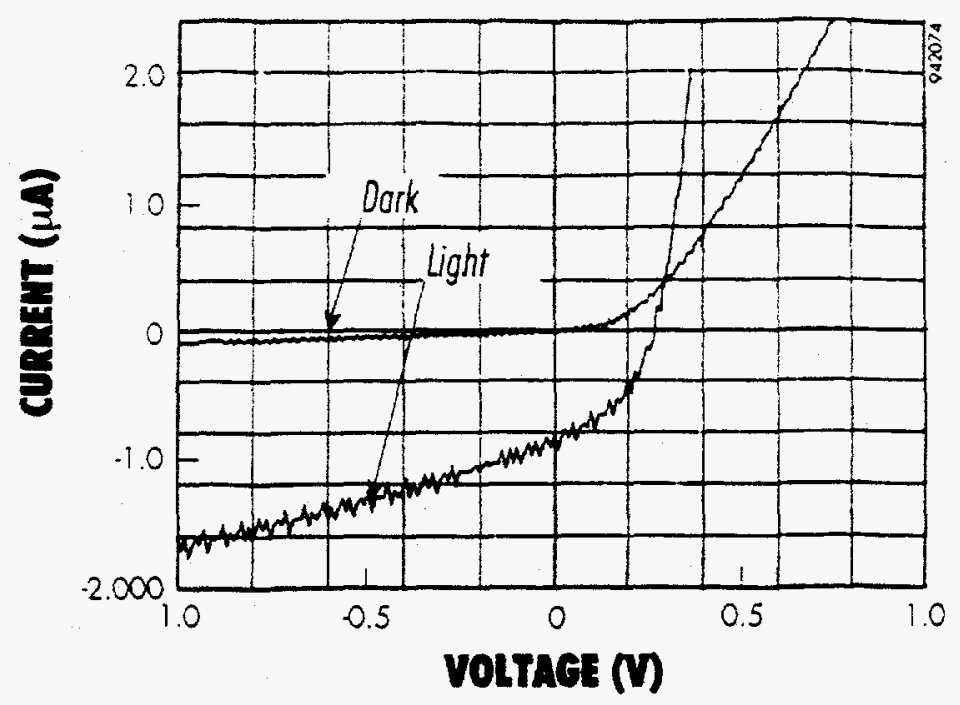

Figure 3 Current-voltage characteristics of porous Si diode exhibiting photovoltaic characteristics.

dependent, and that space charge injection effects may be present. An increase in photocurrent with reverse bias often occurs if there is a barrier to carrier collection, which can be overcome by reverse bias. Also, with the low-doped $\mathrm{Si}$, photogenerated carriers may be modulating the series resistance.

\section{Cells With Porous Si Surface Layer; $p / n$ Junction in Crystalline Si}

Boron was ion implanted into $\mathrm{N}$-type crystalline $\mathrm{Si}$ wafers; junction depth is $\approx 0.75 \mu \mathrm{m}$. After annealing, a simple shadow mask was used to form ohmic dot contacts ( $1.5 \mathrm{~mm}$ diameter). Next thin porous Si was formed by chemical etching the area between the top contacts to a depth of $=0.25 \mu \mathrm{m}$. The spectral response, being that of bulk $\mathrm{Si}$, confirms that the porous layer is thinner than the junction depth. See Figure 1a. Control cells were formed identically, but with no porous surface layer.

\section{Results - Thin Porous Si Layer on Top of Bulk Si Junction; Metal Dot Contact to Bulk Material}

In these devices, a circular Ti/Pd/Au dot as a non-transparent front ohmic contact was formed to the bulk Si before etching to form a shallow surface layer of porous Si. Dark and illuminated characteristics of the cells were measured before and after the porous Si etch step. I-V data reveal that the metal contact was not disturbed by the etching. We found that the photocurrent had increased markedly after the porous Si had been formed.

Using AM1.5G illumination, the bulk $\mathrm{Si}$ cell has $\mathrm{J}_{\mathrm{sc}}=17.42 \mathrm{~mA} / \mathrm{cm}^{2}$ without antireflection coating. The fill factor is $66.3 \%$, indicating that there are some series resistance problems. The measured efficiency was $6.4 \%$. It is clear that we have not optimized our processing at this stage. When the surface is made porous, the sample has $\mathrm{J}_{\mathrm{se}}=30.07 \mathrm{~mA} / \mathrm{cm}^{2}$, which appears to be related to the light-trapping properties of porous $\mathrm{Si}$. With this $70 \%$ larger photocurrent, we measure $\mathrm{V}_{o c}$ of $0.563 \mathrm{~V}$. However, the fill factor for this cell is reduced to $51.9 \%$. The measured AM1.5G efficiency of the best device with the porous Si surface is $8.79 \%$. 
We measured spectral response and reflectance of the two types of devices (Figure 4). Without AR coating, reflectance is in the 30 to $40 \%$ range. For a device with a porous Si surface, reflectance throughout most of the visible region has been reduced to $10 \%$ or less. Also, the internal quantum efficiency is now at $=90 \%$ from about 600 to $1000 \mathrm{~nm}$. Therefore, we have shown that porous Si makes an effective antireflection coating and front-surface passivation for Si solar cells.

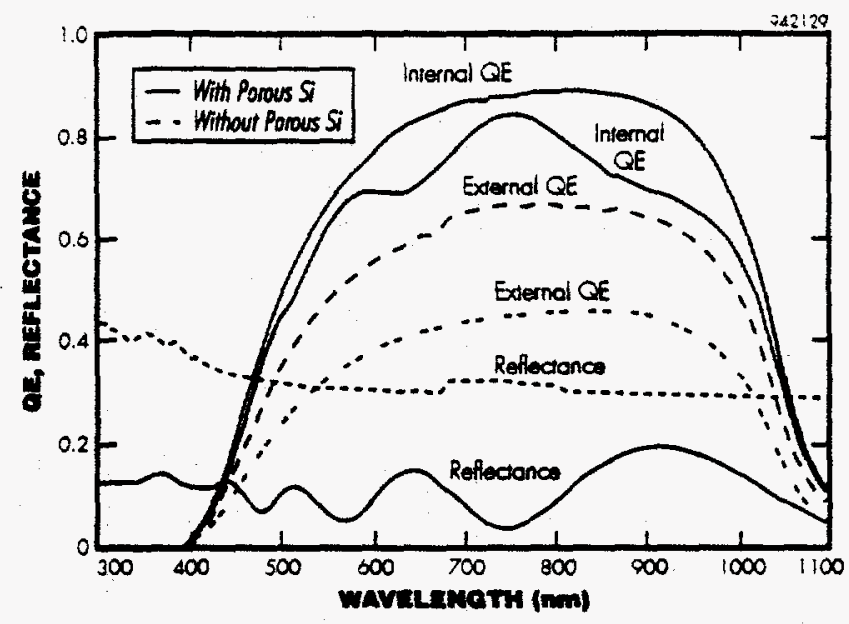

Figure 4 Measured optical reflectance and quantum efficiency spectra for bulk Si solar cell, with and without porous Si surface layer.

It should be noted that the spectral response of the cell with the porous Si surface is basically similar to any bulk Si solar cell. Therefore, most of the current is being generated by light which is scattered under the contact, and absorbed in material below the metal.

\section{Conclusions/Summary}

We have shown that porous $\mathrm{Si}$ is an interesting material for solar cell applications. The antireflection/surface passivation aspects of porous $\mathrm{Si}$ layers on bulk $\mathrm{Si}$ solar cells have been demonstrated, yielding a $70 \%$ increase in photocurrent with no increase in dark current. Photovoltaic effects of porous $\mathrm{Si}$ active layers have been observed. Open-circuit voltage of $\approx 0.3 \mathrm{~V}$ has been measured, with short-circuit current increasing with reverse bias. Spreading resistance problem and charge trapping in porous $\mathrm{Si}$ are the problems to be overcome for future advances.

\section{Publications}

"High Performance Porous Silicon Solar Cell Development," S.M. Vernon, N.M. Kalkhoran, and W.D. Halverson, Proc. of $1^{\text {st }}$ World Conference on Photovoltaic Energy Conversion, Wailkoloa, HI, December 1994.

"High Performance Porous Silicon Based Photovoltaic Cells," N.M. Kalkhoran, S.M.Vernon, H.P. Maruska, and W.D. Halverson, presented at 1994 MRS Meeting, Symposium on Microcrystalline and Nanocrystalline Semiconductors, Boston, MA, December 1994. 


\section{SUBCONTRACTORS}

Title:

Organization:

Contributor:

\section{Thin Film Silicon by Constitutional Supercooling}

State University of New York at Buffalo

Department of Electrical and Computer Engineering

217C Bonner Hall, Amherst, NY 14260

W.A. Anderson, program manager; D.D.L. Chung and K. Etemadi, principal investigators; X. Gu, B. Jagannathan, R. Sridhar, R. Wallace and J. Yi, research assistants.

\section{Objective}

This project is undertaken to fabricate low-cost $\mathrm{Si}$ solar cells utilizing an a-Si:H/poly-Si heterojunction. Computer simulation predicts close to $20 \%$ possible efficiency. A further goal is the production of low-cost, thin-film polycrystalline silicon having a thickness of $20-50 \mu \mathrm{m}$.

\section{Approach}

Thin-film poly-Si is grown onto a Mo or stainless steel substrate by sputter deposition onto a heated substrate after first forming a Sn or In/Ti pre-layer. Growth occurs at about $600^{\circ} \mathrm{C}$ by a liquid epitaxy-type technique. The films may then be improved by laser processing followed by hydrogenation. The solar cell is completed by amorphous silicon deposition to form an a-Si/polySi heterojunction.

\section{Thin Film Si by CS Growth - R. Wallace [1-4]}

Advances have been made in both deposition and characterization over the past year. The most significant change has been in recognizing that the wetting agent used to improve coverage of the substrate by the liquid prelayer typically leaves a conducting residue at the film/substrate interface. Removing the sheet Mo substrate and etching away the wetting agent residue forced conduction through the Si film. This has greatly improved our Hall effect results. Typical carrier concentrations (room temperature) are in the low $10^{16} \mathrm{~cm}^{-3}$ range; typical hole mobilities are 20-30 $\mathrm{cm}^{2} \mathrm{~V}^{-1} \mathrm{~s}^{-1}$.

Hall effect measurements have been improved in two areas. A system incorporating a cryostat is used, allowing carrier concentration to be determined as a function of temperature. A computer controlled data aquisition system is now used to collect Hall voltage data, significantly reducing problems with noise. Activation energy for carrier generation is $\sim 135 \mathrm{meV}$, identical to the activation energy for (dark) conduction.

The photoresponse of the CS film using the Ti wetting agent is usually small, indicating a short lifetime. It is well known that $\mathrm{Ti}$ is a lifetime killer in $\mathrm{Si}$, so efforts have been made to eliminate its use. Films deposited without the Ti show a lower degree of crystallinity by XRD, a smaller apparent grain size by SEM, and a lower carrier mobility by Hall effect (Figure 1). These films are deposited using a Sn prelayer, instead of the In prelayer used along with the Ti wetting agent. Since Sn does not dope Si, the films have been found to be n-type when deposited from an n-type target.The photoresponse is greater, indicating a much longer lifetime. Preliminary lifetime measurements by photoconductive decay show a lifetime of $\sim \mu \mathrm{s}$. 
A microwave hydrogenation system has been built (Figure 2), as hydrogen passivation of grain boundaries is desired. This system uses a magnetron tube from a household microwave oven mounted onto a cylindrical resonant cavity. Results to date indicate an order of magnitude increase in mobility after a 15 minute exposure (CS films using no wetting layer).

\section{Heterojunction Solar Cell Fabrication - B. Jagannathan [5]}

a-Si (amorphous silicon) deposited on crystalline silicon (c-Si) results in a pn heterojunction, which has a distinct advantage of being a low temperature junction. Solar cells with $n^{+} a-S i / i$ a-Si/p c-Si structures were first studied on PC-1D software. The simulations explored the effect of amorphous thickness and substrate (c-Si) resistivity. Simulations indicted that efficiencies in the range of $17-$ $18 \%$ were possible.

The solar cells were fabricated by depositing a-Si on c-Si by rf glow discharge techniques. The back $\mathrm{Al}$ contact on $\mathrm{c}-\mathrm{Si}$ was done first since it required a sintering step. Evaporated Ytterbium formed the front ohmic contact. The initial studies resulted in a solar cell having a $\mathrm{J}_{\mathrm{sc}}$ of 2.7 $\mathrm{mA} / \mathrm{cm}^{2}, \mathrm{a} \mathrm{V}_{\mathrm{oc}}$ of 0.33 volts and a FF of 0.42 . To probe into the low values of $J_{\mathrm{sc}}$ and $F F$ observed in these structures, current-voltage-temperature $(\mathrm{I}-\mathrm{V}-\mathrm{T})$ and capacitance-voltage $(\mathrm{C}-\mathrm{V})$ tests were done. The I-V-T tests on "bad" samples indicated that there were regions of biases were the device was behaving in an ohmic fashion. Also, C-V tests showed that the depletion region was not spreading onto the $\mathrm{C}-\mathrm{Si}$ in these samples. These two factors strongly pointed to some defects states at the interface, which resulted in the Fermi level pinning, and that in turn caused the poor photovoltaic properties. These defects could be a result of plasma bombardment of the interface during a-Si deposition or presence of an oxide film at the interface.

Having recognized that a problem could exist at the hetero-interface, careful attention was paid to substrate preparation. The front face of $\mathrm{C}-\mathrm{Si}$ was cleaned with HF just prior to a-Si deposition. This ensured that the interface would be relatively oxide free. Fig. 3 shows the photoresponse of a $\mathrm{n}^{+} \mathrm{a}-$ $\mathrm{Si} / \mathrm{i}(0.1 \mu \mathrm{m}) \mathrm{a}-\mathrm{Si} / \mathrm{p}(0.1-0.3 \Omega-\mathrm{cm}) \mathrm{c}-\mathrm{Si}$ structure. As can be seen, there is a large improvement in $\mathrm{J}_{\mathrm{sc}}$ and $\mathrm{V}_{\mathrm{oc}}$ compared to the earlier device. Also, the $\mathrm{U}-\mathrm{V}$ response of the cell is enhanced as the a-Si thickness is decreased, probably due to reduced recombination in the amorphous regions as can be seen in Fig. 4.

\section{Laser Processing - N. Sridhar [6-8]}

Intrinsic hydrogenated amorphous silicon thick films ( $7 \mu \mathrm{m}$ thick)deposited by dc glow discharge on a molybdenum substrate were laser crystallized to achieve a polysilicon film having a good photoresponse (necessary for solar cell applications), either by trapping the hydrogen and/or increasing the grain size. The films were annealed using a Nd:glass laser having an emission wavelength of $1.06 \mu \mathrm{m}$. Increasing the laser energy density and/or decreasing the beam scan rate decreased the photoresponse in the film (0.1-0.4), in spite of the increase in the crystallinity. This decrease was attributed to the severe hydrogen evolution taking place in the film at high energy densities and/or decreasing beam scan rate, based on evolved gas analysis. Infrared spectroscopy showed the presence of $\mathrm{SiH}$ and $\mathrm{SiH}_{2}$ bonding at all laser annealing conditions (Fig. 5) and a grain size greater than $0.2 \mu \mathrm{m}$ (as detected from $\mathrm{x}$-ray diffraction). Increasing the substrate temperature during laser annealing did not show any change in the photresponse up to $300^{\circ} \mathrm{C}$, but at $400^{\circ} \mathrm{C}$, the photoresponse increased greatly and then showed a continued decrease with further increase in the substrate temperature. This increase at $400^{\circ} \mathrm{C}$ was due to the release of the weakly bonded hydrogen which occurs at around $365^{\circ} \mathrm{C}$. Presence of a capped layer such as SiO and SiN not only increased the photoresponse, but also gave a moderate improvement in the surface morphology of 
of the film. This improvement was more distinctly seen for SiN capping. Increasing the deposition temperature of the a-Si:H film played a major role in improving the photoresponse (0.15-1.5). This was due to the increase in the amount of $\mathrm{SiH}$ bonding after laser crystallization with increasing a-Si:H film deposition temperature (Fig. 6), as observed by the increase in the $\mathrm{SiH}$ intensity at 2000 and $630 \mathrm{~cm}^{-1}$ in FTIR performed in the reflectance mode. This trend was opposite to that of furnace crystallized films, where crystallinity and grain size were the main factors in the improvement of the photoresponse. In summary, the photoresponse of the laser crystallized films (1.5) was much higher than in the furnace crystallized film (0.08). and this was due to the amount of strongly bonded hydrogen (mainly SiH bonding) present in the films after laser crystallization.

\section{Hydrogenation - X. Gu}

Half micron $(0.5 \mu)$-thick $\mathrm{i} / \mathrm{n}^{+} / \mathrm{Mo}$ films were used for the experiments. Conductivity measurement was carried out after removing the Mo substrate. The standard rf discharge hydrogenation conditions were: power $=25$ Watts; pressure $=300$ mTorr; substrate temperature $=300 \mathrm{C}$; and exposure time $=6-8$ hours. The photo-conductivity/dark conductivity ratio increased to 10 from 0.1 when comparing after to before treatment. The activation energy decreased to $0.43 \mathrm{eV}$ from $0.46 \mathrm{eV}$.

\section{Conclusions}

CS-growth of poly-Si onto Mo results in a film having carrier density of about $1 \times 10^{16} \mathrm{~cm}^{-3}$, Hall mobility of $30 \mathrm{~cm}^{2} / \mathrm{V}-\mathrm{s}$ and high degree of crystallinity.

Laser crystallization may be conducted such that $\mathrm{H}^{+}$is retained in the defects, thus preserving passivation.

a-Si:H/crystalline heterojunctions have been fabricated and show reasonable values of photogenerated current and voltage. Fill factor is reduced due to interface defects. This must be resolved.

\section{References}

1. J. Yi, R. Wallace, W.A. Anderson, et.al., "Crystallized Amorphous Silicon for Low-Cost Solar Cells", Solar Cells, 30, 403-413, May 1991.

2. R.L.Wallace and W.A. Anderson, "Thin Film Polycrystalline Si by A Novel Solution Growth Technique", First World Conference on Photovoltaic Energy Conversion, Waikoloa, Hawaii, Dec. 5-9, 1994.

3. R.L. Wallace and W.A. Anderson, "Thin Film Polycrystalline Si by CS Solution Growth Technique", Materials Research Society Fall Meeting, Boston, Nov. 28-Dec. 2, 1994.

4. J. Yi, R. Wallace, J. Palmer and W.A. Anderson, "Thin Film Amorphous and Microcrystalline Si for Solar Cells", Solar Energy Materials and Solar Cells, 33, 145-155, 1994.

5. B. Jagannathan, J. Yi, R.L. Wallace and W.A. Anderson, "Effect of the Hetero-Interface on the Photoresponse of a-Si/c-Si Solar Cells", Materials Research Society Fall Meeting, Boston, Nov. 28-Dec. 2, 1994.

6. N. Sridhar, D.D.L. Chung, W.A. Anderson, W.Y. Yu, L.P. Fu, A. Petrou and J. Coleman, "Pulsed Laser Annealing of Hydrogenated Amorphous Silicon Thick Films", Materials Research Society Meeting, Boston, Nov. 29-Dec. 3, 1993.

7. N. Sridhar, D.D.L. Chung and W.A. Anderson, "Effect of Deposition Temperature on the Photoresponse of Crystallized Hydrogenated Amorphous Silicon Films", Materials Research Society Fall Meeting, Boston, Nov. 28-Dec. 2, 1994. 
8. D.D.L. Chung, W.A. Anderson, W.Y. Yu, L.P. Fu, A. Petrou and J. Coleman, "Kinetics of Hydrogen Evolution and Crystallization in Hydrogenated Amorphous Silicon Films Studied by Thermal Analysis and Raman Scattering", Materials Research Society Meeting, Boston, Nov. 29-Dec. 3, 1993.

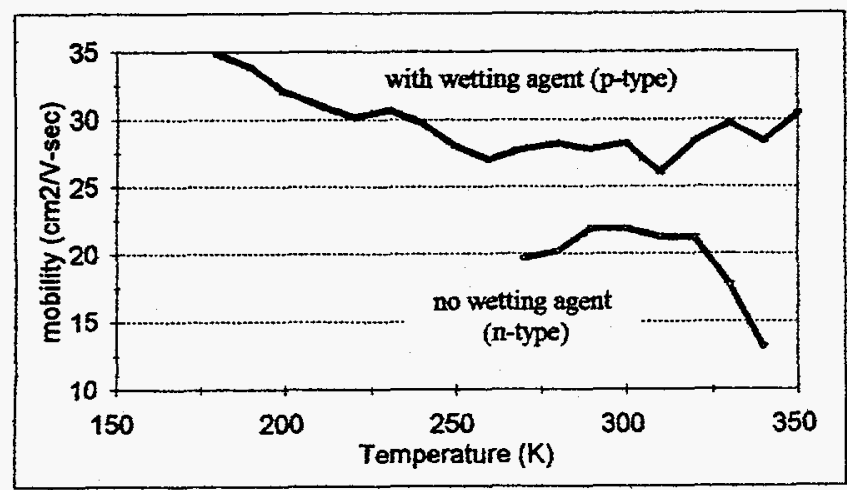

Fig. 1 Comparison of carrier type and mobility for CS films with and without wetting agent.

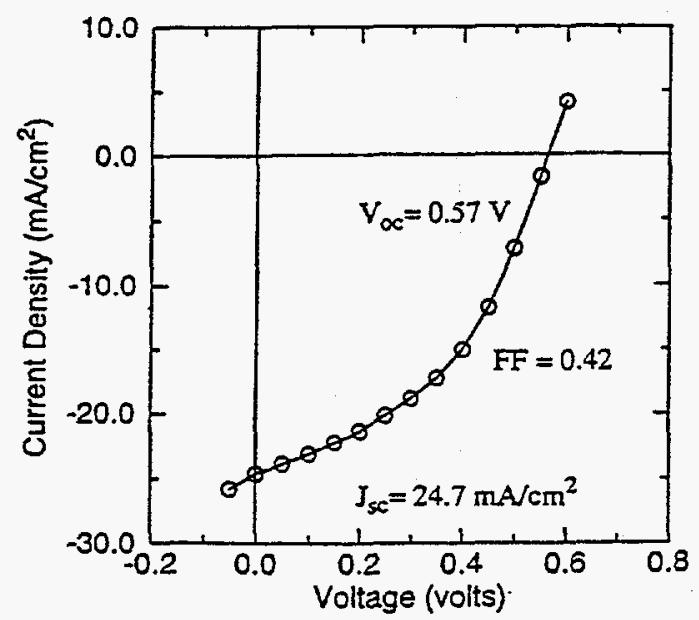

Fig. 3 Photoresponse of $\mathrm{n}^{+} \mathrm{a}-\mathrm{Si} / \mathrm{i}(0.1 \mu \mathrm{m})$ a-Si/p c-Si $(0.1-0.3 \Omega-\mathrm{cm})$ solar cell.

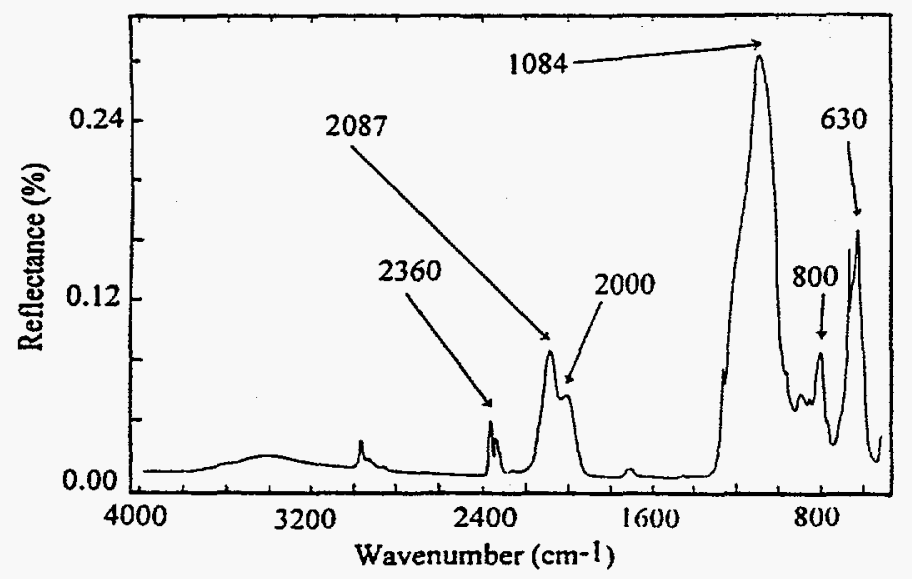

Fig. 5 Infrared spectrum of laser crystallized a-Si:H film at $12 \mathrm{~J} / \mathrm{cm}^{2}$ scanned at $0.45 \mathrm{~mm} / \mathrm{s}$.

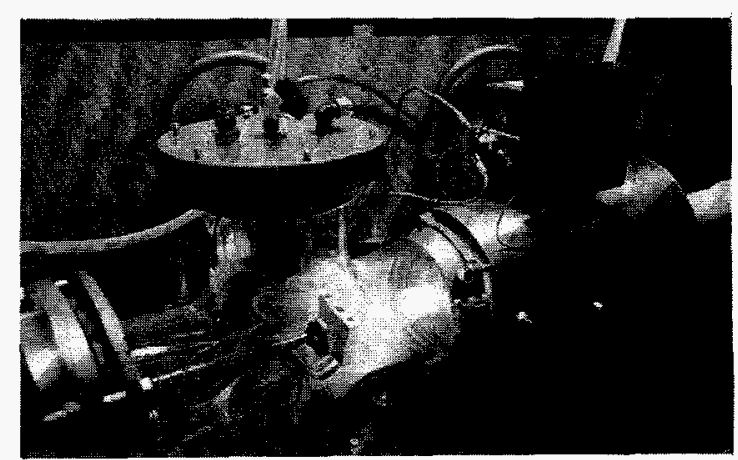

Fig. 2 Microwave hydrogenation system.

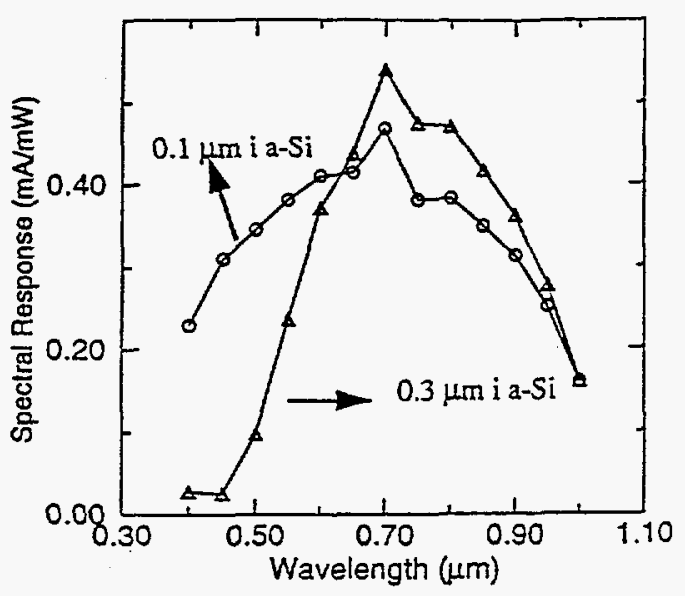

Fig. 4 Effect of a-Si thickness on the spectral response of the solar cells.

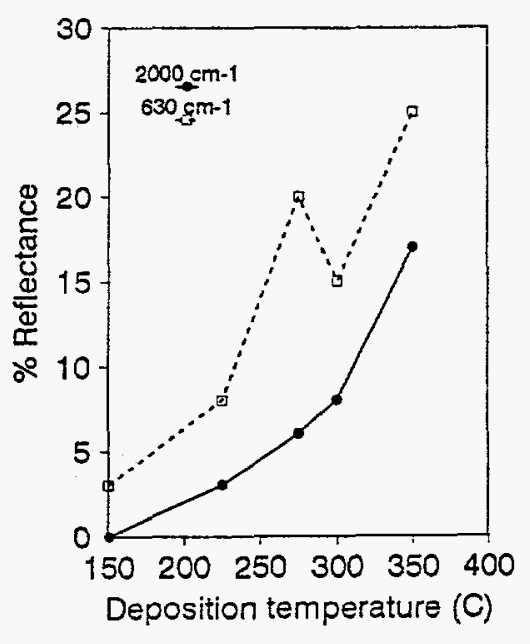

Fig. 6 Effect of a-Si:H deposition temperature on $\mathrm{SiH}$ intensity in the laser crystallized films. 
Title:

\title{
THEORETICAL ANALYSIS OF
} HYDROGEN-VACANCY COMPLEX FORMATION AND DIFFUSION IN Si

\author{
Organization: $\quad$ Physics Department, Texas Tech University \\ Lubbock, TX 79409-1051
}

Contributors:

Stefan K. Estreicher, principal investigator

\section{Objectives}

The diffusion of hydrogen in $S i$ has gained considerable importance in the past few years because of the ability of hydrogen to passivate the electrical activity of many defects which are electron-hole recombination centers. Recent work done at NREL[1-3] has revealed that the diffusivity of hydrogen is greater in vacancy-rich $S i$ material (such as poly-Si) than in others (notably FZ and CZ Si). The objectives of this research is to investigate theoretically the interactions involving vacancies and a range of impurities and defects (dopants, hydrogen, other vacancies, etc.), with emphasis on vacancy-hydrogen complex formation and diffusion. Our goal is to determine microscopic properties of the vacancy in $S i$ and of the following complexes: $\left\{V, H_{n}\right\}, n=1, \cdots, 4,\{V, B\}$ (and other acceptors), $\{V, P\}$ (and other donors), $\{V, V\}$ (the divacancy), and complexes of the type $\{V, X, H\}$, where $X$ is an acceptor, a donor, another vacancy, or an impurity such as carbon. When a defect may be mobile, molecular dynamics simulations are used.

\section{Theoretical Approach}

The equilibrium geometries, electronic configurations, dissociation energies, diffusion paths, and activation energies for diffusion are calculated using approximate $a b$-initio and $a b$-initio Hartree-Fock (HF) methods in molecular clusters. Whenever possible, the $a b$ initio HF calculations are complemented by Møller-Plesset corrections in electron correlation (see $[4,5]$ and references therein). We also use the $a b$-initio tight-binding molecular dynamics (MD) package developed by Sankey et al. [6,7] to study the dynamics of complex formation and dissociation, the vibrational frequencies associated with specific impurities, as well as impurity and complex diffusion. In the MD approach, the host crystal is represented by periodic supercells of about 64 atoms. For details about the theoretical techniques, their strengths and limitations, see Refs. [8].

The HF and MD codes ran on a dedicated IBM RS6000/375 at Texas Tech University as well as on a NEC SX-3/24G at the Swiss Center for Scientific Computing.

\section{The Vacancy and Vacancy-Hydrogen Complexes}

$V^{0}$ in $S i$ does not consist of 4 dangling bonds in $T_{d}$ symmetry but undergoes a slight tetragonal $\left(D_{2 d}\right)$ outward distortion (Fig. 1). This must occur since $V^{0}$ is an orbital triplet in $T_{d}$ symmetry and is therefore Jahn-Teller unstable.[9] Our geometry optimizations at the HF level are consistent with this. The MD technique provides a similar result for finite temperatures. The distortion becomes very small if quenching is allowed down to $T=0 \mathrm{~K}$. 
The distortion results in the formation of two long, covalent bonds. They are stretched by about $60 \%$ relative to a normal $S i-S i$ bond, but the overlap corresponds to nearly $75 \%$ of a two-electron covalent bond. The overlap between $S i$ atoms in different pairs is nearly zero.

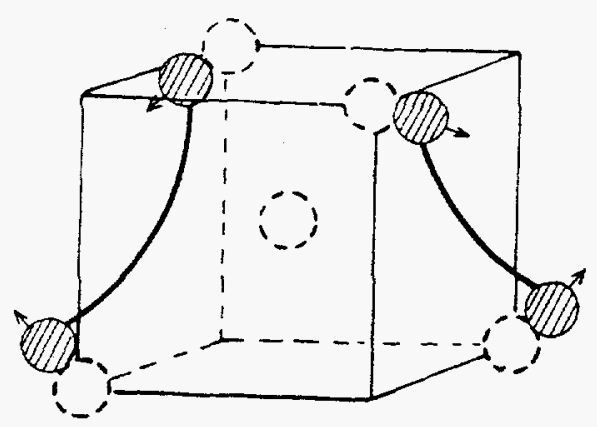

FIG. 1. Reconstructed neutral vacancy in Si. In the reconstructed bonds, the $\mathrm{Si}-\mathrm{Si}$ internuclear distance is $3.759 \AA$, and the amount of covalent (bonding) overlap amounts to $73 \%$ of a two-electron bond. As for Si atoms in different pairs, $\mathrm{Si}-\mathrm{Si}=3.929 \AA$, and there is no covalent overlap. The symmetry is $D_{2 d}$. The dashed circles show the perfect lattice sites.

The optimized configurations of the four $\left\{V, H_{n}\right\}$ complexes are shown in Fig. 2. The figure gives the calculated bond lengths (with degrees of bonding in parenthesis) and the symmetry of the resulting complex. The 'degree of bonding' $[10]$ is a measure of the amount of covalent overlap ( 1.00 is a perfect two-electron covalent bond) but is not a measure of the bond strength.

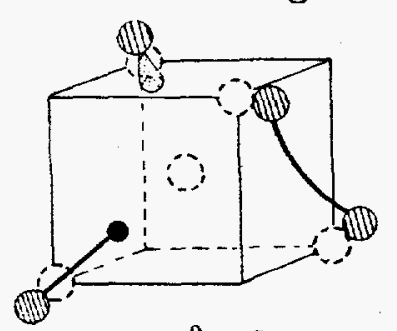

$\left\{\mathrm{V}, \mathrm{H}_{1}\right\}^{0} \quad \mathrm{C}_{1}$ $\mathrm{Si}--\mathrm{Si}=3.880 \AA(0.73)$ $\mathrm{Si}-\mathrm{H}=1.397 \AA(0.91)$

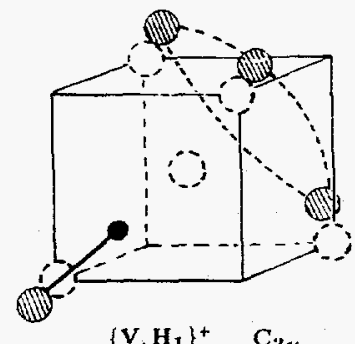

$\left\{\mathrm{V}, \mathrm{H}_{1}\right\}^{+} \quad \mathrm{C}_{3 \mathrm{~V}}$ $\mathrm{Si}--\mathrm{Si}=4.160 \AA(0.28)$ $\mathrm{Si}-\mathrm{H}=1.381 \AA(0.92)$

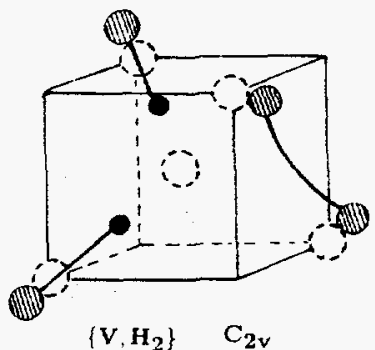
$\begin{aligned} \mathrm{Si}-\mathrm{Si}=3.757 \AA & (0.75) \\ \mathrm{Si}-\mathrm{H}=1.387 \AA & (0.97)\end{aligned}$

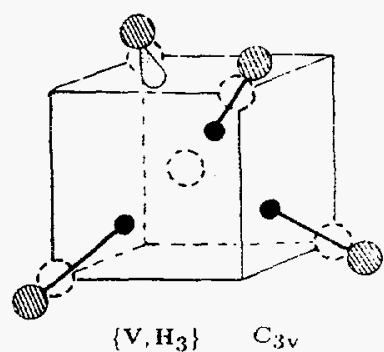

$\mathrm{Si}-\mathrm{H}=1.382 \mathrm{~A}(0.98)$

FIG. 2. Calculated configurations of the neutral $\left\{V, H_{n}\right\}$ complexes in $\mathrm{Si}$, with $n=1, \ldots, 4$, and of $\left\{\mathrm{V}, \mathrm{H}_{3}\right\}^{+}$. The symmetry, internuclear distances (in $\AA$ ) and degrees of bonding (between parentheses) are indicated.

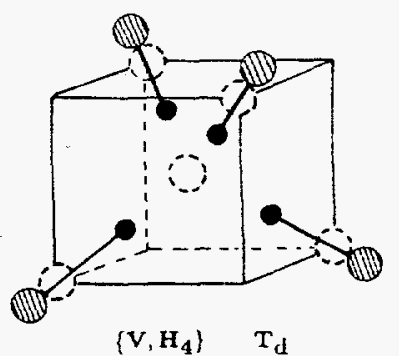

$\left\{V, H_{4}\right\} \quad T_{d}$
$S i-H=1.378 \AA(0.08)$

The stretching vibrational frequencies[11] of $H$ in the four complexes calculated with the MD code at room temperature are $2168,2268,2301$, and $2334 \mathrm{~cm}^{-1}$ for $\left\{V, H_{n}\right\}$ with $n=1,2,3$, and 4 , respectively. These frequencies are in rather good agreement with the most recent (low temperatures) experimental values[12] $2060,2120,2160$, and $2220 \mathrm{~cm}^{-1}$. Note that an error of the order of $5 \%$ is in fact better than the accepted accuracy of most such calculations.

The calculated dissociation energies of the complexes were discussed in the previous report. They are of the order of 2.5 to $3.5 \mathrm{eV}$ per $H$ atom, showing that the vacancy is a strong trap for one to four hydrogen interstitials. 
Finally, we investigated the interactions of an isolated $H$ interstitial with an isolated vacancy in the same supercell. Several MD simulations were performed in the temperature range 1000 to $1600 \mathrm{~K}$ with two possible initial configurations. First, we considered $V^{0}$ and hydrogen at a tetrahedral interstitial site, $H_{T}^{0}$, located $4.8 \AA$ away from $V^{0}$. Then, we started with interstitial hydrogen at a relaxed bond-centered site, $H_{B C}^{0}$, located $8.3 \AA$ away. The result of the $V^{0}+H_{T}^{0}$ case is illustrated in Fig. 3. The trajectory of $H_{T}^{0}$ is shown as the small open circles, the initial position is marked by an (i). Hydrogen hops from $T$ to $T$ site, via the hexagonal interstitial site. It remains in the low-density region of the crystal throughout. The initial position of the vacancy is the large dashed circle. In the final configuration, the vacancy has jumped one step and $H$ saturates one of the dangling bond.

FIG. 3. Trajectories of the neutral vacancy (initially, the dashed circle) and hydrogen (initial $\mathrm{T}$ site marked by the (i)) at $1200 \mathrm{~K}$ in a 64 atom supercell. Pair formation occurs in about 0.25 picosecond. The full circle shows the silicon atom to which hydrogen ends up bound.

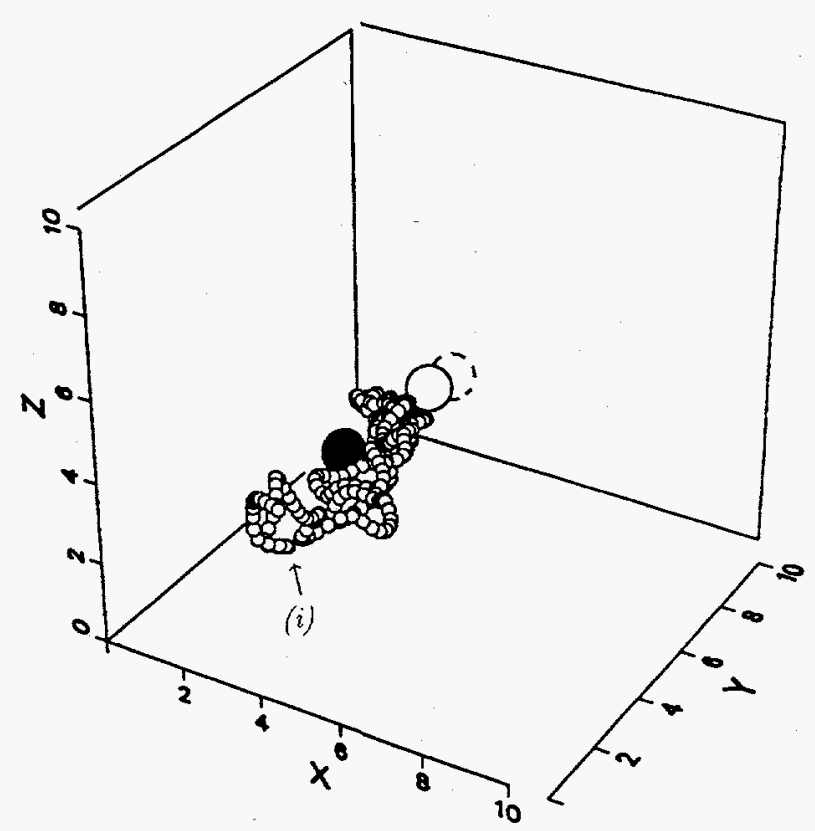

The vacancy and hydrogen attract each other and form the $\left\{V, H_{1}\right\}$ pair in less than $0.25 \mathrm{ps}$. $H$ moves toward $V$ and $V$ moves toward $H$. In the $V^{0}+H_{B C}^{0}$ case, a similar capture process occurs, but $H$ moves slower, jumping from a $\mathrm{BC}$ to another $\mathrm{BC}$ site via a $\mathrm{C}$ site. Pair formation occurs in about $1 p s$.

\section{Diffusion Properties}

The simulation times were too short to obtain the diffusivities of the vacancy and of hydrogen as a function of time. At $300 \mathrm{~K}$, the diffusivity of $V^{0}$ was found to be $1.1 \cdot 10^{-4} \mathrm{~cm}^{2} / \mathrm{s}$, a value consistent with experimental data. Total energy differences calculated at that temperature show an activation energy of the order of $0.5 \mathrm{eV}$, in close agreement with the measured value[9] $(0.45 \pm 0.045 \mathrm{eV})$.

The mobility of the $\left\{V, H_{1}\right\}$ pair was also investigated using the MD code. In 3,000 time steps (which represents only 1 ps real time but about one week of CPU time) at 1000 and $1600 \mathrm{~K}$, the pair did not diffuse. However, one of the $S i$ nearest neighbor to $\left\{V, H_{1}\right\}$ made several attempts to jump into the vacancy. The activation energy for its motion is smaller than the same motion of the same atom without $H$ trapped in the vacancy. This result agrees well with the predictions of the HF calculations reported last year. The rate- 
limiting step in the diffusion of $\left\{V, H_{1}\right\}$ is the break-up of the $S i-H$ bond in the vacancy and the re-formation of a new $S i-H$ bond to the $S i$ atom moving into the vacancy.

It may be that the prefactor is too low or the activation energy too high for this jump to be observed in less than a ps. It is also possible that the diffusion of the pair is enhanced by the capture of an electron or hole.

\section{Vacancy-impurity-hydrogen complexes}

This part of the research is still in progress. Preliminary results are as follows. The geometries of the ionized and neutral acceptors $B, A l$, and $G a$ and donors $P$ and $A s$ have been calculated at the approximated ab-initio HF level. Preliminary configurations, electronic structures, and dissociation energies of the $\{V, B\}$ pair and the $\{V, B, H\}$ complex have also been obtained.

The key result here is that hydrogen substantially increases the dissociation energy of $\{V, B\}$. Thus, $\{V, B, H\}$ remains bound at temperatures much higher than those at which $\{V, B\}$ breaks up. This result may be of importance if the formation of vacancy-dopant pairs plays a role in the nucleation of hydrogen platelets.

\section{References}

1. B.L. Sopori, MRS Proc. 262, 407 (1992).

2. B.L. Sopori, K. Jones, and X.J. Deng, Appl. Phys. Lett. 61, 2560 (1992).

3. B.L. Sopori, X. Deng, J.P. Benner, A. Rohatgi, P. Sana, S.K. Estreicher, Y.K. Park, and M.A. Roberson, Proc. First World PV conf. (IEEE Press, to be published).

4. M.A. Roberson, S.K. Estreicher, L. Korpás, and J.W. Corbett, Mat. Sci. Forum 143147, 1227 (1994)

5. M.A. Roberson and S.K. Estreicher, Phys. Rev. B 49, 17040 (1994).

6. O.F. Sankey and D.J. Niklewski, Phys. Rev. B 40, 3979 (1989).

7. O.F. Sankey, D.J. Niklewski, D.A. Drabold, and J.D. Dow, Phys. Rev. B 41, 12750 (1990).

8. S.K. Estreicher, MRS Proc. 240, 643 (1992). An extensive review is to be published soon: S.K. Estreicher, Mat. Sci. Engr. Reports (in print).

9. G.D. Watkins in 'Deep Centers in Semiconductors', ed. S.T. Pantelides (Gordon and Breach, New York 1986).

10. D.R. Armstrong, P.G. Perkins, and J.J.P. Stewart, J. Chem. Soc. Dalton Trans. (1973), p. 838.

11. Y.K. Park, S.K. Estreicher, C.W.Myles, and P.A. Fedders, submitted to Phys. Rev. B.

12. B. Bech Nielsen, private communication. 


\title{
Title: $\quad$ New Plasma Source of Hydrides for Epitaxial Growth
}

\author{
Organization: Department of Chemical Engineering, University of Florida, \\ Gainesville, Florida
}

Contributors: T. J. Anderson and B. J. Stanbery

\section{Objectives}

The objective of this research effort is to establish that the use of plasma-activated reactants with an electron cyclotron resonance source for semiconductor epitaxy is a viable method and can be used to prepare $\mathrm{CuInSe}_{2}$ films of a quality suitable for high efficiency solar cells.

\section{Approach}

To achieve these goals we have combined a novel plasma-activated selenium source with conventional evaporation sources for copper and indium to enable the development of Electron Cyclotron Resonance Plasma-Assisted Epitaxy (ECR-PAE) of CuInSe ${ }_{2}$. The selenium source is designed to excite and dissociate the polyatomic elemental vapor exiting from the aperture of an effusion cell, and to react that flux with a stream of gas injected separately into the discharge. No other ECR sources reported in the literature, to our knowledge, utilize such a combination of elemental and gas sources. This provides more control of the precursor-formation processes than prior approaches by permitting the use of mixtures of inert gas and hydrogen, so that dissociation, activation, and hydrogenation processes within the plasma may be adjusted relative to one another.

This ECR plasma "cracker" consists of a cavity within an magnetic field-generation and flux shunt assembly which creates a magnetic mirror profile for plasma confinement. Microwave power at $2.45 \mathrm{GHz}$ is coupled to the cavity via a high temperature coaxial microwave cable, and coupled within the cavity to the plasma by a helical antenna [1] designed to couple efficiently to the "Rwave" eigenmode of the coupled electromagnetic wave and plasma system. The reactants are isolated from the cavity walls by a sapphire tube in order to prevent unwanted deposition within the source, and to insure that all of the escaping reactant flux is directed toward the substrate. Thus this ECR source enables the in situ generation of hydride precursors for plasma-assisted epitaxial growth utilizing the safer elemental reactants instead of the hydrides (in this case selenium and hydrogen instead of hydrogen selenide).

\section{Results and Discussion}

Our principal activities during this fiscal year's efforts have been installation and facilitization of the reactor within which the ECR plasma source operates, and further evaluation of the source's operation based on characterization conducted under a prior contract. A CIS deposition system and combined SEM/EDX/WDX analysis system which were donated to the University of Florida have been installed at the MICROFABRITECH ${ }^{\circledR}$ Central Processing Facility in Gainesville. 
CIS film growth will take place in a CHA SE-1000 diffusion-pumped $\mathrm{LN}_{2}$-trapped baseplate system and feedthrough collar with a custom built stainless steel bell-jar built by MDC Vacuum Products Corporation. Inside is a rotating molybdenum disc substrate platen with a capacity of up to nine 3 " diameter substrates revolving above both a dual filament EPI, Inc. copper effusion cell and EPI single modified filament indium effusion cell. Rate control is provided by a Leybold-Hereaus Inficon Sentinal III with both EIES sensors for monitoring and controlling the metals deposition process and quartz crystal oscillators for calibration. The system incorporates custom boron nitride-coated radiant heaters designed for operation to $1000^{\circ} \mathrm{C}$ and used for substrate temperature control, and a load-lock for the improved source stability that results from reduced oxidation and thermal cycling of sources during sample changes. A custom cryoshroud, purchased under this contract, has been added for high selenium pumping speeds, low background pressure, and isolation of the film growth zone from the rest of the system. The reactor can simultaneously grow on up to nine substrates per run, each mounted on a separate, removable sample holder. The stainless steel water-jacketed chamber walls include ports with source shutters (also purchased under this contract) for the copper and indium. A feedthrough adjacent to the plasma source is designed specifically for an EPI, Inc. thermal cracking effusion cell, with its beam flux coincident on the growth surface with the source beam from the ECR plasma cracker source for selenium. The ECR plasma source is mounted onto a water-cooled baffle which improves control and further isolates the group VI sources from the metal evaporation sources. Microwave power is provided by an Astex S-250 power supply. A Leybold-Hereaus Quadrex 200 residual gas analyzer with a separate turbomolecular pump is attached to the load-lock port, and a Balzers QMG 420 quadrapole mass analyzer (cross-beam configuration) is attached to the bell-jar directly above the plasma source for direct measurement of the ion and flux mass distribution.

Dedicated ex-situ analytical support is provided by an Hitachi S-450 scanning electron microscope for characterization of microstructural morphology. It is also equipped with two $\mathrm{x}$-ray analytical instruments, a Princeton Gamm-Tech System 4 EDX elemental analyzer and a Peak high resolution wavelength dispersive $X$-ray crystal spectrometer to resolve the narrowly seperated X-ray emissions of indium and zinc. These instruments will be utilized for quantitative elemental composition analysis of the CuInSe ${ }_{2}$ on ZnTe. A Leitz Phase Contrast Microscope is also available in the laboratory for evaluation of the larger scale microstructure of the films.

Facilitization of the MICROFABRITECH ${ }^{\circledR}$ laboratory space for these systems has required the installation of two additional power lines from the building's central distribution point; extension of the building chilled water system; design and construction of an exhausted pump enclosure to meet ES\&H requirements; purchase and installation of a Novapure exhaust gas scrubber for neutralizing hydrogen selenide; installation and interfacing of hazardous gas detection and emergency shut-off interlocks with the building alarm systems; and the installation of a high purity gas delivery system (which includes a SemiGas Nanochem 3000 point-of-use gas purifier) to the ECR source .

\section{Summary and Conclusions}

The development of the ECR-PAE process for single crystal CuInSe $\mathrm{C}_{2}$ heteroepitaxy on both (111)and (100)-oriented $\mathrm{ZnTe}$ wafers is the starting point of further development. The proposed 
technique, utilizing elemental copper, indium, and selenium from independently controllable sources will enable us to investigate various nucleation options and their consequences during the course of this work. Furthermore, the unique ability of this source to introduce into the plasma both chemically active gases such as hydrogen and inert gases such as helium will be employed to seek insight into the roles of: physical processes such as intramolecular excitation, molecular dissociation, charge and momentum transfer, and UV irradiation; and chemical processes such as surface hydrogenation and hydride precursor formation.

We have constructed an epitaxial growth system for $\mathrm{CuInSe} \mathrm{H}_{2}$ incorporating a novel Electron Cyclotron Resonance (ECR) plasma source to activate mixtures of an injected gas and the selenium vapor flux of a conventional MBE effusion cell. The source is installed in a system ideally suited to atomic layer-by-layer film epitaxial film growth, a deposition mode known as Migration Enhanced Epitaxy (MEE). [2] We will utilize the system during the remainder of this contract to develop a unique, low-temperature Plasma-Assisted Epitaxy (ECR-PAE) technique for the growth of high-quality single-crystal $\mathrm{CuInSe}_{2}$ epilayers for photovoltaic applications.

\section{References}

1. Kraus, J.D., "The Helical Antenna," Antennas, $2^{\text {nd }}$ edition, New York: McGraw-Hill Book Company, 1988.

2. Horikoshi, Y. and M. Kawashima, "Migration-Enhanced Epitaxy of GaAs and AlGaAs," Workbook of the Fifth International Conference on Molecular Beam Epitaxy, Sapporo, Japan: Tokyo Institute of Technology, p. 25-31. Conference held in 1988. 
Title:

Organization:

Contributors:
Fundamental Studies of the Effect of Crystal Defects on $\mathrm{CuInSe}_{2} / \mathrm{CdS}$ Heterojunction Behavior

Department of Materials Science and Engineering University of Illinois

Urbana, IL

J. Benner, program manager; A. Rockett, principal investigator; G. Kenshole, visiting scientist; H.Z. Xiao, post-doctoral research associate; G. Berry, D. Schroeder, L.-C. Yang, graduate research assistants; A. Lee, A. Minervini, K. Guillims, undergraduate research assistants.

\section{Objectives}

The project seeks to determine the source of sample-to-sample variability in $\mathrm{CuIn}_{1-x} \mathrm{Ga}_{\mathrm{x}} \mathrm{Se}_{2}$ (CIGS)-based solar cell performances and the mechanisms restricting carrier collection at large voltages in CIGS/CdS heterojunctions. The detailed objectives involve determining the relative importance of structural and chemical defects in the material and how they affect electronic properties and hence device performance. A byproduct of the project is fundamental data on the properties of CIGS alloys as a function of processing conditions and composition. Because much variability in the devices results from adhesion failures at the back contact, a portion of the research program has been devoted to the development of novel back contacts. Finally, as a university research program the goals include the training of graduate students and post-doctoral researchers and the exposure of as many individuals to research in renewable energy as possible (see participant list, above).

\section{Approach}

The basic approach is to grow the highest quality single crystals of CIGS possible under a variety of conditions and to measure the morphology, microstructure, and properties of the resulting layers. These results are then compared with results on polycrystals grown simultaneously and at other laboratories to determine the relative significance of the observed defects. Novel contact studies have primarily concerned development of metastable alloys containing one or more component which dissolves in the growing film.

The results described were recorded for CIGS deposited in a single step from magnetron sputtered $\mathrm{Cu}, \mathrm{Ga}$ and In fluxes and evaporated molecular Se. Polycrystalline CIS films were deposited on Corning type 7059 borosilicate or common soda-lime glass substrates coated with Mo, Cu-Mo, or Cu-Ga-Mo metastable alloys. The single crystal CuIn $\mathrm{Se}_{5}$ and $\mathrm{CuIn} \mathrm{In}_{1-\mathrm{x}} \mathrm{Ga}_{\mathrm{x}} \mathrm{Se}_{2}$

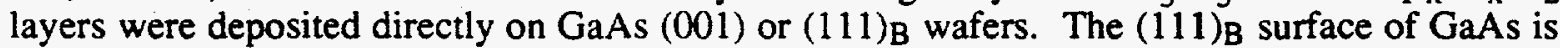
As-terminated and gives the best surface morphology for the CIS epitaxial layers. The substrates were heated to deposition temperatures $\mathrm{T}_{\mathrm{S}}$ of $\geq 550^{\circ} \mathrm{C}$. A mechanical shutter prevented deposition during magnetron and effusion cell start-up and target sputter-cleaning. Targets used were pure $\mathrm{Cu}, \mathrm{In}$, or $\mathrm{Cu}-\mathrm{In}$ or $\mathrm{Cu}-\mathrm{Ga}$ alloys. All targets were cast as solid solutions with the exception of the $>25$ at. \% Ga Cu-Ga targets which were hot pressed from powders.

Microstructural studies were carried out by X-ray diffraction (XRD) and by transmission electron microscopy (TEM) using Philips 400,420 and 430 instruments, two of which were equipped with EDAX energy-dispersive microchemical analysis instruments (EDS). Composition analyses were also carried out by secondary ion mass spectrometry (SIMS), and by EDS during scanning electron microscopy (SEM). Films were formed either as single layers from fixed 
fluxes of constituents (for epitaxy) or as bilayers beginning with a strongly $\mathrm{Cu}$-rich region and finishing with an In-rich layer (for polycrystals). Cathodoluminescence measurements were carried out in a Zeiss SEM at a temperature of $\sim 4 \mathrm{~K}$. Hall-effect measurements were obtained using In or evaporated $\mathrm{Au}$ contacts arranged in a van der Pauw pattern. All contacts were checked for ohmic character and resistivity to assure that the measurements were not influenced by the contacts. Currents and voltages were computer controlled and were recorded as a function of temperature.

\section{Results (10/1/93 - 9/30/94)}

Selected structural defects in single crystals of CIGS were characterized and their effect on the electrical properties determined during this reporting period. Record hole mobilities ranging from 250 at $300 \mathrm{~K}$ to 1500 at $100 \mathrm{~K}$ were recorded in near stoichiometry films. Native point defects were found to order in high resistivity n-type material. These defects are valence compensating and increase the energy gap of the material. Variations in local ordering apparently give rise to subgap optical activity consistent with band tails. In p-type material the defects have concentrations consistent with values predicted from theory and cause doping. Stacking faults appear to be optically active and have a major effect on the growth rate of the CIGS. The mechanisms for improved adhesion in Cu-Mo metastable alloy contacts have been demonstrated to be due to phase separation and partial dissolution of the $\mathrm{Cu}$ during growth as well as by strain relaxation in the deposited films. Each of these points is discussed in more detail below.

\section{Near Stoichiometry CIGS}

Near stoichiometry epitaxial (CIGS) films were grown on semi-insulating GaAs (111) As at temperatures between 600 and $735^{\circ} \mathrm{C}$. The resulting films were 1 to $2.3 \mu \mathrm{m}$ thick. Ga was not intentionally added but was present in the films by outdiffusion from the substrate. The stoichiometry was controlled by adjusting the relative currents to the $\mathrm{Cu}$ and In targets. Excess Se (five times the combined metals flux) was supplied simultaneously. Samples were analyzed by SEM, TEM, and Hall Effect. SEM and TEM showed the samples to be single crystal epitaxial layers devoid of second phases with defects as observed previously in epitaxial layers. Hall effect measurements were carried out at and below room temperature. At the lowest temperatures the samples exhibited composition-dependent type conversion and increases in resistivity which prevented further measurements. Samples grown below $700{ }^{\circ} \mathrm{C}$ showed a decreasing $\mathrm{Ga}$ concentration toward the surface of the films while samples grown at higher temperatures showed no change in $\mathrm{Ga}$ concentration in the films. Single crystal epitaxial layers with $\mathrm{Ga}$ added by replacement of the $\mathrm{Cu}$ target with $\mathrm{Cu}-\mathrm{Ga}$ alloys have been grown on $(111)_{\mathrm{B}}$ and (001) GaAs substrates by the hybrid method. Results show that films containing intentionally added Ga have structures and surface morphologies consistent with those of pure CIS epitaxial layers. Less $\mathrm{Ga}$ outdiffusion from the GaAs substrate was observed in these layers.

All films (ranging from slightly $\mathrm{Cu}$-rich to slightly In-rich) were p-type at room temperature. Samples containing more In converted to highly resistive n-type layers at higher temperatures consistent with the presence of valence compensating point defects (see below). One sample containing the most $\mathrm{Cu}$ remained p-type down to $25^{\circ} \mathrm{C}$ (data in Fig.1). All samples exhibited similar resistivity and hole mobility values beginning at hole concentrations of $\sim 10^{17} \mathrm{~cm}^{-3}$ and mobilities of $200 \mathrm{~cm}^{2} / \mathrm{V}-\mathrm{sec}$ and with hole concentrations decreasing and mobilities increasing as the temperature dropped. The carrier concentration decreased from $10^{17} \mathrm{~cm}^{-3}$ at room temperature to $\sim 10^{15} \mathrm{~cm}^{-3}$ with a characteristic energy of $80 \mathrm{meV}$. At $\sim 10^{15} \mathrm{~cm}^{-3}$ holes the behavior became dominated either by a $40 \mathrm{meV}$ level or by a type conversion to n-type carriers. The results are consistent with the presence of three defects, a deeper acceptor level present at 
$\sim 10^{18} \mathrm{~cm}^{-3}$ and two shallower and composition-dependent levels present at $\sim 10^{15} \mathrm{~cm}^{-3}$ concentrations. A more detailed analysis is currently underway. Cathodoluminescence imaging of the samples showed the presence of triangular bright features which corresponded to the location of triangular pyramidal islands protruding from the surface. Cross-sectional TEM showed that stacking faults commonly underlie the surface but that faults are generally absent from under the islands. We propose that the faults modify the surface chemistry by inverting the surface polarity. The surface then grows rapidly between the faults in order to form facets of the lower energy surface. Further, we conclude that the stacking faults decrease the optical emission intensity and are thus probably minority carrier recombination centers. Stacking faults and twins in the films were shown to be converted to dislocations by rapid thermal annealing (RTA) in $\mathrm{N}_{2}$ at $700^{\circ} \mathrm{C}$ for 60 seconds.
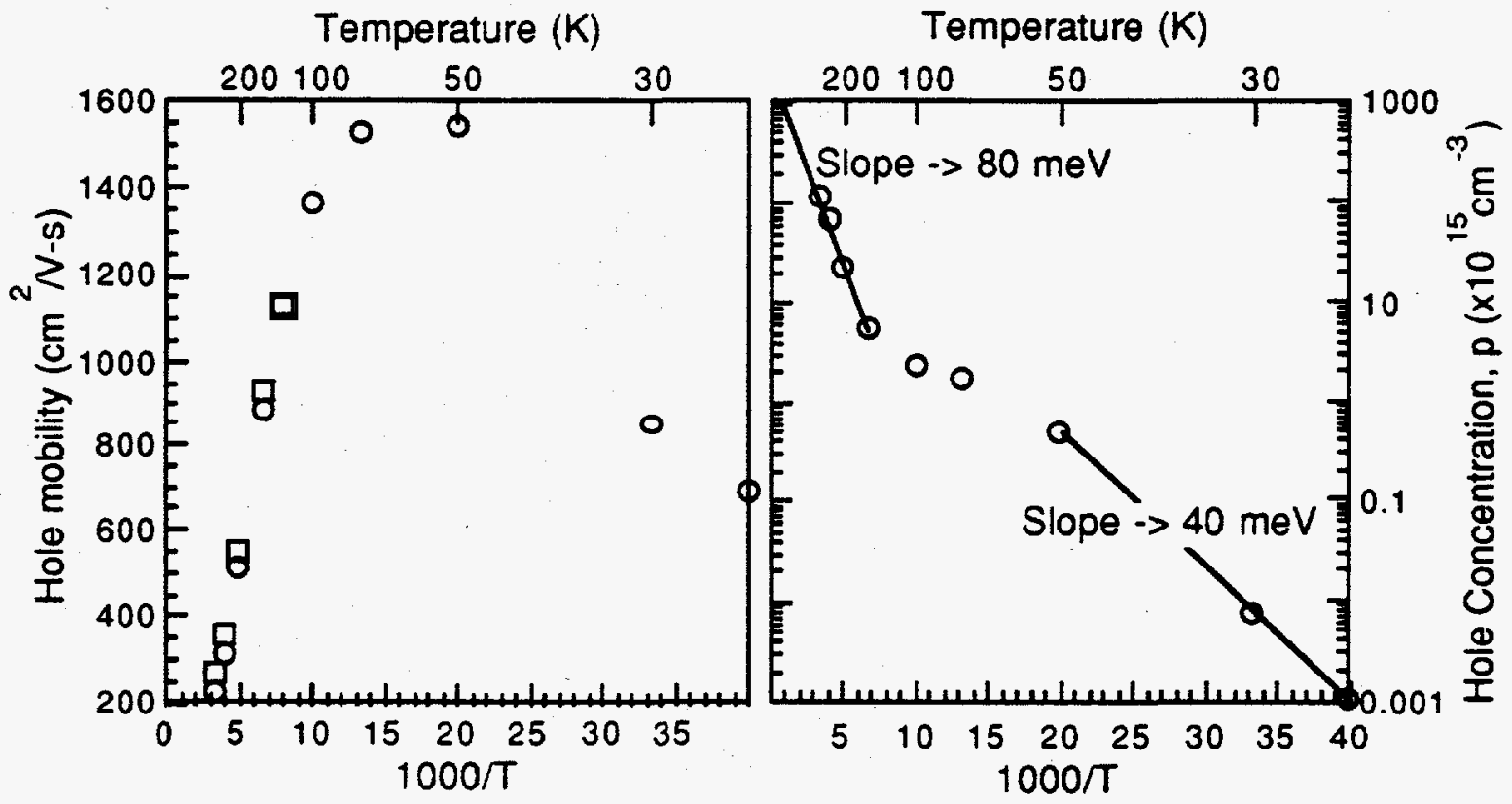

Figure 1. Hall mobility and hole concentrations for two epitaxial CIS layers as a function of temperature. The hole concentration shows evidence for two acceptor states.

\section{In-rich Ordered Vacancy Compounds}

The surface stoichiometry of CIGS as a function of composition is critical to resulting solar cell performance. Only group III-rich CIGS films yield active photovoltaic devices. These are thought to include a strongly In-rich defect-ordered surface phase with a composition of approximately $\mathrm{CuIn}_{3} \mathrm{Se}_{5}$. To study $\mathrm{CuIn}_{3} \mathrm{Se}_{5}$ in more detail, single crystal $\mathrm{CuIn}_{3} \mathrm{Se}_{5}$ epitaxial films were synthesized on $\mathrm{GaAs}(001)$ and $(111)_{\mathrm{B}}$. The microstructure, microchemistry, and selected electrical and optical properties of the films were investigated by SEM, EDS, TEM, cathodoluminescence $(\mathrm{CL})$, optical absorption and reflection, and four-point probe resistivity measurements. The basic growth mechanism and structural defects were found to be similar to those of near stoichiometry CIS. Optical transmission and reflection data were recorded as a function of wavelength and photon energy. An absorption coefficient $\alpha \sim 5 \times 10^{3} \mathrm{~cm}^{-1}$ for 1.25 $\mathrm{eV}$ photons increasing to $\sim 2 \times 10^{4} \mathrm{~cm}^{-1}$ for $1.4 \mathrm{eV}$ light was observed. The absorption coefficient decreased exponentially below $\sim 1.4 \mathrm{eV}$ with a characteristic energy of $\sim 85 \pm 10 \mathrm{meV}$, which is much slower than for polycrystalline stoichiometric CIS. Similar effects were observed by CL recorded at a temperature of $4 \mathrm{~K}$. The broad photon emission spectrum peaked at $\sim 1.18 \mathrm{eV}$ and 
decreased nearly exponentially from an energy just below the maximum with a characteristic energy of $75 \pm 5 \mathrm{meV}$. Four-point probe measurements showed that the epitaxial layers were ntype with resistivities of $>10^{5} \Omega-\mathrm{cm}$, consistent with values for In-rich polycrystalline CIS and much greater than slightly In-rich epitaxial CIS. Electron diffraction patterns from the asdeposited epitaxial $\mathrm{CuIn}_{3} \mathrm{Se}_{5}$ confirmed that $\mathrm{CuIn}_{3} \mathrm{Se}_{5}$ is a defect-ordered chalcopyrite compound in the epitaxial layers. Diffraction points were observed which are forbidden in the chalcopyrite structure but which are consistent with computer simulated ordered point defects on the (001) plane of the chalcopyrite structure.

\section{Improved Contact Materials}

We have continued our previous work on improved contact metallizations with a more detailed analysis of the mechanisms of improved adhesion in Cu-Mo alloy contacts. The as-deposited films are single phase metastable body-centered cubic alloys. Strain relief and grain growth occurred between 200 and $450^{\circ} \mathrm{C}$ and phase separation began above $450^{\circ} \mathrm{C}$. For pure Mo no grain growth would be expected at $450^{\circ} \mathrm{C}$. The film orientation changed during grain growth yielding a strong increase in the Mo (110) XRD reflection. The compositions of CIS layers deposited at $400^{\circ} \mathrm{C}$ on $\mathrm{Cu}$-Mo alloys had compositions similar to layers on pure Mo. At $450^{\circ} \mathrm{C}$, deposition for 30 min resulted in an $\sim 10 \%$ increase in $\mathrm{Cu}$ atom fraction while at $500^{\circ} \mathrm{C}$ greater increases in $\mathrm{Cu}$ content occurred. In no case was all of the $\mathrm{Cu}$ in the substrate found to have diffused into the CIS layer. $\mathrm{Cu}_{0.3} \mathrm{Mo}_{0.7}$ exposed to a flux of only In and Se for $60 \mathrm{~min}$ at $450^{\circ} \mathrm{C}$ produced rough 200-800 nm-thick slightly $\mathrm{Cu}$-rich CIS layers on the back contact, consuming $\sim 10 \%$ of the $\mathrm{Cu}$ in the back contact in the process. It was concluded that $\mathrm{Cu}$ in the back contact could diffuse readily into the overlying layer but was not dissolved so fast that all the $\mathrm{Cu}$ in the back contact was consumed in each deposition. Similar results for CIS deposition on Cu-Ga-Mo metastable alloys were obtained although the increase in adhesion was less significant.

\section{Conclusions}

Results obtained to date indicate the basic nature of native defects in CIGS single crystals. The achievement of record mobilities in p-type CIGS shows that high-quality single crystals can be obtained. By comparison with polycrystal data it is clear that native point defects are common and probably beneficial in both the stoichiometric CIGS inside normal grains and in the ordered defect material typically present on surfaces. Extended defects can be detrimental to optical properties and carriers and should be minimized to the maximum extent possible.

During the remainder of the program we will expand on the results obtained in this contract period and will consider extrinsic point defects. In particular we will examine the effect of alkali metals on the properties of CIGS. In addition, we will attempt to simulate the segregated ordered defect structure in epitaxial layers by the deposition of highly In-rich material on near stoichiometry epitaxial layers. This may permit devices to be fabricated on CIGS single crystals with controlled thicknesses of ordered defect compound. The results should indicate the importance of this layer to the resulting device performance. 
University of Illinois
at Urbana-Champaign

The Engineering Sciences

Building

$1101 \mathrm{~W}$. Springfield Avenue

Urbana, IL 61801
College of Engineering

rocket!@ux1.cso.uiuc.edu

USA - 217-333-0417

USA - 217-244-1631 fax

14 February 1995

Ms. Kathy Summers

Project Assistant

NREL, Photovoltaic Program

1617 Cole Boulevard

Golden, CO 80401, USA

Dear Ms. Summers:

Please find enclosed a copy of my annual report for my NREL Universities Program project.

Please contact me immediately if you require changes. I prefer to communicate by electronic mail as it is fastest and most efficient but you are welcome to call me at work or at home as well. FAX works fine but is less reliable and no faster than email. I would be happy to send you a copy of this document as an attachment to an email message if that would be helpful.

In any case, I hope this report meets your needs. If it does not please let me know and I will modify it. A list of publications related to this project is attached below.

Cheers,

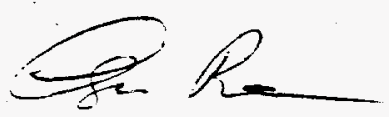

Angus Rockett

rockett@uxl.cso.uiuc.edu

Until August 1, 1995 contact me at:

Teknikum,Uppsala University, Tekniska Högskolan, Box 534, S-751 21 Uppsala, Sweden 011-46-18-18-3039 (office)

011-46-18-40-1772 (home)

011-46-18-55-5095 (FAX)

\section{Publications for this FY:}

L. Chung Yang and A. Rockett, "Cu-Mo Contacts to CuInSe 2 For Improved Adhesion in Photovoltaic Devices," J. Appl. Phys 75(2), 1185 (1994).

H. Xiao, L.-Chung Yang, and A. Rockett, "Structural, Optical and Electrical Properties of Epitaxial Chalcopyrite CuIn3Ses Films," J. Appl. Phys. 76(3) 1503 (1 August 1994).

L.-Chung Yang, H. Xiao and A. Rockett, W. N. Shafarman and R.W. Birkmire, "The Fabrication of Device-quality Bilayer CuInSe 2 Films by the Hybrid Sputtering and Evaporation Method", Solar Energy Materials \& Cells, in press.

H.Z. Xiao, L.-Chung Yang, S.L. Lai, Z. Ma, and A. Rockett, "Structural Properties of Metastable Cu-Mo Solid Solution Thin Films Synthesized by Magnetron Sputtering", Scripta Metallurgica et Materialia, in press.

L. Chung Yang, G. Berry, H.-Z. Xiao, and A. Rockett, "Structure and Optoelectronic Properties of Single Crystal Epitaxial CuIn $1-x \mathrm{Ga}_{x} \mathrm{Se}_{2}$ and Ordered Defect Compounds", Proceedings of the First World Conference on Photovoltaic Energy Conversion, 5-9 Dec, 1994, Waikoloa, Hawaii (Institute of Electrical and Electronics Engineers, New York, 1995) in press. 


\section{Title: Optimization of Gettering Processes for Photovoltaic Silicon}

Organization: Center for Microelectronics Research, University of South Florida, Tampa, Florida

Contributors: $\quad$ L.Jastrzebski, principal investigator, S.Ostapenko, G.Novak, J.Lagowski

\section{Objectives}

Our effort focused on optimization and development of various gettering approaches which were used to improve the diffusion length $(\mathrm{L})$ of commercial poly-Si. Polycrystalline wafers are very inhomogeneous and variations by a factor of ten in $L$ values across the wafer are very common. To generate a statistically valid data base of the diffusion length, 177-point mapping was performed prior to and after gettering using the surface photovoltage (SPV) technique. The details of the gettering treatment and measurement procedures have been described in previous reports.

\section{$\mathrm{P}$ and $\mathrm{Cl}$ gettering}

The study of this gettering approach had been completed. The most important findings are summarized below. It was found that $\mathrm{P}$ and $\mathrm{Cl}$ gettering results in a diffusion length improvement only in long diffusion length regions of the wafer. The improvement of $L$ values was well correlated with the reduction of the dissolved heavy metal concentration. In wafer regions with low diffusion lengths, this type of gettering did not improve the minority carrier lifetime. It appears that grown defects controll a recombination in these regions and their recombination properties are not affected by $\mathrm{P}$ and $\mathrm{Cl}$ gettering.

\section{Al/RTA and furnace gettering}

The effects of $\mathrm{Al}$ gettering during RTA and furnace annealing were investigated as a function of annealing temperature, time and cooling rate for two types of poly-Si: casted from Solarex and EFG from Mobil. The investigated samples exhibited very large variations in the L value across the wafer (up to 10 times), therefore, SPV mapping of the diffusion length prior to and after processing was used to assess changes induced by Al gettering. A typical histogram of the diffusion length distribution before and after RTA of the Solarex samples is shown in Fig. 1a. Fig.1b shows the ratio of diffusion length improvement $\left(\mathrm{L}_{\mathrm{after}} / \mathrm{L}\right.$ before $)$ in different regions of the sample plotted as a function of the initial $\mathrm{L}$ value. The largest improvement of $\mathrm{L}$ (by factor of 3.5) is observed in regions with low $L$ and the smallest in regions with large $L$ values. The magnitude of the effect is a function of the RTA parameters (Fig.2a,b). The effect is clearly associated with $\mathrm{Al}$ since no improvement was observed for samples annealed without $\mathrm{Al}$ (Fig.2b). The best results were obtained : 1) at relatively low temperatures $\left(650^{\circ} \mathrm{C}\right.$ to $\left.800^{\circ} \mathrm{C}\right)$; 2) at short RTA times ( 100 to $200 \mathrm{sec}$ ), and 3) with a slow ramping rate of $30^{\circ} \mathrm{C}$. There is some difference in the optimum annealing conditions for the Solarex and Mobil wafers as shown by a comparison of the data in Fig.2c. In the case of casted poly-Si, the improvement does not deteriorate when the RTA time is extended to 300 sec.

The results of conventional furnace annealing of the EFG samples are presented in Fig.2d. In contrast to the RTA results, the largest improvement is achieved at the highest temperature of $900^{\circ} \mathrm{C}$. At the lower temperature range of $600^{\circ} \mathrm{C}$ to $750^{\circ} \mathrm{C}$, there is also an improvement of $\mathrm{L}$ in samples annealed without $\mathrm{Al}$. A substantial improvement of $\mathrm{L}$, due to $\mathrm{Al}$ gettering, took place only for the samples annealed at the highest temperatures $\left(800^{\circ} \mathrm{C}\right.$ to $\left.900^{\circ} \mathrm{C}\right)$. 


\section{Gettering by ultrasound treatment (UST)}

In the last year we discovered that UST could increase the lifetime of minority carriers in solar-grade poly-Si wafers [1,2]. Fig. 3 shows the diffusion length line scans across a casted poly-Si wafer before and after UST. The improvement effect is substantial in the region with relatively low diffusion length value. This effect is stable versus post-UST holding time. Piezoelectric transducers (PZT-5H ceramics with $\mathrm{Cr} / \mathrm{Au}$ electrodes) were used as a source of ultrasound vibrations on resonance frequency of either a radial mode $(50 \mathrm{kHz})$ or a thickness mode $(590 \mathrm{kHz})$. The samples subjected to UST were tightly bound by the back side to the surface of a transducer. The UST is specified by parameters: 1) the voltage of UST generator, $V_{u s}=1-40 \mathrm{~V}$, which is proportional to the amplitude of an acoustic strain generated by a transducer; 2) the holding time, $\left.t_{u s}=10-60 \mathrm{~min} ; 3\right)$ the temperature of a sample during UST, $\mathrm{T}_{\mathrm{us}}=20-100^{\circ} \mathrm{C}$, and 4) frequency or mode of ultrasound vibrations. Principles of UST technique were presented in previous reports.

Recently we applied the UST technique to commercial polycrystalline Si solar cells. Fig.4 presents a distribution of diffusion length measured in Solarex solar cell before and after UST. As the result of the UST it shows a statistically significant improvement in the diffusion length distribution. The change of the solar cell parameters due to UST are shown in the Table I. The increase of current and efficiency are measured at 0.1 sun light intensity. The increase of solar cell efficiency, $\Delta \eta / \eta$, is about $10 \%$ and attributed to the increase of the current and the fill factor. The magnitude of this improvement induced by the UST is a function of the initial cell efficiency: it is the largest for cells with relatively low efficiency and significantly is decreased for high efficiency cell (Fig.5). The UST effect also depends on illumination intensity: the largest improvement occurs for light intensity lower than 0.1 sun and practically vanishes at the one sun illumination. (Fig.6). The fill factor shows a similar trend versus light intensity (Fig.6). At the present moment we have no solid interpretation of this light intensity dependence for UST effect in solar cells.

\section{Summary}

The classical gettering approaches in poly- $\mathrm{Si}(\mathrm{Cl}$ and $\mathrm{P}$ gettering) provided an improvement of the diffusion length in regions with relatively large $\mathrm{L}$ values, where recombination is controlled by dissolved heavy metals. They were not as effective in wafer regions with short $L$ values. To improve $L$ in these regions, alternative gettering approaches (Al/RTA or furnace annealing and UST) have to be used. Our results demonstrate that the RTA or furnace $\mathrm{Al}$ gettering improved $\mathrm{L}$ values (up to 3.5 times) especially in the low diffusion length regions. A comparison of trends observed during RTA and furnace annealing indicates that different mechanisms are involved in the $\mathrm{Al}$-RTA gettering and in $\mathrm{Al}$ furnace gettering. It seems, that the RTA mechanism involves nonequilibrium, probably, photon assisted phenomena, while the results of a furnace processing are partially related to defect annihilation during annealing, and contribution from Al gettering is only at the elevated temperature range. We also found that UST gettering improved parameters of commercial solar cells measured at 0.1 sun, but this effect vanished at one sun illumination. Future research is needed to understand physical mechanism involved. This could result in optimization of the UST parameters to achieve the effect of ultrasound stimulated improvement of solar cells at the arbitrary light intensities. This would provide an exciting new opportunity to improve of material / device parameters of finishted poly-Si solar cells at low temperatures $\left(<100^{\circ} \mathrm{C}\right)$.

1. S.Ostapenko, L.Jastrzebski, J.Lagowski, and B.Sopori, Appl.Phys.Let.65, 1555 (1994).

2. S.Ostapenko, L.Jastrzebski, and B.Sopori, J.Appl.Phys. (1995), be published. 


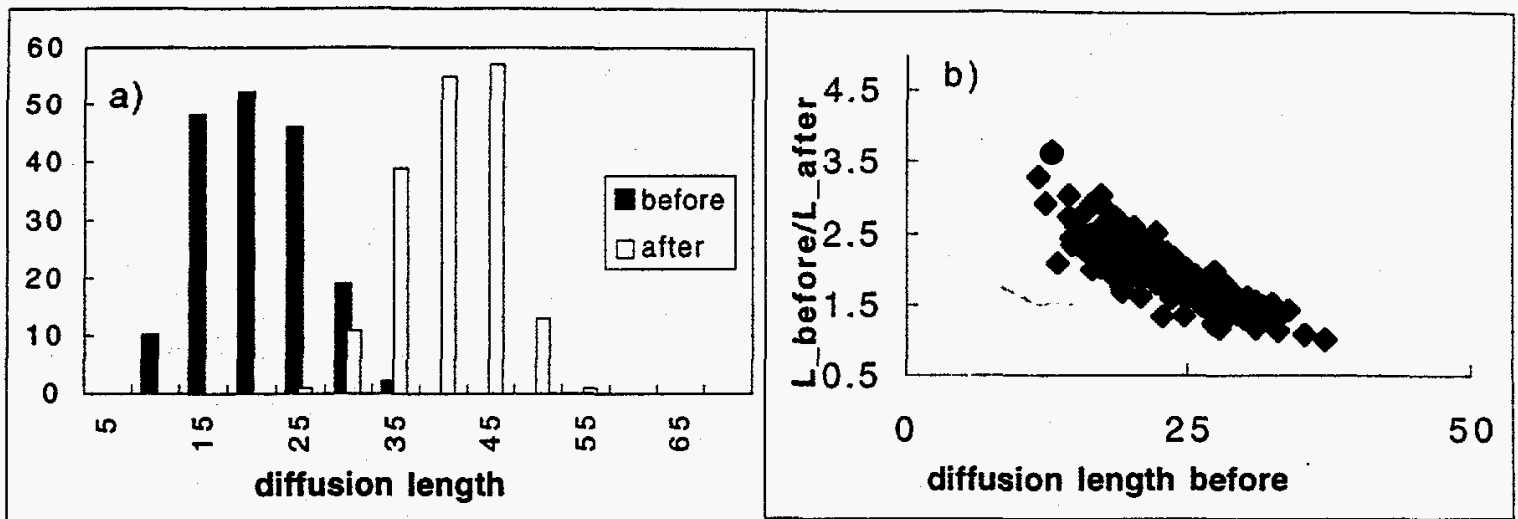

fig.1 a)histogram of diffusion length distribution in casted silicon sample before and after Al/RTA $\left(700^{\circ} \mathrm{C}, 300 \mathrm{~s}, 30^{\circ} \mathrm{C} / \mathrm{s}\right)$

b) ratio of diffusion length increase vs initial $L$ for data in fig.a

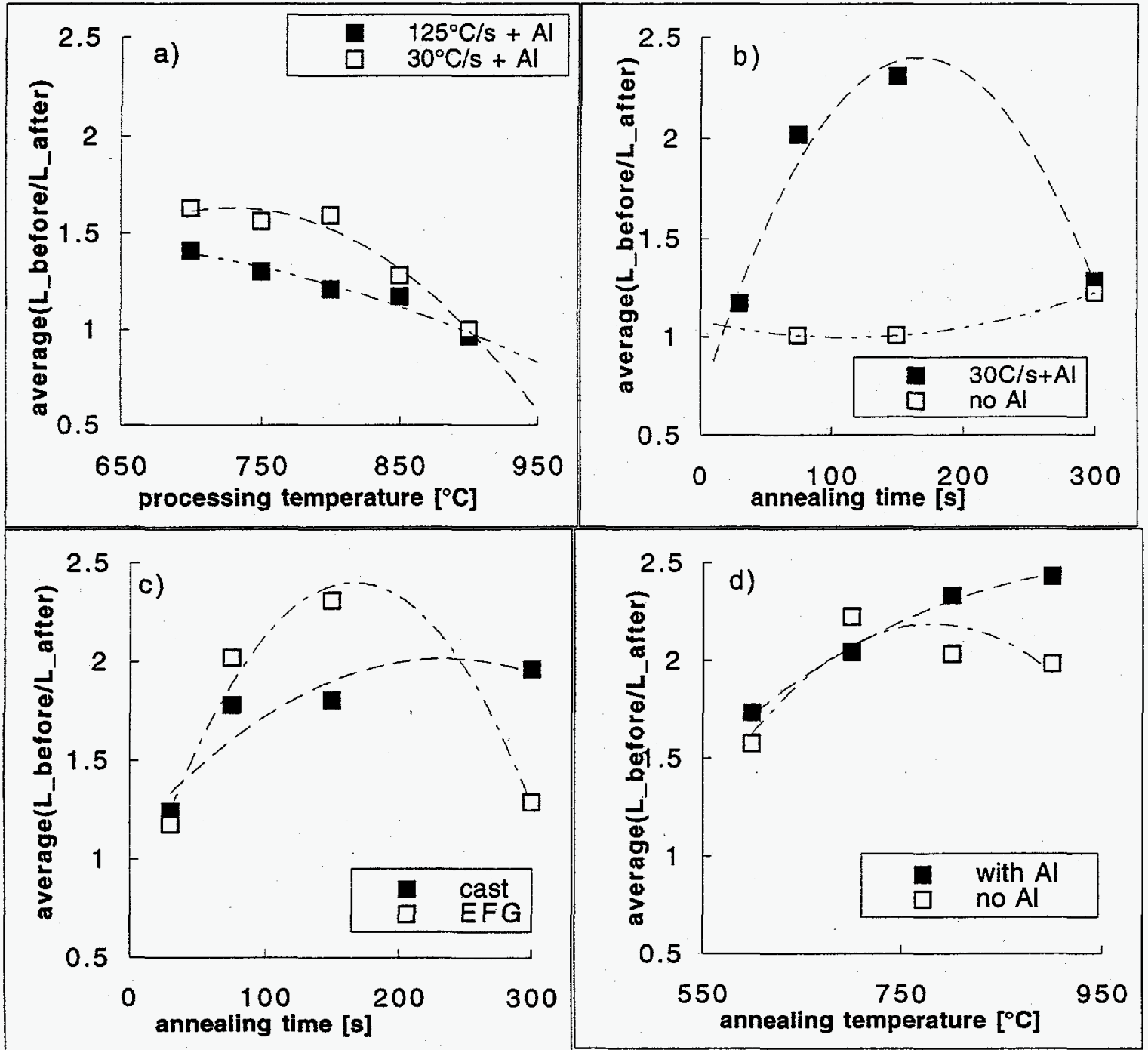

fig.2 Ratio of diffusion length increase averaged over sample area.

a-isohronal RTP of EFG polysilicon for $300 \mathrm{~s}$

b-isothermal RTP of EFG polysilicon at $700^{\circ} \mathrm{C}$

c- comparision of EFG and casted polysilicon Al/RTA results $\left(700^{\circ} \mathrm{C}\right.$ with $\left.\mathrm{Al}, 30^{\circ} \mathrm{C} / \mathrm{s}\right)$

$d$ - results of furnance annealing of EFG polysilicon for $2 h$ 


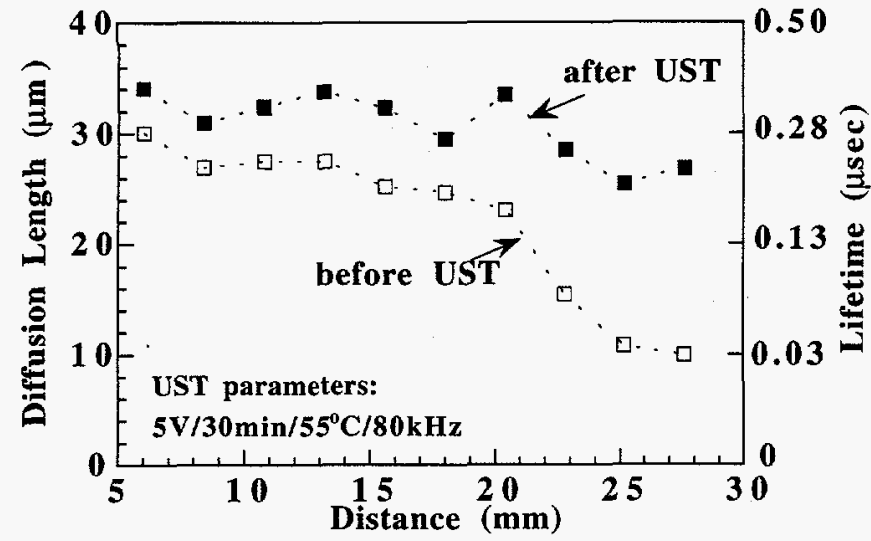

Fig.3. Increase of $\mathrm{L}$ in poly-Si by UST

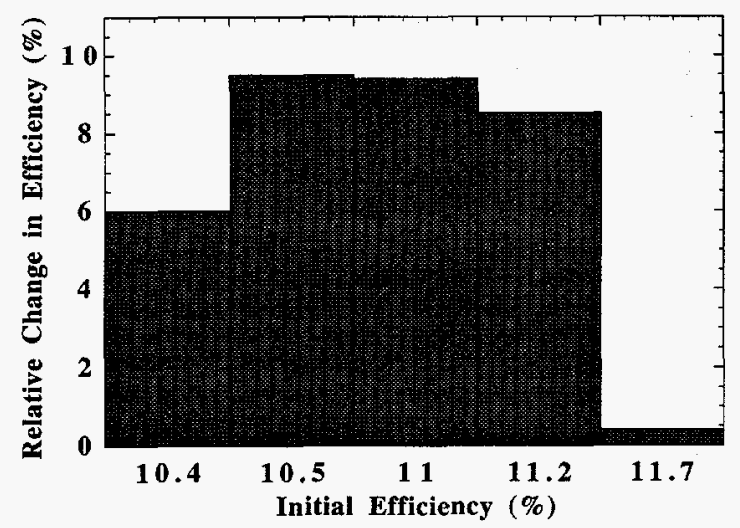

Fig.5 Increase of Solarex cell efficiencies after UST versus initial efficiency (0.1sun).

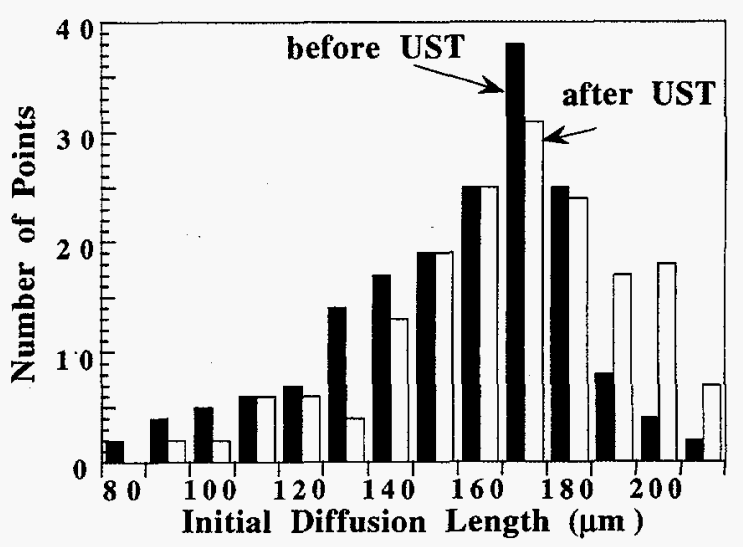

Fig.4 Histogram of $\mathrm{L}$ distribution before and after UST in Solarex solar cell

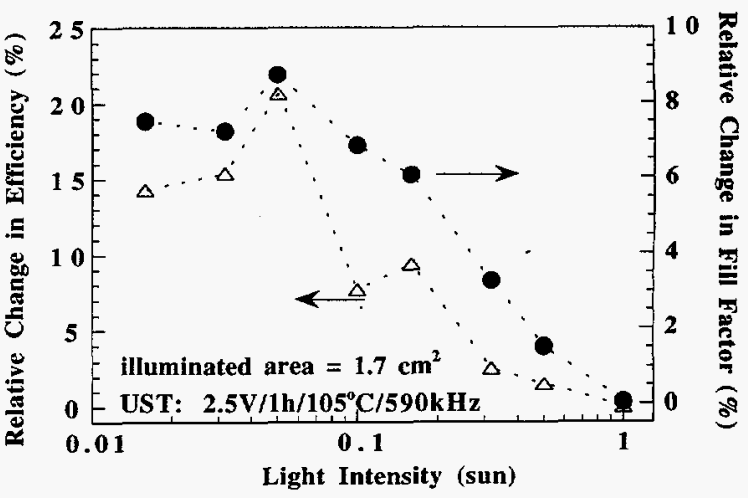

Fig.6 Change of solar cell efficiency and fill factor due to UST versus illumination intensity

Table I. UST of solar cell \#38-1 ("Solarex")

\begin{tabular}{|c|c|c|c|}
\hline Parameters of solar cell & Before UST & UST1 & Relaxation \\
\hline $\mathrm{V}_{\text {oc }}(\mathrm{V})$ & .401 & .411 & 0413 \\
\hline $\mathrm{i}_{\mathrm{sc}}\left(\mathrm{mA} / \mathrm{cm}^{2}\right)$ & 9.175 & 9.38 & 9.40 \\
\hline $\mathrm{V}_{\max }(\mathrm{V})$ & .290 & .300 & .300 \\
\hline $\mathrm{i}_{\max }\left(\mathrm{mA} / \mathrm{cm}^{2}\right)$ & 7.25 & 7.56 & 7.68 \\
\hline Fill factor $(\%)$ & 57.2 & 58.9 & 59.4 \\
\hline$\Delta \eta / \eta(\%)$ & --- & 7.6 & 9.5 \\
\hline
\end{tabular}

${ }^{*}$ Measured by illumination of $1 \mathrm{~cm}^{2}$ of $5 \times 5 \mathrm{~cm}^{2}$ cell with 0.1 sun halogen lamp. 
Title:

Organization:

Contributors:
D. L Morel and C. S. Ferekides

Advanced Processing Technology for High Efficiency Thin-

Film CuInSe ${ }_{2}$ and CdTe Solar Cells; Heterojunction

Development and Optimization in Thin-Film Compound

Semiconductor Solar Cells.

Department of Electrical Engineering

University of South florida,

Tampa, Florida

G. Attar, V. Ceekala, K. Dugan, S. Karthickeyan, L. Killian,

H. Natarajan, D. Niermen, D. Oman, P. Bhethanabolta, and

S. Zafar

\section{CdTe}

\section{Objectives}

The main objectives of this project are to prepare high efficiency CdTe solar cells on soda lime (SL) substrates by the close spaced sublimation process (CSS), evaluate/develop rf sputtering and CSS as alternative processes to the chemical bath deposition (CBD) for the preparation of the $\mathrm{CdS}$ films, and correlate processing to device performance.

\section{Device Fabrication}

Solar cells prepared on soda lime glass substrates utilize CdS prepared by CBD, and a CSS CdTe layer deposited at low temperatures: $460-520^{\circ} \mathrm{C}$. Borosilicate glass substrates are being used for the preparation of devices that utilize CSS or rf sputtered CdS, and CSS $\mathrm{CdTe}$ (typically deposited at temperatures in excess of $550^{\circ} \mathrm{C}$.)

\section{Cadmium Sulfide Films}

Both rf sputtered and CSS CdS films are prepared at substrate temperatures in excess of $150^{\circ} \mathrm{C}$ (rf sput.: $150-400^{\circ} \mathrm{C}$; CSS: $250-500^{\circ} \mathrm{C}$.) The rf sputtered CdS films exhibit small grain size (typically $0.1-0.2 \mu \mathrm{m}$ ), and high dark resistivity $\left(>10^{6} \Omega-\mathrm{cm}\right.$ ). High open-circuit voltages $(>840 \mathrm{mV})$ have been obtained for devices with $\mathrm{CdS}$ thicknesses in excess of $2000 \AA \AA$; smaller thicknesses yield reduced $V_{O C}$ 's. Annealing of the rf sputtered $\mathrm{CdS}$ in $\mathrm{H}_{2}$ was found to influence the film properties and solar cell performance. Figure 1 shows the $\mathrm{SR}$ of several cells utilizing rf sputtered $\mathrm{CdS}$ annealed at different temperatures. In general, device performance was limited by low fill factors (mid 60's.)

Cadmium sulfide films prepared by CSS were also highly resistive. The grain size is a strong function of the deposition conditions (films with grain size in excess $1 \mu \mathrm{m}$ can be easily obtained.) Annealing in $\mathrm{H}_{2}$ was also found to influence the properties of the CSS CdS films (and to a lesser extent the properties of solar cells). Open-circuit voltages in excess of 840 $\mathrm{mV}$ and fill factors over $70 \%$ have been attained for film thicknesses of about $800-1000 \AA$. Solar cell efficiencies of $13 \%$ have been achieved.

\section{Soda Lime Substrates}

Inexpensive soda lime glass substrates are preferred (for the fabrication of solar cells) 
over the expensive borosilicate type. The properties of soda lime glass (softening point, linear expansion coefficient, impurity content etc.) make it incompatible with high temperature processing. Cadmium telluride films and devices prepared by CSS are being optimized at lower processing temperatures $\left(460-520^{\circ} \mathrm{C}\right)$ for the purpose of fabricating efficient solar cells on soda lime substrates. At high temperatures $\left(>550^{\circ} \mathrm{C}\right) \mathrm{CdTe}$ films prepared on SL substrates exhibit "blistering" and "peeling". As the temperature is lowered adhesion improves but the density and structure of the films change; device performance degrades due to a decrease in $\mathrm{V}_{\mathrm{OC}}$. Obtaining denser CdTe films was crucial in improving the performance of CdTe devices prepared on SL substrates, and achieving efficiencies over 13\%. Figure 2 shows the light (AM1.5) I-V characteristic of a CdTe solar cell with efficiency of $13.5 \%$ verified at the National Renewable Energy Laboratory.

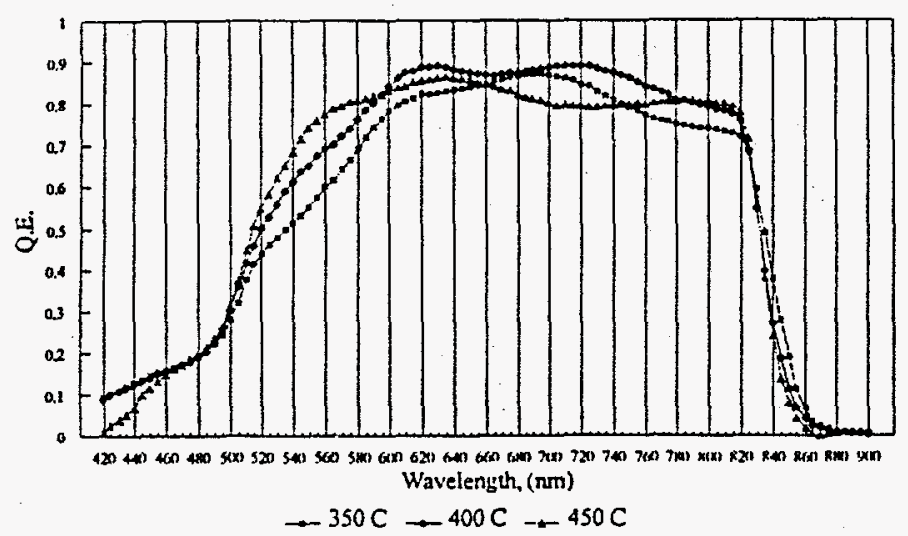

Fig. 1. The SR of solar cells prepared with rf sputtered CdS annealed in $\mathrm{H}_{2}$ ambient.

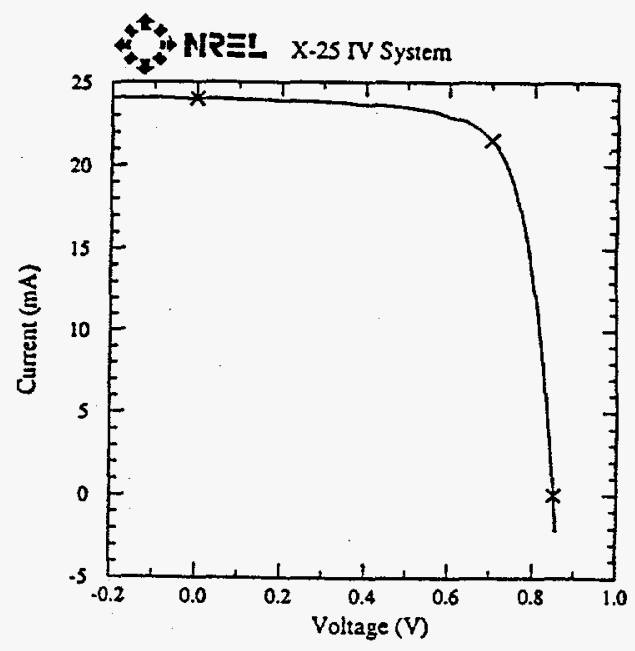

Fig. 2. Illuminated I-V characteristics of a $13.5 \%$ cfficient CdTe solar cell prepared on a soda lime glass substrate.

\section{CIS}

\section{Objectives}

The objectives of this project are to develop manufacturing-friendly processes for CIS solar cells, to correlate performance and processing mechanisms, and to develop reactively sputtered $\mathrm{ZnO}$ as an alternative window layer.

\section{Process Description}

Our approach to processing has been driven by two primary guidelines: 1 . Non- $\mathrm{H}_{2} \mathrm{Se}$ based processing, and 2. Non-critical process control. Our primary process is thus one which we describe as "coat and cook". It involves sequential physical vapor deposition of $\mathrm{Cu}$, In and Se under minimal control, and the anneal cycle runs at atmospheric pressure, features which make it very attractive from a manufacturing perspective. 


\section{CIS Performance Correlations}

Our baseline devices which contain only $\mathrm{CuInSe}_{2}$ and no $\mathrm{Ga}$ currently have an efficiency of about $10 \%$. This represents significant progress for our simplified processing approach. Also, we have developed additional insights to processing variables which are leading to further advancements in performance. In figure 3 we show the dependence of $V_{o c}$ on metal ratio for our baseline process(solid line) and for recent advances in performance $(x$ 's) beyond our baseline due to improved understanding. The data points at $400 \mathrm{mV}$ are due to modifications of our anneal profile. The data point at $450 \mathrm{mV}$ was accomplished recently by etching the surfaces of our devices. Through modeling (see below) we have determined that device performance is limited by excessive recombination at the junction interface. Elimination of the centers responsible for the recombination produced the significant increase in $\mathrm{V}_{\mathrm{oc}}$ to $450 \mathrm{mV}$. This level of performance is now approaching the highest values achieved for non-Ga-containing CIS.

\section{$\underline{\mathrm{ZnO}}$}

$\mathrm{ZnO}$ is an important window layer material for CIS and other solar cell devices. We have been developing a process for depositing $\mathrm{ZnO}$ by reactive sputtering with a zinc target. This promises much higher deposition rates than can be achieved by sputtering from a $\mathrm{ZnO}$ target. Using Al doping we have achieved state-of-the-art opto-electronic properties. Uniform films with resistivities of $2-5 \times 10^{-4} \Omega$-cm and suitable optical properties are routine. In attempting to further improve the performance of these films we have developed new insights to the underlying physical mechanisms which determine performance and the deposition parameters which influence those mechanisms. For example, while we have achieved doping efficiencies of nearly $100 \%$, we find that at doping levels above about $4 \mathrm{x}$ $10^{20} / \mathrm{cm}^{3}$ ionized impurity scattering starts to reduce the mobility thus limiting the conductivity. This, together with the dependence of optical properties on free carrier effects, sets fundamental limits on the electro-optical properties of $\mathrm{ZnO}$. Further advancements in these properties will require novel, non-conventional doping techniques.

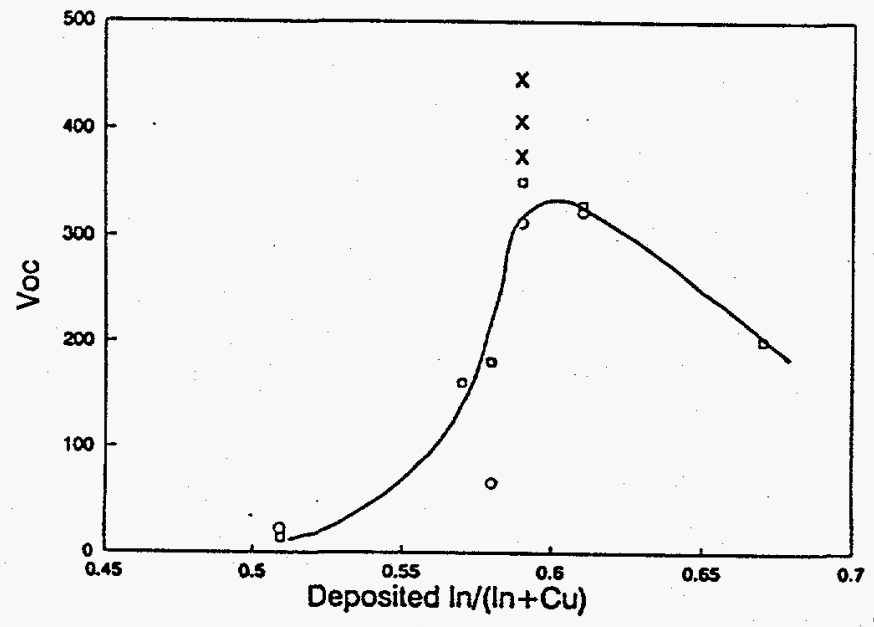

Fig 3. $V_{o c}$ vs. metal ratio for baseline devices $\square$ and $O$, and for advanced processing, $x$.

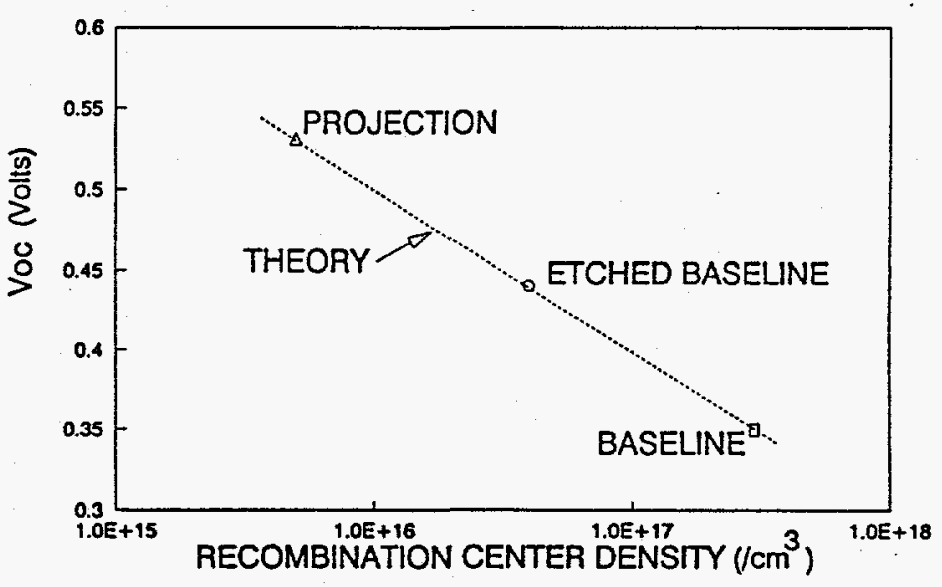

Fig. 4. $V_{\propto}$ vs. recombination center density. $O$, $\square$ - data points, $\Delta$ - projection. 


\section{HETEROJUNCTION MODELING AND DEVELOPMENT}

\section{Objectives}

The objectives of this project are to develop a phenomenological model to aid the understanding of the heterojunction in CdTe and CIS devices and to use the model to guide the further development of the technologies.

\section{Results and Discussion}

After careful examination of the ability of classical models to fit critical sets of data we have determined that a recombination-based model is the most suitable choice for both CIS and CdTe. The model follows the standard equation

$$
\mathrm{I}=\mathrm{I}_{\mathrm{o}}\left(\mathrm{e}^{\mathrm{q}(\mathrm{V}-\mathrm{IR}) / A k T)}-1\right)+(\mathrm{V}-\mathrm{IR}) / \mathrm{R}_{\mathrm{sh}}-\mathrm{I}_{\mathrm{L}}
$$

where $R$ is the series resistance, $R_{s h}$ is the shunt resistance and $I_{L}$ is the light generated current. The diode factor A may range between 1 and 2 depending upon the location of the recombination sites within the gap. The prefactor $I_{0}$ may be written as

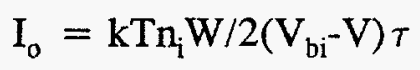

where k-Boltzmann's constant, T-temperature, $n_{i}$-intrinsic carrier concentration, W-space charge width, $\mathrm{V}_{\mathrm{bi}}$-built-in voltage, $\tau$-lifetime. For both $\mathrm{CdTe}$ and CIS we find self-consistent fits to dark and light $I-V, I_{s c}-V_{o c}$, the one sun power curve, and $V_{o c}-T$. Since several of the parameters can be measured directly, the key parameters to be determined by the fits are A and $\tau$ which are not necessarily independent.

In taking and interpreting data with regard to this model special care has to taken to properly account for extraneous effects. For example, we have found that the commonly observed crossover phenomenon in CdTe is due to a region of the CdS outside of the space charge layer. Once it is accounted for, good fit to the model can be realized.

For typical CdTe devices we find $A$ values in the range $1.4-1.8$, with the better devices exhibiting the lower $\mathrm{A}$ values. We also find that $\mathrm{A}$ is often a complex function of device structure and the exposure history of the device. In particular we observe transient behavior in $V_{o c}$ which is largely explainable in terms of fluctuations in A. Key correlations to process variables are being observed and are helping guide our fabrication efforts.

Similar observations are being made with CIS confirming our supposition that the common CdS heterojunction would provide leveraged opportunities to study junction mechanisms. In the case of CIS we are commonly observing higher A values in the 1.8-2.0 range due to enhanced recombination resulting from smaller lifetimes. This has kept our $\mathrm{V}_{\text {oc }}$ values low and has reduced fill factors. These insights led to the etching procedures described above. Briefly, modeling indicated that the responsible centers were at the junction interface. The intent of the etch was to simply reduce these centers to "turn off" the dark I-V curve which would then result in a higher $V_{o c}$. This is what was observed experimentally, and in figure 4 we show additional projections from the model. As seen in the figure the advance in $\mathrm{V}_{\text {oc }}$ from $400 \mathrm{mV}$ to $450 \mathrm{mV}$ was due to a reduction of these centers to the mid $10^{16} / \mathrm{cm}^{3}$ range, and further reduction to below the $10^{16} / \mathrm{cm}^{3}$ range is expected to yield $500+\mathrm{mV}$. Experiments are currently under way to continue these advancements in performance. 
Title:

Organization:

Contributors:

\section{Electronic Processes in Thin Film PV Materials}

Department of Physics, University of Utah, Salt Lake City, Utah

P.C. Taylor, principal investigator; G.A. Williams, W.D. Ohlsen,

S.Q. Gu, J.M. Viner, K. Gaughan, S. Hershgold, D. Chen, P. Hari

The major objectives of this subcontract are (1) to identify metastabilities caused by carrier recombination or by frozen-in departures from equilibrium, and to determine if the metastabilities that plague devices are "intrinsic" and therefore not correctable or "extrinsic" and therefore capable of being eliminated in future devices, (2) to characterize the important defects and impurities in the bulk and at surfaces and interfaces because interfaces are becoming more important with the advent of tandem cells with several heterojunctions, and (3)to measure the motion of hydrogen in a-Si:H and related alloys on a microscopic scale because the motion of hydrogen is thought to play an important role in many of the metastable effects that occur in a-Si:H and related alloys and the microscopic mechanism for this motion is not known. A recent review is available elsewhere. ${ }^{1}$

\section{Approaches}

Two new approaches, that have not been employed in the past, have been emphasized: (1) below-gap spectroscopy, such as optical absorption, photoluminescence (PL), PL excitation, electron spin resonance and other spectroscopies using primarily a tunable Ti sapphire laser system to excite carriers well below the optical gap (down to $-1.1 \mathrm{eV}$ ). (2) novel nuclear magnetic resonance (NMR) techniques such as measurements of the spin-lattice relaxation in a dipolar field $\left(T_{1 D}\right)$, to probe local hydrogen motion in doped and intrinsic a-Si:H and selected alloys.

\section{Research Results}

The subcontract is divided into several tasks. One task is the growth and characterization of doped and undoped a-Si:H and related alloys. Tertiarybutylphosphine (TBP) and ditertiarybutylsilane (DTBS) have been employed in a standard rf plasma reactor to produce pure and doped a-Si:H and a-Si $\mathrm{C}_{1-\mathrm{x}}: \mathrm{H}$ alloys. $^{2,3,4}$ Because they are less toxic and less pyrophoric, many of these liquid organic sources may be preferable to the usual gases employed in film growth. Ditertiarybutylsilane has been added to silane to vary the bandgap of $\mathrm{a}-\mathrm{Si}_{\mathrm{x}} \mathrm{C}_{1-\mathrm{x}}: \mathrm{H}$ alloys from $\mathrm{E}_{04}$ of about 1.9 to about $3 \mathrm{eV}{ }^{4}$ High doping levels of TBP in $\mathrm{SiH}_{4}(>1$ at. \%) generate samples with reduced doping efficiencies, increased PL efficiencies and other properties consistent with the alloying of phosphorus in the a-Si:H network. ${ }^{2,3}$ Samples of a-SiS ${ }_{x}: H$ have been made using mixtures of silane and hydrogen sulfide. ${ }^{5,6}$ Samples with large sulfur concentrations $(>1$ at. \%) exhibit the phenomenon of persisitent photoconductivity.

A second task concerns below gap spectroscopy in a-Si:H and related alloys. We have been using Photoluminescence (PL) excitation spectroscopy (PLE), time resolved PL and optically detected magnetic resonance (ODMR) to probe defects which produce absorption below the gap in a-Si:H. ${ }^{7,8,9}$ Photoluminescence in a-Si: $\mathrm{H}$ has been investigated using optical excitation energies $\mathrm{E}_{\mathrm{x}}$ varying from 1.27 to $2.0 \mathrm{eV}$. A model of phonon-assisted absorption has been proposed to account for the experimental results. $^{7}$ The ODMR results ${ }^{8,9}$ indicate that dramatic changes in the lineshapes occur when $\mathrm{E}_{\mathrm{x}}$ is less than about $1.5 \mathrm{eV}$ as shown in Fig. 1. Recent measurements on the PL that occurs at energies above the exciting-light energy indicate that two-step excitation processes through the silicon dangling-bond states are important, even in device-quality material. ${ }^{10}$ 
A third task involves studies of local diffusion of hydrogen in a-Si:H using NMR techniques. We measured $T_{1 D}$, the dipolar spin lattice relaxation time, whose inverse $\left(T_{1 D}^{-1}\right)$ gives a measure of the local motion of hydrogen. ${ }^{11,12,13}$ The $T_{1 D}$ values (times at which the decay curves in Fig. 2 reach $\mathrm{e}^{-1}$ of their initial values) for $10^{-4} \mathrm{~B}$-doped, $10^{-5} \mathrm{~B}$-doped and undoped a-Si:H are, respectively, $1.7 \mathrm{~ms}, 11 \mathrm{~ms}$ and $22 \mathrm{~ms}$ at $300 \mathrm{~K}$. These trends are similar to the variation of the macroscopic diffusion of hydrogen with respect to various doping levels, but the details of the local motion are very different from those of the macroscopic diffusion. 13

A fourth task involves the study of interfaces, defects and impurities in a-Si:H and related alloys, including the metastabilities that are related to such interfaces, defects and impurities. The measurement of (1) PL, (2) PL fatigue, or decay, under the application of band-gap light, (3) the bleaching, or restoration, of the fatiqued PL with below-gap light, and (4) the temperature dependence of the dark ESR in nitrogen-rich hydrogenated amorphous silicon nitride $\left(\mathrm{a}-\mathrm{SiN}_{1.6}: \mathrm{H}\right)$ films ${ }^{14,15}$ has provided a probe of optically-induced metastabilities in these films. These experiments have helped to identify some of the important defect levels in a-SiN ${ }_{1.6}: \mathrm{H}$ films. ${ }^{15}$

Although they do not fall directly under any of the tasks of the present subcontract, we have measured PL in $\mu \mathrm{c}$-Si:H samples as a function of boron doping, ${ }^{16}$ and NMR lineshapes in a-Si:H made in a very high frequency $(70 \mathrm{MHz})$ glow discharge reactor. ${ }^{17}$

\section{Conclusions}

Major accomplishments of the previous year include (1) the characterization of, $\mathrm{P}$-doped a-Si:H, a-Si $\mathrm{C}_{1 \text { - }}$ $\mathrm{x}: \mathrm{H}$ alloys, and a-SiS $: \mathrm{H}$ alloys which have been grown using liquid organic sources mixed with silane, (2) the measurement of PL, PLE, time-resolved PL and ODMR spectra in a-Si:H using excitation energies down to $1.1 \mathrm{eV}$, (3) correlation of local motion of hydrogen in B-doped, P-doped, compensated and intrinsic a-Si:H using an NMR dipolar echo technique, and (4) the measurement of PL, PL fatigue, PL bleaching, and dark ESR in a-Si $\mathrm{N}_{1-\mathrm{x}}: \mathrm{H}$ alloys. Future directions for the research include (1) measurements to obtain a better understanding of the role of small amounts of $\mathrm{S}$ in a-Si:H and of the persistent photoconductivity at higher S concentrations, (2) continuation of below-gap spectroscopy of a$\mathrm{Si}: \mathrm{H}$ to identify the important absorption and degradation mechanisms.

\section{References}

1. "Photoluminescence in Hydrogenated Amorphous Silicon" (P.C. Taylor), Brazilian J. Phys. 23, 132 (1993).

2. "Doping Efficiency of n-Type a-Si:H Doped with a Liquid Organic Source" (K. Gaughan, Zhaohui Lin, J.M. Viner, P.C. Mathur and P.C. Taylor), in Amorphous Silcion Technology - 1993, edited by E.A. Shiff, M.J. Thompson, A. Madan, K. Tanaka and P.G. LeComber (Materials Research Society, Pittsburgh, 1993), Vol. 297, p. 503.

3. "Electronic and Optical Properties of n-Type a-Si:H" (K. Gaughan, Z. Lin, J.M. Viner, P.C. Taylor, P.C. Mathur and R.M. Mehra), J. Non-Cryst. Solids 164-166, 343 (1993).

4. "Electronic and Optical Properties of $\mathrm{a}-\mathrm{Si}_{1-\mathrm{x}} \mathrm{C}_{\mathrm{x}}: \mathrm{H}$ Films Produced from Admixtures of Silane and Ditertiarybutylsilane" (K. Gaughan, J.M. Viner and P.C. Taylor), in Amorphous Silicon Technology - 1994, edited by E.A. Schiff, M. Hack. A. Madan, M. Powell and A. Matsuda (Materials Research Society, Pittsburgh, 1994), in press.

5. "Persistent Photoconductivity in $\mathrm{a}-\mathrm{Si}_{1-\mathrm{x}} \mathrm{S}_{\mathrm{x}}: \mathrm{H}$ at Low Sulfur Concentration" (S.L. Wang, J.M. Viner, M. Anani and P.C. Taylor), J. Non-Cyrst. Solids (1993). 
6. "Electronic and Optical Properties of a-Si:H Films Alloyed with Sulfur" (S.L. Wang, Z.H. Lin, J.M. Viner and P.C. Taylor), in Amorphous Silicon Technology - 1994, edited by E.A. Schiff, M. Hack. A. Madan, M. Powell and A. Matsuda (Materials Research Society, Pittsburgh, 1994), in press.

7. "Excitation Spectroscopy of Thin-Film Disordered Semiconductors" (S.Q. Gu, P.C. Taylor, J.T. McKinley, A. Ueda, X. Wang and N.H. Tolk), SPIE Conf. Proc. 1854, 77 (1993).

8. "ODESR Studies of a-Si:H with Subgap Excitation" (D. Mao, S.Q. Gu and P.C. Taylor), in Amorphous Silcion Technology - 1993, edited by E.A. Shiff, M.J. Thompson, A. Madan, K. Tanaka and P.G. LeComber (Materials Research Society, Pittsburgh, 1993), Vol. 297, p. 333.

9. $\quad$ "Optically Detected ESR Studies of a-Si:H" (D. Mao and P.C. Taylor), J. Non-Cryst. Solids 164166, 367 (1993).

10. "Photoluminescence Above the Excitation Energy in a-Si:H" (X. Yin, J.M. Viner, S.Q. Gu, M.E. Raikh and P.C. Taylor), Phys. Rev. B 49, 5073 (1994).

11. "Doping Dependence of Local Hydrogen Motion in Hydrogenated Amorphous Silicon" (P. Hari, P.C. Taylor and R.A. Street), in Amorphous Silcion Technology - 1993, edited by E.A. Shiff, M.J. Thompson, A. Madan, K. Tanaka and P.G. LeComber (Materials Research Society, Pittsburgh, 1993), Vol. 297, p. 297.

12. "Microscopic Motion of Hydrogen in the Dilute and Clustered Phases of Hydrogenated Amorphous Silicon" (P. Hari, P.C. Taylor and R. A. Street), J. Non-Cyrst. Solids 164-166, 313 (1993).

13. "Effect of Light Soaking on the Local Motion of Hydrogen in Hdyrogenated Amorphous Silicon" (P. Hari, P.C. Taylor and R.A. Street), in Amorphous Silicon Technology - 1994, edited by E.A. Schiff, M. Hack. A. Madan, M. Powell and A. Matsuda (Materials Research Society, Pittsburgh, 1994), in press.

14. "Temperature Dependence of the Electron Spin Resonance in Nitrogen-rich Amorphous Silicon Nitride" (D. Chen, J.M. Viner, P.C. Taylor and J. Kanicki), Phys. Rev. B (1994), submitted.

15. "Photobleaching of PL and Temperature Dependence of ESR in Nitrogen-Rich Amorphous Silicon Nitride Films" (D. Chen, J.M. Viner, P.C. Taylor and J.Z. Kanicki), in Amorphous Silicon Technology - 1994, edited by E.A. Schiff, M. Hack. A. Madan, M. Powell and A. Matsuda (Materials Research Society, Pittsburgh, 1994), in press.

16. "Photoluminescence in B-Doped $\mu \mathrm{c}-\mathrm{Si}: H "$ (S.Q. Gu, J.M. Viner, P.C. Taylor M.J. Williams, W.A. Turner and G. Lucovsky), in Microcrystalline Semiconductors - Materials Science and Devices, Y. Aoyagi, L.T. Canham, P.M. Fauchet, I. Shimizu and C.C. Tsai, eds. (MRS, Pittsburgh, 1992), Vol. 283.

17. "Nuclear Magnetic Resonance Studies on Hydrogenated Amorphous Silicon Prepared by Very High Frequency Glow Discharge" (Q. Li, P.C. Taylor and F. Finger), Phil. Mag. B 69, 169 (1993). 


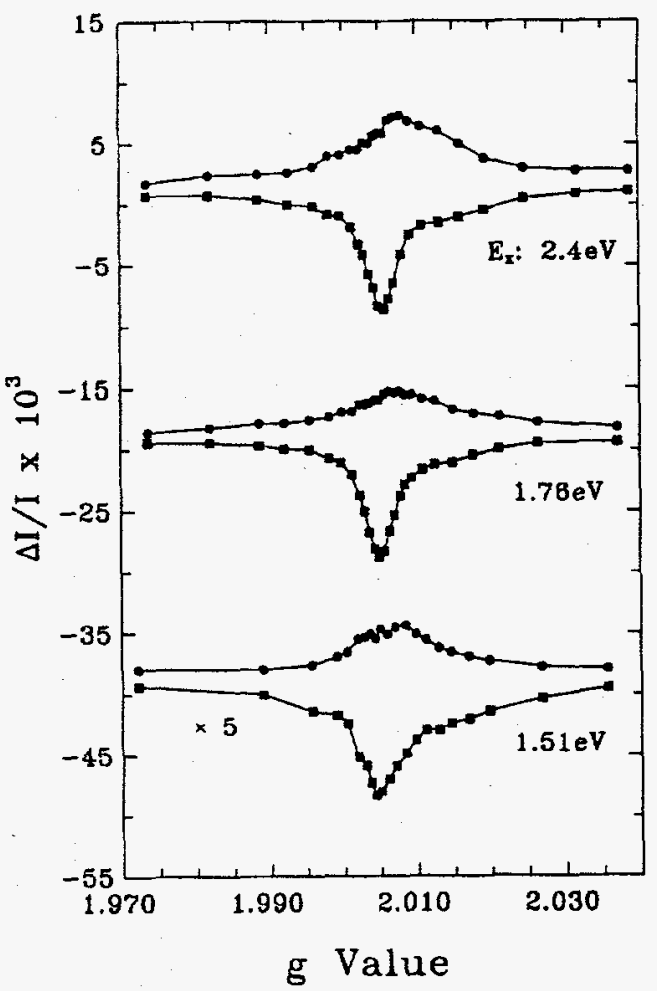

Fig. 1. ODESR lineshapes obtained at a microwave frequency of $16 \mathrm{GHz}$ under various excitation conditions. The energy of the exciting light is labelled below each trace. The electron-hole-pair creation rate for each trace is as follows (from top to bottom): $7.8 \times 10^{21}, 4.7 \times 10^{20}$, and $7.1 \times 10^{19} \mathrm{~cm}^{-3} \mathrm{~s}^{-1}$, respectively.

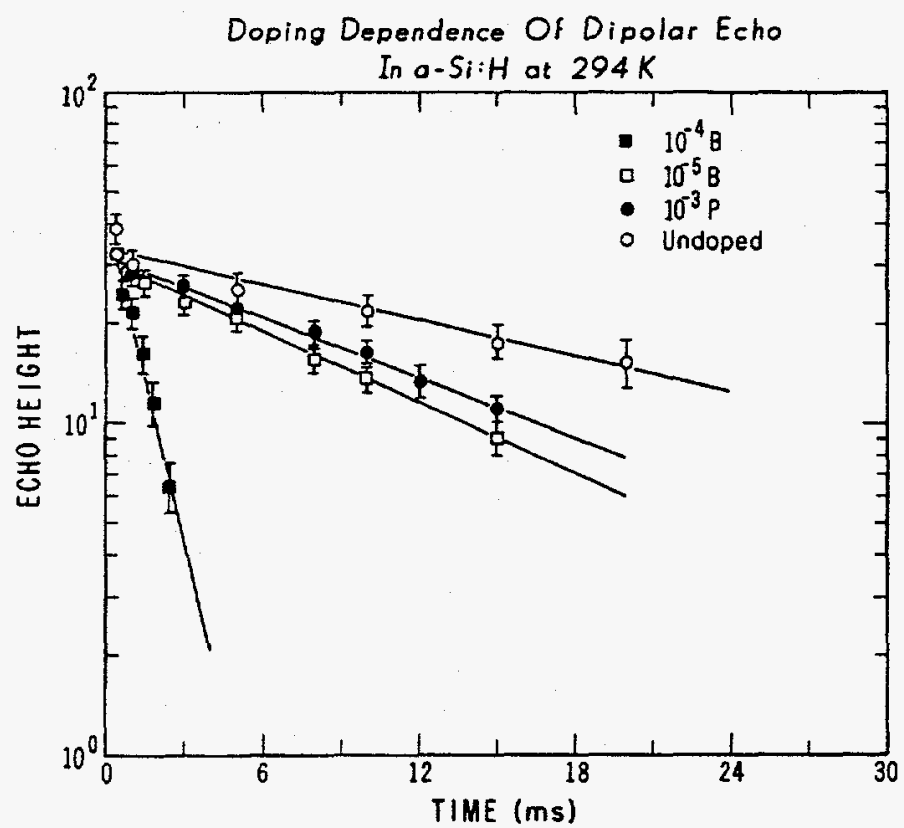

Fig. 2. Doping dependence of dipolar echo in a-Si:H at $294 \mathrm{~K}$. The $10^{-4} \mathrm{~B}$-doped a-Si:H sample has the shortest $T_{1 D}$ of $1.7 \mathrm{~ms}$. Values of $T_{1 D}$ for $10^{-5} \mathrm{~B}$-doped, $10^{-3} \mathrm{P}$-doped and undoped a-Si:H are $8 \mathrm{~ms}, 11$ $\mathrm{ms}$ and $22 \mathrm{~ms}$, respectively. 


\title{
3.0 THIN-FILM TECHNOLOGIES PROJECT
}

\author{
Ken Zweibel, Manager
}

During FY 1994, NREL reorganized thin-film research into one project-the Thin-Film Technologies Project. Work within the project encompasses a-Si and polycrystalline thin films of CIGS, CdTe, and Si. Also during the year, the project initiated the Thin-Film Partnership Program, which was begun through a letter of interest (LOI) solicitation for new industrial and university subcontracts. In addition, work within the partnership organized teamed research with other organizations around working groups in the various thin films. The Partnership competition was completed during FY 1994, although contracts were not scheduled to begin until FY 1995. The new teams were formed in CIGS, CdTe, and ES\&H. They joined the existing "model" team in a-Si, which has existed for 2 years.

Progress in improved cell, submodule, and module efficiencies continued during the year. Figure 3-1 shows the ongoing progress in cell efficiencies. NREL once again raised the world record for cell efficiency above its previous high, now to $16.8 \%$-a level of performance starting to approach the record efficiency $(17.8 \%)$ for polycrystalline $\mathrm{Si}$. Such efficiencies were once thought impossible for thin films. Meanwhile, submodule and module efficiencies also improved (Table 3-1), as did power output and size. Table 3-1 shows the first $10 \%$ (stabilized) a-Si submodule, made by United Solar Systems Corporation (USSC). This module symbolized the revitalization of a-Si technology, which had been experiencing a period of consolidation. By the end of FY 1994, it was clear that a-Si thin film modules can be manufactured at about $7 \%-8 \%$ efficiency (as opposed to previous highs near 5\%), making them viable for existing crystalline Si PV markets. Companies such as USSC and Solarex-Enron announced plans for significant new production of a-Si modules (greater than 20-MW planned capacity).

Solar Cells, Inc. (SCI) of Toledo, $\mathrm{OH}$, was responsible for aggressively improving the efficiency of CdTe PV modules, and SCI produced an 8- $\mathrm{ft}^{2}$ module with more than $60-\mathrm{W}$ output (Table 3-1) and a total-area efficiency of about $8.4 \%$, well above previous results. This continued the trend of efficiency enhancements in CdTe power modules. Meanwhile, Golden Photon moved rapidly toward production at its new 2-MW facility in Golden, Colorado. They installed all the necessary equipment and began shakedown tests and early module deployment.

In CIGS, companies moved to meet the significant challenges of CIGS scale-up. Siemens Solar Industries (SSI) of Camarillo, CA, worked intensively on smaller-area (about $100 \mathrm{~cm}^{2}$ ) submodules to solve critical yield issues. SSI successfully improved efficiencies, significantly tightened yield, and developed a new, more-forgiving set of processing techniques. SSI's best power module efficiency also improved-to 10.2\% measured at NREL (Table 3-1). Meanwhile, Energy PV of Princeton, NJ, moved rapidly from cell experimentation to submodule and module fabrication, produced decent cell/submodule results $\left(9 \%\right.$ on $\left.200 \mathrm{~cm}^{2}\right)$, and then began larger-area $\left(6 \mathrm{ft}^{2}\right)$ module experiments. 


\begin{tabular}{|l|l|l|l|l|}
\hline \multicolumn{5}{|c|}{$\begin{array}{c}\text { Table 3-1. Best Thin-Film Modules } \\
\text { after preliminary light-soaking for a-Si) }\end{array}$} \\
\hline \multicolumn{1}{|c|}{ Company } & \multicolumn{1}{|c|}{ Material } & \multicolumn{1}{c|}{ Area $\left(\mathbf{c m}^{2}\right)$} & \multicolumn{1}{c|}{ Efficiency } & \multicolumn{1}{c|}{ Power } \\
\hline SCI & CdTe & 7200 & $8.4 \%$ & $60.3 \mathrm{~W}$ \\
\hline APS & a-Si/a-Si & 11522 & $4.6 \%$ & $53.0 \mathrm{~W}$ \\
\hline SSI & CIS & 3832 & $11.2 \% 1.2$ & $43.1 \mathrm{~W}$ \\
\hline SSI & CIS & 3859 & $10.2 \%$ & $39.3 \mathrm{~W}$ \\
\hline BP Solar & CdTe & 4540 & $8.4 \%$ & $38.2 \mathrm{~W}$ \\
\hline ECD & $\begin{array}{l}\text { a-Si/a-Si/a- } \\
\text { SiGe }\end{array}$ & 3906 & $7.8 \%$ & $30.6 \mathrm{~W}$ \\
\hline Golden Photon & CdTe & 3528 & $7.7 \%$ & $27.5 \mathrm{~W}$ \\
\hline USSC & a-Si/a-Si & 3676 & $6.2 \%$ & $22.8 \mathrm{~W}$ \\
\hline Fuji & a-Si/a-Si & 1200 & $8.9 \%$ & $10.7 \mathrm{~W}$ \\
\hline SSI & CIS & 938 & $11.1 \%$ & $10.4 \mathrm{~W}$ \\
\hline Matsushita Battery & CdTe & 1200 & $8.7 \% 1$ & $10.0 \mathrm{~W}$ \\
\hline USSC & $\begin{array}{l}\text { a-Si/a-Si/a- } \\
\text { SiGe }\end{array}$ & 903 & $10.2 \%$ & $9.2 \mathrm{~W}$ \\
\hline BP Solar & CdTe & 706 & $10.1 \%$ & $7.1 \mathrm{~W}$ \\
\hline
\end{tabular}

Note: Updated August 1994

${ }^{1}$ Not measured at NREL

${ }^{2}$ Unencapsulated

${ }^{3}$ Not monolithic

Numerous technical achievements occurred during the year. Perhaps the most promising was the initial development of hot-wire a-Si cells at NREL. These cells are made with a new method-hot wire deposition-designed to alleviate and possibly remove the well-known Staebler-Wronski Effect (SWE) in a-Si; that is, to make a-Si cells stable. Initial tests on lowefficiency cells (about 5\%) showed very promising results (Figure 3-2). This apparent stability is yet unproven, as stability must be demonstrated in cells of reasonable efficiencies (greater than $10 \%$ ) before industry can begin to make the needed investment in scaling up this new production method. However, removing the SWE instability in a-Si, if achieved, would be perhaps the most significant technical achievement of the DOE PV Program. FY 1995 should be a year of intense effort to develop hot-wire a-Si technology at NREL. 
Other information concerning stability also became available during the year. Selected achievements in cooperative R\&D are shown in Table 3-2. The first 300-1000-W arrays of CIS and CdTe were delivered to NREL for outdoor testing. Figures 3-3 and 3-4 show the results of these tests on an SSI CIGS $1-\mathrm{kW}$ array and an SCI 300-W array tested at NREL. They show excellent stability. The CdTe is the first of its kind to show such robust output.

\begin{tabular}{|c|c|c|}
\hline Company & Achievement & Results \\
\hline $\begin{array}{l}\text { PV43 } \\
\text { USSC }\end{array}$ & $\begin{array}{l}\text { (Jan 1994) Produced the first }>10 \% \text { - } \\
\text { efficient stabilized a-Si } 1-\mathrm{ft}^{2} \text { module }\end{array}$ & $\begin{array}{l}\text { Demonstrated that a-Si (a } \\
\text { commercial thin film) has the } \\
\text { potential to reach reasonable } \\
\text { efficiencies; breathed new life } \\
\text { into a-Si as PV option }\end{array}$ \\
\hline $\begin{array}{l}\text { PV43 } \\
\text { SCI }\end{array}$ & $\begin{array}{l}\text { Rapid progress in improving } \\
\text { efficiency/output of large }\left(7200 \mathrm{~cm}^{2} \text {, }\right. \\
\left.8 \mathrm{ft}^{2}\right) \text { CdTe modules to } 8.4 \%, 60 \mathrm{~W} \\
\text { delivered } 1 \mathrm{~kW} \text { of modules to NREL } \\
\text { for testing }\end{array}$ & $\begin{array}{l}\text { Near-commercial thin film with } \\
\text { a size and power output in } \\
\text { excess of all others }\end{array}$ \\
\hline $\begin{array}{l}\text { PV43 } \\
\text { NREL }\end{array}$ & $\begin{array}{l}\text { Pushed world record for all thin films } \\
\text { from previous best ( } 15.5 \%) \text { to } 16.8 \% \\
\text { on a CIGS thin-film cell; shared } \\
\text { technology with U.S. CIGS industry } \\
\text { through on-site extended visits (e.g., } \\
\text { pushed Solarex CIGS from } 11 \% \text { to } \\
14.5 \% \text { through hands-on tech transfer) }\end{array}$ & $\begin{array}{l}\text { Shows that long-term } \\
\text { efficiencies needed by thin } \\
\text { films to be competitive with } \\
\text { conventional electricity can be } \\
\text { met; allowed full transfer of } \\
\text { DOE-funded breakthrough }\end{array}$ \\
\hline $\begin{array}{l}\text { PV43 } \\
\text { NREL }\end{array}$ & $\begin{array}{l}\text { Reached baseline capability of } 10 \% \\
\text { cells (best, } 12 \% \text { ) }\end{array}$ & $\begin{array}{l}\text { Allows NREL's relatively new } \\
\text { CdTe program to meet the } \\
\text { needs of the CdTe R\&D } \\
\text { community }\end{array}$ \\
\hline $\begin{array}{l}\text { PV43 } \\
\text { NREL }\end{array}$ & $\begin{array}{l}\text { Initial encouraging stability results on } \\
\text { hot-wire a-Si:H (no degradation } \\
\text { observed in first set of cells) }\end{array}$ & $\begin{array}{l}\text { If validated by further work, } \\
\text { could demonstrate the first } \\
\text { stable a-Si:H material suitable } \\
\text { for high-efficiency cells (this is } \\
\text { the "holy grail" of a-Si } \\
\text { research) }\end{array}$ \\
\hline
\end{tabular}




\begin{tabular}{|l|l|l|}
\hline $\begin{array}{l}\text { PV43 } \\
\text { USF }\end{array}$ & $\begin{array}{l}\text { Produced best CdTe cell on low-cost } \\
\text { sodalime glass (13.5\% efficient) }\end{array}$ & $\begin{array}{l}\text { Indicates that high efficiencies } \\
(15.8 \%) \text { seen for CdTe on } \\
\text { more costly borosilicate glass } \\
\text { should be reproduced on } \\
\text { manufacturable substrates }\end{array}$ \\
\hline $\begin{array}{l}\text { PV43 } \\
\text { SSI }\end{array}$ & $\begin{array}{l}10.2 \% \text { encapsulated CIGS power } \\
\left.\text { module (4 } \mathrm{ft}^{2}\right) ; \text { delivered world's first } \\
1-\mathrm{kW} \text { CIS array to NREL for testing }\end{array}$ & $\begin{array}{l}\text { First thin-film power module to } \\
\text { exceed 10\% efficiency }\end{array}$ \\
\hline $\begin{array}{l}\text { PV43 } \\
\text { Photon }\end{array}$ & $\begin{array}{l}\text { Introduced first commercial U.S. } \\
\text { CdTe products (demonstration scale); } \\
\text { built 2-MW production plant (first } \\
\text { CdTe plant in the world) }\end{array}$ & $\begin{array}{l}\text { Introduction of a new } \\
\text { generation of potentially lower- } \\
\text { cost thin-film products }\end{array}$ \\
\hline $\begin{array}{l}\text { PV43 } \\
\text { Energy }\end{array}$ & $\begin{array}{l}\text { Reached 9\% on 200-cm }{ }^{2} \text { CIGS; built } \\
\text { equipment for depositing 7-ft } \\
\text { modules }\end{array}$ & $\begin{array}{l}\text { Emergence of a new, more } \\
\text { dynamic force in CIGS } \\
\text { commercialization }\end{array}$ \\
\hline $\begin{array}{l}\text { PV43 } \\
\text { NREL }\end{array}$ & $\begin{array}{l}\text { Carried out Thin-Film Partnership } \\
\text { solicitation on schedule; formed } \\
\text { Partnership's CIS, CdTe, and ES\&H } \\
\text { R\&D teams }\end{array}$ & $\begin{array}{l}\text { Strengthens the momentum of } \\
\text { thin-film development; adds } \\
\text { R\&D focus to joint } \\
\text { NREL/university/industrial } \\
\text { efforts }\end{array}$ \\
\hline
\end{tabular}




\section{Thin-Film Technologies In-House Research}



Title:

\section{Development of Polycrystalline Thin-Film CdTe Solar Cells Deposited by Close Spaced Sublimation}

Organization: National Renewable Energy Laboratory

Golden, Colorado

Contributors: $\quad$ P. Sheldon, Principal Investigator

T.A. Gessert, Senior Scientist

D.S. Albin, Staff Scientist

R.G. Dhere, Staff Scientist

X. Li, Staff Scientist

D. Rose, Graduate Student

\section{Objectives:}

- Implement a focused research program which integrates materials fabrication, device development and characterization expertise, with an emphasis on a team approach.

- Work towards developing a better understanding of the limiting factors in CdTe device structures deposited by close-spaced sublimation (CSS), leading to optimized processing sequences and improved structures necessary to realize enhanced efficiencies.

- Develop the infrastructure necessary to support a competitive CdTe research program. This will include developing the capability to deposit high-quality transparent conducting oxides (TCO's) and barrier layers as well as completing a second generation CSS deposition system with enhanced versatility and control.

\section{Technical Approach:}

In FY94 the CdTe task focused on the chemical bath deposition (CBD) technique for CdS deposition and CSS for CdTe deposition. Work on sputter deposited CdTe material was suspended due to a lack of resources and a desire to focus our research efforts. The in-house $\mathrm{CdTe}$ research program consists of an integrated effort, focused on three key areas: (i) $\mathrm{CdS} / \mathrm{CdTe}$ device development - development of optimized processing sequences, focusing on an improved understanding of precontact application etching chemistries; (ii) CdTe ohmic contact development - development of an alternate high-performance, manufacturable ohmic contact; and (iii) An infrastructure development plan to develop deposition hardware which will improve process control and productivity. Progress in each of these areas is outlined below.

\section{Results:}

CdS/CdTe Device Optimization and Process Development:

Improvements in device performance over our baseline CBD CdS/CSS CdTe process were realized in three areas, which led to a $12.0 \% 0.5 \mathrm{~cm}^{2} \mathrm{SnO}_{2} / \mathrm{CdS} / \mathrm{CdTe}$ device $\left(\mathrm{V}_{\mathrm{oc}}=827 \mathrm{mV}, \mathrm{J}_{\mathrm{sc}}=20.5\right.$ $\mathrm{ma} / \mathrm{cm}^{2}, \mathrm{FF}=70.8 \%$ ). These improvements included: (i) incorporating an rf-sputtered insulating $\mathrm{SnO}_{2}$ layer in between the conductive $\mathrm{SnO}_{2}$ and the $\mathrm{CdS}$ window layer. We find that by using a $1500 \AA$ thick insulating $\mathrm{SnO}_{2}$ layer (sheet resistance $>1 \mathrm{M} \Omega /$ square), we can significantly enhance the device open circuit voltage. Using this technique we have been able to achieve $V_{o c}$ 's in excess of $800 \mathrm{mV}$; (ii) reducing the CdTe pin-hole density by the using modified deposition profiles and improved preparation techniques; and (iii) optimization of the back contact, yielding reductions in the series resistance and improved fill factors. 
Significant effort has gone into characterizing and optimizing our baseline contact procedure. The baseline process consists of a surface pre-treatment used to modify the surface chemistry of the CdTe film to facilitate ohmic contact formation. The three etchants explored were $0.1 \%$ $\mathrm{Br} / \mathrm{Methanol}(\mathrm{BM}), \mathrm{HNO}_{3}: \mathrm{H}_{3} \mathrm{PO}_{4}(\mathrm{NP})$ and $\mathrm{K}_{2} \mathrm{Cr}_{2} \mathrm{O}_{7} / \mathrm{H}_{2} \mathrm{SO}_{4}(\mathrm{KD})$. Our results indicate that the $\mathrm{HNO}_{3}: \mathrm{H}_{3} \mathrm{PO}_{4}(\mathrm{NP})$ etchant yields the lowest $\mathrm{R}_{\mathrm{s}}$ and the highest $\mathrm{R}_{\text {sh }}$ compared to both the $0.1 \%$ $\mathrm{Br} / \mathrm{Methanol}$ and $\mathrm{K}_{2} \mathrm{Cr}_{2} \mathrm{O}_{7}: \mathrm{H}_{2} \mathrm{SO}_{4}: \mathrm{H}_{2} \mathrm{O}$ etches. Therefore, we have critically evaluated the effect the NP etch $\left(2.5 \mathrm{ml}-\mathrm{HNO}_{3}: 100 \mathrm{ml}-\mathrm{H}_{3} \mathrm{PO}_{4}: 40 \mathrm{ml}-\mathrm{H}_{2} \mathrm{O}\right)$ has on both the surface chemistry and critical device parameters.

Detailed surface analyses results (AES and XPS) indicate that the surface modified layer, obtained after the NP etch, consists of a Te-rich film. Figure 1 is a plot of the Te surface concentration as a function of etch time in the NP etch (as measured by Auger electron spectroscopy). We find that the Te concentration increases with etching time. After about one minute etch in the NP solution, the surface of CdTe film is nearly covered with pure Te. To help understand the effect a Te-rich surface layer has on the on the properties of the CdTe thin-film, a very thin Te layer was evaporated on a CSS-deposited CdTe film. The Te/CdTe heterojunction properties and the electronic structure was monitored by UPS at the Synchrotron Radiation Center at the University of Wisconsin-Madison. The important results of this experiment were: (i) with as little as $100 \AA$ pure Te deposited on the CdTe surface, the surface is converted to a strong p-type material; and (ii) the valence band offset, $\Delta \mathrm{E}_{\mathrm{V}}=0.26 \mathrm{eV}$ between $\mathrm{p}-\mathrm{CdTe}$ and Te layer is small, making hole flow from the p-CdTe into the Te layer possible. This work shows that a pure Te layer pins the Fermi level close to the CdTe valence band maximum. This layer facilitates ohmic contact formation by reducing or eliminating the barrier to hole flow. Furthermore, making a good ohmic contact to the Te layer should be easy, because the band gap of Te is small (only $0.33 \mathrm{eV}$ ). These results indicate that the NP etch aids back contact formation in CdS/CdTe devices.

Cross-sectional samples were prepared and examined by SEM to analyze bulk film morphology following the chemical etch. One sample was kept as control sample, and the three remaining pieces were etched in the NP etchant for 1,2, and 5 minutes, respectively. Samples were then cleaved for cross-sectional examination. The cross-sections of the control sample reveal a very dense film with grain boundaries which are difficult to differentiate (see for example, Figure 2a). Once the samples are etched by the NP acid, the grain boundaries are clearly visible. The grain boundary delineation evolves with increased etching time. For longer times $(5$ minutes, see for example, Figure $2 \mathrm{~b}$ ) the grain boundary openings extend all the way to the CdS/CdTe interface. These results indicate that even at low nitric acid concentrations there is significant preferential etching along grain boundaries. Clearly, the Te-rich layer on the back surface is desirable for low-resistance ohmic contact formation. However, the conductive Te sheath covering grain boundaries (left by the NP etch) could be detrimental to device performance (shunting problems).

In order to investigate this effect, I-V measurements were performed on samples etched for various times. The series resistance $\left(R_{s}\right)$ change with etch conditions are shown in Figure 3 . The series resistance decreases as the etch time increases for etch times of less than 80 seconds. For etch times of greater than 80 seconds the series resistance begins to increase. This can be explained by the opening of grain boundaries producing voids which extend down to the CdS/CdTe interface. Therefore, although the NP etchant produces the most promising device parameters, this step must be carefully controlled (both etchant concentration and etchant time) to prevent device shunting problems. 
Alternative Ohmic Contacts to CdTe (Ni/ZnTe:Cu/CdTe):

$\mathrm{Cu}$ diffusion from the back contact to the CdS/CdTe interface is generally considered to be a degradation mechanism in these devices. However, historically, $\mathrm{Cu}$ has been included in many of the best II-VI solar cell devices in order to achieve low-resistance contacts. With this in mind we have continued work on contacts that include a $\mathrm{Cu}$ doped $\mathrm{ZnTe}$ interfacial layer between the metal and the CdTe. In this structure; only a small amount of $\mathrm{Cu}$ is required to dope the $\mathrm{ZnTe}$, and it should be bound in the ZnTe lattice. This structure has the potential of both enhanced performance and stability over conventional $\mathrm{Cu}$ based contacts. The general design criteria of the Metal/Cu-doped $\mathrm{ZnTe} / \mathrm{p}-\mathrm{CdTe}$ contact requires that the $\mathrm{ZnTe}$ is doped sufficiently high to allow quantum-mechanical tunneling at the metal/ZnTe interface. This tunneling will thereby afford low specific contact resistance $\left(r_{c}\right)$ at this interface, while the $\mathrm{ZnTe} / \mathrm{CdTe}$ interface allows hole current flow by the close alignment of the CdTe and $\mathrm{ZnTe}$ valence bands. We have demonstrated that the $\mathrm{Ni} / \mathrm{ZnTe}: \mathrm{Cu}$ interface has a very low contact resistance $\left(\mathrm{r}_{\mathrm{c}}=\sim 5 \times 10^{-3} \mathrm{Ohm}\right.$ $\mathrm{cm}^{2}$ for 4 at.\% $\mathrm{Cu}$ ). This result strongly suggests that the required tunneling is indeed proceeding, and that the $r_{c}$ at this interface of the contact stack will not limit the device efficiency. In fact, contact resistance measurements of $\mathrm{Ni} / \mathrm{ZnTe} \mathrm{Cu} / \mathrm{CdTe}$ deposited on glass substrates yield specific contact resistances of $<0.10 \mathrm{Ohm}-\mathrm{cm}^{2}$ (the total device $r_{c}$ can be as high as $0.1 \mathrm{Ohm}-\mathrm{cm}^{2}$ without noticeably affecting device efficiency). We have also applied the $\mathrm{Ni} / \mathrm{ZnTe}: \mathrm{Cu}$ contact to $\mathrm{CdTe} / \mathrm{CdS} / \mathrm{SnO}_{2}$ /glass devices where the CdTe was deposited by CSS. Preliminary results indicate that these contacts perform at least as well as the conventional graphite based contacts we are presently using. Work in this area is ongoing.

\section{Infrastructure Development:}

A significant effort has been undertaken by the CdTe group to enhance our deposition capabilities in conjunction with the move to the SERF. We completed the first of these projects with the commissioning of a new state-of-the-art CSS deposition system. This system has several enhancements not found on our previous deposition system which include:

- Fully automated process control system for improved run-to-run repeatability and temperature profiling.

- Fully automated and modular gas introduction system.

- Complete data logging capability for all temperature and pressure process variables.

- Significantly improved source and substrate temperature uniformity over a 25 $\mathrm{cm}^{2}$ area.

This system was commissioned on 8/30/94 and we have spent the later part of FY94 transferring our process to this new system, producing devices with efficiencies as high as $11.7 \%$. In FY95 additional deposition capabilities will be established in our SERF laboratory, these include: i) a second CSS deposition system; ii) a vapor $\mathrm{CdCl}_{2}$ deposition system; and iii) a CVD $\mathrm{SnO}_{2}$ deposition system.

\section{Future Directions:}

The emphasis will be towards developing optimized processing sequences for improved device performance and developing a fundamental understanding of what is happening at the critical $\mathrm{CdS} / \mathrm{CdTe}$ interface in CSS deposited material. This will include developing a tight baseline process, which will allow us to evaluate several alternate processing schemes (for example, vapor $\mathrm{CdCl}_{2}$ treatments and CSS CdS) and alternate back contact structures. We will also complete our infrastructure development plan which will include developing the capability to deposit highquality transparent conducting oxides and barrier layers. 


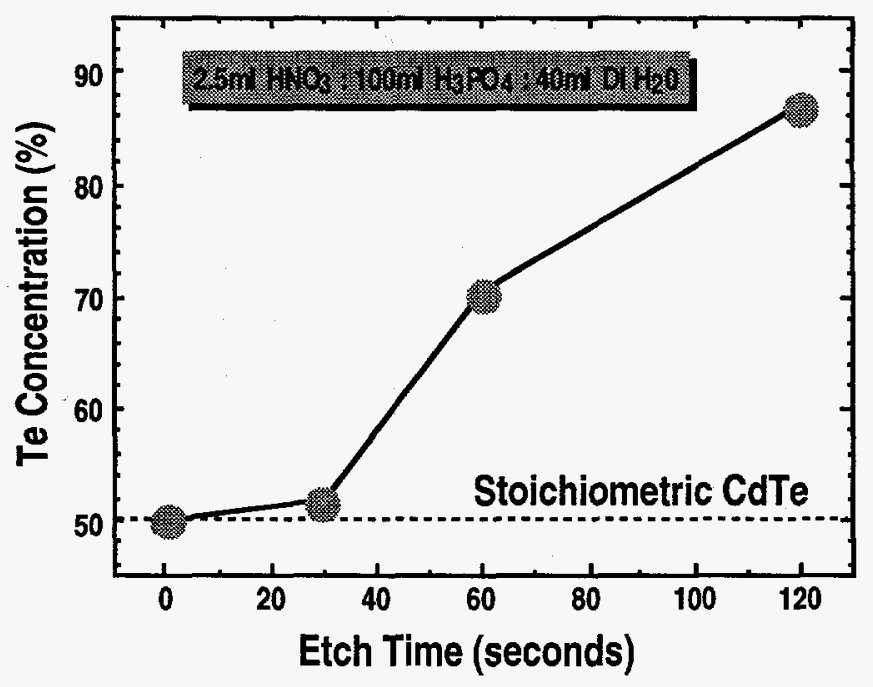

Figure 1: Te concentration as a function of time for surfaces etched in the nitric phosphoric acid etchant.

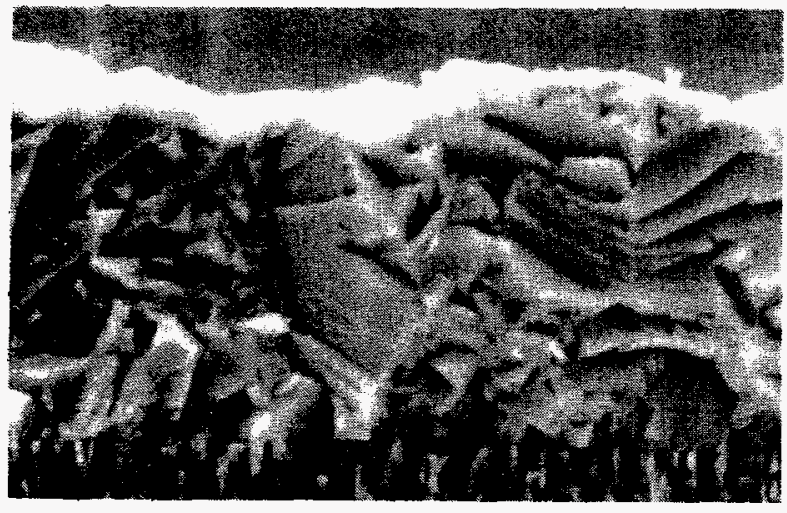

Figure 2a: Unetched CdTe film

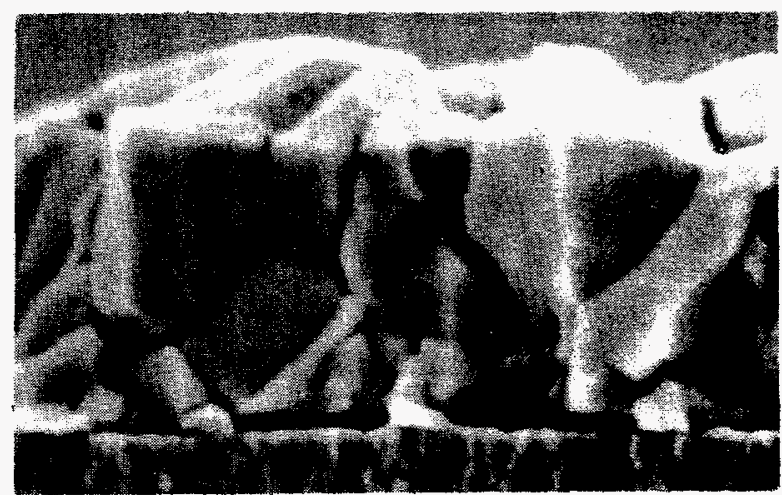

Figure 2b: CdTe film etched for $5 \mathrm{~min}$. in NP etchant

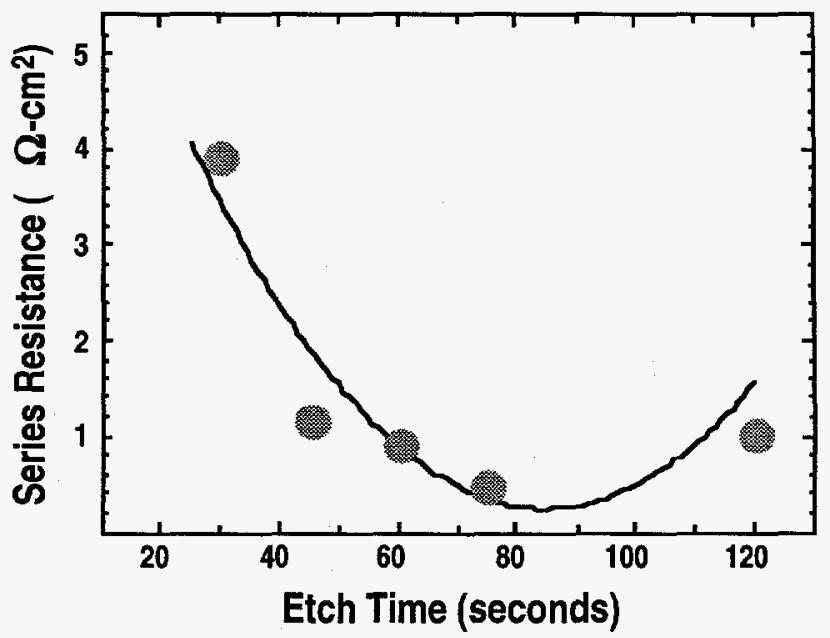

Figure 3: Series resistance for CdS/CdTe devices etched in the nitric phosphoric etchant prior to contact application. $\mathrm{R}_{\mathrm{s}}$ obtained from light $\mathrm{I}-\mathrm{V}$ measurements. 


\section{Development of Polycrystalline Cu(In,Ga)Se 2 Thin Films and Devices}

\section{Contributors:}

Full time

R. Noufi, Principal Investigator and Task Leader

J.R. Tuttle, Senior Scientist

K. Ramanathan, Senior Scientist

M. Contreras, Staff Scientist

A. Tennant, Assoc. Staff Scientist

J. Dolan, Master Technician

J. Keane, Master Technician

A. Gabor, Graduate Student, CU

L. Wang, Visiting Professional, CSM

A. Johnson, Graduate student, CSM

J. Scofield, Visiting Professional, Oberlin College

\author{
Part time \\ S. Asher, Senior Scientist \\ R. Matson, Senior Scientist \\ M. Bode, Senior Scientist \\ D. Niles, Staff Scientist \\ B. Keyes, Staff Scientist \\ A. Franz, Assoc. Staff Scientist \\ A. Mason, Master Technician
}

\section{Objective}

Advance the development of the CIS-based Photovoltaics by:

(1) Improving the materials quality and exploring new device structures to obtain high conversion efficiency.

(2) Develop different deposition processes to present options for manufacturing.

(3) Addressing key technical issues in partnership with industry which will support their plans for near-term commercialization.

\section{Technical Approach}

We take the following approaches:

(1) Improve the performance of devices (efficiency) by enhancing the quality of the material and exploring new device structures. This will be accomplished by optimizing the processes involved in the different layers of the cells including the absorber.

(2) Guided by device modeling, engineer the band gap by alloying with Ga according to prescribed compositional profiles.

(3) Develop road maps for the reaction pathways used to fabricate the absorber to guide the development of manufacturing processes.

(4) Work with industry on technology transfer.

\section{Results}

Device Development. In FY93, we described the development of four deposition processes to fabricate high efficiency solar cells based on $\mathrm{Cu}(\mathrm{In}, \mathrm{Ga}) \mathrm{Se}_{2}$ (See NREL PV Program Annual Report FY 1993). This period we focused on the one process (the three-stage process) which demonstrated the highest efficiency. The absorber formation in the three-stage process is a serial combination of ( $\left.\operatorname{In}_{0.75} \mathrm{Ga}_{0.25}\right)_{2} \mathrm{Se}_{3}$ delivery, CIGS compound formation and recrystallization by exposure to $\mathrm{Cu}_{\mathrm{x}} \mathrm{Se}$ at $\mathrm{T}_{\text {sub }}=550^{\circ} \mathrm{C}$, and surface modification by exposure to (In,Ga)-Se activity until termination. Details of the microstructure evolution in the three-stage process and additional discussion of variable band gap absorber structures are described in the references given below.

Figure 1 shows a summary of performance for devices of different total areas. The $16.8 \%$ total area efficiency represents a world-record for any thin-film solar cell. It is also worth noting here the performance of the CIGS device under moderate concentration. A one-sun $15.1 \%$ efficient 
device improved to $17.2 \%$ under 22 -sun concentration. The reader is referred to ref. ( ) for details.

Absorber development. We have developed three simple and scalable processes to fabricate device quality thin-film CIS and CIGS. We utilize three approaches which result in large grain absorber films. The processes features allow for: (In, Ga) profiling into the absorber; do not require precise control of the $\mathrm{Cu} /(\mathrm{In}+\mathrm{Ga})$ ratio; and allow for in-line/in-situ diagnostics and endpoint detection. The advantages of these processes are: (1) reproducible formation chemistry, (2) wider tolerance window for composition variation relative to other processes, (3) precursor material can be produced by a variety of deposition techniques, (4) processes can produce homogeneous and/or graded CIS and CIGS, and (5) processes produce highest efficiency solar cells due to improved crystallinity and smooth surfaces.

In FY93, we described a universal growth model for the formation dynamics of the thin-film CIS absorber. Here, we present a concise growth model for the process responsible for the highest efficiency (referred to here as the 3-stage process). This model is consistent with the universal growth model from FY93. Fig. 2 depicts graphically the growth mechanisms as described below.

1. Precursor formation - Codeposition of $\mathrm{In}+\mathrm{Ga}+\mathrm{Se}$ forms amorphous or crystalline (In,Ga)2Se3 with an hexagonal crystal orientation. Annealing the film up to $560^{\circ} \mathrm{C}$ more fully crystallizes the film into $\gamma(\mathrm{In}, \mathrm{Ga})_{2} \mathrm{Se}_{3}$. The $\mathrm{Ga}$-In distribution is fairly uniform. The film is specularly smooth.

2. Evolution to $\mathrm{Cu}(\mathrm{In} . \mathrm{Ga}) \mathrm{Se}_{2}-$ Addition of $\mathrm{Cu}+\mathrm{Se}$ at $\mathrm{T}_{\mathrm{S}} \sim 560^{\circ} \mathrm{C}$ causes an evolution of the film through intermediate phases on the $\mathrm{Cu}_{2} \mathrm{Se}-(\mathrm{In}, \mathrm{Ga})_{2} \mathrm{Se}_{3}$ pseudobinary tie line. The kinetics of the reactions are sufficiently fast such that the process is not rate limited by $\mathrm{Cu}$ diffusion but is rather rate limited at a Cu delivery of $2.0 \AA / \mathrm{s}$. The progression goes through a) mixed (In,Ga) ${ }_{2} \mathrm{Se}_{3}$ (hexagonal) $+\gamma \mathrm{Cu}-\mathrm{In}-\mathrm{Ga}-\mathrm{Se}$ (hexagonal); b) $\gamma \mathrm{Cu}-\mathrm{In}-\mathrm{Ga}-\mathrm{Se}$ (hexagonal) $+\beta \mathrm{Cu}-$ In-Ga-Se (cubic); c) $\alpha \mathrm{Cu}-\mathrm{In}-\mathrm{Ga}-\mathrm{Se}$ (tetragonal); d) stoichiometric $\alpha \mathrm{Cu}(\mathrm{In}, \mathrm{Ga}) \mathrm{Se}_{2}$ (tetragonal). The grain surfaces are modeled as reservoirs of $\mathrm{Cu}$ with the $\mathrm{Cu}$ in the form of $\mathrm{Cu}(\mathrm{s}), \mathrm{Cu}_{\mathrm{x}} \mathrm{Se}$ (s or $\ell$ ), and/or $\mathrm{Cu}-\mathrm{In}-\mathrm{Ga}-\mathrm{Se}$ phases of higher $\mathrm{Cu}$ concentration than the bulk. The progression from $\beta \mathrm{Cu}-\mathrm{In}-\mathrm{Ga}-\mathrm{Se}$ to $\mathrm{Cu}(\mathrm{In}, \mathrm{Ga}) \mathrm{Se}_{2}$ is modeled as diffusion of $\mathrm{Cu}$ from the $\mathrm{Cu}$ reservoir into the bulk of the grains and diffusion of In and $\mathrm{Ga}$ from the bulk toward the top surface where the In and $\mathrm{Ga}$ can react with material in the reservoir to form new unit cells. In this way, the film increases in thickness. Out diffusion of In is energetically favorable over out diffusion of $\mathrm{Ga}$, and thus the surface region becomes progressively depleted of $\mathrm{Ga}$ relative to the rest of the film. The films have grains which on average are $<1 \mu \mathrm{m}$ in size.

3. Recrystallization by $\mathrm{Cu}_{\mathrm{x}} \mathrm{Se}$ - Upon adding enough $\mathrm{Cu}+\mathrm{Se}$ to make the film $\mathrm{Cu}-\mathrm{rich}$, the excess $\mathrm{Cu}$ forms a secondary $\mathrm{Cu}_{\mathrm{x}} \mathrm{Se}(\ell)$ phase which acts as a flux to encourage grain growth. No more than $26 \%$ total $\mathrm{Cu}$ ( $1 \%$ beyond stoichiometry) is necessary for effective recrystallization which increases the grains on average to widths close to the film thickness down to $1 \mu \mathrm{m}$. At $560^{\circ} \mathrm{C}$, this recrystallization can occur within 2 minutes. The film exists as a two-phase mixture of stoichiometric CIGSe and $\mathrm{Cu}_{\mathrm{x}} \mathrm{Se}$.

4. Consumption of $\mathrm{Cu}_{\mathrm{x}} \mathrm{Se}-$ Deposition of $\mathrm{In}+\mathrm{Ga}+\mathrm{Se}$ to the $\mathrm{Cu}$-rich film converts the $\mathrm{Cu}_{\mathrm{x}} \mathrm{Se}$ to additional stoichiometric CIGSe.

5. Evolution to Cu-poor CIGSe - Further deposition of In+Ga+Se to the stoichiometric CIGSe causes a diffusion of $\mathrm{Ga}$ and In into the bulk to make these grains $\mathrm{Cu}$-poor. This indiffusion is accompanied by an out diffusion of $\mathrm{Cu}$, and this $\mathrm{Cu}$ reacts with the additional $\mathrm{In}+\mathrm{Ga}+\mathrm{Se}$ to form new unit cells at the surface. Preferential indiffusion of $\mathrm{Ga}$ over In can occur.

Other Developments. In addition to process development and optimization of the materials quality to achieve high efficiency, we studied fundamental issues related to the interdiffusion of 
In and Ga during the absorber growth, and understanding and shaping the properties of the Mo film back contact. The following is a list of some of the topics explored:

(1) The structure, chemistry, and growth mechanisms of CIGS films.

(2) In and $\mathrm{Ga}$ interdiffusion in alloy structures of CIGS.

(3) Band gap engineering in CIGS thin films.

(4) Understanding the interaction between the glass substrate and the Mo back contact.

(5) Microstructural effects associated with the deposition conditions of Mo.

A complete account of the results can be found in the following references:

\section{References}

1. "Progress Towards Higher Efficiency CuInSe 2 and CuInGaSe 2 Solar Cells by Se-vapor Selenization," by D.S. Albin, J.R. Tuttle, M. Contreras, A.M. Gabor, A. Mason, and R. Noufi (NREL) and P. Singh (Villanova). Submitted to Solar Energy Materials and Solar Cells.

2. "Characterization of variable-band-gap thin-film $\mathrm{Cu}(\mathrm{In}, \mathrm{Ga}) \mathrm{Se}_{2}$ : A Simple Model for the Interdiffusion of In and $\mathrm{Ga}$ in Alloy Structures," by J.R. Tuttle, D.S. Albin, A. Tennant, A.M. Gabor, M. Contreras, R. Noufi. Solar Energy Materials and Solar Cells 35 (1994), 193-201, Elsevier.

3. "16.4\% Total area Conversion Efficiency Thin-film Polycrystalline $\mathrm{MgF}_{2} / \mathrm{ZnO} / \mathrm{CdS} / \mathrm{Cu}(\mathrm{In}, \mathrm{Ga}) \mathrm{Se}_{2} / \mathrm{Mo}$ Solar Cell," by M.A. Contreras, A.M. Gabor, A.L. Tennant, S. Asher, J. Tuttle, R. Noufi. Progress in Photovoltaics: Research and Applications, , Vol. 2, 287-0292 (1994).

4. "Graded band-gap $\mathrm{Cu}(\mathrm{In}, \mathrm{Ga}) \mathrm{Se}_{2}$ Thin-film Solar Cell Absorber with enhanced Open-circuit Voltage", by M. Contreras, J. Tuttle, D. Du, Y. Qi, A. Swartzlander, A. Tennant, and R. Noufi, Appl. Phys. Lett 63 (13), 27 Sept. 93.

5. "High Efficiency $\mathrm{Cu}(\mathrm{In}, \mathrm{Ga}) \mathrm{Se}_{2}$-Based Thin-film Solar Cells Fabricated by Simple, Scalable Processes," by J.R. Tuttle, A/.M. Gabor, D.S. Albin, A. Tennant, M. Contreras, and R. Noufi, Proceedings of the 7th Sunshine Workshop on Thin-Film Solar Cells, Tokyo, Japan.

6. "The Structure, Chemistry, and Growth Mechanisms of Photovoltaic Quality Thin-Film $\mathrm{Cu}\left((\mathrm{n}, \mathrm{Ga}) \mathrm{Se}_{2}\right.$ Grown from a Mixed-Phase Precursor," by J.R. Tuttle, M. Contreras, M.H. Bode, D. Niles, D.S. Albin, R. Matson, A.M. Gabor, A. Tennant, A. Duda, and R. Noufi, Journal of Applied Physics, in press.

7. "Sodium Diffusion, Selenization, and Microstructural Effects Associated with Various Molybdenum Back Contact Layers for CIS-based Solar Cells," by J.H. Scofield, S. Asher, D. Albin, T. Tuttle, M. Contreras, D. Niles, R. Reedy, A. Tennant, and R. Noufi, Proceedings of the 1st World Conference on Photovoltaic Energy Conversion, Waikoloa, Hawaii, Dec. 1994 (in press).

8. "High Efficiency $\mathrm{Cu}(\mathrm{In}, \mathrm{Ga}) \mathrm{Se}_{2}$-Based Solar Cells: Processing of Novel Absorber Structures," by M.A. Contreras, J. Tuttle, A. Gabor, A. Tennant, K. Ramanathan, S.Asher, A. Franz, J. Keane, L. Wang and R. Noufi, Proceedings of the 1st World Conference on Photovoltaic Energy Conversion, Waikoloa, Hawaii, Dec. 1994 (in press).

9. "High-efficiency Cu(In,Ga)Se 2 -Based Thin-film Solar Cells: $16.8 \%$ Total-area 1-Sun and $17.2 \%$ Total-area 22-sun Device Performance," by J.R. Tuttlem, M.A. Contreras, J.S. Ward, A.M. Gabor, K.R. Ramanathan, A.L. Tennant, L. Wang, J. Keane, and R. Noufi, Proceedings of the 1st World Conference on Photovoltaic Energy Conversion, Waikoloa, Hawaii, Dec. 1994 (in press).

10. "Band-Gap Engineering in $\mathrm{Cu}(\mathrm{In}, \mathrm{Ga}) \mathrm{Se}_{2}$ Thin Films Grown from (In,Ga) 2 Thin Films Grown from (In,Ga) $2 \mathrm{Se}_{3}$ Precursors," by A.M. Gabor, J.R. Tuttle, A. Schwartzlander, A.L. Tennant, M.A. Contreras, and R. Noufi, Proceedings of the Ist World Conference on Photovoltaic Energy Conversion, Waikoloa, Hawaii, Dec. 1994 (in press). 


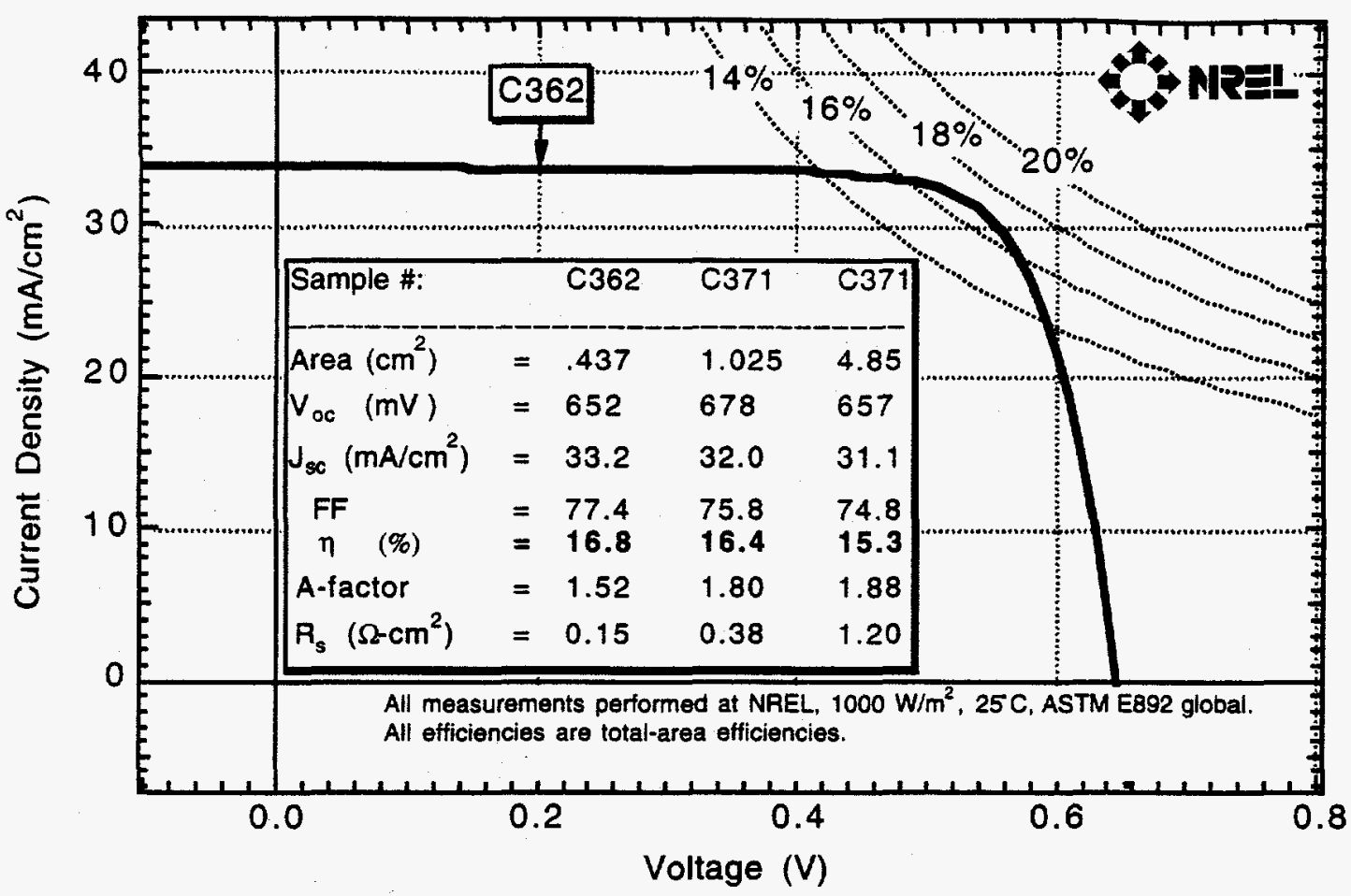

Fig. 1. I-V characteristic of $16.8 \%$ total-area efficient CIGS-based solar cell. The device parameters of other champion cells are shown for comparison

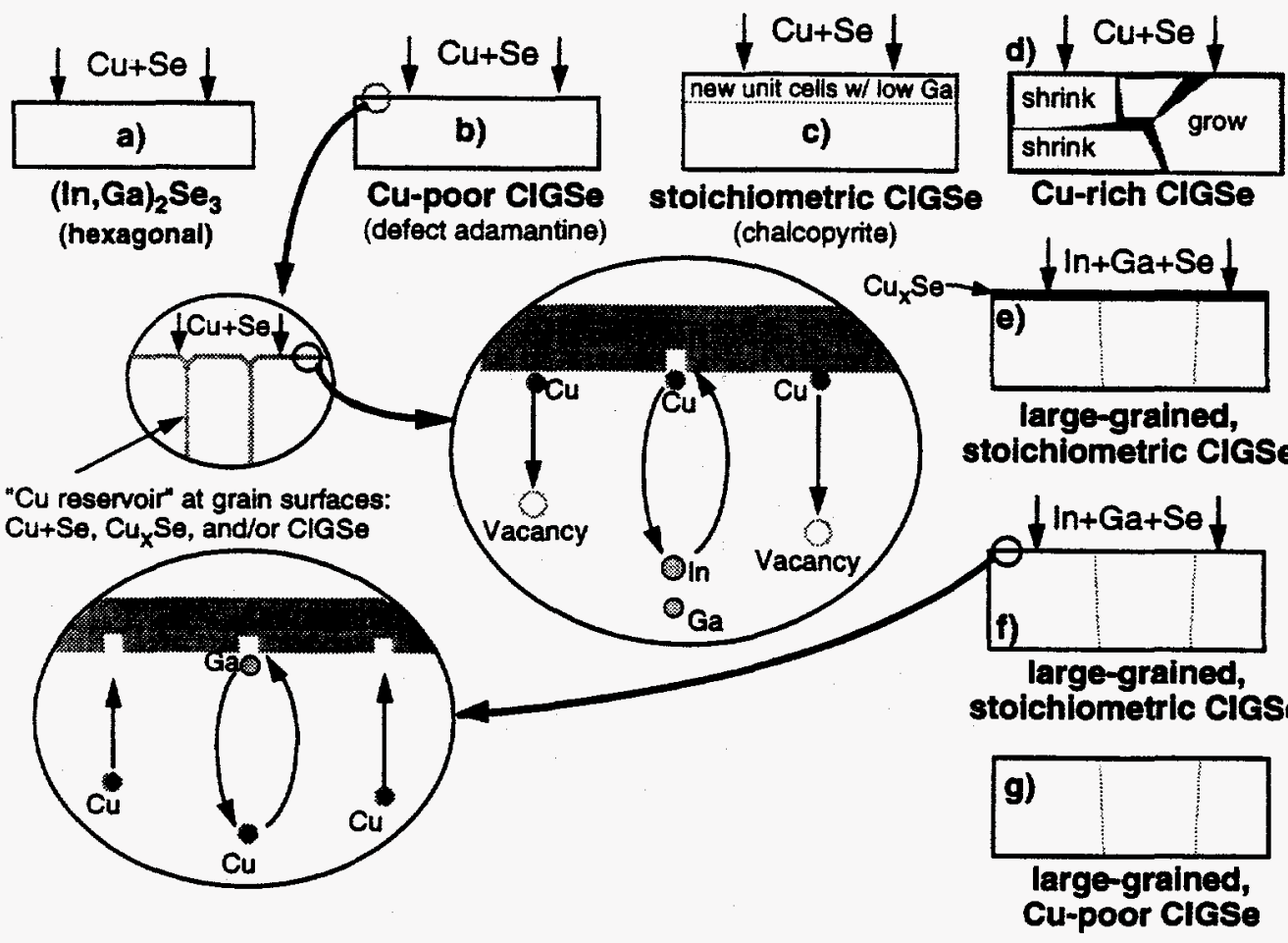

Fig. 2. A growth model for the 3-stage recipe. 
Title:

Connection between Dangling Bond

Relaxation and Metastability in

Hydrogenated Amorphous Silicon

Organization:

National renewable Energy Laboratory, Golden CO

Contributers:

Richard Crandall, principal investigator, Marten Carlen, and Yueqin Xu

\section{Objective}

To find the source, understand and ameliotate the effects of light induced degradation in hydrogenated amorphous silicon (a-Si:H).

\section{Approach}

Metastable light-induced degradation (Staebler-Wronski effect) in a-Si:H has plagued the material since its discovery nearly 20 years ago. Although much progress has been made in reducing the effect through solar cell engineering, it still limits the ultimate efficiency of the material. Several models have been proposed to explain the Staebler-Wronski effect and then point to ways to reduce it. However, the most popular model pointing to hydrogen as being the source of the metastability has not been experimentally verified and does not suggest ways to cure the effect and while maintaining material quality. The model suggesting that charged dangling bonds are the source of the Staebler-Wronski effect does point to ways of reducing the effect by reducing these defects through structural improvements. However, the central thesis of this model that charged dangling bonds, $\mathrm{D}^{+}$and $\mathrm{D}^{-}$, can be converted to neutral dangling bond, $\mathrm{D}^{0}$, has not been shown. To try and verify this model we make transient capacitance experiments on p-type hydrogenated amorphous silicon where we can control the charge state of the dangling bond.

\section{Experimetal details}

The a-Si:H samples are fabricated using radio-frequency glow discharge of silane and doping gases. Trimethylboron (TMB) and phosphine, respectively, are used for the p-and n-type doping. The $\mathrm{H}$ content of the samples is about $10 \%$. The films are grown on $1 \times 1$ in. 7059 glass substrates coated with $\mathrm{SnO}_{2}$ that serves as a back contact to the $\mathrm{p}$ layer. To probe $\mathrm{p}$-type samples we deposited the following sequence on the $\mathrm{SnO}_{2}$ coated glass; a $20 \mathrm{~nm}$ thick 1 vol\% (TMB/SiH 4$)$ $\mathrm{p}^{+}$layer; a thick lightly doped p layer, about $0.05 \mathrm{vol} \%(\mathrm{TMB} / \mathrm{SiH}) ;$ a $40 \mathrm{~nm}$ thick $1 \mathrm{vol} \%$ $\left(\mathrm{PH}_{3} / \mathrm{SiH}_{4}\right)$ doped $\mathrm{n}$-type layer and a metallic contact. The thick, lightly doped $\mathrm{p}$ layer varies between $420 \mathrm{~nm}$ and $1.5 \mu \mathrm{m}$ in different samples. The sample area is about $600 \mathrm{~mm}^{2}$ and the area of the $\mathrm{Al}$ top contact is $5 \mathrm{~mm}^{2}$. The capacitance is measured by a standard lock-in technique using a frequency of $10 \mathrm{kHz}$ and an ac test voltage of $30 \mathrm{mV}$ rms. The measuring bias is $-2 \mathrm{~V}$. [1] The devices are mounted in an evacuated liquid nitrogen Dewar capable of maintaining a stable temperature. Measurements are performed at different temperatures between $299 \mathrm{~K}$ and $470 \mathrm{~K}$. The trapped charge, $\mathrm{N}(\mathrm{t})$, using the depletion width approximation, is related to the capacitance by: $N(t) \propto C^{2}(-0)-C^{2}(t)$, where the steady state reference capacitance, $C(-0)$ is measured prior to the voltage pulse. We use this depletion width approximation and a built in voltage of $1.3 \mathrm{~V}$ to calculate $N(t)$.

\section{Results}


In this section we present examples of charge transients and the method of data analysis for the devices described above. As discussed in [1], dangling bond relaxation and annealing of metastability are often observed during the same capacitance transient. However, at low temperature and for short majority carrier pulses one observes only a pure dangling bond relaxation exhibiting a power law decay of the form, $\mathrm{N}(\mathrm{t})=0.5 \mathrm{~N}_{\text {rel }}\left(\mathrm{t} / \tau_{\mathrm{rel}}\right)^{-\mathrm{c}}$, where $\mathrm{c}$ is the power-law exponent, $\mathrm{N}_{\text {rel }}$ is the number of defects and $\tau_{\text {rel }}$ the time for $\mathrm{N}(\mathrm{t})$ to decrease to $0.5 \mathrm{~N}_{\text {rel }}$. At high temperature the transient shows mainly metastability annealing described by stretched-exponential decay of the form, $N(t)=N_{\text {met }} \exp \left(-\left(t / \tau_{\text {met }}\right)^{\beta}\right)$, where the characteristic time, $\tau_{\text {met }}$, for this decay exhibits an Arrhenius behavior with a characteristic energy that depends on sample type.[2-5]

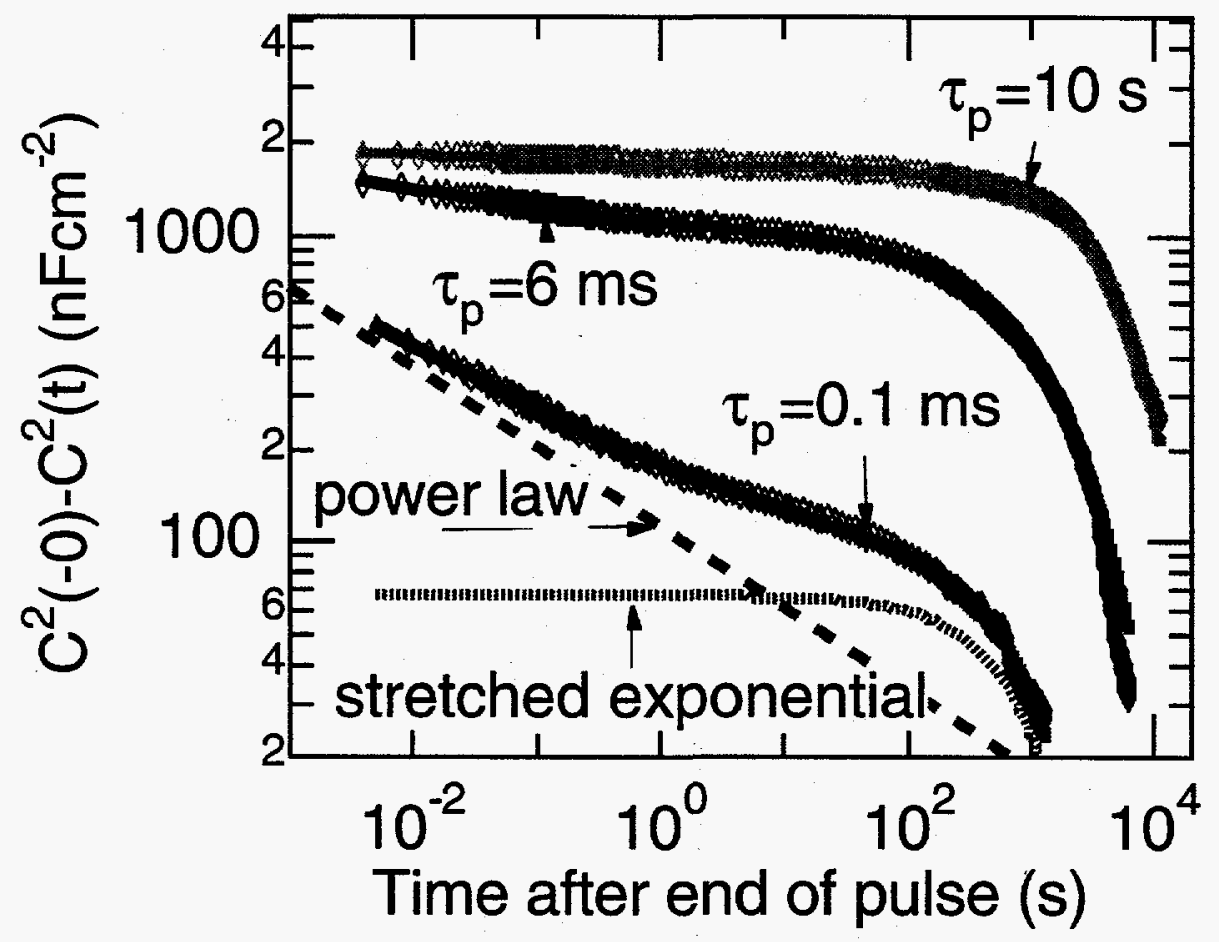

Figure 1. Decay transients for sample 90 exhibiting both relaxation and metastability annealing at $\mathrm{T}=364 \mathrm{~K}$. The data are represented by symbols and the solid lines are the best fits to the data using a sum of power law and stretched exponential functions.

Figure 1 shows capacitance transients representing annealing of metastable trapped holes as well as power law relaxation behavior at short time. The data in Fig. 1 clearly show the mixing of the two decay modes. The emission transient for the shortest pulse $(0.1 \mathrm{~ms})$ decays like a power law for short times and then a stretched exponential for longer times. For the longest pulse $(10 \mathrm{~s})$, the decay exhibits mostly a stretched exponential time dependence. However, the initial part is a fast power law decay indicative of relaxation. At lower temperature only a pure power law decay with exponent $c=0.3$ is observed.[1] The longer $\tau_{p}$ (time of pulse) also result in longer times for the metastable trapped holes to thermally anneal. At $\tau_{\mathrm{p}}=0.1 \mathrm{~ms}$, metastability annealing contributes less than $5 \%$ to the measured emission transient. For the $6 \mathrm{~ms}$ pulse roughly half the observed transient corresponds to dangling bond relaxation and half to metastability annealing. For this pulse length, $\tau_{\mathrm{met}}$ is five orders of magnitude longer than $\tau_{\text {rel }}$. However, at $\tau_{\mathrm{p}}=10 \mathrm{~s}$, trapped 
hole annealing contributes about $75 \%$ to the transient. Metastable trapped hole annealing transients depend weakly on $\tau_{\mathrm{p}}$ in contrast to relaxation which shows a linear variation of $\tau_{\text {rel }}$ with $\tau_{\mathrm{p} \text {.[1] }}$

The data presented in Fig. 1 is for a temperature high enough that the metastability anneals during the time of measurement. At lower temperatures the metastability anneals so slowly that measurements of $\mathrm{D}^{0}$ relaxation can be made during a time where the change in metastable trapped holes is imperceptible. For example, complete annealing requires over $10^{5} \mathrm{~s}$ at room temperature.[1] We exploit this opportunity to demonstrate that the number of $\mathrm{D}^{0}$ decrease as the number of metastable trapped holes increase. The increase in the number of these metastable trapped holes can be measured by the decrease in the dc capacitance.

The experiment to probe these changes in the density of $D$ defects proceeds as follows: We apply a series of progressively longer preparatory $-0.5 \mathrm{~V}$ pulses to trap the holes in the depletion width. After the fast charge transient( due to $\mathrm{D}$ defect relaxation) following each preparatory pulse has died away, a short ( $\tau_{\mathrm{p}}=6 \mathrm{~ms}$ ) probe voltage pulse produces a charge transient due to the $D$ defects that can undergo relaxation. The amplitude of these probe transients measures the number of remaining $\mathrm{D}^{0}$. Figure 2 compares the decrease in $\mathrm{D}^{0}$ with the increase in trapped positive charge for samples 90 and 20. The data show the remarkable result that the number of holes trapped during degradation equals the number of $\mathrm{D}^{0}$ that are lost during degradation. For this temperature $20 \%$ of the total $\left(1.2 \times 10^{18} \mathrm{~cm}^{-3}\right) \mathrm{D}^{0}$ are converted to $\mathrm{D}^{+}$.

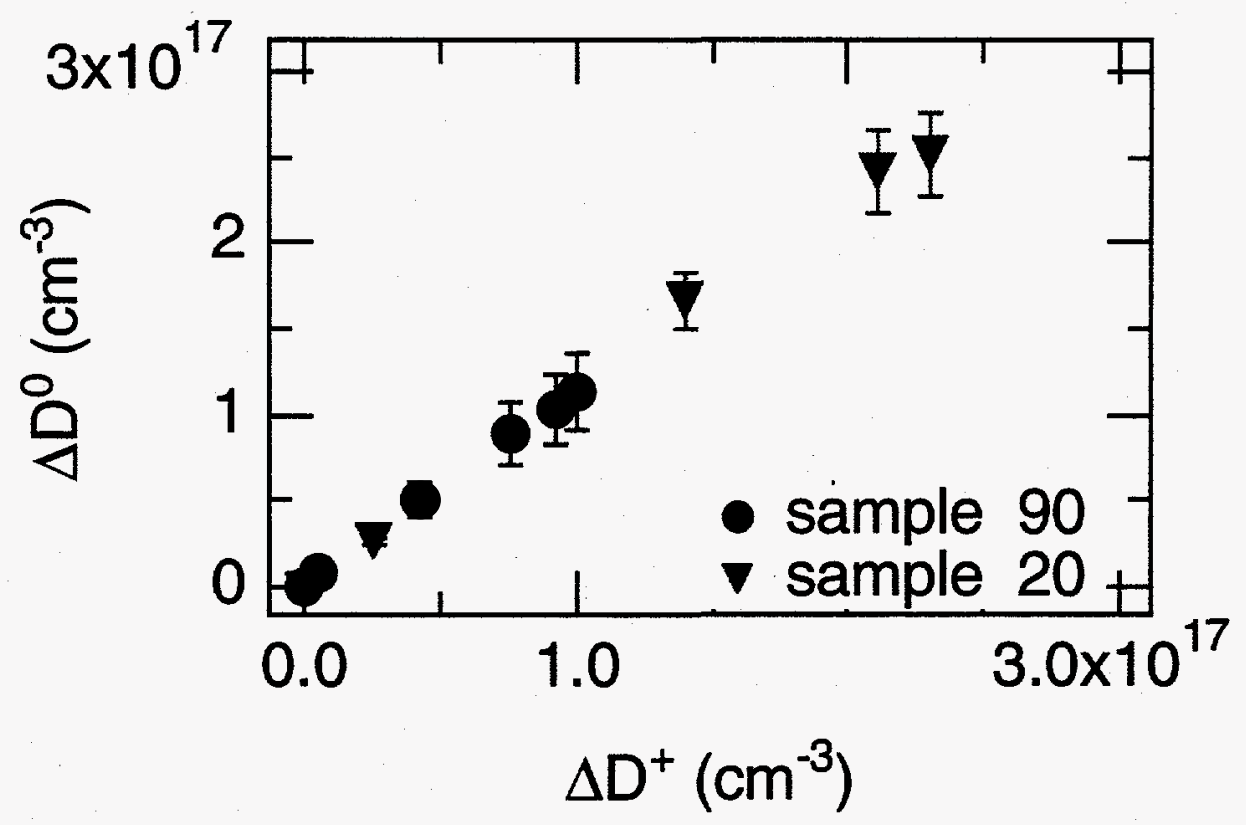

Figure 2. The change in neutral defects, labeled $\Delta \mathrm{D}^{0}$ determined from probe pulse amplitude plotted versus positive charged defects, labeled $\Delta \mathrm{D}^{+}$, determined from the change in $\mathrm{dc}$ capacitance. Measured at $310 \mathrm{~K}$ for sample 90 and at $327 \mathrm{~K}$ for sample 20.

The result that $\mathrm{D}^{0}$ can trap holes and convert to positive $\mathrm{D}^{+}$in a metastable configuration has a natural explanation in terms of the Adler mechanism. The Adler model implies that the reaction $\mathrm{D}^{0}+\mathrm{h} \underset{\mathrm{D}}{\leftarrow} \mathrm{D}^{+}$might also produce metastable charge trapping. This reaction is applicable to the experiments described above where holes are trapped on $\mathrm{D}^{0}$ during an injection pulse. If the 
barrier to conversion of the $\mathrm{D}^{0}$ in configuration $\mathrm{Q}^{0}$ to $\mathrm{D}^{+}$in configuration $\mathrm{Q}^{+}$can be surmounted, a metastable $\mathrm{D}^{+}$results. If not the hole is quickly thermally emitted. Our experiments show a significant temperature activated barrier for this reaction as well as the inverse reaction that anneals the metastable $\mathrm{D}^{+}$back to $\mathrm{D}^{0}$.

The connection between relaxation and metastability, described in the above experiments now becomes clear in terms of the Adler mechanism[6] and the structural memory model for relaxation proposed by Branz and Fedders.[7] Relaxation is just the deepening of the transition level due to bond angle change in response to the charge trapped on the $\mathrm{D}^{0}$. However, if this change proceeds far enough, the elasticity of relaxation is lost and the system remains frozen in the configuration consistent with the charge on the $D$.

\section{Summary}

The data show that relaxation and metastability are intimately related. In the charge emission transients one can observe both relaxation and annealing of metastability. Nevertheless, they can be easily distinguished because they decay on different time scales and each has its own unique time dependence. Near room temperature the transients show mostly relaxation and at higher temperature they show mostly metastability annealing. The following important features are observed.

(1) Hole injection converts neutral dangling bonds to metastabily trapped positive charge. One neutral dangling bond converts into one positively charged dangling bond.

(2) The reverse reaction one positively charged dangling traps an electron and converts to one neutral dangling bond is just the Adler Model [6] of metstability.

Therefore, our experiments demonstrate that this is the model most appropriate to describe the Staebler-Wronski effect. Its cure then is to reduce the number of charged dangling bonds in the $i$ layer of a solar cell.

\section{References}

1. M. W. Carlen, Y. Xu, and R. S. Crandall, Observation of Slow Dangling-Bond Relaxation in p-type Hydrogenated Amorphous Silicon., Phys. Rev. B 51, 2173 (1995).

2. R. A. Street, J. Kakalios, C. C. Tsai, and T. M. Hayes, Thermal equilibrium processes in amorphous silicon., Phys. Rev. B 35, 1316 (1987).

3. R. A. Street, M. Hack, and W. B. Jackson, Mech of thermal equilibration in doped., Phys. Rev. B 37, 4209 (1988).

4. R. S. Crandall, Defect Relaxation in Amorphous Silicon - Stretched Exponentials, the Meyer-Neldel Rule, and the Staebler-Wronski Effect., Phys. Rev. B 43, 4057 (1991).

5. R. S. Crandall, Metastable defects in a-Si:H., Phys. Rev. B 36, 2645 (1987).

6. D. Adler, Origin of the Photo-Induced Changes in Hydrogenated Amorphous Silicon., Solar Cells 9, 133 (1983).

7. H. M. Branz and P. Fedders, Structural Relaxation, in Amorphous Silicon Technology1994, edited by A. Madan, M. J. Thompson, P. G. LeComber, Y. Hamakawa, and P. C. Taylor (Materials Research Society, Pittsburgh, 1994), p. 129. 


\title{
Title: Studies of $\mathbf{H}$ Diffusion and $\mathbf{H}$ Bonding in Device Quality a-Si:H
}

\section{Organization: National Renewable Energy Laboratory, 1617 Cole Blvd., Golden, $\mathrm{CO} 80401$}

\author{
Contributors: A. H. Mahan, principal investigator; E. J. Johnson, research \\ technician; H. M. Branz, senior scientist; E. Francisco, MAERC student \\ trainee
}

We have examined the effusion of $\mathrm{H}$ from device-quality glow discharge deposited a-Si:H films. In particular, we measured the relative decrease in the amount of Si- $\mathrm{H}$ infrared absorption as pieces of an a-Si:H sample were incrementally annealed at fixed temperatures between 425$500^{\circ} \mathrm{C}$. After each anneal, a room temperature IR spectrum was taken, and the relative decrease in the amount of $\mathrm{SiH}$ absorption was determined by measuring the area under the absorption curve. The anneal time increments were adjusted as needed to progressively evolve a large fraction (typically $>90 \%$ ) of the bonded $\mathrm{H}$. The composite data were fit first to the rate equation for loss of $\mathrm{SiH}$ bonds, and then to the diffusion equation for $\mathrm{H}$ in bulk material to determine the activation energies needed to break the $\mathrm{SiH}$ bonds and the diffusion prefactors for bulk $\mathrm{H}$ transport in the a-Si:H lattice. We observed the presence of two distinct rate processes, a fast process corresponding to effusion of $\sim 70 \%$ of the $\mathrm{H}$ contained in the sample, and a slower process corresponding to effusion of the remainder. An Arrhenius plot yielded an activation energy of 1.4-1.5 eV for the fast (majority $\mathrm{H}$ ) loss process; this is the dominant loss process previously examined by $\mathrm{H}$ evolution and deuterium tracer diffusion measurements. A similar analysis for the slow (minority $\mathrm{H}$ ) loss rate process yielded an activation energy of $\sim 2.1 \mathrm{eV}$ and a diffusion constant prefactor higher than that for the fast loss process. The latter value represents the first determination (1) by any method of the bonding depth of the "isolated" $\mathrm{H}$ component previously observed in nuclear magnetic resonance experiments. These results are important from two points of view. First, from a basic science standpoint, they shed light on the fundamental question of how much bond reconstruction occurs after the rupture of an isolated $\mathrm{SiH}$ bond. Bond reconstruction has played an integral part in the successful interpretation of the clustered $\mathrm{H}$ evolution data, but has not been seriously considered for the isolated $\mathrm{H}$ component, partly due to the lack of experimental data. And secondly, recent annealing experiments have suggested that the Staebler-Wronski effect may be $\mathrm{H}$ site specific (2), so it is important to have an established experimantal method available to examine not only the presence, but also the bonding of both types of $\mathrm{H}$.

(1) A. H. Mahan et. al., to be presented at Spring MRS Conference, San Francisco, CA, April 1995.

(2) M. Vanecek and A. H. Mahan, J. non-Cryst. Solids (M. Silver Memorial Issue), 1995, in press. 
Title:

Organization:

Contributors:
Amorphous Silicon Solar Cells Incorporating Hot-Wire Deposited Intrinsic Layers

National Renewable Energy Laboratory (NREL), 1617 Cole Blvd., Golden, CO 80401 , USA

* Universiteit Utrecht, Debye Institute, Department of Atomic and Interface Physics, P. O. Box 8000, 3508 TA Utrecht, The Netherlands

Brent P. Nelson, Eugene Iwaniczko, Ruud E. I. Schropp*, Harv Mahan, Edith C. Molenbroek, Stan Salamon, and Richard S. Crandall
We make our most stable intrinsic materials by flowing silane over a hot filament using a process known as filament assisted chemical vapor deposition (CVD), or the hot-wire technique. By using substrate temperatures higher than the typical temperature regime for depositing device quality a-Si:H, the hot-wire technique produces a-Si:H with very low hydrogen concentrations. Materials with hydrogen concentrations in the range of 2-3 atomic \%, the material exhibits remarkable stability to light exposure and thermal quenching is absent.

In Table 1 we summarize typical values for solar cell characteristics for both allPECVD and devices where the $\mathrm{p}+$ and $\mathrm{n}+$ layers are made by PECVD and the i-layer is made by the hot-wire technique (hybrid devices). The best efficiency values for our all-PECVD devices are $10 \%$ and $7.3 \%$ for our hybrid cells. Note that we did not make $\mathrm{i}$-layers in the hybrid devices at the higher temperatures necessary to make the very low hydrogen content material. This is a first step in making cells replicating the conditions of our PECVD devices.

\begin{tabular}{|l|c|c|c|c|}
\hline $\begin{array}{c}\text { Device } \\
\text { Descrip- } \\
\text { tion }\end{array}$ & $\begin{array}{c}\text { Voc } \\
\text { (V) }\end{array}$ & $\begin{array}{c}\text { Jsc } \\
(\mathrm{mA} / \\
\left.\mathrm{cm}^{2}\right)\end{array}$ & ff $^{*}$ & $\begin{array}{c}\text { Eff. } \\
(\%)\end{array}$ \\
\hline PECVD & 0.84 & 16.79 & 0.68 & 9.53 \\
\hline PECVD* $^{*}$ & 0.80 & 15.94 & 0.56 & 7.14 \\
\hline hybrid & 0.80 & 14.24 & 0.54 & 6.16 \\
\hline hybrid* & .078 & 12.30 & 0.48 & 4.62 \\
\hline
\end{tabular}

Table 1: Initial and light-soaked solar cell data for an all-PECVD device and a hybrid device. Those marked with an asterisk are light-soaked for 140 hours
In Figure 1 we show the normalized efficiency of the two cells in Table 1 as a function of illumination time. The data in Figure 1 are quite striking in that the stability of our all-PECVD device is virtually identical to that of our hybrid device. The efficiency of both devices show 25\% degradation after 140 hours. Our light source is an ELH bulb producing $100 \mathrm{~mW} / \mathrm{cm}^{2}$.

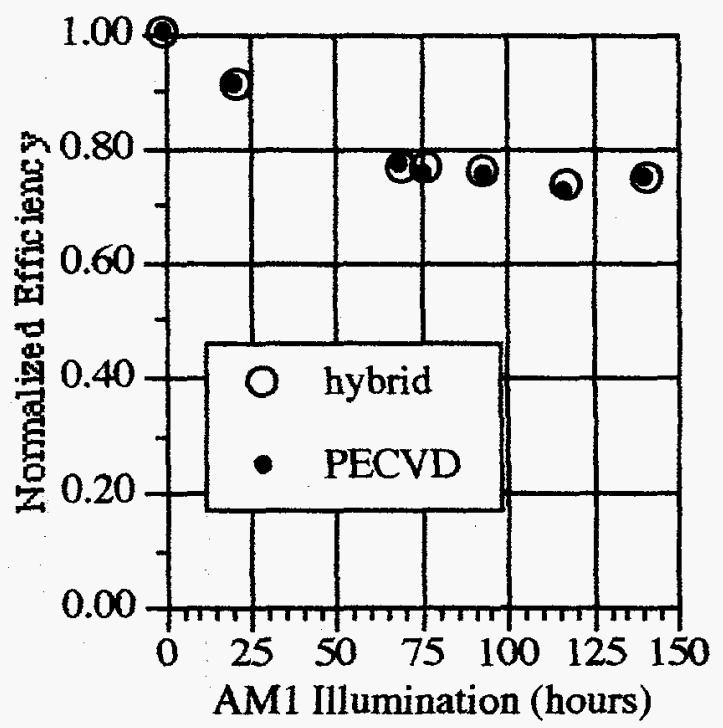

Figure 1: Normalized solar cell efficiency vs. illumination time for a hybrid device and an all-PECVD device.

We demonstrate that we can make high quality $i$-layers with the hot-wire deposition technique. We also demonstrate that we can incorporate $\mathrm{i}$-layers made by hot-wire into $\mathrm{p}-\mathrm{i}-\mathrm{n}$ solar cell devices.

Currently we are incorporating hot-wire $\mathrm{n}+$ and $\mathrm{i}$-layers into $\mathrm{n}-\mathrm{i}-\mathrm{p}$ structures on stainless steel substrates. Based our preliminary results, this work shows great promise. 


\section{Thin-Film Technologies Subcontracts}



Title:

Organization:

Contributors:
Development of Large Area, Monolithically Integrated Silicon-Film ${ }^{\text {TM }}$ Photovoltaic Modules

AstroPower, Inc.

Newark, Delaware

R.B Hall, program manager; J.A. Rand and J.E. Cotter, principal investigators; Y. Bai, D.H. Ford, A.E. Ingram, T.R. Ruffins, C.J. Thomas, A.M. Barnett

\section{OBJECTIVE}

The objective of this program is to develop the technologies leading toward a $1200 \mathrm{~cm}^{2}$, $18 \%$ efficient (AM1.5G), monolithically interconnected thin-film silicon solar cell sub-module. The device structure, called Product III, is a thin $(<100 \mu \mathrm{m})$ polycrystalline layer of silicon on a durable, insulating, ceramic substrate. High efficiency with imperfect material is possible through the use of a thin-film solar cell design which includes light-trapping and surface passivation. The insulating substrate allows the silicon layer to be electrically isolated into small sub-elements, then metallized to form a series interconnected sub-module.

\section{BACKGROUND AND TECHNICAL APPROACH}

The scope of the four phase program is organized around five key sub-objectives, addressing the key material and device issues related to the development effort (see Figure 1). These key areas are: (1) developing the film growth process, (2) developing the large-area ceramic substrate, (3) developing the metallurgical barrier material and deposition technique, (4) developing improved sub-element solar cell processing, and (5) demonstrating the feasibility of the monolithic sub-module concept. Work during the last year focused on improving the electrical quality of the photovoltaic layers and demonstrating improved sub-element solar cell processing.

(3) Metallurgical Barrier

(4) Sub-Element Processing
(5) Monolithic Interconnections

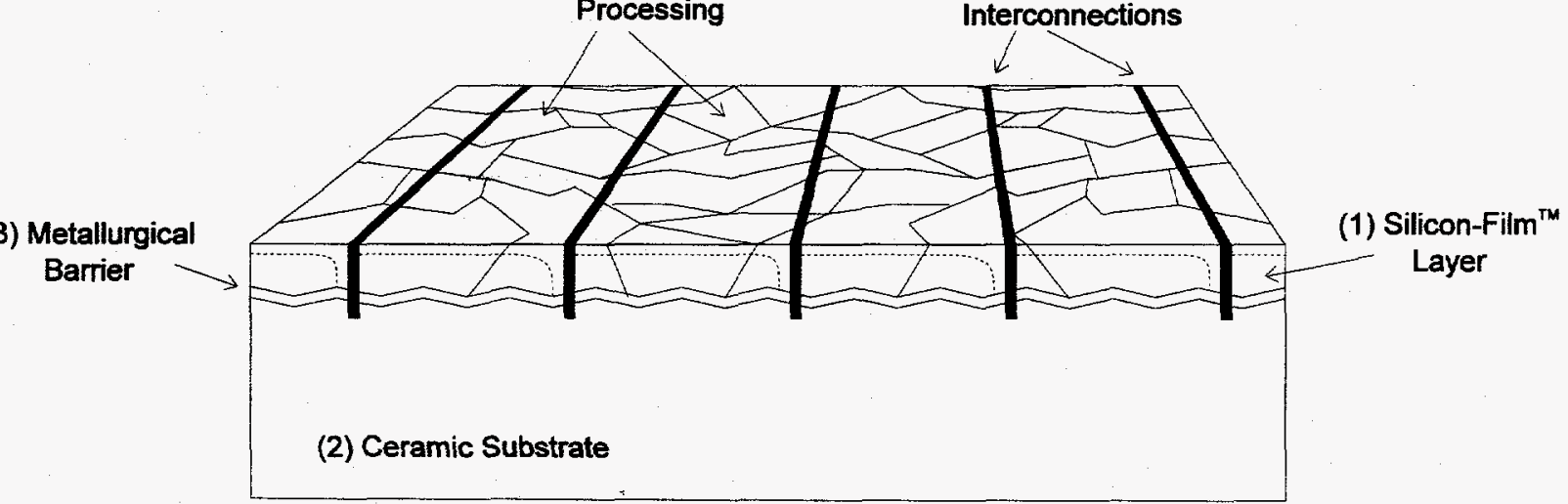

Figure 1. Illustration of the Product III structure showing the key material and device features. 


\section{RESULTS -- PHOTOVOLTAIC MATERIALS}

The technologies for the fabrication of thermally-matched ceramic substrates, and the deposition and growth of silicon layers less than $100 \mu \mathrm{m}$ thick have been developed.. Barrier materials and deposition technologies have also been developed to isolate the silicon active layer from the ceramic. The key limit to device performance has been the electrical quality of the polycrystalline silicon layers, specifically minority carrier diffusion length. Minority carrier diffusion lengths are limited to less than $10 \mu \mathrm{m}$, resulting in low currents and poor fill factors for solar cells formed on them. The Phase II program concluded that trace impurities originating from the ceramic substrate were primarily responsible for the degradation of material quality [ANNUAL SUBCONTRACT REPORT, 1993].

The Phase III program determined that the contamination was caused primarily by the diffusion of impurity species into the photovoltaic layer during the film growth process. Several approaches have been developed to solve this problem. These include (1) upgrading the existing ceramic substrate utilizing higher-purity precursor materials, (2) the development of alternate substrates (such as graphite-fiber-based cloth), and (3) the development of a metallurgical barrier interlayer with low diffusivity for contaminates at the growth conditions.

The precursor materials originally used in the ceramic substrate formulation are 98-99.5\% pure, containing oxides of transition metals such as iron, titanium and chromium. Upgrading one component from 98 to $99.9 \%$ purity, resulted in diffusion lengths as high as $18 \mu \mathrm{m}$. The metallurgical barrier has also led to some improvement in material quality when used on the original ceramic formulation. A two layer barrier, consisting of a thick dielectric layer sprayed onto the ceramic and a thin layer to facilitate wetting during the layer growth, has been successful in improving material quality slightly; diffusion lengths as high as $18 \mu \mathrm{m}$ have been measured.

An alternate substrate explored during this program is a cloth material formed from graphite filaments. Woven graphite cloth is available commercially from a variety of vendors at a modest cost. Graphite-based fabrics make an excellent substrate for the film growth process because they are electrically conductive, and are compatible with the photovoltaic layer growth process. They have excellent wetting characteristics, are very durable during the growth process, and result in the high quality material necessary for high efficiency devices. However, the graphite cloth does not have the capability to form the monolithic sub-module without the addition of an insulating barrier layer.

Like the ceramic substrate, trace metallic impurities found in the graphite cloth are the key factor limiting device performance. These impurities diffuse into the photovoltaic layer during the growth process. The impact on cell characteristics vary according to the specific impurity element. Impurities such as $\mathrm{C}$ and $\mathrm{O}$ can be tolerated in relatively high concentrations $(\geq 10 \mathrm{ppm}$ ), while others, such as $\mathrm{Fe}$ or $\mathrm{Cr}$ can have significant detrimental effects at concentrations of only $0.1 \mathrm{ppm}$. Other impurities, such as B or $\mathrm{P}$, can alter the doping concentrations or conductivity type of the grown film. 
A variety of techniques were applied to improve the purity of the cloth material, including vacuum heat treating, chemical washing to remove trace impurities, and the application of a metallurgical barrier to block the transport of impurities into the photovoltaic layer. The most successful technique utilized a dielectric layer to encapsulate the graphite filaments. Graphite cloth substrates which had filaments intentionally coated with a dielectric resulted in silicon layers with higher average diffusion length. Initially, the encapsulation improved the average diffusion length from $20 \mu \mathrm{m}$ up to $40 \mu \mathrm{m}$. With further refinements, the encapsulation resulted in average diffusion lengths of over $80 \mu \mathrm{m}$ and as high as $100 \mu \mathrm{m}$.

\section{RESULTS - SUB-ELEMENT SOLAR CELL DEVELOPMENT}

The solar cell sub-element fabrication and processing techniques are required to be compatible with the ceramic substrate, optimized for high efficiency and developed to enhance material quality. Throughout the program, $1.0 \mathrm{~cm}^{2}$ solar cell conversion efficiencies for ceramicbased devices were limited by the contamination problem. The best solar cell was tested at an open circuit voltage of $519 \mathrm{mV}$, a short circuit current of $23.4 \mathrm{~mA} / \mathrm{cm}^{2}$, and a fill factor of $59 \%$. An efficiency of $7.2 \%$ (unverified) was achieved. Major efforts at sub-element process development were postponed during a majority of the program until material of sufficient quality grown on the ceramic substrate was available. However, when the quality of the material grown on graphite cloth substrates improved significantly (at the end of Phase III), solar cell process development effort was able to proceed.

Solar cell processes were developed based on existing fabrication technologies. All chemical surface preparation steps, diffusion sequences, passivation techniques, and metallizations are similar to those used in standard silicon solar cell processes. This is made possible by the fact that the cloth substrate is compatible with all high temperature and wet chemical processes. Several processes were optimized specifically for material grown on the graphite cloth substrate: phosphorous gettering, emitter diffusion, hydrogenation, emitter etchback, emitter passivation, contact pattern and anti-reflection coating. The fabrication process was developed for direct transfer to ceramic-based structures' [ANNUAL SUBCONTRACT REPORT, 1994].

$1.0 \mathrm{~cm}^{2}$ solar cells were fabricated on graphite cloth substrates aperiodically throughout the program whenever advances were achieved in the material growth or device processing. Figure 2 describes the progress made in efficiency during the last two years of the program. Initially, solar cell performance was limited by the quality of the silicon film. Trace impurities and contamination from the graphite cloth limited film quality. After the cloth preparation techniques were well developed, further advances in efficiency were achieved through the development and optimization of the solar cell fabrication processes. The final improvements were made by improving the grain structure by optimizing the growth conditions and by optimizing the doping levels within the base layer.

The culmination of these efforts is a $13.4 \%$ (AM1.5G, verified at NREL) efficient $1.0 \mathrm{~cm}^{2}$ solar cell. All of the 41 solar cells fabricated in the final lot of solar cell had an average efficiency of $11.6 \%$, with a standard deviation of $0.86 \%$ absolute. 


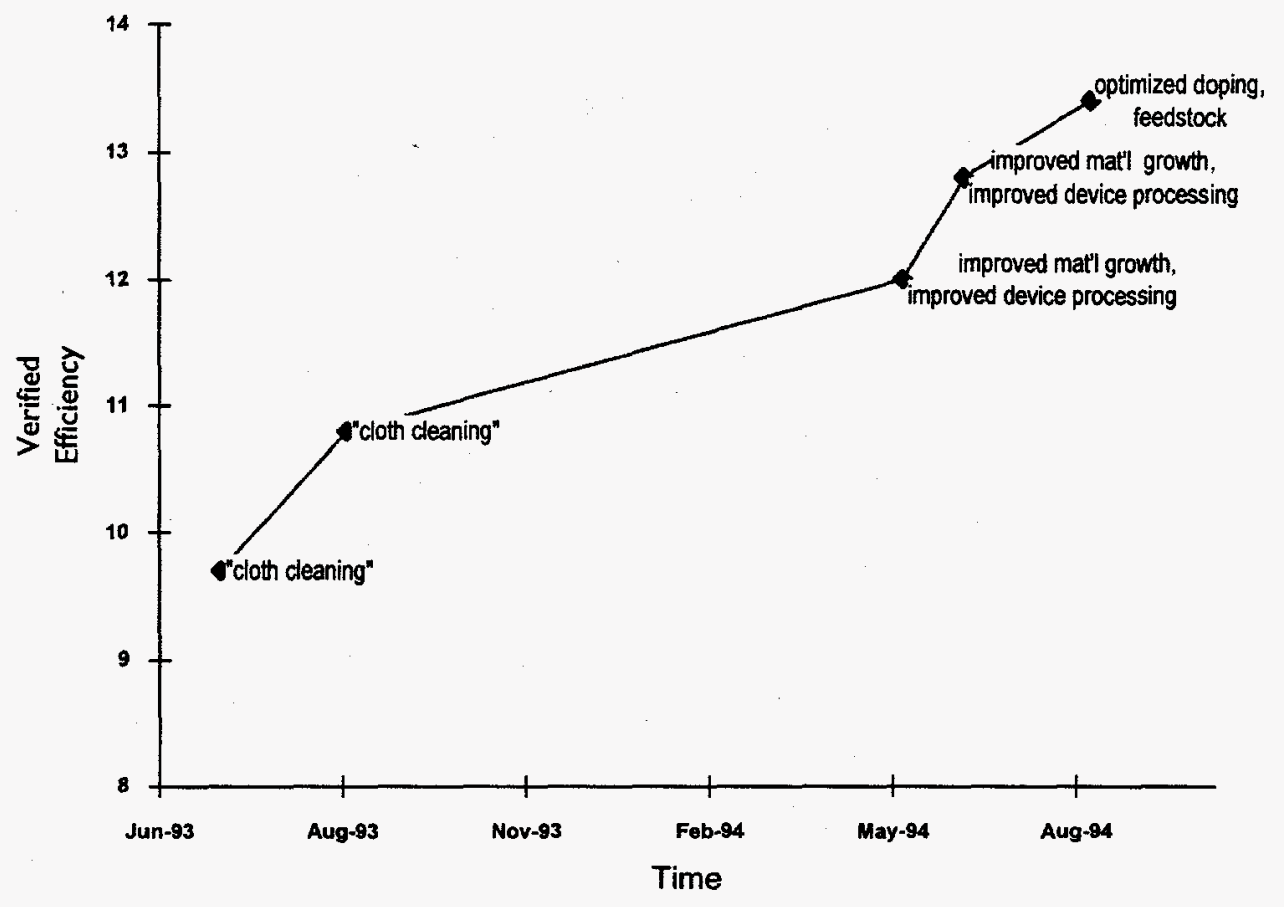

Figure 2 Progress of -on-graphite-cloth solar cell process development efforts.

\section{CONCLUSIONS}

The Phase IV program focused on improving the electrical quality of the photovoltaic layer and developing and demonstrating the sub-element solar cell processing. Contamination from the low-cost ceramic substrate presently limits solar cell performance. A greater understanding of impurity sources and transport methods has been achieved. Our efforts to solve this problem by upgrading the ceramic and developing barrier layers have had some success.

Graphite-fiber-based cloth has been developed as an alternate to the ceramic substrate. A method to upgrade the quality of films grown on this substrate has been developed which results in an improvement in minority carrier diffusion length from about $20 \mu \mathrm{m}$ to over $100 \mu \mathrm{m}$. Solar cell processing techniques have been developed and optimized on material grown on the graphite cloth substrate. A $13.4 \%$ efficient $1.0 \mathrm{~cm}^{2}$ solar cell has been demonstrated.

\section{ACKNOWLEDGMENT}

This work was funded by DOE through the National Renewable Energy Laboratory under contract \#ZR-1-11064.

\section{REFERENCES}

ANNUAL SUBCONTRACT REPORT, (1993), NREL /TP-410-5335, p. 103-106 Available NTIS Order No. DE93000092 ANNUAL SUBCONTRACT REPORT, (1994), To be published. 
Qrganization:

Department of Physics, Colorado School of Mines

Golden, Colorado 80401

Contributors:

J.U. Trefny, T.E. Furtak, D.L. Williamson, T. Ohno, D. Kim, and D. Mao, co-principal investigators; L. Feng, S. Pozder, B. Qi, and Y. Zhu.

\section{Objectives}

The objective of this project is to develop improved processes for the fabrication of $\mathrm{CdTe} / \mathrm{CdS}$ polycrystalline thin film solar cells. The technique we use for the formation of CdTe, electrodeposition, is a non-vacuum, low-cost technique that is attractive for economic, large-scale production.

\section{Technical Approach}

(1) Optimization of CdTe electrodeposition conditions.

(2) Improvement of the chemical bath deposition technique for CdS.

(3) Optimization of $\mathrm{CdCl}_{2}$ treatment and annealing processes and studies of recrystallization and residual stress resulting from heat treatment.

(4) Investigation of $\mathrm{ZnTe}$ for improving the back contact and performance stability.

\section{Results}

Eabrication of CdS/CdTe and CdS/CdTe/ZnTe cells: Soda lime glass coated with $\mathrm{SnO}_{2}$ (Nippon Sheet Glass) was used as the substrate for cell fabrication. The CdS layers $(0.1-0.3 \mu \mathrm{m}$ thick) were deposited by chemical bath deposition (CBD). The CdTe layers ( $2 \mu \mathrm{m}$ thick) were deposited electrochemically from an aqueous solution containing $\mathrm{CdCl}_{2}$ and $\mathrm{TeO}_{2} . \mathrm{CdCl}_{2}$ mist treatment and subsequent annealing were performed following both the $\mathrm{CdS}$ and $\mathrm{CdTe}$ deposition. The highest annealing temperature during the cell processing did not exceed $450^{\circ} \mathrm{C}$. Prior to the deposition of the back contact, the CdTe was etched in $\mathrm{Br}_{2}-\mathrm{MeOH}$. The back contact layers were either $\mathrm{Au}$, without a $\mathrm{Cu}$ interlayer, or $\mathrm{Cu}$-doped $\mathrm{ZnTe}$. In the latter case, the $\mathrm{Cu}$ content was kept low so that the total equivalent thickness of $\mathrm{Cu}$ was less than $1 \mathrm{~nm}$.

Optimization of CdTe electrodeposition parameters. We have performed systematic investigations to optimize the growth conditions of CdTe. Among the parameters adjusted are the deposition current density, anode current ratio, water jet position, and Te concentration. High-quality CdTe films are consistently obtained as a result of this optimization effort. The resulting $\mathrm{CdS} / \mathrm{CdTe} / \mathrm{Au}$ cell (without anti-reflection coating) show $\mathrm{V}_{\mathrm{oc}}$ of $0.76 \mathrm{~V}, \mathrm{~J}_{\mathrm{sc}}$ of $22.0 \mathrm{~mA} / \mathrm{cm}^{2}$. (under $1000 \mathrm{~W} / \mathrm{m}^{2}$ global), FF of 0.66 , and efficiency of $11.0 \%$ (measured at NREL).

Effect of $\mathrm{CdCl}_{2}$ treatment and annealing on the microstructure and residual stress of the electrodeposited CdTe film. $\mathrm{CdCl}_{2}$ treatment and subsequent annealing induce recrystallization and grain growth in electrodeposited CdTe. This recrystallization and grain growth can be studied by 
examining changes in the X-ray diffraction (XRD) patterns. The intensity ratio of the (220) peak and the (111) peak of the XRD pattern can be used as a measure of the degree of texture in the film. In Fig.1, we have plotted this intensity ratio, normalized to that of $\mathrm{CdTe}$ powders, as a function of annealing time. For shorter times, the crystalline orientation of the film moves toward a random state, presumably because of recrystallization. If we choose $R_{\text {normalized }}=0.1$ and plot the times corresponding to this ratio for different temperatures (Fig.2), we obtain an Arrhenius plot for the recrystallization. The activation energy obtained from this analysis, $2.5 \mathrm{eV}$, matches well with $\mathrm{E}_{\mathrm{a}}$ for $\mathrm{Cd}$ diffusion in $\mathrm{CdTe}$ (2.67 eV at maximum $\mathrm{Cd}$ vapor pressure and $2.44 \mathrm{eV}$ at minimum $\mathrm{Cd}$ vapor pressure). This indicates that the recrystallization of $\mathrm{CdTe}$ during annealing is $\mathrm{Cd}$-diffusion limited.

Heat treatment induces stress in CdTe films because of differences in the thermal expansion coefficients of $\mathrm{CdTe}$ and the substrates. We studied the annealing-induced stress in the electrodeposited CdTe as a function of annealing temperature and times, based on XRD measurements. The lattice constants were measured along the direction normal to the surface. A decreased lattice constant in the surface normal direction is a result of an increase of in-plane lattice spacing which in turn is a result of an in-plane tensile stress. Fig.3 shows the changes of lattice constant as a function of annealing temperature and time. Fig. 4 shows the corresponding residual stress, calculated based on the measured lattice constants. The magnitude of the stress shows a maximum at approximately $50 \mathrm{MPa}$. Because the critical resolved shear stress of CdTe is less than $10 \mathrm{MPa}$, our results suggest that the residual stress may introduce crystalline defects such as stacking faults and dislocations in the crystallites of the film.

Effects of $\mathrm{CdCl}_{2}$ treatment on cell performance were studied using Auger depth profiling and electron beam-induced current (EBIC). Auger depth profile studies indicate that a Cd-rich surface layer may be formed after annealing, which affects both the back contact and the current collection efficiency. EBIC studies also indicate the existence of dead regions within $0.5 \mu \mathrm{m}$ of the back contact. These results signify the role of $\mathrm{CdCl}_{2}$ treatment and its optimization.

Studies of $\mathrm{Cu}$-doped $\mathrm{ZnTe}$ back contact layer. $\mathrm{Cu}$-doped $\mathrm{ZnTe}$ was studied as a promising material for forming stable, low-resistance contacts to the p-type CdTe. The $\mathrm{ZnTe}$ was formed by vacuum co-evaporation of $\mathrm{Cu}$ and $\mathrm{ZnTe}$. The electrical properties were studied systematically as a function of $\mathrm{Cu}$ concentration and annealing temperature. The dark resistivity of the as-deposited $\mathrm{ZnFe}$ decreased by more than 3 orders of magnitude as the $\mathrm{Cu}$ concentration was increased from 4 to 8 atomic percent. The resistivity decreased to less than $1 \mathrm{ohm}-\mathrm{cm}$ after annealing at $260^{\circ} \mathrm{C}$ for $30 \mathrm{~min}$ in vacuum. A decrease of conductivity was also observed during annealing at $150-200^{\circ} \mathrm{C}$. The activation energy of the dark conductivity of annealed ZnTe was found to be less than $50 \mathrm{meV}$. At this stage, the processing conditions for cell applications have not yet been optimized. Preliminary experiments have, however, yielded cells with fill factors consistently approaching (often exceeding) 0.70 and energy conversion efficiencies consistently higher than $10 \%$. We have also succeeded in depositing $\mathrm{ZnTe}$ using electrodeposition as an alternative method.

Exploration of modified cell structures. The open-circuit photovoltage obtained with electrodeposition at low processing temperatures is in general lower than the $V_{o c}$ values obtainable with techniques that involve processing at higher temperatures (e.g., close-spaced sublimation). To compensate for this limitation and to obtain higher $\mathrm{V}_{o c}$, we have explored several ways of modifying the cell structure. We found that the addition of a fresh, unannealed CdS layer on annealed CdS substrates enhances the $V_{o c}$ by an average of $50 \mathrm{mV}$. Other methods, e.g., the addition of an 
undoped $\mathrm{ZnO}$ layer between $\mathrm{CdS}$ and $\mathrm{SnO}_{2}$, are less effective in terms of improving $\mathrm{V}_{\mathrm{oc}}$. Effects of different types of conducting glass substrates have also been investigated.

\section{Summary}

We have investigated several issues that are critical to the fabrication of low-cost, high-efficiency $\mathrm{CdTe} / \mathrm{CdS}$ thin film solar cells. The conditions for $\mathrm{CdTe}$ electrodeposition have been optimized. The effects of $\mathrm{CdCl}_{2}$ treatment and annealing have been studied in terms of recrystallization, residual stress, and their impact on cell performances. Studies of $\mathrm{ZnTe}$ indicate that this is a potentially stable, low-resistance contact material for the CdTe. Small area, $11 \%$ efficient cells have been produced from this work. Addition of the $\mathrm{ZnTe}$ back contact layer is expected to yield even higher efficiencies.

\section{Publication/Presentations}

"Thin Film CdS/CdTe Solar Cells Fabricated by Electrodeposition", D. Kim, S. Pozder, B. Qi, Y. Zhu, T.E. Furtak, D.L. Williamson, and J.U. Trefny, 12th NREL Photovoltaic Program Review, AIP Conference Proceedings 306, R. Noufi and H.S. Ullal, ed. p.320.

"Effect of Annealing on Microstructure, Residual Stress, and Photovoltaic Characteristics of Electrodeposited CdTe Films", D. Kim, B. Qi, D.L. Williamson, and J.U. Trefny, The IEEE First World Conference on Photovoltaic Energy Conversion, Kona, Hawaii, December, 1994.

"Polycrystalline Thin Film CdTe Solar Cells Fabricated by Electrodeposition", D. Kim, S. Pozder, Y. Zhu, and J.U. Trefny, The IEEE First World Conference on Photovoltaic Energy Conversion, Kona, Hawaii, December, 1994. 


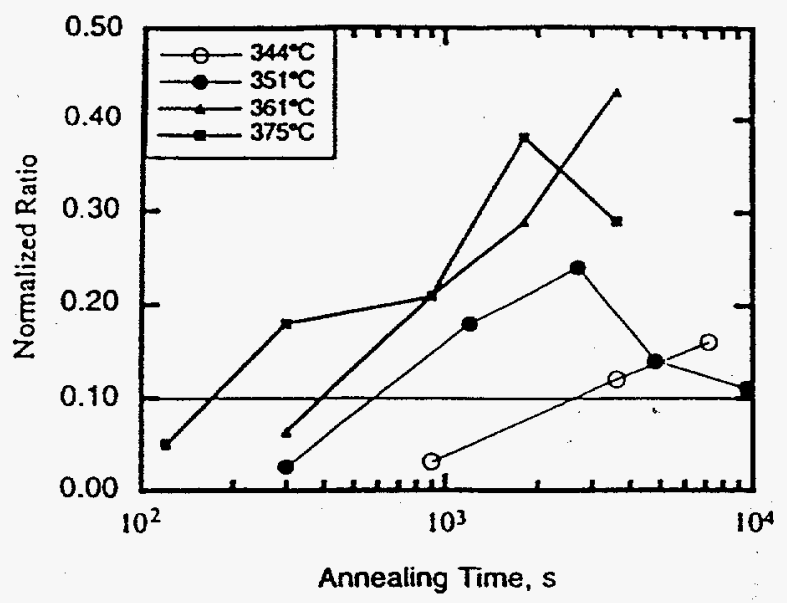

Fig. 1. Normalized intensity ratios of XRD peaks for CdTe films annealed at various temperatures for different times.

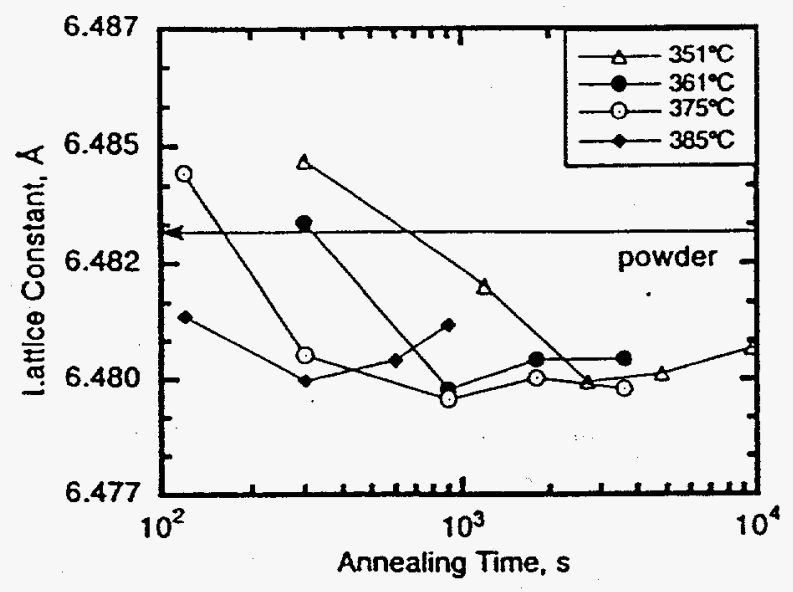

Fig. 3. Change of lattice constant of electrodeposited CdTe according to the annealing temperature and time.

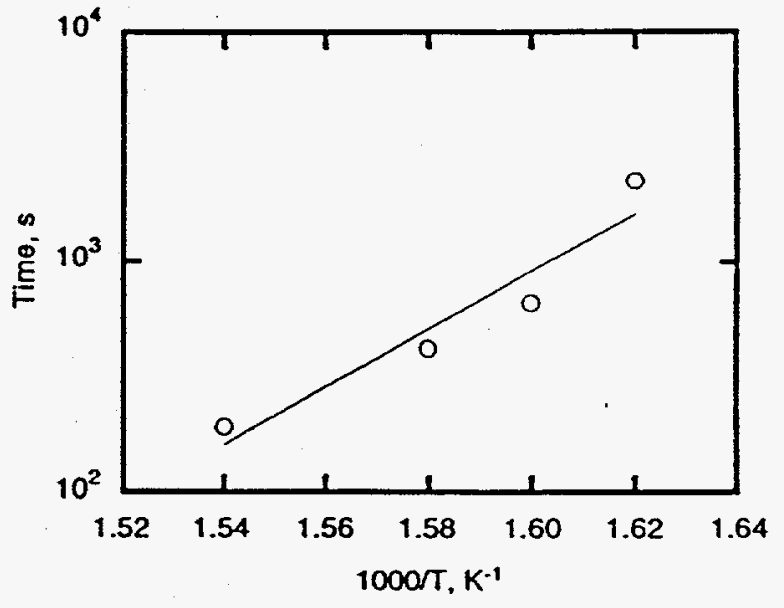

Fig. 2. Relationship between the annealing temperature and the time for $10 \%$ recrystallization.

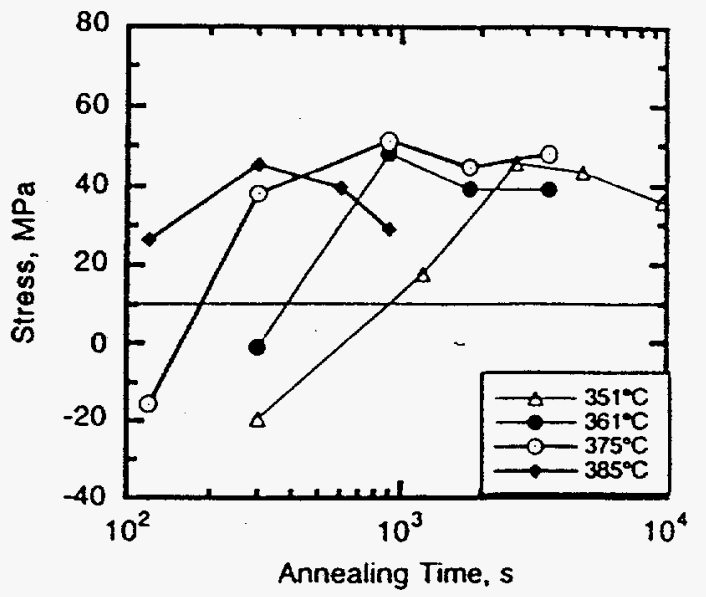

Fig. 4. Residual stress in electrodeposited $\mathrm{CdTe}$ films as a function of annealing temperature and time. 


\section{Title: Microstructure of Amorphous-Silicon-Based Solar Cell Materials by Small-Angle X-ray Scattering}

Organization: Department of Physics, Colorado School of Mines, Golden, Colorado

Contributors: D.L. Williamson, principal investigator; S. Jones, Y. Chen and D. Min

\section{Objectives}

The general goals are to establish details of the microstructure of a-Si:H and related alloys presently being incorporated into solar cells and to help determine whether particular microstructural features are correlated with photovoltaic behavior. If certain features are found to be detrimental to solar cell efficiency and stability, then optimization efforts will be carried out with the film growers and device makers of the various NREL Amorphous Silicon Teams to improve existing deposition methods or consider alternate methods and materials.

\section{Approach}

The direct structural characterization method of small-angle $\mathrm{x}$-ray scattering (SAXS) is being used to study microstructural features ranging in size from the atomic up to approximately 30 nm. Features such as microvoids, hydrogen-rich clusters, columnar-like growth, microcrystallites, surface roughness, and alloy composition fluctuations or segregation can all be detected with a high degree of sensitivity. For example, microvoids of volume fraction $0.1 \%$ are readily seen and their average size can be determined. Atomic-scale structure can be inferred from the x-ray diffuse scattering intensity. Anisotropy in the SAXS, as detected by a sensitivity of the SAXS to sample orientation relative to the $\mathrm{X}$-ray beam, is direct evidence of oriented microstrural features such as columnar-like growth.

The approach involves collaboration with the NREL Low-gap, Mid-gap, and High-gap Teams, particularly the film-making and device-making groups that supply relevant, systematic sets of samples to help address specific issues within the above objectives. We also collaborate with several groups around the world not supported directly by NREL but recognized as experts in the field. SAXS-suitable substrates are supplied by us to all collaborating groups. SAXS measurements made at a central facility allow accurate comparisons of the microstructure of materials prepared by different deposition methods, conditions, and systems. Other in-house measurements complementing our SAXS studies include Raman spectroscopy, $x$-ray diffraction and flotation density. A high-quality annealing system is also available.

\section{Results}

During FY94 several significant results emerged. The discussion will be separated according to materials appropriate to the low-gap, mid-gap, and high-gap components of a multijunction solar cell. The latter is the structure being developed to meet the long-range goal of $15 \%$ stabilized efficiency with the amorphous silicon thin-film technology.

The present focus material for the low-gap component is $\mathrm{a}-\mathrm{Si}_{1-\mathrm{x}} \mathrm{Ge}_{\mathrm{x}}: \mathrm{H}$. The value of $\mathrm{x}$ needed to 
achieve the desired optical band-gap, $\mathrm{E}_{\mathrm{g}}$, of about $1.4 \mathrm{eV}$ is near 0.4 to 0.5 and all alloys studied by SAXS near this composition show much enhanced heterogeniety relative to the a-Si:H used as the mid-gap component $\left(E_{\mathrm{g}}=1.7 \mathrm{eV}\right)$. The microstructure is typically oriented, consistent with columnar-like growth, and the features seen by the SAXS are interpreted as microvoids or Hrich regions located along the column boundaries [1,2]. The use of hydrogen dilution of the source gases silane and germane led to significant improvements in the photoresponse and microstructure of films supplied by a group in India [3]. However, the effect of hydrogen dilution on the microstructure and opto-electronic behavior is not universal: films prepared by United Solar Systems Corporation (USSC) show a much stronger anisotropy than any of the India films and yet yield the best photovoltaic performance of low-gap material at USSC. Detailed quantitative investigation of the SAXS data of this material indicates a low density (average separation of about $200 \mathrm{~nm}$ ) of extremely anisotropic objects that are modeled as ellipsoids with major-to-minor axis ratios in excess of 20 . The microstructure of the material between these rod-like features is nearly free of the spherical-like nanovoids that have been correlated with poorer photovoltaic behavior in a-Si:H [4,5]. The fact that the rod-like features are aligned with the carrier transport direction and that they are sparse in density may explain their apparent lack of a detrimental effect on the solar cell behavior. The possibility that the rodlike features are microcrystallites has been considered since sufficiently high hydrogen dilution is known to produce microcrystalline material. A search by Raman and x-ray diffraction showed no evidence for crystallinity but the very low volume fraction suggested by the SAXS may not allow its detection by these methods. It is interesting to note that the flotation densities of all a$\mathrm{Si}_{1-\mathrm{x}} \mathrm{Ge}_{\mathrm{x}}: \mathrm{H}$ films show no sharp change with $\mathrm{x}$ or hydrogen-dilution conditions. Instead, a steady increase in density deficit relative to $c-\mathrm{Si}_{1-\mathrm{x}} \mathrm{Ge}_{\mathrm{x}}$ is seen, where at $\mathrm{x}=0.6$ it reaches about $6 \%[1]$.

A significant development with low-gap alloys was the preparation of high-quality material at Harvard with $\mathrm{x}$ near 0.7 under conditions of strong ion bombarment. The SAXS shows, for certain hydrogen-dilution conditions, that the microstructure is non-oriented and has void densities nearly as low as that of the best a-Si:H [1].

The mid-gap material, a-Si:H, has achieved record low values of SAXS-detected microstructural features. A film prepared at NREL with PECVD conditions yielding their best solar cell efficiency has a maximum possible void volume fraction of $0.03 \%$. The residual SAXS intensity indicates larger-scale features $(>20 \mathrm{~nm})$ that are likely related to surface roughness or $\mathrm{H}$ concentration fluctuations. The flotation density of this film is $2.25 \mathrm{~g} / \mathrm{cm}^{3}$, yielding a density deficit of $3.4 \%$ relative to $\mathrm{c}-\mathrm{Si}\left(2.33 \mathrm{~g} / \mathrm{cm}^{3}\right)$. This is attributed to the approximately 10 at.\% $\mathrm{H}$ in the amorphous matrix occupying only 30 to $40 \%$ of the atomic volume of Si atoms [1]. Films made by Solarex and Advanced Photovoltaic Systems representing their best photovoltaic material have yielded SAXS intensities only slightly larger than those of the NREL film, corresponding to maximum void fractions at the $0.1 \%$ level.

The SAXS intenities from a-Si:H prepared by the hotwire (HW) method at NREL decrease significantly as the $\mathrm{H}$ content is lowered by the use of elevated substrate temperatures. Using deposition conditions that show evidence of improved stability under light, the SAXS is only slightly larger than the above-discussed PECVD sample from NREL. Also, its density of 2.29 $\mathrm{g} / \mathrm{cm}^{3}$ is consistent with a much lower bonded $\mathrm{H}$ content near 3 at.\%. Our earlier work with HW 
material [6] showed evidence of columnar-like microstructure but that material contained more $\mathrm{H}$ and was prepared at lower substrate temperature. This new $\mathrm{HW}$ material with lower $\mathrm{H}$ content is promising enough that team-work between NREL and USSC is underway to test its capabilities as the intrinsic layer in solar cells.

The use of a cathode heating technique applied to the PECVD method has been explored to improve a-Si:H and results of SAXS and opto-electronic characterization provides addtional evidence that nanovoids are detrimental [5]. In another study, the effects of systematic annealing of a-Si:H films have been examined using the combined techniques of SAXS and infrared absorption spectroscopy to show that above temperatures of $350^{\circ} \mathrm{C}$, the hydrogen begins leaving the Si-H bonding sites and microvoids start increasing in size and number at the same temperature [7]. Interdiffusion of the $\mathrm{Si}$ and $\mathrm{Al}$ occurred at $430^{\circ} \mathrm{C}$ leading to partial crystallization of the a-Si:H. This helps explain the observation of partial crystallization in some of the HW films deposited at elevated substrate temperatures.

The high-gap focus material is a-Si ${ }_{1-\mathrm{x}} \mathrm{C}_{\mathrm{x}}: \mathrm{H}$. Such films prepared by Solarex, Utrecht University, and University of Illinois show much enhanced SAXS intensities compared to a-Si:H, even for $x$ as low as 0.06 [2]. Larger values of $x$ than this are desired for the high-gap component of the multijunction cell $\left(E_{\mathrm{g}}=2.0 \mathrm{eV}\right)$. The microstructural features producing the SAXS from these carbon-containing alloys are much different from those in the a-Si ${ }_{1-\mathrm{x}} \mathrm{Ge} \mathrm{x}_{\mathrm{X}}: \mathrm{H}$. They are at the 1 $\mathrm{nm}$ size scale and show little or no preferred orientation effects, suggestive of spherical-like, nanovoid objects. In addition, their density is at or above the 1 vol. \% level, inferring number densities in excess of $10^{19} \mathrm{~cm}^{3}$. Such defect densities are likely to limit photovoltaic performance. Flotation densities of these films are well below those expected for the ideal tetrahedral, diamond-like bonding that is desireable, but part of this is likely due to the relatively large $\mathrm{H}$ contents ( $>20$ at.\%). Since a wide range of deposition conditions have been tried by PECVD (Solarex and Utrecht) and by reactive magnetron sputtering (Illinois), it seems clear that some new or alternative method needs to be investigated (e.g. HW method).

Alternative high-gap alloys, $\mathrm{a}-\mathrm{Si}_{1-\mathrm{x}} \mathrm{Se}_{\mathrm{x}}: \mathrm{H}$, are being investigated by a group at Texas A\&M University with support from EPRI. SAXS studies of one series of samples [1,8] shows that Se incorporation does not lead to sharp degradation of the microstructure in contrast to $\mathrm{C}$ incorporation. However, the band-gap increase is not yet at the desired level and it remains to be seen whether the good microstructure and opto-electronic quality persist at higher Se contents.

\section{Conclusions and Future Work}

During FY94, further circumstantial evidence has been obtained regarding the mid-gap a-Si:H material: a-Si:H prepared by PECVD or HW with the least amount of SAXS-detected microstructure leads to the best photovoltaic performance. These films contain no detectable microvoids in the nanometer size range but there remain some larger-scale features that are the subject of further investigations.

In the case of the low-gap a-Si ${ }_{1-\mathrm{x}} \mathrm{Ge}_{\mathrm{x}}: \mathrm{H}$ alloys, evidence has been found supporting the concept that reduced amounts of low density columnar-like structure leads to better opto-electronic behavior. In contrast, evidence has also been collected from USSC films that highly-oriented, 
rod-like features are present in their current best device-quality alloys, suggesting that this type of microstructural feature may not be detrimental in solar cells.

Further work is planned to understand more completely the origin of the SAXS in a-Si $i_{1-\mathrm{x}} \mathrm{Ge}_{\mathrm{x}}: \mathrm{H}$ alloys including (1) a joint project with a group at the German synchrotron facility in Hamburg, which will try the method of anomalous SAXS (ASAXS) to look for the possiblilty of Ge composition fluctuations; (2) a systematic study of the diffuse scattering intensity versus $\mathrm{Ge}$ content that could allow the determination of whether the alloys show any short-range (atomicscale) clustering or ordering effects; and (3) high-resolution TEM to look more carefully for possible microcrystallites as the origin of the rod-like features detected by SAXS.

With respect to the high-gap a-Si $i_{1-x} \mathrm{C}_{\mathrm{x}}: \mathrm{H}$ alloys, SAXS demonstrates a high density of nanometer-size features that are nearly spherical or randomly oriented non-spheres. Such features may explain the present difficulty in obtaining stable, device-quality material with the desired bandgap near $2.0 \mathrm{eV}$. Further work with alternative alloys or unexplored deposition methods and conditions is apparently needed.

\section{References}

1. D.L. Williamson, S.J. Jones, and Y. Chen, Final Subcontract Report (July 1994), NREL/TP411-6855, 32 pp, Available NTIS: Order No. DE94011840.

2. D.L. Williamson, AIP Conf. Proc. No. 306, Ed. by R. Noufi and H.S. Ullal (AIP, New York, 1994) p. 442.

3. A.R. Middya, S. Ray, S.J. Jones and D.L. Williamson, J. Appl. Phys. [in press].

4. S. Guha, J. Yang, S.J. Jones, Y. Chen and D.L. Williamson, Appl. Phys. Lett. 61 (1992) 1444.

5. S. Chattopadhyay, S. N. Sharma, R. Banerjee, D.M. Bhusari, S.T. Kshirsagar, Y. Chen and D.L. Williamson, J. Appl. Phys. 76 (1994) 5208.

6. A.H. Mahan, Y. Chen, D.L. Williamson and G.D. Mooney, J. Non-Cryst. Solids $137 \& 138$ (1991) 65.

7. J. Shinar, H. Jia, R. Shinar, Y. Chen and D.L.Williamson, Phys. Rev. B50 (1994) 7358.

8. M. Z. He, H.G. Lin, J. O'M. Bockris, Y. Chen and D.L. Williamson (unpublished). 
Title:

Organization:

Contributors:
Device Physics of Thin-Film Polycrystalline Cells and Modules

Department of Physics, Colorado State University, Fort Collins, Colorado

J. R. Sites, principal investigator; X. X. Liu, I. L. Eisgruber, G. Stollwerck, J. E. Granata, J. A. Sharp, B. Murphy

\section{Objectives}

The objectives of this program are (1) the separation and quantification of individual losses in specific thin-film solar cells, (2) the detailed characterization of small modules, and (3) the presentation of a viable model for the forward-current loss mechanism.

\section{Cell Analysis}

Major progress was made in the separation of photocurrent losses. The quantum efficiency apparatus was rebuilt to incorporate a small monochrometer light sources. White-light bias is furnished from the same source as used for current-voltage measurements, so cells need not be moved. At the same time, collaborators have supplied cells terminated at each layer step for optical measurements. The result is a complete and accurate accounting of photocurrent losses [1], as illustrated in Fig. 1. $\mathbf{J}_{\mathrm{SC}}$ is the integrated quantum efficiency weighted by the appropriate spectrum. The individual losses are the integrals over the individual loss regions, again weighted by the spectrum. The photocurrent plus losses sum to the maximum value allowed by the CdTe bandgap.

Other cell-analysis techniques have included capacitance and conductance measurements as a function of frequency and voltage [2], fits of light and dark forward currents to a model incorporating grain-boundary recombination [3], separation of features due to a second diode barrier [1], investigation of time-dependent features in current-voltage measurements, and the anticipated variations in current-voltage characteristics as a function of conduction band offset. Altogether 25 report individual analysis reports were prepared based on cells from nine different collaborating organizations.

\section{Module Analysis}

Several features of module analysis have been investigated using data taken both at Colorado State and with the laser-scanning apparatus at NREL [4-6]. These experiments have utilized small $(10 \times 10 \mathrm{~cm})$ modules consisting of 6-8 series-connected thin-film cells. Two features are illustrated in Fig. 2, which shows laser scans across a CdTe module. In general, one only knows the bias across the entire module, and thus the scan can be distorted by cell-to-cell variations. Figure $2 \mathrm{a}$ shows a scan with no light bias. The large variations in response from different cells is due to variations in shunt resistance among the cells. When these resistances are measured independently, the calculated scan response tracks the measured response 
reasonably well. Similarly in Fig. $2 \mathrm{~b}$, there are large changes in the cell-to-cell response when white-light bias with a small intentional variation in intensity is used. Again, however, the response can be calculated by incorporating the intensity as well as the shunting variations, and it shows reasonable agreement with the measured scan.

Other module features investigated have included the signatures of different types of defects, the changes in cell-analysis related to the typical cell geometry found in thin film modules, the corrections required for a highly focused scanning beam, and the time-dependent response related to scan rates and chopping frequencies. Modules from two collaborating organizations were studied and five reports prepared in addition to references 4-6.

\section{Status of Thin-Film Polycrystalline Cells}

A continuing attempt is made to place thin-film polycrystalline cell device physics in the context of crystalline cells and ideal photovoltaic diodes $[7,8]$. The primary difference is the significantly larger forward current of the polycrystalline cells attributed to electron-hole recombination at grain boundaries in the depletion region. Although this loss mechanism has been steadily decreasing over time, it remains an open question whether there are inherent limitations that will prevent it from approaching single-crystal values.

\section{References}

1. "Quantitative Separation of Photon and Back-Contact Losses in CdTe Solar Cells." G. Stollwerck, M.S. Thesis, Colorado State University, December 1994, unpublished.

2. "Admittance Measurements on $\mathrm{Cu}(\mathrm{In}, \mathrm{Ga}) \mathrm{Se}_{2}$ Polycrystalline Thin-Film Solar Cells," J. H. Scofield, M. Contreras, A. M. Gabor, R. Noufi, and J. R. Sites, WCPVEC-1 (1994).

3. "Current Transport With and Without Grain-Boundary Recombination for Polycrystalline $\mathrm{CuInSe}_{2}$ Solar Cells," X. X. Liu, Ph.D. Thesis, Colorado State University, August 1994, unpublished.

4. "The Large-Scale Laser Scanner: Milli-Characterization of Photovoltaic Devices and Modules," R. J. Matson, K. A. Emery, I. L. Eisgruber, and L. L. Kazmerski, EPVSEC-12, 1222 (1994).

5. "Effect of Thin-Film Module Geometry on Solar Cell Current-Voltage Analysis," I. L. Eisgruber and J. R. Sites, WCPVEC-1 (1994).

6. "Interpretation of Laser Scans from Thin-Film Polycrystalline Photovoltaic Modules, " I. L. Eisgruber, R. J. Matson, J. R. Sites, and K. A. Emery, WCPVEC-1 (1994).

7. "Status of Polycrystalline Thin-Film Solar Cells, " I. L. Eisgruber and J. R. Sites, AIP Conf. Proc. 208, 407 (1994).

8. "Six-Year Efficiency Gains for CdTe and CuIn ${ }_{1-\mathrm{x}} \mathrm{Ga}_{\mathrm{x}} \mathrm{Se}_{2}$ Solar Cells: What Has Changed?" J. R. Sites and X. X. Liu, WCPVEC-1 (1994). 
CdTe solar cell

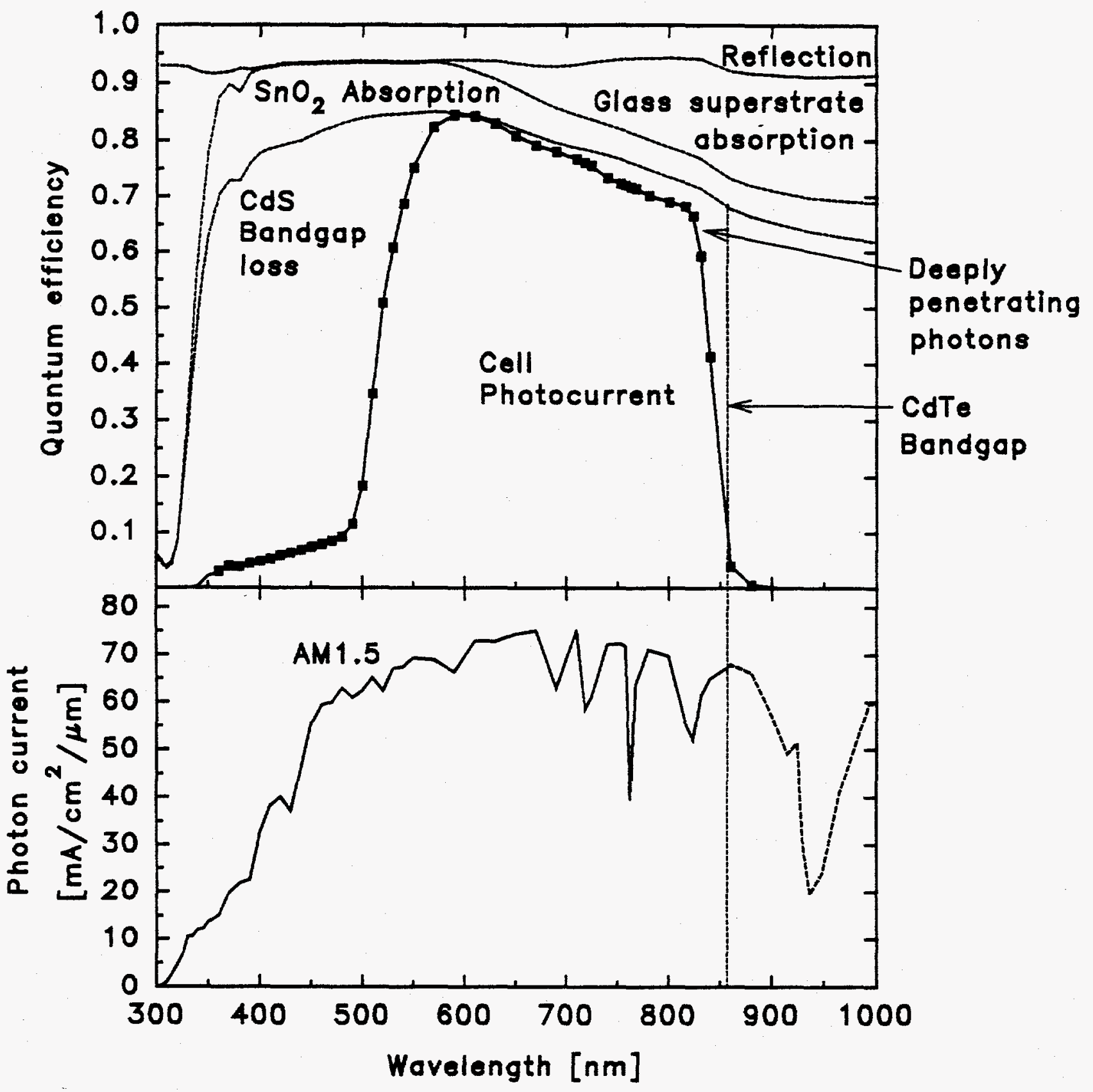

\begin{tabular}{|c|c|c|c|c|c|c|}
\hline $\mathrm{J}_{s c}$ & $\begin{array}{c}\text { Reflection } \\
\text { loss }\end{array}$ & $\begin{array}{c}\text { Glass } \\
\text { absorp. }\end{array}$ & $\begin{array}{c}\mathrm{SnO}_{2} \\
\text { absorp. }\end{array}$ & $\begin{array}{c}\text { CdS } \\
\text { loss }\end{array}$ & $\begin{array}{c}\text { Deep } \\
\text { penetr. }\end{array}$ & $\begin{array}{c}\text { Max. photo } \\
\text { current }\end{array}$ \\
\hline 17.6 & 1.7 & 2.0 & 2.4 & 5.8 & 1.1 & 30.6 \\
\hline
\end{tabular}

all currents in $\mathrm{mA} / \mathrm{cm}^{2}$

Fig. 1. Photon accounting in a CdTe solar cell. 

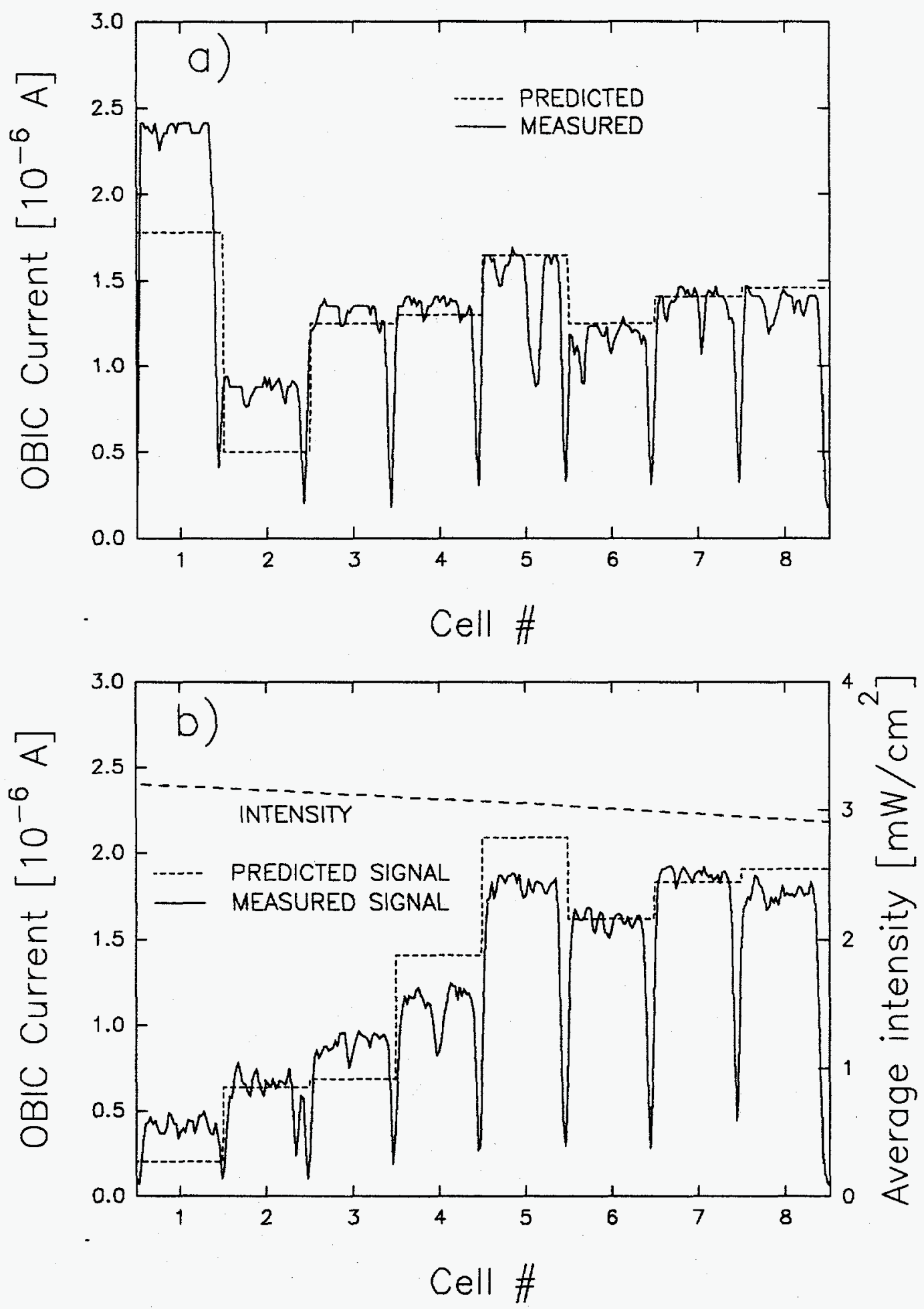

Fig. 2. Measured and calculated laser-scan response from a $10 \times 10 \mathrm{~cm}$ CdTe module at short circuit. (a) No light bias. (b) White light bias. 
Title:

Organization:

Contributors:

\section{Development of High, Stable-Efficiency \\ Triple-Junction a-Si Alloy Solar Cells}

Energy Conversion Devices, Inc.

Troy, Michigan 48084

X. Deng, Principal Investigator; M. Izu, S. J. Jones,

K. L. Narasimhan, J. Evans, and S. R. Ovshinsky

\section{Objectives}

The overall objective of this three-year, cost-shared, amorphous silicon research program is to develop advanced technologies and to demonstrate stable 14\% efficient triple-junction a-Si alloy solar cells. The technologies developed in this program will then be incorporated into ECD's continuous roll-to-roll deposition process to further enhance ECD's photovoltaic manufacturing technology.

\section{Technology}

In ECD's solar cell design, triple-junction a-Si alloy solar cells are deposited onto stainless steel substrates coated with $\mathrm{Ag} / \mathrm{ZnO}$ back-reflector layers. This type of cell design enables us to use a continuous roll-to-roll deposition process to manufacture a-Si PV materials in high volume at low cost. Using this cell design, ECD has previously achieved 13.7\% initial solar cell efficiency [1] using the following features: 1) a triple-junction two-bandgap, spectrum-splitting solar cell design; 2) a microcrystalline silicon p layer; 3) a bandgap profiled a-SiGe alloy as the bottom cell $\mathrm{i}$ layer; 4) a high performance $\mathrm{Ag} / \mathrm{ZnO}$ back-reflector; and 5) a high performance tunnel junction between component cells.

\section{Approaches}

In this program, we are further advancing ECD's existing PV technologies to meet the goal of $14 \%$ stable solar cells by performing the following four tasks:

\section{Improving the Stability of the Intrinsic a-Si Alloy Materials}

Incorporating fluorine into a-Si material improves its stability. It is believed that a small amount of fluorine in a-Si alloy material can be beneficial in terms of passivating dangling or weak bonds. In particular, we have demonstrated previously that the a-Si:H:F material shows low defect density after prolonged light soaking compared to the unfluorinated a-Si:H made in the same system [2]. Under this task, we are optimizing the deposition process of a-Si:H:F material and demonstrating the improved stability in solar cell devices.

\section{Improving the Quality of Low Bandgap a-SiGe Alloy}

Under this task, we are optimizing the deposition of a-SiGe alloy to further improve the material quality, especially for low bandgap a-SiGe alloys. The new deposition process we are 
investigating includes a-SiGe deposition using a cathode plate with a controlled bias and ion bombardment in an rf PECVD system. The improved narrow bandgap material will be demonstrated in the device.

\section{Improving $\mathrm{p}^{+}$Window Layers}

ECD has previously developed a proprietary a-Si $\mathrm{p}^{+}$material for the $\mathrm{p}$ layer of a-Si solar cells. Compared to its amorphous counterpart, this a-Si $\mathrm{p}^{+}$offers high built-in potential due to its small activation energy; low optical loss due to its wide bandgap, and low electrical loss due to its low resistivity. In this program, we further improved the $\mathrm{p}^{+}$layer by appropriately alloying the a-Si $\mathrm{p}^{+}$material with oxygen to widen the bandgap. The optimized $\mu \mathrm{c}-\mathrm{SiO}_{\mathrm{x}} \mathrm{p}^{+}$is expected to further improve the transmission of the $\mathrm{p}$ layer and $\mathrm{V}_{\mathrm{oc}}$ of the device.

\section{Development of High Stable Efficiency Triple-junction a-Si Alloy Solar Cells}

Under this task, we will incorporate the developments made under Tasks 1 through 3 into triple-junction solar cells to achieve a high stable efficiency. We will further optimize other device-related aspects including the tunnel junction, back-reflector, top conductor, interface layer and component cell matching.

\section{Major Accomplishments:}

Since the starting of this program in July, 1994, we have made the following major accomplishments:

1. We have demonstrated, in an n-i-p solar cell, the improved stability of a-Si:H:F materials when deposited using a new fluorine precursor. n-i-p single junction solar cells with 3000 to $3500 \AA$ a-Si:H:F intrinsic layers were deposited on $\mathrm{Ag} / \mathrm{ZnO}$ back-reflector in one of our multichamber deposition systems (LL). The initial efficiency of the a-Si:H:F device is around $9 \%$, which is the same as the baseline unfluorinated a-Si:H n-i-p solar cell deposited in this machine. After 384 hours of one sun light soaking at $50^{\circ} \mathrm{C}$, the a-Si:H:F devices showed degraded efficiency of around $8 \%$, as seen in Table 1 . The degradation in efficiency with light soaking is $10-11 \%$, which is lower than the $17 \%$ drop for the baseline a-Si:H n-i-p device with a $3000 \AA$ thick i layer after the same amount of light soaking.

2. We have developed an a-Si(n)/a-Si alloy/Pd Schottky barrier device for the effective evaluation of photovoltaic performance of a-Si alloy materials [3]. In this device, the intrinsic a$\mathrm{Si}$ alloy materials to be studied are deposited onto a heavily doped $\mathrm{n}^{+}$crystalline silicon wafer and a thin layer of $\mathrm{Pd}$ is subsequently evaporated on top to complete the device structure. The fill factor of such a device under weak red-light reflects the minority carrier transport properties, just as in a real solar cell. Figure 1 shows the J-V curve of a typical c-Si/a-Si/Pd device. We have systematically studied the interface effect at the c-Si//a-Si alloy, and the a-Si alloy//Pd interfaces, as well as the handling procedures in fabricating these interfaces to achieve reliable and consistent results. A comparison between these Schottky barrier devices and complete n-i-p 
solar cells for a series of samples have indicated good correlation in the measurements. The c-Si $\left(\mathrm{n}^{+}\right) / \mathrm{a}-\mathrm{Si}$ alloy/Pd device, offers a convenient technique to reliably evaluate the material performance in a solar cell device.

3. We have optimized the deposition of the intrinsic a-Si layer and the boron doped a-Si $\mathrm{p}^{+}$ layer to improve $\mathrm{V}_{\mathrm{oc}}$. As a result, $\mathrm{V}_{\mathrm{oc}}$ of $1.04 \mathrm{eV}$ was achieved for a single junction $\mathrm{a}-\mathrm{Si}: \mathrm{H} n-\mathrm{i}-\mathrm{p}$ solar cell [4], as shown in the J-V curve in Figure 2. The high $\mathrm{V}_{\text {oc }}$ is achieved by depositing the intrinsic a-Si layer with high hydrogen dilution and at relatively low temperature.

4. We designed a novel structure for the $\mathrm{p}-\mathrm{n}$ tunnel junction (recombination layer) in a multiple junction solar cell and demonstrated the improvement in the solar cell $\mathrm{V}_{0 \mathrm{oc}}$ and FF. We tested this improved $\mathrm{p}-\mathrm{n}$ tunnel junction using an $\mathrm{n}-\mathrm{i}-\mathrm{p}-\mathrm{n}$ device structure, which is a standard single-junction n-i-p device with a tunnel junction stacked on top. Table 2 lists the characterists of a standard n-i-p-n device and an improved n-i-p-n device. The $V_{o c}$ and FF are imporved. The slight decrease in $J_{\text {sc }}$ will become negligible when additional $i-p$ layers of the top cell are deposited, as in a complete multiple-junction solar cell.

Table 1. The J-V performance data of two a-Si:H:F n-i-p devices before and after 384 hours of one sun light soaking at $50^{\circ} \mathrm{C}$

\begin{tabular}{|c|c|c|c|c|c|c|}
\hline Device & $\begin{array}{l}\text { Light } \\
\text { Soaking }\end{array}$ & $\begin{array}{l}\mathrm{V}_{\mathrm{oc}} \\
(\mathrm{V})\end{array}$ & $\begin{array}{l}\mathrm{J}_{\mathrm{sc}} \\
(\mathrm{mA} / \mathrm{cr}\end{array}$ & $\begin{array}{l}\text { FF } \\
\mathrm{m} 2 \text { ) }\end{array}$ & $\begin{array}{l}\text { Eff } \\
(\%)\end{array}$ & $\begin{array}{l}\text { Drop in } \\
\text { Efficiency }\end{array}$ \\
\hline LL944 & $\begin{array}{l}0 \text { hrs } \\
384 \text { hrs }\end{array}$ & $\begin{array}{l}0.935 \\
0.908\end{array}$ & $\begin{array}{l}14.37 \\
14.07\end{array}$ & $\begin{array}{l}0.636 \\
0.601\end{array}$ & $\begin{array}{l}8.55 \\
7.68\end{array}$ & $10.1 \%$ \\
\hline LL945 & $\begin{array}{l}0 \mathrm{hrs} \\
384 \mathrm{hrs}\end{array}$ & $\begin{array}{l}0.931 \\
0.897\end{array}$ & $\begin{array}{l}15.02 \\
14.91\end{array}$ & $\begin{array}{l}0.638 \\
0.592\end{array}$ & $\begin{array}{l}8.93 \\
7.91\end{array}$ & $11.4 \%$ \\
\hline
\end{tabular}

Table 2. The J-V performance data of n-i-p-n devices with standard and improved p-n tunnel junction.

$\begin{array}{llllc}\text { Sample } & \begin{array}{l}\mathrm{V}_{\mathrm{oc}} \\ (\mathrm{V})\end{array} & \mathrm{FF} & \mathrm{R}_{\mathrm{s}} & \begin{array}{c}\mathrm{J}_{\mathrm{sc}} \\ \left(\mathrm{mA} / \mathrm{cm}^{2}\right)\end{array} \\ \text { Standard } & 0.906 & 0.560 & 12.1 & 10.8 \\ \text { Improved } & 0.938 & 0.571 & 13.0 & 9.36\end{array}$




\section{References}

1. J. Yang, R. Ross, T. Glatfelter, R. Mohr, G. Hammond, C. Bernotaitis, E. Chen, J. Burdick, M. Hopson and S. Guha, Proceedings 20th IEEE PV Spec. Conf., 241 (1988).

2. X. Deng, E. Mytilineou, R.T. Young and S.R. Ovshinsky, MRS Proceedings. 258, 491 (1992).

3. X. Deng, S.J. Jones, K.L. Narasimhan, J. Evans, M. Izu and S.R. Ovshinsky, to be presented in MRS Spring Meeting, April, 1995.

4. X. Deng, K.L. Narasimhan, J. Evans, M. Izu and S.R. Ovshinsky, Proceedings of 1st World Conf. on Photovoltaic Energy Conversion (1994).
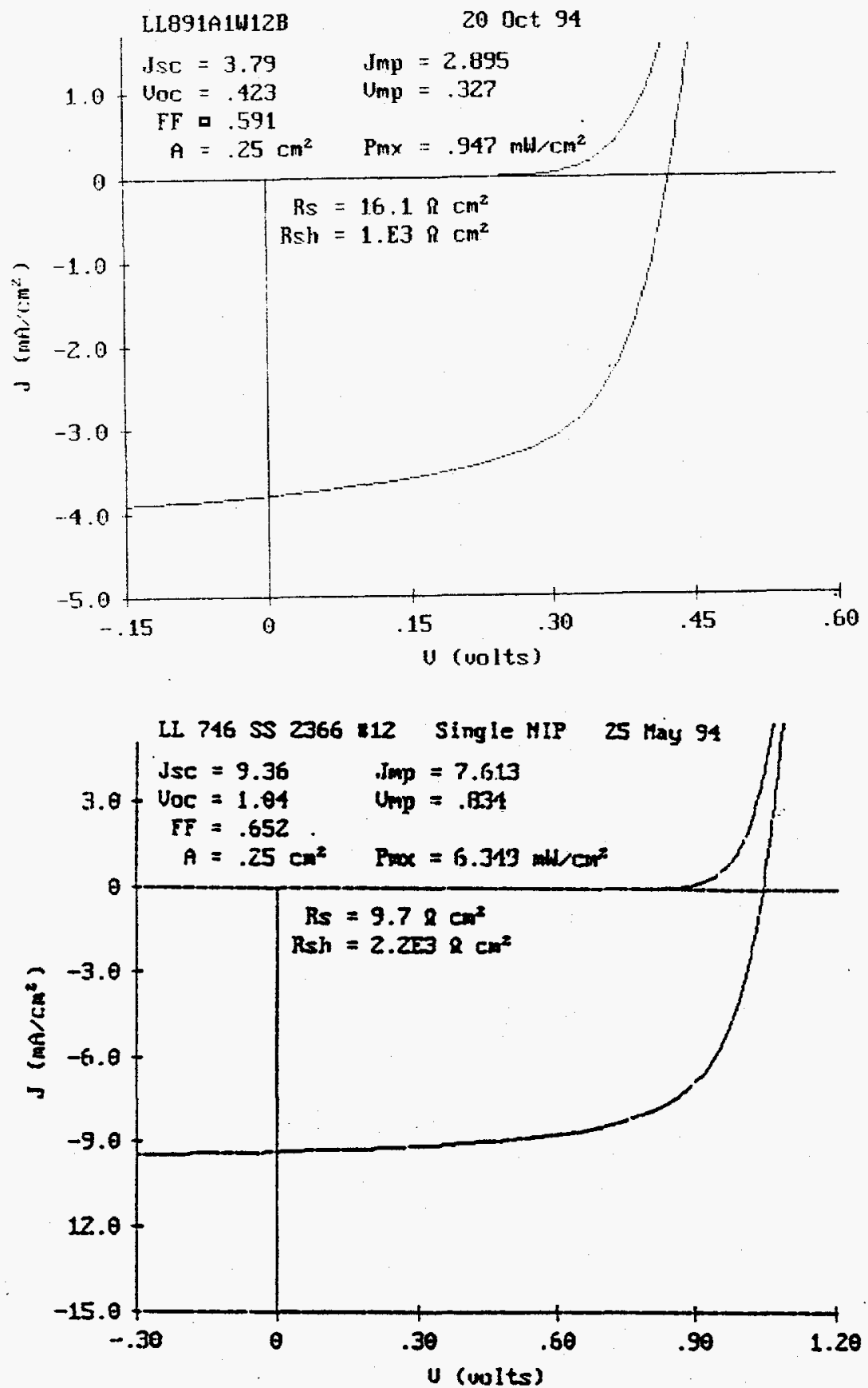

Figure 1 .

$\mathrm{J}-\mathrm{V}$ curve of a c-Si $\left(\mathrm{n}^{+}\right) / \mathrm{a}-\mathrm{Si}$ alloy/Pd Schottky barrier device.
Figure 2.

$\mathrm{J}-\mathrm{V}$ curve of a $\mathrm{n}-\mathrm{i}-\mathrm{p}$ solar cell showing $1.04 \mathrm{~V} \mathrm{~V}_{\text {oc }}$ 
Title: $\quad$ Non- $\mathrm{H}_{2} \mathrm{Se}$, Ultra-Thin CIS Devices

Organization: Energy Photovoltaics, Inc. (EPV)

Princeton, NJ

Contributors: Z. Kiss, program manager; A.E. Delahoy, principal investigator; J. Britt, G. Butler, F. Faras, A. Muthaiah, A. Sizemore, F. Ziobro.

\section{Objectives}

The overall objective of this subcontract is the demonstration of large area, thin film CulnSe photovoltaic modules using methods applicable to non-hazardous, high throughput manufacturing. Specific goals include the fabrication of a $12 \% 1 \mathrm{~cm}^{2}$ cell, a $7 \mathrm{~W} 1 \mathrm{ft}^{2}$ module, and a $50 \mathrm{~W}$ submodule.

\section{Approach}

EPV's approach to the formation of CIS is based on inherently scalable methods of vacuum deposition and selenization, including magnetron sputtering and extended source evaporation. For reasons of safety, selenium is delivered by evaporation of the element, and not via use of hydrogen selenide. Module formation is accomplished using a novel and reliable interconnect process. Scale up of all processing steps has been completed to allow the coating and patterning of substrates $0.5-0.6 \mathrm{~m}^{2}$ in area. A unique combination of equipment features has been developed to permit high temperature selenization of these larger substrates without glass warpage.

\section{Results}

In FY93 we reported $12.5 \%$ cells and development of a new module patterning technology $[1,2]$. The emphasis in FY94 has been on improving module efficiency, exploring a variety of methods of preparing $\mathrm{CIS}$, and on characterizing the behavior of $\mathrm{CIS}$ modules under various conditions of temperature and irradiance $[3,4]$.

To improve module efficiency, the following tasks were accomplished: improvement of CIS uniformity via improved substrate temperature uniformity, incorporation of gallium at low levels by initiation of its delivery during the second half of the selenization process, and reduction of module shunt conductance. Table I shows the dependence of PV parameters on the Ga introduction temperature. The I-V curve of an unencapsulated module fabricated using these new techniques is shown in Figure 1. The aperture area is a little over $200 \mathrm{~cm}^{2}$ and the corresponding efficiency is $9.0 \%$. With improved ZnO uniformity, a CIGS module was produced with an aperture area of $741 \mathrm{~cm}^{2}$, and an efficiency of $8.1 \%$ (see Fig. 2). The power produced by this module in sunlight is 6.0 watts. It was noticed that the introduction of gallium made it easier to obtain a high quality absorber over larger areas.

Various aspects of the operating temperature dependence of CIS modules have been investigated. Some of the results have already been published, for example, the measured $\mathrm{dV}_{\mathrm{oc}} \mathrm{dT}$ for $\mathrm{CIS}$ modules $\left(-1.9 \mathrm{mV} /{ }^{\circ} \mathrm{C}\right)$, and the calculated temperature coefficient of the 
efficiency $\left(-0.62 / 0.68 \% /{ }^{\circ} \mathrm{C}\right)$ [3]. A recent outdoor measurement of the latter coefficient gave $-0.75 \% 1^{\circ} \mathrm{C}$ referenced to a module temperature of $25^{\circ} \mathrm{C}$. Figure 3 shows the intensity dependence of the conversion efficiency of (unalloyed) CIS cells and modules. The shape of the curves is determined by competition between a rising fill factor and a falling open circuit voltage (dominant) as the irradiance is reduced (plus shunting effects, if present), and the influence of series resistance at high irradiance levels.

The stability of CIS modules is being monitored through 24 hour/day outdoor exposure of glass-glass laminated $1 \mathrm{ft}^{2} \mathrm{CIS}$ modules on an outdoor test rack and measurement station. Figure 4 shows the power output of such an EPV module (normalized to the power of a reference module) for over 3500 hours of outdoor exposure. The modules so far appear to be durable and stable.

\section{Conclusions and Future Plans}

A key result of this phase was the achievement of a CIGS module approaching $1 \mathrm{ft}^{2}$ in area that produces 6.0 watts in sunlight. Outdoor weathering tests have so far demonstrated the stability of encapsulated CIS modules for over 3500 hours. Equipment is in place to fabricate modules up to about $0.6 \mathrm{~m}^{2}$ in area. EPV exhibited a CIS module of this size at the First World Conference on Photovoltaics in Hawaii in December 1994.

Under the upcoming Thin Film Partnership Program we anticipate optimizing both the metallic precursor route to $\mathrm{CIS}$ and an alternative Se-containing precursor route. We will also strive to develop wider gap CIGS in order to reduce the temperature sensitivity of modules. Our goals are to produce CIGS modules generating in excess of 50 watts, to introduce new technology that will enable manufacturing cost reductions to be made, and to demonstrate qualified, userready CIGS modules.

\section{References}

1. A.E. Delahoy, J. Britt, F. Faras, F. Ziobro, A.Sizemore, G. Butler, and Z. Kiss, "Progress in CulnSe $\mathrm{S}_{2}$ and Culn(Se,S), Module Development" 12th NREL PV Program Review Meeting, Oct. 13-15, 1993, Denver, CO. AIP Conf. Proc. Vol. 306 (AIP, NY, 1994) pp. 370-381.

2. A.E. Delahoy, J. Britt, F. Faras, and Z. Kiss, Annual Subcontract Report FY 1993, NRELTP-413-7165, Sept. 1994. Available NTIS: Order No. DE94011894.

3. A. Delahoy, J. Britt, G. Butler, F. Faras, A. Sizemore, F. Ziobro, and Z. Kiss, "Recent Advances in Manufacturing Technology for CulnSe,-Based Power Modules" Presented at the 12th European Photovoltaic Solar Energy Conference and Exhibition, Amsterdam, The Netherlands, 11-15 April 1994, pp. 1612-1615.

4. J. Britt, A. Delahoy, G. Butler, F. Faras, A. Muthaiah, A. Sizemore, F. Ziobro, and Z.

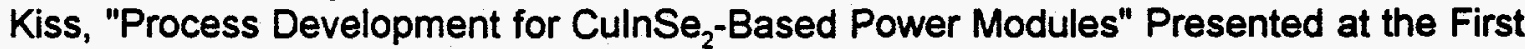
World Conference on Photovoltaic Energy Conversion, Waikoloa, Hawaii, Dec. 5-9, 1994. 
Table 1. Photovoltaic parameters as a function of $\mathrm{Ga}$ introduction temperature

\begin{tabular}{|c|c|c|c|c|}
\hline Temperature & $\begin{array}{c}\mathrm{V}_{\mathrm{oc}} \\
\text { (volts) }\end{array}$ & $\begin{array}{c}\mathrm{J}_{\mathrm{sc}} \\
(\mathrm{mAvcm})\end{array}$ & $\begin{array}{c}\text { Efficiency } \\
(\%)\end{array}$ \\
\hline $\mathrm{No} \mathrm{Ga}$ & 0.442 & 34.7 & 0.697 & 10.7 \\
\hline $370^{\circ} \mathrm{C}$ & 0.463 & 33.9 & 0.709 & 11.1 \\
\hline $420^{\circ} \mathrm{C}$ & 0.451 & 32.0 & 0.656 & 9.5 \\
\hline
\end{tabular}

EPV CdS/Cu(In,Ga)Se,

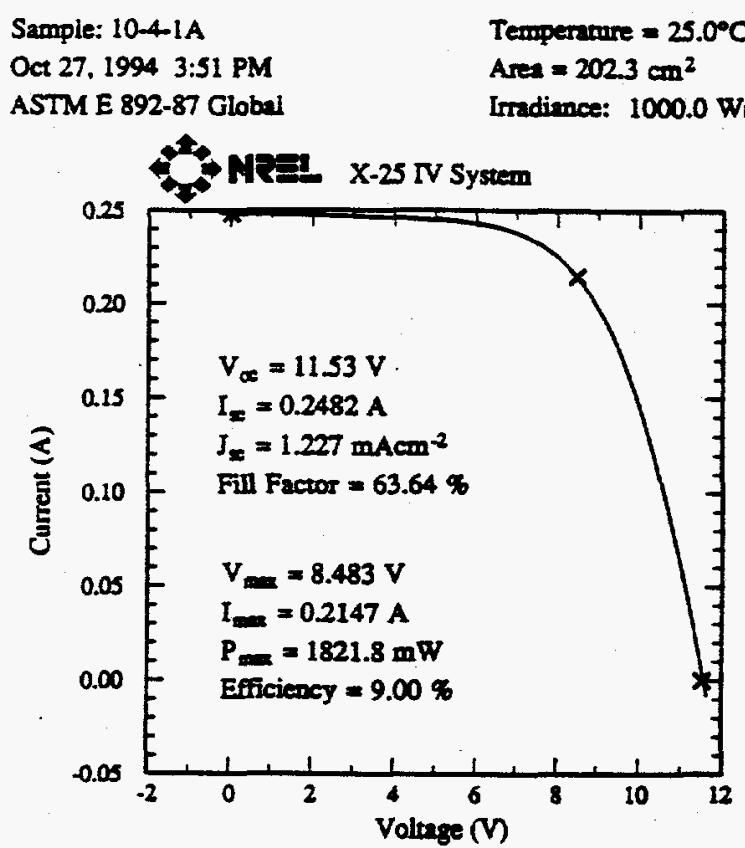

Fig. 1. Illuminated $\mathrm{l}-\mathrm{V}$ curve for a $202 \mathrm{~cm}^{2}$ CIGS submodule with an aperture area efficiency of $9.0 \%$

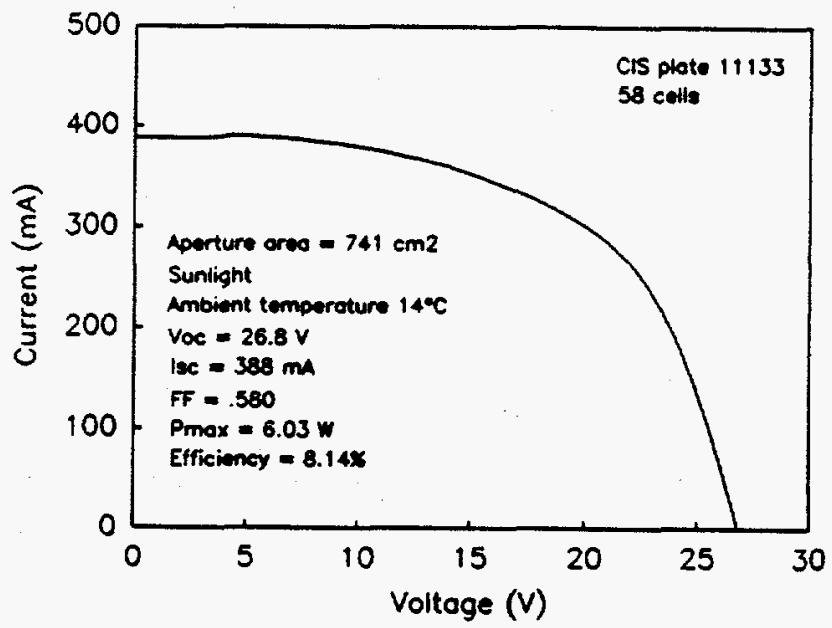

Fig. 2. I-V curve taken in sunlight for a 58 cell CIGS module producing 6.0 watts (8.1\% aperture area efficiency, 462 $\mathrm{mV} /$ cell $\mathrm{V}_{\infty}$ ) 


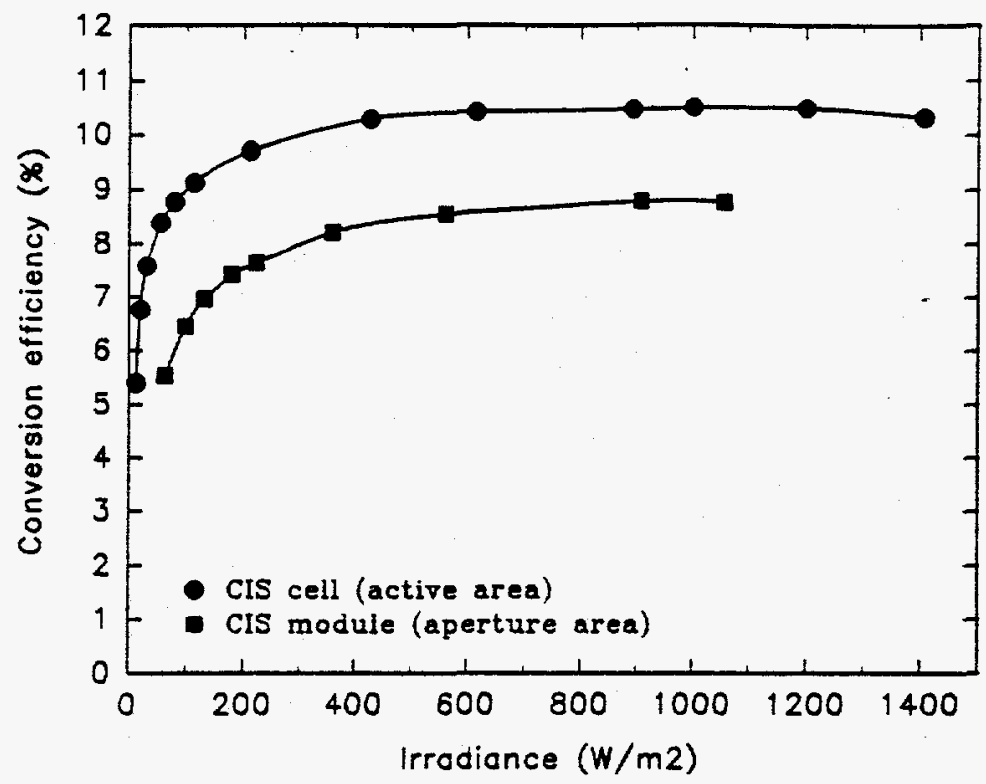

Fig. 3. Conversion efficiency versus irradiance for CIS cells and modules.

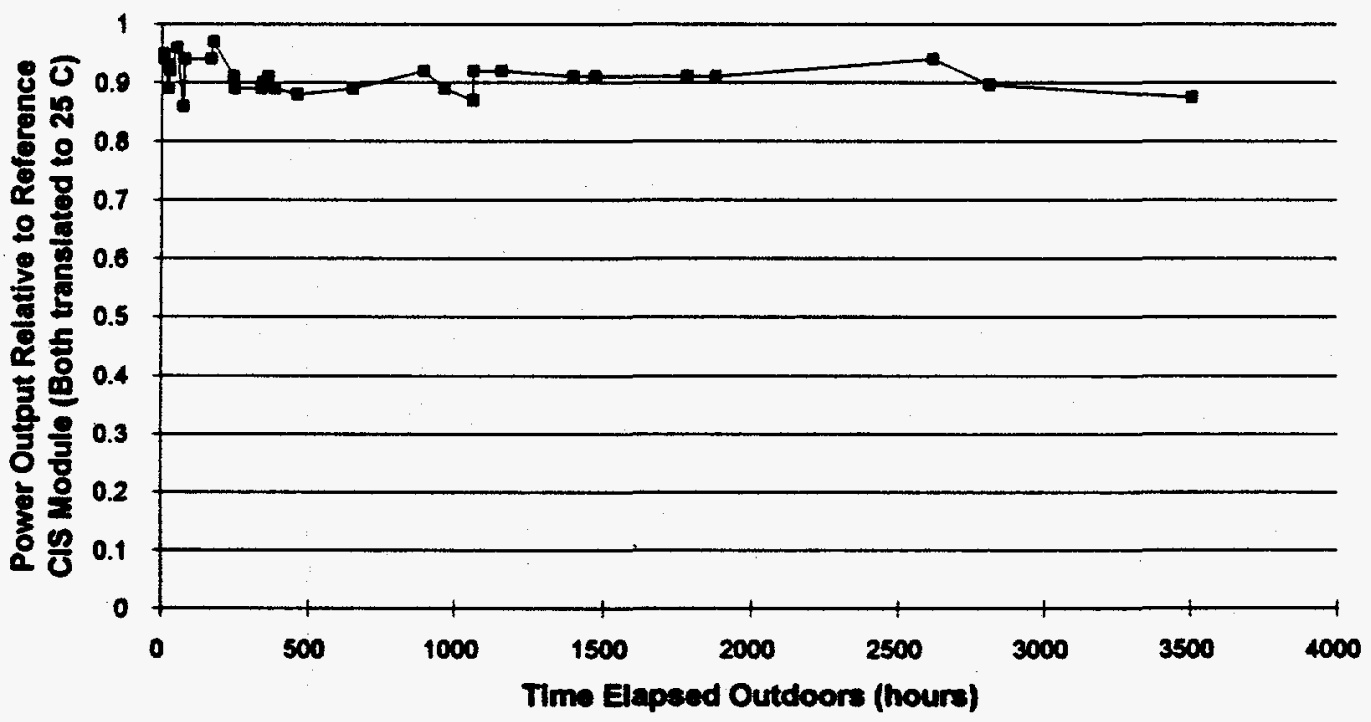

Fig. 4. Outdoor performance of an EPV encapsulated $1 \mathrm{ft}^{2} \mathrm{CIS}$ module throughout 3500 hours of exposure. 
Title:

Organization:

Contributors:
Polycrystalline CuInSe $e_{2}$ \& CdTe PV Solar Cells

Florida Solar Energy Center, University of Central Florida, Cape Canaveral, Florida

Neelkanth G. Dhere, Principal Investigator, Kevin Lynn, Mark Wollam, Shanker Kuttath, Lucy Orozco, and George Freeman

\section{Objectives}

The principal objective of the research project is to develop novel and low-cost processes for the fabrication of stable and efficient copper-indium-gallium-diselenide, $\mathrm{CuIn}_{1-x} \mathrm{Ga}_{\mathrm{x}} \mathrm{Se}_{2}$ and cadmiumtelluride, CdTe polycrystalline-thin-film solar cells using reliable techniques amenable to scaledup for economic, large-scale manufacture.

\section{Technical Approach}

Two-stage selenization and magnetron sputtering from $\mathrm{Cu}-\mathrm{Ga}$ alloy targets have been developed for the preparation of well-adherent, large-compact-grain $\mathrm{CuIn}_{1-\mathrm{x}} \mathrm{Ga}_{\mathrm{x}} \mathrm{Se}_{2}$ thin films and solar cells, by non-toxic Se vapor selenization of $\mathrm{Cu}-\mathrm{In}-\mathrm{Ga}$ thin films. Magnetron-sputtered $\mathrm{Cd}$ and $\mathrm{Te}$ layers are being studied for CdTe-solar-cell fabrication.

\section{$\mathrm{CuIn}_{1-x} \mathrm{Ga}_{x} \mathrm{Se}_{2}$ Polycrystalline-Thin-Film Solar Cells}

Magnetron-sputter-depositions of Mo and Cu-In-Ga metallic precursor films on soda-lime float glass substrates were carried out in a $18^{\prime \prime}$ diameter and 24 " height bell jar pumped with a cryopump. The Cu-In-Ga films were subjected to an in situ heat treatment at $80-90^{\circ} \mathrm{C}$ for 10 min. A novel two-stage selenization process developed at FSEC improved the adhesion to Mo back-contact layer considerably. Addition of gallium to prepare higher bandgap $\mathrm{CuIn}_{1-x} \mathrm{Ga}_{\mathrm{x}} \mathrm{Se}_{2}$ thin films improved adhesion still further. The two-stage Se vapor selenization of magnetron sputtered metallic precursors and $\mathrm{Ga}$ incorporation using single $\mathrm{Cu}-\mathrm{Ga}(22$ at.\%) alloy target are being used routinely for preparation of well-adherent, large-grain CuIn $\mathrm{Cu}_{1-\mathrm{x}} \mathrm{Ga}_{x} \mathrm{Se}_{2}$ thin films having near-optimum composition $\mathrm{Cu}: \mathrm{In}: \mathrm{Ga}: \mathrm{Se}$ of 22.95:25.03:1.40:50.63. The process sequence is shown in figure 1 . Very small sub-grain features were studied for the first time by atomic force microscopy (AFM), and quantitative data on rms roughness and 3-d images were obtained. Morphology of precursors was found to change from initial very smooth layers with 200-300 $\AA$ size features and a rms surface roughness of $<10 \AA$, to 3-d $\sim 9000 \AA$ size islands, coalescing grains with a fine sub-grain structure, and finally to compact, well-faceted, large-grain $\mathrm{CuIn}_{1}$. ${ }_{x} \mathrm{Ga}_{x} \mathrm{Se}_{2}$ thin films with rms roughness in the range 950-1500 $\AA$. AFM images of good quality $\mathrm{CuIn}_{1-\mathrm{x}} \mathrm{Ga}_{\mathrm{x}} \mathrm{Se}_{2}$ samples showed large, compact, well-faceted grains with crystallographic shapes (Fig. 2). The factors which improved morphology of $\mathrm{CuIn}_{1-x} \mathrm{Ga}_{x} \mathrm{Se}_{2}$ thin films and efficiency of solar cells were in situ homogenization of $\mathrm{Cu}$-rich precursors prior to the first selenization, $\mathrm{Se}$ vapor incidence rate of $50 \AA \mathrm{sec}^{-1}$, and a maximum selenization temperature of $550-560^{\circ} \mathrm{C}$ which provided beneficial fluxing action of copper selenide [1]. Several cells were completed at NREL and IEC using glass $\mathrm{MolCuIn}_{1-x} \mathrm{Ga}_{x} \mathrm{Se}_{2}$ samples prepared at FSEC. $\mathrm{V}_{o c}$ of solar cells was in the 
$370-400 \mathrm{mV}$ range. The best cells obtained with films prepared using precursors homogenized in situ prior to selenization showed $\mathrm{J}_{\mathrm{sc}}$ of $31.5 \mathrm{~mA}$, fill factor of $45.1 \%$ and the total area efficiency of $5.8 \%$ (fig. 3) and active area efficiency of $8.2 \%$. Spectral response was fairly constant over the entire spectral range (Fig. 4) [2].

\section{CdTe Polycrystalline-Thin-Film Solar Cells}

Chemical bath deposition (CBD) technique has been well-developed at FSEC and CdS layers for $\mathrm{CdTe}$ solar cells are being prepared routinely from acetate reactants. $\mathrm{Cd}$ and $\mathrm{Te}$ layers were magnetron-sputtered in a 6-way 6" diameter cross type chamber pumped with a turbomolecular pump. Vacuum melted targets and adequate thermal and electrical contact between the magnetron sputtering source and Te target and efficient water cooling were found to be essential for well-controlled $\mathrm{Te}$ deposition at high deposition rates. It is estimated that using this technique, higher and controllable deposition rates could be employed to allow deposition of elemental $\mathrm{Cd} / \mathrm{Te}$ layers required to form $>4 \mu \mathrm{m}$ thick $\mathrm{CdTe}$ film in $<4 \mathrm{~min}$. Elemental stacks consisted of first and last $\mathrm{Te}$ layers so as to obtain better adhesion and to minimize $\mathrm{Cd}$ reevaporation. Experiments were conducted with stoichiometric $\mathrm{Cd} / \mathrm{Te}$ layers and elemental excesses ranging from $10 \%$ Cd-rich to $30 \%$ Te-rich. Most experiments were carried out with small excesses of Te to promote p-type conductivity. XPS analysis showed the presence of traces of $\mathrm{CdTe}$ even in as-deposited $\mathrm{Cd} / \mathrm{Te}$ layers on unheated substrates. Complete $\mathrm{CdTe}$ phase formation was observed by XRD and XPS analysis after heat treatment at temperatures $<300^{\circ} \mathrm{C}$ for 30 minutes of $\mathrm{Cd} / \mathrm{Te}$ elemental layers with a small excess $\mathrm{Cd}$ or Te. CdSiCdTe interface and junction formation was promoted by heat treatments at $450-475^{\circ} \mathrm{C}$ and by using thicker $\mathrm{CdS}$ layers. A semi-closed graphite crucible and 5-30\% Te excess with a Te top layer were used to minimize $\mathrm{Cd}$ and even Te reevaporation at temperatures over $440^{\circ} \mathrm{C}$. Tellurium oxide and cadmium chloride were detected by XPS analysis at considerable depths in the $\mathrm{CdCl}_{2}$-treated Terich samples and heated in oxygen-containing ambients at $350-450^{\circ} \mathrm{C}$. Well-adherent and mostly pinhole-free $\mathrm{CdTe}$ layers on glass $\mathrm{SnO}_{2}: \mathrm{FCdS}$ samples are being prepared routinely by optimization of the heat treatment process. CdTe cells were completed after $\mathrm{CdCl}_{2}$ treatment by applying doped graphite paste, and silver paste contacts. CdTe thin-films Solar cells prepared after low-temperature $\left(\leq 400^{\circ} \mathrm{C}\right.$ ) heat-treatments showed $\mathrm{V}_{\mathrm{oc}}$ of $320-380 \mathrm{mV}, \mathrm{J}_{\mathrm{sc}}$ of $\sim 5 \mathrm{~mA}$ and a poor FF values of $\sim 30 \%$. Solar cells prepared using high-temperature-treated CdTe layers showed $V_{\mathrm{oc}}$ of $>580 \mathrm{mV}$, low $\mathrm{J}_{\mathrm{sc}}$ and poor fill factor probably resulting from blocking contacts or junction formation away from the CdTelCdS interface.

\section{References}

1. N. G. Dhere, S. Kuttath, H. R. Moutinho, "Morphology of Precursors and CuIn $\mathrm{Cu}_{1-\mathrm{x}} \mathrm{Ga}_{\mathrm{x}} \mathrm{Se}_{2}$ Thin Films Prepared by Two-Stage Selenization Process", J. Vac. Sci. \& Technol. A (Accepted).

2. N. G. Dhere, S. Kuttath, K. W. Lynn, R. W. Birkmire and W. N. Shafarman "Polycrystalline CuIn $\mathrm{Ca}_{1-x} \mathrm{Ge}_{2}$ Thin Film PV Solar Cells Prepared by Two-stage Selenization Process Using Se Vapor", To be published in the the Proc. of the First World Conference on Photovoltaic Energy Conversion held at Waikoloa, Hawaii during Dec. 5$9,1994$. 


\begin{tabular}{|c|c|}
\hline \multicolumn{2}{|c|}{ Total thickness } \\
\hline CuGa & $3682 \AA$ \\
\hline In & $3924 \AA$ \\
\hline
\end{tabular}

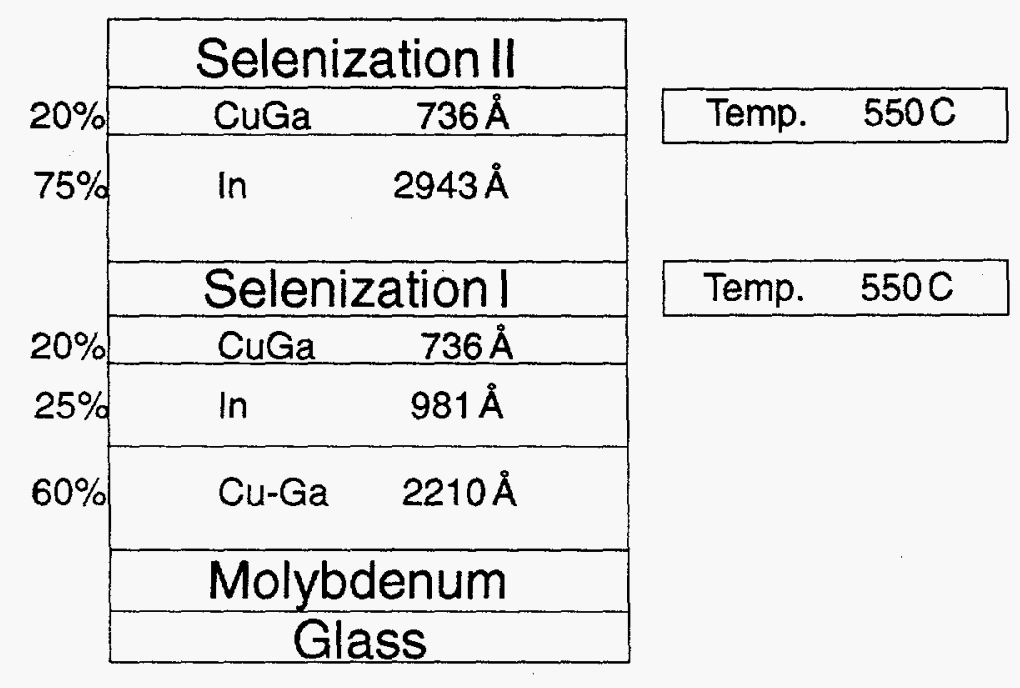

Fig. 1. Two-selenization process sequence for preparation of $2.5 \mu \mathrm{m} \mathrm{CuIn} \mathrm{Cl}_{1-\mathrm{x}} \mathrm{Ga}_{\mathrm{x}} \mathrm{Se}_{2}$ layer.

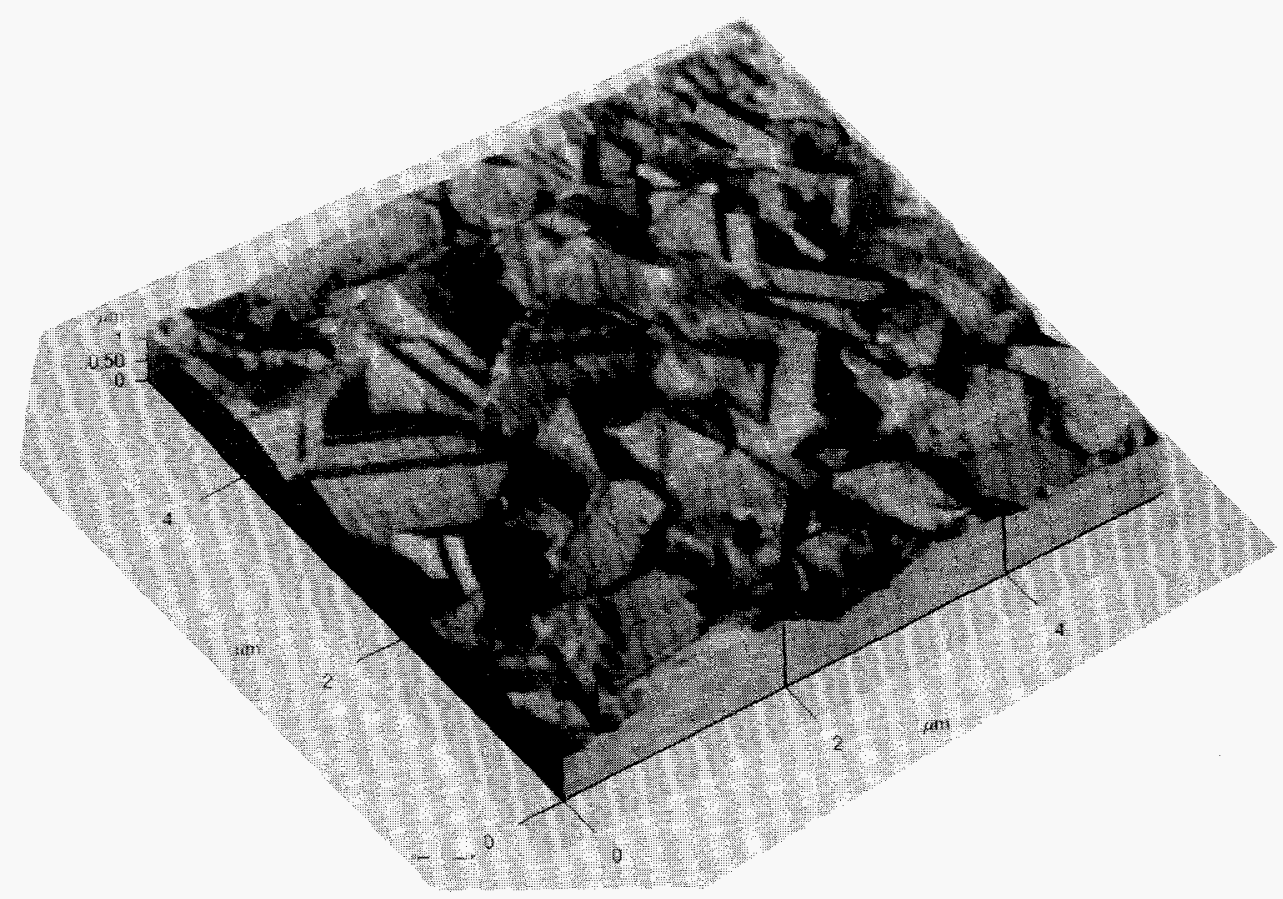

Fig. 2. 3-d AFM image of good quality $\mathrm{CuIn}_{1-x} \mathrm{Ga}_{\mathrm{x}} \mathrm{Se}_{2}$ samples showing large, compact, wellfaceted grains with crystallographic shapes. 


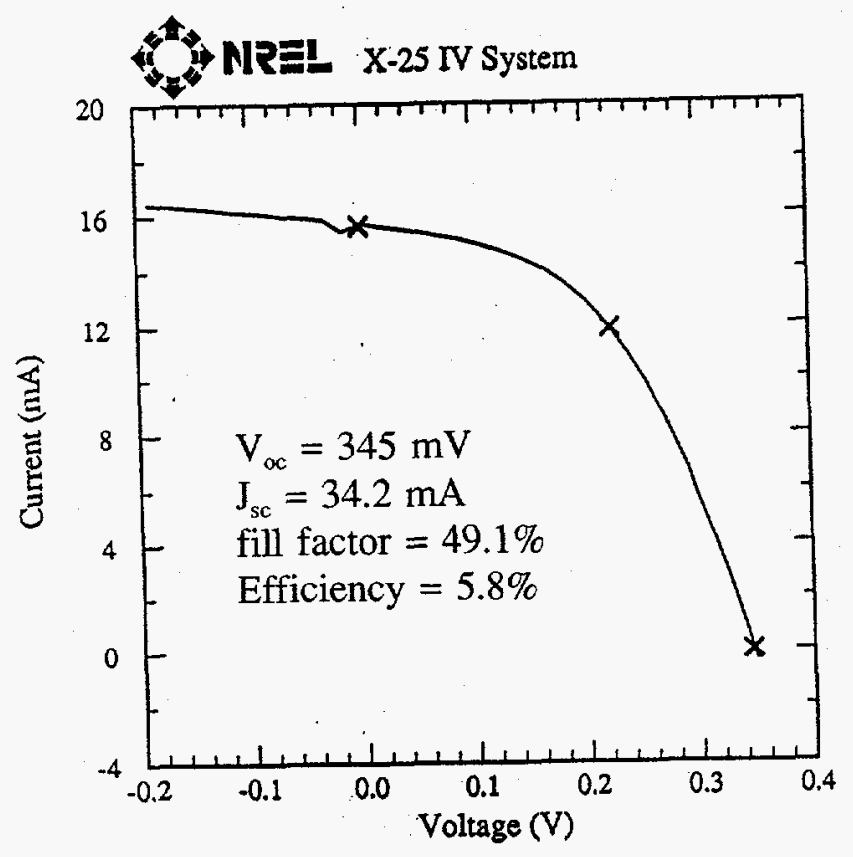

Fig. 3. ASTM E $892-87$ Global IxV characteristic of a $0.4571 \mathrm{~cm}^{2} \mathrm{CuIn}_{1-\mathrm{x}} \mathrm{Ga}_{\mathrm{x}} \mathrm{Se}_{2}$ thin-film solar cell

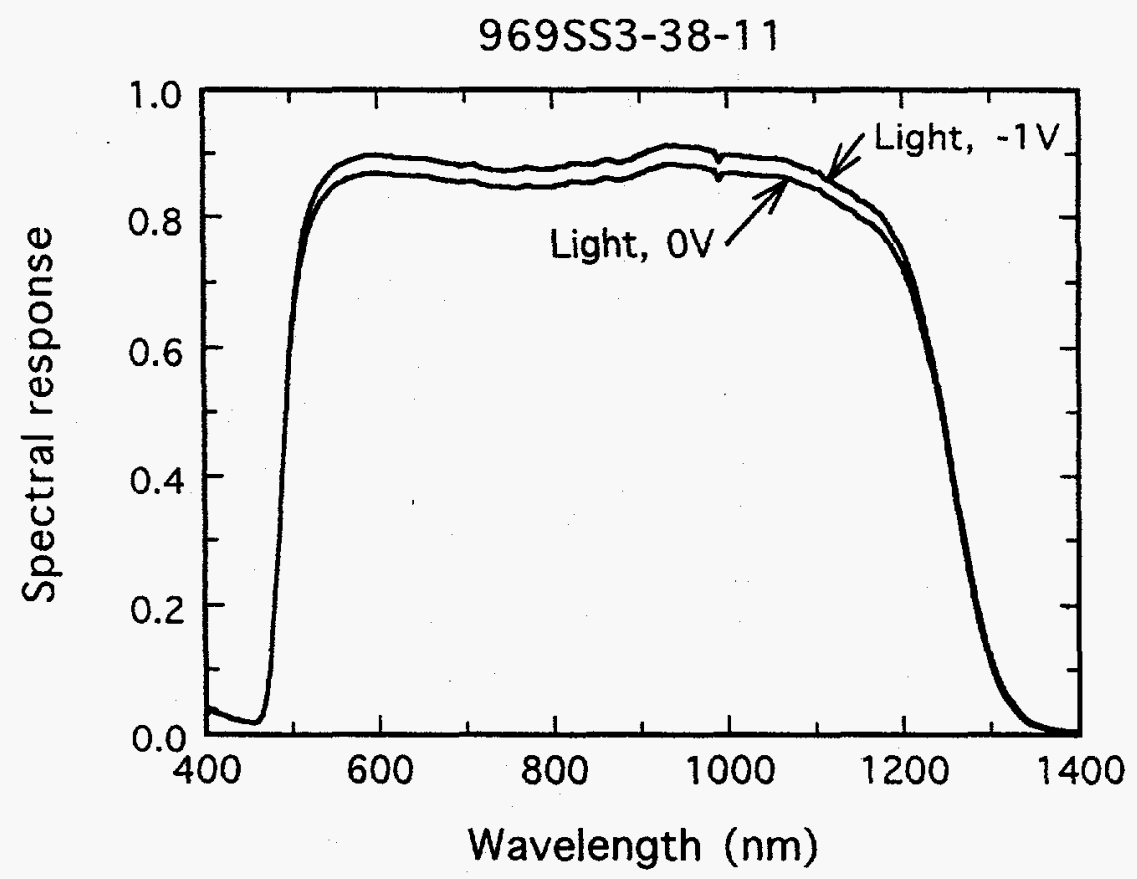

Fig. 4 Spectral response of $\mathrm{CuIn}_{1-\mathrm{x}} \mathrm{Ga}_{\mathrm{x}} \mathrm{Se}_{2}$ thin-film solar cell 
Title:

Organization:

Contributors:

\section{Development of High Efficiency CdTe Solar Cells}

\author{
School of Electrical and Computer Engineering,
} Georgia Institute of Technology, Atlanta, Georgia.

\section{Objective}

The objective of this program is to improve the basic fundamental understanding of efficiency limiting mechanisms in polycrystalline CdTe cells, fabricate high efficiency CdTe cells, and provide guidelines for achieving higher efficiency CdTe cells.

\section{Film Growth and Cell Fabrication}

CdS films (1000-2000 $\AA$ ) were deposited on textured $\mathrm{SnO}_{2}$ /glass substrates, using solution growth starting from $\mathrm{CdCl}_{2}$, thiourea, $\mathrm{NH}_{4} \mathrm{Cl}$, and $\mathrm{NH}_{4} \mathrm{OH}$. The CdS/SnO$/$ glass substrates were treated with a $\mathrm{CdCl}_{2}: \mathrm{CH}_{3} \mathrm{OH}$ solution and annealed in the furnace at $450^{\circ} \mathrm{C}$ in $\mathrm{N}_{2}$ ambient for $50 \mathrm{~min}$. Polycrystalline CdTe films with a thickness of $2.6 \mu \mathrm{m}$ were grown by MOCVD on the annealed $\mathrm{CdS} / \mathrm{SnO}_{2}$ /glass substrates at a temperature of $400^{\circ} \mathrm{C}$ in $\mathrm{N}_{2}$ ambient for $70 \mathrm{~min}$. After the CdTe deposition, $\mathrm{CdTe} / \mathrm{CdS}$ structures were treated in a $\mathrm{CdCl}_{2}: \mathrm{CH}_{3} \mathrm{OH}$ solution followed by an air anneal at $400^{\circ} \mathrm{C}$ for $30 \mathrm{~min}$. This treatment is perfomed to enhance the grain growth which also improves the cell performance. Ohmic back contacts were formed on the CdTe surface by sequential evaporation of $100 \AA \mathrm{Cu}$ and $400 \AA \mathrm{Au}$. After the metallization, a contact anneal was perfomed at $150^{\circ} \mathrm{C}$ in $\mathrm{Ar}$ ambient for $90 \mathrm{~min}$. Cell fabrication was completed by a $0.1 \% \mathrm{Br}_{2}: \mathrm{CH}_{3} \mathrm{OH}$ etch, followed by a DI water rinse and $\mathrm{N}_{2}$ blow-dry. The final CdTe solar cell structure consists of $\mathrm{Au} / \mathrm{Cu} / \mathrm{CdTe} / \mathrm{CdS} / g l a s s$ layers. Also, we have recently started fabrication of single crystal $\mathrm{CdTe} / \mathrm{CdS}$ films.

\section{Material and Device Characterization}

Measurements techniques, such as SIMS, C-V, and I-V were performed to probe the Cu migration and its influence on doping density, shunts and cell performance. XRD measurements were performed on $\mathrm{CdTe} / \mathrm{GaAs}$ interface to assess lattice mismatch-induced changes in lattice constant and defects.

\section{Results and Discussion}

In the past we have reported $12 \%$ efficient furnace annealed and $10.7 \%$ RTP annealed Te rich MOCVD grown CdTe solar cells at Georgia Tech. It was also shown that a Te-rich growth ambient allows more interdiffusion at the CdS-CdTe interface, and increases carrier lifetimes in the CdTe. However, in order to further enhance our cell performance we need to understand and optimize some other efficiency limiting mechanisms which are discussed below:

\section{Analysis of Photocurrent Loss Due to Reflectance and Absorption in CdTe Solar Cells} The CdS and $\mathrm{SnO}_{2}$ thickness have a significant impact on the CdTe solar cell performance. Reflectance measurements were made using an Optronics Laboratory phase sensitive detection system. The reflectance was modeled as a function of wavelength by utilizing CAMS packeged 
program written by Optikos Corporation. The effects of CdS thickness on the reflectance and photocurrent loss were investigated and it was found from simulation that the reduced reflectance could increase $J_{s c}$ by about $1 \mathrm{~mA} / \mathrm{cm}^{2}$, if the CdS thickness is reduced to $600 \AA$, Fig. 1 . It was found that the optimum CdS thickness is about $600-700 \AA$ for achieving high $\mathrm{J}_{\mathrm{sc}}$ in a practical CdTe solar cell, because at this thickness, it benefits both reduced absorption and reflectance without the risk of pinhole formation. Solar cell data for the $\mathrm{CdTe} / \mathrm{CdS}$ cells grown with different thicknesses is presented in Table I, and the measured reflectance from them is shown in Fig. 2. It was also found that the control of $\mathrm{CdS}$ film thickness is more critical than that of the $\mathrm{SnO}_{2}$ because the reflectance does not change much when the $\mathrm{SnO}_{2}$ thickness exceeds $4000 \AA$ which is used by most investigators today to minimize series resistance, Fig. 3.

\section{Effects of Cu on CdTe/CdS Solar Cells with Au/Cu Contacts}

$\mathrm{Cu}$ plays a dual role in the $\mathrm{CdTe} / \mathrm{CdS}$ solar cells with $\mathrm{Au} / \mathrm{Cu}$ contact. It helps in the formation of better ohmic contact to $\mathrm{CdTe}$ and increases the acceptor doping concentration, but excess $\mathrm{Cu}$ diffuses all the way to $\mathrm{CdTe} / \mathrm{CdS}$ interface, forms recombination centres and shunt paths, and degrades cell performance, Table II. Both SIMS and C-V measurements confirm the incorporation of $\mathrm{Cu}$ into the bulk of the CdTe films, Figs. 4 \& 5. Monotonically decreasing $\mathrm{Cu}$ concentration toward the $\mathrm{CdTe} / \mathrm{CdS}$ interface suggests that diffusion is the most probable mechanisms for $\mathrm{Cu}$ incorporation. The $\mathrm{Cu}$ thickness and deposition rate during the $\mathrm{Cu}$ deposition process, rather than the subsequent heat treatment, influences the $\mathrm{Cu}$ incorporation. Both $\mathrm{R}_{\mathrm{s}}$ and $\mathrm{R}_{\mathrm{sh}}$ decrease with the increase $\mathrm{Cu}$ thickness, Fig. 6. SIMS measurements showed that the increase in $\mathrm{Au} / \mathrm{Cu}$ deposition rate results in $\mathrm{Cd}$ pileup or diffusion towards the CdTe surface, leaving more $\mathrm{Cd}$ vacant sites below the surfaces, Fig. 7. This causes an increase in dopant concentration but could also result in higher defect density and reduced cell performance. Finally, a combination of optimum thickness and a slower deposition rate of $\mathrm{Cu}$ resulted in the highest efficiency $\mathrm{CdTe}$ solar cell in our laboratory.

\section{Development of Single Crystal CdTe and CdTe/CdS Structures by Selective Etching}

A comparison between single and polycrystalline CdTe structures and cells can significantly improve the fundamental understanding of the role of grain boundaries, and provide guidelines for what to expect from grain boundry passivation or elimination. We have sucessfully fabricated thin film CdTe/CdS device structures using an epitaxial lift-off (ELO) process. Single crystal CdTe and $\mathrm{CdTe} / \mathrm{CdS}$ epitaxial layers were seperated from the GaAs substrate by selective etching and were the transferred and bonded to a $\mathrm{SnO}_{2}$ /glass substrate, Fig. 8. SIMS analysis of $\mathrm{CdTe} / \mathrm{CdS}$ cells, with $\mathrm{Au} / \mathrm{Cu}$ ohmic contacts to $\mathrm{CdTe}$, showed much less $\mathrm{Cu}$ diffusion in the single crystal $\mathrm{CdTe}$ films due to the absence of grain boundaries, Fig. 9. X-ray diffraction measurements showed that the $\mathrm{CdTe} / \mathrm{CdS}$ lattice structure and quality does not change appreciably after the lift-off process. A new methodology was developed, using XRD and the lift-off technique, to assess the lattice mismatch induced strain and defects at the heterojunction interface. The lattice constant of CdTe at the interface with GaAs was found to be $6.317 \AA$, which is smaller than the ideal lattice constant of $6.481 \AA$ for the CdTe bulk.

\section{Conclusions}

We have made an attempt to identify and understand the efficiency limiting mechanisms that effect the $\mathrm{CdTe} / \mathrm{CdS}$ solar cell performance. We have found that $\mathrm{Cu}$ plays a dual role in CdTe cells and grain boundaries tend to enhance the $\mathrm{Cu}$ diffusion in $\mathrm{CdTe}$ films. 


\section{References}

(1) H.C.Chou, et. al., "An Investigation of Photocurrent Loss Due to Reflectance and Absorption in CdTe/CdS Heterojunction Solar Cells", J.Electron.Mater., 23, p.68 1, 1994.

(2) H.C.Chou, et. al., "Effects of $\mathrm{Cu}$ on $\mathrm{CdTe} / \mathrm{CdS}$ Heterojunction Solar Cells with $\mathrm{Cu} / \mathrm{Au}$ Contacts", J.Electrochem. Soc., 142, p. 254, 1995.

(3) H.C.Chou, et. al., "Approach Toward High Efficiency CdTe/CdS Heterojunction Solar Cells", IURMIS Int. Conference on Electronic Materals, Hsinchu, Taiwan, 1994.

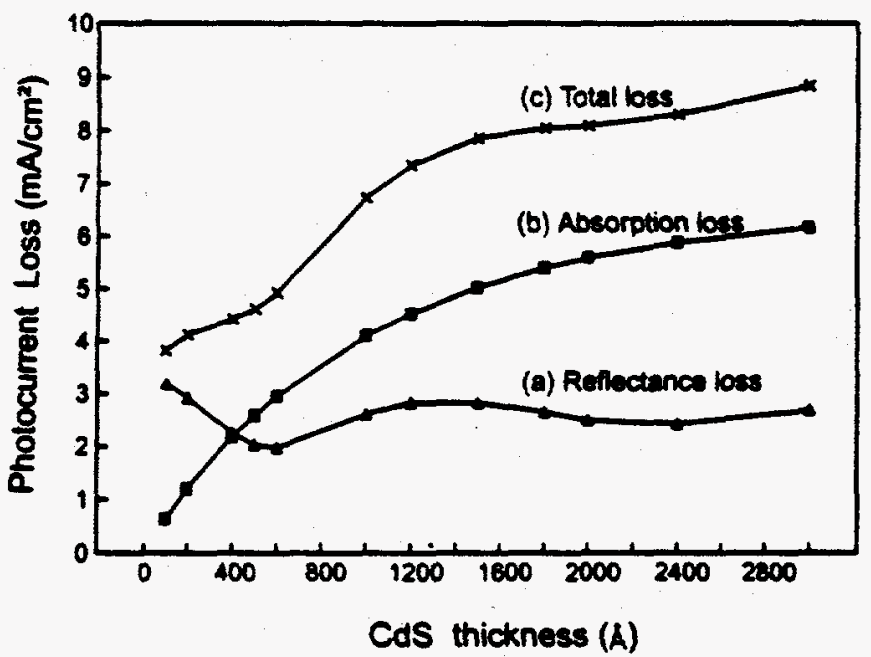

Fig. 1. Photocurrent loss in CoTe solar cells due to the absorption in CdS window layer and the reflection with varying CoS thicknesses.

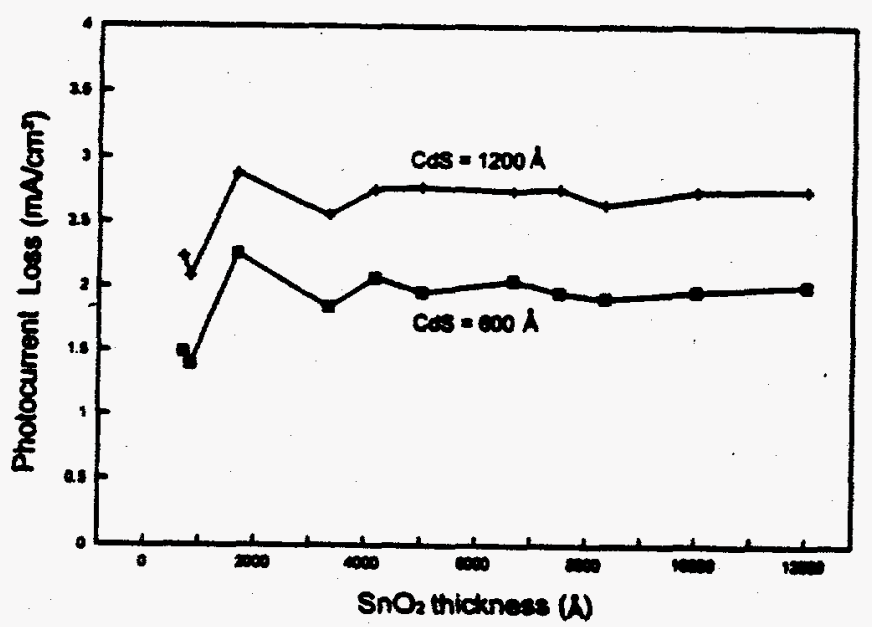

Fig. 3. Photocurrem lose in CdTe solur colls dus to the relluction with varying $\mathrm{SnO}_{2}$ thicknesess.

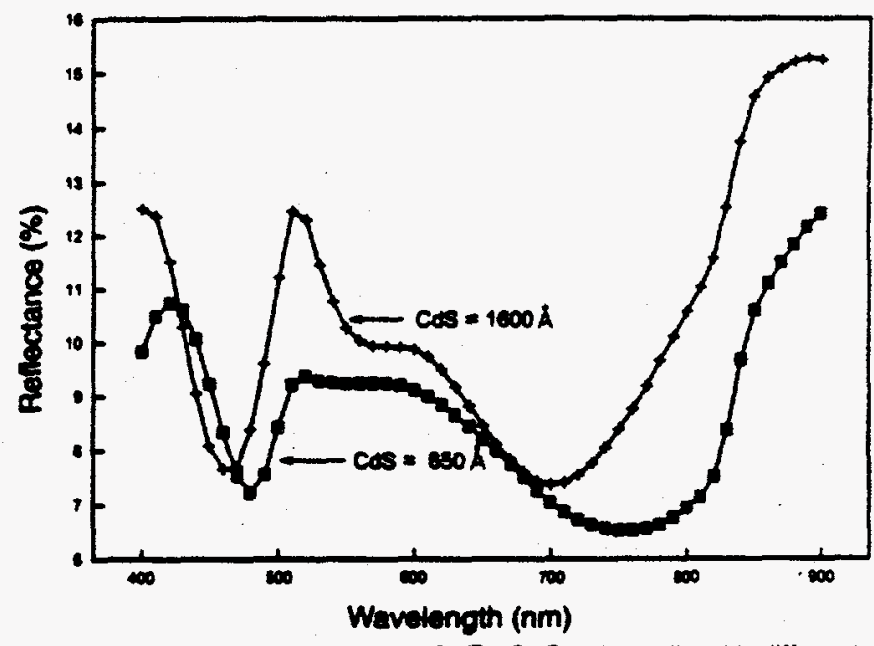

Fig. 2. Measured reflectance for CoTe/CdS solar cells with differint Cos thicknases.

Table I. Solar Cell Data for the CdTe/CdS Cello Grown with Different CdS Thicheasee

\begin{tabular}{|c|c|c|c|c|c|c|}
\hline $\begin{array}{l}\text { CdB } \\
\text { Thich }\end{array}$ & $V_{m}$ & $J_{\infty}$ & R. & $\mathbf{R}_{\mathbf{w}}$ & $\mathbf{F F}$ & EAI \\
\hline $\begin{array}{l}650 \mathrm{~A} \\
1600 \mathrm{~A}\end{array}$ & $\begin{array}{l}0.71 \\
0.74\end{array}$ & $\begin{array}{l}25.01 \\
23.58\end{array}$ & $\begin{array}{l}6.12 \\
4.17\end{array}$ & $\begin{array}{r}556 \\
1108\end{array}$ & $\begin{array}{l}0.55 \\
0.61\end{array}$ & $\begin{array}{r}9.80 \\
10.62\end{array}$ \\
\hline
\end{tabular}

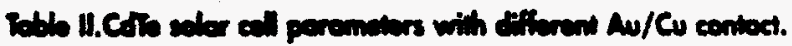

\begin{tabular}{|c|c|c|c|}
\hline & $300 \AA A n$ & $\begin{array}{l}100 \& \mathrm{Au} \\
100 \times \mathrm{Cu}\end{array}$ & $\begin{array}{l}200 \\
300\end{array}$ Au \\
\hline 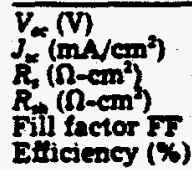 & $\begin{array}{c}0.7075 \\
22.846 \\
20.46 \\
530.5 \\
0.576 \\
9.32\end{array}$ & $\begin{array}{c}0.7091 \\
23.465 \\
2.80 \\
395.06 \\
0.604 \\
10.08\end{array}$ & $\begin{array}{l}\text { All cells } \\
\text { are shorted }\end{array}$ \\
\hline
\end{tabular}




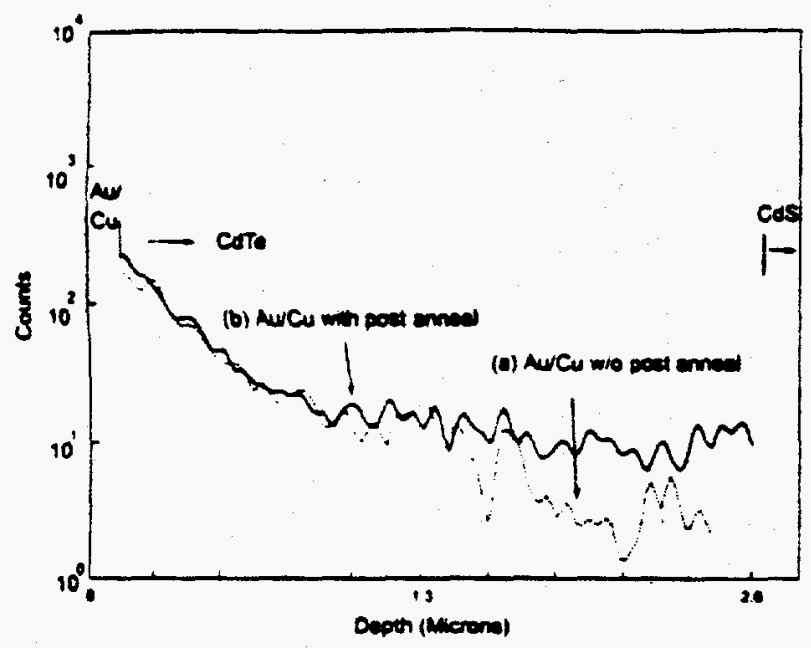

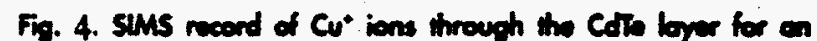

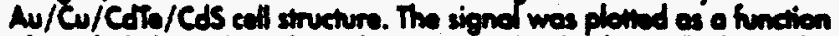
of eredad depth bosed on the measund Cote byor thickness by

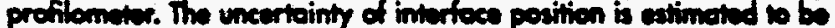
ce. $50 \mathrm{~nm}$ dw to cellisiond etom mixing ond surfece roughnmes.

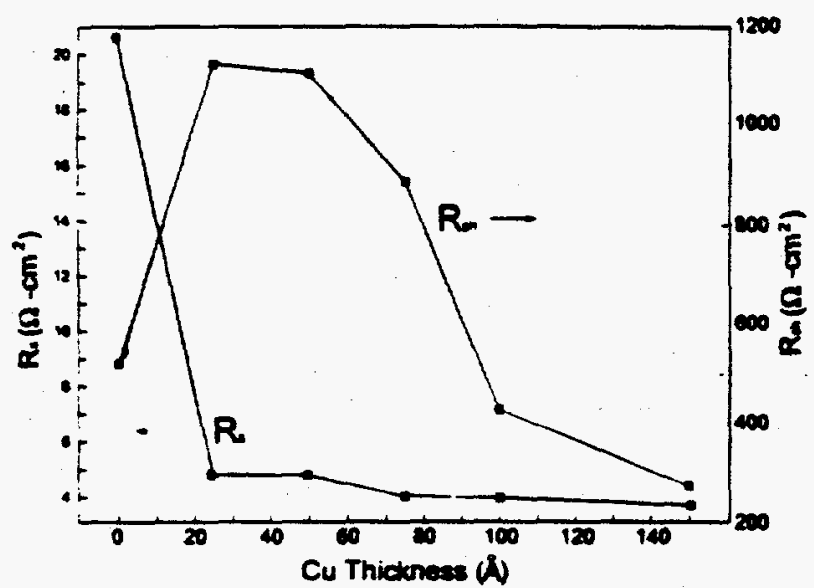

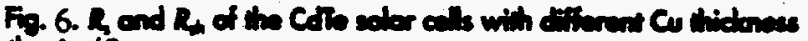
in the Aw/Cu conted.

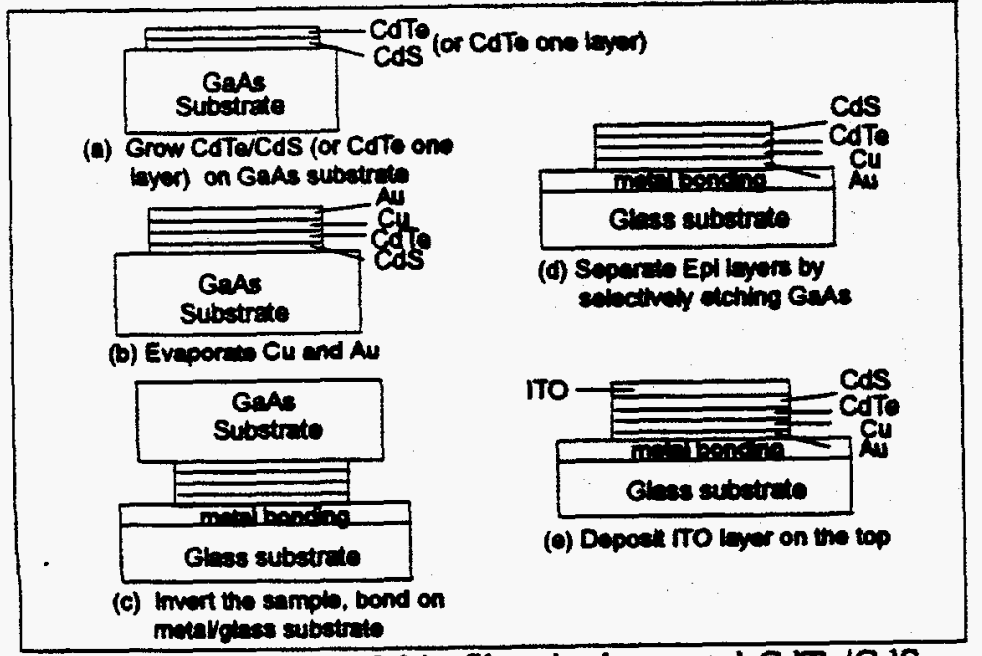

Fig. 8 Separation of thin film single crystal CdTe/CdS structures from the GaAs growth substrate.

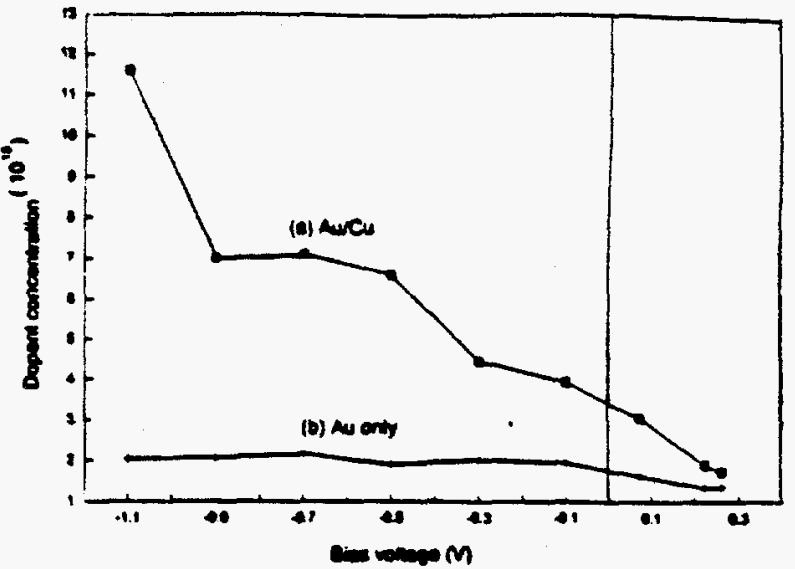

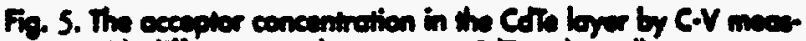
uroment with different medel contect en CdTe soler cells.

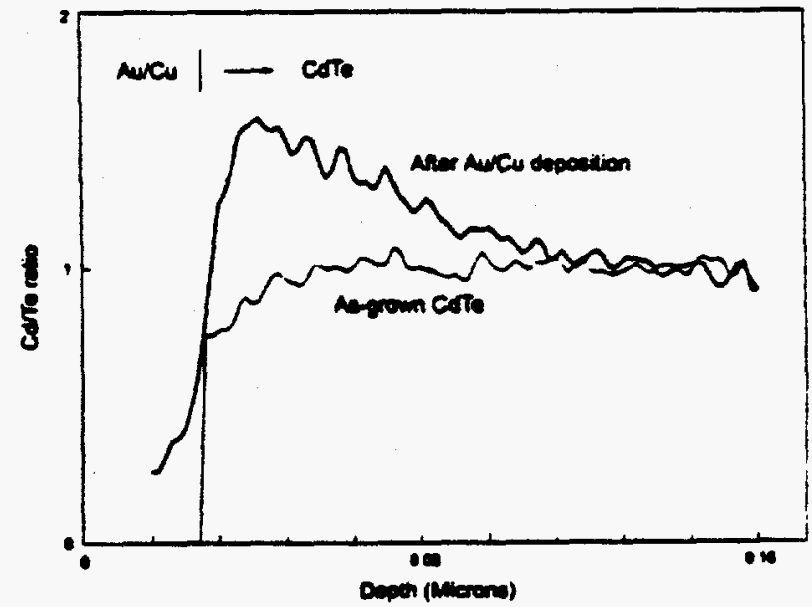

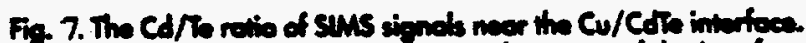
The widith of the Cdrich region is does to the renges of the intertece

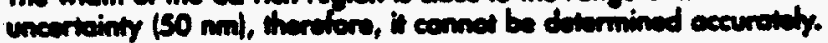

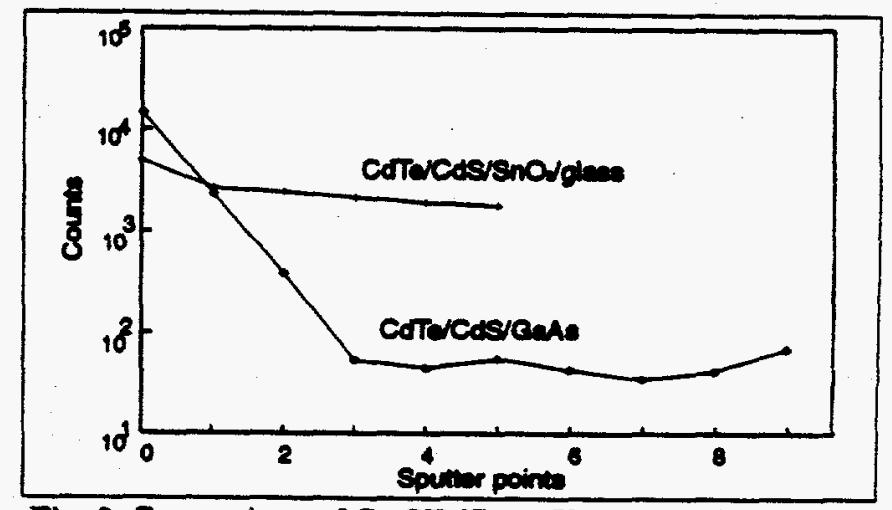

Fig. 9 Comparison of Cu SIMS profiles in the CdTe layer in the ELO single crystal and the conventional polycrystalline solar cells 
Title:

Organization:

Contributors:
Optimization of Transparent and Reflecting Electrodes for Amorphous Silicon Solar Cells

Department of Chemistry, Harvard University, Cambridge, MA.

R. G. Gordon, Principal Investigator;

J. Hu, H. Liang, J. Thornton

\section{Summary of Current Project Objectives}

Transparent conducting materials are essential components of many kinds of solar cells, in which they serve as front-surface electrodes. In tandem cells, back surface electrodes also need to be transparent. Finally, most designs for highly reflective back contacts also call for a transparent conducting layer. The compositions of these transparent conducting layers are usually based on oxides of tin, indium and/or zinc, and are hence referred to as transparent conducting oxides (TCO). In addition to having low electrical resistance and low optical absorption, the structure of a TCO must minimize reflection losses. The TCO must also resist degradation during cell fabrication and use. Finally, the method for making the TCO must be inexpensive and safe.

Our general objectives are to improve the performance of TCO materials and the methods for their production. We aim to reduce their electrical resistance, optical absorption and reflection losses, and to lower the deposition temperature to avoid thermal degradation of other cell components. For the production method, the prime consideration is to deposit the TCO layers at a high rate with relatively simple apparatus. The method chosen is chemical vapor deposition at atmospheric pressure (APCVD), since it has been demonstrated in the glass-coating industry to be the most cost-effective method for making large areas of TCO coatings.

Task 1. Develop improved methods for depositing and using transparent conductors of fluorinedoped zinc oxide in amorphous silicon solar cells

Task 2. Deposit and evaluate tin nitride as a reflection-enhancing diffusion barrier between amorphous silicon and an aluminum or silver back-reflector.

Task 3. Deposit and evaluate tantalum-doped titanium oxide as a transparent conducting layer on which more efficient and more stable cells can be deposited.

About one-third of the current project resources are allocated to each of these three objectives.

An amorphous silicon solar cell incorporating these new materials will have the following structure:

\begin{tabular}{l}
\hline glass \\
\hline $\mathrm{ZnO}: \mathrm{F}$ \\
\hline $\mathrm{TiO}_{2}: \mathrm{Ta}$ or $\mathrm{SnO}_{2}: \mathrm{F}$ \\
\hline $\mathrm{a}-\mathrm{Si}$ \\
\hline $\mathrm{Sn}_{3} \mathrm{~N}_{4}$ \\
\hline $\mathrm{Al}$ or $\mathrm{Ag}$ \\
\hline
\end{tabular}


Task 1. Develop improved methods for depositing and using transparent conductors of fluorine-doped zinc oxide in amorphous silicon solar cells

Why use $\mathrm{ZnO}: \mathrm{F}$ ? It has the lowest absorption loss of any known transparent conductor. $\mathrm{ZnO}$ :F was discovered under our previous NREL contract, and its superior properties documented.

New samples were prepared with several thicknesses of textured $\mathrm{ZnO}: \mathrm{F}$ in order to find the thickness which provides the most efficient cells. Samples were sent to the NREL team members Steven Hegedus at the University of Delaware and Vic Dalal at Iowa State University, who will fabricate amorphous silicon solar cells on these samples.

In discussions with NREL technical personnel and guidance team members, we decided to redirect some of the efforts under this task toward the problem of improving the reproducibility of ZnO:F production, and its use in amorphous silicon solar cells. In particular, pilot production at Solarex had problems reproducing the quality (conductivity and haze level) of $\mathrm{ZnO}: \mathrm{F}$ produced by their Watkins-Johnson belt furnace. Although some samples had outstanding properties, lack of consistent quality hampered optimization of the use of this material.

Our initial analysis of this problem is that the deposition reaction is very sensitive to oxygen and water vapor, both of which are expected to be common contaminants in the atmosphere inside the belt furnace, which ideally should be pure nitrogen. Therefore, a more robust manufacturing process must be based on reactants which are less sensitive to oxygen and water.

One less reactive zinc source is zinc acetylacetonate. It does not react with oxygen or water at room temperature, although it does readily adsorb water of hydration from a moist atmosphere. Thus we began a study of its use as a source for the more reproducible CVD of $\mathrm{ZnO}: \mathrm{F}$. Commercially available zinc acetylacetonate is only partially volatile because it contains water of hydration. We removed the water of hydration by recrystallization from methanol solution, followed by heating. The resulting anhydrous material is easily evaporated from a bubbler containing its melt at about $130^{\circ} \mathrm{C}$.

Zinc acetylacetonate vapor was found to deposit films on substrates heated to temperatures around $500{ }^{\circ} \mathrm{C}$. However, these films were not pure zinc oxide, for they showed strong absorption of visible light. Water vapor was found to react with zinc acetylacetonate vapor at substrate temperatures of about 400 to $450^{\circ} \mathrm{C}$ to deposit highly transparent films of pure zinc oxide. Oxygen was found to react with zinc acetylacetonate vapor at substrate temperatures of about 500 to $550^{\circ} \mathrm{C}$ to deposit highly transparent films of pure zinc oxide.

In the next contract period, we will concentrate our efforts on finding ways to dope these zinc oxide films with fluorine, using either water or oxygen as co-reactants.

Task 2. Deposit and evaluate tin nitride as a reflection-enhancing diffusion barrier between amorphous silicon and an aluminum or silver back-reflector.

A diffusion barrier is needed between the amorphous silicon and the back metal (aluminum or silver) electrode, for the following reasons:

1) The barrier should prevent the diffusion of the metal into the amorphous silicon and the consequent degradation of its electronic properties by the introduction of traps, scattering centers and recombination centers.

2) The barrier should enhance the reflectivity to red light of the metal/silicon interface. To function effectively in this role, the barrier should have a high real part to its refractive index, and a low imaginary part, at optical frequencies corresponding to red light. 
3) The barrier material should have low contact resistances to silicon and to the metal (which requires a suitable work function and a sufficiently high free electron concentration).

4) The barrier material should produce a low recombination velocity at its interface with silicon (which requires a low concentration of recombination centers at the interface).

5) The barrier material must be chemically and mechanically stable, and, in particular, must not react with silicon or the reflective metal.

The most commonly used back diffusion barrier is zinc oxide, which seems to meet these requirements. However, its refractive index is not particularly high (around 2), so the reflectivity enhancement is not as high as it might be with a material of higher refractive index.

\section{Possible improved back diffusion barrier material}

Tin nitride, $\mathrm{Sn}_{3} \mathrm{~N}_{4}$, is a material which we are investigating as a possible improvement over zinc oxide as a back diffusion barrier. The refractive index of tin nitride is very high, over 3 , and therefore its reflectivity enhancement may be greater than from zinc oxide. Tin nitride thin films were successfully prepared in an atmospheric pressure chemical vapor deposition reactor from tetrakis(dimethylamido)tin(IV) and ammonia in the temperature range between $200{ }^{\circ} \mathrm{C}$ and $475^{\circ} \mathrm{C}$. Film thicknesses and refractive indices were determined with an ellipsometer. For thick films, a profilometer was used to measure thickness directly. Forward recoil spectrometry showed that hydrogen concentration in the film decreases with increasing deposition temperature. Rutherford Backscattering spectrometry showed that carbon and oxygen concentrations for films deposited at temperatures above $200^{\circ} \mathrm{C}$ are below the instrument detection limits. Film resistivity and electron concentration were determined with Van der Pauw's methods. Optical measurements showed that film has strong absorption, especially in the short wavelength region. The band gap of tin nitride film was found to be in the range between $2.7 \mathrm{eV}$ and $3.7 \mathrm{eV}$. A damped Lorenz oscillator was used to describe the dispersion of dielectric functions in the wavelength range between $0.5 \mu \mathrm{m}$ and $0.8 \mu \mathrm{m}$. The calculated optical spectra agree well with those obtained from experiments. These optical and electrical properties should provide the basis for simulation to see if tin nitride offers the possibility of improved performance relative to the currently used zinc oxide layers.

Task 3. Deposit and evaluate tantalum-doped titanium oxide as a transparent conducting layer on which more efficient and more stable cells can be deposited.

There are several potential advantages to inserting a transparent, conductive layer of titanium dioxide between the zinc oxide and silicon layers of a solar cell:

1) The titanium dioxide is stable enough so that microcrystalline p-silicon could be deposited directly on it. Cells with this structure are expected to have increased voltage and current.

2) The titanium dioxide layer would reduce the reflection of light, because it has a refractive index (about 2.3) which is intermediate between that of zinc oxide (1.9) and silicon (about 3). This effect should provide increased current.

3) One source of instability in amorphous silicon solar cells is the possible reduction of the transparent metal oxide by the silicon. Such a reduction is possible for tin oxide and zinc oxide, but not for titanium oxide, which should thus provide increased stability.

\section{Thermodynamic stability of the metal oxide to silicon interfaces}

It is possible for silicon to reduce a metal oxide to free metal by reactions such as the following:

$$
\begin{aligned}
& \mathrm{Si}+\mathrm{SnO}_{2} \rightarrow \mathrm{SiO}_{2}+\mathrm{Sn} \\
& \mathrm{Si}+2 \mathrm{ZnO} \rightarrow \mathrm{SiO}_{2}+2 \mathrm{Zn} \\
& \mathrm{Si}+\mathrm{TiO}_{2} \rightarrow \mathrm{SiO}_{2}+\mathrm{Ti}
\end{aligned}
$$

$$
\begin{aligned}
\Delta \mathrm{G} & =-340.5 \mathrm{~kJ} / \mathrm{mol} \\
\Delta \mathrm{G} & =-215.0 \mathrm{~kJ} / \mathrm{mol} \\
\Delta \mathrm{G} & =+32.5 \mathrm{~kJ} / \mathrm{mol}
\end{aligned}
$$


The free metal may then diffuse into the amorphous silicon and degrade its semiconductor properties. The silicon dioxide thus created at the interface may also increase the electrical resistance losses of the solar cell. Both of these effects would lower its efficiency. The calculated free energy changes, $(\Delta G)$, shown for each of these possible reactions, are negative for tin oxide and zinc oxide, showing that such reductions can happen spontaneously. On the other hand, the free energy change for titanium dioxide is positive, which shows that free titanium metal cannot be formed spontaneously at the interface. Instead, titanium silicide may form spontaneously by the reaction

$$
3 \mathrm{Si}+\mathrm{TiO}_{2}-\mathrm{SiO}_{2}+\mathrm{TiSi}_{2} \quad \Delta \mathrm{G}<0
$$

Any titanium silicide thus formed is very stable, and does not permit free titanium to diffuse into the silicon. Thus amorphous silicon solar cells with titanium dioxide transparent conductors should be more stable than those formed on tin oxide or zinc oxide. Additional evidence for the extraordinary stability of titanium dioxide, comes from our studies of CVD of amorphous silicon from disilane at $500^{\circ} \mathrm{C}$. When either tin oxide or zinc oxide films were tried as substrates, these deposition conditions completely destroyed the oxide film, whereas titanium dioxide substrates were completely stable to these extreme conditions.

\section{Formation of TiO2:Ta Films}

Atmospheric pressure chemical vapor deposition was used to deposit films from vapors of titanium isopropoxide and tantalum ethoxide, along with 2-cyclohexen-1-one. These reactants were evaporated from heated bubblers, through which nitrogen carrier gas flowed. Because of the high boiling points of the precursors, special valves were used, which could operate at temperatures up to $200^{\circ} \mathrm{C}$. The vapor mixtures were combined in a Watkins-Johnson gas dispersion nozzle $10 \mathrm{~cm}$ wide. The substrates were carried on a heated plate in a BTU Engineering furnace. The reaction proceeds rapidly (about 20 Angstroms per second) at substrate temperatures of about $480{ }^{\circ} \mathrm{C}$.

The presence of 2-cyclohexen-1-one as a reactant has two important effects on the CVD process: 1) In the absence of cyclohexenone, CVD using titanium isopropoxide as a reactant produces titanium dioxide films, but their nucleation is not reproducible or uniform over the substrate surface. When the cyclohexenone is added, then the titanium dioxide films nucleate uniformly over the substrate surface.

2) In the presence of cyclohexenone, the electrical resistances of tantalum-doped titanium dioxide films are reduced by more than two orders of magnitude.

\section{Properties of TiO2:Ta Films}

The doping level was about 5\% tantalum (compared to titanium). Sheet resistances of two hundred ohms per square were found for films about a half micron thick, corresponding to an effective "bulk" resistivity of $0.01 \mathrm{ohm}-\mathrm{cm}$. At thicknesses corresponding to the antireflection thickness, the films on glass are clear in transmission and have a specular, silvery reflection. In order to test how thick a titanium dioxide layer might be needed for protection, films of various thicknesses of undoped titanium oxide were deposited on top of zinc oxide films. A quick test for complete coverage was to dip the glass $/ \mathrm{ZnO} / \mathrm{TiO}_{2}$ into hydrochloric acid, which rapidly etches zinc oxide, but does not dissolve titanium oxide. We found that layers of titanium oxide about 70 nanometers thick protected the zinc oxide from dissolution, while thinner titanium oxide layers allowed at least portions of the zinc oxide to dissolve. This thickness also corresponds to the antireflection condition for titanium oxide films. Thus we will use titanium oxide layers about 70 nanometers thick to protect the zinc oxide from hydrogen plasma attack during formation of microcrystalline players. 
Title:

Organization:

Contributors:
Processing and Modeling Issues for Thin Film Solar Cell Devices

Institute of Energy Conversion

University of Delaware

United States Department of Energy

University Center of Excellence for

Photovoltaic Research and Education

Newark, DE 19716-3820

R.W. Birkmire, J.E. Phillips, W.A. Buchanan,

S.S. Hegedus, H. Hichri, R. Klenk, B.E.

McCandless, P.V. Meyers, N. Orbey, Y. Qu,

W.N. Shafarman

\section{Objectives/Technical Highlights}

The overall mission of the Institute of Energy Conversion (IEC) is the development of thin film photovoltaic cells, modules and related manufacturing technology and the education of students and professionals in photovoltaic technology. The objectives of this four year NREL subcontract are to advance the state-of-the-art and acceptance of thin film PV modules in the areas of improved technology for thin film deposition, device fabrication and material and device characterization and modeling relating to solar cells based on $\mathrm{CdTe}$, on $\mathrm{CuInSe}_{2}$ and its alloys, and on a-Si and its alloys. Accomplishments during this second phase of a four year contract include: development of equations and reaction rates for the formation of $\mathrm{CuIn}_{1-\mathrm{x}} \mathrm{Ga}_{\mathrm{X}} \mathrm{Se}_{2}$ films by selenization; fabrication of a $15 \%$ efficient $\mathrm{CuIn}_{1-\mathrm{x}} \mathrm{Ga}_{\mathrm{x}} \mathrm{Se}_{2}$ cell; development of a reproducible, reliable, $\mathrm{Cu}$-diffused contact applicable to many CdTe technologies; demonstration of improved plasma CVD process control for a-Si devices; development of analytical and modeling procedures adapted to devices produced with each material system; and significant collaboration with many industrial and university organizations.

\section{Results:}

\section{CuInSe 2 Reaction Chemistry}

Understanding the mechanism of the growth of $\mathrm{CuInSe}_{2}$ by obtaining the pertinent kinetic data is essential for the effective design and real time control of processing equipment to produce $\mathrm{CuInSe}_{2}$ modules. Species concentrations as a function of time and temperature were obtained at IEC from experiments in a tubular chemical vapor deposition (CVD) selenization reactor and a physical vapor deposition (PVD) selenization reactor.

Using the time progressive concentration profiles obtained in the tubular CVD reactor at $400^{\circ} \mathrm{C} \mathrm{a}$ set of chemical equations was proposed and the specific rate constants for these chemical equations at $400^{\circ} \mathrm{C}$ were obtained by solving the species conservation equations. By using the species concentrations measured at the end of the experimental runs in the PVD reactor, the specific rate constants at $250^{\circ} \mathrm{C}$ and $300^{\circ} \mathrm{C}$ were estimated. The activation energies $\mathrm{E}_{\mathrm{i}}$ and the frequency factors $\mathrm{k}_{\mathrm{oi}}$ for the proposed reactions were estimated from the rate constants at $250^{\circ} \mathrm{C}, 300^{\circ} \mathrm{C}$ and $400^{\circ} \mathrm{C}$ using the Arrhenius relation.

A chemical reaction analysis was carried out for both reactors (1). From the time progressive concentration profiles in the tubular CVD reactor at $400^{\circ} \mathrm{C}$, we obtained a simple set of chemical equations and the specific rate constants (2). From the runs in the PVD reactor at 250 and $300^{\circ} \mathrm{C}$, calculated concentrations were in good agreement with data for $\mathrm{Cu} / \mathrm{In}$ ratios of 0.8 and 0.9 (1). Thus, we have combined results from these two different deposition methods to obtain a better 
analysis of the reaction pathways, activation energies and rate constants pertinent to formation of CuInSe 2 films.

\section{CuIn ${ }_{1-x} G a_{x}$ Se Selenization}

The $\mathrm{CuIn}_{1-\mathrm{x}} \mathrm{Ga}_{\mathrm{x}} \mathrm{Se}_{2}$ films used for all high efficiency $(>15 \%)$ solar cells have been typically deposited by PVD. Most approaches to fabricate modules based on CuInSe 2 and its alloys, however, react metal precursor layers in a Se atmosphere. IEC investigated the processing and characterization of the stacked $\mathrm{Cu}-\mathrm{In}-\mathrm{Ga}$ layers and the resulting films obtained by reacting the precursors with $\mathrm{H}_{2} \mathrm{Se}(3)$ or elemental Se at $400^{\circ} \mathrm{C}$ and $500^{\circ} \mathrm{C}(4)$. The precursors were processed in sequences $\mathrm{Cu} / \mathrm{In} / \mathrm{Ga}$ and $\mathrm{Cu} / \mathrm{Ga} / \mathrm{In}$ for $\mathrm{Ga} /(\mathrm{Ga}+\mathrm{In})$ ratio ranging from 0.2 to 1.0. The precursors, before and after a 1 hour heat treatment in an Ar atmosphere, were found to contain only elemental and binary phases. After reaction in a Se atmosphere, the precursors contained mixtures of binary phases $\mathrm{Cu}_{2-x} \mathrm{Se}, \mathrm{GaSe}, \mathrm{Ga}_{2} \mathrm{Se}_{3}$ and $\mathrm{Cu}-\mathrm{Ga}$, and, ternary phases $\mathrm{CuInSe}_{2}$ and $\mathrm{CuGaSe}_{2}$. The reacted precursors were heat treated in an $\mathrm{Ar}$ atmosphere and an $\mathrm{H}_{2} \mathrm{Se}$ atmosphere. The heat treatment in an Ar atmosphere resulted in a single phase $\mathrm{CuIn}_{1-\mathrm{x}} \mathrm{Ga}_{\mathrm{x}} \mathrm{Se}_{2}$ film, whereas heat treatment in $\mathrm{H}_{2} \mathrm{Se}$ atmosphere improved only the crystallinity of the phases present and does not effect homogenization.

\section{High Efficiency $\mathrm{CuIn} 1-x \mathrm{Ga}_{\underline{x}} \underline{S e}_{2}$ Device Performance}

A $0.42 \mathrm{~cm}^{2}$ solar cell fabricated with $\mathrm{CuIn}_{1-\mathrm{x}} \mathrm{Ga}_{\mathrm{x}} \mathrm{Se}_{2}$ deposited by multisource evaporation achieved $14.9 \%$ efficiency in testing at NREL with $\mathrm{V}_{\mathrm{OC}}=0.62 \mathrm{~V}, \mathrm{~J}_{\mathrm{Sc}}=32.8 \mathrm{~mA} / \mathrm{cm}^{2}$, and FF = 73.1\%. The configuration of this cell was: soda lime glass / Mo ( $1 \mu \mathrm{m}$ thick) $/ \mathrm{CuIn} \mathrm{C}_{1-\mathrm{x}} \mathrm{Ga}_{\mathrm{x}} \mathrm{Se}_{2}$ $(2.5 \mu \mathrm{m}, \mathrm{x}=0.29) / \mathrm{CdS}(50 \mathrm{~nm}) / \mathrm{ZnO}: \mathrm{Al}(0.5 \mu \mathrm{m}) / \mathrm{Ni}-\mathrm{Al}$ grid / $\mathrm{MgF}_{2}(130 \mathrm{~nm})$. The CdS was deposited by CBD. The CuIn $1-x \mathrm{Ga}_{x} \mathrm{Se}_{2}$ was a bi-layer evaporation: first layer $=\mathrm{Cu}$-rich $\mathrm{CuIn}_{1-\mathrm{x}} \mathrm{Ga}_{\mathrm{x}} \mathrm{Se}_{2}$ deposited at $\mathrm{T}_{\mathrm{ss}}=450^{\circ} \mathrm{C}$; second layer $=\mathrm{In} / \mathrm{Ga} / \mathrm{Se}$ deposited at $\mathrm{T}_{\mathrm{ss}}=600^{\circ} \mathrm{C}$

\section{CdTe Devices}

A new method was developed for forming low resistance contacts to the CdTe surface of $\mathrm{CdTe} / \mathrm{CdS}$ thin-film solar cells (5). It produces fill factors that are equivalent or superior to those obtained with other contacting methods. The process has been demonstrated on CdTe/CdS devices deposited by different methods and is compatible with module fabrication technologies. After $\mathrm{CdCl}_{2}$ processing, the CdTe surface is coated with a thin layer of copper which is heated and then reacted. Next the structure is immersed in a bromine methanol solution to remove excess $\mathrm{Cu}$ and produce a Te-rich CdTe surface. The heat treatment diffuses $\mathrm{Cu}$ into $\mathrm{CdTe}$, doping it p-type while the etching produces a conductive surface which is easily contacted using different materials such as $\mathrm{Ni}, \mathrm{Cr}, \mathrm{Pt}, \mathrm{Mo}$, carbon paste, and ITO. The general applicability of the contacting process to devices was tested using $\mathrm{CdTe} / \mathrm{CdS}$ films fabricated at other laboratories (5). Current-voltage (JV) parameters obtained applying the diffused $\mathrm{Cu}$ contact process are shown in Table 1.

We have commonly observed a loss in $V_{o c}$ with decreasing thickness of evaporated CdS $(<120$ $\mathrm{nm}$ ). Chemical bath deposited (CBD) CdS films were found to have a significantly lower pinhole density than evaporated CdS films but are harder to control chemically. Various heat treatments were performed on CBD CdS films in an effort to improve the optical and structural properties. Treatments in air, hydrogen, argon, $\mathrm{O}_{2}$ in $\mathrm{Ar}$, and $\mathrm{H}_{2}$ in $\mathrm{Ar}$ at temperatures between 415 and $550^{\circ} \mathrm{C}$ all degraded the optical transmission. It was found, however, that treatments in $20 \% \mathrm{O}_{2}$ in $\mathrm{Ar}$ in the presence of $\mathrm{CdCl}_{2}$ (IEC's "standard $\mathrm{CdCl}_{2}$ pre-treatment" used on evaporated $\mathrm{CdS}$ films(6)) sharpened the band edge making the optical quality of the films comparable to $\mathrm{CdCl}_{2}$ treated evaporated CdS. The same qualitative results were found regardless of whether the solution used to grow the film was based on a chloride, sulfate, or acetate salt. 
Table 1. J-V parameters of $\mathrm{CdTe} / \mathrm{CdS}$ devices made with the diffused $\mathrm{Cu}$ contact process using the conductors and CdTe deposition techniques shown.

\begin{tabular}{|c|c|c|c|c|c|c|}
\hline \multicolumn{2}{|c|}{$\begin{array}{l}\text { CdTe Sample } \\
\text { Method (Group) }\end{array}$} & Conductor & $\begin{array}{l}\mathrm{V}_{\mathrm{oc}} \\
(\mathrm{mV})\end{array}$ & $\begin{array}{l}\mathrm{J}_{\mathrm{sc}} \\
(\mathrm{mA} / \mathrm{cm})\end{array}$ & & $\begin{array}{c}\text { Eff } \\
(\%)\end{array}$ \\
\hline \multirow[t]{2}{*}{ PVD (IEC) } & 40821.11 & $\mathrm{Cr}$ & 811 & 20.7 & 66 & 11.1 \\
\hline & 40826.32 & $\mathrm{C}$ & 830 & 19.4 & 73 & 11. \\
\hline \multirow[t]{2}{*}{ EVD (SCI) } & $10304 \mathrm{D} 2.2$ & $\mathrm{Ni}$ & 804 & 18.0 & 77 & 11.0 \\
\hline & $10304 \mathrm{D} 2.3$ & $\mathrm{C}$ & 82 & 18.1 & 74 & 11. \\
\hline \multirow[t]{2}{*}{ CSS (USF) } & 1075 & $\mathrm{Ni}$ & 792 & 22.4 & 73 & 12.9 \\
\hline & 1075 & $\mathrm{C}$ & 864 & 22.4 & 73 & 14. \\
\hline CVD (GIT) & GT1 & C & 757 & 20.6 & 61 & 9.5 \\
\hline
\end{tabular}

Investigations of alternative halide treatments to replace the liquid $\mathrm{CdCl}_{2}$ treatment were continued and focused on post deposition heat treatments in $\mathrm{CdCl}_{2}, \mathrm{Cl}_{2}$ and $\mathrm{HCl}$ vapors. The $\mathrm{CdCl}_{2}$ vapor was found to be very effective at promoting grain growth, left no detectable surface residue on the $\mathrm{CdTe}$, and produced devices with uniform performance at the $10 \%$ efficiency level. Treatment in chlorine was destructive to the CdTe film integrity. Promising structural modifications and device results were obtained with $\mathrm{HCl}$ treatments. It is believed that the experience and results obtained investigating vapor treatments will lead to a CdTe deposition technology in which the halide treatment can be conducted in situ (during the CdTe deposition) thereby reducing the number of processing steps required.

\section{a-Si Devices}

Our goals are to investigate the effect of processing on device performance and to support the NREL Multijunction Device Design and Interface Team. We established reproducibility and repeatability following major changes to the internal reactor geometry and configuration, leading to devices deposited from 10 a-Si runs with efficiencies between 7.8 and $8.1 \%$ (Ti/Ag contacts). This year our main effort in support of the a-Si Research Teams was to investigate devices with different front and back TCO contacts for improved optical enhancement. We deposited devices on textured TCO from several groups, including $\mathrm{SnO}_{2}$ and $\mathrm{ZnO}$ layers as well as $\mathrm{ZnO} / \mathrm{SnO}_{2}$ and $\mathrm{SnO}_{2} / \mathrm{ZnO}$ bilayers. Analysis of $\mathrm{ZnO}$ and $\mathrm{SnO}_{2}$ absorption indicates a gain of $1 \mathrm{~mA} / \mathrm{cm}^{2}$ will occur for a triple junction device with $\mathrm{ZnO}$ compared to $\mathrm{SnO}_{2}$. Regarding the $\mathrm{ZnO} / \mathrm{Ag}$ back reflector contact, the improved $\mathrm{J}_{\mathrm{sc}}$ due to enhanced red response was found to be independent of $\mathrm{ZnO}$ thickness, suggesting that the main effect of the $\mathrm{ZnO}$ is to prevent interaction between the $\mathrm{Ag}$ and a-Si leading to parasitic absorption losses at the back contact. IEC also provided materials, devices, special test structures, and analysis to other Team members (APS, Solarex, NREL, Harvard, Penn State).

\section{a-Si and Polycrystalline Device Anaiysis}

A procedure has been developed at IEC (7) for accurately representing the J-V characteristic of a-Si solar cells. The measured J-V data is separated into the forward diode current and photocurrent. The forward diode current is characterized to obtain standard diode parameters A, $\mathrm{J}_{0}$, and R. The AM1.5 photocurrent is characterized using the uniform field approximation to determine the ratio of collection length to thickness $L_{C} D$, flat band potential $V_{B}$, and total photocurrent $J_{L}$. Very good agreement is found between measured and calculated currents from reverse bias to $V_{o c}$ in a variety of cells (7). We find that degradation of FF under light soaking from $69 \%$ to $49 \%$ can be explained largely by a decrease in a single parameter $L_{c} / D$, from 14 to 3 . Future plans include applying this 
technique to the JV data of component cells of multijunctions tested under appropriate illumination, then comparing the predicted multijunction performance to actual results.

Solar cells based on CdTe, CuInSe 2 or the alloys of $\mathrm{Cu}(\mathrm{In}, \mathrm{Ga}) \mathrm{Se}_{2}$ have grain sizes on the order of one micron. Materials with these size grains could have an exponential distribution of localized states within the bandgap similar to amorphous materials such as a-Si:H. The current voltage characteristics of $\mathrm{CdTe}, \mathrm{CuInSe} 2$ and $\mathrm{Cu}(\mathrm{In}, \mathrm{Ga}) \mathrm{Se}_{2}$ based solar cells made by various processes were analyzed for evidence of this amorphous like behavior (8). Most high efficiency polycrystalline thin film solar cells such as $\mathrm{CdTe}, \mathrm{CuInSe} 2$ and its alloys operate with the diode current being controlled by recombination within the active light absorbing material. If the recombination is assumed (8) to occur through an exponential distribution of recombination centers localized near midgap with a characteristic energy of the distribution equal to $\mathrm{kT}^{*}$, the diode or recombination current can be expressed in closed form as:

$$
\mathrm{J}_{\mathrm{d}}=\mathrm{J}_{\mathrm{rec}}=\mathrm{J}_{0} \cdot \exp (\mathrm{qV} / \mathrm{AkT}) \text { for } \mathrm{T} \gg \mathrm{T}^{*}
$$

where the diode quality factor $(\mathrm{A})$ becomes slightly temperature dependent and not necessarily equal to one or two, i.e. :

$$
\mathrm{A}=2 /\left(1+\mathrm{T} / \mathrm{T}^{*}\right) \quad 1<\mathrm{A}<2 .
$$

To determine whether this type of model can account for the observed $\mathrm{J}-\mathrm{V}$ behavior in polycrystalline thin film solar cells, 14 different devices made by various processes and manufacturers were examined (8). J-V data was measured over a range of temperatures and light intensities. Values of $\mathrm{T}^{*}$ ranged from 800 to $4000 \mathrm{~K}$ corresponding to A factor values at $300 \mathrm{~K}$ from 1.5 to 1.9. These results suggest that the diode characteristic of CuInSe 2 and $\mathrm{CdTe}$ based materials can be described by recombination through a continuous exponential-like distribution of centers within the bandgap of the light absorbing material. This behavior is similar to that of photovoltaic devices made from amorphous semiconductors suggesting the crystalline order in these polycrystalline thin films is part way between that of crystalline and amorphous material.

\section{Collaborations}

During this year, IEC had substantive collaboration with the following organizations: Advanced Photovoltaic Systems, A.D Plating, International Solar Electric Technology (ISET), Martin Marietta, Solar Cells Inc., Solarex Thin Film, National Renewable Energy Laboratory (NREL), Harvard University, University of South Florida, University of Illinois, Penn State University, Georgia Institute of Technology, and Florida Solar Energy Center.

\section{References}

1. TWF Russell, N. Orbey, R. W.Birkmire, 1st WCPEC, 1994 (to be published).

2. TWF Russell, S. Verma, R. W. Birkmire, 12th Euro. PVSEC, 1994, 633.

3. S. Verma, TWF Russell and R.W. Birkmire , Proc. of the 23rd IEEE PVSC, 1993, p. 431.

4. M. Marudachalam, TWF Russell, R.W.Birkmire, 1st WCPEC, 1994 (to be published)

5. B. E. McCandless, et al., 1st WCPVEC, 1994 (to be published).

6. R. W. Birkmire, B. E. McCandless, S.S. Hegedus, Int. J. Solar Energy, 1992, p. 145.

7. S.S. Hegedus, J.E. Phillips 1st WCPEC, 1994 (to be published).

8. J.E. Phillips, W. N. Shafarman, E. Shan, 1st WCPEC, 1994 (to be published). 
Title: $\quad$ Novel Two-Stage Selenization Methods for Fabrication of Thin Film CIS Cells and Submodules

Organization: International Solar Electric Technology (ISET), Inglewood, California

Contributors: V. K. Kapur, program manager; B. M. Basol, principal investigator; A. Halani, C. R. Leidholm, and A. J. Minnick

The objective of this project is the development of CuInSe ${ }_{2}$ (CIS) submodules of $1 \mathrm{ft}^{2}$ area using selenization techniques. In our FY 93 report and previous publications we presented results on the fabrication of $7 \%$ efficient $1 \mathrm{ft}^{2}$ size modules and a newly developed non-vacuum technique for CIS film formation $[1,2]$. During this research period our efforts concentrated on the factors that affect process yield.

\section{Substrate effects}

Nature of the glass/Mo substrates used in CIS solar cell structures influences the performance of the devices [3]. To study such effects, various glass samples, glass/Mo structures and glass $/ \mathrm{Mo} / \mathrm{Cu}$-In stacks were selenized in $\mathrm{H}_{2} \mathrm{Se}$ atmosphere at around $400{ }^{\circ} \mathrm{C}$. Auger depth profiling was used to determine the degree of selenization near the surface regions of the Mo layers deposited on different types of glass substrates. XPS measurenients were employed to investigate the reaction products on the Mo surfaces. Solar cell performance was correlated with the type of substrate used.

After the selenization step a visual inspection of the various glass samples indicated that the sodalime glass surface was covered with a thin layer of reaction products. The surfaces of all the other glasses including the soda-lime sheet with the $\mathrm{SiO}_{2}$ barrier layer were totally clear of any residues or reaction products. The explanation for the observed phenomenon lies in the reaction of sodium on the surface of the soda-lime glass substrate with the $\mathrm{H}_{2} \mathrm{Se}$ atmosphere at $400{ }^{\circ} \mathrm{C}$ in accordance with the favorable reaction of

$\mathrm{Na}_{2} \mathrm{O}(\mathrm{s})+\mathrm{H}_{2} \mathrm{Se}(\mathrm{g})-\mathrm{Na}_{2} \mathrm{Se}(\mathrm{s})+\mathrm{H}_{2} \mathrm{O}(\mathrm{g})$

The structural and electrical properties of sputtered Mo layers play an important role in determining certain characteristics of the glass/Mo substrates and solar cells fabricated on them. Adhesion of the Mo film to the glass surface and the sheet resistance of the solar cell back contact are two factors that need to be taken into consideration. However, the properties of the Mo layer measured right after deposition may not necessarily be the same as they are in the finished device structure because of the chemical interaction of Mo with the CIS growth environment.

The Auger surveys and depth profiles obtained from two different Mo layers selenized in $\mathrm{H}_{2} \mathrm{Se}$ are given in Fig.1. These films were both deposited on soda-lime glass substrates. Data taken from 
sample Mo-60 display about four times the $\mathrm{Na}$ signal observed at the surface of Mo-127. There is also a larger $\mathrm{O}$ signal. Auger depth profiles of Fig. 1 indicate that $\mathrm{Mo}-127$ has a thin layer of selenide (about $1500 \AA$ ) on its surface, whereas, the selenized surface of Mo-60 is about five times thicker. Two point resistance measurements made on the two selenized films showed resistance values of 2-10 K-ohms for Mo-60 and 10-300 ohms for Mo-127.

The XPS survey spectra of the two Mo films are shown in Fig. 2a. This-data indicates about 5 times more $\mathrm{Na}$ on the surface of Mo-60 compared to Mo-127. The Mo 3d spectra of the two samples (Fig. 2b) shows the double peaks associated with MoSe. But in the spectra of Mo-60 there is clearly a third peak shifted by $3.8 \mathrm{eV}$ that can be attributed to $\mathrm{Na}_{2} \mathrm{MoO}_{4}$. The Se $3 \mathrm{~d}$ spectra of Fig. 2c display the expected $\mathrm{MoSe}_{2}$ peak for both samples. The data of Sample Mo-60, however, has additional peaks shifted by $4.6 \mathrm{eV}\left(\mathrm{Na}_{2} \mathrm{SeO}_{3}\right.$ and/or $\left.\mathrm{SeO}_{2}\right)$ and $8.9 \mathrm{eV}$ (possibly higher order Se oxides).
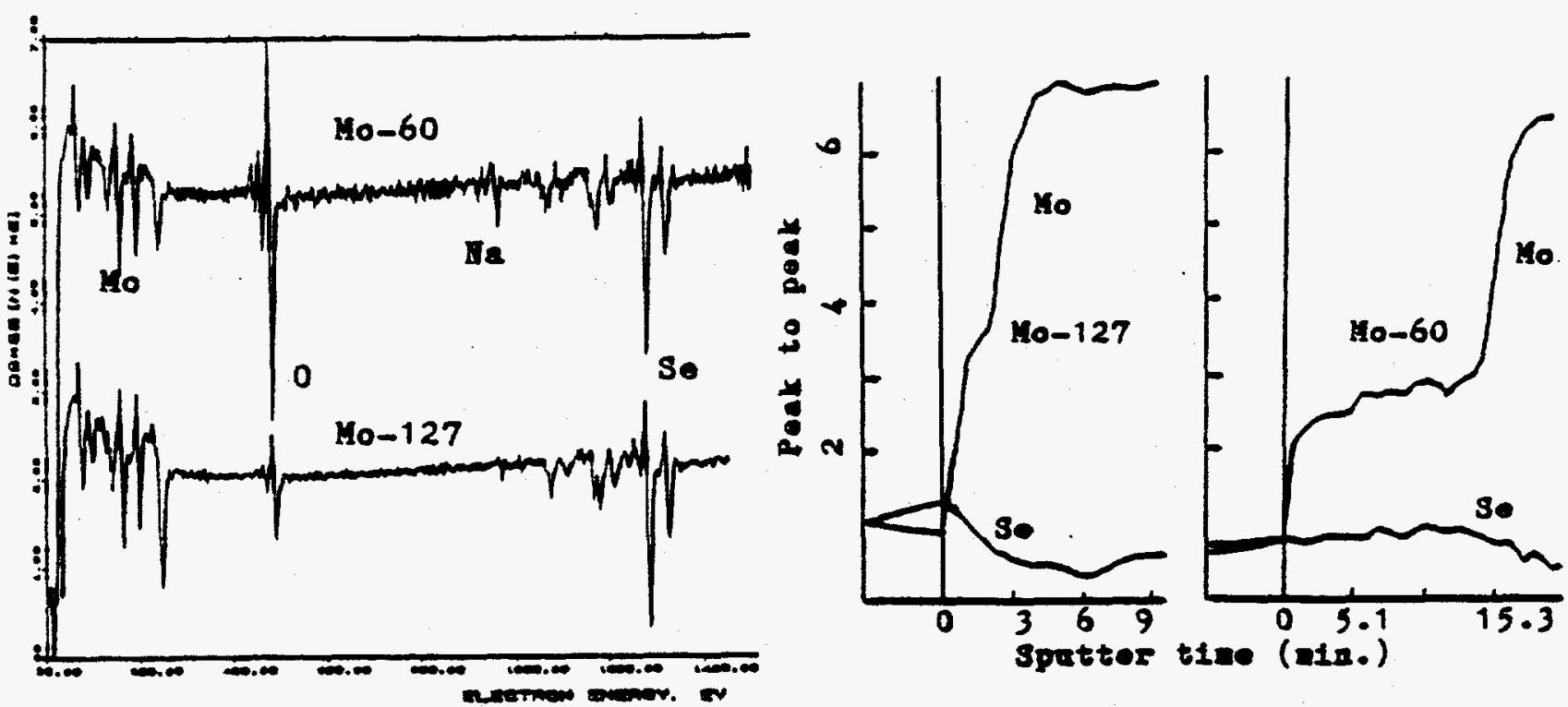

Fig. 1 Auger survey spectra and depth profiles of the Mo-60 and Mo-127 samples.

All the results discussed above point to the fact that two different Mo layers on the same sodalime glass substrate may selenize differently. Excessive reaction yields a dull surface layer with high resistivity which is associated with the appearance of a strong $\mathrm{Na}$ signal as observed from the Auger as well as XPS data. Therefore, reactivity of the Mo surface is related to Na diffusion from the glass substrate to the Mo surface region. The degree of $\mathrm{Na}$ diffusion, on the other hand, depends on the nature of the Mo film itself.

To further correlate the surface reactivity of Mo film with Na diffusion, the selenization experiments were repeated on glass/Mo samples using glass sheets of various compositions. Mo films equivalent to sample Mo-60 did not show excessive reaction with the selenizing atmosphere when they were deposited on Corning 7044 and Corning 7059 glass substrates. Similarty, excessive reaction was not observed for Mo layers deposited on soda lime glass substrates with a surface barrier layer of $3000 \mathrm{~A}$ thick $\mathrm{SiO}_{2}$. 

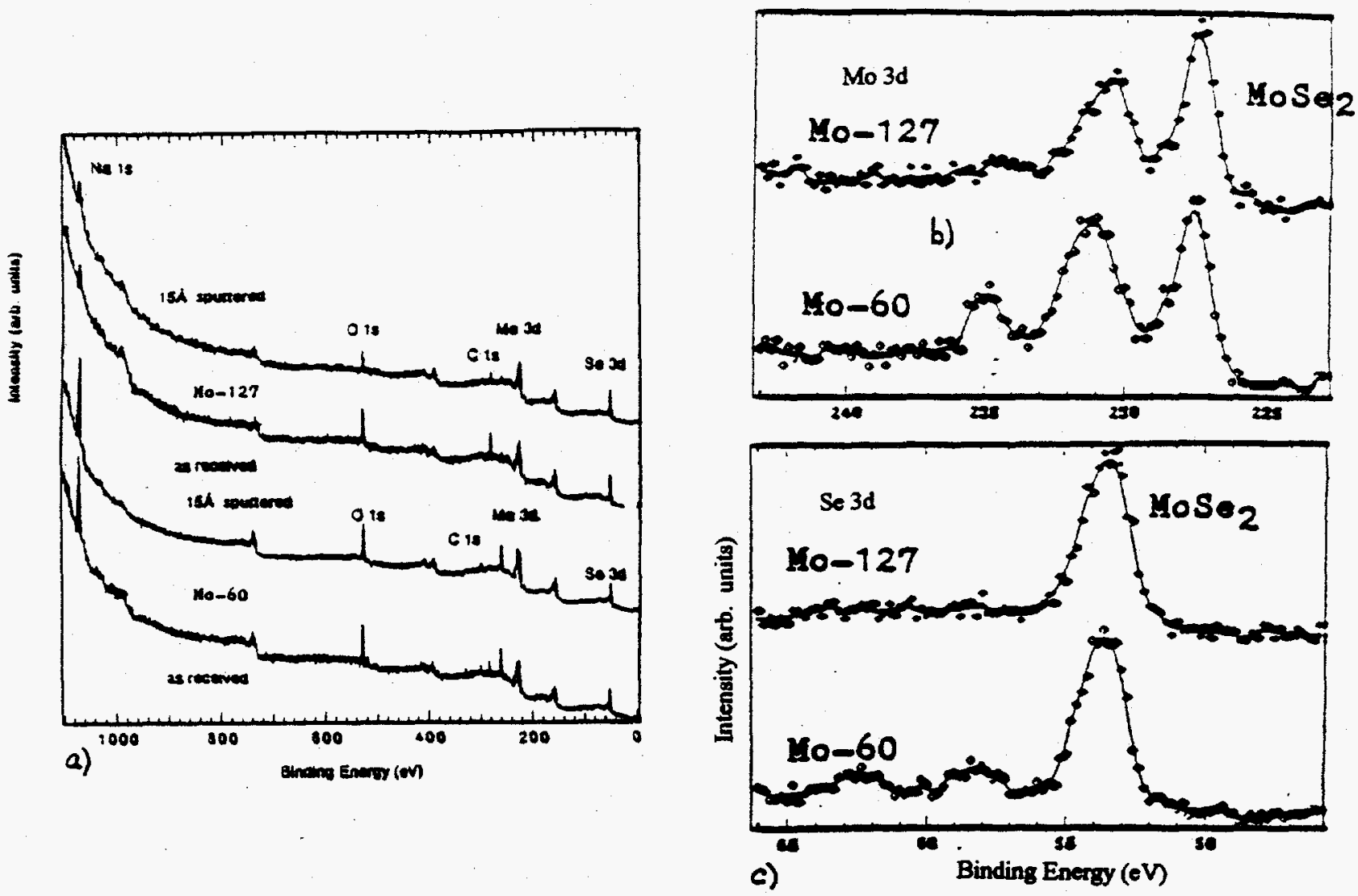

Fig. 2 a) XPS survey spectra, b) Mo 3d and c) Se 3d spectra taken from samples Mo-60 and Mo-127.

Influence of the excessive reaction of the Mo surface on CIS cell performance was studied in an experiment on two cells fabricated on soda-lime glass/Mo substrates. The illuminated I-V characteristics of the devices showed that the one fabricated on the reactive Mo layer suffered from a poor fill factor value which can be attributed to the presence of a high resistivity region at the $\mathrm{Mo} / \mathrm{CIS}$ interface.

The above example demonstrated the deleterious effect of excessive $\mathrm{Na}$ diffusion through the Mo layer on the cell characteristics. Na diffusion into the CIS film at moderate levels, however, can improve the device efficiency as shown in Fig. 3 which shows the I-V characteristics of a set of devices fabricated on soda-lime (A), Corning 7740 (B) and Corning 7059 glass substrates. Device $\mathrm{D}$ was prepared on a soda-lime glass substrate with a $\mathrm{SiO}_{2}$ surface barrier layer. This experiment was carried out for $\mathrm{Cu} / \mathrm{In}$ stoichiometric ratios of $0.88,0.92$ and 0.98 . The general observations from the data of Fig. 3 and the other experiments can be listed as follows:

i) The efficiencies of cells fabricated on the soda-lime glass substrate (without the $\mathrm{SiO}_{2}$ barrier layer) are higher than those on other glass substrates.

ii) The difference between the efficiency of soda-lime glass based device and the others is the largest for the lowest CIS stoichiometric ratios. For films with $\mathrm{Cu} / \mathrm{In}$ ratios of 0.92 and 0.98 the differences between the fill factor values of various cells are not as dramatic as they are in Fig. 3.

iii) A thin layer of $\mathrm{SiO}_{2}$ deposited on the soda-lime glass surface before Mo deposition lowers the fill factor of the CIS cells. 
The above results suggest that $\mathrm{Na}$ diffusing through the Mo layer into the CIS film increases the hole concentration in the CIS layer. Consequently, good devices can be obtained at low $\mathrm{Cu} / \mathrm{In}$ ratios with $\mathrm{Na}$ doping. Presence of $\mathrm{Na}$ actually increases the processing window of solar cells in terms of usable CIS stoichiometries.

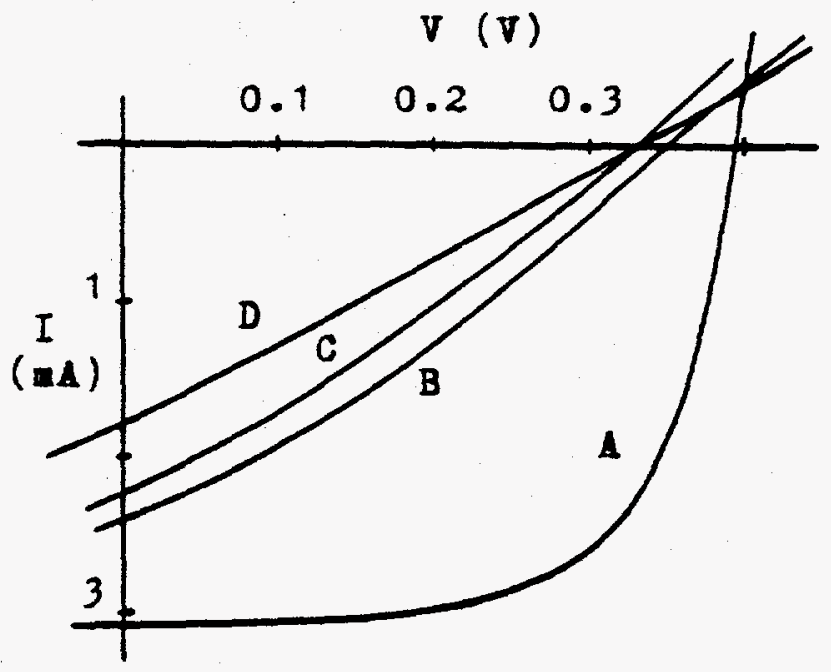

Fig. 3 Iluminated I-V characteristics of $0.09 \mathrm{~cm}^{2}$ CIS cells fabricated on soda-lime glass (A), Corning 7740 glass (B), Corning 7059 glass (C), and soda-lime glass coated with $\mathrm{SiO}_{2}$ (D). The Cu/In ratio for the CIS film was 0.88 .

\section{Novel CIS deposition approach}

During this period, work was carried out to improve the yields obtained in the non-vacuum CIS growth approach that ISET had reported previously [1]. As a result, the processing steps have been streamlined and a large number of cells with conversion efficiencies in the $8-11 \%$ range have been fabricated. Sub-modules of around $5 \%$ efficiency have also been demonstrated. The difference between the cell and sub-module efficiencies suggests that the standard integration method is not well suited for the fabrication of modules based on the non-vacuum process. Work is in progress to address this issue.

\section{References}

1. Annual Report Photovoltaic Program FY 1993. (September 1994). NREL/TP-4106285. $106 \mathrm{pp}$.

2. B.M.Basol, V.K.Kapur, C.R. Leidholm, A. Minnick and A. Halani, "Development of lowcost thin film CIS cells and modules", 12th NREL. PV Program Review. AIP Conf. Proc. 306, 1993 p. 79.

3. B.M.Basol, V.K.Kapur, C.R. Leidholm, A. Minnick and A. Halani, "Studies on substrates and contacts for CIS films and devices", First World Conf. PV Energy Conversion (WCPEC). Waikolon Hawaii. December 5-9, 1994 in press. 
Title:

Organization:

Contributors:
Research on stability and electronic properties of $\mathrm{a}-\mathrm{Si}: \mathrm{H}$ and $\mathrm{a}-(\mathrm{Si}, \mathrm{Ge}): \mathrm{H}$ devices

Iowa state University

Microelectronics Research Center

Ames, Iowa 50011

Vikram L. Dalal, PI

Ralph Knox, Kay Han, Mohan Bhan, Mark Leonard, Greg Baldwin, Sanjeev Kaushal, Scott DeBoer, E. X. Ping, J. Xu and Aziz Ahmed

\section{Objective}

The major objective of the program is to develop alternative growth techniques for growing higher quality and more stable a-Si:H and a$(\mathrm{Si}, \mathrm{Ge}): \mathrm{H}$ materials and devices. An additional objective is to improve the design of $\mathrm{a}-\mathrm{Si}: \mathrm{H}$ and $\mathrm{a}-(\mathrm{Si}, \mathrm{Ge}): \mathrm{H}$ devices by using graded gaps.

\section{I.Material Growth}

\section{I.1. Amorphous materials:}

We have concentrated on growing a-Si:H and a-(Si,Ge):H materials using electron-cyclotron-resonance(ECR) remote-plasma techniques. A remote ECR plasma allows the deposition of films and devices at very low pressures $(5-10 \mathrm{mT})$, and also allows the use of energetic beams of $\mathrm{H}$ or $\mathrm{He}$ to allow controlled ion bombardment of the surface during growth. The use of energetic $\mathrm{H}$ beam can lead to controlled etching during growth, thereby altering the morphology of the film during growth. By increasing the amount of $H$ etching, we can smoothly change the microstructure from amorphous to polycrystaliine, thereby changing both the optical and electronic properties.

Using such plasma beams, we have been able to deposit high quality $\mathrm{a}-(\mathrm{S} i, \mathrm{Ge}): \mathrm{H}$ films in the Tauc bandgap range from $1.3 \mathrm{eV}$ to $1.67 \mathrm{eV}$. The electronic properties of these films are superior to the properties of the films deposited using only glow discharge deposition. As an example, in Fig. 1, we show the Urbach energy of valence band tails, and the subgap absorption coefficient $\alpha$, as functions of Tauc gap, for both ECR and glow-discharge deposited films. The lower values of subgap $\alpha$ and Urbach energy are signatures of higher electronic quality of the ECR-deposited films.

\section{I.2. Microcrystalline Si:H material}

Using the same ECR technique, but with significant $H$ etching, we

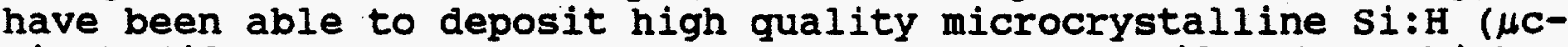
$\mathrm{Si}: \mathrm{H})$ films at low temperatures $(300 \mathrm{c})$. These films have higher grain sizes, appear hazy to the eye, and show Raman spectra which are characteristic of poly and micro-crystalline $s i$, and not of aSi:H. The absorption coefficient of these films is similar to that 
of c-Si. The presence of significant amounts of $\mathrm{H}$, presumably at the grain boundaries, means that these films also have a low subgap absorption, as shown in Fig. 2. The sub-gap absorption was measured using standard two-beam photo-conductivity techniques. The doping density in these films is in the order of $10^{16} / \mathrm{cm}^{3}$. The importance of this work lies in being able to use $\mu \mathrm{C}-\mathrm{Si}: \mathrm{H}$ material as a low-gap alloy in a tandem solar cell with a-Si:H. Analysis indicates that a $2.5 \mu \mathrm{m}$ film of low defect density $\mu \mathrm{C}-\mathrm{Si}: \mathrm{H}$ may lead to a solar conversion efficiency of about $9 \%$ when deposited on a textured substrate, and provided with appropriate reflecting contacts. When this film is combined with a top a-Si:H cell of $1.75 \mathrm{eV}$ tauc gap, the overall tandem cell efficiency may be in the $14 \%$ range. Since the $\mu \mathrm{c}-\mathrm{Si}: \mathrm{H}$ material is stable against light degradation, such a tandem, or even a triple junction cell incorporating $\mu \mathrm{C}-\mathrm{Si}$ :H may offer the advantages of a highperformance, high stability solar cell system based entirely in Group IV materials.

\section{Device stability}

We have been able to significantly improve the stability of a-Si:H devices by using the ECR growth technique for fabricating $p-i-n$ solar cells on textured tin oxide substrates. In Fig. 3, we show the degradation of two solar cells, one made using ECR techniques, and one using glow-discharge techniques. Each cell had similar voltages and fill factors. Each cell started out with fill factors in the range of 0.68 , and voltages in the range of $0.75 \mathrm{~V}$. Each cell had similar thicknesses of the $i$ layer (about $0.4 \mu \mathrm{m}$ ).

The figure shows that the cell made using ECR technique was much more stable against light degradation than its companion glowdischarge cell. This is a significant result, because it shows that changes in growth chemistry lead to improvements in stability of the devices. The result on devices agrees with the improvements in the stability of materials grown using ECR which was reported in last year's report. 

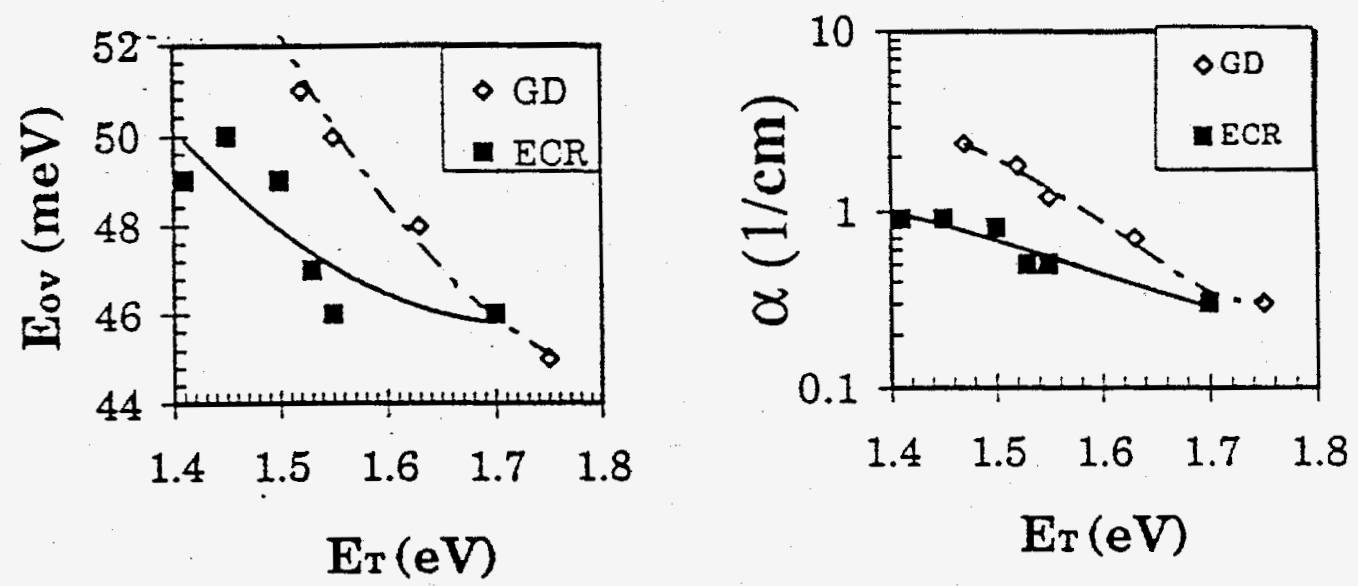

Fig. 1 Urbach energy and subgap $\alpha$ of ECR and glowdischarge deposited a- $(S i, G e): H$ films

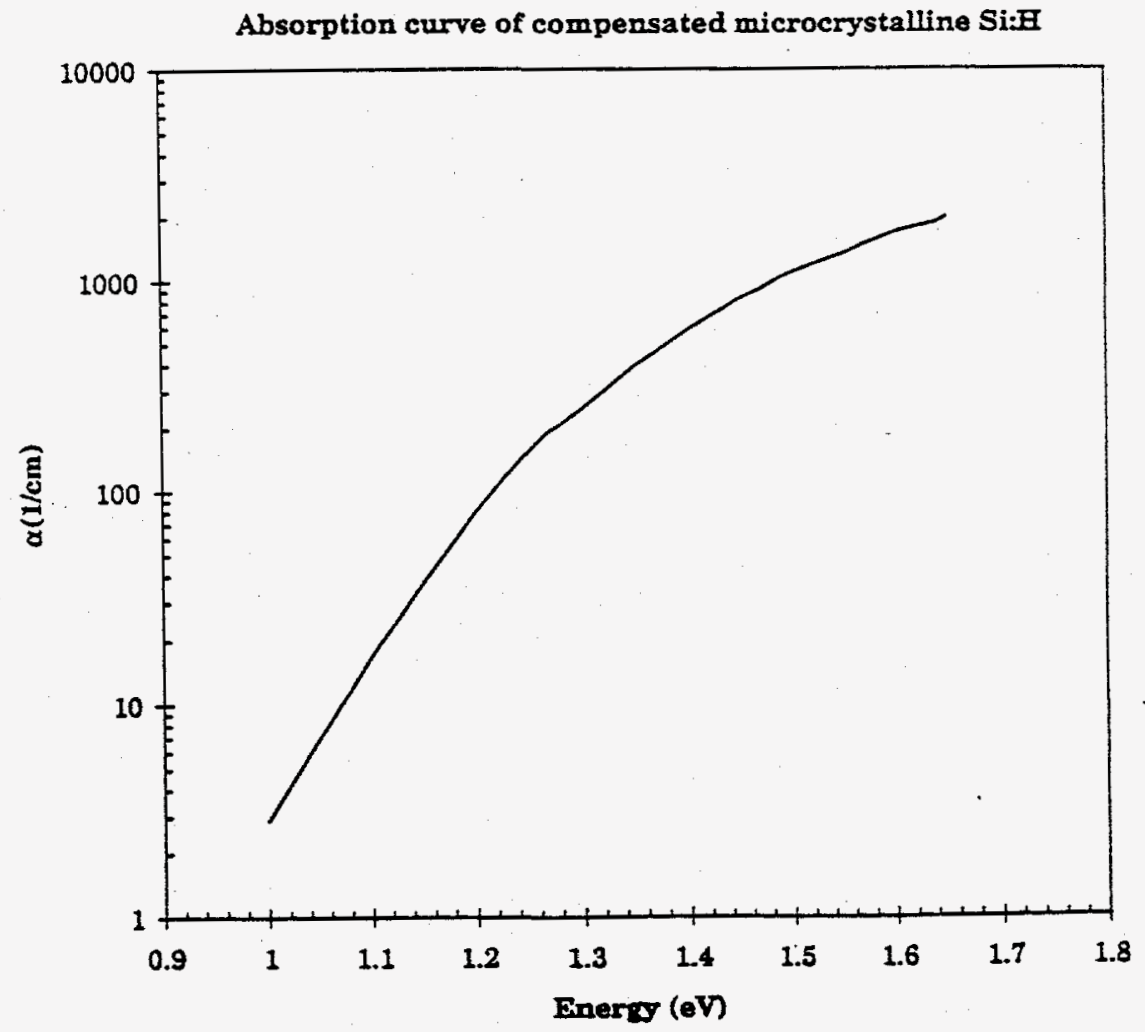

Fig. 2 Absorption coefficient of microcrystalline Si:H film deposited at $300 \mathrm{C}$ using ECR technique 


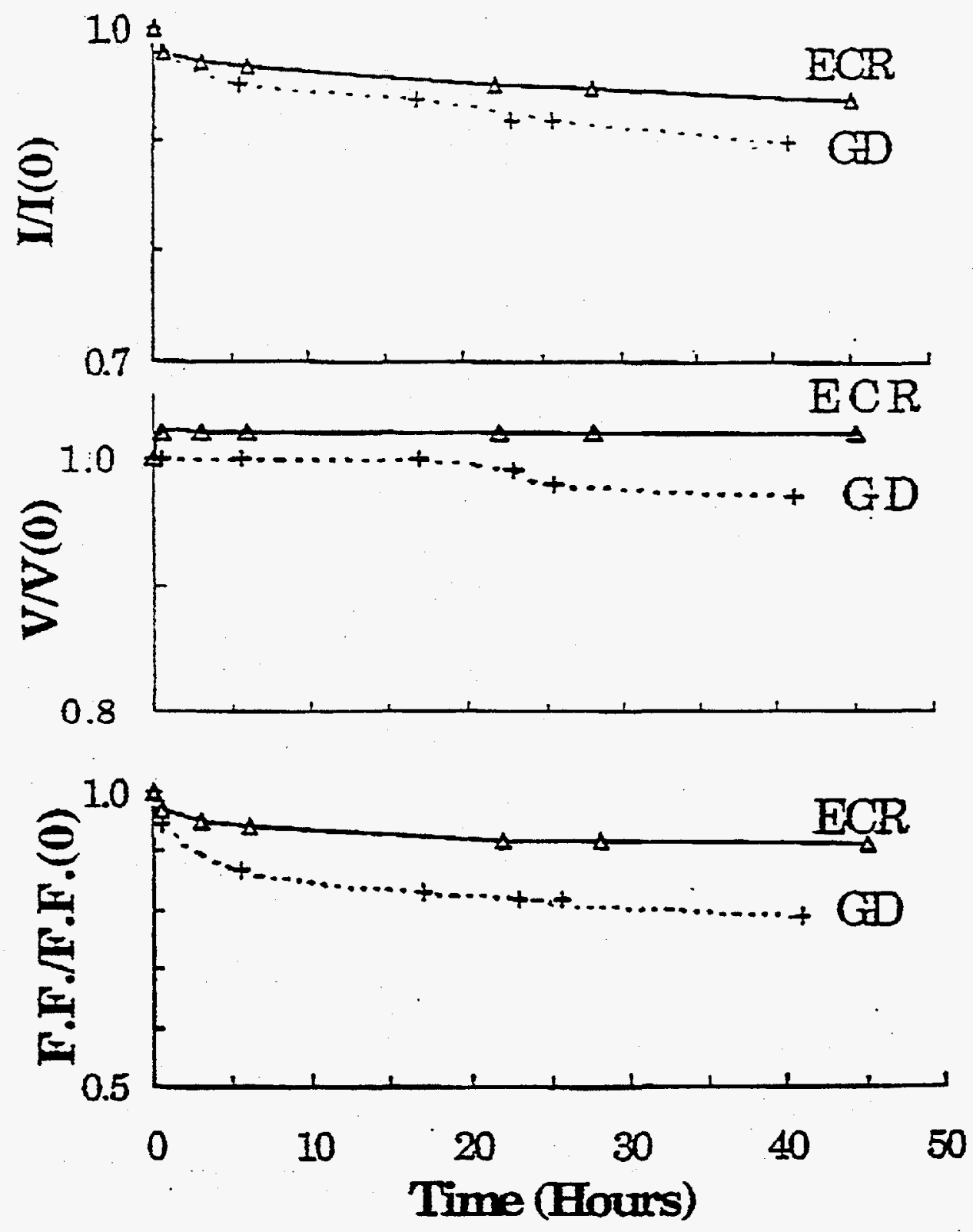

Fig. 3

Comparison of stability of two similar $p-i-n$ solar cells, one deposited using ECR technique, and one deposited using glow discharge technique, under $300 \mathrm{~mW} / \mathrm{cm}^{2}$ illumination. 
Title:

Organization:

Contributors:
Atomic Scale Characterization of Hydrogenated Amorphous Silicon Films and Devices

National Institute of Standards and Technology (NIST)

Boulder, Colorado

A.Gallagher, principal investigator, D.Tanenbaum, A.Laracuente, P.Kalra

\section{Objective}

The overall objective is to assist the development of a cost-effective, U.S. amorphous silicon (a-Si:H) PV technology. This is done by improving understanding of the mechanisms that limit the efficiency of current PV devices, and devising ways to overcome these limitations.

\section{Technical Approach}

Three approaches are being pursued to attain this overall objective; all utilize a scanning tunneling microscope (STM) to measure film and device properties with atomic scale resolution. The first approach is to measure the surface topologies of deposited films, to understand the forms and causes of inhomogeneities believed responsible for loss of efficiency. The second approach is to measure the electrical characteristics of cross sectioned PV cells, as a function of illumination and light induced degradation, to establish where losses and degradation are occurring. The third approach is directed toward measuring, and finding methods to diminish, the growth of small particulates in the discharge, and their incorporation into the growing PV film.

\section{1)Film surface topology}

The measurements of surface topologies are conducted on intrinsic a-Si:H films, deposited from silane rf glow discharge (PECVD) in our deposition chamber and transferred into our STM without exposure to air. Deposition conditions are matched as well as possible to those used for device production at industrial facilities. Typical topologies observed on films of increasing thickness are shown in Fig. 1. These surfaces are uniformly lumpy, with roughness height that increases slowly and a roughness width scale that increases rapidly with film thickness. The surface slopes are relatively gentle, indicating an absence of voids for these relatively thin films. This is a valuable property of these films, from a device context. However, thicker films are rougher and sometimes contain void like features, suggesting that films are not uniform versus depth, as is assumed in device models.

\section{2) Cross sectioned PV cells}

Hydrogenated amorphous silicon PV cells contain many layers and junctions of a-Si:H plus its alloys with Ge and C. Efficiency and stability depend on many factors, and efficient cell design requires models that incorporate these as correctly as possible. This part of our measurements is directed toward obtaining a better understanding of the distributed 
contributions to photocurrent, photovoltage and light induced degradation in the cells of U.S. manufacturers. We intend to cleave cells in our vacuum chamber and use the STM to measure photovoltages and band properties versus depth through the cell. This experiment is under construction and development; STM manipulation, optical and cleaving components are constructed and development of a low current STM underway.

\section{3) Particulate incorporation during cell growth}

In recent years many measurements in silane discharges have shown that silicon particulates form, and are suspended in the plasma due to acquiring a negative charge. There is reason to suspect that as these suspended particles grow in size, but have not yet exceeded $\sim 10 \mathrm{~nm}$ radius, they may be temporarily neutralized. If this occurs they may diffuse to the substrate and be incorporated into the film, and it is very probably that this would cause voids and carrier traps, with deleterious consequences on PV efficiency. But such particle incorporation had not been observed, nor had particle growth in the plasma been clearly identified as an important contributor to film quality. We are studying both of these questions, and have shown for the first time that such small particles are indeed incorporated into films grown in our deposition system. These particles are too small to be observed in the plasma or on the surface by light scattering; we have detected them in our STM measured surface topologies. Fig. 2 shows some of these detected particles; note that they stand out very clearly above the general pattern of surface roughness. We observe a particle volume in the films that represents about $0.1 \%$ of the film volume. It is not known if such silicon particles are also incorporated in the photovoltaics films of PV manufacturers, but it appears reasonably likely. If so, then their elimination may allow achieving significantly better PV efficiencies.

A number of changes in discharge arrangement may mitigate particle growth or incorporation, and we have started to investigate these. The easiest way to detect particulates growth and behavior in the discharge is by light scattering, so we are using this method to investigate dependencies on deposition parameters and particle elimination tricks. However, light scattering does not detect the very small particulates shown in Fig. 2, so this must be complemented with STM studies of the film surface topology.

\section{Conclusions}

We have shown that the growing surface of PECVD a-Si:H films start out as uniformly hilly with relatively small roughness, and with increasing thickness the roughness and characteristic width grows continually. This suggests that film properties may be more depth dependent than is normally considered in PV cell models. However, this typical topology does not contain steep sided canyons or holes, indicative of growing voids, for films of less than $-200 \mathrm{~nm}$ thickness. Some surface contours contain an isolated bump that stands out above the general topology, and clearly results from a particulate deposited from the plasma during film growth. These bumps represent particles of 3-9 $\mathrm{nm}$ diameter, where the lower limit results from our inability to distinguish smaller particles from the general surface roughness. The detected particles represent $\sim 0.1 \%$ of the film volume. It is exceedingly likely that film voids and carrier traps occur around these particles as they are 
covered by film. Thus, their elimination by modification of plasma parameters may allow significantly better material and PV efficiencies to be achieved.

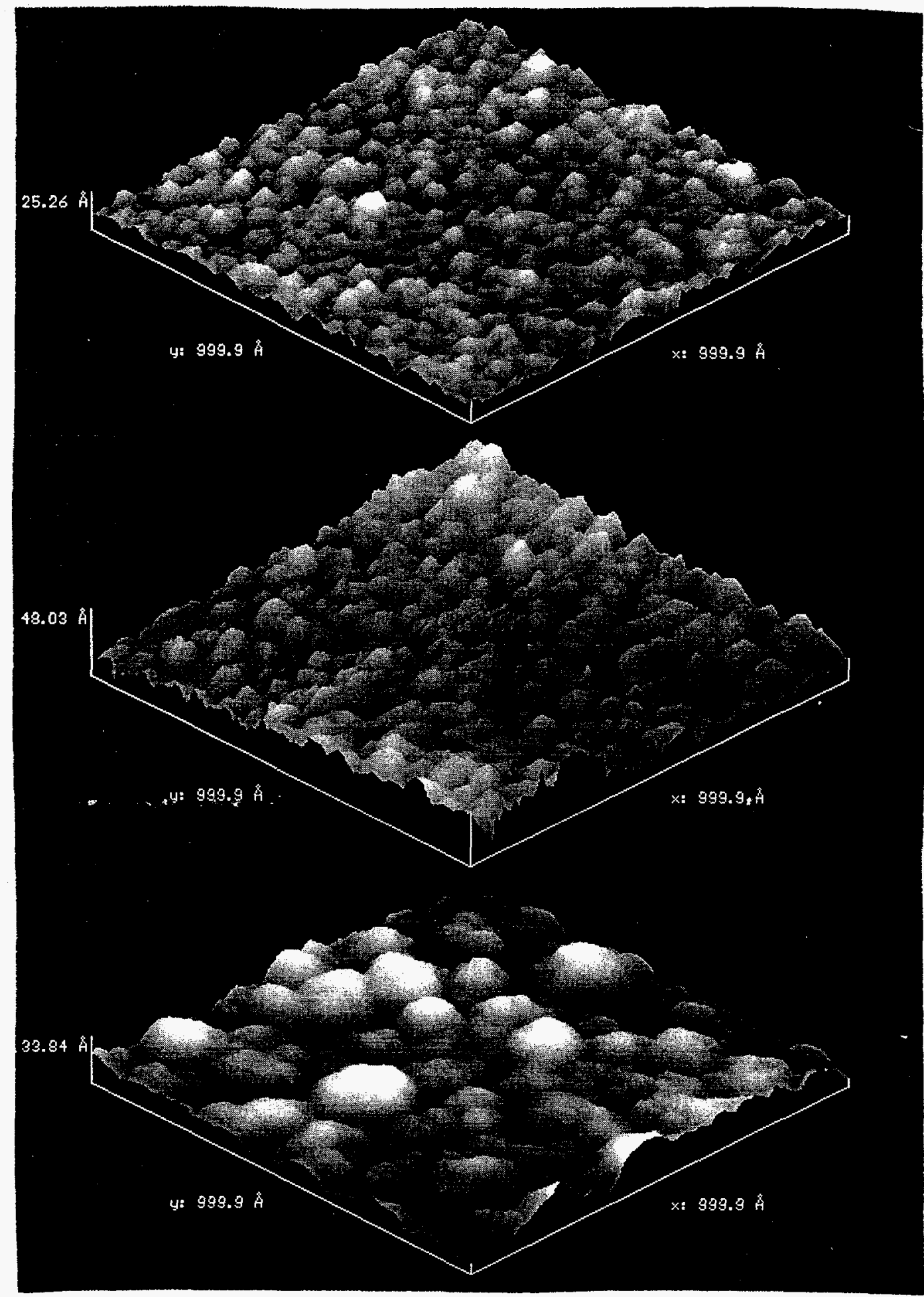

Fig.1 STM images of $1.9 \mathrm{~nm}$ (top), $4.5 \mathrm{~nm}$ (middle) and $50 \mathrm{~nm}$ (bottom) thick a-Si:H films. In all figures a $100 \mathrm{~nm}$ square is shown with the vertical scale expanded $\mathrm{x} 3$ for clarity. 


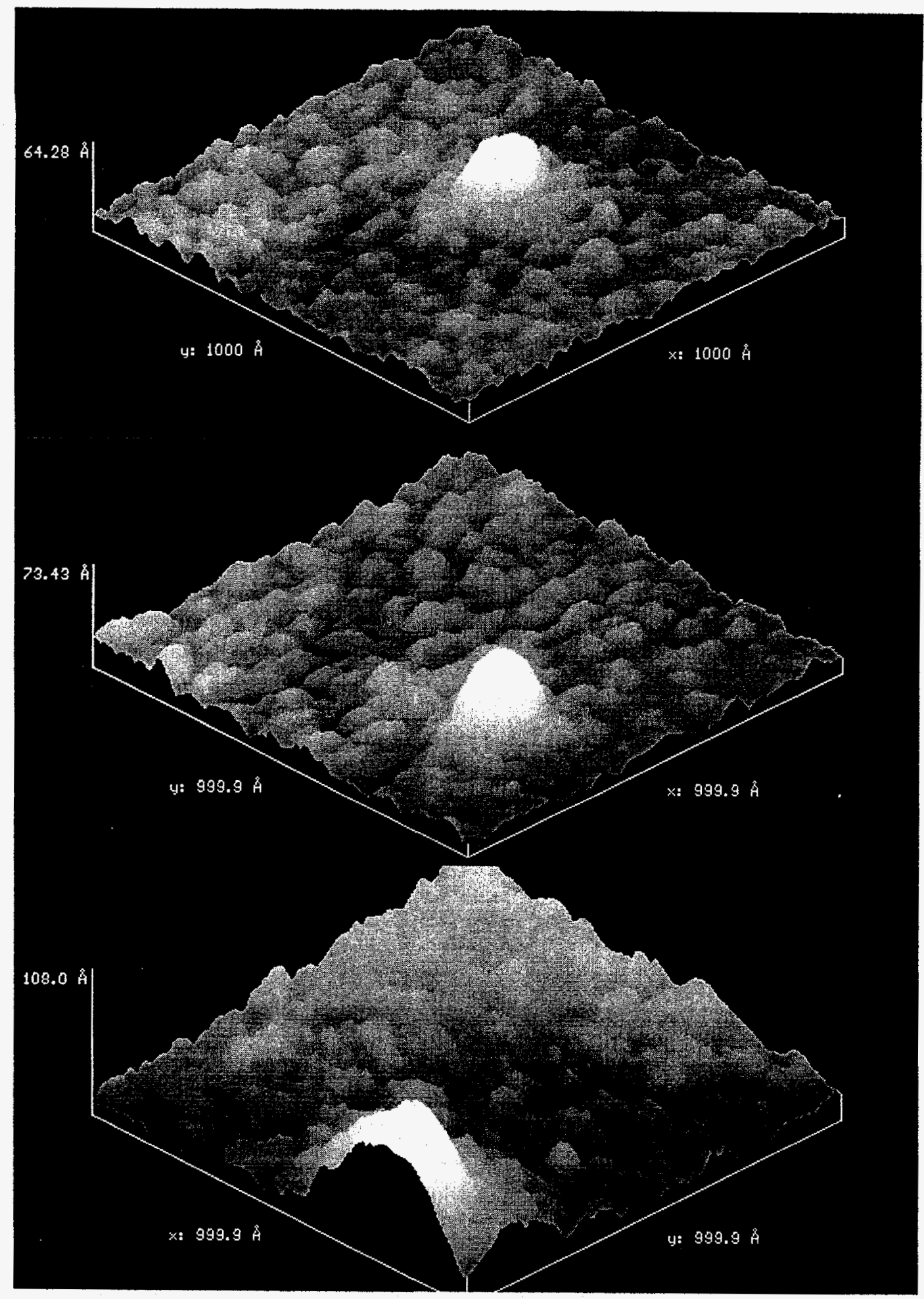

Fig.2 STM images of $100 \mathrm{~nm}$ squares, $\mathrm{x} 3$ vertically expanded, showing large bumps that stand out relative to the surrounding surface roughness. The average film thickness is 1.9 $\mathrm{nm}, 4.5 \mathrm{~nm}$ and $14.5 \mathrm{~nm}$ from top to bottom. 
Title: $\quad$ Wide Bandgap Solar Cells with High Stabilized Performance

Organization: The Pennsylvania State University, University Park, PA.

Contributors: R.W. Collins and C.R. Wronski, Principal Investigators; Ing-shin Chen, Sangbo Kim, Yeeheng Lee, and Yiwei Lu, Graduate Assistants.

\section{Objectives}

The objectives of this research are to: (i) develop intrinsic materials for improved wide-bandgap solar cells through an improved understanding of the growth process; (ii) establish linkages among material and solar cell parameters for wide-bandgap amorphous silicon and silicon-carbon alloys; (iii) optimize $2.2 \mathrm{eV}$ bandgap amorphous silicon-carbon alloys, high hydrogen-content amorphous silicon, and/or microcrystalline p-layers; (iv) develop TCO/p-layer and $\mathrm{p} / \mathrm{i}$-interface formation procedures for optimum solar cell performance; and (v) optimize stabilized performance of cells deposited with real time control of i-layer grading. The broader objectives of the research are to: (i) develop a cost effective amorphous silicon (a-Si) photovoltaics (PV) technology; (ii) to foster a viable a-Si PV industry in the United States, ensuring that this industry remains a world leader in a-Si technology; and (iii) assist the U.S. a-Si PV industry achieve the U.S./DOE PV Program milestone of $15 \%$ stable efficiency multijunction a-Si modules for large scale utility use by 2005.

\section{Introduction and Experimental Details}

In the preparation of a-Si:H-based solar cells, the properties of very thin layers may differ from those of thick layers prepared under the same conditions [1]. The differences arise from the evolution of structure with thickness or from size effects on the electronic properties [2]. In addition, the layers of a structure may interact during deposition or in subsequent processing [3]. Such effects may not be easily identified in a post-process analysis of the completed device. With real time measurements of device fabrication, however, small differences or changes in layer properties can be detected and traced to specific processing steps. Here, the results of a real time spectroscopic ellipsometry (RTSE) study of a-Si:H-based solar cell preparation will be described. With the multichannel ellipsometer used here, $\sim 100$ data pairs in the ellipsometric angles $(\psi, \Delta)$ are collected over a spectral range of $1.5-4.2 \mathrm{eV}$, using a photodiode array detection system [4]. In monitoring the thin $(-200 \AA) \mathrm{p}$-layer as well as the $\mathrm{p} / \mathrm{i}$ interface of the $\mathrm{SnO}_{2}: \mathrm{F} / \mathrm{p}-\mathrm{i}-\mathrm{n} / \mathrm{metal}$ solar cell structure, typical acquisition and repetition times for the 100-point spectra are 0.16 and $1 \mathrm{~s}$, respectively. In monitoring the development of the thicker $i$-layer after formation of the $\mathrm{p} / \mathrm{i}$ interface, slower acquisition and repetition times are employed, 3.2 and $15 \mathrm{~s}$.

The a-Si:H solar cells were prepared in a single-chamber system and co-deposited on three types of substrates: textured and specular $\mathrm{SnO}_{2}: \mathrm{F}$-coated glass for device measurements, and a specular $\mathrm{SnO}_{2}: \mathrm{F}$-coated $\mathrm{Si}$ wafer for RTSE at enhanced sensitivity. The substrate temperature was fixed at $240^{\circ} \mathrm{C}$. The $\mathrm{p}$ - and $\mathrm{i}$ - layers were prepared with an if power flux of $0.07 \mathrm{~W} / \mathrm{cm}^{2}$. The p-layer was a-Si $0.91 \mathrm{C}_{0.09}: \mathrm{H}$ doped with $B$ using flow ratios of $\left[\mathrm{SiH}_{4}\right]:\left[\mathrm{CH}_{4}\right]:\left[\mathrm{B}_{2} \mathrm{H}_{6}\right]:\left[\mathrm{H}_{2}\right]=$ 6:4:0.01:0.99 (in sccm). With this mixture, formation of metallic $\mathrm{Sn}$ via the reduction of the $\mathrm{SnO}_{2}: \mathrm{F}$ substrate surface by the hydrides is below detectivity limits $(<1 \AA)$ [3]. A $55 \mathrm{~min} \mathrm{Ar}$ flush was performed after p-layer and before a-Si:H i-layer deposition. For the latter, pure $\mathrm{SiH}_{4}$ was used. In order to complete the device, $200 \AA$ of P-doped $\mu \mathrm{c}-\mathrm{Si}: \mathrm{H}$ was deposited on the i-layer using a power of $0.5 \mathrm{~W} / \mathrm{cm}^{2}$ and a flow ratio of $\left[\mathrm{SiH}_{4}\right]:\left[\mathrm{PH}_{3}\right]:\left[\mathrm{H}_{2}\right]=0.99: 0.01: 100$ (in sccm). Finally, a $1000 \AA \mathrm{Cr}$ top contact was evaporated onto the n-layer. Because the objective was to characterize the the top junctions of the cell, the deposition of the n-layer and the top contact were not monitored by RTSE. 


\section{Results and Discussion}

Figure 1 shows the structural evolution deduced from RTSE data for p-layer growth by PECVD, for $p / i$ interface layer formation during Ar flushing, and for the initial stage of $\mathrm{i}-\mathrm{layer}$ growth by PECVD. Also shown in Fig. 1 are the dielectric functions $\varepsilon=\varepsilon_{1}+i \varepsilon_{2}$ (insets) and the optical gap determinations for the three layers. The gap is obtained by plotting $\left(\varepsilon_{2}\right)^{1 / 2}$ versus energy, and extrapolating the linear behavior to $\varepsilon_{2}=0$.

In the RTSE data analysis for a-SiC:H p-layer growth on $\mathrm{SnO}_{2}: \mathrm{F}$, we use a three-layer optical model consisting of a surface roughness layer, a bulk layer, and an interface roughness layer that arises from the surface roughness on the $\mathrm{SnO}_{2}: \mathrm{F}$ (see Figure 2). Thus, we determine (i) the dielectric function of the bulk a-SiC:H p-layer, (ii) the thickness $d_{b}$ of the bulk p-layer, (iii) the thickness $d_{s}$ of the surface roughness on the p-layer, (iv) the thickness $d_{i}$ of the roughness on the $\mathrm{SnO}_{2}: \mathrm{F}$, which fills in with p-type a-SiC: $\mathrm{H}$ to become interface roughness, and (v) the volume fraction $\mathrm{f}_{\mathrm{i}}$ of $\mathrm{p}$-type a-SiC:H filling the $\mathrm{SnO}_{2}: \mathrm{F}$ roughness layer. Assumptions in the analysis are that the surface roughness layer of thickness $d_{s}$ has a composition of $\mathrm{a}-\mathrm{SiC}: \mathrm{H}: \mathrm{B} /$ void given by $0.5 / 0.5$, and that the interface roughness layer of thickness $\mathrm{d}_{\mathrm{i}}$ has a composition of $\mathrm{SnO}_{2}: \mathrm{F} / \mathrm{a}-\mathrm{SiC}: \mathrm{H}: \mathrm{B} /$ void given by $0.5 / \mathrm{f}_{\mathrm{i}} /\left(0.5-\mathrm{f}_{\mathrm{i}}\right)$. Figure 2 summarizes the structural parameters used in the analysis. The analyses of $\mathrm{p} / \mathrm{i}$ interface layer formation and i-layer growth are similar, the only difference being the nature of the substrate onto which the deposition occurs (and thus the interface roughness thickness, $\mathrm{d}_{\mathrm{j}}$ ). For the $\mathrm{p} / \mathrm{i}$ interface layer, the thickness on top of the p-layer is so small, that no distinct bulk density layer can develop.

Figure 1 shows that in the first $55 \mathrm{~s}$ of p-layer growth, the modulations in the $98 \AA$ roughness layer on the $\mathrm{SnO}_{2}: \mathrm{F}$ surface fill with p-type a-SiC:H $\left(\mathrm{f}_{\mathrm{i}} \rightarrow 0.5\right)$. After about $55 \mathrm{~s}$, a distinct bulk layer forms and increases linearly at a rate of $1.5 \AA / \mathrm{s}$. A smoothening effect is observed whereby the roughness thickness on the final p-layer surface is less than that on the $\mathrm{SnO}_{2}: \mathrm{F}$. Upon termination of the plasma, $\mathrm{d}_{\mathrm{s}}=67 \AA, \mathrm{d}_{\mathrm{b}}=111 \AA$, and $\mathrm{d}_{\mathrm{i}}=98 \AA$. This leads to a final "mass" thickness of $d_{\text {mass }}=0.5 d_{s}+d_{b}+f_{i} d_{i}=194 \AA$, which is close to the intended value of $200 \AA$. During Ar flushing, a contaminant layer develops on the p-layer surface. The contaminant material, a Si-B alloy [5], is somewhat arbitarily partitioned as either falling into the modulations of the $67 \AA$ p-layer roughness (which increases $f_{i}$ from 0 to 0.035 ), or forming a distinct roughness layer of thickness $d_{s}$ (which increases to $3 \AA$ ). This partitioning is not critical; however, the important parameter is the final mass thickness, given in this case by $d_{\text {mass }}=f_{i} d_{i}+$ $0.5 \mathrm{~d}_{\mathrm{s}}=3.8 \AA$. Figure 1 shows that in the first $45 \mathrm{~s}$ of $\mathrm{i}$-layer growth, the $\mathrm{i}$-layer fills the $67 \AA$ modulations of the p-layer, trapping the contaminant material at the modulated $\mathrm{p} / \mathrm{i}$ interface. The bulk layer growth rate is $1.0 \AA / \mathrm{s}$, and the roughness on the i-layer decreases with time. After $5000 \AA$ of $i$-layer growth, the roughness thickness has decreased to $21 \AA$ (not shown).

The optical gaps in Fig. 1 provide essential input for modeling solar cell performance. It should be recalled, however, that the gaps in Fig. 1 are characteristic of a temperature of $240^{\circ} \mathrm{C}$, and are smaller than the room temperature Tauc gaps by $\sim 0.20 \mathrm{eV}$. Figure 1 shows that by incorporating $\mathrm{C}$, a p-layer gap $0.10 \mathrm{eV}$ wider than the i-layer has been achieved. However, the $\mathrm{p}$ - $\mathrm{i}$ interface contaminant layer exhibits an absorption onset that is $\sim 0.25 \mathrm{eV}$ lower than the i-layer. We cannot interpret this onset as a gap in the usual sense, but rather an indication of a high density of states below the band edges of the p-and i- layers.

The growth and flushing procedures applied here are designed to simulate those used in our multichamber deposition. Thus, we conclude that solar cell efficiency improvements are possible through changes in the procedures that may either passivate the contaminant layer or avoid its formation. The solar cell co-deposited onto textured $\mathrm{SnO}_{2}: \mathrm{F}$ along with the structure of Fig. 1 exhibited an open circuit voltage $\left(\mathrm{V}_{\mathrm{oc}}\right)$ of $0.74 \mathrm{~V}$, a short-circuit current $\left(\mathrm{J}_{\mathrm{sc}}\right)$ of $13.7 \mathrm{~mA} / \mathrm{cm}^{2}$, and a fill-factor (FF) of 0.65 (see Table I). As a reference point, similarly-prepared solar cells fabricated in the multichamber system exhibited $\mathrm{V}_{\mathrm{OC}}=0.85 \mathrm{~V}, \mathrm{~J}_{\mathrm{Sc}}=12.3 \mathrm{~mA} / \mathrm{cm}^{2}$ and $\mathrm{FF}=0.67$. Interface passivation treatments using atomic $H$ have been designed based on RTSE results [6]. Such a treatment has led to improvements in the performance of cells prepared by multichamber deposition, as shown in Table I. Although the improvements are modest, this work suggests that insights into the monolayer growth processes can guide device fabrication. 


\section{References}

1. Luft, W., and Tsuo, Y., Hydrogenated Amorphous Silicon Deposition Processes, New York: Marcel Dekker, 1993.

2. Nguyen, H.V., and Collins, R.W., Phys. Rev. B 47, 1911 (1993).

3. Lu, Y., An, I., Gunes, M., Wakagi, M., Wronski, C.R., and Collins, R.W., Mater. Res. Soc. Symp. Proc. 297, 31 (1993).

4. An, I., Li, Y.M., Nguyen, H.V., and Collins, R.W., Rev. Sci. Instrum. 63, 3842 (1992).

5. Collins, R.W., Appl. Phys. Lett. 53, 1086 (1988).

6. An, I., Li, Y.M., Wronski, C.R., and Collins, R.W., Phys. Rev. B 48, 4464 (1993).
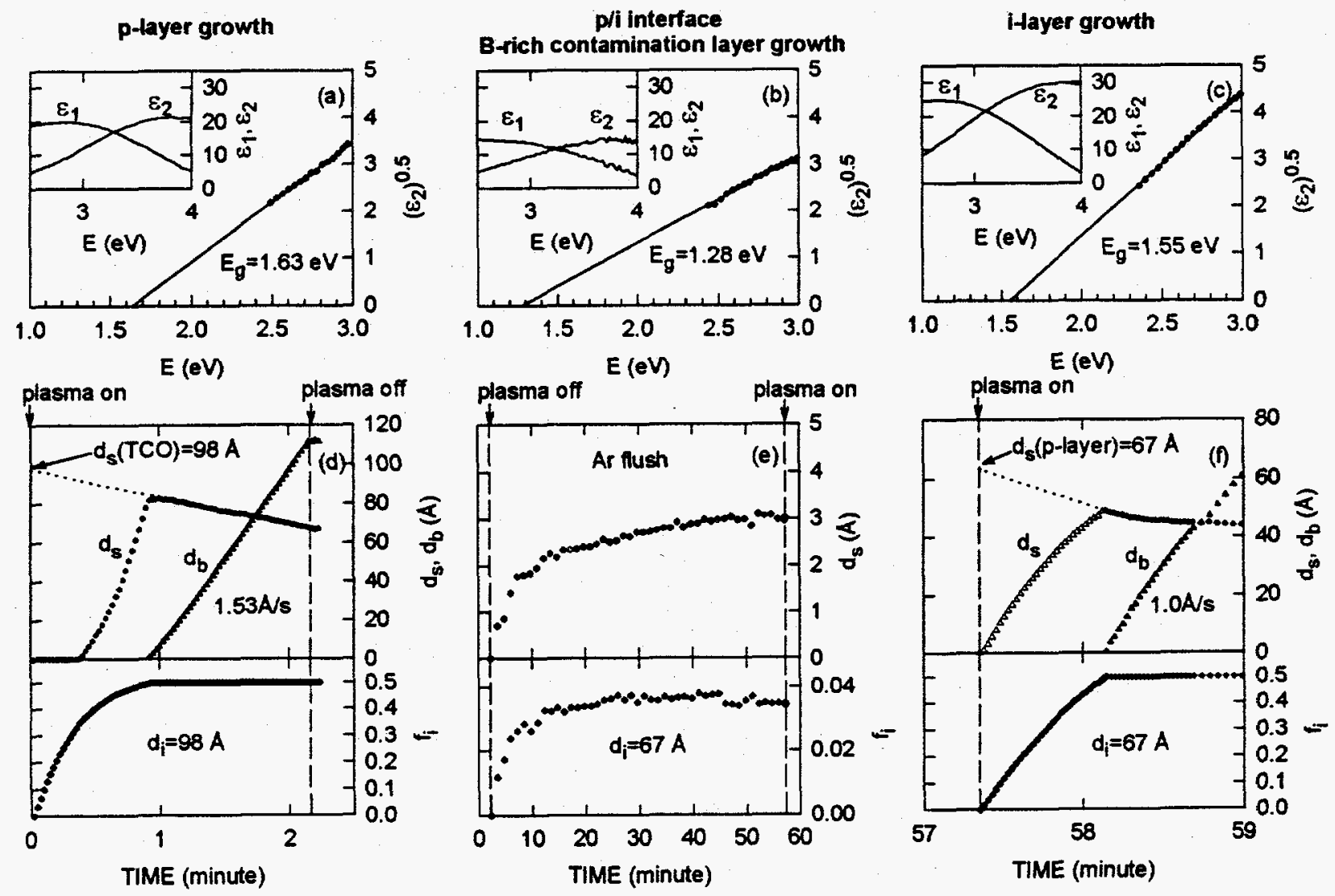

Figure 1 Optical data at $240^{\circ} \mathrm{C}$ (top panels) along with thicknesses and void volume fractions versus time for the PECVD p-layer (left), the $\mathrm{p} / \mathrm{i}$ interface contaminant layer (center), and the PECVD i-layer (right) that make up a $\mathrm{SnO}_{2}: \mathrm{F} / \mathrm{p}-\mathrm{i}-\mathrm{n} / \mathrm{Cr}$ a-Si:H solar cell structure. The optical gap is determined by an extrapolation of the linear trend in $\left(\varepsilon_{2}\right)^{1 / 2}$ to zero ordinate, where $\varepsilon=\varepsilon_{1}+i \varepsilon_{2}$ is the dielectric function of the material. The parameters $\mathrm{d}_{s}, \mathrm{~d}_{\mathrm{b}}$, and $\mathrm{d}_{\mathrm{i}}$ are the thicknesses of the surface roughness, bulk, and interface roughness layers, respectively. In addition, $\mathrm{f}_{\mathrm{i}}$ is the volume fraction of the film that forms within the modulations of the roughness layer on the substrate material. The substrate roughness is completely filled in when $f_{i}=0.5$, and once this occurs, a distinct bulk layer forms. Figure 2 shows a schematic of the multilayer optical model for the case of p-layer growth on $\mathrm{SnO}_{2}: \mathrm{F}$. 


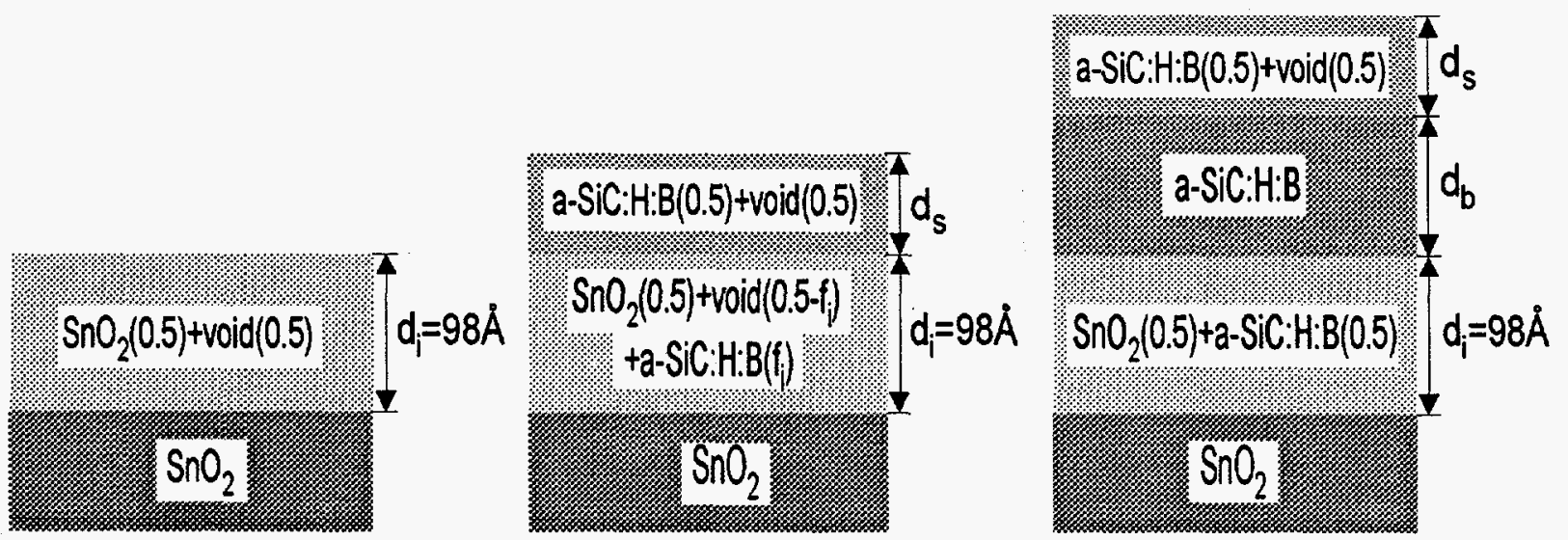

Figure 2 Microstructural evolution for p-type a-SiC:H growth on specular $\mathrm{SnO}_{2}$. A three-layer model for the a-SiC:H:B film is required to interpret the RTSE data. This model consists of (i) a fixed-thickness $\left(\mathrm{d}_{\mathrm{i}}=98 \AA\right.$ ) interface roughness layer that develops from the $\mathrm{SnO}_{2}$ surface roughness, (ii) a bulk-density a-SiC:H:B layer (thickness: $d_{b}$ ), and (iii) a roughness layer on the a-SiC:H:B (thickness: $d_{s}$ ). The $\mathrm{SnO}_{2} / \mathrm{a}-\mathrm{SiC}: \mathrm{H}: \mathrm{B}$ interface layer is modeled during regime (2) as a physical mixture of $\mathrm{SnO}_{2}$ (volume fraction: 0.50 ), a-SiC:H:B (volume fraction: $\mathrm{f}_{\mathrm{i}}$ ), and void. The a-SiC:H:B surface roughness layer is modeled as a physical mixture of bulk a-SiC:H:B (volume fraction: 0.50 ) and void. With this simple model of surface roughness, a good correlation of RTSE analysis results with those of ex situ atomic force microscopy has been obtained.

\begin{tabular}{|c|c|c|c|c|c|c|}
\hline $\begin{array}{c}\text { SOLAR CELL } \\
\text { SYSTEM }\end{array}$ & $\begin{array}{c}\text { STRUCTURE } \\
\text { TCO/pin/Cr }\end{array}$ & $\begin{array}{c}\text { dp/i } \\
(\mathbf{A})\end{array}$ & $\begin{array}{c}\text { Jsc } \\
\left(\mathbf{m A} / \mathbf{c m}^{2}\right)\end{array}$ & $\begin{array}{c}\text { Voc } \\
\text { (Volt) }\end{array}$ & FF & EFF. \\
\hline single-chamber & standard & 4 & 13.7 & 0.74 & 0.65 & $6.6 \%$ \\
\hline multi-chamber & standard & $?$ & 12.4 & 0.82 & 0.67 & $7.1 \%$ \\
\hline multi-chamber & H-treat. & $?$ & 14.9 & 0.86 & 0.67 & $8.6 \%$ \\
\hline
\end{tabular}

Table I Initial characteristics for a-Si:H solar cells prepared in a single-chamber system and monitored by RTSE to determine top junction structure. Also shown are the corresponding characteristics for two cells prepared under similar conditions in a multichamber system. One cell is a control and the other is prepared using an atomic H-passivation treatment of the p-layer and $\mathrm{p}-\mathrm{i}$ interface region. This treatment is performed after $100 \AA$ of the $\mathrm{i}$-layer is deposited. Here $d_{p / i}$ is the mass thickness of the contaminant layer at the $p / i$ interface which is determined for the single-chamber deposition using RTSE. 


$\begin{array}{ll}\text { Title: } & \begin{array}{l}\text { Development and Application of a } \\ \text { Computer Model for CdTe and CuInSe2 } \\ \text { Based Solar Cells }\end{array}\end{array}$

Organization: $\quad$ School of Electrical Engineering

Purdue University

West Lafayette, IN 47907

Contributors: $\quad$ J. L. Gray and R. J. Schwartz, principal investigators;

Y. J. Lee, graduate student

\section{Objective}

The purpose of this research program is to develop an accurate numerical model for $\mathrm{CuInSe}_{2}$ (CIS) and CdTe based solar. The model, ADEPT (A Device Emulation Program and Tool), is used to analyze and aid in the design of CIS and CdTe based solar cells and is being released to the photovoltaic community. An additional objective is to use the model to analyze existing devices and to predict performance.

In addition to development of the computer model, ADEPT, recent efforts have focused on examination of the so-called second junction effects [1,2] in CIS solar cells, and more recently, due in great part to the high efficiencies reported by NREL, on graded band gap CIGS devices [3].

\section{Approach}

The high efficiency reported recently by NREL for CIGS solar cells demonstrates the potential of band gap grading in producing high efficiency thin film solar cells. In order to reap the full benefits of this design strategy, a clear understanding of the fundamental device physics of these structures is needed. ADEPT has been demonstrated to be a useful tool for interpreting the performance of existing solar cells. The accuracy of ADEPT in predicting cell performance depends on several factors. In well understood material systems, such as crystalline, the predictive ability of detailed device simulation codes is quite good. In thin film devices such as CIS and CdTe, the predictive ability of these codes is not as good. Basic characterization of these materials is still an active area and is complicated by variations in the material parameters resulting from different are even more severe fabrication methods and even different facilities. In a graded band gap structure these difficulties are multiplied.

ADEPT generates a numerical solution to a set of three coupled partial differential equations commonly referred to as the semiconductor equations. These are Poisson's equation and the hole and electron continuity equations and are shown below for steady state isothermal conditions.

$$
\begin{gathered}
\nabla \cdot D=q(p-n+N) \\
\nabla \cdot J_{p}=q(G-R) \\
\nabla \cdot J_{n}=-q(G-R)
\end{gathered}
$$

Coupled with the following auxiliary equations, these are solved for the electrostatic potential, $\mathrm{V}$, and the hole and electron concentrations, $\mathrm{p}$ and $\mathrm{n}$, on a domain defined by the device structure subject to boundary conditions (at the contacts, for one-dimensional simulations) and the device operating conditions (the applied bias, operating temperature, and incident light flux). 


$$
\begin{gathered}
D=-\varepsilon \nabla V \\
J_{p}=-q p \mu_{p} \nabla\left(V-V_{p}\right)-k T \mu_{p} \nabla p \\
J_{n}=-q n \mu_{n} \nabla\left(V+V_{n}\right)+k T \mu_{n} \nabla n
\end{gathered}
$$

It is here, in the transport equations, that a significant assumption is made. Carrier transport within the device is assumed to be based on drift-diffusion mechanisms only. Tunneling and thermionic emission are neglected. Tunneling and thermionic emission at the contacts is modeled using effective carrier recombination velocities for the boundary conditions.

As can be seen by examining the preceding equations, there are only a modest number of parameters to be set in order model any device constructed from any semiconductor material, so long as the assumptions regarding transport are not violated. These parameters are: $\mathrm{N}$, the trapped charge density; $G$, the optical generation rate; $R$, the net recombination rate; e, the electric permittivity; $\mu_{p}$ and $\mu_{n}$, the carrier mobilities; and $V_{p}$ and $V_{n}$, the band parameters. While this is a convenient way to express the model equations, it is a somewhat misleadingly simple representation. All the information regarding device structure and material characterization is contained in these terms. Accurate modeling of any device depends on detailed knowledge of these parameters. Even for a relatively simple CdS/CIS structure, there is debate about the exact structure of the device (i.e. presence of an OVC layer, interface states, contact characteristics, etc.) and the correct material characteristics (i.e. absorption coefficients, mobilities, trap/recombination centers, etc.). In a graded band gap structure, these difficulties are compounded.

Even in CIS, there is presumably some variation in absorption coefficient with position within the CIS layer since the ratios of copper to indium to selenium tends vary (i.e. Cu-poor near the CdS interface). The addition of gallium would, of course, change the optical band gap as some function of gallium content. Any other material (such as sulfur) would have a similar effect. Characterization of the absorption coefficient as a function of gallium content is needed in order to produce accurate simulations.

The effective doping and carrier lifetimes in CIS cells are thought to be a result of native defects in the material. Detailed modeling of trapping and recombination can be quite complicated. However, reasonable modeling results have been obtained by assuming a fixed trapped charge density (effective doping) and by modeling recombination as a mid gap single level trap. Improved models are needed for a full understanding of trapping and recombination. ADEPT can be used to examine proposed models, but even a simple model presents a challenge in a graded band gap CIGS solar cell. How do the effective doping and lifetimes depend on band gap (gallium content)? How sensitive is device performance to these parameters?

As with the other parameters, carrier mobility is sure to be a function of gallium content and represents another area of material characterization that must be addressed.

The most explicit effect of band gap grading appears in the band parameters.

$$
\begin{gathered}
q V_{p}=-\left(\chi-\chi_{\text {ref }}\right)-\left(E_{G}-E_{G r e f}\right)+k T \ln \frac{N_{v}}{N_{V_{r e f}}}+k T \ln \frac{F_{1 / 2}\left(\eta_{v}\right)}{e \eta_{v}} \\
q V_{n}=\left(\chi-\chi_{\text {ref }}\right)+k T \ln \frac{N_{C}}{N_{\text {cref }}}+k T \ln \frac{F_{1 / 2}\left(\eta_{C}\right)}{e \eta_{C}}
\end{gathered}
$$


Here, $\chi$ is the electron affinity, $E_{G}$ is the band gap, and $N_{y}$ and $N_{C}$ are valence and conduction band densities of state. The last term in each band parameter expression accounts for degenerate (FermiDirac) statistics. The "ref" subscript refers to an arbitrary reference material. Note that we have assumed parabolic bands.

The band parameters are what allow the modeling of heterostructures and, specifically, graded band gap structures. The electron affinity, band gap, and densities of state can all vary with gallium content. The precise relationship must be determined experimentally.

\section{Results}

Examination of band parameter equations can give some insight into the effect the grading of gallium content may have on cell performance. Neglecting degeneracy, the band parameters are constant with position in a uniform material and hence have no effect on carrier transport. In a graded structure, however, the effect is significant and can sometimes lead to nonintuitive results. Better intuition can be achieved through the use of "effective" energy band diagrams [3]. Conventional energy band diagrams cannot show the effect of positional variations in the conduction band and valence band densities-of-state. This effect is illustrated in Figures 1 and 2, where the conduction band density of states was chosen to vary with position in such a way as to exactly cancel the effect of the graded band gap.

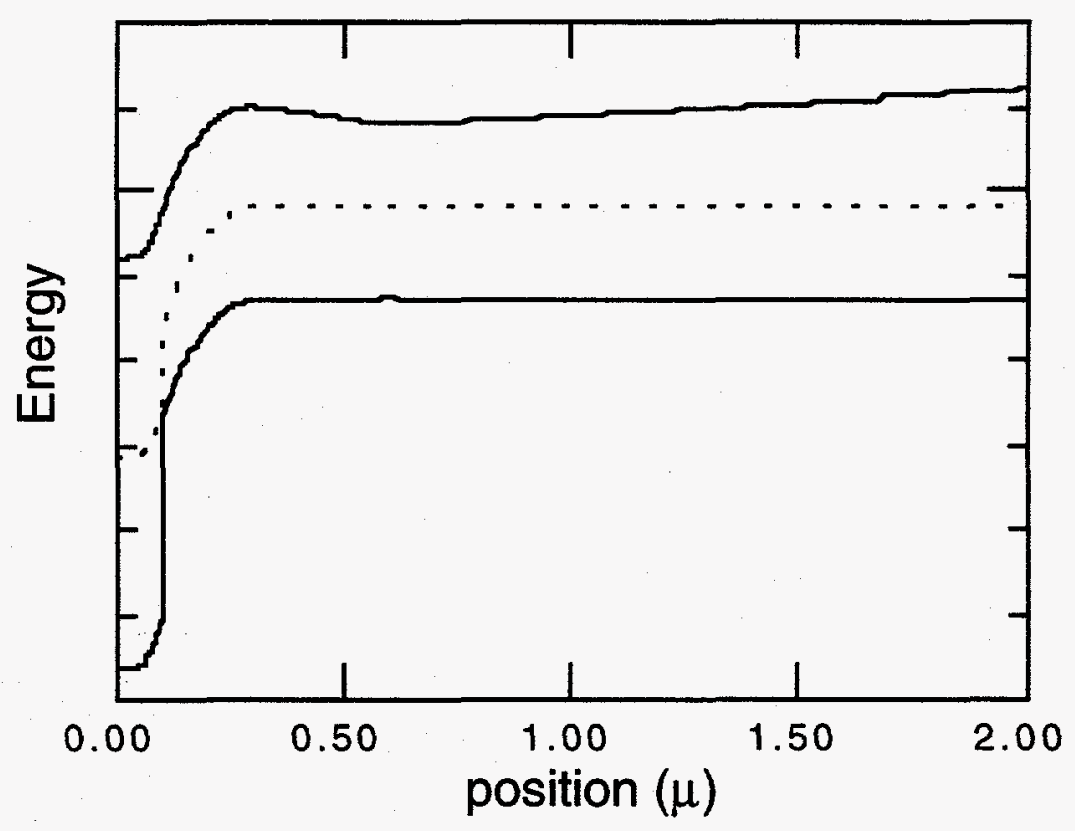

Figure 1. Energy band diagram of a hypothetical CdS/CIGS graded band gap solar cell. 
Figure 1 shows the conventional energy band diagram of a simple CdS/CIGS solar cell. Examination of this plot might lead one to conclude that the band gap grading tends to keep minority carriers away from the CdS interface and the back contact. This would be true if only the band gap changed with position. In this ADEPT simulation, however, that is not the case, as shown in Figure 2. In fact, this device has a somewhat lower efficiency than a comparable CdS/CIS cell ( $14.1 \%$ versus $14.8 \%$ simulated efficiencies).

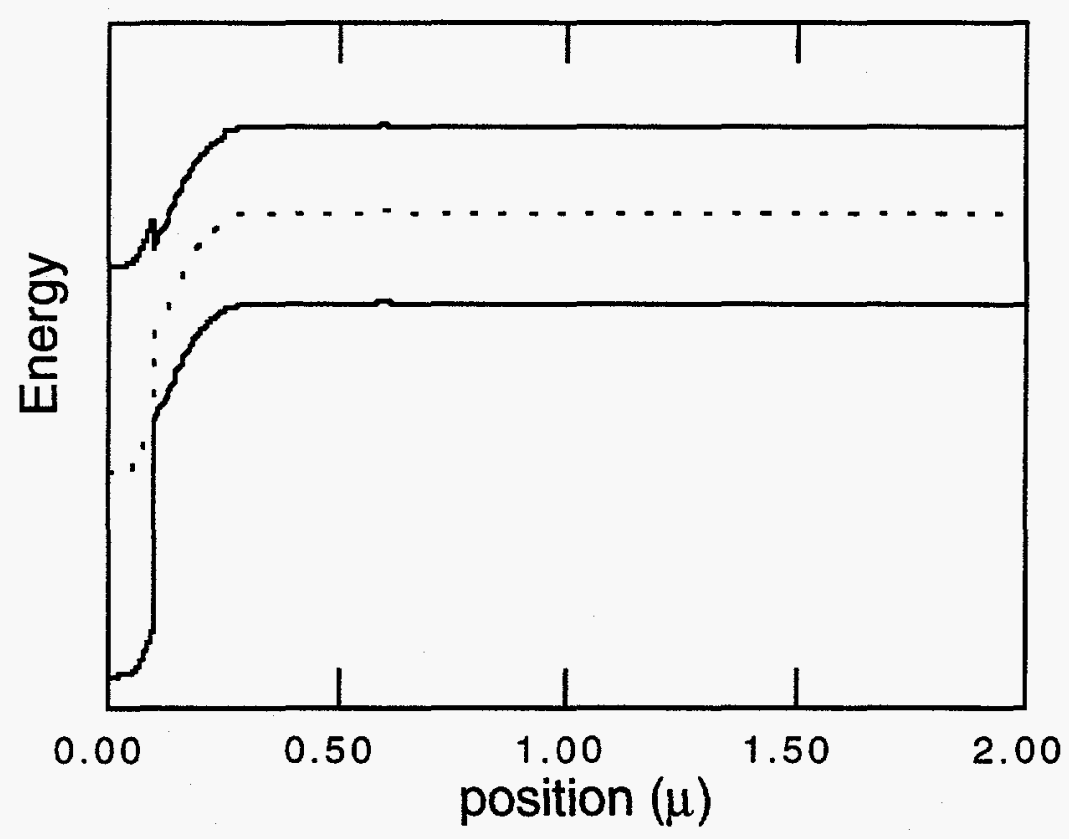

Figure 2. Effective energy bad diagram of same graded band gap cell as used in Figure 1.

\section{Conclusions}

ADEPT has been demonstrated to be a useful tool for analyzing CdTe and CIS based solar cells and for building insight into device operation. For detailed numerical modeling to fulfill its potential as an analysis and design tools, a better understanding of material properties and the relevant device physics is needed. ADEPT can be used to help develop this understanding.

\section{References}

1. Y. J. Lee and J. L. Gray, "Numerical modeling of the Temperature and Illumination Dependent Performance of CIS Solar Cells," Proceedings of the Twelfth European Photovoltaic Solar Energy Conference, Amsterdam, The Netherlands, pp. 1561-1563, April 1994.

2. Y. J. Lee and J. L. Gray, "The Effects of Band Bending Caused by Interface States in CdTe and CIS Solar Cells," presented at the First World Conference on Photovoltaic Energy Conversion, Waikoloa, Hawaii, December, 1994.

3. J. L. Gray and Y. J. Lee, "Numerical Modeling of Graded Band Gap CIGS Solar Cells," presented at the First World Conference on Photovoltaic Energy Conversion, Waikoloa, Hawaii, December, 1994. 
Title:

Organization:

Contributors:

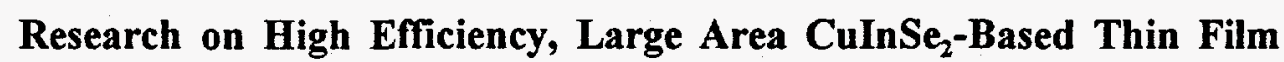
Modules

Siemens Solar Industries

Camarillo, California

J. Bauer, R. Dearmore, D. Delaney, M. Dietrich, W. Frausto, C. Fredric,

R. Gay (Program Manager), C. Jensen, K. Knapp, A. McGee, A. Ramos,

M. Romo, N. Rose, J. Schmitzberger, D. Tarrant (Principal Investigator),

R. Wieting, D. Willett

\section{Introduction}

Siemens Solar Industries (SSI), formerly ARCO Solar, has pursued the research and development

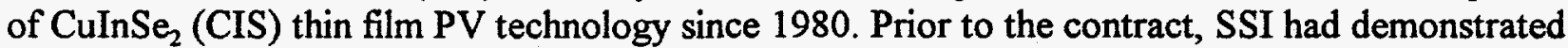
a $14.1 \%$ efficient $3.4 \mathrm{~cm}^{2}$ active-area $\mathrm{ZnO} /$ thin $\mathrm{CdS} / \mathrm{CIS} / \mathrm{Mo} /$ glass cell, unencapsulated integrated modules with aperture efficiencies of $11.2 \%$ on $940 \mathrm{~cm}^{2}$ and $9.1 \%$ on $3900 \mathrm{~cm}^{2}$ and an encapsulated module with $8.7 \%$ on $3883 \mathrm{~cm}^{2}$ (verified by NREL)[1]. During the present phase of this contract, record unencapsulated mini-module efficiencies of $12.7 \%$ on $69 \mathrm{~cm}^{2}$ (verified by NREL) [2] and encapsulated $12.8 \%$ on $68.9 \mathrm{~cm}^{2}$ have been demonstrated. This result meets the efficiency goal for the contract and is the highest module efficiency for any thin film technology. Large area (3860 $\mathrm{cm}^{2}$ ) encapsulated module aperture area efficiency of $10.3 \%$ has also been demonstrated (verified by NREL). Outdoor testing of SSI CIS modules at NREL over a period of more than six years has demonstrated excellent intrinsic module stability.

\section{Objective}

The objective of this research effort is to achieve progress toward the polycrystalline thin-film milestones of the DOE Five Year Plan. The overall project goal is to fabricate a large area, stable, $12.5 \%$ aperture efficient encapsulated CIS module by scalable, low-cost techniques on inexpensive substrates.

\section{Approach}

In Phase 1, the emphasis of the research effort was on establishing the baseline large area CIS module fabrication processes including detailed characterization of the cells and interconnects. Environmental durability of laminated and framed large-area CIS modules was also demonstrated [3].

In Phase 2, the initial focus was to implement the processing and characterization methods demonstrated in Phase 1 to meet the contract deliverables schedule and to produce statistically significant quantities of large area modules for outdoor and accelerated environmental testing. Over $23001 \times 4$ foot CIS modules were fabricated. Although the process produced high-efficiency cells and modules, average performance was not adequate for fabrication of representative prototype products. The origins of module reproducibility problems were unclear. Large area module fabrication was temporarily suspended to determine the critical issues affecting yield losses in module fabrication by using small area test devices $\left(3.3 \mathrm{~cm}^{2}\right.$ active area cells) and modules $\left(60 \mathrm{~cm}^{2}\right.$ 
aperture area) as the experimental test bed. This shift greatly enhanced the ability to identify root causes of yield loss in module fabrication. The most critical issues determining module yield were grouped into three major categories:

1. The uniformity and reproducibility of the absorber formation process dominates the fundamental performance of the material over a large area.

2. The interaction of the substrate with the films requires appropriate selection criteria and preparation techniques for minimizing defects that lead to shunting and areas of poor photoresponse.

3. Performance losses near interconnects can reduce module performance and can cause inadequate performance through module durability testing.

The issues in categories one and two were addressed through the adoption and further refinement of a graded absorber formation process in which the surface layer of CIS incorporates sulfur [4], combined with the appropriate substrate preparation. Performance losses near interconnects were addressed by developing alternate techniques for fabricating interconnects. Interconnects were made after the formation of the absorber by application of insulating and/or conducting materials [1]. Prior to development of these alternate techniques, "classic" interconnects incorporated a pattern in the back electrode prior to the reaction of precursors to form CIS [3]. The alternate patterning techniques culminated in the demonstration of a CIS record $11.7 \%$ aperture area efficiency integrated mini-module [1].

In Phase 3, the three critical issues defined in Phase 2 have been continued and extended. Substrate and base electrode preparation techniques have been developed which minimize the number of defects that lead to shunting, areas of poor photoresponse and losses near interconnects. These Phase 3 techniques are distinct from the techniques developed in Phase 2. Understanding the impact of interactions between the substrate, base electrode, precursors and the reaction process has eliminated the need for the alternate patterning techniques developed in Phase 2. This has allowed the return to "classic" patterning which is a more production capable interconnect formation method and has resulted in record module efficiencies. Current efforts are focused on reduction of variation using statistical process control methods, characterization of performance changes due to thermal and light-induced effects and definition of the strategy for next generation large area reaction systems.

\section{Results and Discussion}

OBIC images of gridded test structure devices are shown in Figure 1 for the substrate and base electrode preparation techniques used in Phases 1 and 2 versus Phase 3 . Each image includes three devices. Three dark horizontal lines are visible on each device due to grid shadowing. A scratch which crosses the lower two devices in each image simulates the affect of patterning the base electrode for a module. For the preparation techniques used in Phases 1 and 2, the affect of the scratch is clearly visible as a linear feature with degraded performance. A large number of randomly located low performance dark spots are also visible which are due to defects in the base electrode or substrate [5]. Similar effects around actual interconnects dictated the development of the post absorber formation techniques for fabricating interconnects during Phase 2. For the preparation 
techniques used in Phase 3, the scratch has minimal affect on performance; there are few defects related to the base electrode or substrate. Development of this electrode preparation technique was expedited by process screening based on observation of alternative base electrodes after oxidation in moist air.

Modifications of the reaction process were necessary to avoid loss of adhesion with the Phase 3 substrate and back electrode preparation techniques. SIMS depth profiles indicate a significantly reduced degree of reaction of the base electrode, particularly with selenium. The combined effect of improvements in substrate/base electrode preparation techniques and reaction process have allowed the return to the more production capable "classic" patterning, reduced the number of substrate-induced defects, and resulted in record module efficiencies with an encapsulated $12.8 \%$ efficient minimodule on $68.9 \mathrm{~cm}^{2}$ and a NREL-verified $12.7 \%$ efficient unencapsulated circuit on $69 \mathrm{~cm}^{2}$.

The major challenge to move from development to production is to improve reproducibility rather than to improve champion device performance. Therefore, emphasis has been placed on reduction of process variation. The reduction of variation efforts are based on the application of the statistical process control (SPC) discipline, improved diagnostics, and thorough protocols. Using statistical analysis has demonstrated that the largest source of variation in the present process is due to the block of process steps from CdS dip through final patterning (Figure 2). The second largest source of variation is from the block of process steps prior to the reaction process. The reaction process and testing introduce relatively low variation for this data set.

Reduction of variation efforts also accelerate progress in other tasks by providing a consistent and well defined baseline process. This will have a positive impact on the two other current major tasks, characterization of thermal and light induced effects [6] and definition of the strategy for next generation large area reaction systems.

\section{Conclusions and Future Directions}

The foundations have been laid to meet the polycrystalline thin-film milestones of the DOE Five Year Plan. The efficiency goal of the contract has been met with the demonstration of a record encapsulated $12.8 \%$ efficient module on $69 \mathrm{~cm}^{2}$. Outdoor testing has demonstrated excellent intrinsic module stability. Future plans include scaling these results to larger areas and emphasizing the reduction of variation methodology to lay the foundation for demonstrating the potential of CIS as a future commercial product.

\section{References}

1.) K. Knapp, et al, "Research on High Efficiency, Large-Area CuInSe - Based Thin-Film Modules", Anmual Report, NREL Photovoltaic Program FY 1993, NREL/TP-410-6585, pp 110-113.

2.) R. Gay, et al, "Efficiency and process Improvements in CuInSe ${ }_{2}$-Based Modules", 12th European Photovoltaic Solar Energy Conference, April 1994, pp 935-938.

3.) C. Eberspacher, et al, Annual Report: Photovoltaic Subcontract Program FY 1992, 
NREL/TP-410-5335, March 1993, pp. 138a-138d.

4.) D. Tarrant, J. Ermer, "I-III- $\mathrm{VI}_{2}$ Multinary Solar Cells Based on CuInSe ${ }_{2}$ ", 23rd IEEE PVSC, 1993, pp. 372-378.

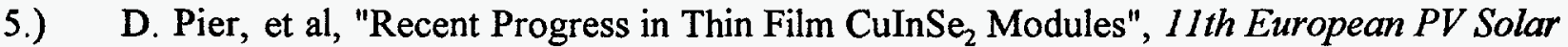
Energy Conference, Oct 1992, pp. 107-111.

6.) D. Willett, S. Kuriyagawa, "The Effects of Sweep Rate, Voltage Bias and Light Soaking on the Measurement of CIS-Based Solar Cell Characteristics", 23rd IEEE PVSC, May 1993, pp. 495-500.
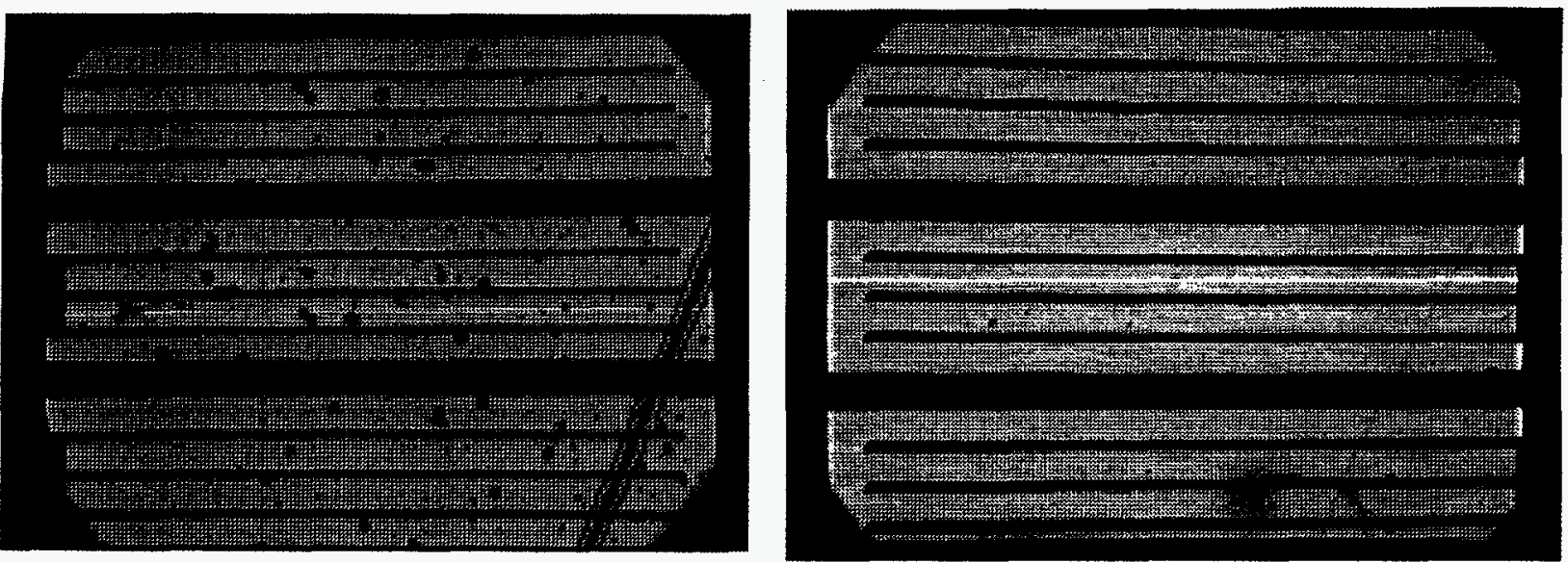

Figure 1.) OBIC images representative of Phases 1 and 2 (left) and Phase 3 (right) substrate and base electrode preparation techniques.

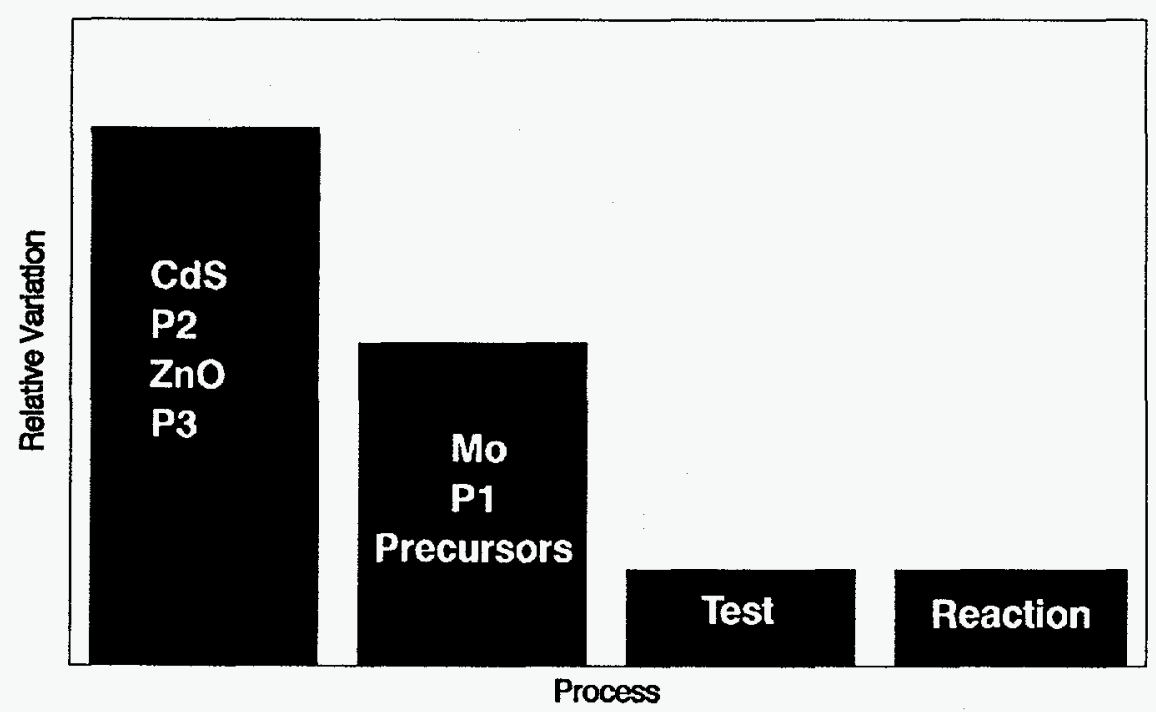

Figure 2.) Relative variation for groups of process steps (P1, P2 and $\mathrm{P} 3$ refer to patterning steps.) 
Title:

Organization:

Contributors:
Fabrication of Stable Large Area Thin Film Cadmium Telluride Photovoltaic Modules

Solar Cells, Inc. Toledo, Ohio

Theodore X. Zhou, Principal Investigator; R.C. Powell, R. Sasala, K. Bihn, L. Deutsch, M. Steele, R. Harju, N. Reiter, G. Rich

\section{Objective}

The overall objective of this project is to demonstrate fabrication of stable large area thin film CdTe photovoltaic modules. Goals for FY 1994 include improvement of device and module efficiency and stability, verification of product life time and further optimization of the process to meet the need of high throughput manufacturing.

\section{Approaches}

Two main tasks have been addressed in FY 1994. In the earlier stage of this project SCI recognized that $\mathrm{CdTe} / \mathrm{CdS}$ device stability could be strongly affected by the choice of back contact materials and process. Various back contact schemes have been tested and improved. The effort of process optimization has been largely focused on seeking alternatives to the conventional heat treatment of $\mathrm{CdTe}$ with $\mathrm{CdCl}_{2}$.

\section{Results}

During FY 1994 both module and cell efficiency have increased. The best NRELconfirmed $0.72 \mathrm{~m}^{2}$ module output at STC is $60.3 \mathrm{~W}$, corresponding to $8.4 \%$ total area efficiency. The best confirmed $1.1 \mathrm{~cm}^{2}$ cell is $11.9 \%$, with Voc $=0.82 \mathrm{~V}, \mathrm{Jsc}=19.6$ $\mathrm{mA} / \mathrm{cm}^{2}, \mathrm{FF}=74.4 \%$. Individual IV parameters such as Voc $\sim 0.85 \mathrm{~V}, \mathrm{FF}>75 \%$ have been reached on different devices. The improvement was achieved mainly by reducing the CdS layer thickness. As indicated by the IV characteristics (low Jsc) and QE measurements the conversion efficiency is still limited by optical losses in the CdS/TCO window layers.

A second 1kW array was installed near SCI's Toledo, Ohio facilities in September, 1994. The new array consists of 24 modules, the same as in array \#1, with a different panel design. Fig. 1 shows the array I-V curve measured at approximately $817 \mathrm{~W} / \mathrm{m}^{2}$ and $25^{\circ} \mathrm{C}$ module temperatue. Total area efficiency is $7.4 \%$, equivalent to an output of $1286 \mathrm{~W}$ at $1 \mathrm{~kW} / \mathrm{m}^{2}$. This represents a $38 \%$ improvement in average module efficiency over array \#1 which was measured $931 \mathrm{~W}$ when installed in Spring 1993.

Various back contact schemes have been studied. Modules and cells prepared with different processes have been subject to a wide range of stress conditions including light intensity, bias voltage and temperature. Fig. 2 plots the efficiency history of one of the 
longest stress-tested ( $>13,000$ hours) $8 \mathrm{~cm} \times 8 \mathrm{~cm}$ mini-modules under Xenon lamp at intensity $\sim 80 \mathrm{~mW} / \mathrm{cm}^{2}$ with resistive load near $P_{\max }$. The sample temperature varies between 50 to $70^{\circ} \mathrm{C}$, similar to outdoor conditions in the summer. This test is equivalent to field operation of more than 6 years, assuming a factor of 5 acceleration. Several SCI modules monitored at NREL also demonstrate good stability (see Fig. 3).

In process optimization SCI has successfully developed alternative methods to replace the conventional $\mathrm{CdCl}_{2}$ heat treatment ${ }^{1}$. The conventional method usually includes: 1) Application of $\mathrm{CdCl}_{2}$ in methanol solution; 2) Heat treatment in air at about $400{ }^{\circ} \mathrm{C}$; 3) Removal of $\mathrm{CdCl}_{2}$ residue from the treated film. In an alternative method, vaporized $\mathrm{CdCl}_{2}$ is applied to $\mathrm{CdTe}$ surface maintained at $\sim 400{ }^{\circ} \mathrm{C}$ in $\mathrm{N}_{2}$ immediately after deposition and the residue can be removed by evaporation following the treatment. Other alternative methods include applying hydrogen chloride $(\mathrm{HCl})$ or chlorine gas to heated $\mathrm{CdTe}$ surface in a controlled atmosphere. The $\mathrm{HCl}$ treatment appears to be easier to control and will be further pursued and incorporated into the manufacturing process.

\section{Future Work}

SCI plans to improve cell and module efficiency by reducing the CdS window thickness from $>2000 \AA$ to about $1000 \AA$. Quantum Efficiency measurements will be used to estimate the CdS thickness. Deposition parameters will be adjusted to ensure uniform coating of thin CdS over $0.72 \mathrm{~m}^{2}$ superstrate. It is also anticipated that the postdeposition process, particularly the chloride treatment, will be further optimized to accommodate films with thinner CdS layers.

In order to issue a meaningful product warranty, it is SCI's goal to demonstrate the module lifetime with the $90 / 90 / 10$ formula, that is, $90 \%$ of modules should retain $90 \%$ of the initial power output performance after 10 years of field operation. This will be accomplished by a combination of data extrapolation and accelerated testing. Possible temperature and light intensity dependence of aging will be investigated in order to better quantify stress acceleration.

\footnotetext{
${ }^{1}$ Proceedings, First World Conference of Photovoltaic Energy Conversion, to be published.
} 

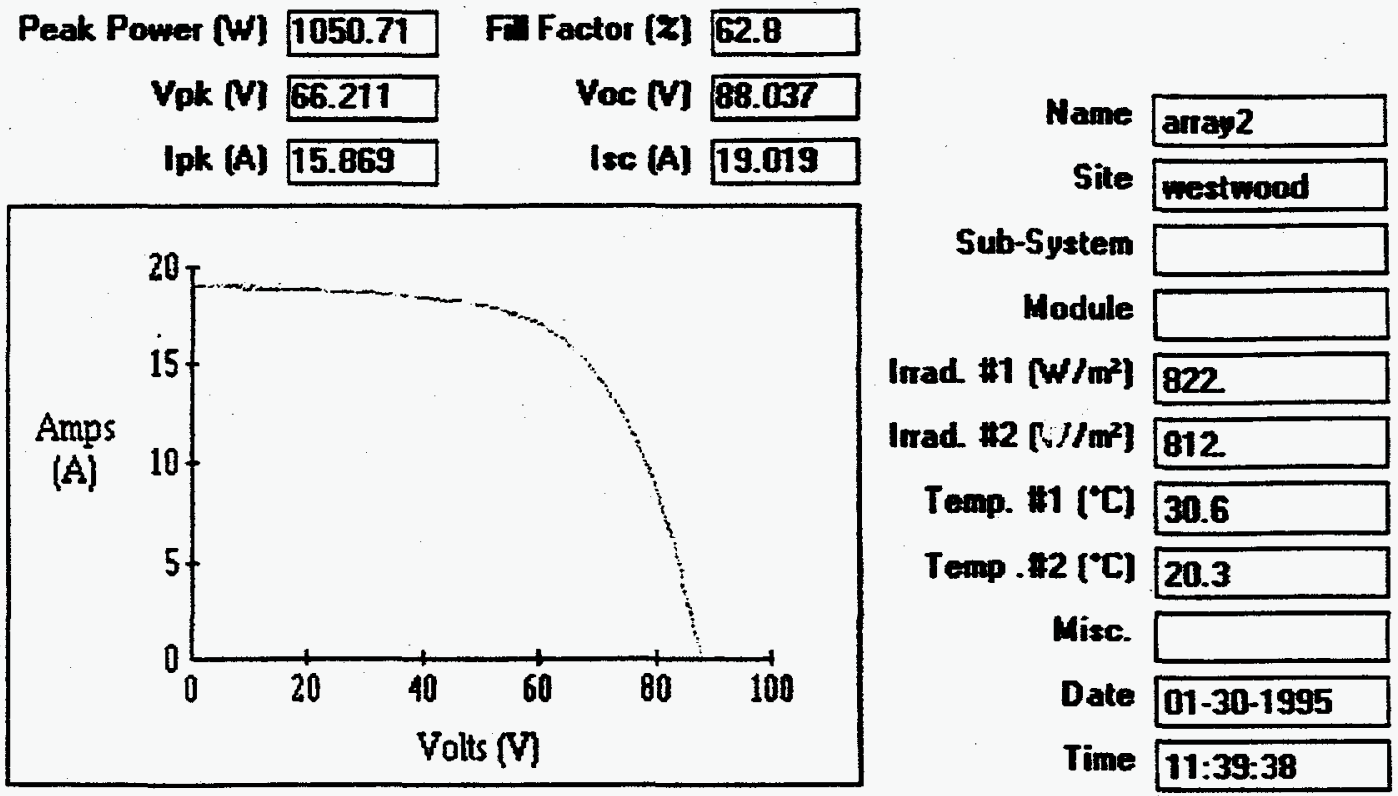

Figure 1: I-Vcharacteristics of SCI outdoor array \#2. The average total area efficiency $=7.4 \%$, equivalent to $1286 \mathrm{~W}$ output at $1 \mathrm{~kW} / \mathrm{m}^{2}$ illumination.

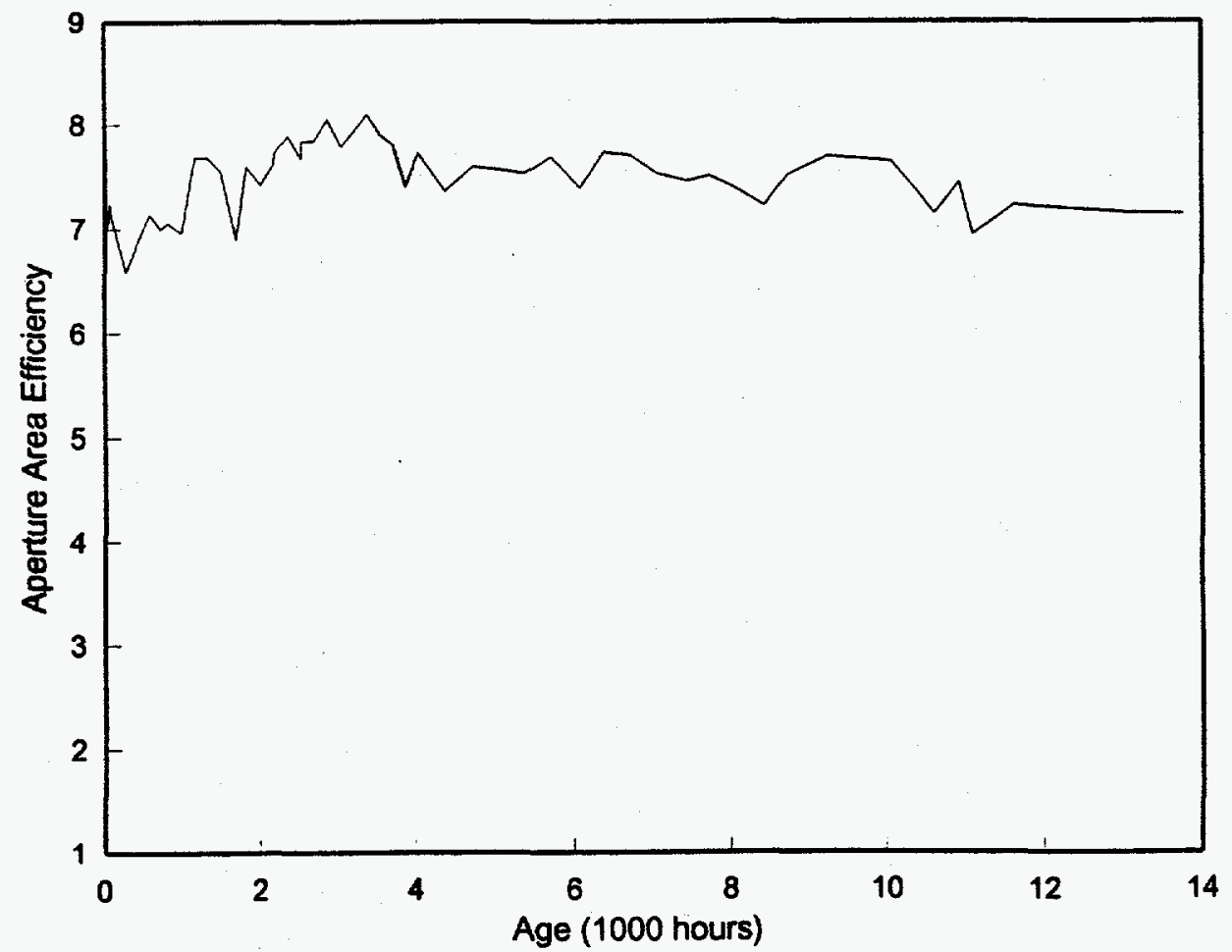

Figure2: Efficiency history of an $8 \mathrm{~cm} \times 8 \mathrm{~cm}$ mini-module under continuous light soak with reisistive load at maxmimum power point. 


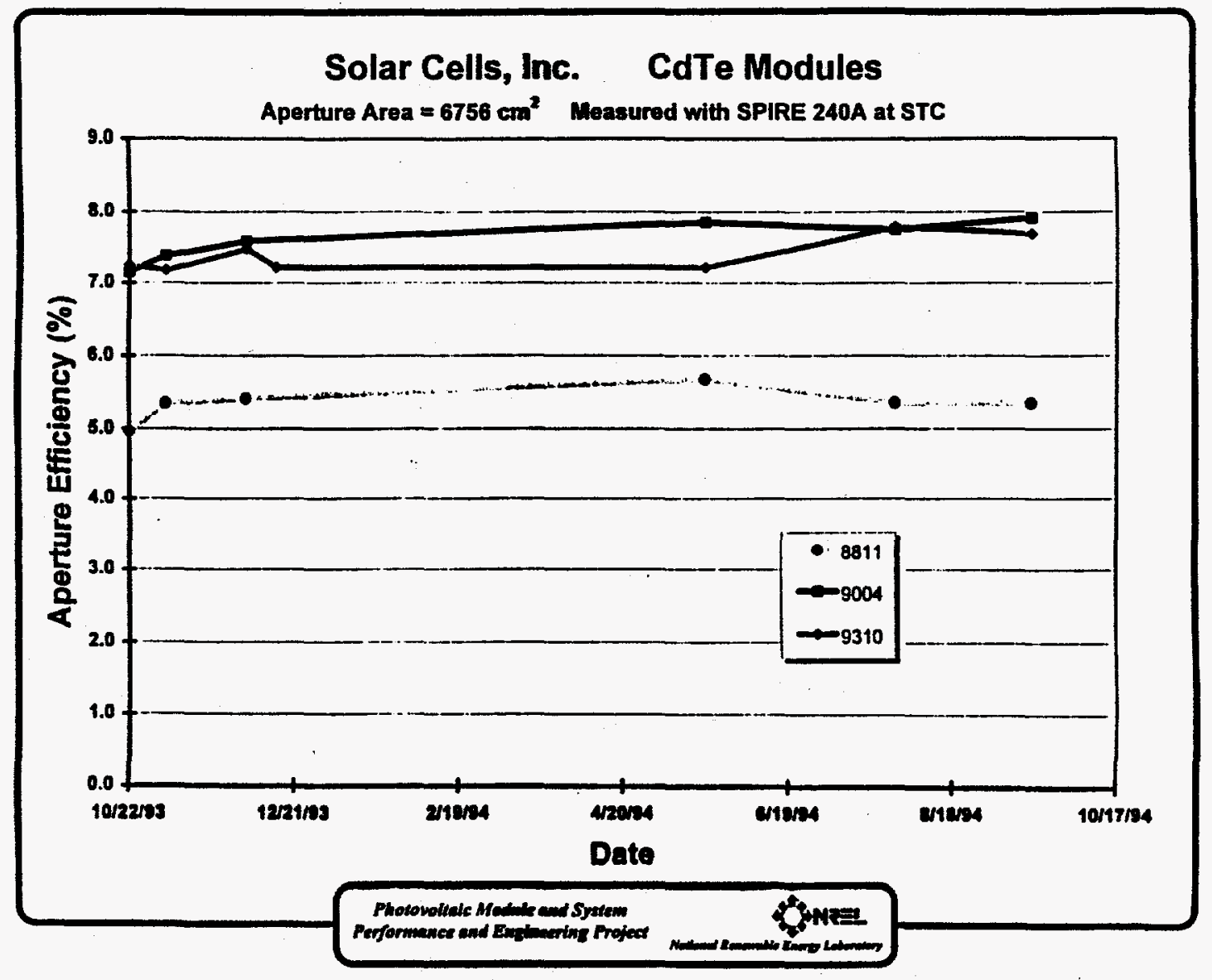

Figure 3: Efficiency history of SCI modules monitored at NREL. 
Title:

Organization:

Contributors:
Research on Stable, High-Efficiency Amorphous Silicon Multijunction Modules

United Solar Systems Corp.

Troy, Michigan

S. Guha, principal investigator; A. Banerjee, E. Chen, R. Clough, T. Glatfelter, G. Hammond, K. Hoffman, M. Hopson, N. Jackett, M. Lycette, J. Noch, T. Palmer, I. Rosenstein, D. Wolf, X. Xu, J. Yang, and K. Younan.

\section{Objective}

The principal objective of the three-phase program is to conduct research on semiconductor materials and non-semiconductor materials to enhance the performance of multi-bandgap, multijunction, large-area amorphous silicon-based alloy modules. The goal is to demonstrate stabilized module efficiency of $10 \%$ for multijunction panel of area greater than $900 \mathrm{~cm}^{2}$.

\section{Approach}

Double-junction and triple-junction cells are made on $\mathrm{Ag} / \mathrm{ZnO}$, back reflector deposited on stainless steel substrates. a-SiGe alloy is used for the i-layer in the bottom and the middle cells; the top cell uses a-Si alloy. After evaporation of antireflection coating, silver grids and bus bars are put on the top surface, and the panel is encapsulated in a EVA/Tefzel structure to make a one-square-foot monolithic module.

\section{Status/Accomplishments}

In the final phase of the program which ended August, 1994, we have continued to make progress drawing upon the advances made during the earlier phases. The major thrust was on the fabrication of triple-junction one-square-foot modules. Five modules were sent to NREL for evaluation; two unexposed and three after one-sun light soaking for 1000 hours at $50^{\circ} \mathrm{C}$. Results from the indoor and outdoor (corrected for spectral mismatch) measurements are shown in Table I. Both sets of measurements are in good agreement. The initial efficiency of $11.75 \%$ is the highest for any thin film module as confirmed by NREL. The stable efficiency of $10.17 \%$ (Fig. 1) is also the highest stabilized efficiency reported for a-Si alloy module. Needless to mention, the achievements were made possible only through the systematic and innovative work carried out during the earlier phases. These include (i) optimization of component cells through innovative material development and cell design, (ii) improvement of the "tunnel" junctions between the component cells, (iii) obtaining better uniformity of the deposited layers over onesquare-foot area and (iv) reducing module losses.

Achieving $10 \%$ stable module efficiency has been considered to be a milestone for thin-film photovoltaic. In order to make further improvement in module efficiency, it is necessary to improve material and device properties to obtain higher component cell efficiency. We have used 
hydrogen dilution to improve the material characteristics and have made significant improvement in the initial performance of the component cells. The results are shown in Table II where the previous best results (that went into the development of the $10 \%$ stable module) are also shown. The component cells were measured under the appropriate illumination conditions for the triplecell configuration. We note significant improvement in efficiency for all the component cells. Efforts are under way to translate the improvements in small-area cell efficiency to large-area modules.

\section{References}

1. S. Guha, J. Yang, A. Banerjee, T. Glatfelter, K. Hoffman, S.R. Ovshinsky, M. Izu, H.C. Ovshinsky, and X. Deng, Mat. Res. Soc. Symp. Proc. 336, 645 (1994).

2. J. Yang, X. Xu, and S. Guha, Mat. Res. Soc. Symp. Proc. 336, 687 (1994).

3. S. Guha, X. Xu, and J. Yang, Mat. Res. Soc. Symp. Proc. 336, 675 (1994).

4. A. Banerjee, J. Yang, T. Glatfelter, K. Hoffman, and S. Guha, Appl. Phys. Lett. 64, 1517 (1994).

5. A. Banerjee, K. Hoffman, X. Xu, J. Yang, and S. Guha, First World Conf. on PV Energy Conversion Proc., 1994 (in press).

6. J. Yang, A. Banerjee, T. Glatfelter, K. Hoffman, X. Xu, and S. Guha, First World Conf. on PV Energy Conversion Proc., 1994 (in press).

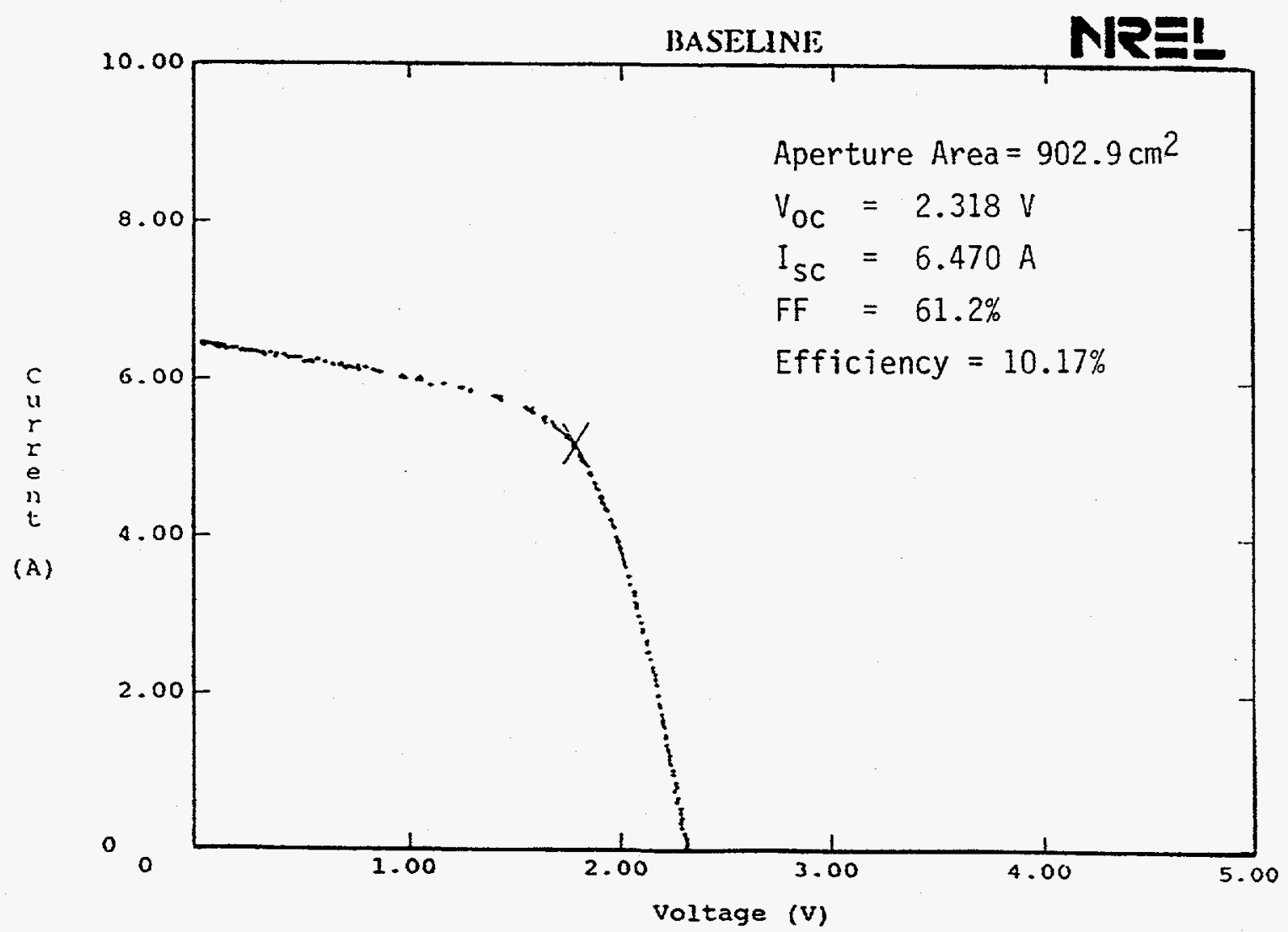

Fig. 1. Current-Voltage Characteristic of the $10.2 \%$ Module. 
Table I. Summary of Triple-junction Module Results as Measured by NREL.

\begin{tabular}{|c|c|c|c|c|c|c|c|}
\hline $\begin{array}{l}\text { Module } \\
\text { No. }\end{array}$ & $\begin{array}{l}\text { Exposure } \\
\text { Condition }\end{array}$ & $\begin{array}{l}\text { Aporture } \\
\text { Area }\left(\mathrm{cm}^{2}\right)\end{array}$ & $\begin{array}{l}V_{\infty} \\
(V)\end{array}$ & $\begin{array}{l}I_{\infty} \\
\text { (A) }\end{array}$ & $\mathbf{F F}$ & $\begin{array}{l}\text { Efficiency } \\
(\%)\end{array}$ & $\begin{array}{l}\text { Outdoor } \\
\text { Corrected } \\
\text { Efficiency } \\
(\%)\end{array}$ \\
\hline 2452 & $\begin{array}{l}\text { Unexposed } \\
\text { (Initial) }\end{array}$ & 905.9 & 2.400 & 6.568 & 0.675 & 11.75 & 11.6 \\
\hline 2465 & $\begin{array}{l}\text { Unexposed } \\
\text { (Initial) }\end{array}$ & 902.9 & 2.395 & 6.757 & 0.652 & 11.69 & 11.9 \\
\hline 2437 & $\begin{array}{l}1000 \text { hours } \\
\text { (Stabilized) }\end{array}$ & 902.9 & 2.354 & 6.196 & 0.629 & 10.16 & 10.4 \\
\hline 2445 & $\begin{array}{l}1000 \text { hours } \\
\text { (Stabilized) }\end{array}$ & 899.9 & 2.349 & 6.337 & 0.607 & 10.04 & 10.2 \\
\hline 2447 & $\begin{array}{l}1000 \text { hours } \\
\text { (Stabilized) }\end{array}$ & 902.9 & 2.318 & 6.470 & 0.612 & 10.17 & 10.3 \\
\hline
\end{tabular}

Table II. Initial Photovoltalc Characteristics for Improved and Prevlously Reported Component Cells Measured under Conditions Described in the Text.

$\begin{array}{cccc}\underset{\mathrm{J}_{\infty c}}{\left(\mathrm{~mA} \mathrm{~cm}^{2}\right)} & V_{\infty} & F F & \begin{array}{c}P_{\max } \\ (\mathrm{mW})\end{array}\end{array}$

\begin{tabular}{llllll}
\hline & A & 8.65 & 1.014 & 0.780 & 6.84 \\
& B & 7.3 & 1.01 & 0.75 & 5.53 \\
& A & & & \\
Middle* & B & 9.51 & 0.757 & 0.719 & 5.18 \\
& B & 7.02 & 0.77 & 0.65 & 3.51 \\
& A & & & & \\
& B & 9.91 & 0.692 & 0.646 & 4.43 \\
& 7.8 & 0.67 & 0.64 & 3.34 \\
\hline
\end{tabular}

* deposited on stainless steel substrates without back reflector

A improved characteristics

B previous characteristics 
Title:

Organization:

Contributors:
Photocharge Transport and Recombination Measurements in Amorphous Silicon Films and Solar Cells by Photoconductive Frequency Mixing

Physics Department, University of California at Los Angeles, Los Angeles, CA 90024-1547

R. Braunstein, Principle Investigator; Yi Tang and Shirun Dong

\section{Program Outline}

The objective of this program is to separately determine the lifetime and mobility in photovoltaic materials and solar cells by the technique of photoconductive frequency mixing. Measurements are performed on as-grown materials and materials under light soaking conditions on high and mid-bandgap alloys and solar cell devices. These measurements separately determine the effects of recombination time, and mobility, which determine the optimization of the materials for photovoltaic devices and contributes to our understanding of the Staebler-Wronski instability in these materials and devices. The photomixing technique consists of heterodyning two monochromatic laser beams when a dc bias is applied which results in a photocurrent current composed of a dc and microwave photomixing current; these two photocurrents allow a determination of the drift mobility $\left(\mu_{d}\right)$ and lifetime $(\tau)$ of the dominant photo-generated carriers [1-5]. A-Si:H, a-SiC:H and a-SiGe:H films obtained from various "round robin" sources are characterized by the photomixing technique and selected layers are in situ light-degraded and a detailed quantitative analyses of the photoconductive parameters as a function of light induced degradation are carried out to be used as input to modeling studies of solar cell performance.

This report covers the period from $5 / 15 / 94$ to $12 / 31 / 94$.

\section{Accomplishments:}

The photoconductivity, lifetime, and drift mobility in intrinsic a-Si:H and a-SiC:H prepared by varies glow techniques while light soaking were determined using the photomixing technique. In addition to the decay of the photoconductivity and lifetime, continuous decay of the electron drift mobility was found during the light soaking process, which reveals a new phenomenon associated with the Staebler-Wronski effect. The experimental decay data can be fitted to stretched exponential laws; different stretched-exponential parameters for the photoconductivity, lifetime and mobility were obtained, which indicates the production of defects with different generation kinetics upon light soaking. Figures 1 and 2 show the decay of the lifetime and the mobility for a typical glow discharge produced a-Si:H, and Figures 3 and 4 show the decay of the mobility of glow discharge and hot wire samples. A detailed discussion of these results can be found in FY1994 Report [6] and in a paper submitted for publication to the Phys. Rev. [7]. The most widely accepted explanation of the Staebler-Wronski degradation of the photocurrent has been that the production of midgap defects which decrease the recombination lifetime but not the mobility. The present results suggests that charged defects are produced and can become quasi-stable through some relaxation processes and can form certain long-range potential 
fluctuations which become the dominant scattering mechanism for the decay of the drift mobility upon light soaking.

Extensive studies on the effects of the deposition conditions, such as deposition temperature and hydrogen dilution ratio on the structural and electronic properties of a-Si:H and a-SiC:H films have been performed in an attempt to improve the quality of these films for photovoltaic applications. The experimentally available transport data for these films, however, are normally limited to conductivities and $\mu \tau$ product which are convolutions of a few physical parameters. With the unique advantage of the photomixing technique, we were able to experimentally determine the drift mobility $\left(\mu_{d}\right)$ and lifetime $(\tau)$ of a-Si:H and a- SiC:H films and conduction band Urbach energy as a function of deposition temperature and hydrogen dilution ratio. Figure 5 shows the results for a-Si:H while Figure 6 shows the results for a-SiC:H; the details of this work has been reported in [8].

We have observed the electric field dependence of the drift mobilities and lifetime in annealed and light degraded a-Si:H produced by glow discharge and hot wire processes for fields in the range from $2000 \mathrm{~V} / \mathrm{cm}$ to $9000 \mathrm{~V} / \mathrm{cm}$. The mobilities were found to increase and the lifetimes was found to decrease with dc bias, while the $\mu \tau$ products remained essentially independent of dc bias. These results indicate the existence of long range potential fluctuations [9]. The decrease of lifetime with increasing $\mathrm{dc}$ bias and the independence of the $\mu \tau$ product on the dc bias indicate diffusion limited recombination in these samples. It was observed that the increase of mobility is much less significant in the light soaked state than in the annealed state. Measurements of the electric field dependence on annealed and light soaked samples are summarized in Figure (7). The $(\mu \tau)_{\mathrm{LS}} /(\mu \tau)_{\mathrm{AN}}$ of the abscissa represents the ratio of the $\mu \tau$ product in the light soaked state after 4 hours at 4 sun intensity to the product in the initial annealed state. Consequently the smaller this ratio the less stable the sample is against light-soaking. The $\mathrm{d} \mu / \mathrm{dE} / \mu$ of the ordinate represents the normalized initial slope of the electric field dependence of the mobility. It should be noted that in general the samples have a greater field dependence in the annealed state compared the lightsoaked state. It should be noted that the sample THD15, the hot wire NREL sample with $1 \%$ hydrogen dilution is more stable than sample THD16 (HW) with $10 \%$ hydrogen dilution against light degradation. The electric field dependence as shown from the ordinate values indicate that the smaller field dependence in the annealed state would predict a more stable sample. The above results may provide a method to characterize stability without going through the time consuming long light soaking process! However, this observation should be regarded as preliminary until a more systematic series of samples have been evaluated.

\section{Conclusions}

The photoconductive frequency mixing technique with its capability of separately determining the drift mobility and lifetime in photo-voltaic materials is enabling further insights to be gained in the microscopic understanding of transport in these materials. This has served as a powerful technique to evaluate the optimum processes for preparing efficient and stable materials for photovoltaic devices. The major task for future work is to perform measurements on systematic series of samples to indicate the optimum preparation process. In addition we plan to extend the present photoconductive frequency measurements to solar cell devices. 


\section{References}

1. E. R. Giessinger, R. Braunstein, S. Dong, and B. G. Martin, J. Appl. Phys. 69,1469 (1991).

2. Yi Tang, R. Braunstein, and B. von Roedern, Mat. Res. Soc. Symp. Proc. 258, 735 (1992).

3. Yi Tang, R. Braunstein, B. von Roedern, and F. R. Shapiro, Mat. Res. Soc. Symp. Proc. 297. 407 (1993).

4. R. Braunstein and Yi Tang, Proceedings of the 21st International Conference on the Physics Semiconductors, (August 1992), Beijing, China (World Scientific, Singapore, 1992), Vol. 1, P. 269.

5. Yi Tang and R. Braunstein, and B. von Roedern, Appl. Phys. Lett. 63(17), 2393 (1993).

6. First Quarterly Report on Subcontract \#- XAN-4-13318-10 15 May 1994 - 15 August 1994.

7. Yi Tang and R. Braunstein, Submitted to Phys. Rev.

8. Yi Tang and R. Braunstein, J. Appl. Phys. Lett. 66(6), 721 (1994).

9. H. M. Branz and M. Silver, Phys. Rev. B 427420 (1990).

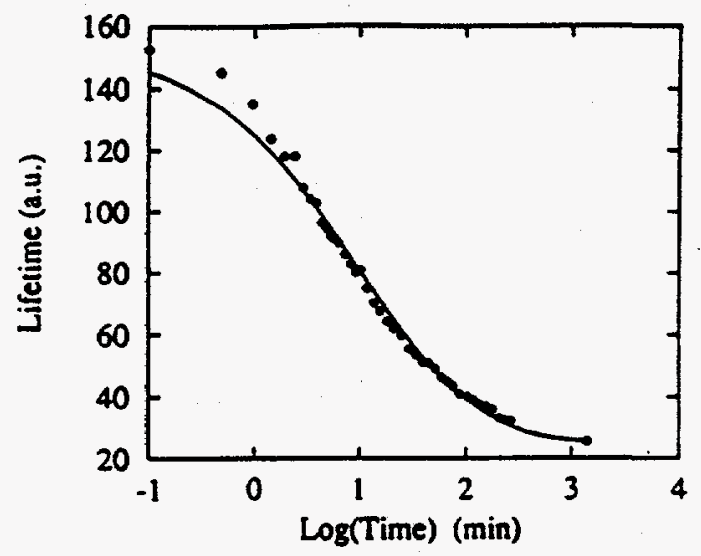

Figure 1. Lifetime for an intrinsic a-Si:H sample versus light soaking time.

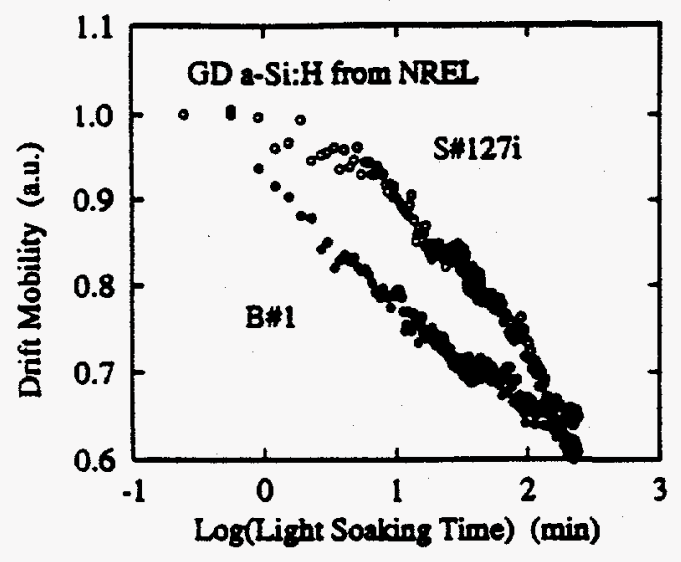

Figure 3. Mobility for glow discharge a-Si:H sample versus light soaking time.

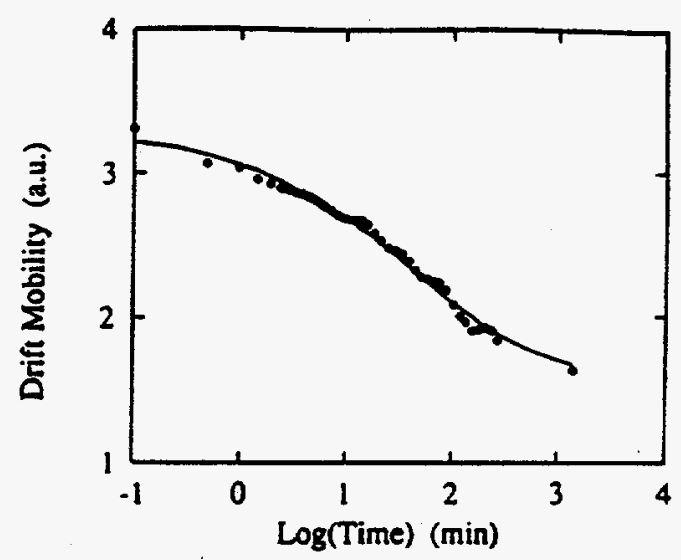

Figure 2. Mobility for an intrinsic a-Si:H sample versus light soaking time.

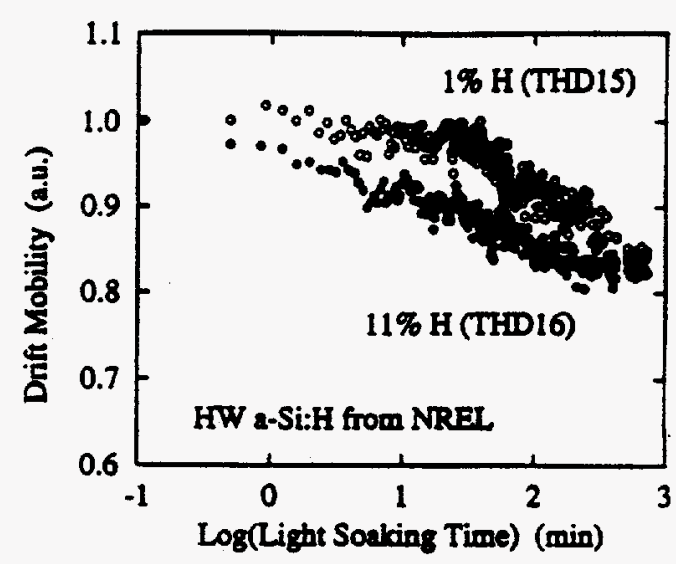

Figure 4. Mobility for hot wire a-Si:H sample versus light soaking time. 


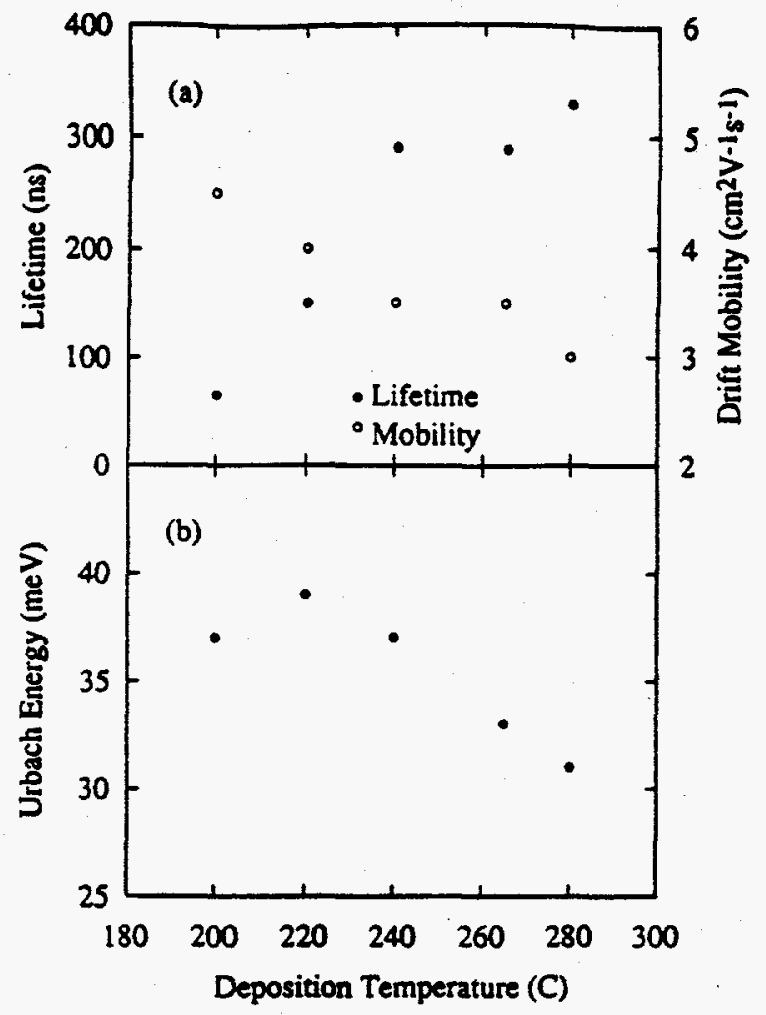

Figure 5. Electron lifetime (a), drift mobility (a) and conduction band Urbach energy (b) of a-Si:H films as a function of deposition temperature.

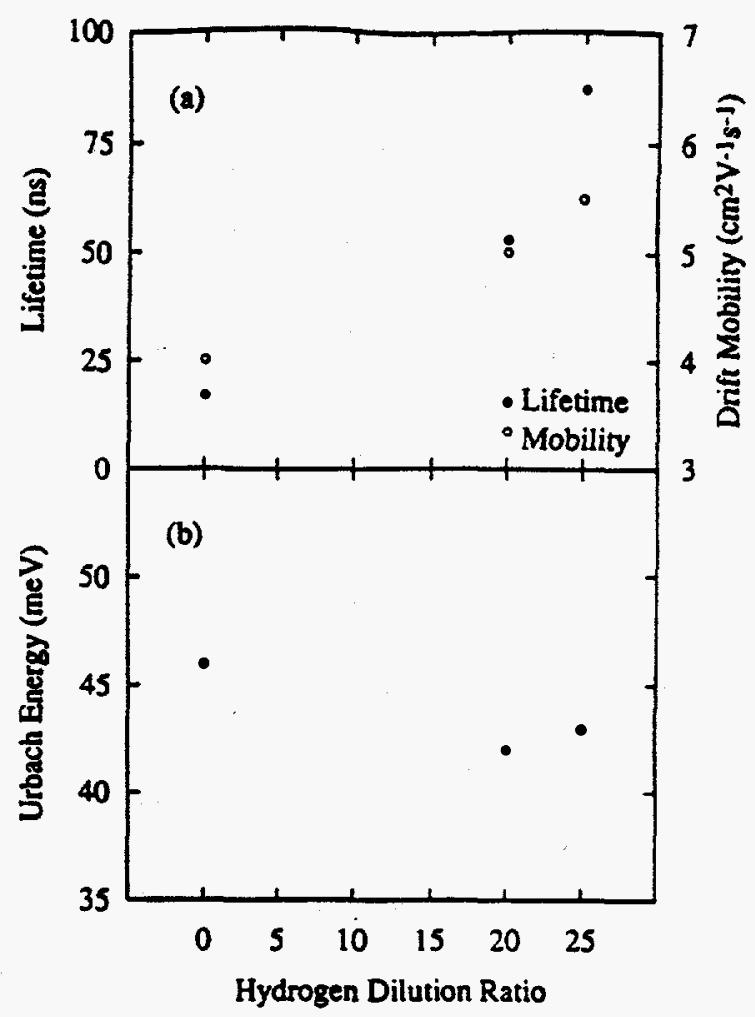

Figure 6. Electron lifetime (a), drift mobility (a) and conduction band Urbach energy (b) of a-SiC:H films as a function of hydrogen dilution ratio.

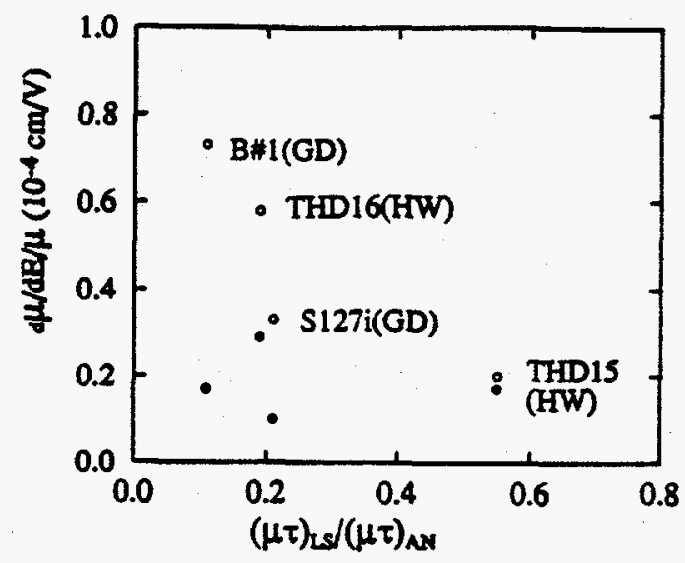

Figure 7. Field dependance versus stability for glow discharge (GD) and hot wire (HW) a-Si:H samples.Open circles for the annealed state and solid circles for light soaked state (for 4 hours and 4 sun). 
Organization:

Department of Physics

University of Colorado

Boulder, CO 80309-0390

Contributors:

A.M. Hermann (principal investigator), Arturo Fernandez, Scott Morrison and Jerry Wagner

Background: The work carried out under this subcontract focussed during 1993 on the use of thermally evaporated precursors (principally in the deposition sequence $\mathrm{In}+\mathrm{Ga}+\mathrm{Se} / \mathrm{Cu}+\mathrm{Se} / \mathrm{In}+\mathrm{Ga}+\mathrm{Se}$ ) to form $\mathrm{Cu}(\mathrm{In}, \mathrm{Ga}) \mathrm{Se}_{2}$ (CIGS) films for CIGS-based solar cells. The experimental work was carried out on-site at NREL (in Rommel Noufi's group) by Andrew Gabor who achieved a world-breaking efficiency of $15.9 \%$ total area efficiency reported in the NREL PV Program Review for 19931. During 1994, Andrew Gabor continued his dissertation research under the direction of the P.I. and was supported by the Associated Western Universities (AWU). The results are therefore reported separately in this Program Review.

The emphasis for the continuation research supported by NREL during 1994 related to development of a non-vacuum process -- namely, the use of electrodeposition -- which uses binary precursors, similar to those established in the vacuum process used for the high efficiency $\mathrm{CdS/CIGS} \mathrm{cell} \mathrm{fabrication.} \mathrm{Electrodeposition} \mathrm{is} \mathrm{an} \mathrm{inexpensive,} \mathrm{non-vacuum} \mathrm{process,} \mathrm{scalable} \mathrm{in}$ principle to large areas. The program was conducted both at NREL (by Arturo Fernandez with input from Rommel Noufi and Raghu Battacharya) and on-site at the University of ColoradoBoulder laboratories of the P.I. (by Arturo Femandez, Scott Morrison and Jerry Wagner).

Objectives: The major objective of the electrodeposition work during 1994 was to deposit pin-hole free smooth films of $\mathrm{Cu}, \mathrm{In}, \mathrm{Cu}+\mathrm{Se}$, and $\mathrm{In}+\mathrm{Se}$ which will provide the base of knowledge needed for a multilayer deposition sequence similar to that used in the successful vacuum evaporation experiments. The formation of CIS and CIGS will then be accomplished in a post-deposition thermal treatment.

Technical Approach: A PAR 371 potentiostat/ integrated-circuit voltage follower was used at CU to deposit $\mathrm{Cu}$ and In films onto Mo-coated soda-lime glass substrates and onto Mo foil substrates at room temperature. The typical cathode area was one square centimeter, and the distance between the anode (Pt mesh) and the cathode was two-three centimeters. Sulfate salts of the metals were used in aqueous solution in concentrations of 1-100 millimolar. A calomel electrode served as the reference electrode. The use of a stirrer minimized bubbling due to the hydrolysis of water in the potentiometric mode (-1 volt vs $S C E$ ). Smooth mirror-like copper films were deposited; indium films were significantly rougher (white in appearance). Current experiments are aimed at the deposition of In $2 \mathrm{Se} 3$ using indium sulfate/selenous acid mixtures onto Mo-coated glass and onto $\mathrm{Cu}$ films which have been electrodeposited onto Mo-coated glass.

In the NREL labs we have electrodeposited smooth $500 \mathrm{~A}$ Cu layers onto Mo-coated glass sub- 
strates with a $\mathrm{Cu}^{+2}$ concentration of $0.05 \mathrm{M}$ at -0.6 (vs SCE) in 60 seconds. We have also deposited $\mathrm{Cu}-\mathrm{Ga}$ alloy layers with $\mathrm{Cu}^{+2}$ and $\mathrm{Ga}^{+3}$ in concentration of $0.01 \mathrm{M}$. EMPA analysis has showed the presence of some oxygen in the films. The $\mathrm{In}_{2} \mathrm{Se}_{3}$ layers were electrodeposited onto substrates composed of glass $/ \mathrm{Mo} / \mathrm{Cu}(500 \mathrm{~A})$ using two different chemical baths. Tables 1 and 2 show the composition of the baths.

Results: Tables 3 and 4 show the results of the compositional analysis for different samples prepared at different experimental conditions. The analysis techniques used were EMPA, SEM and ICP. In these tables one can see the tendency of saturation in the composition of $\operatorname{In}_{2} \mathrm{Se}_{3}$. Analysis using XPS and Auger are in progress.

$\mathrm{Cu}$-Se layers were electrodeposited directly onto Mo-coated glass substrates. The experimental conditions are shown in Table 5. Also in this table are the results of the EMPA. The studies made with EMPA and SEM indicate the morphology varies if the $\mathrm{pH}$ of the solution is changed.

Figure 2 shows the SEM photos for the samples described in Table 5. Note that the morphology differs if the $\mathrm{pH}$ of the solution is changed. At $-0.4 \mathrm{~V}$ (vs SCE) the film surfaces have more crystallites.

Conclusions: Excellent progress has been made toward the electrodeposition of binary (In-Se,CuSe) film precursors for subsequent thermal processing to form CIS and CIGS films for the fabrication of high efficiency CdS/CIS and CdS/CIGS photovoltaic devices. Future work will target the thermal post-deposition annealing and its corresponding materials science issues for fabrication of photovoltaic-quality films.

Table 1

Composition of chemical bath using $\mathrm{InCl}_{3}$

$$
\begin{gathered}
\mathrm{InCl}_{3}=0.025 \mathrm{M}, \\
\mathrm{H}_{2} \mathrm{SeO}_{3}=0.025 \mathrm{M},
\end{gathered}
$$

The $\mathrm{pH}$ is 1.5 (adjusted with $\mathrm{HCl} 10 \%$ )

The temperature of deposition is $22 \infty \mathrm{C}$

Table 2

Composition of chemical bath using $\mathrm{In}_{2}\left(\mathrm{SO}_{4}\right)_{3}$

$$
\begin{gathered}
\mathrm{In}_{2}\left(\mathrm{SO}_{4}\right)_{3}=0.025 \mathrm{M}, \\
\mathrm{H}_{2} \mathrm{SeO}_{3}=0.025 \mathrm{M},
\end{gathered}
$$

The $\mathrm{pH}$ is 1.5 (adjusted with $\mathrm{H}_{2} \mathrm{SO}_{4} 10 \%$ ). 
The temperature of deposition is $22 \infty \mathrm{C}$.

Table 3

Composition Analysis for the experimental conditions shown for electrodeposition of $\operatorname{In}_{2} \mathrm{Se}_{3}$ using the chemical bath described in Table 1 .

\begin{tabular}{lllllll} 
Sample No. & $\begin{array}{c}\mathrm{V} \\
\text { (vs SCE) }(\mathrm{sec})\end{array}$ & \multicolumn{2}{c}{ (Q) } & In & $\begin{array}{c}\text { Se } \\
(\%)\end{array}$ & $\begin{array}{l}\text { EMPA } \\
\text { results }\end{array}$ \\
94121605 & -0.76 & 300 & 1.84 & 22.1872 & 50.7033 & $\mathrm{In}_{1.31} \mathrm{Se}_{3}$ \\
94121601 & -0.8 & 300 & 1.88 & 28.6454 & 48.6689 & $\mathrm{In}_{1.76} \mathrm{Se}_{3}$ \\
94112107 & -0.9 & 300 & 2.19 & 34.0322 & 49.0904 & $\mathrm{In}_{2.07} \mathrm{Se}_{3}$ \\
94110403 & -1.05 & 300 & 3.44 & 37.2481 & 53.0771 & $\mathrm{In}_{2.01} \mathrm{Se}_{3}$
\end{tabular}

Table 4

Composition analysis for the experimental conditions shown for electrodeposition of $\mathrm{In}_{2} \mathrm{Se}_{3}$ using the chemical bath described in Table 2 .

$\begin{array}{rlllccl}\begin{array}{r}\text { Sample No. V } \\ \text { (vs SCE) }\end{array} & \begin{array}{l}\text { time } \\ (\mathrm{sec})\end{array} & \begin{array}{c}\text { charge } \\ (\mathrm{Q})\end{array} & \begin{array}{l}\text { In } \\ (\%)\end{array} & \begin{array}{c}\text { Se } \\ (\%)\end{array} & \begin{array}{l}\text { EMPA } \\ \text { results }\end{array} \\ 94121613 & -0.76 & 300 & 1.839 & 19.3541 & 61.9836 & \mathrm{In}_{0.94} \mathrm{Se}_{3} \\ 94121614 & -0.8 & 300 & 1.93 & 30.0797 & 48.5920 & \mathrm{In}_{1.86} \mathrm{Se}_{3} \\ 94121610 & -0.9 & 300 & 2.25 & 31.7758 & 47.5415 & \mathrm{In}_{2.0} \mathrm{Se}_{3} \\ 94121609 & -1.05 & 300 & 5.23 & 37.6679 & 58.8537 & \mathrm{In}_{1.93} \mathrm{Se}_{3}\end{array}$

Figure 1 shows the surface of the samples of Table 4.

Note that the grains are dense and compact.

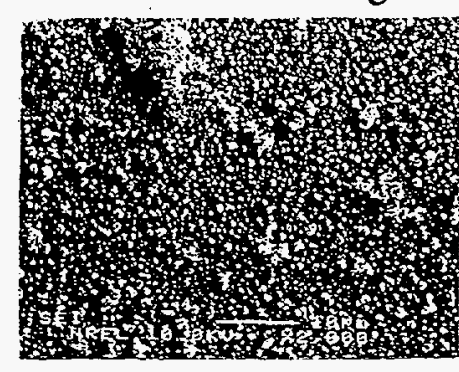

94121613

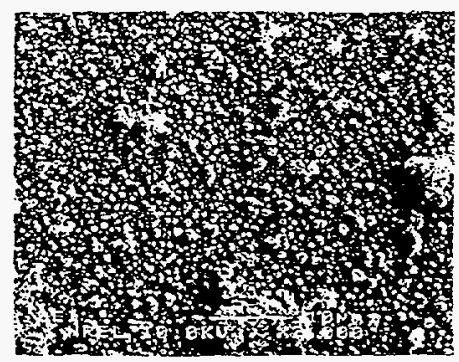

94121610

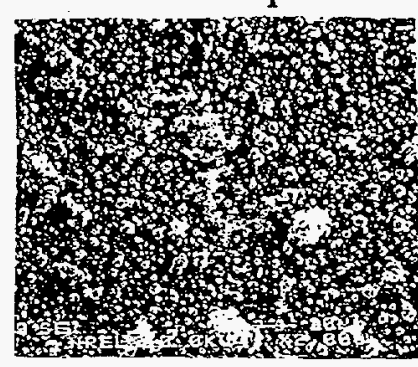

3? ?:514

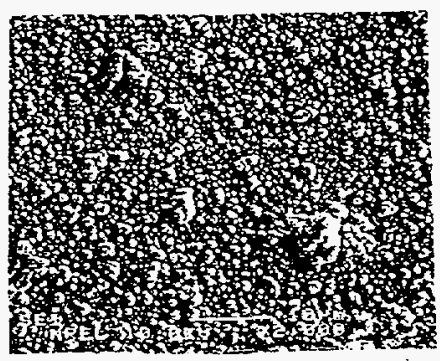

34121609 
Figure 1. SEM photos of films of $\mathbb{I N}_{2} \mathrm{Se}_{3}$ described in Table 4. The magnification is $2000 \mathrm{X}$.

Table 5

Composition analysis of $\mathrm{Cu}$-Se electrodeposited onto Mo-coated glass substrates. The chemical bath consisted of ions of $\mathrm{Cu}^{+2}$ and $\mathrm{Se}^{-4}$. The $\mathrm{pH}$ was adjusted with a solution of $\mathrm{H}_{2} \mathrm{SO}_{4}(10 \%)$.

$\begin{array}{lllllllll}\begin{array}{l}\text { Sample } \\ \text { No. }\end{array} & \begin{array}{l}\text { (vs SCE) } \\ \text { time }\end{array} & \text { (sec) } & \text { (Q) } & \text { (Q) } & \begin{array}{l}\mathrm{pH} \text { of } \\ \text { solution }\end{array} & \begin{array}{l}\mathrm{Cu} \\ (\%)\end{array} & \begin{array}{l}\mathrm{Se} \\ (\%)\end{array} \\ 94122902 & -0.1 & 300 & 3.05 & 1.7 & 57.6978 & 42.2175 & \mathrm{Cu}_{1} \mathrm{Se}_{0.73} \\ 94122901 & -0.4 & 300 & 3.49 & 1.7 & 48.4920 & 51.4476 & \mathrm{Cu}_{1} \mathrm{Se}_{1.06} \\ 94122802 & -0.6 & 300 & 4.72 & 1.7 & 47.1953 & 52.7643 & \mathrm{Cu}_{1} \mathrm{Se}_{1.11} \\ 94122905 & -0.2 & 300 & 1.26 & 2.4 & 49.4743 & 26.6462 & \mathrm{Cu}_{2} \mathrm{Se}_{1.07} \\ 94122904 & -0.4 & 300 & 2.09 & 2.4 & 51.6189 & 49.1945 & \mathrm{Cu}_{1} \mathrm{Se}_{0.95} \\ 94122906 & -0.6 & 300 & 1.762 & 2.4 & 62.4580 & 26.9877 & \mathrm{Cu}_{2} \mathrm{Se}_{0.86} \\ 94122907 & -0.8 & 300 & 1.611 & 2.4 & 62.4580 & 26.9877 & \mathrm{Cu}_{2} \mathrm{Se}_{0.99}\end{array}$

Figure 2. These SEM photos correspond to samples of $\mathrm{Cu}-\mathrm{Se}$. The experimental conditions are listed in Table 5. The magnification is $2000 \mathrm{X}$.
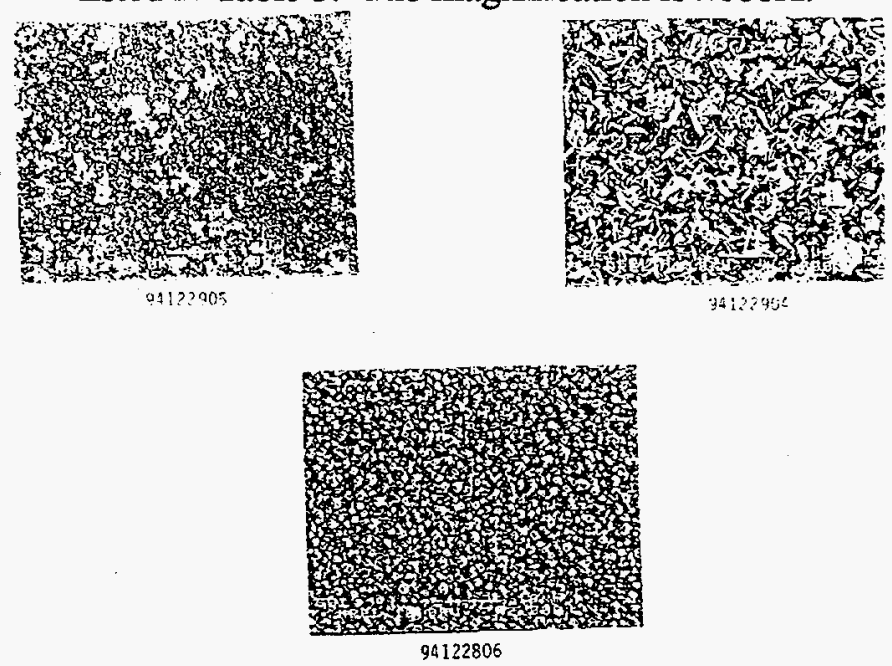

References:
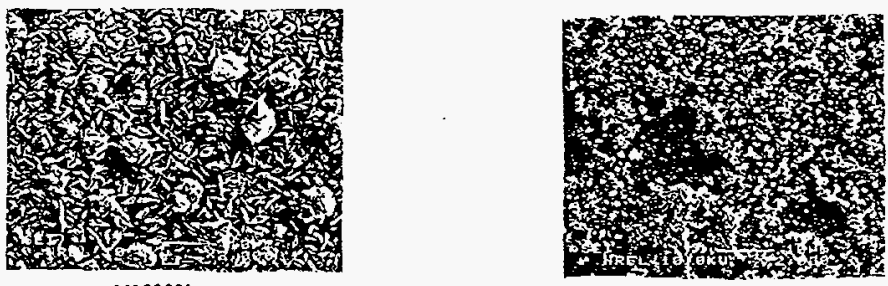

94122901

94122906

1. Annual Report, Photovoltaic Program. FY 1993. (Sept., 1994). NREL/TP-410-6285. 318 pp. 
Title: Experimental Study of Photodegradation in a-Si:H Solar Cells*

Organization: Department of Physics \& Astronomy, University of North Carolina at Chapel Hill, Chapel Hill, North Carolina

Contributors: $\quad$ D. X. Han, principal investigator; L. E. McNeil, co-principal investigator; K. D. Wang, research associate

\section{Objective and Outline}

Our objective is to understand recombination and metastability in amorphous silicon alloys, especially in p-i-n device structures. Our research project is primarily aimed at determining the position and precursor states involved in the SWE, and to gain a better understanding of the relationship between material characteristics and solar cell performance.

It is well-know that the SWE increases the defect density in the intrinsic i-layer and decreases the mobility-lifetime product, $\mu \tau$. Contrary to expectations, film properties obtained from $I R, S A X S$, and $C P M$ experiments do not correlate well with cell performance ${ }^{1}$ To understand this, we measured and characterized the light-induced effects (SWE) in device structures.

During the contract year, March 1, 1994 - Feb. 28, 1995, we continued our experiments on electroluminescence (EL) and transient forward bias current as well as photogain measurements before and after light soaking. We have collected the key equipment (a pulse dye laser and an optical table) for electronic profile measurements. We are pursuing vigorous collaborative efforts : With Dr. Vanderhahgen on $\mu \tau$-product from photogain as a function of junction properties, with Prof. Adriaenssens, on the SWE on charge transport in p-i-n cells by transient forward current. with Prof. McNeil, we studied bulk and/or interface effects by comparing PL to EL spectroscopy, and with Prof. Wu, we worked on H-configuration in hot-wire material by NMR. Five papers have been accepted for publication as a result of this last year's research. ${ }^{2-6}$

\section{The Most Interesting \& Important Research Results}

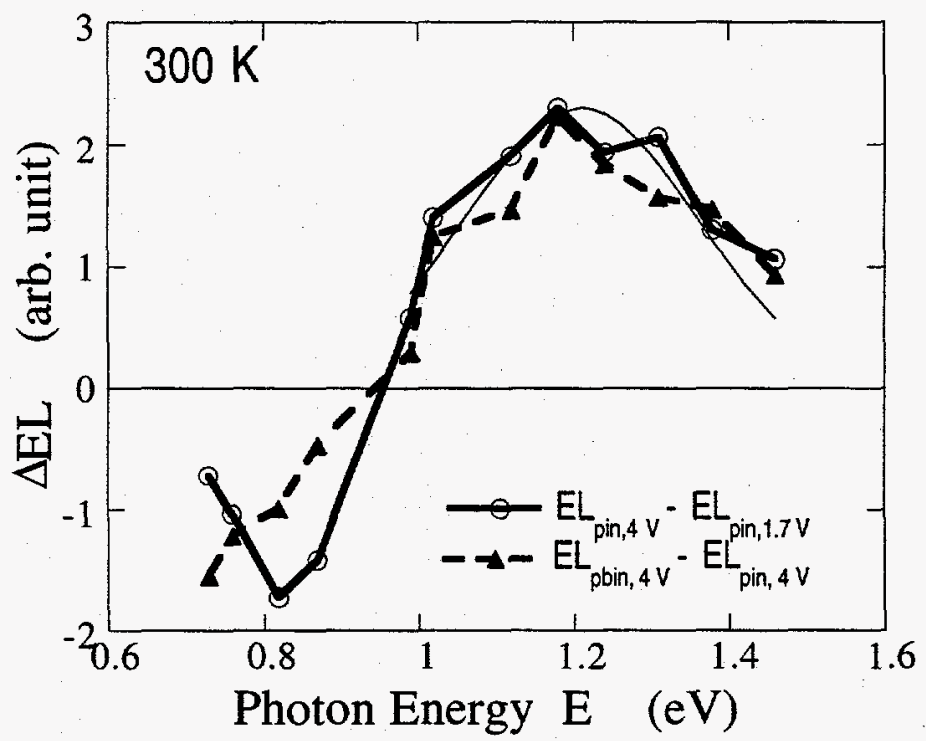

Fig 1 Compares the EL spectral difference at $300 \mathrm{~K}$. EL spectral difference, $\triangle E L$, between SLX26 and SLX-25 cells under $4 \mathrm{~V}, \mathrm{EL}_{\mathrm{pbin}, 4 \mathrm{~V}}-\mathrm{EL}_{\mathrm{pin}, 4 \mathrm{~V}}$, is denoted by triangles; $\triangle \mathrm{EL}$ under 4 $\mathrm{V}$ and $1.7 \mathrm{~V}$ in a p-i-n cell, EL4V - EL $1.7 \mathrm{~V}$, is denoted by open circuits.

*NREL Subcontract No. Xan-4-13318-09 
EL is a result of carrier recombination both in the bulk and junction region. We have studied the function of buffering layer by EL energy spectra. Under the same electric field across the i-layer in both p-i-n SLX-25 and p-b-i-n SLX-26 cells, the EL spectra show an increase of the mainband luminescence in the buffered cells. This is related to improved open circuit voltage, $V_{O c}$ (see table I). Previously, we found that the EL main-band luminescence was enhanced by increasing the applied voltage. ${ }^{7}$ More recently, we found that an increase of $V_{\text {appl }}$ on SLX-25 can give the same EL spectrum as that from SLX-26. Fig. 1 shows that the required applied voltage is just sufficient to shift the quasi-Fermi level position by an amount equal to the $\Delta V_{\text {oc }}$. The increase of applied voltag rom $1.7 \mathrm{~V}$ to $4 \mathrm{~V}$ corresponds to a shift of quasi-Fermi level position of $0.045 \mathrm{eV}$ that is the ifference of $\mathrm{V}_{\text {oc }}$ between SLX-26 and -25 (see table I). These results tell us that the value of $V_{o c}$ is closely related to the quasi-Fermi level splitting obtainable in the i-layer and that the buffer layer serves to increase this splitting.

We further found that the line shape of EL energy spectrum is sensitive to the sample preparation conditions. Under the same conditions, at $0.8 \mathrm{~V}, 300 \mathrm{~K}$, state A, the high conversion efficiency cells ECD-522 show a narrower luminescence band than any other SLX and IEC cells. These results in Fig. 2 tell us that for the same density of deep states, $1016 / \mathrm{cm}^{3}$, the state distribution, then the cell performance, are different.

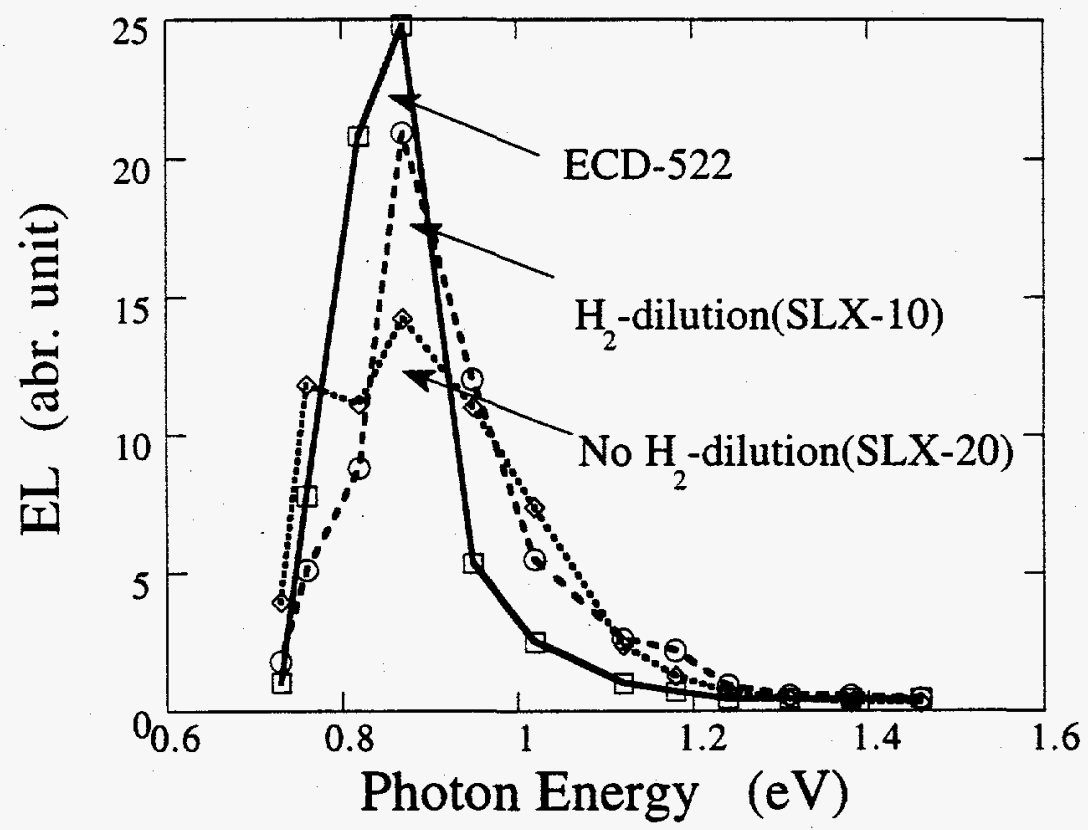

Fig. 2 Comparison of EL spectra between solar cells made at Solarex and ECD. One can see that the EL spectrum of the ECD sample shows a narrow luminescence band around $0.9 \mathrm{eV}$. While the EL spectra show a rather wide band around $0.9 \mathrm{eV}$ for both $\mathrm{H} 2$-dilution and non-dilution cells made at Solarex.

One of the goals of our team is to find the relation between the material parameters and the solar cell performance. We found that the room temperature EL effective efficiency, EL/J $\mathrm{J}_{\mathrm{F}}$ is proportional to the solar cell energy conversion efficiency regardless of preparation conditions. Fig. 3 plots the EL efficiency as a function of the solar cell conversion efficiency for the cells made at Solarex, IEC and ECD. These cells are at State $A$, the $\mu \tau \approx 10^{-5} \mathrm{~cm}^{2} / \mathrm{V}$ for all of the cells, so $\mathrm{EL} / \mathrm{J}_{\mathrm{F}}$ is the effective efficiency of EL. The solar cell parameters are listed in table $\mathrm{I}$. 


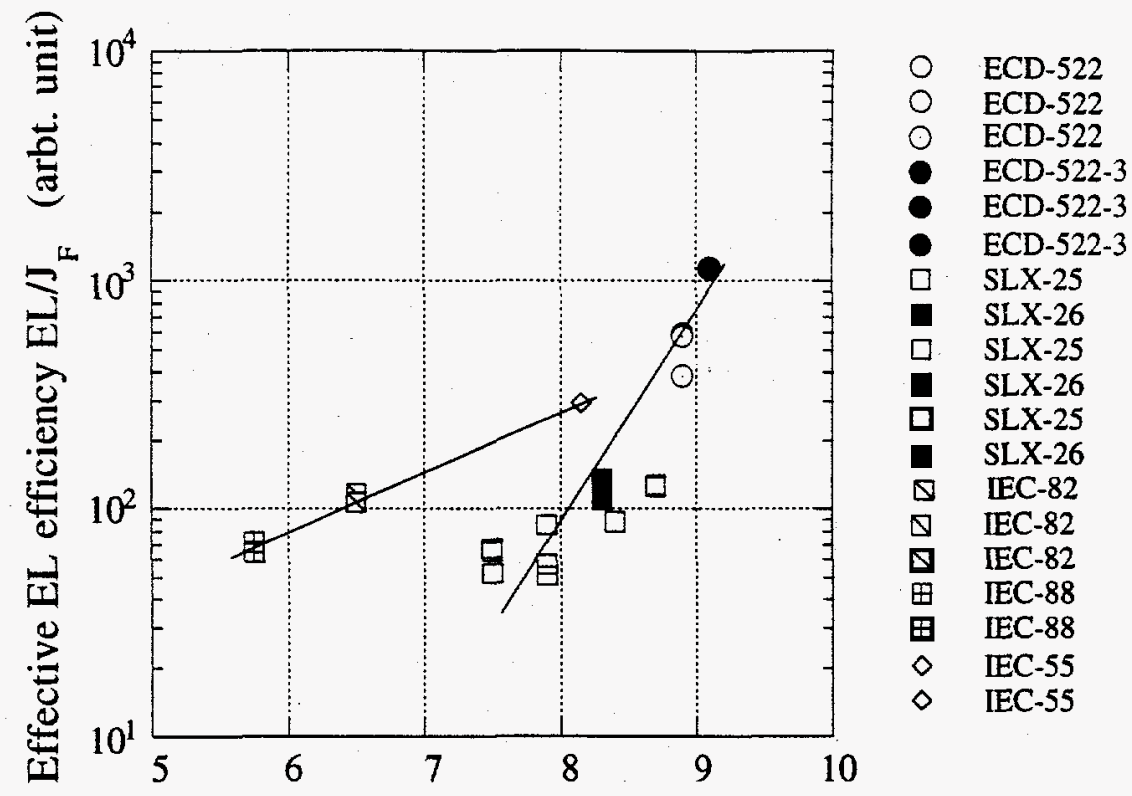

Solar cell energy conversion efficiency

(\%)

Fig. 3 EL efficiency as a function of the solar cell conversion efficiency for the cells listed in Table I. One can see that the data from cells made by PECVD are following the same line. The data from cells made by Photo-CVD are following another line.

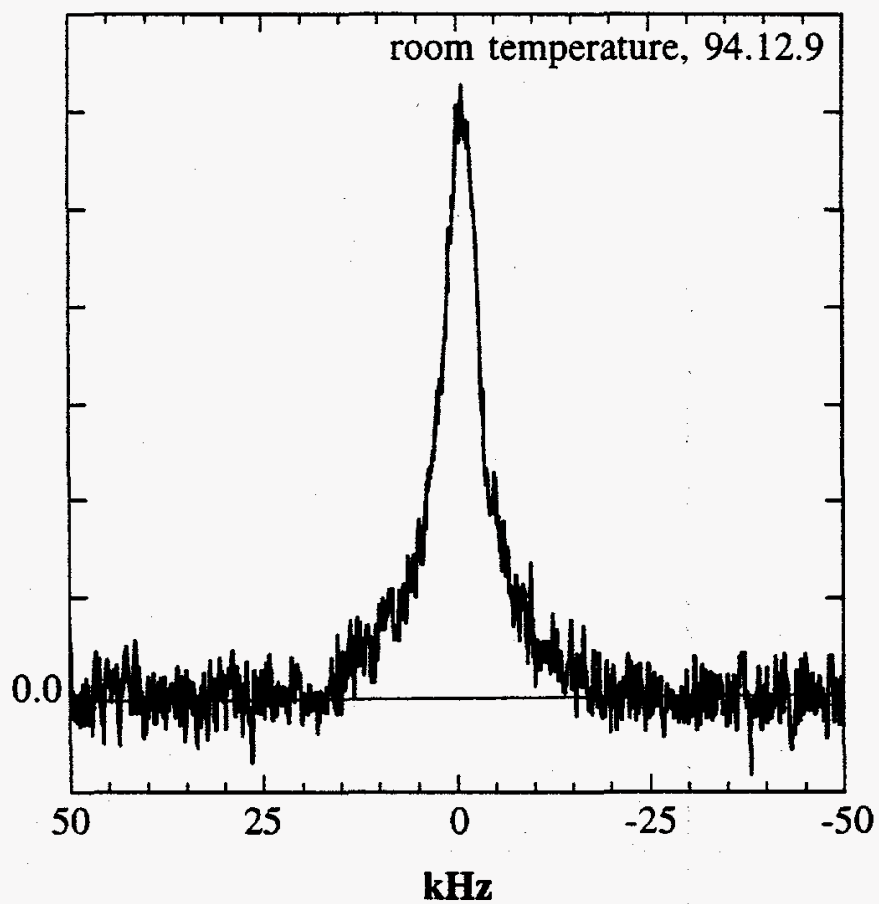

Fig. 4 the NMR spectrum in a hot-wire sample.

Concerning the new material studies, we have preliminary NMR results for the structure of hot- 
wire material that has shown improved stability. Hot-wire film TH125 was deposited on AL at NREL. The film was peeled off by DI water diluted-HCl. A proton-free probe was used for the NMR measurements. Fig. 4 shows the NMR spectrum in a hot-wire sample. A two-component lineshape is ubiquitous, as observed for all a-Si:H films. ${ }^{8}$ The narrow component associates with randomly distributed monohydride groups, the broad component associates with the clustered monohydride groups. Other hydrogenated local bonding configurations also contribute to the broad component. The NMR lineshape in a conventional PECVD a-Si:H contains about $70 \%$ broad component. However, in the hot-wire sample, the percentage of the broad band was as low as $10 \%$ of the total resonance signal. This means $r: 11$ ch less clustered $-H$, dihydrides, trihydrides, and polymeric $\left(\mathrm{Si}-\mathrm{H}_{2}\right)_{\mathrm{n}}$ in the hot-wire fill: This is an experimental evidence that the hydrogen local bonding configuration plays an impurtant role in photodegrada-tion effects. And the randomly distributed monohydride group is more stable than the other group of hydrogenated bonding configurations.

\section{Future Study}

Future work will focus on (1) photodegradation effect on EL spectral line shape in highperformance cells; (2) the H-bonding configurations for new materials.

\section{References}

1. Final Report Photovoltaic Program. Jan. 91-Aug. 94. (Oct. 1994), NREL/TP-411-7190, p.34.

2. Keda Wang, Daxing Han, and M. Silver, "The Power Law Dependence of EL Intensity on Forward Current in a-Si:H p-i-n Devices", in Amorphous Silicon Technology edited by E. A. Schiff, M.Hack, A. Madan, M. Powell, A. Matsuda, MRS Symp. Proc. 336861 (1994).

3. Daxing Han and Keda Wang, "A distinct recombination regime in amorphous silicon diodes under double injection", to be published in Appl. Phys. Lett.(1995.2).

4. Daxing Han and Keda Wang, "Electroluminescence from hydrogenated amorphous silicon $\mathrm{p}-\mathrm{i}-\mathrm{n}$ diodes" to be published in J. Non-Cryst. Sol.

5. Regis Vanderhaghen and Daxing Han, "Interface effects on double injection current and photocurrent in a-Si:H n-i-p and p-i-n diodes", to be published in J. Non-Cryst. Sol.

6. B. Yan, G.J. Adrianenssens, A. Eliat, and D. Han, "Forward current transients in amorphous silicon p-i-n structures", to be published in J. Non-Cryst. Sol.

7. Final Report Photovoltaic Program, Jan. 91-Aug. 94. (July 1994) NREL/TP-451-6491, p.6.

8. J. A. Reimer and M. A. Petrich, in Amorphous Silicon and Related Materials edited by $\mathrm{H}$. Fritzsche (World Scientific, Singapore) p.3.

Table I Sample Preparation Conditions and Cell Performance

\begin{tabular}{|c|c|c|c|c|c|c|c|c|}
\hline $\begin{array}{c}\text { Sample } \\
\text { ID. }\end{array}$ & $\begin{array}{c}\text { Thidkness } \\
(\AA)\end{array}$ & structure & $\begin{array}{c}\text { illayer } \\
\text { Deposition } \\
\end{array}$ & $\begin{array}{c}\text { Hcontent } \\
\text { (at \%) }\end{array}$ & $\begin{array}{l}V_{\infty c} \\
\text { (volt) }\end{array}$ & $\begin{array}{c}\mathrm{J}_{\mathrm{sc}} \\
\left(\mathrm{mAlm}^{2}\right)\end{array}$ & $\begin{array}{c}\text { Fill } \\
\text { Factor }\end{array}$ & $\begin{array}{c}\text { Efficiency } \\
(\%)\end{array}$ \\
\hline ECD-522 & 4,000 & nip & H-dilution & & 0.95 & 17 & 0.56 & 9.0 \\
\hline SLX-25 & 4,000 & $\mathrm{p}-\mathrm{i}-\mathrm{n}$ & puresilane & & 0.81 & $\sim 14$ & 0.66 & 7.5 \\
\hline SLX-26 & 4,000 & $p-b-i n$ & puresilane & & 0.85 & 14.8 & 0.66 & 8.3 \\
\hline SLX-10 & 5,000 & $p-i n$ & H-dilution & & 0.888 & 14.3 & 0.686 & 8.7 \\
\hline SLX-20 & 5,000 & $p-i n$ & pure silane & & 0.862 & 14.2 & 0.684 & 8.4 \\
\hline IEC-82 & 5,300 & $\mathrm{p}-\mathrm{in}_{\mathrm{n}}$ & PhotoCVD & $11 \% \mathrm{H}$ & $0.805-0.815$ & 12 & $0.65-0.67$ & 6.5 \\
\hline EC-88 & 5,000 & $p-i n$ & PhotoCVD & $7 \% \mathrm{H}$ & $0.798-0.800$ & $11.4-124$ & $0.59-0.61$ & $5.5-6$ \\
\hline ECC-55 & 5,500 & $\mathrm{p}-\mathrm{b}-\mathrm{in}$ & PhotoCVD & $7 \% \mathrm{H}$ & $0.840-0.845$ & $13.5-14.5$ & $0.67-0.69$ & $8-8.3$ \\
\hline
\end{tabular}


Title:

Organization:

Contributors:
Microscopic Origins of Metastable Effects in a-Si:H and

Deep Defect Characterization in a-Si,Ge:H Alloys

J. David Cohen, principal investigator; Fan Zhong, Daewon

Kwon, and Chih-Chiang Chen

The primary research goals of this program are to elucidate the basic mect nisms by which a-Si:H degrades with light exposure, and to study the defect structure in low bandgap a-Si,Ge:H alloys. During the past year we have carried out the following studies: (1) A study of glow discharge a-Si,Ge: $\mathrm{H}$ samples produced by the Harvard group encompassing a range of Ge fractions between 0.5 to 1.0 , to determine their deep defect concentrations to compare to a-Si,Ge:H from other sources; (2) A study of light induced degradation in a-Si:H grown under conditions of high hydrogen dilution; and (3) The evaluation of capacitance methods to study deep defect distributions directly in $\mathrm{p}-\mathrm{i}-\mathrm{n}$ device structures.

Approach and Results

Our research related to the Low Gap Team has been focussed on the evaluation of a new series of a-Sil-x $\mathrm{Ge}_{\mathrm{x}}: \mathrm{H}$ alloy samples. This set of 6 samples was obtained from $\mathrm{W}$. Paul's group at Harvard (courtesy of Paul Wickboldt) and had Ge alloy fractions, $x$, ranging between 0.5 to 1.0. Silicon-Germanium alloy samples from the Harvard group were recently found to exhibit superior microstructure as determined by SAXS measurements [1]. Thus it seemed important to compare their electronic properties with a-Si ${ }_{1-\mathbf{x}} \mathrm{Ge}_{\mathbf{x}}: \mathrm{H}$ material from the USSC and IEC groups which we had characterized previously [2,3,4].

We are still at a fairly early stage in carrying out our measurements on this series of samples. However, our examination of the overall deep defect densities using drive-level capacitance profiling suggests electronic properties that may be superior to samples obtained from previous sources in this alloy range. In Fig. 1 we plot a comparison of the drive-level determined mid-gap defect densities between these Harvard samples and the USSC and IEC a-Si ${ }_{1-x} \mathrm{Ge}_{\mathbf{x}}: \mathrm{H}$ samples obtained previously. Here we see almost an order of magnitude improvement in deep defect density compared to either of these earlier series of samples for the $50 \%$ Ge fraction samples, and an even greater improvement for the higher alloy samples.

One is tempted to link this improvement with the superior microstructure that is exhibited by the Harvard samples. However, in Fig. 2 we compare the photocapacitance and photocurrent spectra taken at 330K for a Harvard and a USSC grown a-Si,Ge:H sample with similar compositions. This measurement temperature is sufficiently high that minority carrier transport plays an important role in these spectra $[2,3,4]$. Such transport leads to a different magnitude of the two types of spectra in the bandtail region relative to the deep defect region. It also can lead to a significantly different shape of the defect band signal (region for optical energies less than 1.2eV). Both these characteristics are clearly observed for the spectra of the USSC sample shown in Fig. 2(a). The marked dip in the photocapacitance spectra near $1.0 \mathrm{eV}$ has been attributed to an optical transition between filled valence band states and $\mathrm{D}^{+}$states within the gap.[4] Such a feature has been present in almost all of the a-Si,Ge:H samples we have obtained from USSC and from IEC Delaware. 
The photocurrent spectrum for the Harvard sample shown in Fig. 2(b) is almost identical to the photocurrent spectrum for the USSC sample. Both show a Urbach tail region with a characteristic energy of roughly $50 \mathrm{meV}$. Both also show a defect band shoulder (the region below $1.1 \mathrm{eV}$ ) at nearly the same magnitude. This is in spite of the much lower defect density obtained for the Harvard sample from the drive-level capacitance profiling measurements.

On the other hand, the photocapacitance spectrum appears nearly identical to the photocurrent spectrum for the Harvard sample, quite unlike the USSC ease. The spectra for the USSC sample do become nearly identical for lower temperature as they do for all of our samples) because the escape distance for minority carriers out of $t$ e depletion region is suppressed. Therefore, it appears that minority carrier transport is significantly impeded in this Harvard sample compared to the USSC samples of similar composition. However, since at this point we have obtained detailed sub-band-gap spectra on only a single Harvard sample, we still regard such a conclusion as somewhat preliminary.

FIG. 1. Comparison of drive-level determined deep defect densities for Harvard samples and a-Si,Ge:H samples obtained from other sources. The error bars indicate the extent of the spatial variation in the defect profiles over distances of roughly $0.7 \mu \mathrm{m}$.
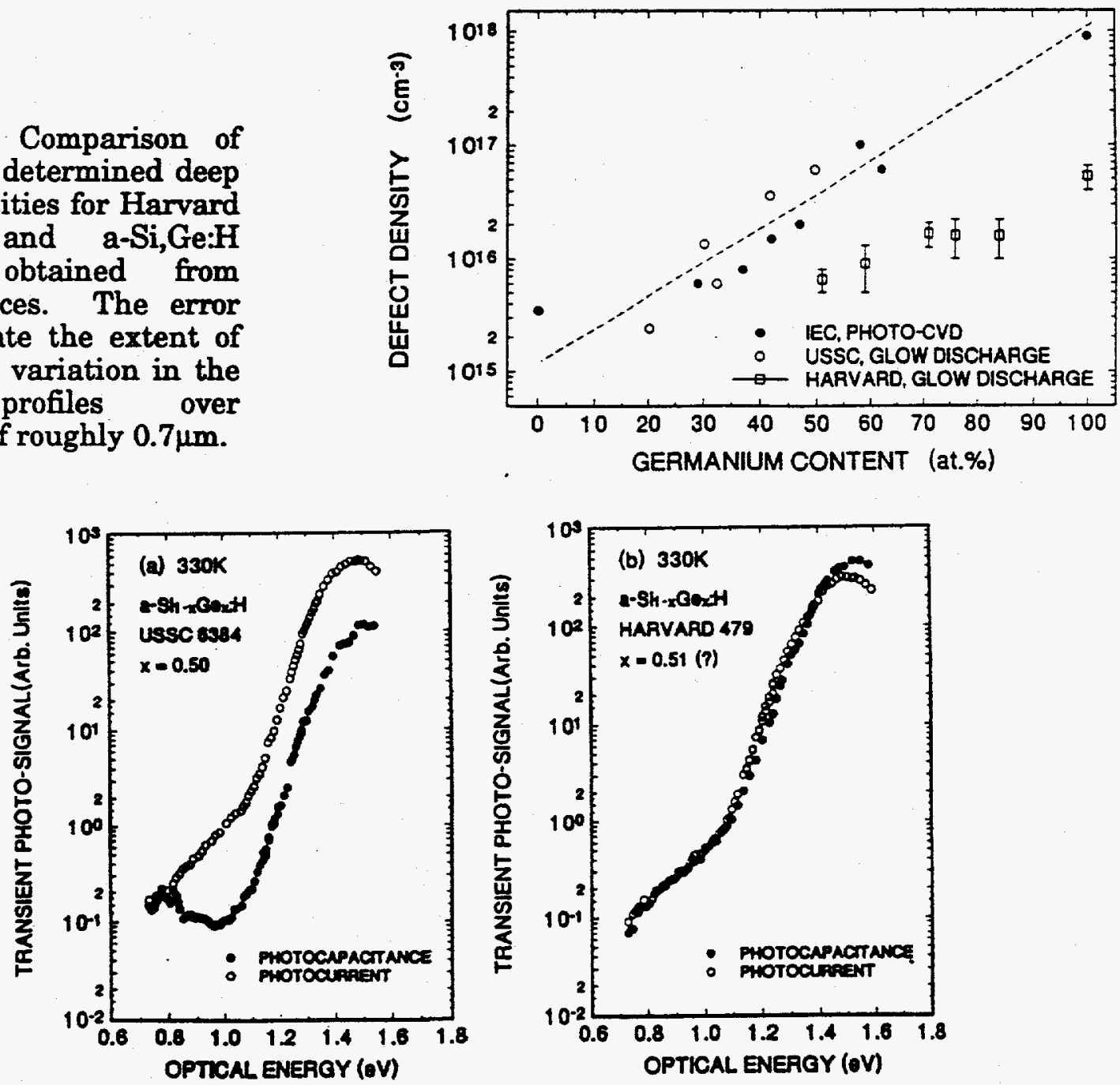

FIG. 2. Pairs of photocapacitance and photocurrent transient spectra for two samples taken at $330 \mathrm{~K}$. These spectra have been aligned to coincide at the lowest optical energies. The marked difference between the two spectra in (a) is attributed to minority carrier processes. 
Our research related to the Mid Gap (and Metastability) Team has been focussed on two issues. First, we have examined some of the effects of $\mathrm{H}$ dilution has on a-Si:H and how this affects stability with respect to prolonged light exposure. Many industrial groups have reported that they can achieve significantly higher stabilized cell efficiencies by utilizing i-layers grown at lower temperature with high hydrogen dilution. Second, we have been trying to apply some of our characterization methods directly to $p$-i-n device structures to see whether we can deduce materials properties in actual working solar cells.

We obtained two samples from Solarex Corporation (courtesy of Liyou Yang), one grown in the normal manner in their glow discharge reactor, and the other grown under high hydrogen dilution. We evaluated these samples using two methods: our drive-level profiling technique, to obtain quantitative values of the deep defect densities after light soaking, and also transient photocapacitance spectroscopy, to compare the sub-band-gap optical spectra and thus verify any differences in deep defect densities. Both samples were light soaked 90 hours at $400 \mathrm{~mW} / \mathrm{cm}^{2}$ before evaluation. Results from the drive-level profiling method are shown in Fig. 3 and those from the photocapacitance are shown in Fig. 4. Both measurements indicate that the hydrogen diluted sample degraded to a deep defect density that was nearly a factor of 2 lower than the normal a-Si:H film. This implies that the hydrogen diluted material is more stable against light-induced deep defect formation. Additional studies which make use of hydrogen modulated a-Si:H films were also begun to help identify the manner in which hydrogen dilution helps stabilize this material [5].

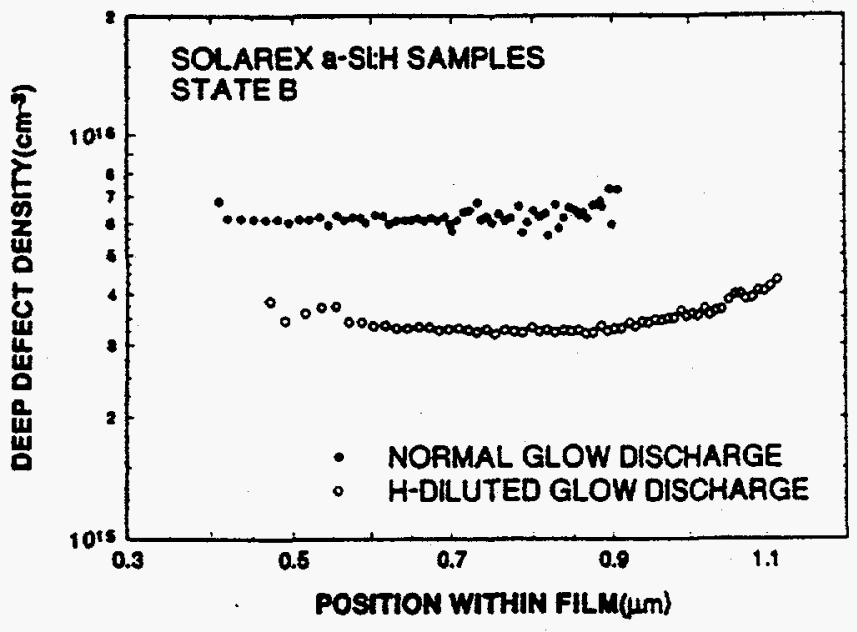

FIG. 3. Drive-level deep defect profiles for two a-Si:H samples in light-degraded states. The sample grown with hydrogen dilution shows a significantly lower deep defect density.

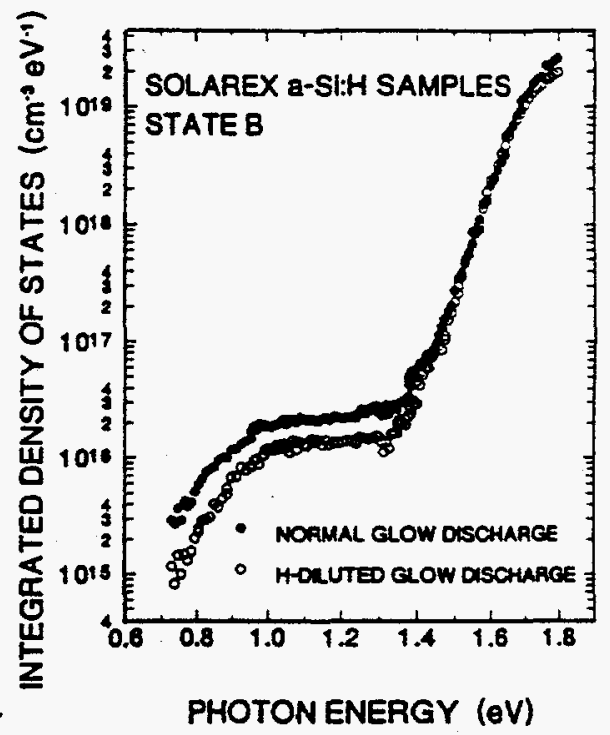

FIG. 4. Photocapacitance sub-bandgap spectra for the same two samples in their light-degraded states. The difference in magnitudes of the deep defect portion of these spectra agrees with that indicated in Fig. 3. 
Finally, we have begun studies to assess the applicability of our capacitance profiling measurements to actual p-i-n solar cell structures. Our best test sample for this purpose is a $2 \mu \mathrm{m}$ thick i-layer device obtained from $\mathrm{Dr}$. Xi at APS. This i-layer is thick enough that, within quite a range of profiling bias, we are fairly certain to be well inside a uniform intrinsic region.

FIG. 5. Series of drive-level capacitance profiling measurements on thick i-layer $p-i-n$ device obtained at $5 \mathrm{kHz}$ and a series of measurement temperatures. The uniform region for the higher temperature profiles indicates an i-layer deep defect density of about $8 \times 10^{15} \mathrm{~cm}^{-3}$. These profiles also exibit a feature, indicated by the arrows, which we can associate with defects at the $p-i$ or $n-i$ interface.

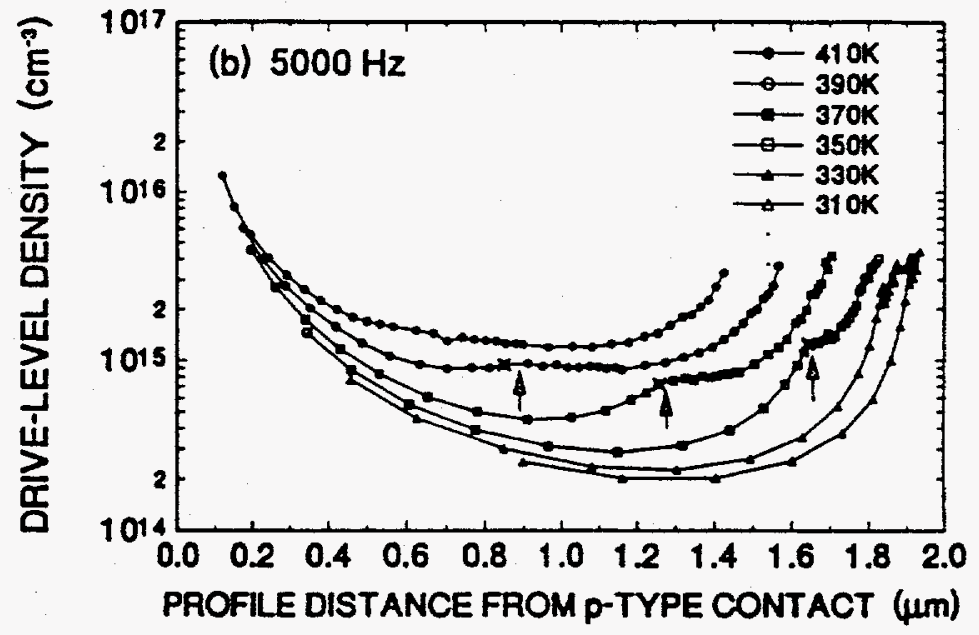

A set of drive-level capacitance profiling measurements for a series of increasing temperatures is shown in Fig. 5 for a measurement frequency of $5 \mathrm{kHz}$. At higher temperatures the profiles are higher because a greater fraction of the deep defect states can respond. These profiles are fairly uniform for temperatures above $390 \mathrm{~K}$ where deep states within the i-layer are contributing to the ac response. Using the algorithm we have previously established to estimate total defect densities from drive-level capacitance profiling measurements on Schottky devices [6], we assign a total deep defect density of $8 \times 10^{15} \mathrm{~cm}^{-3}$ to the i-layer within this sample. One other interesting aspect concerns the bump in several profiles apparent in the $5 \mathrm{kHz}$ data. (This feature has been marked by an arrow). We can fairly definitely assign this feature to the presence of interface states near the $p-i$ or $n-i$ boundaries. Sophisticated modeling of such experimental profiles is now underway to extract more detailed information about the defect distributions in such devices. Comparison studies for this device in a light-degraded state are also to be carried out soon.

\section{References}

1. Don Williamson, S.J. Jone and Y. Chen, Final Subcontract Report: NREL/TP-4116855 (1994).

2. T. Unold, J.D. Cohen, and C.M. Fortmann, Appl. Phys. Lett. 64, 1714 (1994).

3. Cohen, Final Subcontract Report: NRELTP-451-7163 (1994).

4. . Fan Zhong, J.D. Cohen, J. Yang, and S. Guha, Mat. Res. Soc. Symp. Proc. 336, 493 (1994).

5. D. Kwon and J.D. Cohen, Mat. Res. Soc. Symp. Proc. 336, 305 (1994).

6. T. Unold, J. Hautala, and J.D. Cohen, Phys. Rev. B50, 16985 (1994). 
Title:

Organization:

Contributors:

\section{L Morel and C. S. Ferekides}

Advanced Processing Technology for High Efficiency ThinFilm CuInSe, and CdTe Solar Cells; Heterojunction Development and Optimization in Thin-Film Compound Semiconductor Solar Cells.

\author{
Department of Electrical Engineering \\ University of South florida, \\ Tampa, Florida
}

G. Attar, V. Ceekala, K. Dugan, S. Karthickeyan, L. Killian, H. Natarajan, D. Niermen, D. Oman, P. Bhethanabolta, and S. Zafar

\section{CdTe}

\section{Objectives}

The main objectives of this project are to prepare high efficiency CdTe solar cells on soda lime (SL) substrates by the close spaced sublimation process (CSS), evaluate/develop If sputtering and CSS as alternative processes to the chemical bath deposition (CBD) for the preparation of the $\mathrm{CdS}$ films, and correlate processing to device performance.

\section{Device Fabrication}

Solar cells prepared on soda lime glass substrates utilize $\mathrm{CdS}$ prepared by CBD, and a CSS CdTe layer deposited at low temperatures: $460-520^{\circ} \mathrm{C}$. Borosilicate glass substrates are being used for the preparation of devices that utilize CSS or rf sputtered CdS, and CSS $\mathrm{CdTe}$ (typically deposited at temperatures in excess of $550^{\circ} \mathrm{C}$.)

\section{Cadmium Sulfide Films}

Both rf sputtered and CSS CdS films are prepared at substrate temperatures in excess of $150^{\circ} \mathrm{C}$ (rf sput.: $150-400^{\circ} \mathrm{C}$; CSS: $250-500^{\circ} \mathrm{C}$.) The rf sputtered CdS films exhibit small grain size (typically $0.1-0.2 \mu \mathrm{m})$, and high dark resistivity $\left(>10^{6} \Omega-\mathrm{cm}\right.$ ). High open-circuit voltages $(>840 \mathrm{mV}$ ) have been obtained for devices with CdS thicknesses in excess of $2000 \AA$; smaller thicknesses yield reduced $V_{\text {OC }}$ 's. Annealing of the rf sputtered $\mathrm{CdS}$ in $\mathrm{H}_{2}$ was found to influence the film properties and solar cell performance. Figure 1 shows the SR of several cells utilizing if sputtered $\mathrm{CdS}$ annealed at different temperatures. In general, device performance was limited by low fill factors (mid 60's.)

Cadmium sulfide films prepared by CSS were also highly resistive. The grain size is a strong function of the deposition conditions (films with grain size in excess $1 \mu \mathrm{m}$ can be easily obtained.) Annealing in $\mathrm{H}_{2}$ was also found to influence the properties of the CSS CdS films (and to a lesser extent the properties of solar cells). Open-circuit voltages in excess of 840 $\mathrm{mV}$ and fill factors over $70 \%$ have been attained for film thicknesses of about $800-1000 \AA$. Solar cell efficiencies of $13 \%$ have been achieved.

\section{Soda Lime Substrates}

Inexpensive soda lime glass substrates are preferred (for the fabrication of solar cells) 
over the expensive borosilicate type. The properties of soda lime glass (softening point, linear expansion coefficient, impurity content etc.) make it incompatible with high temperature processing. Cadmium telluride films and devices prepared by CSS are being optimized at lower processing temperatures $\left(460-520^{\circ} \mathrm{C}\right)$ for the purpose of fabricating efficient solar cells on soda lime substrates. At high temperatures $\left(>550^{\circ} \mathrm{C}\right) \mathrm{CdTe}$ films prepared on SL substrates exhibit "blistering" and "peeling". As the temperature is lowered adhesion improves but the density and structure of the films change; device performance degrades due to a decrease in $\mathrm{V}_{O C}$. Obtaining denser CdTe films was crucial in improving the performance of CdTe devices prepared on SL substrates, and achieving efficiencies over $13 \%$. Figure 2 shows the light (AM1.5) I-V characteristic of a CdTe solar cell with efficiency of $13.5 \%$ verified at the National Renewable Energy Laboratory.

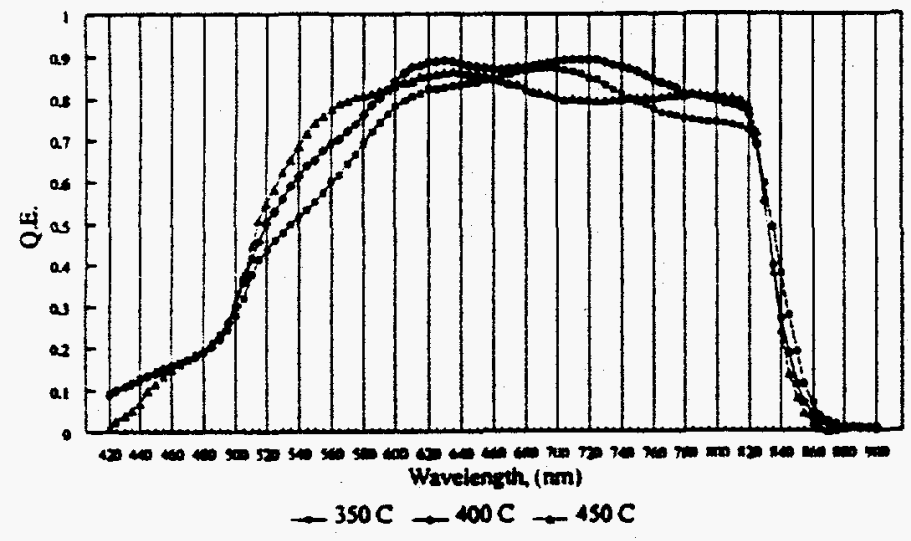

Fig. 1. The SR of solar cells prepared with if sputtered $\mathrm{CdS}$ annealed in $\mathrm{H}_{2}$ ambient.

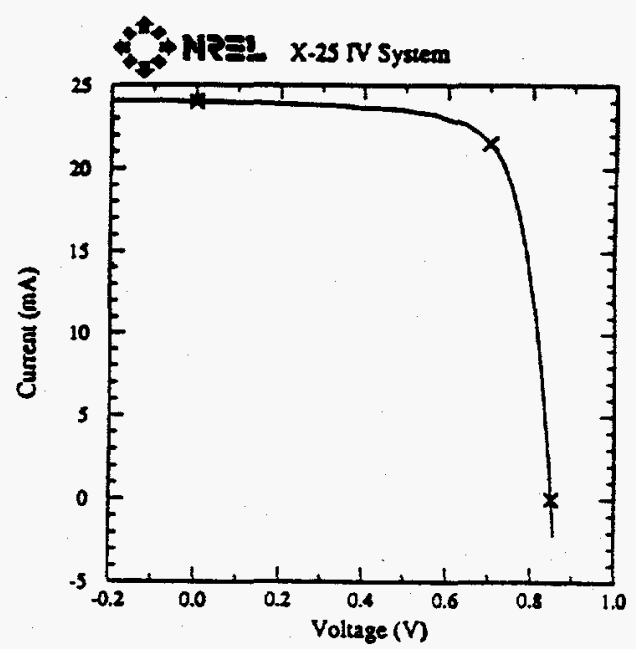

Fig. 2. Illuminated I-V characteristics of a 13.5\% efficient CdTe solar cell prepared on a soda lime glass substrate.

\section{CIS}

\section{Objectives}

The objectives of this project are to develop manufacturing-friendly processes for CIS solar cells, to correlate performance and processing mechanisms, and to develop reactively sputtered $\mathrm{ZnO}$ as an alternative window layer.

\section{Process Description}

Our approach to processing has been driven by two primary guidelines: 1 . Non- $\mathrm{H}_{2} \mathrm{Se}$ based processing, and 2. Non-critical process control. Our primary process is thus one which we describe as "coat and cook". It involves sequential physical vapor deposition of $\mathrm{Cu}$, In and Se under minimal control, and the anneal cycle runs at atmospheric pressure, features which make it very attractive from a manufacturing perspective. 


\section{CIS Performance Correlations}

Our baseline devices which contain only $\mathrm{CuInSe}_{2}$ and no Ga currently have an efficiency of about $10 \%$. This represents significant progress for our simplified processing approach. Also, we have developed additional insights to processing variables which are leading to further advancements in performance. In figure 3 we show the dependence of $V_{o c}$ on metal ratio for our baseline process(solid line) and for recent advances in performance( $x$ 's) beyond our baseline due to improved understanding. The data points at $400 \mathrm{mV}$ are due to modifications of our anneal profile. The data point at $450 \mathrm{mV}$ was accor uplished recently by etching the surfaces of our devices. Through modeling (see below) we $\mathrm{h}$ ve determined that device performance is limited by excessive recombination at the junction interface. Elimination of the centers responsible for the recombination produced the significant increase in $V_{o c}$ to $450 \mathrm{mV}$. This level of performance is now approaching the highest values achieved for non-Ga-containing CIS.

\section{$\underline{\mathrm{ZnO}}$}

$\mathrm{ZnO}$ is an important window layer material for CIS and other solar cell devices. We have been developing a process for depositing $\mathrm{ZnO}$ by reactive sputtering with a zinc target. This promises much higher deposition rates than can be achieved by sputtering from a $\mathrm{ZnO}$ target. Using Al doping we have achieved state-of-the-art opto-electronic properties. Uniform films with resistivities of $2-5 \times 10^{-4} \Omega-\mathrm{cm}$ and suitable optical properties are routine. In attempting to further improve the performance of these films we have developed new insights to the underlying physical mechanisms which determine performance and the deposition parameters which influence those mechanisms. For example, while we have achieved doping efficiencies of nearly $100 \%$, we find that at doping levels above about $4 \mathrm{x}$ $10^{20} / \mathrm{cm}^{3}$ ionized impurity scattering starts to reduce the mobility thus limiting the conductivity. This, together with the dependence of optical properties on free carrier effects, sets fundamental limits on the electro-optical properties of $\mathrm{ZnO}$. Further advancements in these properties will require novel, non-conventional doping techniques.

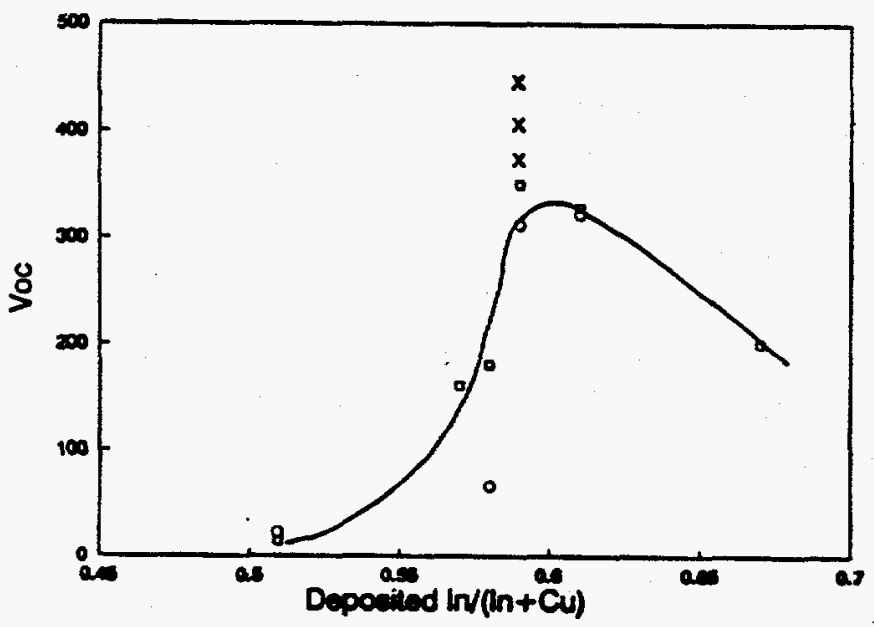

Fig 3. $V_{\propto}$ vs. metal ratio for baseline devices and $O$, and for advanced processing, $x$.

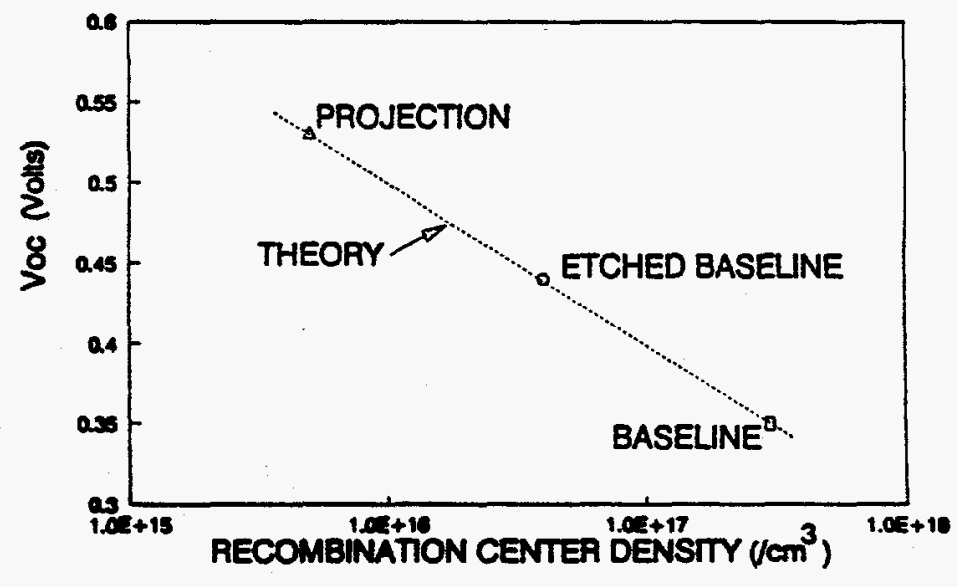

Fig. 4. $v_{\propto}$ vs. recombination center density. $O$, $\square$ - data points, $\Delta$ - projection. 


\section{HETEROJUNCTION MODELING AND DEVELOPMENT}

\section{Objectives}

The objectives of this project are to develop a phenomenological model to aid the understanding of the heterojunction in CdTe and CIS devices and to use the model to guide the further development of the technologies.

\section{Res!lts and Discussion}

After careful examination of the ability of classical models to fit critical sets of data we have determined that a recombination-based model is the most suitable choice for both CIS and CdTe. The model follows the standard equation

$$
\mathrm{I}=\mathrm{I}_{\mathrm{o}}\left(\mathrm{e}^{\mathrm{q}(\mathrm{V}-\mathrm{IR}) / \mathrm{AkT})}-1\right)+(\mathrm{V}-\mathrm{IR}) / \mathrm{R}_{\mathrm{sh}}-\mathrm{I}_{\mathrm{L}}
$$

where $R$ is the series resistance, $R_{s h}$ is the shunt resistance and $I_{L}$ is the light generated current. The diode factor A may range between 1 and 2 depending upon the location of the recombination sites within the gap. The prefactor $\mathrm{I}_{0}$ may be written as

$$
\mathrm{I}_{\mathrm{o}}=\mathrm{kTn}_{\mathrm{i}} \mathrm{W} / 2\left(\mathrm{~V}_{\mathrm{bi}}-\mathrm{V}\right) \tau
$$

where k-Boltzmann's constant, T-temperature, $\mathrm{n}_{i}$-intrinsic carrier concentration, W-space charge width, $\mathrm{V}_{\mathrm{bi}}$-built-in voltage, $\tau$-lifetime. For both $\mathrm{CdTe}$ and $\mathrm{CIS}$ we find self-consistent fits to dark and light $I-V, I_{s c}-V_{o c}$, the one sun power curve, and $V_{o c}-T$. Since several of the parameters can be measured directly, the key parameters to be determined by the fits are A and $\tau$ which are not necessarily independent.

In taking and interpreting data with regard to this model special care has to taken to properly account for extraneous effects. For example, we have found that the commonly observed crossover phenomenon in CdTe is due to a region of the CdS outside of the space charge layer. Once it is accounted for, good fit to the model can be realized.

For typical CdTe devices we find $\mathrm{A}$ values in the range $1.4-1.8$, with the better devices exhibiting the lower $\mathrm{A}$ values. We also find that $\mathrm{A}$ is often a complex function of device structure and the exposure history of the device. In particular we observe transient behavior in $\mathrm{V}_{\mathrm{oc}}$ which is largely explainable in terms of fluctuations in $\mathrm{A}$. Key correlations to process variables are being observed and are helping guide our fabrication efforts.

Similar observations are being made with CIS confirming our supposition that the common CdS heterojunction would provide leveraged opportunities to study junction mechanisms. In the case of CIS we are commonly observing higher A values in the 1.8-2.0 range due to enhanced recombination resulting from smaller lifetimes. This has kept our $\mathrm{V}_{\mathrm{oc}}$ values low and has reduced fill factors. These insights led to the etching procedures described above. Briefly, modeling indicated that the responsible centers were at the junction interface. The intent of the etch was to simply reduce these centers to "turn off" the dark I-V curve which would then result in a higher $V_{o c}$. This is what was observed experimentally, and in figure 4 we show additional projections from the model. As seen in the figure the advance in $V_{o c}$ from $400 \mathrm{mV}$ to $450 \mathrm{mV}$ was due to a reduction of these centers to the mid $10^{16} / \mathrm{cm}^{3}$ range, and further reduction to below the $10^{16} / \mathrm{cm}^{3}$ range is expected to yield $500+\mathrm{mV}$. Experiments are currently under way to continue these advancements in performance. 
Title: $\quad$ Research on Wide Bandgap, Amorphous Silicon Based Solar Cells.

Organization: Department of Physics, Syracuse University, Syracuse, New York 132441130.

Contributors: Eric A. Schiff, principal investigator; Qing Gu and Qi Wang.

In 1994 our research has focused on wide bandgap materials, in conjunction with our participation in the wide bandgap team of the Amorphous Silicon Research Project. This research was supported by two NREL subcontracts, including the present one named above and the predecessor, "Research on Defects and Transport in Amorphous Silicon Based Solar Cells." We are working on the following topics:

- Development of novel thin films as possible alternatives to current amorphous and microcrystalline $p^{+}$(boron doped) silicon films. The goal of this research is to develop materials which will increase the open circuit voltage $V_{o c}$ of wide bandgap a-Si based solar cells.

- Electroabsorption studies of a-Si based solar cells to probe their built-in potential. We seek a tool to permit us to directly assess $V_{\mathrm{bi}}$ as it varies with differing $n^{+}$and $p^{+}$layers.

- Electron and hole mobility measurements in a-Si based materials. We are particularly interested in finding out how bandgap modification of a-Si materials (by alloying or by changes in the hydrogen microstructure) affects these mobilities.

In the present report we shall primarily discuss the electroabsorption project and the origin of built-in potentials in a-Si. Our research contract was acknowledged in the following publications in 1994:

"Hole Drift Mobility Measurements in Amorphous Silicon Carbon Alloys," Qing Gu, Qi Wang, Eric A. Schiff, Yuan-Min Li, and Charles T. Malone, J. Appl. Phys. 76, 2310--2315 (1994).

"Hole Drift Mobility in a-SiC," Qing Gu, Qi Wang, E. A. Schiff, Y.-M. Li, and C. T. Malone," in Amorphous Silicon Technology - 1994, edited by E. A. Schiff, M. Hack, A. Madan, A. Matsuda, and M. Powell (Materials Research Society, Pittsburgh, 1994), pp. 523--528.

"Built-in Potentials Via Electroabsorption Measurements in a-Si:H pin Solar Cells: A Critical Assessment," Qi Wang, E. A. Schiff, and S. Hegedus, in Amorphous Silicon Technology 1994, edited by E. A. Schiff, M. Hack, A. Madan, A. Matsuda, and M. Powell (Materials Research Society, Pittsburgh, 1994), pp. 365--370.

"Recent Progress in Amorphous Silicon PV Technology," W. Luft, H. M. Branz, V. L. Dalal, S. S. Hegedus, and E. A. Schiff, collected in AIP Conference Proceedings No. 306: 12th NREL Photovoltaic Program Review, edited by Rommel Noufi and Harin S. Ullal (American Institute of Physics, New York, 1994), pp. 31--45. 


\section{Electroabsorption and Built-in Potentials}

In Fig. 1 we present some data [1,2] on the relationship of the open-circuit voltage in a-Si based $\mathrm{p}-\mathrm{i}-\mathrm{n}$ solar cells to the bandgap of the intrinsic layer in these cells (estimated using the "Tauc" procedure). Although it is well known that many aspects of a solar cell's fabarication affect $V_{o c}$, the overall trend of the measurements seems fairly clear. For lower bandgap intrinsic layers, there is essentially a one-to-one relationship between the open-circuit voltage and the bandgap: a 0.1 $\mathrm{eV}$ change in the bandgap produces fairly close to a $0.1 \mathrm{~V}$ change in $V_{\mathfrak{o c}}$. This trend, however, is not continued for cells with larger bandgaps, for which $V_{o c}$ saturates near $1.05 \mathrm{~V}$. If this saturation effect can be overcome one can anticipate a significant improvement in $V_{o c}$ for wider bandgap cells.

The wide bandgap cells of Fig. 1 were prepared by Yuan-Min Li at Solarex Corp., who suggested that the saturation effect may occur because $V_{\mathrm{oc}}$ is approaching the built-in potential $V_{\mathrm{bi}}$ of the cells [1]. Although the intrinsic layers vary greatly for Fig. 1, the doped layers which determine $V_{b i}$ do not -- which is consistent with Li's hypothesis. On the other hand, first principles estimates of $V_{\mathrm{bi}}$ are usually much larger than $1.05 \mathrm{~V}$.

We have been developing the electroabsorption method [3] for determining $V_{\mathrm{bi}}$ in a-Si based solar cells for the last two years. In this method one passes a light beam through the solar cell, and measures the effect of the cell's bias voltage $V$ on the transmitted light. Without delving into the details, it is sufficient to note that an electroabsorption signal $S$ can be measured which depends linearly on $V: S=a\left(V_{0}-V\right)$. This relationship is easily understood if the parameter $V_{0}$ is interpreted as the built-in potential $V_{\mathrm{bi}}$ of the cell, as was originally proposed by the Osaka University group over ten years ago [3].

The problem which we are working on is that $V_{0}$ depends upon the measuring wavelength. In Fig. 2 we show measurements on three different a-Si cells for 3 different wavelengths. Although the quantitative details vary, the value of $V_{0}$ increases with increasing measuring wavelength. This behavior was not considered in the original Osaka University research.

We believe that this effect is due to the relatively small part of the built-in potential of the solar cell which is dropped in the $p^{+}$layer of the cell; the wavelength dependence is then due to the differing electroabsorption properties of the $p^{+}$and the $i$ layers of these cells. We have proposed a procedure for determining $V_{\mathrm{bi}}$ from the wavelength-dependent measurements. If the electroabsorption coefficient $\alpha^{\prime \prime}$ of the $p^{+}$layer and the $i$ layer are measured independently, $V_{0}$ can be plotted as a function of the ratio $\alpha^{\prime \prime} / \alpha^{\prime \prime}{ }_{\mathrm{i}} . V_{\mathrm{bi}}$ is estimated as the value of $V_{0}$ when this ratio is unity (ie. when the $p$ and $i$ layers have the same electroabsorption properties).

We have made a preliminary estimate of $V_{\mathrm{bi}}$ using this procedure for a cell/film pair deposited at the Institute for Energy Conversion (University of Delaware). The results are presented as Fig. 3, from which we estimate $V_{\mathrm{bi}} \approx 0.96 \mathrm{~V}$ for this cell. Our procedures for measuring electroabsorption in doped films have significant limitations in their accuracy; we are presently working to improve these electroabsorption spectra and to extend them to the $p^{+}$materials present in the cells from other laboratories. 


\section{References}

1. Li, Yuan-min, in Amorphous Silicon Technology-1993, edited by Eric A. Schiff, Malcolm, $J$. Thompson, Arun Madan, and Kazunobo Tanaka, (Materials Research Society, Pittsburgh, 1993), p. 803.

2. Qi Wang, Homer Antoniadis, E. A. Schiff, and S. Guha, in Amorphous Silicon Technology1992, edited by M. J. Thompson, et al (Materials Research Society, Pittsburgh, 1992), p. 881.

3. S. Nonomura, H. Okamoto and Y. Hamakawa, 'mn. J. Appl. Phys. 21, 464 (1982).

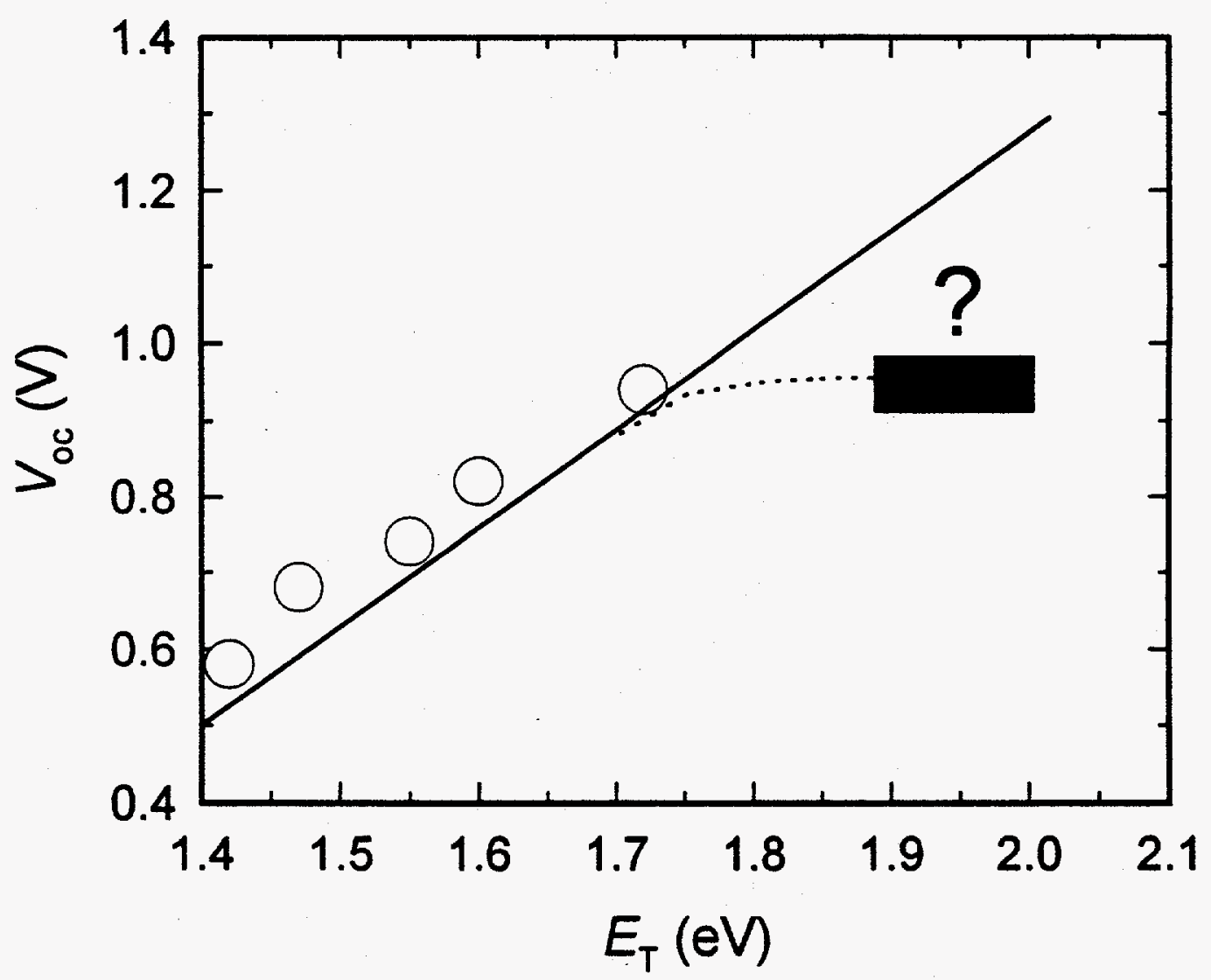

Fig. 1: Correlation of the open-circuit voltage $V_{\mathrm{oc}}$ and the intrinsic layer optical bandgap $E_{\mathrm{T}}$ for amorphous silicon based pin solar cells. Points for $E_{\mathrm{T}}<1.70 \mathrm{eV}$ are a-SiGe alloys [1]; data for $E_{\mathrm{T}}>1.80 \mathrm{eV}$ are a-SiC alloys [2]. 


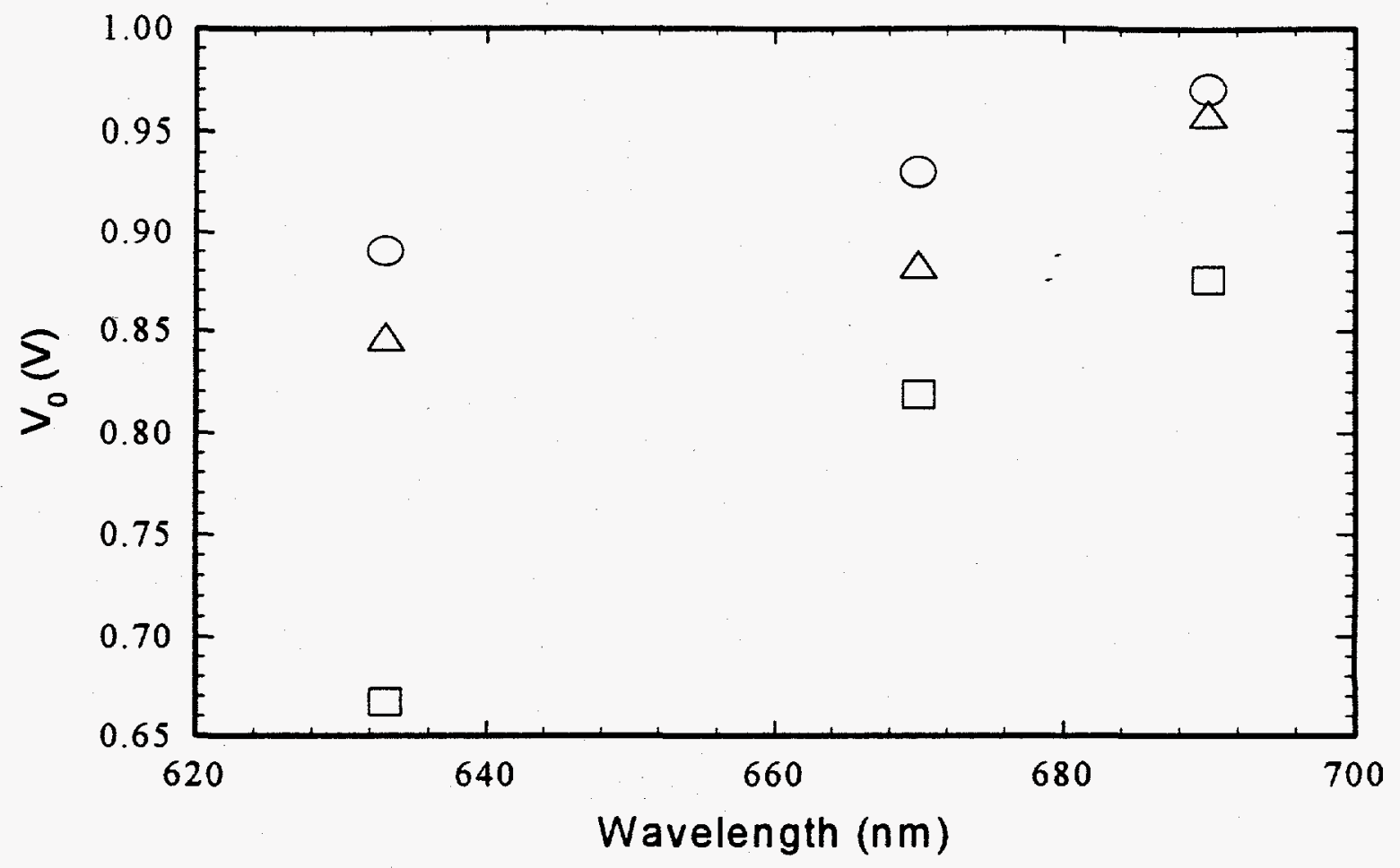

Fig. 2: Electroabsorption parameter $V_{0}$ measured at three wavelengths for 3 types of a-Si solar cell. Circles: photoCVD a-Si solar cell, a-SiC $\mathrm{p}$ (Institute for Energy Conversion). Triangles: PECVD a-Si solar cell, microcrystalline $p$ (United Solar Systems Corp.). Squares: PECVD a-Si solar cell, a-SiC p (Solarex Corp.).

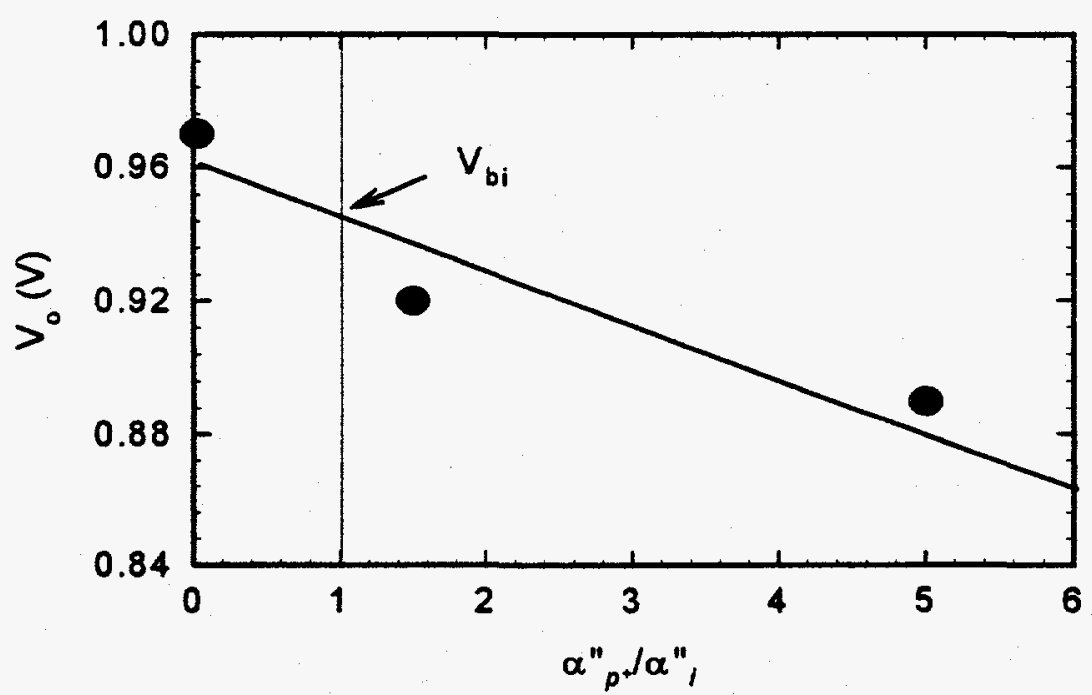

Fig. 3: Illustration of proposed procedure for obtaining $V_{\mathrm{bi}}$ from the wavelength dependent values of $V_{0}$. At each wavelength the electroabsorption parameter $\alpha^{\prime \prime}$ is obtained independently for the $p$ and $i$ layers. $V_{\mathrm{bi}}$ is estimated by the value of $V_{0}$ when the two layers have the same electroabsorption parameters. 
Title: $\quad$ High Efficiency Thin Film Cadmium Telluride Photovoltaic Cells

Organization: Department of Physics and Astronomy

The University of Toledo

Toledo, OH 43606

Contributors: Alvin D. Compaan, principal investigator, Randy G. Bohn and Yasantha Rajakanunanayake co-investigators; Charles N. Tabory, Meilun Shao, Andreas Fischer, Zhirong Feng, Faming Shen, Chitra Narayanswami, and Ilvydas Matulionis

\section{Objectives}

This project is focussed on improvements in the fundamental understanding of rf sputtering for the deposition of CdTe-related polycrystalline thin films with the goal of raising the CdS/CdTe if sputtered cell efficiencies well beyond the $10.4 \%$ (AM1.5) already demonstrated.

\section{Technical Approaches}

The University of Toledo effort emphasizes if sputtering from compound semiconductor targets. Presently both CdS and CdTe layers are being sputter deposited. A major focus during the past year was the improved understanding and control of the plasma and its interaction with the growing film surface. Other work utilized laser physical vapor deposition for initial studies of temary alloy materials related to CdS and CdTe. Additionally we developed new non-contact in-situ measurements of glass substrate temperature.

During the past year we have continued to use the film characterization techniques of Hall effect, optical absorption, photoluminescence, Raman scattering, $x$-ray diffraction, SEM, and SEM/EDS. Device characterization included $\mathrm{J}-\mathrm{V}, \mathrm{C}-\mathrm{V}$, and spectral quantum efficiency measurements. We also initiated studies of optical emission from the if plasma as well as Langmuir probe measurements of the plasma electrical characteristics and their dependence on if power, magnetic field, substrate bias, and gas pressure.

We have continued collaboration with Solar Cells Inc and other NREL-supported groups.

\section{A two-gun sputtering system with in situ optical beam monitoring}

We have previously demonstrated that if sputtering can be used for the deposition of both semiconductor layers in the CdS/CdTe system yielding efficiencies above $10 \%{ }^{1}$ This work was done in a simple onegun sputtering system with target changes between CdS and CdTe depositions. During the past year we have constructed a new two-gun system ${ }^{2}$ in which it is possible to do a sequential deposition of $\mathrm{CdS}$ followed by CdTe without exposure of the film to air and without interruption of the substrate heating. The system is sketched in Fig. 1. This chamber incorporates four small windows at $45^{\circ}$ which permit laser beam access for in situ glass temperature monitoring and film thickness monitoring described below. ${ }^{3}$ The two sputter guns are two-inch diameter magnetrons with different magnetic field configurations. We have analyzed extensively the differences in the plasma generated by each of these guns and in the properties of films grown by these two guns. Some of our results are presented below.

In situ optical measurements of glass temperature and film thickness

As shown in Fig. 1, the four optical access ports at $45^{\circ}$ to the sputter guns permit reflection and 
transmission measurements of the glass substrate and the growing films. In reflection from the back side, interference effects allow one to obtain the glass temperature. The observed fringe shift as a function of temperature, shown in Fig. 2, arises from two effects. The first is the thermal expansion of the glass and the second is the change in index of refraction of the glass. The two effects are of comparable magnitude and calibration of this technique required us to measure accurately the index of refraction change with temperature for the various glasses which might be used as substrates or superstrates for PV materials. ${ }^{3}$ Such data are not available in the literature with the precision needed for this measurement. Our results are given in Fig. 3. We have concluded that this optical method can easily yield temperature measurements with an error of less than $\pm 5 \mathrm{C}$.

This new sputtering chamber also allowed us to monitor film thicknesses suring growth via the transmission of either a HeNe laser line at $633 \mathrm{~nm}$ (for CdTe films) or an argon ion laser line at $458 \mathrm{~nm}$ (for CdS). This optical measurement yields high accuracy and is not affected by the if fields and ionization present in the chamber during film growth.

\section{Magnetic field effects on rf-magnetron sputtering of CdTe and CdS}

Magnetron sputtering as a film deposition technique admits the possibility of exploiting electron, ion and neutral atom fluences to improve the film characteristics. To study the range of effects which are possible, we have installed two very different magnetron sputter guns. One gun utilizes an approximately balanced magnetic field configuration and the other gun uses a "type II" unbalanced configuration. ${ }^{4}$ Our measurements of the field configurations are sketched in Fig. 3. An example of one of the several optical emission lines we have studied is shown in the bottom panel of Fog. 3. This neutral $\mathrm{Cd}$ line at $508.6 \mathrm{~nm}$ was obtained with a fiberoptically coupled $1 / 4$ meter spectrometer and a vidicon. ${ }^{2}$ Note that the plasma from the unbalanced magnetron shows much stronger light emission from Cd atoms several centimeters away from the sputter target surface implying the presence of more energetic electrons with the unbalanced gun. This is confirmed with the Langmuir probe data.

A number of measurements were made using a Langmuir probe located $1 \mathrm{~cm}$ in front of the substrate. $^{2}$ In addition, we measured the self bias which appears on the electrically isolated substrate and its holder as well as the deposition flux as obtained from the final film thickness. The results are summarized in Table 1 . Note that the electron density and temperature together with the ion flux on the substrate all are substantially higher for the unbalanced magnetron.

We believe that one or all of these effects help to explain the major differences observed in the photoluminescence (PL) signal from CdS films grown from these two guns. The CdS film

\begin{tabular}{|c|c|c|}
\hline \multicolumn{3}{|c|}{$\begin{array}{c}\text { Table 1. Unbalanced vs. Balanced } \\
\text { Magnetron Characteristics } \\
\left(\mathbf{P}_{\mathbf{R F}}=75 \mathrm{~W}, \mathrm{P}_{\mathrm{Ar}}=18 \mathrm{mT}, \mathrm{CdS} \text { Target }\right)\end{array}$} \\
\hline${ }^{2}$ & UNBAL. & BALANCED \\
\hline $\begin{array}{l}\text { substr. self bias } \\
\mathrm{V}_{\text {sb }}\end{array}$ & $-2.2 \mathrm{~V}$ & $+8 V$ \\
\hline $\begin{array}{c}\text { plasma potential, } \\
\mathbf{V}_{p}\end{array}$ & $+19 V$ & $+26 \mathrm{~V}$ \\
\hline $\begin{array}{c}\text { substr. sheath, } \\
V_{p}-V_{s b}\end{array}$ & $+21 \mathrm{~V}$ & $+18 V$ \\
\hline $\begin{array}{l}\text { ion flux, } F_{I} \\
\left(\mathrm{~cm}^{-2} \sec ^{-1}\right)\end{array}$ & $1.8 \times 10^{15}$ & $1.0 \times 10^{15}$ \\
\hline $\begin{array}{l}\text { deposition flux, } \\
\mathbf{F}_{\mathrm{Cd}+\mathrm{S}}\left(\mathrm{cm}^{-2} \sec ^{-1}\right)\end{array}$ & $2.4 \times 10^{15}$ & $2.6 \times 10^{15}$ \\
\hline $\mathbf{F}_{\mathrm{Y}} \mathbf{F}_{\mathrm{Cd}+\mathrm{S}}$ & 0.75 & 0.4 \\
\hline $\begin{array}{l}\text { electron temp., } \\
\mathrm{T}_{e}\end{array}$ & $5.3 \mathrm{eV}$ & $4.0 \mathrm{eV}$ \\
\hline $\begin{array}{l}\text { electron density, } \\
n_{e}\left(\mathrm{~cm}^{-3}\right)\end{array}$ & $7.6 \times 10^{10}$ & $3.9 \times 10^{10}$ \\
\hline
\end{tabular}


grown with the unbalanced magnetron shows very sharp and strong band-edge PL (See Fig. 4.), whereas the film grown with the balanced magnetron has a PL peak shifted about $70 \mathrm{~nm}$ to the red and weaker by a factor of $10^{3}$. (Similar PL effects are observed for CdTe films grown with the different sputter guns.) We suggest that the film quality is improved substantially by the higher ion and/or electron bombardment which occurs during growth with the unbalanced magnetron. Further work is in progress to understand these effects and to optimize the sputter gun parameters for photovoltaic properties. We have found, for example, that high cell efficiency requires that the CdTe layer be deposited with the unbalanced magnetron. The CdS layer appears less sensitive to the type of sputter gun used.

\section{Other work in progress}

Additional efforts have been applied to understanding the if sputter growth of ZnTe films doped either with copper or reactively sputtered with nitrogen. ${ }^{5}$ Copper doping has yielded resistivities as low as $10^{-2}$ $\Omega$-cm and $N$ doping has given resistivities as low as $25 \Omega-\mathrm{cm}$.

We have begun studies of CdZnTe films for the absorber layer. Initial studies used laser physical vapor deposition for film growth. One of the first cells ${ }^{6}$ obtained using $\mathrm{Cd}_{.85} \mathrm{Zn}_{.15} \mathrm{Te}$ as the absorber layer gave $\mathrm{V}_{\mathrm{OC}}=0.677 \mathrm{~V}, \mathrm{~J}_{\mathrm{SC}}=16.5 \mathrm{~mA} / \mathrm{cm}^{2}$, and an $\mathrm{AM} 1.5$ efficiency of $6 \%$. Further work is in progress to explore the use of these ternary alloys for eventual use in band gap grading in CdTe based cells.

During the year we also demonstrated a new $780 \mathrm{~nm}$ diode-laser-excited resonant Raman and photoluminescence technique for CdTe materials characterization. ${ }^{7}$

Conclusions and future directions

The magnetic field and related ion/atom/electron bombardment effects on film quality are being examined extensively. We are acquiring a second unbalanced magnetron sputter gun to replace the balanced magnetron which appears to produce inferior film quality. Additional work is being done on the optimization of deposition conditions for alloy films related to CdS and CdTe.

\section{References}

1. A. D. Compaan and R. G. Bohn, Final Subcontract Report: Thin Films Cadmium Telluride Photovoltaic Cells, 1 Nov 1992 - 1 Jan. 94 (Sept. 1991) NREL/TP-451-7162. Available NTIS: Order No. DE9401186.

2. A. D. Compaan, M. Shao, C. N. Tabory, Z. Feng, A. Fischer, I. Matulionis, and R. G. Bohn, Proc. First World Conf. on Photovoltaic Energy Conversion, Dec. 5-9, 1994, Hawaii (to be published).

3. Z. Feng, C. N. Tabory, \& A. D. Compaan, Proc. First Worid Conf. on Photovoltaic Energy Conversion, Dec. 5-9, 1994, Hawaii (to be published).

4. B. Window and N. Savvides, J. Vac. Sci. Tech. A 4, 196-202 (1986).

5. R. G. Bohn, C. N. Tabory, C. Deak, M. Shao, A. D. Compaan, \& N. Reiter, Proc. First World Conf. on Photovoltaic Energy Conversion, Dec. 5-9, 1994, Hawaii (to be published).

6. A. D. Compaan, M. Pearce, P. M. Voyles, D. Spry, Z. Feng, A. Fischer, M. Shao, \& R. G. Bohn, Laser Induced Thin Film Processing (SPIE Photonics West Symposium, Feb. 8-10, 1995 (to be published).

7. A. Fischer, A. Compaan, A. Dane, \& A. Aydinli, Materials Science Forum, vol. 173-174, 349-354 (1995). 


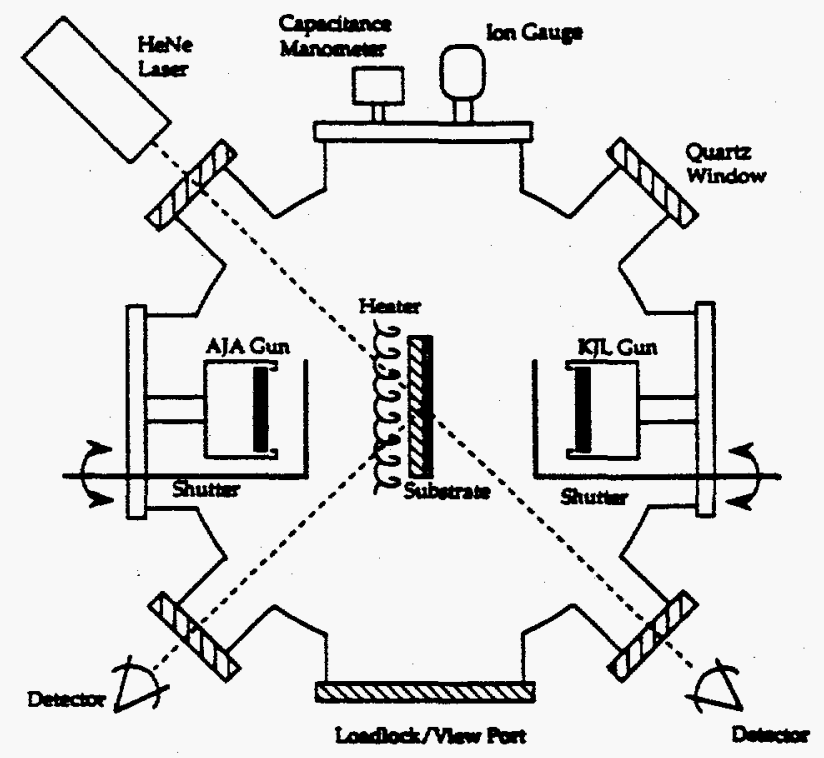

Fig. 1 Top view sketch of two-gun sputter chamber.

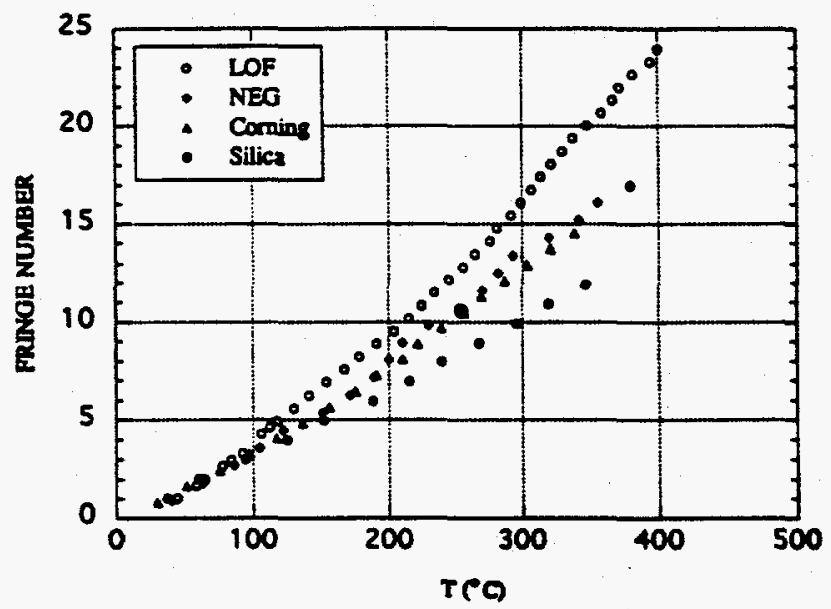

Fig. 2 Optical interference fringe shift at $633 \mathrm{~mm}$ vs. temperature for four glasses. Data have been normalized to a glass thickness of $1 \mathrm{~mm}$.

Fig. 4 (right) PL at 77K from CdS films grown with balanced and unbalanced magnetrons.
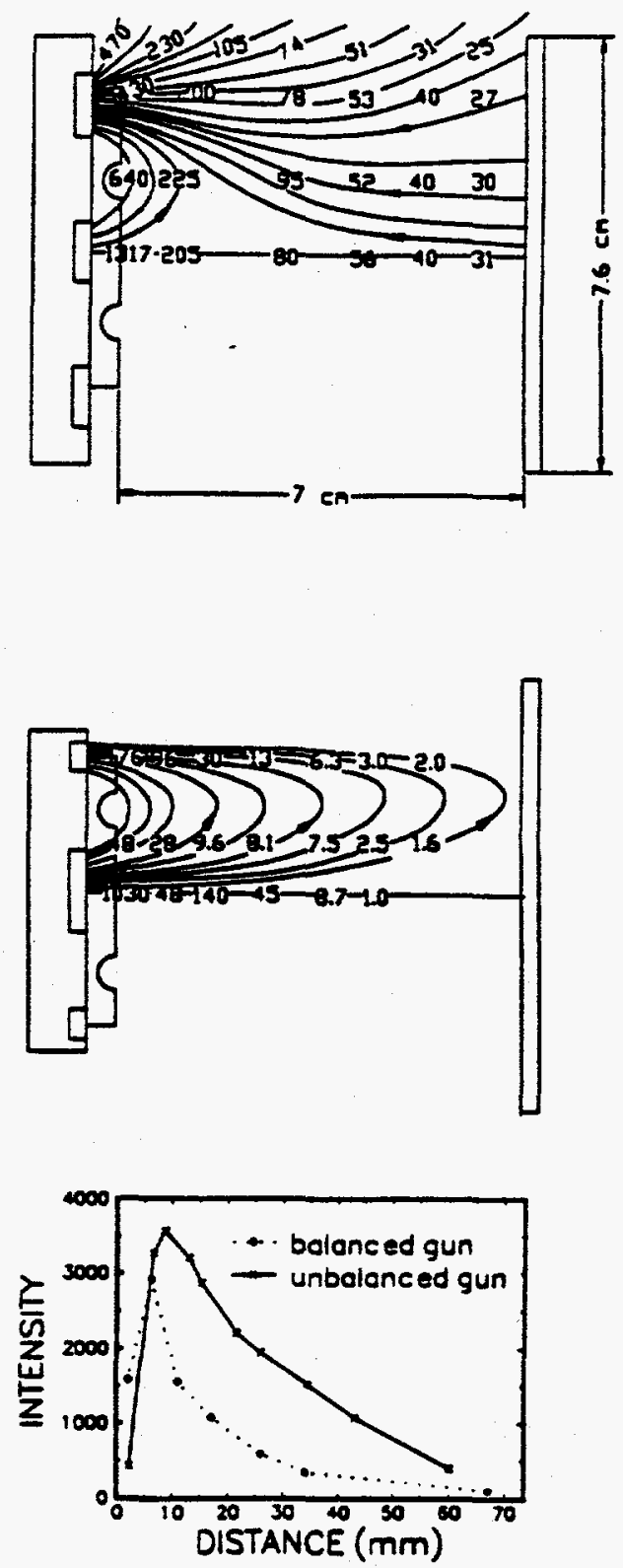

Fig. 3 Magnetic fields of unbalanced (top) and balanced (middle) magnetrons and $508.6 \mathrm{~mm}$ CdI emission (bottom) from plasma region.

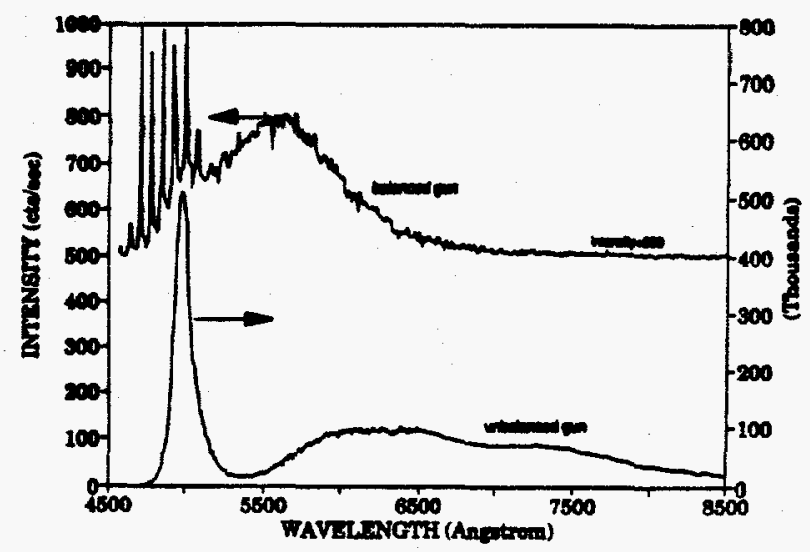


Title:

Organization:

Contributors:
Investigation Of Polycrystalline Thin Film CulnSe2 Solar Cells Based On ZnSe Windows

Electronic Materials Laboratory

Washington State University / Tri-Cities

Richland, Washington

Larry C. Olsen, Principal Investigator;

F.W. Addis, Dave Greer, Phillip Lei and Heriberto Aguilar

\section{OBJECTIVES}

The primary objective of this program is to determine if $\mathrm{ZnSe}$ and/or $\mathrm{ZnO}$ are viable alternatives to $\mathrm{CdS}$ as window materials for CIS-based and CIGS-based solar cells. A discussion of the technical approach used in these studies follows.

\section{TECHNICAL APPROACH}

The program is structured into three tasks: (1) CIS and CIGS cells with $\mathrm{ZnSe}$ and $\mathrm{ZnO}$ windows; (2) Material and device characterization; and, (3) Device modeling. Task 1 comprises the major thrust of the program. During FY 94 efforts on this task mainly involved studies of $\mathrm{ZnSe} / \mathrm{CIS}$ and direct $\mathrm{ZnO} / \mathrm{CIS}$ cells based on Siemens CIS substrates. $\mathrm{ZnSe}$ and $\mathrm{ZnO}$ are grown by MOCVD. Devices are characterized by carrying out I-V analyses and external photoresponse measurements. Physical characterization involves collaboration with NREL. Device modeling studies involves investigation of current loss mechanisms with emphasis on correlation of device performance with cell processing.

\section{MOCVD Growth Of ZnSe And ZnO}

Growth of $\mathrm{ZnSe}$ and $\mathrm{ZnO}$ are carried out in the WSU MOCVD system which consists of a SPIRE $500 X T$ reactor with added gas handing capabilities. $\mathrm{ZnSe}$ is grown by reacting a zinc adduct with $\mathrm{H}_{2} \mathrm{Se}$. Conductive n-type $\mathrm{ZnSe}$ is grown by using iodine as a dopant. Ethyliodide was mixed with helium and installed on one of the gas lines to the system. The zinc adduct is formed by reacting triethylamine and $\mathrm{DMZn}$. Growth rates of $\mathrm{ZnSe}$ are typically $1 \AA / \mathrm{s}$ and CIS substrate temperatures are between $100^{\circ} \mathrm{C}$ and $250^{\circ} \mathrm{C}$. The WSU SPIRE reactor has been modified for $\mathrm{ZnO}$ growth. Tetrahydrofuran (THF) is utilized as a source of oxygen, while the zinc adduct used for the growth of $\mathrm{ZnSe}$ is also utilized for $\mathrm{ZnO}$ growth.

\section{CIS Substrates}

Siemens Substrates -- This work has been conducted using two kinds of CIS substrates from Siemens Solar, namely, Siemens 'standard' CIS material and the so-called 'graded absorber' material [1]. CIS substrates consist of a CIS layer deposited onto Mo coated glass. As discussed in Reference 1, the standard material is essentially CuInSe2 throughout the film except near the CIS-Mo interface where $\mathrm{Ga}$ is added. This material is denoted in this report as simply CIS. The Graded absorber material has sulfur near the front face and also higher concentration of Ga near the Mo interface. We refer to the graded absorber material is referred to as CIS(GA). It is important to note that the photoresponse reported in Reference 1 for the CIS(GA) material is very similar to that for the standard CIS material. The main advantage of the CIS(GA) material appears to result 
from the sulfur at the front surface providing a larger bandgap in that region which appears to reduce current losses.

Physical Characterization -- Two approaches were used to characterize the CIS surface, photoluminescence and thermoelectric probe analyses. The PL measurements clearly establish that a segregated phase or phases exist at the CIS surface, and thermoelectric probe studies indicate that the surface layer is n-type. PL measurements were conducted at NREL. Optical excitation was provided using $835 \mathrm{~nm}$ line of a diode laser. By varying the angle of incidence of the laser beam, it is possible to measure the PL emission from different depths from the sample surface. Figure 1 shows a PL emission spectrum for a CIS sample that had been degreased and etched with KCN. The PL data were taken with an incident laser beam power of $20 \mathrm{~mW}$, an angle of incidence of $25^{\circ}$ and at a temperature of $15^{\circ} \mathrm{K}$. Emissions due to the bulk CIS and a segregated phase(s) at the surface are evident. If the angle of incidence is decreased to $15^{\circ}$, the emission due to the surface phase(s) increases relative to the CIS emission. PL emission spectra measured for as-received CIS material (prior to any surface preparation) were very similar to that described by Figure 1.

Thermoelectric probe analyses involved the measurement of the potential between two points on the surface of a CIS sample while heat is applied at one end of the sample. With the probe nearest the heated side grounded, the potential at the probe furthest from the heat became negative with respect to ground. This result is obtained consistently, and indicates that electrons are the dominant carrier at the surface. If the probes are allowed to penetrate into the material, p-type conductivity is indicated. Results of the PL and thermoelectric probe analyses are consistent with a buried heterojunction model.

\section{ZnSe/CIS SOLAR CELLS}

Al/ZnSe/CIS Test Cells -- ZnSe/CIS structures have been studied by depositing an array of aluminum circular areas $2.8 \mathrm{~mm}$ in diameter onto the $\mathrm{ZnSe}$ film to serve as contacts. Al films are deposited with a thickness of 100 to $150 \AA$ and at a rate $\geq 15 \AA /$ sec so that light can pass through the film and to minimize the sheet resistance. Illuminated and dark characteristics for a test cell (defined as a single Al circular area) allow one to estimate the potential performance of CIS cells based on $\mathrm{ZnSe}$ windows. Illuminated characteristics of test cells are measured by illuminating the device until $\mathrm{J}_{\mathrm{Sc}} \approx 40 \mathrm{~mA} / \mathrm{cm}^{2}$. The cell efficiency may be regarded as an estimated active area, AM1.5 efficiency, assuming that one can add a top contact layer and collector grid without degrading the junction properties. A plot of test cell efficiency versus $\mathrm{ZnSe}$ thickness is shown in Figure 2. As indicated by Figure 2, test cell efficiencies can exceed $14 \%$ as long as the $\mathrm{ZnSe}$ thickness is in the range of $100 \AA$ to $250 \AA$, where the thickness is a value determined by reflection analysis on a silicon witness (during MOCVD growth of $\mathrm{ZnSe}$ ). Generally, it is found that for ZnSe thicknesses $>250 \AA$, inflected I-V curves result and values of J $\mathrm{Sc}$ approach zero.

The reduction of photocurrent can be interpreted to be a result of the formation of a significant conduction band spike at the ZnSe-CIS interface, as predicted $(0.85$ to $0.9 \mathrm{eV})$ in Reference 2 for an interface between relaxed, bulk layers of ZnSe and CIS. T-I-V analyses have been carried out for several test cells. Typically, I-V data can be interpreted in terms of two current mechanisms acting in parallel. At larger voltages ( 0.4 to 0.6 Volts), the dominant current mechanism appears to be due to depletion layer recombination with $\mathrm{n}=1.5$, and $\mathrm{J}_{\mathrm{O}}=5 \times 10^{-8} \mathrm{~A} / \mathrm{cm}^{2}$ at room temperature, and the lower voltage mechanism ( 0.2 to 0.4 Volts) appears to be due to multiple step tunneling.

PL studies were also conducted on $\mathrm{ZnSe} / \mathrm{CIS}$ structures by NREL in the same manner as done for CIS substrates. The PL spectrum due to emission from the top surface layer is dominated by three emissions of $1.26 \mathrm{eV}, 1.22 \mathrm{eV}$ and $1.15 \mathrm{eV}$. Low energy emission from a deeper point shows the typical emission from a bulk CIS film. Considering the PL results for CIS substrates and for 
$\mathrm{ZnSe} / \mathrm{CIS}$ structures, it appears that the MOCVD growth of ZnSe on CIS does not alter the segregated phase(s) on the CIS substrates.

Completed ZnSe/CIS Solar Cells -- Solar cells based on the ZnSe buffer layers were fabricated by having highly conducting $\mathrm{ZnO}$ deposited onto $\mathrm{ZnSe} / \mathrm{CIS}$ structures by other laboratories. Top contact layers of $\mathrm{ZnO}$ were deposited by sputtering and CVD processes. In every case, degradation of cell properties relative to the original $\mathrm{ZnSe} / \mathrm{CIS}$ junction properties occurred. $\mathrm{ZnSe} / \mathrm{CIS}$ cells were fabricated that incorporate a layer of MOCVD ZnO as a protective layer (for the $\mathrm{ZnSe}$ ). A cell fabricated with this procedure was measured by NREL to have a total area eficiency of $8.74 \%$. To further test the potential of the ZnSe/CIS junction, a $\mathrm{ZnSe} / \mathrm{CIS}$ cell was f. bricated with a CdS protective layer. In this case, a total area efficiency of $11.0 \%$ was achieved.

\section{DIRECT ZnO/CIS SOLAR CELLS}

Studies of direct $\mathrm{ZnO} / \mathrm{CIS}$ cells were initiated once the approach to MOCVD growth of $\mathrm{ZnO}$ was developed. Although this effort is relatively new, significant progress has been made. To date the resistivity of $\mathrm{ZnO}$ films has been controlled by varying the substrate temperature as described by Figure 3. Additional control of the resistivity will soon be added by including a dopant in the growth process. Solar cell studies based on MOCVD $\mathrm{ZnO}$ buffer layers have involved the use of Siemens CIS $(G A)$ material. The approach to cell fabrication is described by Figure 4. After surface preparation, a thin layer of high resistivity $\mathrm{ZnO}$ is grown by $\mathrm{MOCVD}$ for the buffer layer. The structure is then sent to Siemens for deposition of a low resistivity $\mathrm{ZnO}$ top contact layer, and then returned to WSU for deposition of a Ni/Ag collector grid. After adding a capability for doping $\mathrm{ZnO}$, the top contact layer will also be grown at WSU. The properties of the cell shown in Figure 4 are possibly the best yet reported for a direct $\mathrm{ZnO} / \mathrm{CIS}$ cell. Note that the open circuit voltage is $492 \mathrm{mV}$ which is much higher than values reported by other workers.

\section{CONCLUSIONS}

These studies have resulted in the following key results: (1) Siemens standard CIS material has an n-type segregated phase at the surface; (2) ZnSe/CIS test cells exhibit high voltages, and estimated active area efficiencies on the order of $14 \%$; (3) To date, deposition of a top contact layer of $\mathrm{ZnO}$ has consistently caused degradation of $\mathrm{ZnSe} / \mathrm{CIS}$ cells; (4) Efficient direct $\mathrm{ZnO} / \mathrm{CIS}(\mathrm{GA})$ cells have been fabricated using MOCVD grown $\mathrm{ZnO}$ buffer layers.

\section{REFERENCES}

1. D.Tarrant and J. Ermer, "I-III-VI2 Multinary Solar Cells Based On CuInSe2", 23rd IEEE PVSC. 1993, pp 372 - 378.

2. Art J. Nelson, et al., "Theoretical and Experimental Studies of the $\mathrm{ZnSe} / \mathrm{CuInSe} 2$ Heterojunction Band Offset", Appl. Phys. Lett. 62 , 2559 (1993). 


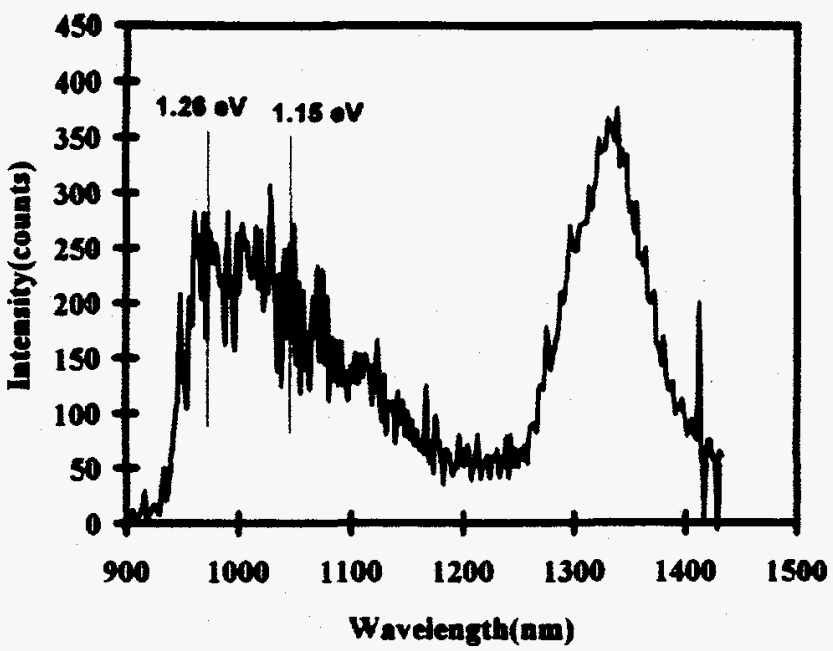

Figure 1. PL emission for Siemens CIS material.

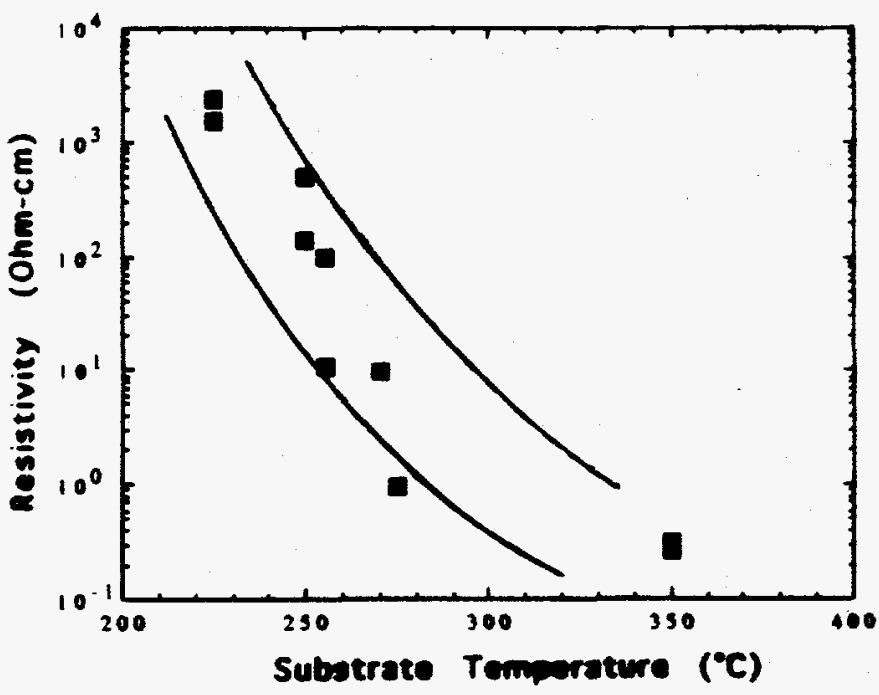

Figure 3. Resistivity of $\mathrm{ZnO}$ films grown on quartz by MOCVD.

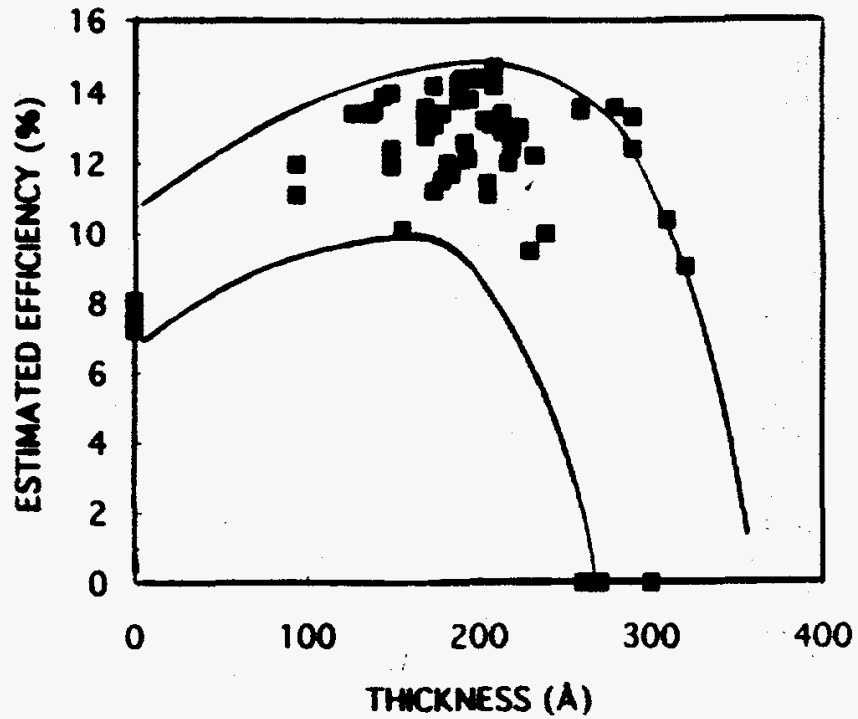

Figure 2. Test Cell efficiency vs $\mathrm{ZnSe}$ Thickness.
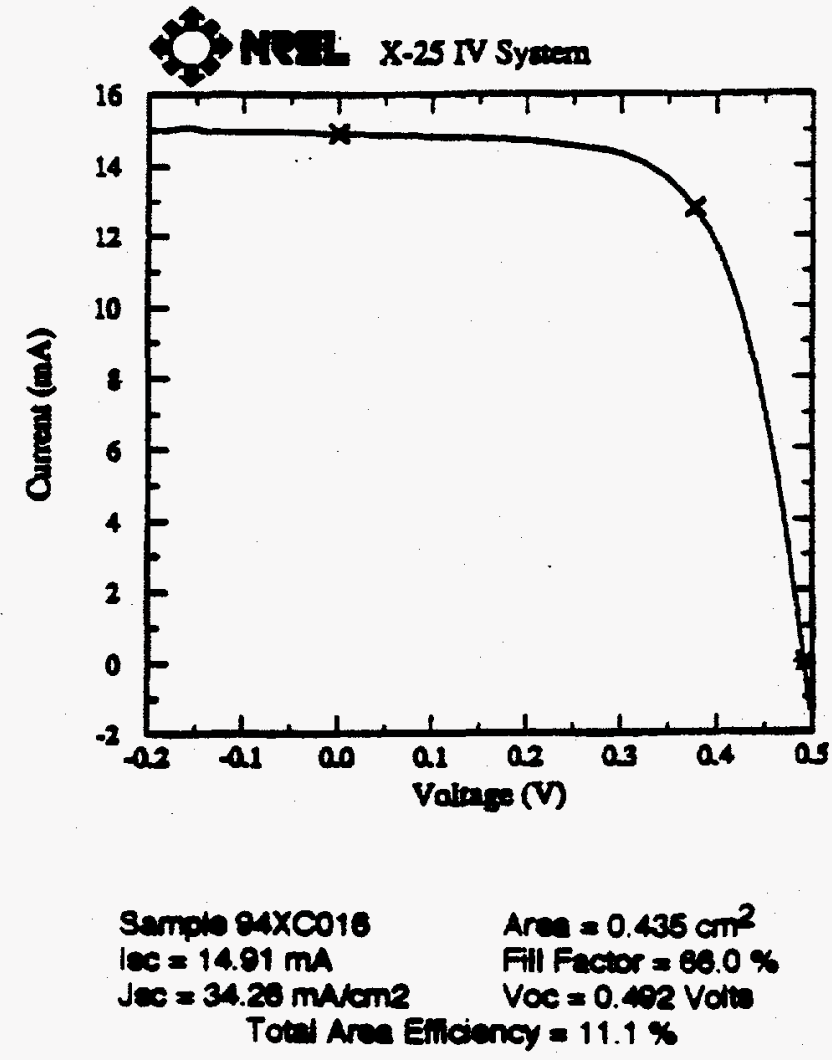

Figure 3. Illuminated I-V for direct $\mathrm{ZnO} / \mathrm{CIS}(\mathrm{GA})$ cell. 


\section{2}




\title{
4.0 PHOTOVOLTAIC MANUFACTURING TECHNOLOGY (PVMaT) PROJECT
}

\author{
C. Edwin Witt, Manager
}

The PVMaT project was initiated in 1990 to help the U.S. PV industry extend its world leadership role in manufacturing and commercially developing PV modules and systems. It is designed to accomplist this by helping the U.S. PV industry improve manufacturing processes, accelerate manufactur ing cost reductions for PV modules, improve commercial product performance, and lay the groundwork for a substantial scale-up in the capacity of U.S.-based PV manufacturing plants.

PVMaT is a government/industry R\&D partnership between the federal government (through DOE) and members of the U.S. PV industry, staggered in phases to support industry progress. The program is being carried out in four separate phases, each of which is aimed at achieving the PVMaT goals and coordinated to support the specific needs of the PV industry as it progresses toward module cost reduction and production capacity scale-up. Each phase is designed to address separate $R \& D$ requirements.

\section{Phase 1. Identified Problems}

During Phase 1-in about a 3-month period-the status and needs of the U.S. PV manufacturing industry were identified, and a Phase 2 procurement responsive to the industry's needs was developed. The Phase 1 subcontracts were completed in 1991.

Phase 2. Addresses Process-Specific Module Manufacturing Problems of Individual Manufacturers

Phase 2 actually includes two phases, Phase 2A and Phase 2B, that are discussed below.

Phase 2A. Solicitation Open Only to Organizations That Received Awards in Phase 1

Seven 3-year subcontracts were implemented in early 1992. The Phase 2A subcontracts through NREL were cost-shared between government and PV industry participants. A description of subcontracted R\&D activities and technical accomplishments under Phase $2 \mathrm{~A}$ is presented in Table 4-1. These subcontracts are completing their third and final year of research.

AstroPower, Inc. (API) is enhancing its Silicon-Film ${ }^{\mathrm{TM}} \mathrm{PV}$ manufacturing production capabilities, optimizing its solar cell processing, and improving the polycrystalline Silicon-Film ${ }^{\mathrm{TM}}$ module assembly portion of its manufacturing line. API's goals under the PVMaT project are to:

1. Design, construct, and demonstrate a production machine that generates sheets of SiliconFilm $^{\mathrm{TM}}$ at a wafer production rate equivalent to $3 \mathrm{MW} / \mathrm{yr}$

2. Develop a low-cost fabrication process for 3.15 -W solar cells that are $15 \mathrm{~cm} \mathrm{x} 15 \mathrm{~cm}$ 


\begin{tabular}{|l|l|l|}
\hline \multicolumn{3}{|c|}{$\begin{array}{c}\text { Table 4-1. PVMaT Phase 2A Subcontract Awards } \\
\text { (includes subcontractor's cost share) }\end{array}$} \\
\hline \multicolumn{1}{|c|}{ Subcontractor } & \multicolumn{1}{|c|}{ Research Emphasis } & \multicolumn{1}{c|}{$\begin{array}{c}\text { Principal } \\
\text { Investigators }\end{array}$} \\
\hline AstroPower, Inc. & Si-film PV manufacturing technology & -S. Collins, R. Hall \\
\hline $\begin{array}{l}\text { Energy Conversion } \\
\text { Devices, Inc. }\end{array}$ & $\begin{array}{l}\text { Continuous roll-to-ro a-Si PV } \\
\text { manufacturing technoiogy }\end{array}$ & M. Izu \\
\hline ENTECH, Inc. & $\begin{array}{l}\text { PV manufacturing technology } \\
\text { improvements for ENTECH's } \\
\text { concentrator module }\end{array}$ & M. O'Neill \\
\hline $\begin{array}{l}\text { Siemens Solar } \\
\text { Industries }\end{array}$ & $\begin{array}{l}\text { PV Cz Si manufacturing technology } \\
\text { improvements }\end{array}$ & T. Jester \\
\hline Solarex Corporation & $\begin{array}{l}\text { Large-area, multi-junction a-Si allow } \\
\text { production scale-up }\end{array}$ & R. Oswald \\
\hline Utility Power Group & a-Si PV manufacturing technology & M. Stern, G. Duran \\
\hline
\end{tabular}

3. Develop a large-area module production line that produces $113-\mathrm{W}_{\mathrm{p}}, 0.9-\mathrm{m}^{2}$ modules and $170 \mathrm{~W}_{\mathrm{p}}, 1.4-\mathrm{m}^{2}$ modules.

After $2 \frac{1}{2}$ years of this subcontract, API has accomplished the following:

- Fabricated the first $15-\mathrm{cm} \times 45-\mathrm{cm}$ Silicon-Film ${ }^{\mathrm{TM}}$ cell (the largest solar cell ever produced)

- $\quad$ Reduced Silicon-Film ${ }^{\mathrm{TM}}$ wafer cost by $53 \%$ and module fabrication costs by $42 \%$

- Demonstrated a $1000 \%$ increase in Silicon-Film ${ }^{\mathrm{TM}}$ wafer production capacity at 4.4 MW/yr (47\% higher than its third-year project goals)

- Increased $238-\mathrm{cm}^{2}$ (AP-225) cell fabrication capacity

- Demonstrated an NREL-verified $11.8 \%$ efficiency for the Silicon-Film ${ }^{\mathrm{TM}}$ AP-225 cell at $2.82 \mathrm{~W}_{\mathrm{p}}$

- Assembled a $93-\mathrm{W}_{\mathrm{p}}$ module into $0.9 \mathrm{~m}^{2}$ with 36 of these AP-225 Silicon-Film ${ }^{\mathrm{TM}}$ cells.

Energy Conversion Devices, Inc. (ECD) has directed its research efforts toward developing the continuous roll-to-roll a-Si PV manufacturing technology. ECD's overall goals under this subcontract are to: 
1. Support production of $0.3-\mathrm{m} \times 1.2-\mathrm{m}(1-\mathrm{ft} \times 4-\mathrm{ft})$ triple-junction a-SiGe alloy modules with $10.2 \%$ stable efficiency

2. Enhance the effective production throughput by developing advanced serpentine deposition techniques

3. Reduce material and labor costs.

Successful implementation of this project will allow ECD to work toward an ultimate goal of building a $100-\mathrm{MW} / \mathrm{yr}$ roll-to-roll, automated a-Si module manufacturing facility. At this anticipated production capacity, ECD expects to reduce the cost of PV modules to less than $\$ 1.00 / \mathrm{W}_{\mathrm{p}}$.

After the second phase of this subcontract, ECD has accomplished the following:

- Demonstrated production of triple-junction solar cells with $11.1 \%$ initial conversion efficiency

- Demonstrated $8 \%$ stabilized efficiency on a $0.37-\mathrm{m}^{2}, 31-\mathrm{W}_{\max }$ triple-junction a-Si module

- Demonstrated full production runs (762 m) with $99.7 \%$ subcell yield and high uniformity

- $\quad$ Reduced material costs by $77 \%$ for germane, $58 \%$ for disilane, and $30 \%$ for module assembly

- $\quad$ Completed improvements to the ECD a-SiGe alloy deposition system

- Established the first roll-to-roll a-SiGe production line facility

- Produced a triple-junction, small-area cell that uses a new serpentine deposition technology for the intrinsic a-Si layer, resulting in an initial conversion efficiency of $9.5 \%$ with improved gas utilization of $20 \%-30 \%$

- Developed an improved textured $\mathrm{Ag} / \mathrm{ZnO}$ back-reflector system that demonstrates a $26 \%$ gain in short-circuit current over the previous textured Al back-reflector system

- Developed a new grid/bus-bar design using thin wire grids that improve relative module efficiency by $3 \%-4 \%$ and reduce grid/bus-bar material cost by about $50 \%$

- $\quad$ Reduced material costs by $56 \%$ through cumulative improvements.

ENTECH, Inc., has directed its research under its PVMaT subcontract at PV manufacturing technology improvements for ENTECH's $21 \mathrm{X}$ linear concentrator module. The specific ENTECH goals under this project are to:

1. Enhance its concentrator module quality 
2. Increase the ENTECH concentrator module performance

3. Improve product durability

4. Expand the ENTECH manufacturing capacity to $10-\mathrm{MW} / \mathrm{yr}$

5. Address environmental, safety, and health (ES\&H) issues

6. Reduce ENTECH module manufacturing costs

7. Increase ENTECH manufacturing line automation.

Successful completion of this research is expected to result in a linear concentrator module manufacturing plant that can produce PV modules at a rate of $10 \mathrm{MW} / \mathrm{yr}$.

After $2 \frac{1}{2}$ years of this subcontract, ENTECH has accomplished the following:

- Worked with $3 \mathrm{M}$ to develop an improved prismatic solar cell lens cover tape

- $\quad$ Reduced material and labor costs by $90 \%$

- Identified four separate sources of process-compatible advanced cells, three of which provide $19 \%$-efficient cells

- Worked with $3 \mathrm{M}$ to develop a continuous, prelaminated, rolled Fresnel lens that reduced materials cost by $20 \%$ and eliminated solvent use in the ENTECH process

- Developed ribbon solder techniques that reduced labor and materials costs by $80 \%$

- Worked with Columbia Aluminum to develop the world's largest extruded heat sink

- Developed a semi-automated work station to align and attach prism covers to solar cells

- Developed new dry-film processes for attaching cell strings to the heat sink and encapsulating the cell assembly

- $\quad$ Established a team that can produce lenses, heat sinks, and cells at rates well above 10 $\mathrm{MW} / \mathrm{yr}$, with capability for a quick scale-up to $100 \mathrm{MW} / \mathrm{yr}$

- Developed a new fourth-generation module with a power output of greater that $430 \mathrm{~W}$.

Siemens Solar Industries (SSI) is investigating improvements to its Czochralski (Cz) Si PV manufacturing technology. Its goals for this research are to:

1. Improve $\mathrm{Cz} \mathrm{Si}$-ingot quality 
2. Increase the materials use efficiency for crystalline-Si (c-Si) wafer sawing

3. Investigate improvements in device processing

4. Investigate the introduction of automation to a significant portion of its $\mathrm{c}-\mathrm{Si}$ module manufacturing lines

5. Reduce the amount of hazardous waste generated.

The successful completion of this project was expected to result in a decrease in SSI's c-Si module costs of more than half, while doubling its U.S. manufacturing capability.

After $2^{1 / 2}$ years under this subcontract, SSI has accomplished the following:

- Increased wire-saw production capacity by $>30 \%$ due to reduced kerf loss and thinner wafer processing

- Increased crystal growth yields by $3 \%$ as a result of studies on polysilicon feedstock

- Eliminated Freon use and incorporating a no-clean solder paste in SSI's module production in April 1993

- Incorporated graphite design changes into the SSI crystal growers, which has resulted in a $30 \%$ savings of $\$ 300 \mathrm{~K} / \mathrm{yr}$

- Increased crystal growers' capacity by $20 \%$

- $\quad$ Devised a new lower-cost junction box

- Increased average cell yield by $4 \%$ since 1992

- Concentrated plant waste streams, which reduced waste volume by $10 \%$ and waste costs by $20 \%$.

Solarex Corporation is focusing on the large-area, multi-junction, a-Si alloy production scale-up. The goals of this effort are to:

1. Improve the deposition and quality of its transparent front contact

2. Optimize its laser patterning process

3. Scale up the semiconductor deposition process

4. Improve the back contact deposition

5. Scale up and improve the encapsulation and testing of its a-Si:H modules. 
Successful completion of this 3-year project will result in Solarex producing a-Si:H modules of $\sim 0.37 \mathrm{~m}^{2}\left(4 \mathrm{ft}^{2}\right), 10 \%$ stabilized efficiency, $50 \mathrm{~W}$, and a total overall module yield of $\geq 75 \%$. At a proposed production capacity of $10 \mathrm{MW} / \mathrm{yr}$, Solarex believes this will reduce the module cost from the present price of $\$ 11.05 / \mathrm{W}_{\mathrm{p}}$ to $\$ 1.14 / \mathrm{W}_{\mathrm{p}}$.

After $2 \frac{1}{2}$ years of this subcontract, Solarex has accomplished the following:

- Improved the manufacturing process

- $\quad$ Reduced manufacturing costs by developing front-contact deposition equipment, which uses a simpler and more cost-effective chemistry for front-contact deposition

- Increased laser processing throughputs by factors of 1.5 to 7.5

- $\quad$ Reduced area losses caused by laser interconnects on large-area modules by $50 \%$

- Demonstrated the Solarex a-Si deposition equipment capable of film thickness uniformity of $\pm 3 \%$ over an area of $0.74 \mathrm{~m}^{2}\left(8 \mathrm{ft}^{2}\right)$

- Increased by $40 \%$ the rate of use for the raw material in the a-Si deposition process

- $\quad$ Decreased by $70 \%$ the raw material cost for rear-contact deposition

- Developed a new $0.37-\mathrm{m}^{2}$ monolithic a-Si multi-junction module product with an initial conversion efficiency of $8.9 \%$.

Utility Power Group (UPG) and its major lower-tier subcontractor, Advanced Photovoltaic Systems, have focused their research on a-Si PV manufacturing technology. UPG's goals under the PVMaT project are to:

1. Significantly advance its PV manufacturing technologies

2. Reduce module production costs

3. Increase average module performance

4. Increase the production capacity of its existing manufacturing facility.

After identifying two specific process steps with the greatest potential for cost reduction in PVMaT Phase 1, UPG chose to concentrate its research efforts on encapsulating (with consideration given to approaches that do not require a second glass layer) and automating electrical termination.

After $2 \frac{1 / 2}{2}$ years of this subcontract, UPG has accomplished the following: 
- Eliminated the back glass and developed a new scratch-resistance silicone back encapsulant that is impregnated with 100 - $\mu \mathrm{m}$ glass spheres, reducing encapsulation costs by $72 \%$

- Developed an advanced electrical termination system that takes advantage of the enhanced mechanical characteristics of the new encapsulant, reducing costs by more than $81 \%$

- Eliminated 30 steps from the Powerglass PV module termination and encapsulation manufacturing processes

- Increased the average Powerglass module power rating

- Increased the level of automation associated with encapsulating and terminating

- Completed Powerglass module qualification testing for candidate encapsulation materials

- Increased the total power rated capacity of its manufacturing facility by a factor of almost five

- Increased throughput on the production line and decreased overall Powerglass module production costs by $28 \%$.

Advanced Photovoltaic Systems (APS), the major lower-tier subcontractor to UPG, has as its major goals to:

1. Greatly improve its PV manufacturing technology, primarily by improving process control

2. Increase automation of the manufacturing line

3. Improve the quality of its product.

After $2 \frac{1}{2}$ years of work as a lower-tier subcontractor under the UPG/NREL subcontract, APS has accomplished the following:

- Completed the installed equipment automation in the new Eureka production factory

- Developed a new procedure for wire connection from the PV films, which significantly cut manufacturing costs by reducing processing time $200 \%$

- Increased manufacturing capacity by reducing EVA curing time $64 \%$

- Improved Si deposition uniformity to less than $\pm 10 \%$ over the more than $1.2 \mathrm{~m}^{2}\left(13 \mathrm{ft}^{2}\right)$ of module area, which contributes to an increased module power output of $10 \%-15 \%$

- Designed full-scale Eureka modules, including enhancements developed under the PVMaT project 
- Introduced two new Eureka module products (a $25-\mathrm{W}$ and a $50-\mathrm{W}$ product for $12-\mathrm{V}$ applications).

Phase 2B. Solicitation Open to All U.S. Photovoltaic Firms

Under Phase 2B, the most recently subcontracted phase of the PVMaT Project, NREL awarded four 3-year subcontracts in late 1993. These awards included two in CdTe module manufacturing, one on the manufacture of Spheral Solar ${ }^{\mathrm{TM}}$ cells and modules, anc one on cast polysilicon wafers, cells, and module manufacturing. These subcontracts (described in Table 4-2) represent new technological additions to the PVMaT project and are cost-shared at $58 \%$ by the subcontractors.

Phase 2B was open to all U.S. PV industrial firms. This allowed organizations not ready for the earlier Phase $2 \mathrm{~A}$ procurement cycle, or that did not participate in Phase 1, to ramp on and participate in this process-specific solution phase of the project. These subcontracts represent new technology additions to the PVMaT project. Subcontracts initiated in earlier phases are nearing completion, and their progress is summarized.

Table 4-2. PVMaT Phase 2B Subcontract Awards (includes subcontractor's cost share)

\begin{tabular}{|l|l|l|}
\hline \multicolumn{1}{|c|}{ Subcontractor } & \multicolumn{1}{|c|}{ Research Emphasis } & Principal Investigators \\
\hline Golden Photon, Inc. & $\begin{array}{l}\text { Commercial scale-up of advanced thin- } \\
\text { film PV technologies }\end{array}$ & S. Johnson \\
\hline Solar Cells, Inc. & $\begin{array}{l}\text { High-throughput manufacturing of thin- } \\
\text { film CdTe PV modules }\end{array}$ & D. Sandwisch \\
\hline Solarex Corp. & $\begin{array}{l}\text { Cast polycrystalline Si PV cell and } \\
\text { module manufacturing technology } \\
\text { improvements }\end{array}$ & J. Wohlgemuth \\
\hline Texas Instruments & $\begin{array}{l}\text { PV Manufacturing Technology Phase } \\
\text { 2B-spheral solar technology }\end{array}$ & J. Skelly, R. Schmit \\
\hline
\end{tabular}

Golden Photon, Inc., is concentrating its activities on the commercial scale-up of advanced thinfilm CdS/CdTe PV modules produced using spray deposition. The goals set by Golden Photon for this subcontract include developing and implementing:

1. Improved production processes and subsequent equipment designs that allow incremental scale-up to a $2-\mathrm{MW} / \mathrm{yr}$ manufacturing capacity

2. An employee exposure monitoring plan that will ensure employee safety from Cd exposure 
ES\&H programs that meet the requirements of all local, state, and national authorities.

At the completion of the subcontract, these activities are expected to result in a $2-\mathrm{MW} / \mathrm{yr} \mathrm{CdTe}$ module manufacturing line that can produce at about $\$ 1.50-2.00 / \mathrm{W}_{\mathrm{p}}$.

During the first year of this subcontract, Golden Photon accomplished the following:

- Completed its design for installation and startup of a 2-MW/yr CdTe module manufacturing line

- Initiated a "prove-in" phase of operation.

Solar Cells, Inc. (SCI) has directed its research at developing high-throughput manufacturing of thin-film CdTe PV modules. Its goals under the PVMaT project are to:

1. Advance its PV manufacturing technologies

2. Reduce module production costs

3. Increase module performance

4. Provide the groundwork for SCI to expand its commercial production capacities.

SCI plans to meet these objectives by designing and debugging a $20-\mathrm{MW} / \mathrm{yr}$, automated, continuous PV manufacturing line that produces $60-\mathrm{cm} \times 120-\mathrm{cm}$ CdTe modules.

After the first phase of this subcontract, $\mathrm{SCI}$ has accomplished the following:

- Completed improvements in raw material supply, glass conveyance, pressure control, and temperature control that have increased the SCI pilot system capacity by a factor of 100

- Designed a high-throughput deposition system for the 20-MW line

- Initiated interim qualification testing of $\mathrm{SCI} 60-\mathrm{cm} \times 120-\mathrm{cm}$ CdTe modules

- Increased average module efficiencies by more than $15 \%$ (from $6.3 \%$ to $7.4 \%$ )

- Implemented an ES\&H program to semiannually test and monitor personnel exposure to Cd.

Solarex Corporation (Crystalline Silicon Division) has focused its research under this subcontract on advancing its cast-ingot polycrystalline Si PV module manufacturing technology. Solarex's goals under PVMaT Phase 2B are to:

1. Increase automation for assembling modules and handling larger wafers 
2. Improve the module manufacturing throughput, yield, and process control

3. Reduce PV module production labor and production costs to less than $\$ 1.20 / \mathrm{W}$

4. Increase solar cell performance from $12.8 \%$ to $15 \%$

5. Increase wafer production by using wire saws to reduce center-to-center cut distances from $600 \mu \mathrm{m}$ to $400 \mu \mathrm{m}$

6. Develop larger wafers through increased ingot casting sizes

7. Expand commercial module manufacturing capacity by a factor of 3 .

After the first phase of this subcontract, Solarex has accomplished the following:

- Implemented design modifications to one casting station, which increased production capacity by $73 \%$, and initiated modifications to the remaining stations, which increased their production capacity by $20 \%$ each

- Developed a process for casting larger ingots with a $15-\mathrm{cm} \times 15-\mathrm{cm}$ cross-section and transferred it onto the manufacturing line to replace the previous bricks with a $11.4-\mathrm{cm}$ $\mathrm{x}$ 11.4-cm cross-section

- Integrated a new wire saw for the cutting cast polysilicon bricks

- Demonstrated an eight-brick-at-a-time wire-saw cutting run, which resulted in 2400 wafers in $8 \mathrm{~h}$.

Texas Instruments, Inc. (TI) is directing its subcontract activities under PVMaT toward research on Spheral Solar ${ }^{\mathrm{TM}}$ Si PV technology. TI's goals under this subcontract are to:

1. Optimize existing processes

2. Investigate alternative approaches for reducing material waste, material handling (labor) costs, and cycle times of its Spheral Solar ${ }^{\mathrm{TM}}$ technology to demonstrate a module manufacturing process with costs of $\$ 2.00 / \mathrm{W}$

3. Increase manufacturing yield while reducing cost and improving logistics via sphere fabrication processes

4. Improve cell yields by optimizing bonding pressures, temperatures, and foil configuration parameters

5. Increase Spheral Solar ${ }^{\mathrm{TM}}$ cell efficiencies from $8.5 \%$ to greater than $11 \%$.

After the first phase of this subcontract, TI has accomplished the following: 
- Developed equipment capable of multi-MW production rates for the highest-risk processes

- Identified a new proprietary furnace design to more effectively handle initial melt operations while increasing throughput and reducing capital costs

- Improved the front bond process, resulting in a $>65 \%$ increase in the yield on $>7.0 \%$ efficient unencapsulated cells

- Demonstrated cell yields as high as $90 \%$ on the pilot production line

- Investigated surface passivation using forming gas, resulting in an initial $1.8 \%$ increase in $\mathrm{V}_{\mathrm{oc}}$ and a $2.9 \%$ increase in the $\mathrm{J}_{\mathrm{sc}}$ for $10-\mathrm{cm}^{2}$ cells

- Demonstrated an $8.3 \%$-efficient, $4000-\mathrm{cm}^{2}$ module using a nonconventional encapsulant.

Phase 3. Addresses Research and Development

This phase uses a teamed research approach to address problems common to the PV industry as a whole. Two subcontracts were awarded in January 1993. They focus on the module-related $\mathrm{R} \& \mathrm{D}$ problems shown in Table 4-3.

\section{Phase 3A. Focuses on Common Problems}

The PVMaT Phase 3A subcontracts (described in Table 4-3) both began in early January 1993. They have focused on module-related R\&D problems that are common to several PV manufacturing groups.

\begin{tabular}{|l|l|l|}
\hline \multicolumn{3}{|c|}{$\begin{array}{c}\text { Table 4-3. PVMaT Phase 3A Subcontract Awards } \\
\text { (includes subcontractor's cost share) }\end{array}$} \\
\hline \multicolumn{1}{|c|}{ Subcontractor } & \multicolumn{1}{|c|}{ Research Emphasis } & Principal Investigator \\
\hline Spire Corporation & $\begin{array}{l}\text { Automated solar cell assembly teamed } \\
\text { process research }\end{array}$ & M. Nowlan \\
\hline $\begin{array}{l}\text { Springborn } \\
\text { Laboratories, Inc. }\end{array}$ & PV manufacturing technology & B. Holley \\
\hline
\end{tabular}

Spire Corporation has focused its research on automated solar cell assembly. Spire's overall goal under the PVMaT project is to reduce PV costs by developing high-throughput (5-MW/yr), automated processes for interconnecting thin $(200-\mu \mathrm{m})$ Si solar cells. Significantly more $200-\mu \mathrm{m}$ wafers can be produced from a Si ingot than conventional $350-\mu \mathrm{m}$ to $400-\mu \mathrm{m}$ wafers, thus reducing cell cost. However, an unacceptably high breakage rate in processing normally makes such thin cells impractical. In this project, Spire has attempted to achieve high yields with these 
fragile cells by developing processes that reduce mechanical and thermal stresses and increase the use of automation and higher process throughput. At the completion of this subcontract, an automated processing system for cell tabbing is expected to be available as a commercial product to PV manufacturers.

Spire's accomplishments include developing the prototype, fully automated Spi-Assembler ${ }^{\mathrm{TM}} 5000$ solar cell assembly system for processing standard and thin solar cells with cell-testing capabilities. Spire ssearchers have developed low-stress processes for loading, aligning, and soldering cells of different thicknesses. They have also conducted demonstration tests with the Spi-Assembler ${ }^{\mathrm{TM}} 5000$ in cooperation with SSI, API, Solec, and others, using company-specific cells.

Springborn Laboratories, Inc., has focused its research on understanding the degradation of EVA-based encapsulants in PV modules. Springborn's goals under the PVMaT project are to:

1. Pursue the advanced development of a PV module encapsulation sheet

2. Reformulate the EVA-based elastomer

3. Formulate alternative elastomer-based encapsulants that will more effectively resist degradation when exposed to high temperature and UV insolation in the field. Some EVA-based encapsulants have yellowed or browned at a number of installations worldwide, where both temperature and UV radiation are comparatively high.

After the first phase of this subcontract, Springborn has accomplished the following:

- Established a baseline of information through a complete literature search and survey of case histories of EVA-based encapsulants in PV devices

- Completed accelerated testing on samples of various combinations of glass and encapsulants such as EVA and Tedlar

- Discovered that glass supplied over the past several years by two particular suppliers transmits significantly less radiation in the UV-B region than glass supplied during the 1980s.

\section{Phase 4A. Emphasizes Product-Driven Manufacturing Research and Development}

The intention of Phase 4A is to emphasize improvement and cost reduction in manufacturing fullsystem PV products. The work areas may include, but are not limited to, issues such as improvement of module manufacturing processes; system and system component packaging, integration, manufacturing, and assembly; product manufacturing flexibility; and balance-ofsystem development with the goal of product manufacturing improvements. 
This phase of the PVMaT project is now being initiated. Proposals in response to the Phase 4A solicitation were received in October 1994. They are currently in the evaluation stage, with awards planned for mid-1995.

\section{Progress in the Photovoltaics Industry}

It is important to put into perspective the technical progress being made by the U.S. PV industry. Based on manufacturing process improvements, cost reductions, new product introduction, and product performance improvements, the PV industry would be expected to exhibit both a scale-up of its U.S.-based PV manufacturing capacity and a reduction in its production costs. This has indeed been the case.

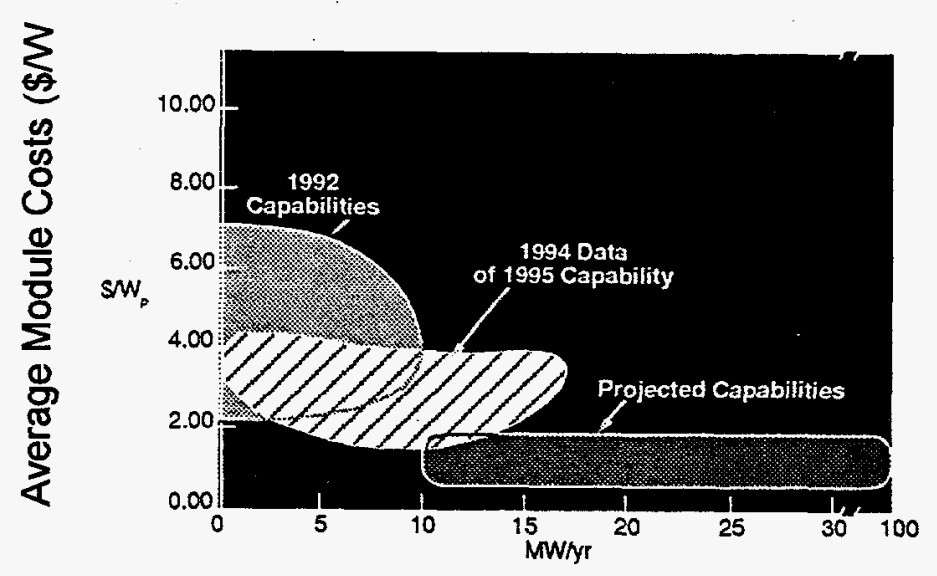

Figure 4-1. PVMaT Phase 2A Initial Manufacturing Cost/Capacity Goals

At the beginning of Phase $2 \mathrm{~A}$ subcontracted research in the PVMaT project, information was collected to establish the status of the current and projected capacities and module costs for the eight subcontractors (APS was included as a lower-tier to UPG). These data, shown in Figure 4-1, represented the diverse status of a still-maturing industry and the optimistic speculation of its members regarding the effects the project would have after PVMaT efforts were allowed to take effect. Additional information was collected as these subcontractors neared the completion of their individual subcontracts in late 1994.

These recent data, shown in Figures 4-1 and 4-2, represent an update of the previous projections regarding these efforts. Data projected in these figures are based on each manufacturer's maximum production capacity during a given year, assuming they were to operate $24 \mathrm{~h} / \mathrm{d}$ and 365 $\mathrm{d} / \mathrm{yr}$. Module cost estimates were then based on these manufacturing levels and included only those costs directly associated with manufacturing the modules (excluding marketing, administration, sales, etc.). The data associated with any particular point in time represent a potential capability. Actual manufacturing production levels may be less (and concomitant costs lower) because of considerations such as market conditions and available labor.

The data for establishing the 1995 module manufacturing costs have a relatively high degree of accuracy, as capital equipment for 1995 plant installations is generally already in place or on order. This lends significant credibility to the trend represented in Figure 4-2 for the Phase 2A subcontractors. When compared to the 1992 "Current Capabilities" and the "Projected 
Capabilities" in Figure 4-1, the Phase 2A subcontractors appear to be on track for achieving their original goals.

In conjunction with the 1994 collection of cost/capacity data for the Phase 2A participants, an initial inquiry of the four PVMaT Phase 2B participants was conducted. The result of this data collection is presented in Figure 4-3. The same diverse initial status and optimistic speculation regarding their future after their PVMaT supported research is represented. However, these participants had a higher ratio of "new technology manufacturers" involved in their group, as can be seen by the size and spread in initial module costs.

Figures 4-1, 4-2, and 4-3 indicate that the U.S. PV industry involved in the PVMaT project has significantly reduced manufacturing costs and increased PV module manufacturing capacity. They also indicate its future cost/capacity date and its technical projections that its optimism for continuing these improvements is high.

An indication of an industry's strength is the relative market share. Figure 4-4 shows the U.S. market share on a world basis declined significantly during the early 1980s. Although the U.S. PV market share began declining in 1982, the marked decline of production in 1985 clearly reflects the end of energy tax credits in the United States. Since that time, the U.S. production has hovered between $30 \%$ and $36 \%$ of the world total. It is promising, however, that the U.S. market share strengthened in 1993, achieving 35\% of the world shipments. We believe an element of this strengthening position is the result of a new generation of PV modules with improved performance and durability, and these modules have been developed with the help of government-industry partnerships that form the cornerstone of the PVMaT project.

The PVMaT project is currently completing its subcontracted efforts under the Phase 2A 3-year solicitation, with research in Phases $2 \mathrm{~B}$ and $3 \mathrm{~A}$ well under way. Proposals for product-driven research under the newest procurement, Phase $4 \mathrm{~A}$, are currently in the evaluation stage, with awards expected later in 1995.

The members of the U.S. PV manufacturing industry that have participated in the DOE PVMaT government/industry R\&D partnership have progressed toward achieving their individual goals. They have successfully increased the performance and reliability of their products, increased the yield and throughput of the production lines, reduced PV product manufacturing costs, introduced new product lines, and scaled up PV manufacturing plant capacity. We believe that these activities, supported by the PVMaT government/ industry R\&D partnership, have helped 


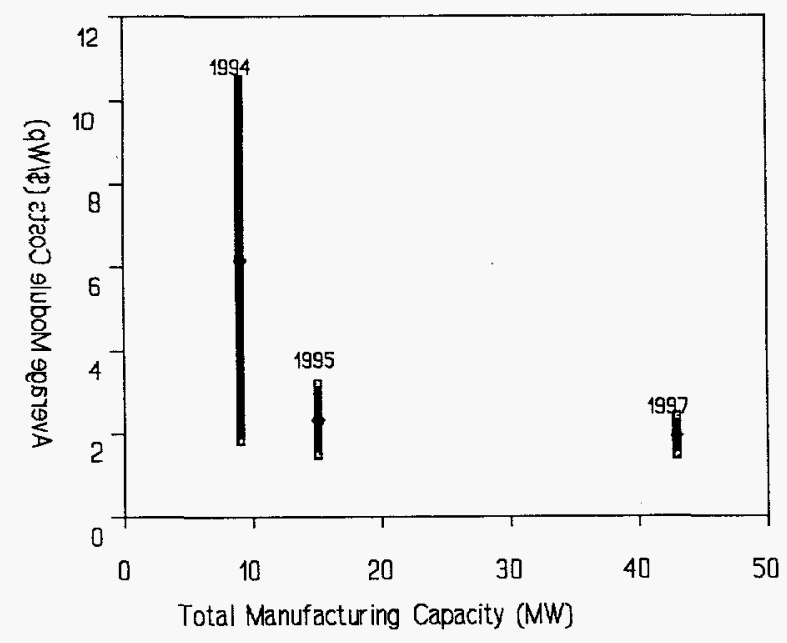

strengthen and stabilize the U.S. PV manufacturing industry as a whole, and this partnership is providing the U.S. industry with a solid base for a significantly improved and less costly renewable energy technology.

Figure 4-3. PVMaT Phase 2B Current

Manufacturing Cost/Capacity Levels

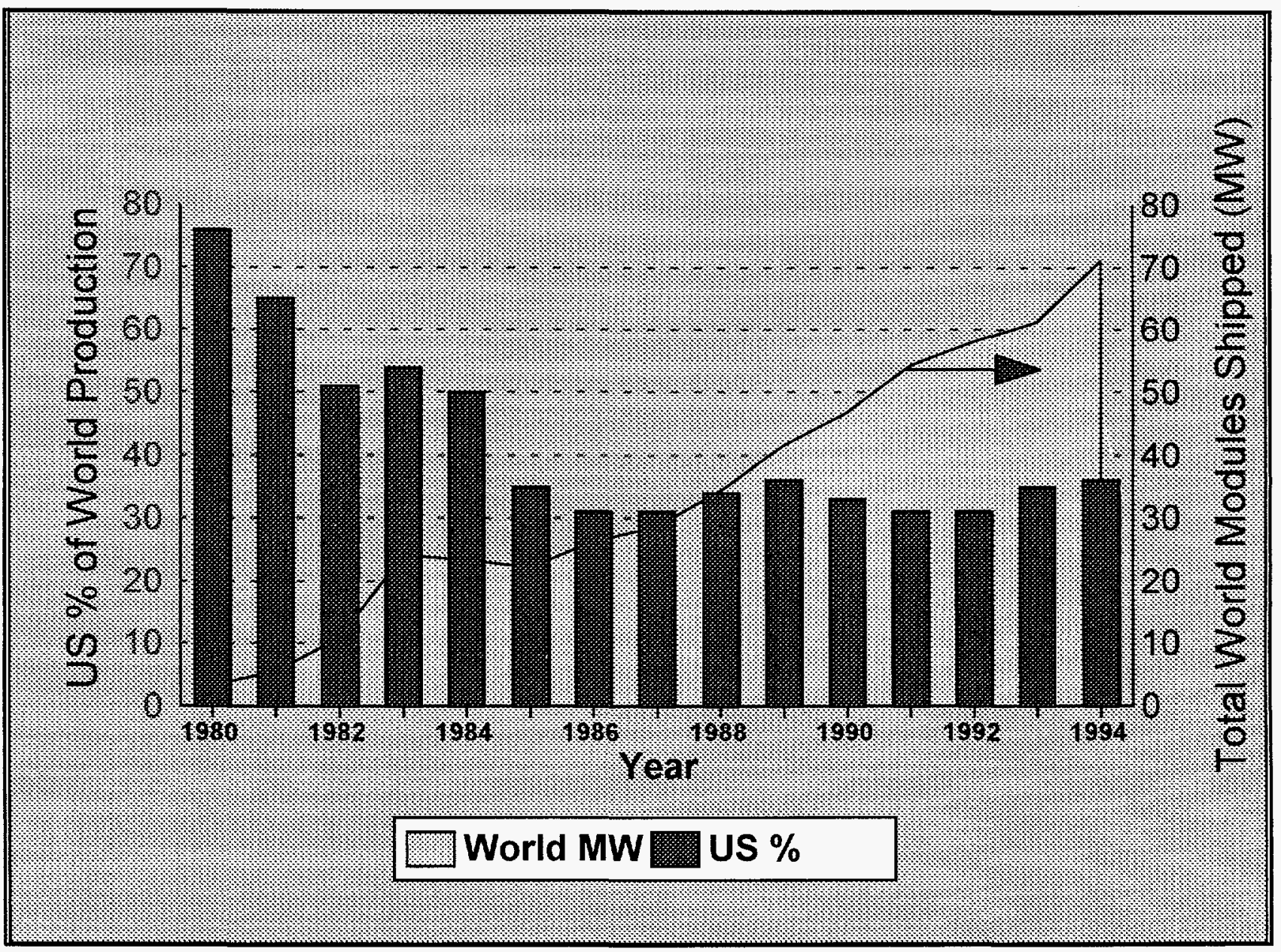

Figure 4-4. U.S. Market Share of World Production 
PVMaT Subcontracts 


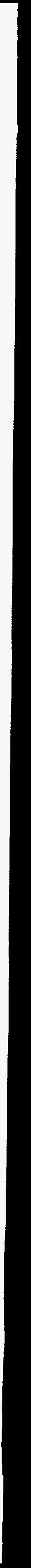




\title{
Amorphous silicon Photovoltaic Manufacturing Technology - Phase II
}

\author{
Advanced Photovoltaic Systems \\ Princeton, New Jersey \\ Fairfield, California
}

\author{
H. N. Volltrauer, program manager \\ A. Roniares, manager Fairfield facility
}

\section{objectives}

The objectives of this program are to significantly advance PV manufacturing technology, reduce module production costs, increase module performance and increase the production capacity of APS. The APS program consists of four separate tasks: new module design, process and quality control, automation of the manufacturing line and information management.

\section{Results}

Four major areas of accomplishment supported by the PVMaT program will be discussed. These are, the new APS manufacturing facility, new products, equipment issues and quality issues.

\section{APS' 12 MW Manufacturing Facility}

The major accomplishment for the past year was the startup of the new 12 MW APS manufacturing facility in Fairfield California. The first a-Si deposition run was made in December of 1993 and production runs followed soon thereafter. While production has not yet reached the desired level of performance (production stopped in May of 1994 because of a court restraining order related to a patent dispute with solarex Corporation), the line is capable of making high quality modules at low cost.

The current status of performance is summarized in Fig 1 , where the as-made output distribution for deposition runs made in Trenton during PVUSA production (EMF, Eureka Manufacturing Facility) is compared to production in the new Facility (FF). and to optimization work carried out in Trenton following the PVUSA production (PD). Additional work needs to be done to increase the average power output of the modules and the yield. The changes needed to bring this about have in large degree been identified and some will be discussed below. Thus much of the yield increases (and a tighter distribution of power output) will be achieved with newly designed box carriers (the enclosure in which the glass is held during silicon deposition) and improved rf cables. Advances in back contact lasering will also help increase the output. 
Based on the extent of automation, the volume of production and the increased power output of the modules, production costs are estimated to be near $\$ 1.00$ per Wp in four years.

\section{New Products}

In addition to the $20 \%$ increase in module output (now at $60 \mathrm{~W}$ ), two other new products were delivered in the past year. The first was a semitransparent module of which prototypes were delivered to NREL, and the second was PV plate delivered to Flachglass Solartechnik (Flagsol) of Koln, Germany. 420 APS a-Si plates rated at $50 \mathrm{~W}$ each were installed by Flagsol in a double-glass structure in the world's largest curtain wall system, a seven-story building of the Joint Research Centre for the European Community in Ispra, Italy.

APS modules were chosen because of low cost and uniform appearance. The much improved uniformity of these modules compared to the product shipped to the PVUSA site in Davis California, made for a very attractive structure. According to Mr. Joachim Benemann, Managing Director of Flagsol, "We estimate the use of PV adds under $20 \%$ to the cost of a curtain wall system, and less than $1 \%$ to the total cost of a typical multi-story building."

\section{Equipment issues}

Several pieces of equipment were modified for the new factory. In addition, more equipment will have to be upgraded to complete the planned level of automation for the line.

In the first category, changes were made to the box carrier for holding the glass during silicon deposition and to the rf cables that supply the rf to the electrodes of the box carrier.

The major causes of non uniform deposition had already been identified and corrections tested and verified. Based on these results, a new box carrier was designed. It is expected to achieve a deposition rate uniformity within plus minus $5 \%$ over the entire active area of the $13 \mathrm{sq}$. ft. plate.

Because this design moves the rf feed point to the bottom center of the box carrier, much longer rf cables are required. The previously used design incorporating a commercial coaxial cable was found to be unreliable, and a new scheme was developed. The new cables are constructed using copper and teflon tubing with terminations made of copper and connections spring mounted at the door of the box carrier. Bench tests of these cables showed them to be capable of carrying 100 Amps at $60 \mathrm{~Hz}$ for five minutes and withstanding 8000 volts.

In the area of automation, work on two pieces of equipment has been initiated. A design had been developed for equipment that will automatically load and unload the laminators. The device for doing this will consist of two swinging arms, one on each side of the 
laminator, connected with a rod on top and pivoted at the floor in the area between the laminator and the conveyor which transports the glass. Attached to the connecting rod will be vacuum lifters for pulling the plate from either the conveyor or laminator and placing it on the other. Counterweights will be used to minimize the motor requirements for driving the device. Calculations have been made to determine the floor plan and work envelope of the device.

For the second piece of equipment, which will automatically open and close the door of the preheat and deposition chambers, $a$ conceptual design has been arrived at. It is expected that both of these designs will be implemented in the next year.

\section{Quality issues}

During initial startup of the factory, several quality related problems were identified and studied. One of these is the shorting introduced during aluminum lasering. Besides reducing the power output of the modules, this shorting, because it is often erratic and variable, makes it difficult to evaluate the rest of the processing steps. Two interesting aspects of this problem will be briefly described. The first is the well defined nature of the problem that is sometimes (but not always) observed. Fig. 2 shows the shunting that is observed as a function of the thickness of the a- Si film. As can be seen the shunting becomes exponentially worse as the thickness becomes less. Some control of the lasering is possible by changing parameters such as laser power level or pulse width, but the general relationship shown is often obtained. The results shown in the figure were obtained in $5 \mathrm{~cm}$ wide test cells on one particular plate, they are rarely that well defined.

In order to evaluate the remainder of the process, techniques were developed to calculate the effect of the shunting on the IV parameters, and mathematically remove them. This was simply done by deliberately shunting cells, measuring the change in Voc and FF as a function of the degree of shunting $g$ and then empirically fitting the shunt resistance to the changes in Voc and FF.

As part of a report format developed for analyzing factory performance, Voc and FF both "corrected" for shunting are printed together with the as-measured values. As a result of these calculations, it appears that up to $5 \%$ of power is lost for typical i-layer thicknesses.

\section{Plans}

Emphasis in the next year will be on further automating the line, improving quality and yield and implementing a factory wide information system. 


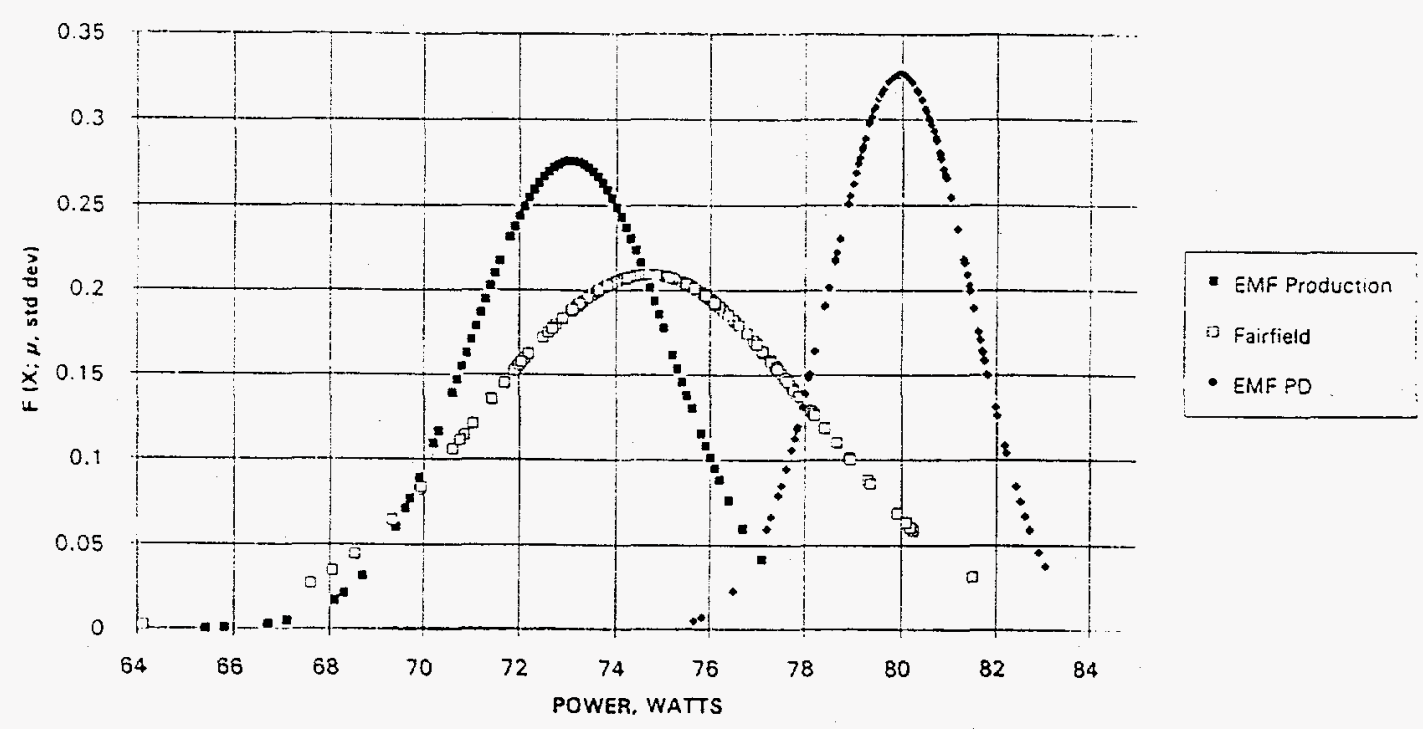

Figure 1. Performance Comparison between Factories

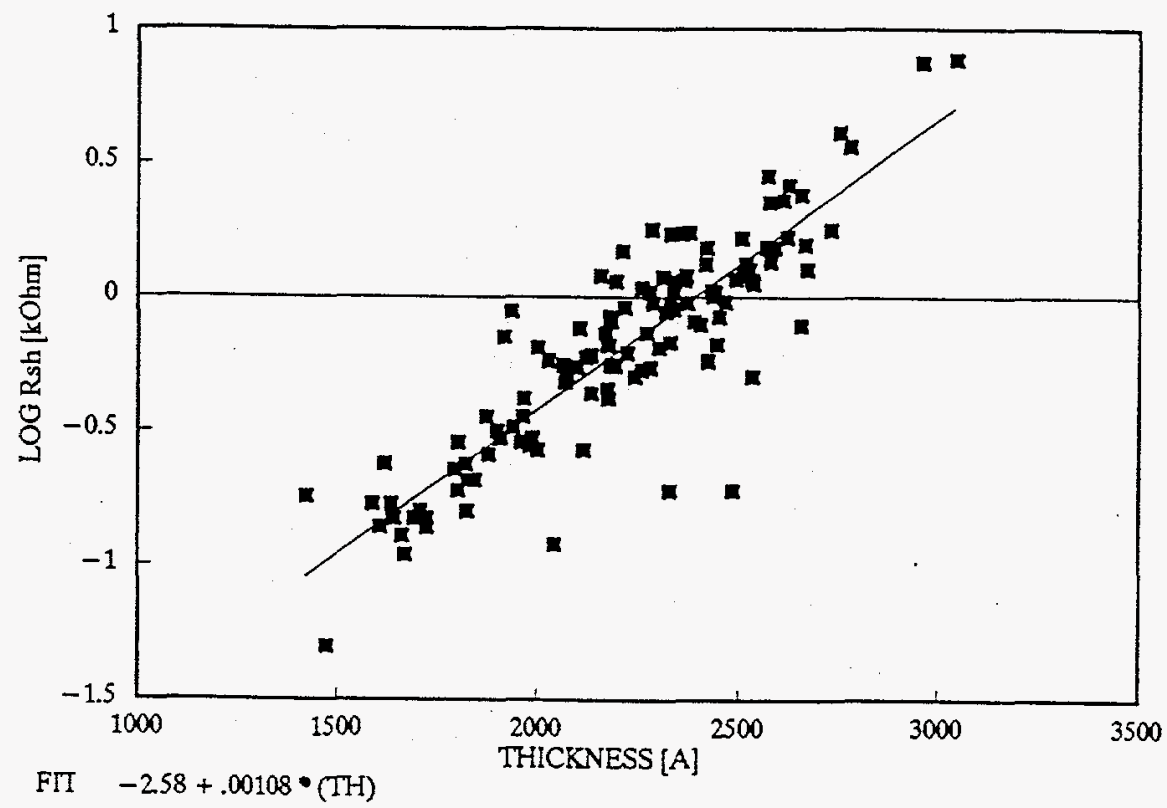

Fig 2. Variation of Shunt Resistance, Rsh, with Thickness 
Title:

$\begin{array}{ll}\text { Organization: } & \text { AstroPower, Inc. } \\ & \text { Newark, Delaware }\end{array}$

Contributors: $\quad$ S.R. Collins and R.B. Hall, principal investigators; J.C. Checchi, C.L. Kendall, S.M. Lampo, D.H. Ford, J.A. Rand, A.M. Trabant, A.M. Barnett

\section{INTRODUCTION}

AstroPower is in the third phase of a three year project to upgrade its facility to produce low cost $1.4 \mathrm{~m}^{2}$ Silicon-Film ${ }^{\mathrm{TM}}$ photovoltaic modules with an output of $170 \mathrm{Wp}$. The key contract milestones are shown in Table I. During the past year our efforts have focused on improving the following aspects of the Silicon-Film ${ }^{\mathrm{TM}}$ process:

* device performance

* wafer throughput

* overall yield through wafer and solar cell production

* wafer machine reliability

We were successful in all four areas: solar cell efficiency was increased from $10.3 \%$ to $11.8 \%$; wafer throughput was improved by $117 \%$; a $62 \%$ improvement was achieved in overall yield through solar cell fabrication; and production runs were extended to 16 continuous hours.

\section{APPROACH}

The Silicon-Film ${ }^{\mathrm{TM}}$ Process generates a continuous sheet of polycrystalline silicon on a low cost substrate. Because these sheets are produced at the desired thickness, ingot sawing and wafer polishing steps are eliminated resulting in a significant reduction in cost. The larger area and resulting higher power of the Silicon-Film ${ }^{\mathrm{TM}}$ products result in less handling and less labor per generated watt. The current project is focused on $16 \mathrm{~cm}$ wide sheets producing $240 \mathrm{~cm}^{2}$ solar cells. Figure 1 is a photograph showing 40 inches of Silicon-Film ${ }^{\mathrm{TM}}$ sheet with a standard 100 $\mathrm{cm}^{2}$ solar cell and $240 \mathrm{~cm}^{2}$ (AP-225) solar cells in the foreground.

\section{RESULTS}

Improvements in solar cell efficiency were achieved through changes in machine component design and developments in post-growth material enhancement processes. The redesign of critical machine components led to significant improvements in material quality uniformity. A typical wafer now has a median diffusion length of 45 microns across its $15.5 \mathrm{~cm}$ width; this is a significant improvement over last year's maximum diffusion length of 50 microns but median diffusion length of 20 microns. Development of a post-growth gettering process led to a two-fold increase in the as-grown diffusion lengths. These increases in median diffusion length have led to the demonstration of $11.8 \%$ efficient $240 \mathrm{~cm}^{2}$ solar cells. 
Improvements in machine component design and machine reliability were key to the advances in the Silicon-Film ${ }^{\mathrm{TM}}$ production process development during Phase III. Areal generation rate was increased by $117 \%$; material quality was preserved at these higher rates by altering machine component designs to maintain the required thermal environment. Wafer yields were increased by $40 \%$ by sequentially increasing operating hours to identify component lifetime and degradation. Component design was then altered to extend lifetime or eliminate degradation.

An $11 \%$ increase in solar cell visual/mechanical yield was achieved by increasing the volume of wafers through the solar cell production line up to 1000 wafers per day for a period of two months. Improvements in the fabrication process in the areas of diffusion, contact printing, contact firing, and anti-reflection coating were required. In addition, feedback from the solar cell fabrication line to wafer production allowed subtle but high impact changes that resulted in further improvements in solar cell yield. With the previously described developments, the overall yield of the Silicon-Film ${ }^{\mathrm{TM}}$ process was improved by $62 \%$ during 1994 . Overall yield includes wafer production yield during 16 hours of operation, solar cell visual/mechanical yield, and solar cell electrical yield.

The objective for Phase III was to demonstrate a continuous process with a production capacity of $3.0 \mathrm{MW} / \mathrm{year}$ and a material use efficiency (MUE) of $90 \%$. Both of these objectives were met. (Production capacity is calculated using the highest efficiency measured by NREL on a solar cell fabricated from representative material. MUE is calculated using applicator yield, geometric yield, and visual and mechanical yield of the wafer production process.) Presently, our operating conditions for production runs 16 hours in duration yield a production capacity of $4.4 \mathrm{MW} / \mathrm{yr}$ with an MUE of $86 \%$.

NREL tested Silicon-Film ${ }^{\mathrm{TM}}$ solar cells and modules fabricated during Phase III. The following list summarizes their findings:

$\Rightarrow 2.8$ Wp solar cell with a total area of $240 \mathrm{~cm}^{2}$ (Figure 2).

$\Rightarrow 93.0 \mathrm{Wp}$ module of $36,240 \mathrm{~cm}^{2}$ solar cells with a total area of $0.95 \mathrm{~m}^{2}$.

$\Rightarrow 7.9$ Wp solar cell with a total area of $676 \mathrm{~cm}^{2}$.

Figure 3 illustrates the progress demonstrated and projected for both overall yield (including wafer and solar cell yields) and production capacity for the Silicon-Film ${ }^{\mathrm{TM}}$ process.

\section{CONCLUSIONS}

During 1994, five critical elements were achieved in the development of a Silicon-Film ${ }^{\mathrm{TM}}$ manufacturing technology. These elements are:

1. parallel improvements in solar cell efficiency, areal generation rate, yield, and machine reliability.

2. production of 2.8 watt, $240 \mathrm{~cm}^{2}$ Silicon-Film ${ }^{T M}$ solar cells, and

3. a 93 watt, 36 cell module fabricated from $240 \mathrm{~cm}^{2}$ Silicon-Film ${ }^{T M}$ solar cells

4. at a standard production rate capable of $4.4 \mathrm{MW} / \mathrm{yr}$.

5. demonstration of a 2.8 times larger solar cell $\left(676 \mathrm{~cm}^{2}\right)$ with a 2.8 times higher power (7.9 watts). 
The focus of our continuing efforts will be to improve machine reliability through continuous 'non-failure' runs, reduce costs by reducing the thickness of the Silicon-Film ${ }^{\mathrm{TM}}$ sheet; improve solar cell performance of the AP-225 to 3.15 watt solar cells and a 56 cell, 170 watt module; and to further increase areal generation rate without sacrificing solar cell performance to achieve an even higher production capacity.

Table I. Silicon-Film ${ }^{\mathrm{TM}}$ Manufacturing Technology Milestones

\begin{tabular}{lccc}
\hline & Phase I & Phase II & Phase III \\
\hline $\begin{array}{l}\text { Wafer Machine } \\
\text { Production Rate }\end{array}$ & $400 \mathrm{~kW} / \mathrm{yr}$ & $1.3 \mathrm{MW} / \mathrm{yr}$ & $3.0 \mathrm{MW} / \mathrm{yr}$ \\
Material Use Efficiency & $75 \%$ & $85 \%$ & $90 \%$ \\
Solar Cell Size* & a) $10 \mathrm{~cm} \times 10 \mathrm{~cm}$ & b) $15 \mathrm{~cm} \times 15 \mathrm{~cm}$ & b) $15 \mathrm{~cm} \times 15 \mathrm{~cm}$ \\
& b) $15 \mathrm{~cm} \times 15 \mathrm{~cm}$ & c) $15 \mathrm{~cm} \times 45 \mathrm{~cm}$ & c) $15 \mathrm{~cm} 45 \mathrm{~cm}$ \\
Solar Cell Power & a) 1.1 watts & b) 2.5 watts & b) 3.15 watts \\
& & & c) 7.0 watts \\
Module Power & $-\cdots-\cdots$ watts & 170 watts \\
& & $(36$ cells) & (56 cells) \\
\hline
\end{tabular}

* Product Identification: a) AP-100, b) AP-225, c) AP-675

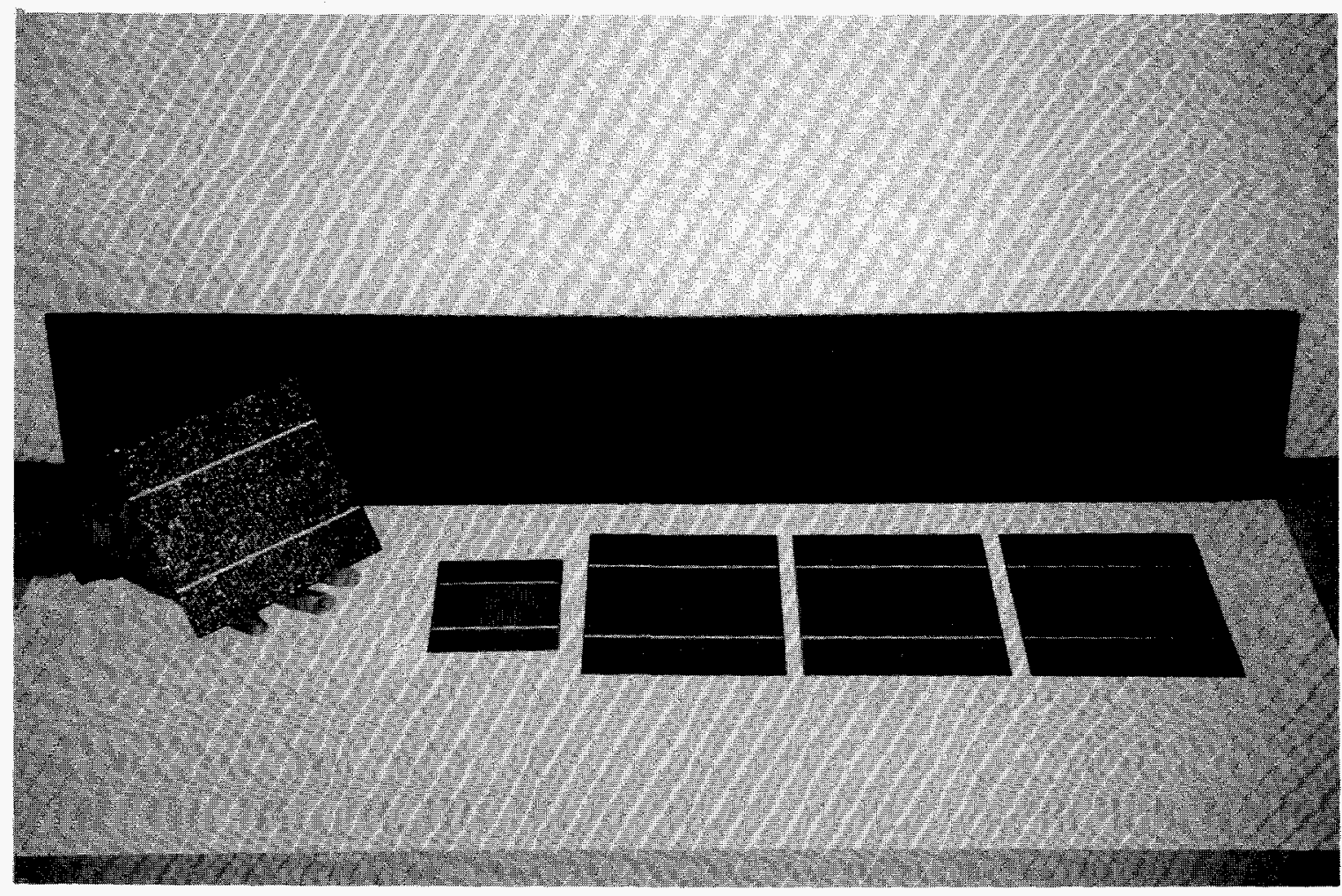

Figure 1. Photograph showing 40 inches of Silicon-Film ${ }^{\mathrm{TM}}$ sheet with a standard $100 \mathrm{~cm}^{2}$ solar cell and $240 \mathrm{~cm}^{2}$ (AP-225) solar cells in the foreground. 
Sample: D21\#85

Oct 18, 1994 12:46 PM

ASTM E 892-87 Global
Temperature $=25.0^{\circ} \mathrm{C}$

Area $=238.7 \mathrm{~cm}^{2}$

Irradiance: $1000.0 \mathrm{Wm}^{-2}$

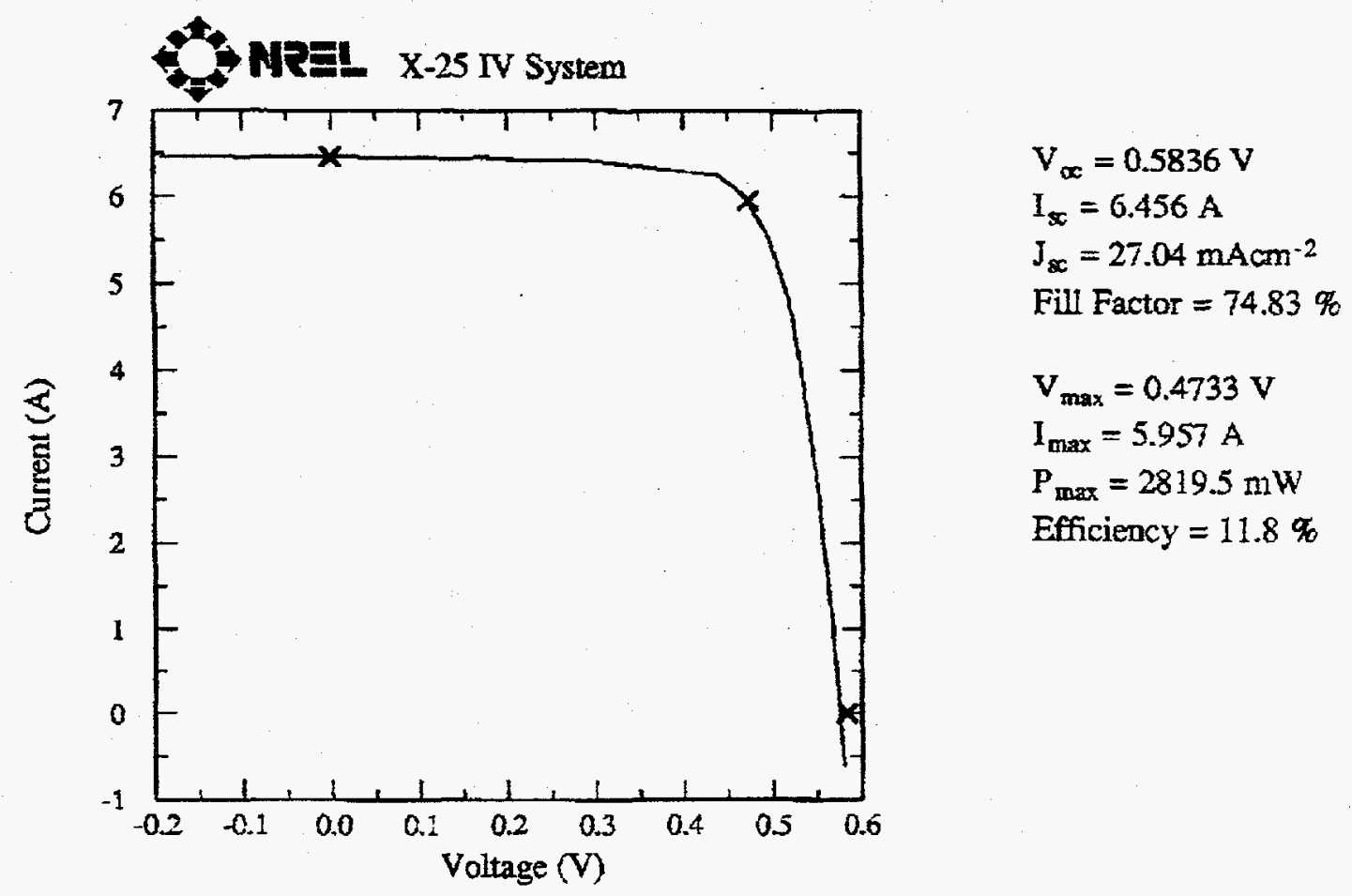

Figure 2. NREL current-voltage characteristic of a 2.82 watt AP-225 Silicon-Film ${ }^{\mathrm{TM}}$ solar cell.

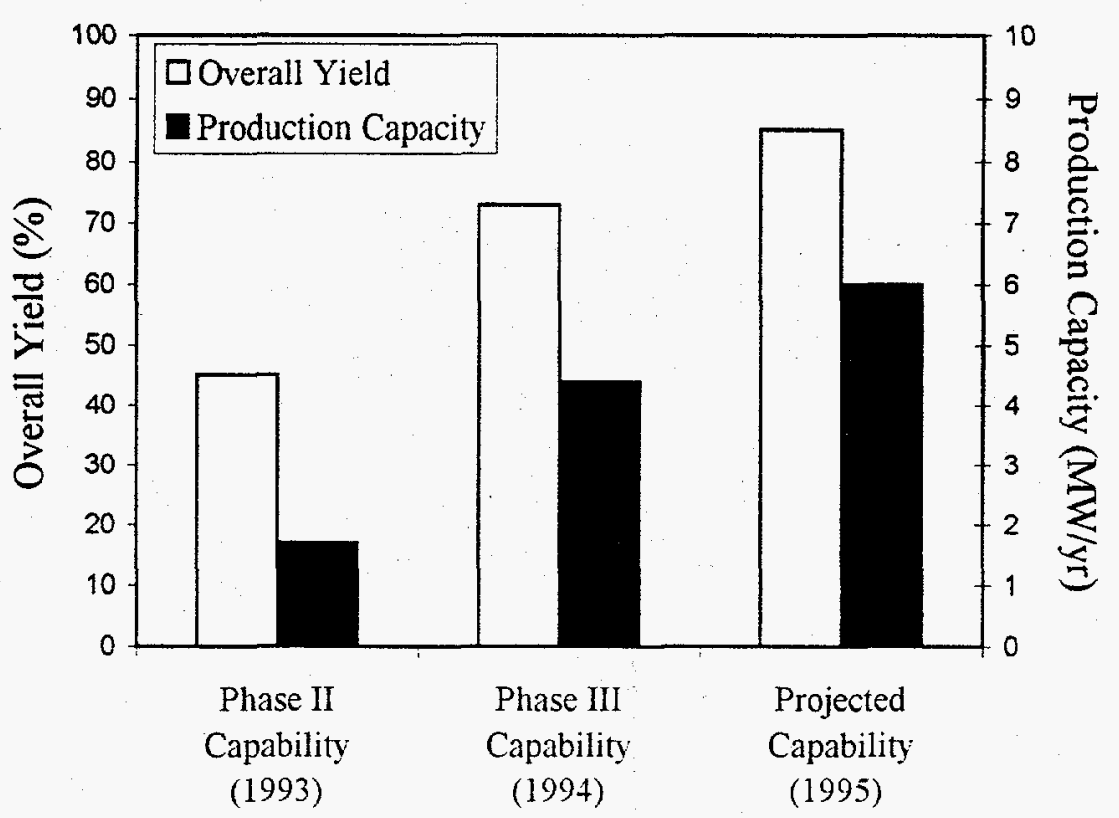

Figure 3. Demonstrated and projected improvements in production yield and capacity. 
Title:

Organization:

Contributors:
Continuous Roll-To-Roll a-Si

Photovoltaic Manufacturing Technology

Energy Conversion Devices, Inc.

Troy, Michigan 48084

M. Izu, Principal Investigator, S.R. Ovshinsky,

X. Deng, A. Krisko, H.C. Ovshinsky, K.L. Narasimhan, and S.J. Jones

\section{Objectives}

ECD's overall objective under this program is to improve its amorphous silicon (a-Si) alloy solar cell manufacturing technology, which uses a continuous roll-to-roll deposition process. The specific objective is to develop a large-scale manufacturing technology with the capability of producing modules with stable efficiencies greater than $10 \%$ in high volume, at a cost of approximately $\$ 1.00$ per peak Watt. Successfully implementing this project supports ECD's ultimate objective of building a roll-to-roll, automated a-Si module manufacturing facility with a production capacity of $100 \mathrm{MW}$ per year.

\section{Technology}

ECD's technology focuses on producing a-Si PV modules, using a continuous roll-to-roll process, to deposit nine layers of a-Si alloys in a single pass onto a $700 \mathrm{~m}$ long stainless steel substrate. The stainless steel substrate is 5 mil thick, $0.35 \mathrm{~m}$ wide, and has many advantages compared to glass substrates, including:

- Stainless steel will not shatter during manufacturing operation and handling

- Stainless steel is lightweight and flexible

- The transport mechanism is simple, reliable, and produces minimal component wear, keeping maintenance costs low

- The thin stainless steel substrate can be heated and cooled quickly during deposition.

The key feature of the ECD continuous roll-to-roll production is the use of triple-junction, multiple bandgap solar cells with high quality, bandgap-profiled a-SiGe as the bottom intrinsic layer. Using this a-Si/a-Si/a-SiGe triple-junction design, ECD has demonstrated 13.7\% initial small-area solar cell energy conversion efficiency. The manufacturing line has been designed and engineered to produce solar cells incorporating this most advanced cell design for obtaining high energy-conversion efficiency modules with good stability.

The encapsulation system for these PV modules comprises ethylene vinyl acetate (EVA)/Tefzel. With this construction, the modules are flexible, lightweight, and shatterproof.

\section{Approaches}

We have performed manufacturing technology development work utilizing our advanced $2 \mathrm{MW}$ continuous roll-to-roll triple-junction amorphous silicon (a-Si) alloy solar cell production line, which was engineered and manufactured by ECD [1-4]. The production line consists of: 1 . A continuous 
roll-to-roll substrate washing machine; 2. A continuous roll-to-roll back-reflector machine; 3 . A continuous roll-to-roll a-Si alloy deposition machine; and 4. A continuous roll-to-roll transparent conductor deposition machine.

The production line produces triple-junction two-bandgap a-Si solar cells consisting of $\mathrm{Si} / \mathrm{Si} / \mathrm{SiGe}$ structure on a 5 mil. thick, 14 inches wide, $700 \mathrm{~m}$ stainless steel roll at a speed of $1 \mathrm{ft} / \mathrm{min}$. This production line represents the world's first commercial production line which produces highefficiency a-Si alloy solar cells using a multi-junction spectrum-splitting cell design and high performance back-reflector.

We have also developed new photovoltaic manufacturing technologies using ECD's multi-purpose continuous roll-to-roll a-Si solar cell deposition machine [5-7]. Using this machine, we further improved the manufacturing process for the deposition of triple-junction triple-bandgap a-Si/a$\mathrm{SiGe} / \mathrm{a}-\mathrm{SiGe}$ solar cells; the deposition of a-Si materials using a serpentine web configuration; and the deposition of back-reflector and top conductor.

\section{Major Accomplishments}

- We have successfully completed $700 \mathrm{~m}$ production runs of a-Si/a-Si/a-SiGe triple-junction solar cells on a $\mathrm{Ag} / \mathrm{ZnO}$ back-reflector using roll-to-roll manufacturing line at production speeds of $1 \mathrm{ft} / \mathrm{min}$., which is required for the 2 Megawatt annual throughput. Subcell yield of $99.7 \%$ was achieved for a full production run.

- The cell efficiency that we have achieved for triple-junction spectrum-splitting a-Si/a$\mathrm{Si} / \mathrm{a}-\mathrm{SiGe}$ solar cells is $11.1 \%$, which is the highest subcell efficiency of a-Si alloy solar cells produced in a high volume manufacturing line at full production speed. We have produced $4 \mathrm{ft}^{2}$ a-Si triple-junction PV modules in a commercial, continuous roll-to-roll production line. These modules have an initial module efficiency of $9.5 \%$, and a stable module efficiency of $8 \%$ after 600 hours of light soaking under one sun at approximately $50^{\circ} \mathrm{C}$ with load. No further degradation was observed when these modules were lightsoaked up to 2400 hours.

- To further improve ECD's manufacturing technologies, we have designed and constructed, at ECD's expense, and completed the initial optimization of a $200 \mathrm{~kW}$ multipurpose continuous roll-to-roll a-Si alloy solar cell deposition machine having upgraded machine and construction specifications. Initial results confirm that solar cells produced in this machine have a higher fill factor which is a result of the improved quality of the intrinsic layers. Figure 1 is a picture of this new continuous roll-to-roll deposition machine.

- We have designed and constructed, at ECD's expense, a serpentine deposition chamber which will be used to demonstrate a compact, low-cost deposition machine design with improved throughput and gas utilization factor.

- We have demonstrated $9.5 \%$ initial small-area efficiency for triple-junction a-Si alloy devices with top cell intrinsic a-Si layer deposited using serpentine technology in the initial start-up experiments. Figure 2 is the $\mathrm{J}-\mathrm{V}$ curve of a triple-junction serpentine solar cell.

- We have developed a new back-reflector evaluation technique using PDS to effectively analyze the optical losses of textured back-reflector. 
- We have developed an improved textured $\mathrm{Ag} / \mathrm{ZnO}$ back-reflector system demonstrating $26 \%$ gain in $\mathrm{J}_{\mathrm{sc}}$ over previous textured $\mathrm{Al}$ back-reflector systems.

- We have developed a new grid/bus-bar design utilizing thin wire grids to improve the efficiency by approximately $3-4 \%$ and reduce the grid/bus-bar cost by approximately $50 \%$.

- As a result of the optimization process to reduce layer thickness and to improve gas utilization, a $77 \%$ material cost reduction for germane and a $58 \%$ reduction for disilane have been achieved. We have achieved a cumulative material cost reduction of $56 \%$.

- We have developed a concept design for a low-cost large-area PV module for utility applications to further reduce manufacturing costs.

- We have developed a concept design for an automated high-volume PV manufacturing plant for producing this low-cost large-area module.

\section{REFERENCES}

1. M. Izu, X. Deng, A.J. Krisko, K. Whelan, R. Young, H.C. Ovshinsky, K.L. Narasimhan, S.R. Ovshinsky, Proceedings of 23rd IEEE PV Specialists Conference, 919, (1993).

2. M. Izu, S.R. Ovshinsky, X. Deng, A.J. Krisko, H.C. Ovshinsky, K.L. Narasimhan, and R. Young, Proceedings of 12th NREL PV Program Review Meeting, AIP Conference Proceedings, 306, (1993)

3. X. Deng, M. Izu, K.L. Narasimhan, S.R. Ovshinsky, Amorphous Silicon Technology, Proceedings of the MRS Spring Meeting, 699 (1994).

4. S. Guha, J. Yang, A. Banerjee, T. Glatfelter, K. Hoffman, S.R. Ovshinsky, M. Izu, H.C. Ovshinsky, X. Deng, Amorphous Silicon Technology, Proceedings of the MRS ring Meeting, 645,(1994).

5. M. Izu, H.C. Ovshinsky, X. Deng, A.J. Krisko, K.L. Narasimhan, R. Crucet, T. Laarman, A. Myatt, and S.R. Ovshinsky, Proceedings of the First World Conference on Photovoltaic Energy Conversion, Hawaii, December, 1994.

6. X. Deng, and K.L. Narasimhan, Proceedings of the First World Conference on Photovoltaic Energy Conversion, Hawaii, December, 1994.

7. X. Deng, K.L. Narasimhan, J. Evans, M. Izu, S.R. Ovshinsky, Proceedings of the First World Conference on Photovoltaic Energy Conversion, Hawaii, December, 1994. 


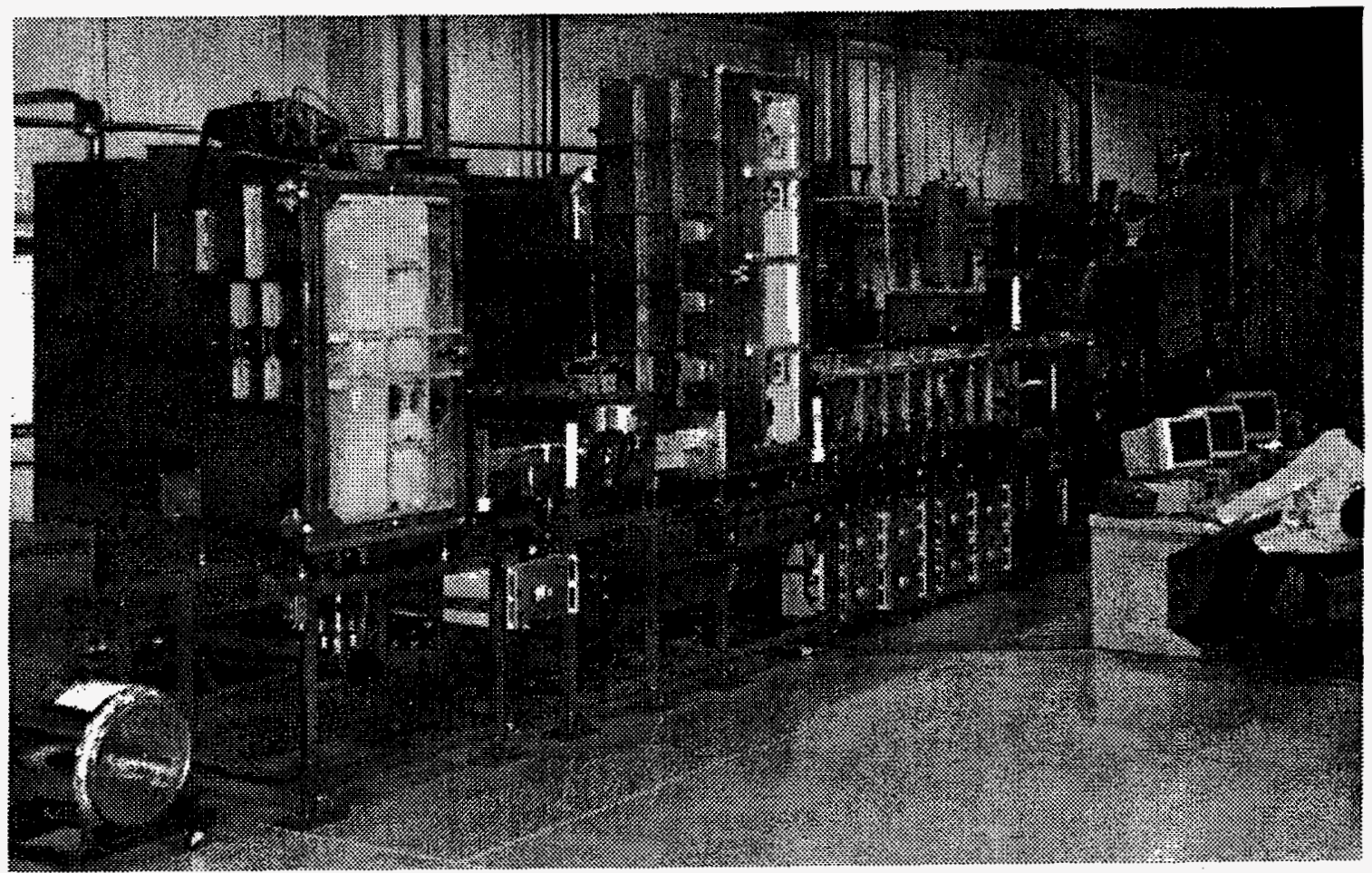

Figure 1. ECD's multipurpose continuous roll-to-roll a-Si alloy solar cell deposition machine.

The tall chamber in the picture is a serpentine chamber for the intrinsic layer deposition.

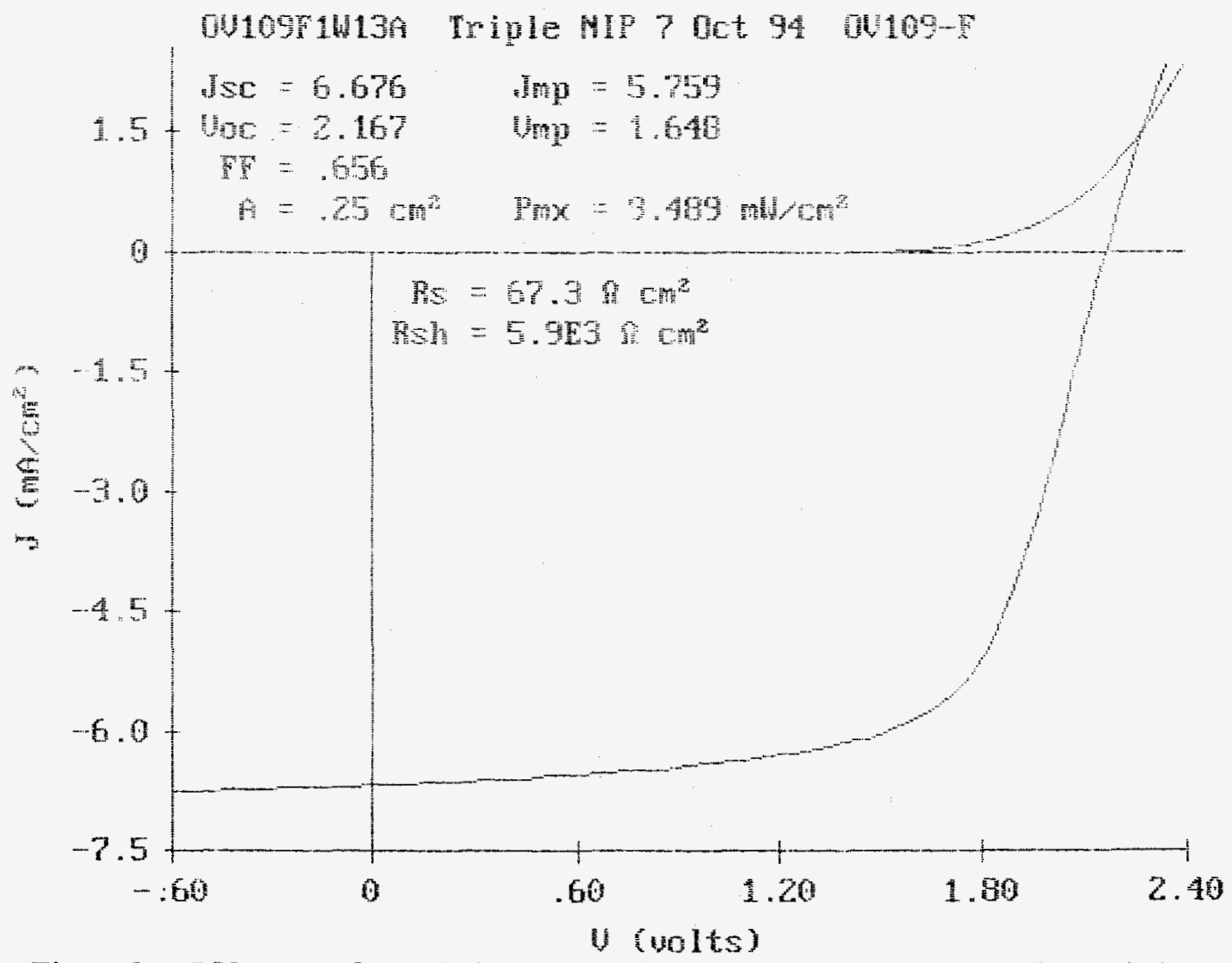

Figure 2. J-V curve of a triple-junction a-Si alloy solar cell with the top intrinsic layer deposited in the serpentine chamber. 
Title: $\quad$ Photovoltaic Manufacturing Technology (PVMaT) Improvements for ENTECH's Concentrator Module

Organization: ENTECH, Inc., Dallas-Fort Worth Airport, Texas

Contributors: M.J. O'Neill, Program Manager

A.J. McDanal, Principal Investigator

\section{Introduction}

With significant assistance from the U.S. Department of Energy (DOE), Sandia National Laboratories (Sandia), and the National Renewable Energy Laboratory (NREL), the ENTECH technical team has been developing, field-testing, refining, and commercializing linear Fresnel lens photovoltaic concentrator technology for the past 17 years. Under this PVMaT project, ENTECH is developing the manufacturing technology required to mass-produce our fourth-generation concentrator module.

\section{Objective}

The key objective of ENTECH's PVMaT Program is to design, develop, and implement improved manufacturing processes for ENTECH's fourth-generation concentrator module. The improved processes are being engineered to simultaneously achieve enhanced product quality (i.e., improved module efficiency, reliability, and field lifetime), higher production volume (10 MW/year initial rate), and lower production costs $\left(\$ 1.25 / \mathrm{W}_{\text {peak }}\right.$ goal).

\section{Approach}

An outstanding international technical team has been assembled to improve the manufacturing processes associated with our fourth-generation linear Fresnel lens concentrator module. This team includes technical personnel from NREL (Ed Witt, Rick Mitchell, John Benner, et al.), Sandia (Alex Maish, Dave King, et al.), 3M (Paul Jaster, DuWayne Radke, Char Thill, et al.), Consumers, Inc. (Arnie Kapitz), BP Solar (Tim Bruton), ASE (Wilfried Schmidt), Siemens Solar (Kim Mitchell and Richard King), Solarex (John Wohlgemuth and Mohan Narayanan), Spire Corporation (Mike Nowlan), AIT (Ed Chalupa and Hugh Wilson), Klos Technologies (Rick Simpson), Columbia Aluminum (Walt Brown), DuPont/Clear Solutions (Stan Levy), and key component suppliers, in addition to ENTECH's in-house staff.

\section{Module Description}

Our line-focus photovoltaic concentrator modules have been described in many previous publications ${ }^{1-7}$. Fig. 1 shows a cross-sectional sketch of our fourth-generation module. The lens aperture is $84 \mathrm{~cm}$ ( $33 \mathrm{in}$.) wide by $366 \mathrm{~cm}$ (144 in.) long, for a total sun-capturing area of $3.1 \mathrm{~m}^{2}$. Our production lenses from $3 \mathrm{M}$ provide a net optical efficiency of $90 \%$ while focussing sunlight onto 37 series-connected solar cells, each having active area dimensions of $4.1 \mathrm{~cm}$ (1.6 in.) wide by 9.65 $\mathrm{cm}$ (3.8 in.) long. The geometric concentration ratio of the module is $85 \mathrm{~cm}$ divided by $4.1 \mathrm{~cm}$, which equals $21 \mathrm{X}$. Each cell is covered with a prismatic cell cover to eliminate gridline shadowing losses. Waste heat from the cell string is rejected via the world's largest extruded aluminum heat sink. 
The module peak efficiency at standard test conditions (STC $=25 \mathrm{C}$ cell temperature and $1 \mathrm{~kW} / \mathrm{m}^{2}$ direct normal irradiance) is equal to the product of lens optical efficiency (90\%), cell-to-receiver packing factor $(98 \%)$, receiver encapsulating layer transmittance $(94 \%)$ and cell efficiency. Three of our cell suppliers (ASE, BP Solar, and Solarex) have already achieved about $19 \%$ peak efficiency for developmental cells delivered under this program, as verified in recent tests at Sandia. With such developmental cells, our peak module efficiency is presently about $16 \%$, corresponding to a module power rating of about $500 \mathrm{~W}_{\text {peak. }}$. For a production run of 21,000 cells during 1994, ASE achieved over $17 \%$ lot-average cell efficiency. With such production cells, our peak module efficiency is presently about $14 \%$, corresponding to a module power rating of about $430 \mathrm{~W}_{\text {peak }}$, as recently verified by Sandia. Clearly, our fourth-generation module is the largest photovoltaic module of any type yet produced, in terms of both aperture area and power output.

\section{Significant Results During FY 1994}

Cell Package Improvements: During FY 1994, we have streamlined our solar cell package design and associated manufacturing process. The new cell package, shown in Fig. 2, incorporates die-cut prism cover tape, with integral pressure-sensitive adhesive (PSA), bonded to the upper surface of the cell to eliminate gridline obscuration losses. The new prism cover tape is delivered by our team member, $3 \mathrm{M}$, on rolls containing 3,500 prism covers each. Each prism cover is aligned and bonded to a solar cell using a semi-automated prism cover application work station, which allows covers to be applied to cells at rates faster than one per minute. Each cell package also includes two integral bypass diodes, for cell shading protection. Each cell package also includes four solder-plated copper ribbons, for carrying the cell current into and out of the cell package.

Dielectric Improvements: During FY 1994, we developed a proprietary new dry-film process for bonding the cell packages to the heat sink, and for encapsulating the resultant cell string. This new process (patent pending) incorporates two mass-produced polymer film layers, the first between the cells and heat sink, and the second between the cells and surrounding atmosphere. The selected film is DuPont Tefzel $(\mathbb{B}$, which provides excellent dielectric strength, high-temperature survivability, outstanding toughness, ease-of-use, and reasonable cost. The new dielectric process uses off-theshelf tape and film dispensing equipment to attach the film layers to the heat sink and completed receiver assembly, respectively. The new process also eliminated over 300 parts per receiver.

Independent Module Testing: During FY 1994, two full-size concentrator modules were delivered to Sandia and NREL under this PVMaT program. Typical operational IV curve measurements for these modules are shown in Fig. 3. Note that the typical peak power output is about $20 \mathrm{~A}$ at $20 \mathrm{~V}$. Sandia thoroughly tested both modules and arrived at a peak power rating of $430 \mathrm{~W}_{\text {peak }}$ for each module. Both modules used 17\%-efficient production cells from ASE.

System Production and Deployment: During FY 1994, 576 modules of the new type were produced for deployment in two $100 \mathrm{~kW}$ power plants for the two largest utility companies in Texas. One plant, owned by Central \& South West (CSW), is deployed near Ft. Davis, Texas. The other plant, owned by TU Electric (TUE), is deployed in Dallas, Texas. These two production runs utilized all of the new manufacturing processes developed under this PVMaT program. Fig. 4 shows a typical SolarRow of 72 modules. Four such SolarRows comprise each $100 \mathrm{~kW}$ power plant. 


\section{Conclusions and Future Plans}

Substantial progress has been made in developing the manufacturing technology needed to produce efficient, reliable, high-quality, low-cost concentrator modules. Unlike many other emerging photovoltaic technologies, our fourth-generation concentrator technology requires no further breakthroughs in materials, efficiency, stability, or basic manufacturing processes, to meet the Department of Energy's electrical energy cost goals. High-volume procurement of vendor-furnished parts, coupled with automated assembly of these parts into concentrator modules, will enable this technology to provide cost-effective electrical power in sunny regions of the world.

\section{References}

1. M.J. O'Neill et al, "Photovoltaic Manufacturing Technology Improvements for ENTECH's Concentrator Module: Phase 1 Final Technical Report," NREL/TP-214-4486, Denver, November 1991.

2. M.J. O'Neill et al., "ENTECH's Fourth-Generation Linear Concentrator Module," 1992 DOE/Sandia Crystalline Photovoltaic Technology Project Review Meeting, SAND92-1454, Albuquerque, July 1992.

3. T.M. Bruton et al., "Recent Developments in Concentrator Cells and Modules Using Silicon Laser Grooved Buried Grid Cells," 11 th European PVSEC, Montreaux, Switzerland, October 1992.

4. M.J. O'Neill, "Fourth-Generation, Line-Focus, Fresnel Lens Photovoltaic Concentrator," 4th Sunshine on Crystalline Silicon Solar Cells, Tokyo, Japan, November 1992.

5. M.J. O'Neill and A.J. McDanal, "Manufacturing Technology Improvements for a Line-Focus Concentrator Module," 23rd IEEE-PVSC, Louisville, May 1993.

6. M.J. O'Neill, "Photovoltaic Manufacturing Technology (PVMaT) Improvements for ENTECH's Concentrator Module," 12th NREL PV Program Review Meeting, Denver, October 1993.

7. M.I. O'Neill and A.J. McDanal, "Fourth-Generation Concentrator System: From the Lab to the Factory to the Field," 1st WCPEC, Hawaii, December 1994.

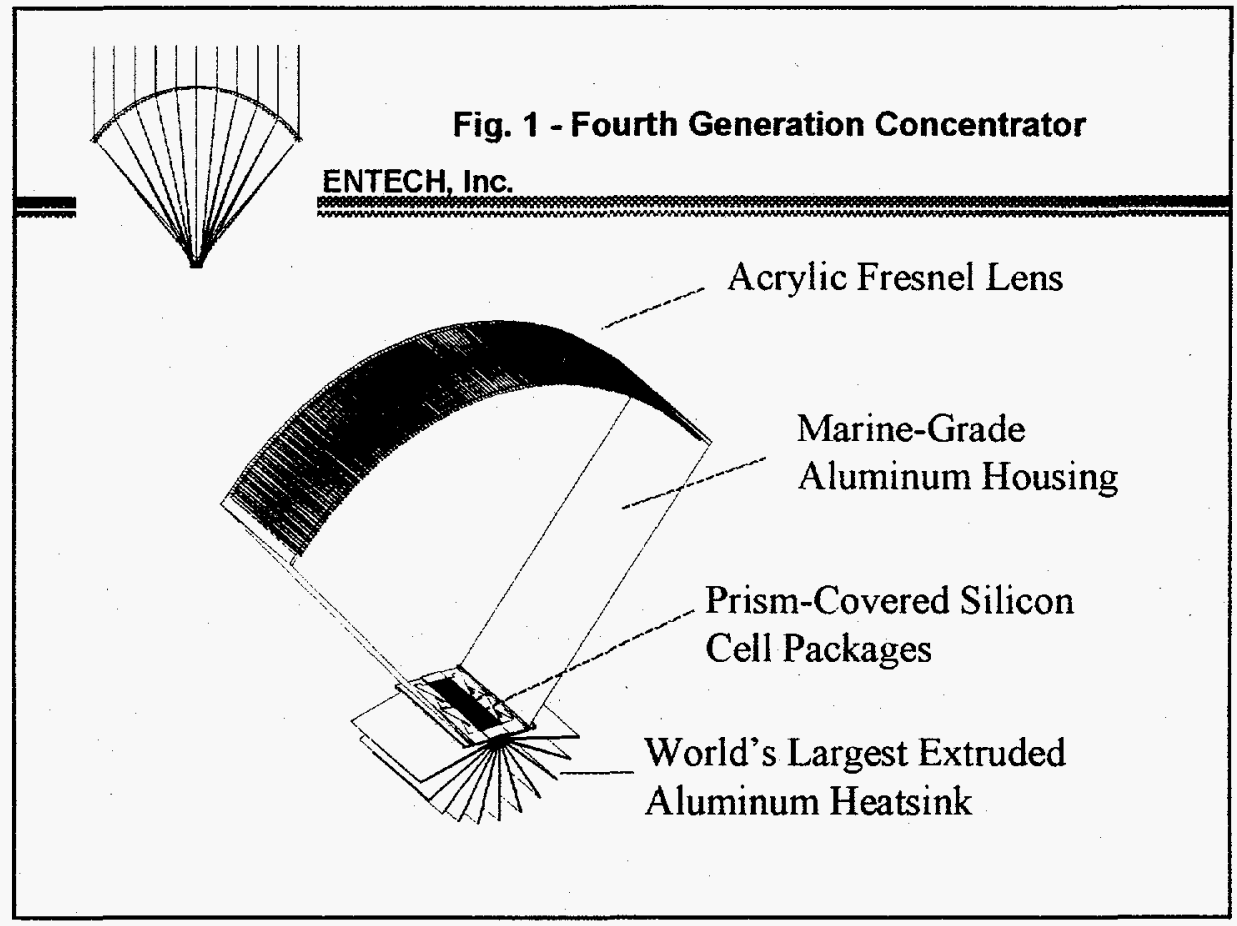




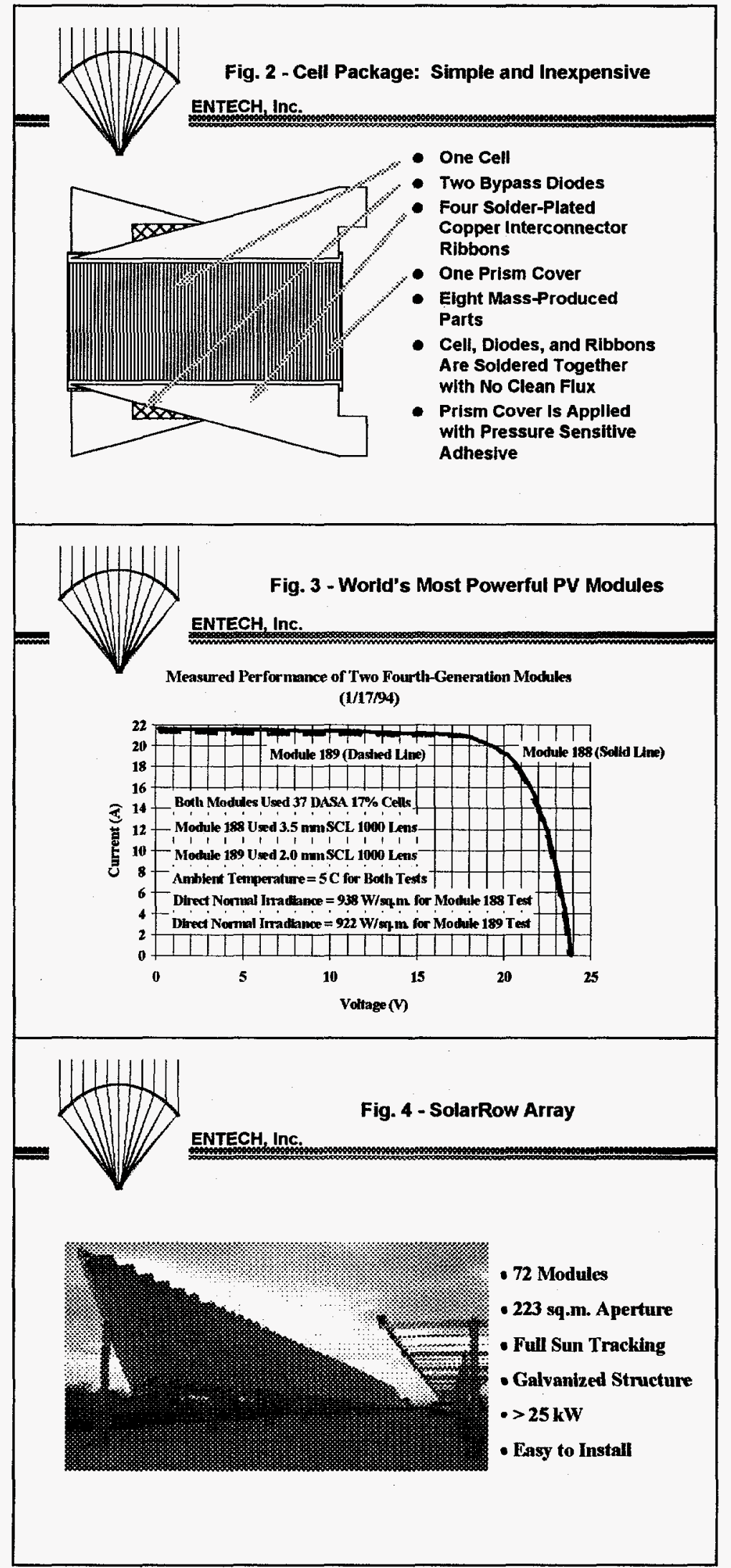


Title: Commercial Production of Thin Film CdTe Photovoltaic
Modules

Organization: Golden Photon, Inc.

P.O. Box 4040

Golden, CO 80401

Contributors: Dan Weisiger, Principal Investigator

Scot Albrigist, Jeff Brines, and Steve Johnson

\section{Objective}

Golden Photon is performing research under the PVMaT program, Phase 2B. The objective of the research is to advance GPI's manufacturing technology, reduce module production costs, increase average module performance, and identify ways to expand production capacity. More specifically, the tasks established for FY 94 were to design and install leasehold improvements for the 2MW production line; to improve and develop product design, efficiency and marketability; to ensure uninterrupted qualified supplies and raw materials for production; to address environmental, health and safety issues encountered during production of PV modules; and to reduce the cost of manufacturing modules.

\section{Accomplishments}

Prior to contract award, GPI was able to demonstrate prototype quantities of CdTe PV modules at the 24 watt average output level using pilot scale equipment at the R\&D facility in El Paso, Texas. PV module and process step specifications developed from successful El Paso facility pilot plant operations were used in the scale up plans for design and installation of a new 2 MW PV manufacturing facility in Golden, Colorado.

In 1994, all of the processing equipment required for start up of a $2 \mathrm{MW}$ production line was installed at the manufacturing facility in Golden, Colorado. This involved moving GPI's facility in El Paso, TX to the Golden location. The $2 \mathrm{MW}$ production line installation and evaluation are complete, and PV modules are being produced. Throughput rates and yields are continually evaluated and are improving.

The "start up" phase included the debugging and design of in house equipment critical to process prove-in. All manufacturing equipment was initiated and each process was determined to be suitable for manufacturing PV modules. Investigations of material handling techniques were completed and will be considered when throughput rates increase to 1500 per week. During the year the production rate increased from 80 panels per week in the second quarter to 350 panels per week in the fourth quarter.

In the first half of the year, the development, design, and debugging of cell interconnection equipment critical to start up was completed. During the second and third quarters, the primary focus was given to the substrate deposition steps (tin oxide, cadmium sulfide, and cadmium telluride) and cell interconnection steps (division). In general, process development, engineering and quality teams have continued to focus on identifying, baselining and improving (through re-design) actual process equipment operation parameters in order to meet the required PV panel specifications and improve process throughput rates and yields. 
Sources for supplies and raw materials required for production of modules have been identified and a specification and qualification system is now in place. Multiple sources have been identified for most materials and supplies, and GPI has established an MRP system.

By the end of the first quarter, all permits necessary for the operation of the $2 \mathrm{MW}$ facility were in place. These permits include air permit, water permit, water permit exemption, storm water permit, construction permits for capital improvements, and building occupation permits. Monthly review and updating of the current permits have taken place throughout the year; no violations or problems were cited.

Once the production line was complete, work began on evaluating and improving the processes. The process development, engineering and quality teams have continued to focus on identifying, baselining and improving (through re-design) actual process equipment operating parameters in order to meet the required PV panel specifications and to improve process throughput rates and yields.

Baseline production rates and yields were established at the El Paso facility before the move to Golden. The following summarizes this baseline:

\begin{tabular}{|ll|}
\hline Process Throughput Yield: & $80 \%$ through encapsulation \\
\hline Process Throughput Rate: & Tin Oxide $-64 \mathrm{~min} . / \mathrm{modul}$ \\
& Cadmium Sulfide $-48 \mathrm{~min} . /$ module \\
& Cadmium Telluride $-2 \mathrm{~min} . /$ module \\
& Division $(5$ steps $)-12 \mathrm{~min} . /$ module \\
& Encapsulation $-60 \mathrm{~min} . / \mathrm{modul}$ \\
& Overall daily rate -2 modules $/$ day \\
\hline
\end{tabular}

Progress toward improving the efficiency and therefor lowering the cost of the manufacturing process was accomplished in Phase I as follows:

\begin{tabular}{|ll|}
\hline Tin Oxide & $4 \mathrm{~min} . /$ module \\
Cadmium Sulfide & $8 \mathrm{~min} . /$ module \\
Cadmium Telluride & $2 \mathrm{~min} . /$ module \\
Division $(5$ steps $)$ & $4 \mathrm{~min} . /$ module \\
Encapsulation & $15 \mathrm{~min} . /$ module \\
\hline
\end{tabular}

During the second quarter, the $\mathrm{CdS}$ oven deposition rates exceeded the goal of six times the original rates; the deposition rate averaged 8 minutes per panel compared to 48 minutes per panel. The tin oxide oven deposition rates exceeded the task goal of three times the "original" rates, averaging 4.0 minutes per panel compared to 53.3 minutes per panel. In addition, the cell interconnection step throughput rate exceeded the task goal of four times the "original" rates, averaging 5 minutes per panel compared to 35 minutes per panel. These significant improvements contribute to the overall optimism that GPI will meet milestones set for the next phase of this program. 
During the fourth quarter, the $\mathrm{CdS}$ thickness uniformity goal was finally achieved. The average panel thickness uniformity across a 24 " by 24 " substrate for the CdS step improved from $18.5 \%$ in May to $4.7 \%$ in October. In addition, the implementation of improved equipment designs for packaging steps was completed.

In the area of Environmental, Health and Safety, occupational and equipment air samples (cadmium and VOC's), as well as "wipe" samples (equipment and floor - cadmium) continue to be monitored as production rates increase. All airborne cadmium monitoring continues to demonstrate that the measured levels are well below OSHA action level of 2.5 micrograms/cubic meter. The "wipe" sampling data evaluated also revealed that consistently low levels of cadmium have been maintained in spite of the increased use of cadmium. GPI also closely monitors all hazardous and non-hazardous waste streams.

The ES\&H plan continues to be refined as production increases. Modifications have addressed various occupational safety procedures, updating the "active" MSDS log book, modifications to the personal protective equipment and the egress decontamination procedures. Waste handling and disposal procedures have been added and clarified.

By the end of the third quarter, the Employee Exposure Monitoring Plan was completed. The plan included implementation of procedures for measuring and controlling potential airborne cadmium dust and VOC (volatile organic compounds) materials, as well as facility and equipment wipe sampling program for potential settled dust exposure. At the beginning of each quarter throughout the year, the Employee Exposure Monitoring Plan has been reviewed and updated as needed.

During Phase I of the PVMaT program, the $2 \mathrm{MW}$ manufacturing line was installed and all of the environmental, health and safety issues have been addressed. Yields for the process steps continue to improve with the highest average batch substrate yields: CdS deposition, $88 \%$; tin oxide, $77 \%$; and interconnection step, 99\%. Module outputs have ranged from 15 to 24 watts (See Figure 1 below), and overall process yields are illustrated in Figure 2.

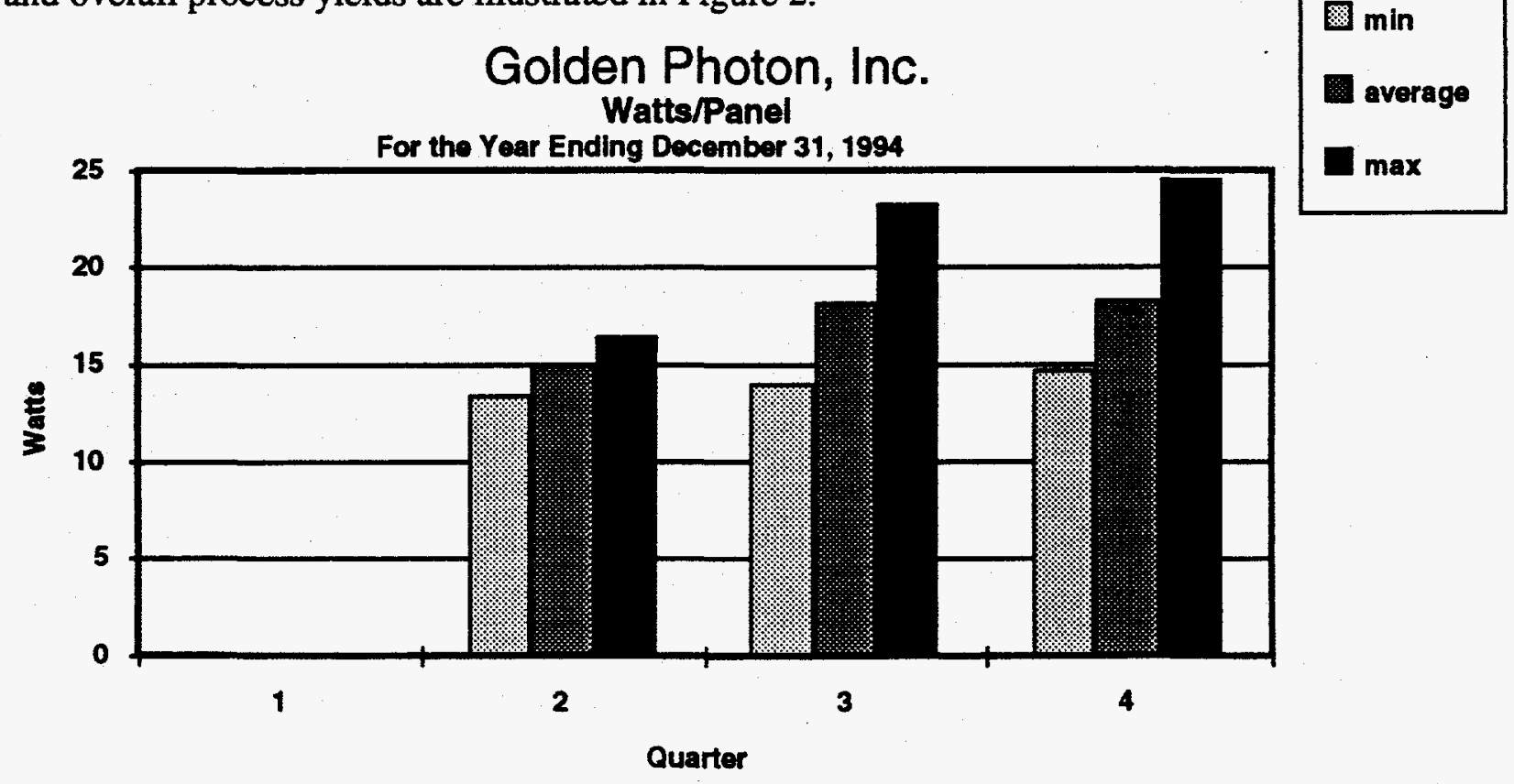

Figure 2: Watts / Panel 


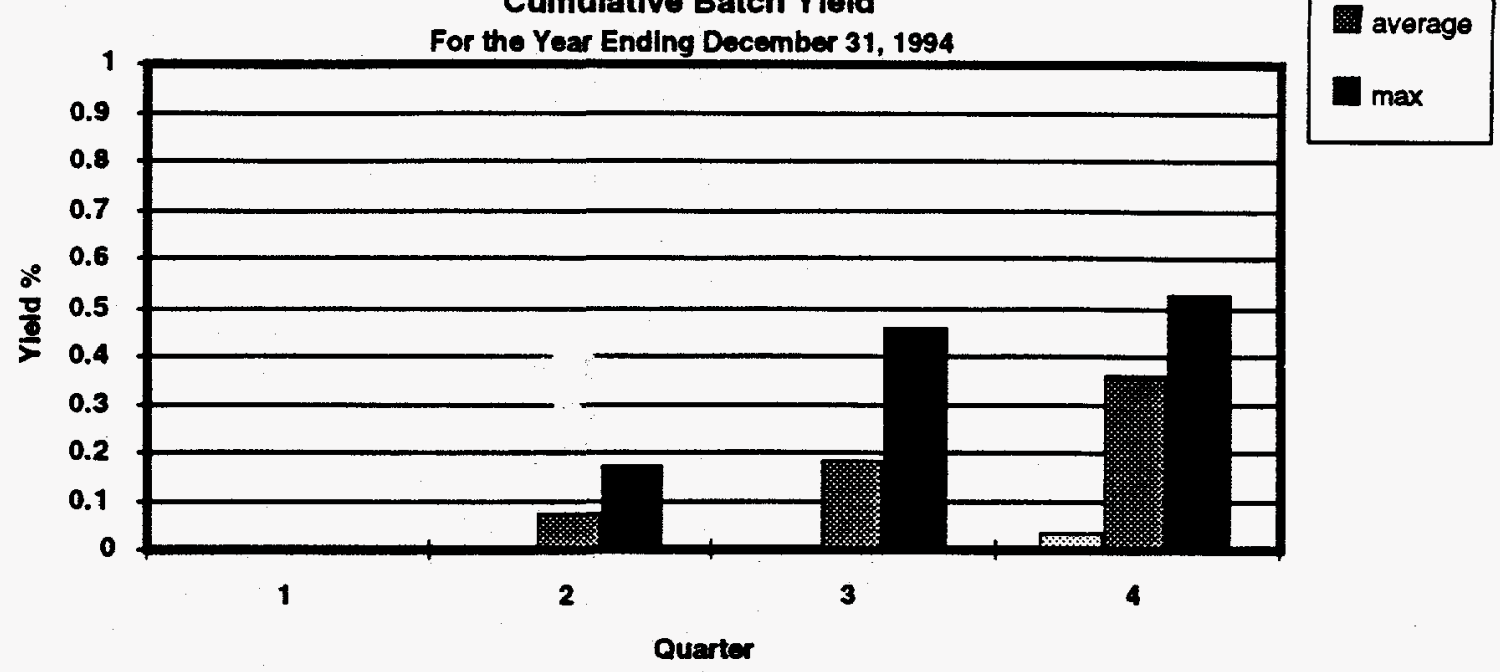

Figure 2:Cumulative Batch Yield

\section{Future Research}

GPI will continue to work on efforts to improve yields, production rates and module output during Phase II of the PVMaT program. The projected work also includes the designing and specifying of the next $4 \mathrm{MW}$ portion of the production capability as a step toward completion of a $10 \mathrm{MW}$ plant. As GPI adds production capability, we will continue to evaluate and debug equipment, the QA/QC and ES\&H programs will be refined, and multiple sources for materials will be identified in order to reduce the cost of raw materials. The data from the $2 \mathrm{MW}$ production line will be evaluated to establish a new baseline, and the process controls will be used to increase rates and improve material utilization. The $6 \mathrm{MW}$ production line should be implemented by the end of Phase II with post CdTe process yields of $95 \%$ at a 24 watt average and $85 \%$ for a 28 watt average output level.

The processing equipment and material handling capabilities required for the next $4 \mathrm{MW}$ of additional production of the $10 \mathrm{MW}$ plant will be identified. Upon completion of equipment design and specification, GPI will develop line installation procedures and conduct machinery start up and process evaluation. Other design requirements include an analysis of the line labor demands and the evaluation of labor minimization methods. The use of automatic traffic controlled conveying at the $6 \mathrm{MW}$ level to connect the conveyorized processes will be explored. This will include the design and debugging of automation equipment, investigation of material handling alternatives, and implementation of material handling automation.

During Phase II, GPI will develop specifications for manufacturing procedures to be utilized in the expansion to a 6-10 MW manufacturing line. Methods to be used toward this effort are verification of control methods, review of production records to identify and quantify process problems, and investigation and implementation of solutions to the identified process problems. GPI is also going to review the results of scaling up the area of the present module while evaluating the impact of increasing the production facility.

In general, GPI will update and document monthly costs, yields, and throughput rates associated with each process step. Each area will be analyzed and averaged to provide the basis of the presentation of relative cost and yield data. This effort is intended to continue to optimize key manufacturing processes and increase production rates and yields. 
Title:

Organization:

Contributors:
PV Cz Silicon Manufacturing Technology

Improvements

Siemens Solar Industries, Camarillo, CA 93011

Theresa Jester, Kim Mitchell, Rob Probst, Sergio Vasquez

\section{Introduction}

Siemens Solar Industries (SSI) began a three-year, three-phase cost shared contract in March 1992 to demonstrate significant cost reductions and improvements in manufacturing technology. The work has focused on near-term projects for implementation in the SSI Czochralski (Cz) manufacturing facility in Camarillo, California. The work has been undertaken to increase the commercial viability and volume of photovoltaic manufacturing by evaluating the most significant cost categories and then lowering the cost of each item through experimentation, materials refinement, and better industrial engineering. The program has concentrated in the areas of crystal growth, wafer technology, module development and environmental, safety and health issues.

\section{Objective}

The objective of the program is to reduce costs in photovoltaic manufacturing by approximately $10 \%$ per year. The program consists of three focused tasks relating to cost reduction. The silicon wafer itself contributes about half of the total module cost and has the most potential for cost reduction. The cell processing costs are about a quarter of the module costs, with cell efficiency results being most important. Module assembly and packaging costs are the balance, with the module design, both materials and labor, contributing significantly.

\section{Approach}

Task 1: Silicon Crystal Growth and Thin Wafer Technology. Crystal growing costs are driven by material growing yields and indirect manufacturing costs such as electricity and machine parts used each time an ingot is fabricated. Wafering costs are driven by labor and the number of good slices yielded per length of crystal processed. This first task of reducing the wafer costs has focused on the polysilicon material used for ingot growth, and the evaluation of wire saw machines to improve the yielded wafers per inch of ingot.

Task 2: Silicon Cell Processing. Cell processing costs are driven by the electrical contacts used, and the labor required for the process steps to clean the wafer, form the semiconductor junction, and the contacts. The cell tasks have been focused on better junction formation and reduced contact resistance. Process automation for lower labor costs have begun.

Task 3: Silicon Module Fabrication and Environmental, Safety, and Health Issues. Module costs are highly sensitive to labor and materials. The module design tasks are driven by high reliability in the field and lower costs. Environmental, safety and health issues govern the ability to 
manufacture products commercially. This program has focused on chlorflorocarbon (CFC) elimination and caustic chemical waste reduction.

\section{Results and Discussion}

During FY 1994 (10/01/93-9/30/94), several significant manufacturing technology improvements were achieved.

The Crystal Growing operation increased production significantly during the last two years. The increase is attributable to several factors including improved polysilicon quality and cleaning, upgrades to the growing equipment, specifically diameter controls, and upgrades to the hotzone graphite parts which have increased reliability and reduced cycle time. A wide range of poly silicon starting materials have been evaluated. Crystal Growing yields, cell performance and impurity analysis have been conducted. Overall crystal growing yields improved by $4 \%$ as a result of the study.

A study of oxygen concentration was conducted. The study included different types of crucibles and different growth parameters such as pull speed and rotation speed to improve the electrical properties of the as grown ingot. The crucible is the major source of the oxygen which is incorporated into a silicon ingot during growth. Crucibles with a denser, bubble free layer on the inside wall have been shown to devitrify less and to contribute less oxygen to the silicon melt. A set of "low bubble" crucibles was compared to standard crucibles and samples of the silicon ingot taken from the top and the bottom of the ingots. Fourier Transform Infrared, FTIR, analytical technique was used to measure the oxygen concentration on each sample. The results show the oxygen concentration for the low bubble crucible to be lower at the bottom of the ingot than for the standard crucible. Larger diameter ingots have been implemented successfully and larger crucibles have also proven effective in reducing crystal growth costs.

A prototype, commercially available wiresaw was tested and a process developed for manufacturing silicon wafers for photovoltaic use. The average number of wafers per inch obtained to date on the wiresaw is 44 , which is a $50 \%$ yield improvement over the results obtained for ID sawn wafers (see Fig. 1). Several critical issues have been identified for high yield of the wiresaw process. These include:

- Wire material specification, such as strength and temper

- Slurry and solvent usage

- Wafer cleaning

- Ingot mounting

The most significant of these has been the combination of wire usage and slurry usage. The yield on the wire saw process has improved from $70 \%$ to over $90 \%$ in a one year time frame through better understanding of these interactions (see Fig. 2).

The quality of wafers sliced varies significantly in thickness control and taper (non-parallel sides) if these parameters are not tightly controlled. The goal is to maximize the number of runs obtained 
with each wire change and slurry batch used. The quantity of these materials consumed per run or per wafer is a very important parameter for cost effectiveness in manufacturing high volumes of wafers.

Cell processing costs are driven by the amount of silicon used per cell (wafer thickness), the electrical performance of the cells, and the labor required for the processing of wafers into active cell devices. Work in this area has been focused on the reduction of labor through automation of various material transfer steps and the improve nt of the electrical output of the cells processed by improving the contact resistance and diffusion. ocesses. The increased yield of wafers per inch with wire saws is a significant improvement in cost. Decreasing wafer thickness in high volume is a more critical process change affecting yield. Optimizing the mechanical yield with thin wafers requires automated wafer and cell transfer mechanisms. Automated upgrades to cell processing equipment have been designed and installed in the manufacturing line.

The cell electrical distribution has improved significantly as a result of improved contact and diffusion processes. Through these process studies, coupled with contact firing process changes, contact coverage re-design and diffusion changes, a significant shift in the electrical performance was effected (see Fig. 3).

Larger area cells have been developed and implemented which have an area improvement of almost $40 \%$.

Significant progress in cost reduction in module design has been made. Several items have been identified and implemented in manufacturing to reduce the final cost per watt of modules manufactured. The most leveraging of these has been the introduction of a larger module with larger cells. The IV characteristics of this new module produces 75 watts at Standard Test Conditions. The final module improvement has been in the development of a new junction box for modules. This improvement in manufacturing has been approved by Underwriters Laboratory.

Safety, health and environment issues have been addressed with the complete elimination of CFC's during May 1993. Significant reduction in costs and volume of caustic chemicals have also been achieved. Costs per cell have been reduced $20 \%$ and volume by over $10 \%$.

\section{Summary}

Reduction in $\mathrm{Cz}$ photovoltaic manufacturing costs are driven by several parameters. Low wafer cost, high electrical and mechanical yields in fabricating cells, low labor in cell fabrication and module fabrication, and materials used for making modules are all significant factors. Siemens Solar has reduced costs by over $25 \%$ in two years by addressing each of these items. Further improvements in each area are planned. 


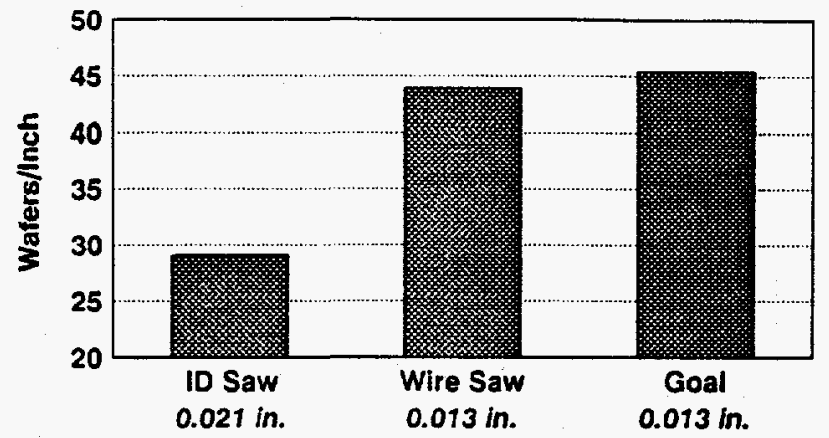

Fig. 1.Wire Saw Evaluation

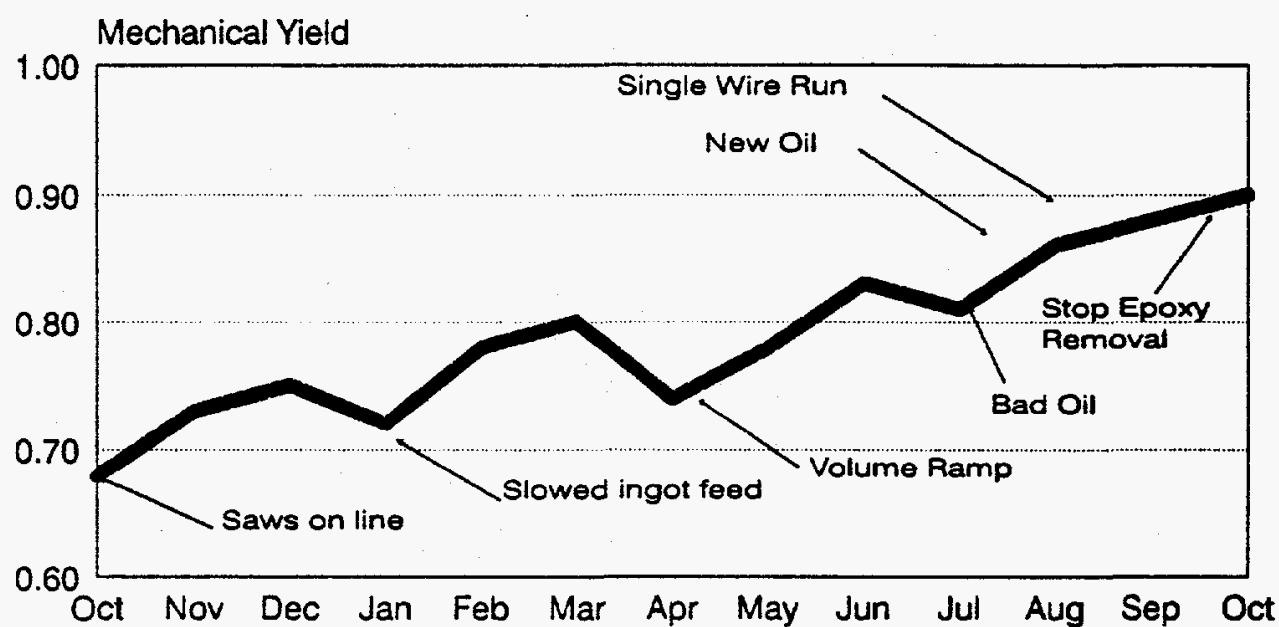

Fig. 2. Wire Saw Yields

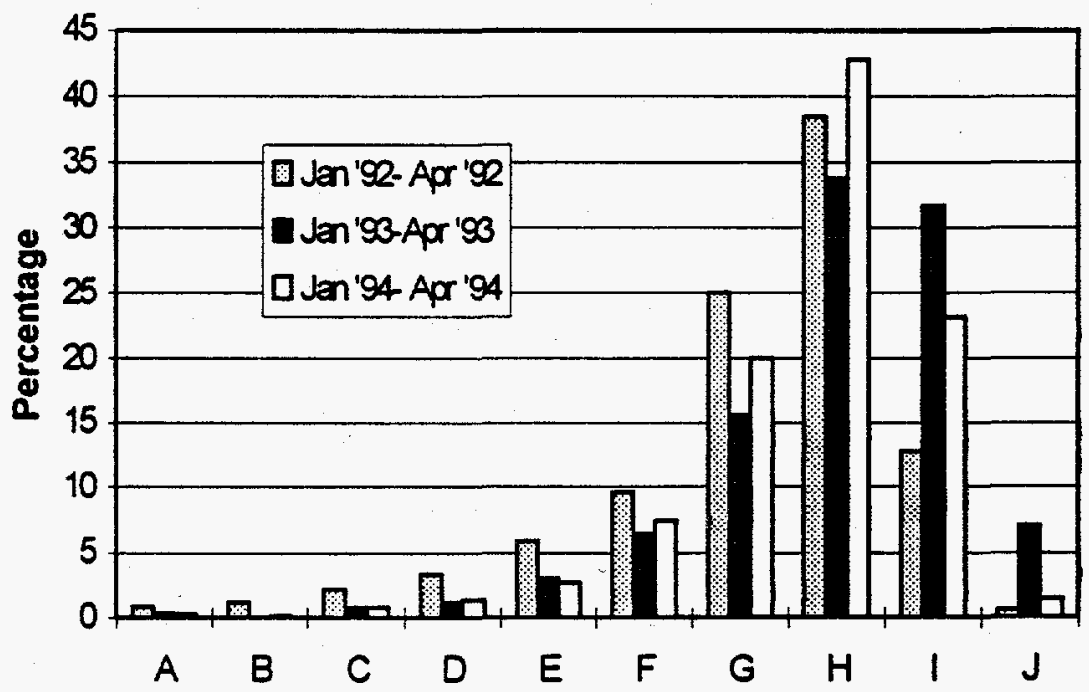

Fig. 3. Cell Electrical Improvement 
Title:

Organization: $\quad$ Solar Cells, Inc.

Contributors:

Toledo, Ohio
Development of CdTe Module Manufacturing

D. Sandwisch, Principal Investigator; S. Cox, L. Crosser, M. Flis, T. Firsdon, J. Foote, S. Kaake, W. Kazan, J. Kern, G. Khouri, D. Malkuian, H. McMaster, K. Miller, R. Nicholson, R. Powell, and N. Reitur

\section{Objective}

The objective of this subcontract over its three year duration is to advance SCI's PV manufacturing technologies, reduce module production costs, increase module performance, and provide the groundwork for SCI to expand it's commercial production capacities. SCI shall meet these objectives by designing, debugging, and operating a $20 \mathrm{MW} / \mathrm{yr}$ automated, continuous PV manufacturing line that produces $60 \mathrm{~cm} \times 120 \mathrm{~cm}$ thin film CdTe PV modules.

During the first year of this effort SCI focused on product definition, process definition, quality control/assurance and $\mathrm{E}, \mathrm{S}$, and $\mathrm{H}$ programs.

\section{Conclusions}

SCI has proven that thin film CdTe provides a technology vehicle by which significant advantages can be obtained over current PV technologies ${ }^{[1]}$. Key results and conclusions are:

1) SCI has produced a 60 Watt, $8.4 \%$ efficient module - the most powerful thin film polycrystalline module on record.

2) SCI has defined and demonstrated cost effective thin-film CdTe modules capable of achieving installed costs of less than $\$ 3.00 / W$ att by 2000 .

3) Module durability has been demonstrated.

4) The process for making these modules has been proven on a $100 \mathrm{~kW}$ pilot line.

5) No technology barriers have been identified including safety, health, and environmental issues.

\section{Product Definition}

Solar Cells, Inc. has developed products to address remote and grid-connected applications. Both of these products incorporate glass/EVA/glass module construction; however, alternative potting and mounting designs are offered to address market requirements. The longterm baseline SCI product is a $60 \mathrm{~cm} \mathrm{x} 120 \mathrm{~cm}$ high-voltage module targeted at grid-connected applications. Development efforts on this product have resulted in greater than a $40 \%$ reduction in product finalization costs. This product has been installed in a $1.2 \mathrm{~kW}$ array near SCI's plant. A similar array was shipped to Tunisia in December 1994 as part of a PV evaluation project sponsored by their national utility. 


\section{Process Definition}

Table 1 outlines the major processes used to manufacture the thin film cadmium telluride modules. These processes are demonstrated daily on a $100 \mathrm{~kW}$ pilot production line. This line has advanced module aperture area efficiency to $8.8 \%$ (see Fig. 1).

Table 1. Major manufacturing processes.

TCO Inspection

TCO Preparation

Scribe 1

Film Deposition

Film Thickness Check

Film Treatments

Scribe 2

Metal Deposition
Scribe 3

Bus Bar

Application

Encapsulation

Potting

Safety Checks

Pmax Test

Final Inspection

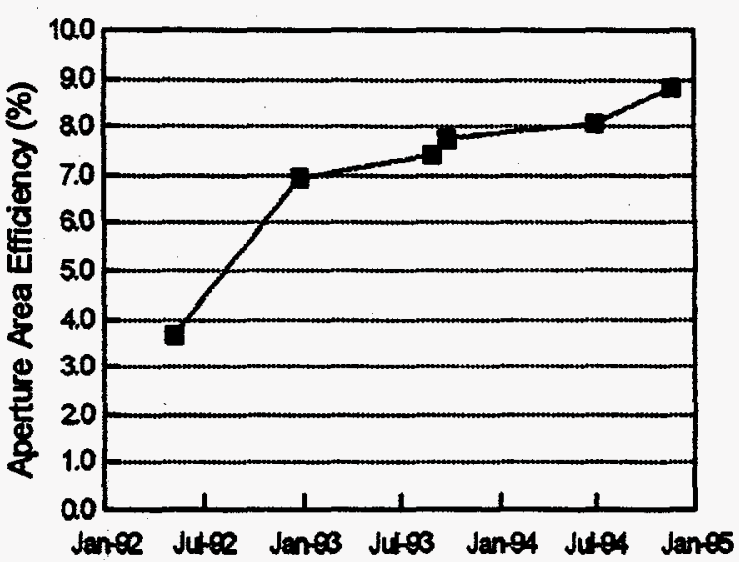

Fig. 1. History of aperture area efficiency on champion $60 \mathrm{~cm} \times 120 \mathrm{~cm}$ modules

(NREL confirmed).

In 1994, SCI built and began to debug a high-throughput deposition system which incorporated several advantages over a pilot deposition system including increased throughput and steady-state, continuous operation. The manufacturing system is designed for throughput of one module/minute. This advanced deposition system will be integrated with approximately 40 other pieces of equipment to produce modules at an annual capacity of 10-20MW.

SCI has also developed a post deposition vapor treatment which eliminates the traditional wet dip $\mathrm{CdCl}_{2}$ treatment, thereby, mitigating the environmental impacts of the traditional process and providing a more efficient process for manufacturing implementation ${ }^{[2]}$.

\section{Quality Control and Assurance}

SCI has initiated a quality assurance program specifically focused on the $20 \mathrm{MW}$ manufacturing line. This program is under evaluation and refinement on a pilot production line. Module performance has steadily improved over a two and one-half year period with significant increases in the past twelve months covering production batches 30-50 (see Fig. 2). Concurrently, the relative standard deviation of the performance decreased substantially to below $10 \%$.

Additional module stress tests are incorporated to understand the effects of these process improvements on long-term durability. The majority of these stress tests are based on the protocols established in the "Interim Qualification Tests and Procedures for Terrestrial Photovoltaic Thin-Film Flat-Plate Modules" (IQT) ${ }^{[3]}$. This testing has demonstrated significant improvement in overall pass rates from below $10 \%$ in early 1993 to well over $95 \%$ currently. SCI 
also extends certain elements of the IQT to evaluate module lifetime. For instance, three modules have undergone over 650 thermal cycles and demonstrated less than a $10 \%$ decrease in performance. This result is one indication that the current module design has a 30 -year lifetime ${ }^{[4]}$.

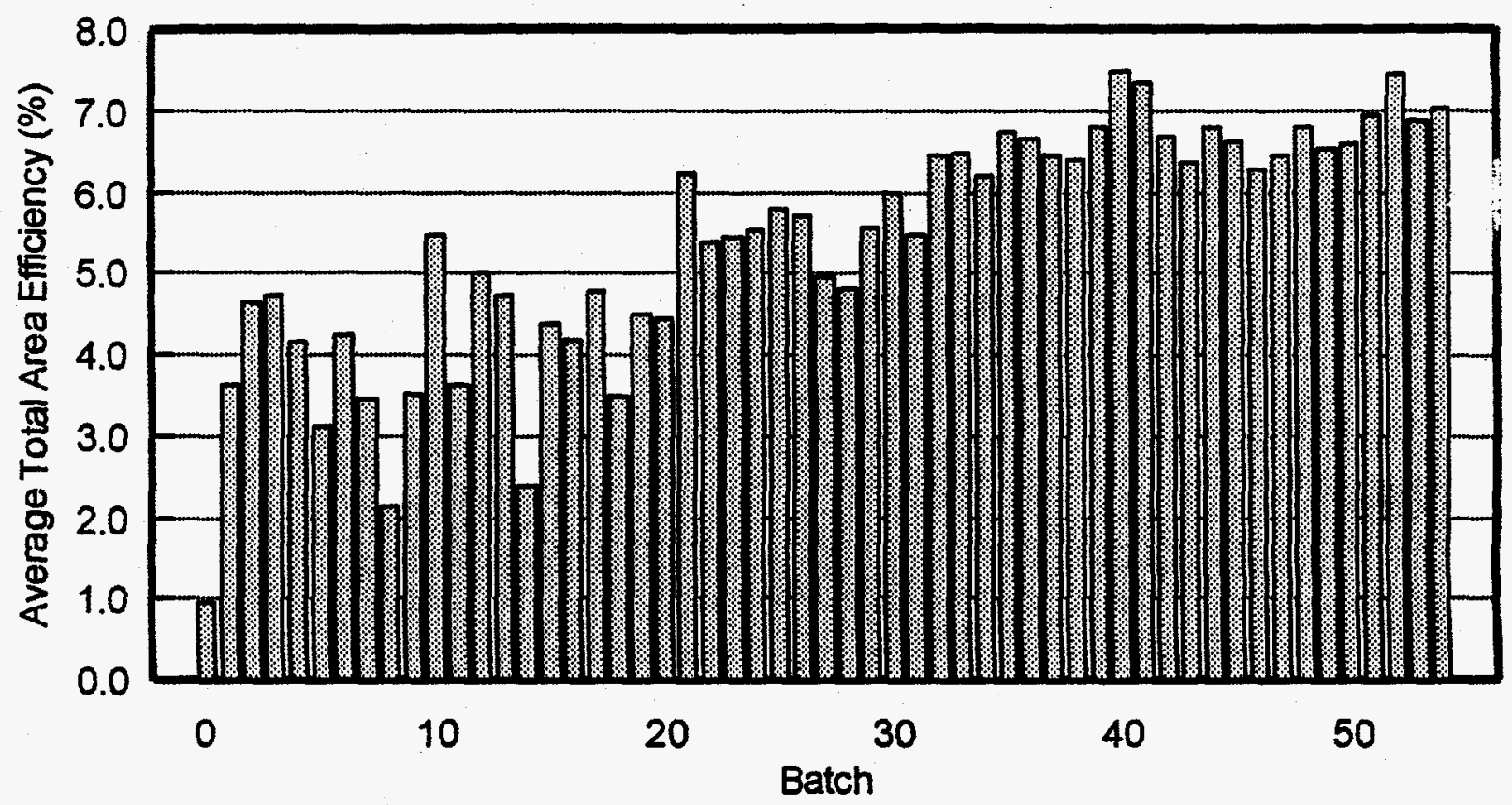

Fig. 2. Average total area efficiency vs. production batch.

\section{Environmental, Safety, and Health Programs}

Production of PV modules by the process outlined above involves regulated materials including cadmium. An important part of the development effort is to establish programs which effectively handle safety, health and environmental issues that accompany the production, deployment and disposal of these modules. SCI has engaged outside agencies and consultants to conduct safety and health audits of the manufacturing facilities and to formulate appropriate programs and corrective actions. These programs include basic training programs as well as specific operational plans such as industrial hygiene and biological monitoring.

Environmental development has focused on process waste minimization and product

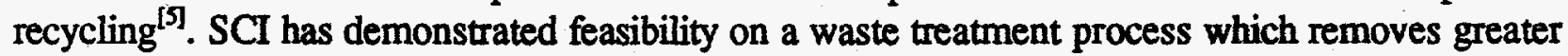
than $95 \%$ of cadmium from low concentration liquid wastes. SCI has also demonstrated product recycling by shipping modules to a raw material supplier for reintroduction into the smelting process. 
Next Steps

During the next two years, SCI will focus on commercialization of this product. These efforts will include:

1) SCI will expand its current pilot line to $200 \mathrm{~kW}$ while installing a multi-megawatt manufacturing facility.

2) SCI will increase module testing efforts including product certification.

3) Several test arrays are planned for installation including a $100 \mathrm{~kW}$ grid-connected field in Toledo, Ohio and 0.5kW array at Southwest Technology Development Institute in Las Cruces, New Mexico.

4) SCI will test its recycling strategy in several established markets.

\section{Acknowledgments}

The author is pleased to acknowledge the efforts of the SCI development team. The extraordinary capability and dedication of this team was essential in achieving the results described above.

\section{References}

[1] D. Sandwisch, "Development of CdTe Module Manufacturing", Proceedings of First World Conference on Photovoltaic Energy Conversion, 1994.

[2] T. Zhou, et. al., "Vapor Chloride Treatment of Polycrystalline CdTe/CdS Films", Proceedings of First World Conference on Photovoltaic Energy Conversion , 1994.

[3] "Interim Qualification Tests and Procedures for Terrestrial Photovoltaic Thin-Film Flat-Plate Modules", SERI/TR-213-3624, 1990.

[4] J.H. Wohlgemuth, "Testing for Module Warranties", Proceedings of the Photovoltaic Performance and Reliability Workshop, NREL/CP-410-6033, pp. 200-205, 1993.

[5] R. Sasala, et. al., "Environmentally Responsible Production, Use, and Disposition of Cd Bearing PV Modules", Proceedings of First World Conference on Photovoltaic Energy Conversion, 1994. 
Title:

Organization:

Contributors:
Large-Area, Multi-junction

a-Si Alloy Production Scale-Up

Solarex Corporation, Thin Film Division

826 Newtown-Yardley Road

Newtown, Pa. 18940

(215) 860-0902

R. Oswald, principal investigator; J. Morris, R. Arya,

W. Ashenfelter, M. Bennett, K. Jansen, B. Johnson,

E. Twesme, P. Weiss, F. Willing, and L. Yang

\section{OBJECTIVE:}

In this paper we report the significant advances that have emerged from the jointly sponsored Solarex/NREL development program under contract number ZM-2-11040-2. The objectives of this project are to scale-up photovoltaic(PV) module production capability to large-areas, advance PV production technology, reduce module production costs, and increase large-area module performance.

\section{APPROACH:}

Our work under this effort is divided into seven areas or tasks. These areas are: Front Contact Development, Laser Scribing Process Development, Amorphous Silicon Based Semiconductor Deposition, Rear Contact Deposition, Bus/Wire/Encapsulate, Material Handling, and Environmental Test/Yield/Performance Analysis.

\section{RESULTS:}

Notable developments include: the scale-up of uniform tin oxide deposition to $0.37 \mathrm{~m}^{2}\left(4 \mathrm{ft}^{2}\right)$ size substrates, the improvements in laser scribe capability resulting in an area utilization of over $95 \%$ on $0.37 \mathrm{~m}^{2}\left(4 \mathrm{ft}^{2}\right)$ size modules, the scale-up of module fabrication processes to attain an total area efficiency of $9.08 \%$ on a large-area module, and the development of several low cost rear contact deposition processes. Taken together, these accomplishments have culminated in a process run of 180 large-area modules which when yielded to $80 \%$, have an average initial aperture area conversion efficiency of $8.75 \%$.

In the area of Front Contact Development our work has been concentrated in two major areas-tin oxide deposition process development and injector development. We use Atmospheric Pressure Chemical Vapor Deposition (APCVD) to deposit highly transmitting, textured tin oxide films over large areas. We have explored several feed stock materials for depositing tin oxide films including tin IV chloride and organotin materials. The tin IV chloride material yielded a process to deposit uniform and textured tin oxide at deposition rates in the range of 200 to 300 Angstroms per second with good optical and electronic properties. Typical film properties are: $14 \mathrm{ohms} / \mathrm{sq}$., $80 \%$ transmission, and $17 \%$ haze. We have also demonstrated uniform and textured tin oxide films using the organotin feed stocks. Typically we obtain films with $16.3 \mathrm{ohms} / \mathrm{sq} ., 80.3 \%$ transmission, 
and $15.8 \%$ haze with this material. The organotin feed stocks achieve the desired properties on relatively thin layers--approximately 5600 Angstroms thick, with a material utilization significantly higher than that of the tin IV oxide, resulting in a lower cost per square meter of coating. In addition to our investigations of feed stock materials. we have made a series of improvements to our injector design resulting in improved uniformity of tin oxide films deposited over large areas. Both the average non-uniformity and the range of variation in uniformity have been improved to less than $+/$ $8 \%$.

In the area of L ser Scribe Process Development, our efforts have been focused on both improving the active area utilization of large-area modules and on increasing the speed of laser processes. We have reduced the total interconnect scribe width from $0.676 \mathrm{~mm}(0.026 \mathrm{in}$.) to $0.401 \mathrm{~mm}(0.0158$ in.), resulting in an area utilization of over $95 \%$ on a $0.37 \mathrm{~m}^{2}$ size module. In addition, we have demonstrated a laser scribe process throughput rate of 30 plates per hour. Our approach to both these goals has been to investigate the effect of scribe parameters on scribe quality and to install a series of upgrades to both the optical and positioning components. We installed dual wavelength optical components on our large-area laser system, and upgraded the mechanical positioning components to achieve tighter beam positioning tolerances and to minimize the variation in beam location at the beginning and end of a scribe line. These mechanical improvements in combination with the capability to alter the wavelength of the laser have enabled us to achieve a significant increase in area utilization on large-area modules.

In the area of Amorphous Silicon Based Semiconductor Deposition, we have reduced the deposition time of these layers by $25 \%$ while making steady improvements in the performance of large-area modules. The semiconductor deposition process was critically examined to determine the changes that might be made to reduce deposition time without sacrificing stabilized module performance. An intensive study was initiated to introduce these changes into the process. Over the course of several months, the deposition time was gradually reduced by about $25 \%$ with no detectable loss in performance.

In parallel with our studies to decrease deposition time, we have continued to make steady improvements in the performance of large area modules. The best module to date has an initial total area conversion efficiency of $9.08 \%$ as measured by NREL. The equivalent aperture area efficiency of this module is $9.75 \%$. Several improvements to the module fabrication process have led to increasing module performance. First, we have used high transmission tin oxide deposited in-house on the large-area furnace as the front contact. Second, we have continued to improve the opto-electronic properties of the front junction p-layer resulting in an increase in both open circuit voltage and in current. Finally, we have made steady progress in improving the material properties of the narrow bandgap SiGe alloy back junction.

Our fourth major effort is in the area of Rear Contact Deposition Process Development. We have developed several low-cost methods of depositing a highly reflecting zinc oxide/aluminum rear contact. Good quality zinc oxide films suitable for use in a rear contact have been deposited over large areas by two different methods: reactive sputtering from a zinc metal target and low pressure chemical vapor deposition(LPCVD). The first method to be scaled up to large areas was the reactive sputtering technique. In this method there is no controlled doping of the zinc oxide films, 
consequently critical deposition parameters must be identified and carefully controlled. Zinc oxide film properties attainable by reactive sputtering on a 700 Angstrom thick film are typically 500 ohms/sq. and $87 \%$ integrated transmission. These films have been used in rear contacts of both $0.1 \mathrm{~m}^{2}$ and $0.37 \mathrm{~m}^{2}$ size modules with good results. Comparable film quality has been obtained using LPCVD to deposit zinc oxide. Typical films have a sheet resistance of $437 \mathrm{ohms} / \mathrm{sq}$. with an integrated optical transmission of $85 \%$. Currently, LPCVD of zinc oxide is our standard deposition process.

Under the bus/wire/encapsulate/frame portion of our effort, we have continued to investigate alternative delivery systems for the bus material and to evaluate low-cost materials systems. We have carried out preliminary studies using a positive displacement pump to meter the delivery of bus material to the substrate. In several trials with this pump we found that the repeatability of the quantity of material dispensed to the substrate is superior to that of our present system. Discussions with the pump manufacturer are continuing in an effort to more fully develop the capabilities of this dispensing system. In addition to working with the dispensing system, we have identified several alternative bus formulations that are lower in cost than our standard formulation. In preliminary trials several formulations have been evaluated for dispensing characteristics and pull strengths after environmental stress testing. The results of these trials clearly indicate that at least three different formulations, all lower in cost than our present system, show promise as a bus material.

Under our Materials Handling effort we have begun to explore concepts to accomplish equipment integration that would be required for a large-area module production facility. Station - to - station transport of work-in-process and universal indexing system concepts are under consideration. Station-to-station transport designs involve the movement of substrates from one processing station to the next where the movement is external to an integrated machine. The purpose is to accomplish total integration of the production process. To this end several vendors of automated material transport equipment have been contacted. A number of transport schemes have been identified including systems based on air-float principles, conveyor rollers, and ball transfer systems. An important consideration is the extent to which these systems may be integrated with the indexing systems that would be used at several points in the production process. These indexing systems are precise and repeatable locating mechanisms that would be required for certain processing steps. A universal system - one that could be used at several points on a line-offers the advantages of part inter-changability and one-time integration.

In the area of Environmental Test, Yield and Performance Analysis, environmental stress testing and yield analysis are ongoing efforts. As we refine encapsulation techniques and introduce new candidate encapsulation materials we routinely subject samples of large-area modules to environmental stress tests. These tests include 50 and 200 thermal cycles, humidity-freeze cycling, and dry and wet hi-pot testing. Our yield analysis data are summarized in Figure 1. To date we have produced roughly 200 large-area multi-junction modules in our process development effort. Figure 1 is a histogram of the efficiency data at a yield of about $80 \%$. At this yield the average efficiency of the modules was $8.75 \%$ with a standard deviation of about $0.30 \%$. 


\section{CONCLUSION :}

Significant advances have been made in the scale-up of large-area multi-junction amorphous silicon alloy photovoltaic modules. Advances have been made both in reducing the module fabrication costs and in increasing the conversion efficiency of the modules. A production-scale run of 180 modules using low cost materials and processes and yielded to $80 \%$ resulted in an average initial aperture area conversion efficiency of $8.75 \%$ on $0.37 \mathrm{~m}^{2}$ modules. The highest efficiency module observed in this run was $9.75 \%$. Further reductions in cost and gains in module efficiency are expected.

This work has been jointly funded by NREL and Solarex under Subcontract Number ZM-2-1040-2.

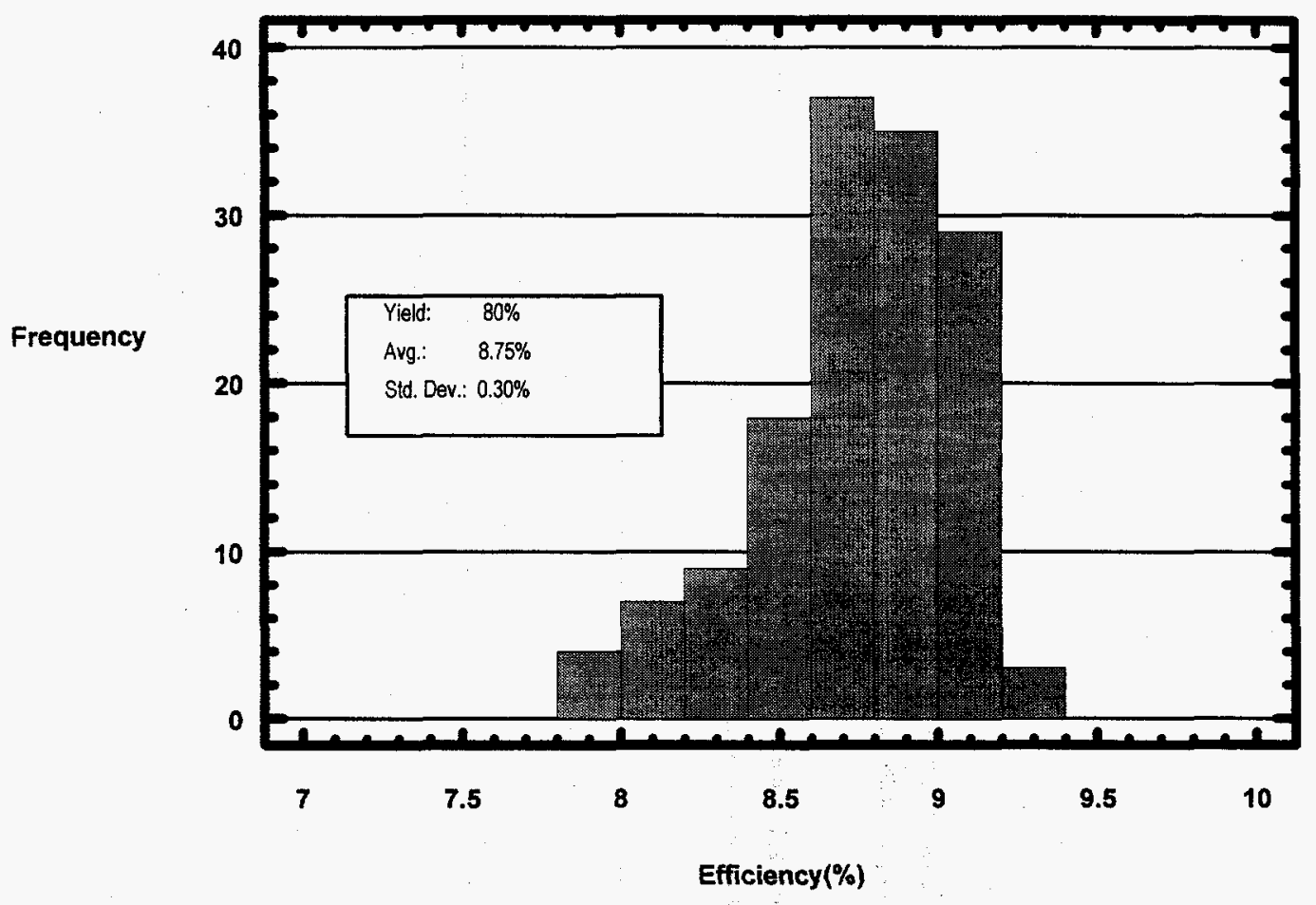

Figure 1: Histogram of $0.37 \mathrm{~m}^{2}$ module efficiencies at $80 \%$ yield 
Title

Organization Solarex, A Business Unit of Amoco/Enron Solar

Contributors J. H. Wohlgemuth, Program Manager; F. Artigliere, M. Conway, J. Creager, T. Duquaine, J. Ellis, T. Koval, T. Tomlinson and D. Whitehouse of Solarex; B. Huff and D. Vanecek of ARRI

\section{Objectives}

The objectives of this three-year program are to advance Solarex's cast polycrystalline silicon manufacturing technology, reduce module production cost, increase module performance and expand Solarex's commercial production capacities. Two specific objectives of this program are to reduce the manufacturing cost for polycrystalline silicon PV modules to less than $\$ 1.20 /$ watt and to increase the manufacturing capacity by a factor of three.

\section{Approach and Accomplishments}

\section{Casting}

The goal of the casting task is to develop the ability to cast ingots that yield four bricks with a cross-section of $15 \mathrm{~cm}$ by $15 \mathrm{~cm}$ with at least equivalent material quality as now achieved for 11.4 $\mathrm{cm}$ by $11.4 \mathrm{~cm}$ bricks. This represents a $73 \%$ increase in the useable silicon obtained from each casting.

During the first year of the program, Solarex has designed and fabricated new larger ceramic pieces, designed and implemented modifications to a casting station and designed and implemented modifications to the sizing saws in order to be able to cast and size larger ingots. A $20 \%$ increase in charge weight was achieved by redesigning the ceramic crucible to have an elliptical rather than a cylindrical cross-section to fit around the heating elements. This change has been successfully demonstrated and already transferred to manufacturing to cast ingots with $33 \%$ larger cross-sectional area with equivalent yields and average cell efficiencies. To further increase the size of silicon charge that the station can melt, an extension collar was design and fabricated. The crucible was then enlarged by adding an insert to the top. The extension collar, a new insulation package and new heating elements were designed, fabricated and installed in one station. This station has been used to cast experimental full size PVMaT ingots with $73 \%$ larger volume.

\section{Wire Saw}

The goal of the wire saw task is to develop the wire saw technology for cutting $15 \mathrm{~cm}$ by $15 \mathrm{~cm}$ polycrystalline wafers on $400 \mu \mathrm{m}$ centers at lower cost per cut than achieved today 
on the ID saws. This represents a $50 \%$ increase in the useable silicon obtained from each cast and a $50 \%$ increase in the yield of wafers per purchased kilogram of Si feedstock.

Solarex identified four vendors of wire saws (Meyer-Berger, HCT, M. Setek and Nippei Toyama), visited each vendor and evaluated the performance, specification and cost of the 4 saws. Solarex then selected and purchased an HCT wire saw. The HCT wire saw has been operational since July, 1994. The saw has performed well, having cut nearly 400 bricks ( $>125,000$ wafers) with an average yield of $93 \%$ ( $97 \%$ if you don't count aborts). Other important technical accomplishments are:

- Demonstrated the ability to cut $8-11.4 \mathrm{~cm}$ by $11.4 \mathrm{~cm}$ bricks at one time, resulting in the cutting of 2400 wafers in one 6.5 hour run.

- Defined a wire saw process and operated it in a trial production mode. This process produces wafers with an estimated wafer cost that is $5 \%$ below the program goal for the wire saw and $13 \%$ below the ID saw production cost.

- Demonstrated the ability to cut both $11.4 \mathrm{~cm}$ by $11.4 \mathrm{~cm}$ and $15 \mathrm{~cm}$ by $15 \mathrm{~cm}$ wafers on $400 \mu \mathrm{m}$ centers with high cutting yields.

- Thousands of wire saw wafers have been processed through the Solarex cell line. The wire saw cells had a $3.6 \%$ higher mechanical yield and a $4.4 \%$ total yield than the coprocessed ID wafers. In addition, there was no statistical difference between the wire saw and the ID saw cell efficiencies.

\section{Cell Process}

The goal of the cell task is to increase cell efficiencies to $15 \%$, while decreasing the cost per watt at the module level. The developed process must be compatible with automated manufacturing at large volumes.

The initial area of effort was in the development of a cost effective back surface field (BSF). Aluminum pastes have been used successfully to improve the efficiency of solar cells for years. Table 1 compares the results for non-BSF cells with those made using $\mathrm{Al}$ paste from two different vendors. Back surface fields usually increase cell efficiency by 5 to $6 \%$. The issue is that of cost. Program efforts have involved development of a paste and a process that does not require post fire clean-up. In addition, we have worked with the paste vendors in an effort to reduce the cost of the material and the amount required per cell. Both of these efforts have been successful.

The second area of effort was in the development of improved optical coupling, since polycrystalline silicon can not be textured with an alkaline etch as most single crystal solar cells are. A new method for evaluating the effectiveness of optical coupling techniques was developed [2]. The method was applied to a number of different surface textures. The resulting analysis showed that a variety of texturing methods including chemical etching, texturing with a tapered blade and texturing with a mechanical tool can increase the short circuit current of an unencapsulated solar cell by 7 or $8 \%$. However, when 
encapsulated the current gain from texturing is reduced to 3 to $4 \%$, with the low cost texturing tool performing as well as chemical texturing and as well as tapered blades.

\section{Module Assembly}

The goal of the module assembly task is to modify Solarex's present module assembly system to increase throughput by $100 \%$ and decrease the labor requirement by $50 \%$. The Automation and Robotics Research Institute at the University of Texas at Arlington (ARRI) is to work with Solarex to model the present automated module assembly system and to recommend modifications to increase throughput and reduce labor.

As a first step a detailed flow chart of the module assembly system was prepared. A simulation model of the Solarex module manufacturing process was developed using the MPX queuing analysis tool. The model was used to identify resource limits that prevent additional output capacity. The model was able to identify those process stations that limit the factories ability to produce a larger quantity of modules. The model was also useful in developing "what-if" scenarios, to determine what additional resources were necessary to increase the manufacturing capacity by $50 \%$. FactoryFlow simulation was used to determine materials handling costs and to identify potential bottlenecks in the factory. Combined with a resource capacity study, these models lead to proposed changes in a number of processes and lay-outs. Several of these changes have been implemented. One of these changes resulted in an improvement in the cycle time of the matrix robot, so that it is no longer a limit on factory output.

\section{Frameless Module Development}

The goal of the frameless module task is to develop and qualify a frameless module design incorporating a lower cost back sheet material (less than $\$ 0.05 /$ square foot) and user friendly, low cost electrical termination (less than $\$ 1.00 /$ module). Since PVMaT is designed for large systems, modules can be designed to mount directly onto the support structure without integral frames.

The first step has been the design of a compatible support structure and the identification of $3 \mathrm{M}$ Very High Bond Tape for mounting the modules to the structure. A sample array structure has been constructed and modules mounted using the tape. So far the tape has proven to be an excellent adhesive candidate, having withstood winds in excess of 60 miles per hour from the north or back of the array, the worst case condition.

A module backsheet specification has been prepared and utilized in discussion with potential vendors. Three experimental back sheet materials have been selected and all of these materials have successfully passed environmental qualification to IEC-1215, "Crystalline Silicon Terrestrial Photovoltaic Modules - Design Qualification and Type Approval". All three materials also successfully passed an in-house simulated UL fire test and wet high-pot tests. Mini-modules with these backsheets are now undergoing 
accelerated outdoor exposure tests with the backsheet exposed directly to concentrated sunlight.

\subsection{Automated Cell Handling}

The goal of the automated cell handling task is to develop automated handling equipment for $200 \mu \mathrm{m}$ thick $15 \mathrm{~cm}$ by $15 \mathrm{~cm}$ polycrystalline silicon wafers and cells with a high yield (less than $0.1 \%$ breakage per process handling step) at a throughput rate of at least 12 cells or wafers per minute. This task will begin in the second year.

\section{Conclusions}

The results achieved during the first year of the program include:

- Larger ingots can be cast in the Solarex casting stations, although work on modifications to the insulation package and process program will be required to achieve equivalent material quality.

- The wire saw has the potential to significantly reduce cost. Work in the second year will center on developing a manufacturing process and the design and development of a process for demounting and cleaning the wafers after they are cut.

- Various cost effective efficiency improvements have been identified. An integrated high efficiency process must be selected and verified during the second year.

- The modeling tools available at ARRI will be utilized to guide Solarex's expansion of the module assembly area.

- Lower cost backsheets and electrical termination have been identified and will be environmentally qualified in the module package designed for use with the frameless module support structure developed during the first year.

\section{References}

[1] J. H. Wohlgemuth, D. Whitehouse, T. Koval, J. Creager, F. Artigliere and M. Conway, "Solarex Crystalline PVMaT Program", First World Conference on Photovoltaic Energy Conversion, 1994.

[2] J. H. Wohlgemuth, T. Koval, D. Whitehouse and J. Creager, "Evaluating the Effectiveness of Optical Coupling Techniques", First World Conference on Photovoltaic Energy Conversion, 1994.

Table 1

Al Paste Results

\begin{tabular}{|ccccc|}
\hline Paste & $\begin{array}{c}\text { Efficiency } \\
(\boldsymbol{\%})\end{array}$ & $\begin{array}{c}\text { Voc } \\
(\mathbf{m V})\end{array}$ & $\begin{array}{c}\text { Jsc } \\
\left(\mathbf{m A} / \mathbf{c m}^{2}\right)\end{array}$ & $\begin{array}{c}\text { FF } \\
(\boldsymbol{\%})\end{array}$ \\
\hline None & 13.13 & 580.9 & 29.4 & 76.8 \\
Vendor 1 & 13.92 & 592.2 & 31.2 & 74.8 \\
Vendor 2 & 13.99 & 589.6 & 31.6 & 75.1 \\
\hline
\end{tabular}


Title:

Organization:

Contributors:
Automated Solar Cell Assembly Teamed Process Research

Spire Corporation, Bedford, Massachusetts

G. Darkazalli, Program Manager, M.J. Nowlan, Principal Investigator, S.J. Hogan, S.F. Sutherland, J.M. Murach, W.F. Breen, and J. Patterson

\section{Introduction}

The objective of this research is to develop new processes for high throughput, high yield, fully automated interconnection of standard and thin silicon solar cells. Improvements in ingot slicing (wire sawing) techniques are now enabling the production of $\mathrm{Si}$ wafers that are substantially thinner $(\sim 200 \mu \mathrm{m})$ than conventional wafers $(350$ to $500 \mu \mathrm{m})$. The use of these thin wafers can significantly reduce module manufacturing costs, provided that processing yields remain as high as they are now for standard thickness wafers.

1994 was the second year of this 30-month program, supported by NREL's Photovoltaic Manufacturing Technology (PVMaT) project. During this period we completed the design, fabrication, subassembly integration, and control software for a new highly automated cell interconnecting system, the SPI-ASSEMBLER ${ }^{\mathrm{TM}}$ 5000. A photograph of the Assembler is provided in Figure 1.

The Assembler achieved high yield (98.5\%) in initial evaluations through the use of low mechanical stress cell handling and low thermal stress soldering processes. Throughputs up to 900 cells per hour were obtained; at this rate, more than $5 \mathrm{MW}$ of cells (with an area of $100 \mathrm{~cm}^{2}$ and an efficiency of $12 \%$ ) can be processed annually in a 3 shift/day, 5 day/week operation. Additional work is planned to evaluate the Assembler with larger quantities of cells in a production setting and to evaluate its suitability with various novel cell designs.

\section{Approach}

The Assembler automatically produces strings of interconnected solar cells from stacks of cells, reels of copper ribbon, and liquid flux. Interconnect ribbon is transported through a flux applicator that coats the ribbon with flux, a die that forms a stress-relief bend, and a knife set that cuts the ribbon to length. Linear motors drive a ribbon shuttle assembly at high speed to cut and form ribbons to user-specified dimensions. Two ribbons are processed side-by-side, resulting in a pair of cut, bent, and flux coated ribbons, or "tabs."

Round or square cells up to $15 \mathrm{~cm}$ across can be processed. Cells are loaded from stack carriers rather than slotted cassettes to reduce the volume taken up by cells in process and to reduce the amount of operator handling. A two-stack shuttle system allows continuous cell feeding without interruptions for operator loading. A special vacuum pick-up was designed with a floating mount for handling thin cells with low mechanical force. The pick-up is mounted on a 2-axis $(x, z)$ linear actuator for picking up the top cell in the stack and placing it on an aligner stage. 
At the aligner state, a machine vision system inspects the cell and determines its location in $\mathrm{x}$, $y$, and $\theta$. A 4-axis robotic manipulator arm picks up the cell, aligns it, and places it on a pair of tabs or, if the cell fails the vision inspection, in a reject stack. A unique conveyor belt with vacuum and heating capabilities holds the tabs and cell in alignment, preheats them to reduce thermal shock, and indexes them under a solder head. The solder head's high intensity lamps heat the cell with visible and infrared radiation, avoiding the thermal and mechanical shocks generated by conduction heating processes. Both the top and bottom tabs are soldered to the cell in a single operation, unlike conventional methods in which tabs are soldered to the cell's top contact in one heating cycle and to the back contact in another.

Series-connected cell strings are built up on the conveyor belt by repeating the tab fabrication and placement sequence, the cell inspection and alignment sequence, and the soldering process until the desired number of cells have been assembled together. Cell strings up to two meters long can be fabricated.

The conveyor belt positions the completed string under a string transfer mechanism, which has separately floating vacuum pick-ups for each cell in the string. The mechanism picks up the string and places it on an I-V tester that measures the string's performance under simulated sunlight. If the string fails to meet user-selected criteria for $\mathrm{V}_{o c}, \mathrm{I}_{s c}$, or $\mathrm{P}_{\max }$ it is placed on a reject carrier. Strings that pass are placed in sequence on a module carrier in the proper seriesparallel arrangement selected for the particular module design.

\section{Results Obtained in 1994}

Mechanical and electrical design, subassembly fabrication and testing, system integration, and software development were completed in 1994. Initial processing evaluations were done at Spire using $10 \mathrm{~cm}$ square $200 \mu \mathrm{m}$ thick cells from Solec International and $10 \mathrm{~cm}$ square $325 \mu \mathrm{m}$ thick cells from Siemens Solar Industries. These evaluations identified certain weaknesses in the original design which were subsequently corrected. For example, a vacuum chuck which is transparent to the vision system was devised that prevents cell motion during pick up by the aligner manipulator; a white shutter and improved lighting were added to the cell aligner stage, allowing the vision system to clearly detect cell edges; and tab position accuracy was increased by improving the ribbon guide design and modifying the vacuum conveyor belt to increase its grip on the tabs.

Ribbon flux coating tests successfully identified a no-clean flux which produces good solder joints, leaves no greasy residue on the ribbons, and does not stick the ribbon to the tooling. A soldering process with a 3.2 s cycle time was developed that produces solder joints with good pull strengths. Pulls in the range of 200 to 500 grams at a $90^{\circ}$ angle and 400 to 1000 grams at a $45^{\circ}$ angle are typical. Failure during pull testing is typically at the cell's contact-silicon interface or in the bulk silicon, rather than at the solder joint-contact interface.

A trial run of approximately 1000 Siemens Solar cells was done on the Assembler at Spire in May, 1994. The silicon wafers were nominally $325 \mu \mathrm{m}$ thick by $10 \mathrm{~cm}$ square. Twelve-cell strings were soldered with a no-clean flux and the residue was not removed after soldering. Ten modules were fabricated at Spire by encapsulating 30 strings with a SPI-LAMINATOR ${ }^{\text {TM }} 350$. 
The modules were then shipped to Siemens for accelerated environmental testing. Three modules were subjected to 200 thermal cycles (T-200) from $-40^{\circ} \mathrm{C}$ to $+90^{\circ} \mathrm{C}$, three were subjected to 50 thermal cycles (T-50) from $-40^{\circ} \mathrm{C}$ to $+90^{\circ} \mathrm{C}$ followed by 10 humidity-freeze cycles (HF-10) from $85^{\circ} \mathrm{C} / 85 \% \mathrm{RH}$ to $-40^{\circ} \mathrm{C}$ followed by another 10 humidity-freeze cycles (HF-20); three were subjected to 1000 hours of damp heat soaking (DH-1000) at $85^{\circ} \mathrm{C} / 85 \% \mathrm{RH}$; one was a control module. Module performance was measured before and after environmental testing. The results, provided in Table 1, show maximum degradations of $1.5 \%$ after T-200, 3.8\% after T-50 followed by HF-20, and $2.4 \%$ after DH-1000, all well below the $10 \%$ acceptance limit. These tests provide an important verification of the soldering process developed under this program.

A second trial run with Siemens Solar cells was done at Spire in September, 1994, in which 183 strings ( 2196 cells) were processed. The yield was measured to be $98.5 \%$, a full percentage point higher than the yield in May. Improvements in the vision system and the ribbon handling hardware provided more accurate cell alignment and ribbon placement accuracy than was obtained in May. Faster loading system software resulted in cell-to-cell cycle times in the 3.8 to 4.0 second per cell range, nearly one second faster than the cycle time in May. The string-tostring cycle time was measured at approximately $68 \mathrm{~s}$ per 12-cell string. This cycle time is longer than the cell-to-cell cycle time due to the present method of string fabrication sequencing. Sequencing improvements will be investigated to further reduce the cycle time and increase product throughput.

\section{Future Plans}

The Assembler's performance will be evaluated with approximately two thousand thin $(200 \mu \mathrm{m})$ cells fabricated by Solec International. The program was recently modified to allow an evaluation of the Assembler for processing various novel cell designs, such as cells made from ribbon silicon or thin film silicon, and to investigate various methods for joining strings together with bus ribbons. The Assembler will then be shipped to a PV module manufacturer for evaluations in a module production line. System performance data will be documented.

\section{References}

M.J. Nowlan, S.J. Hogan, G. Darkazalli, S.F. Sutherland, W.F. Breen, J.M. Murach, and J.S. Patterson, "Advanced Automation Techniques for Interconnecting Thin Silicon Solar Cells," Proc. Ist World Conf. on Photovoltaic Energy Conversion, Waikoloa, HI, Dec. 5-9, 1994.

S.J. Hogan, M.J. Nowlan, T.L. Jester, and J.J. Hummel, "Performance and Reliability Testing of Crystalline Silicon Cell Strings Fabricated with a SPI-ASSEMBLER 5000 Apparatus," NREL PV Performance and Reliability Workshop, Lakewood, CO, Sept. 21, 1994. 


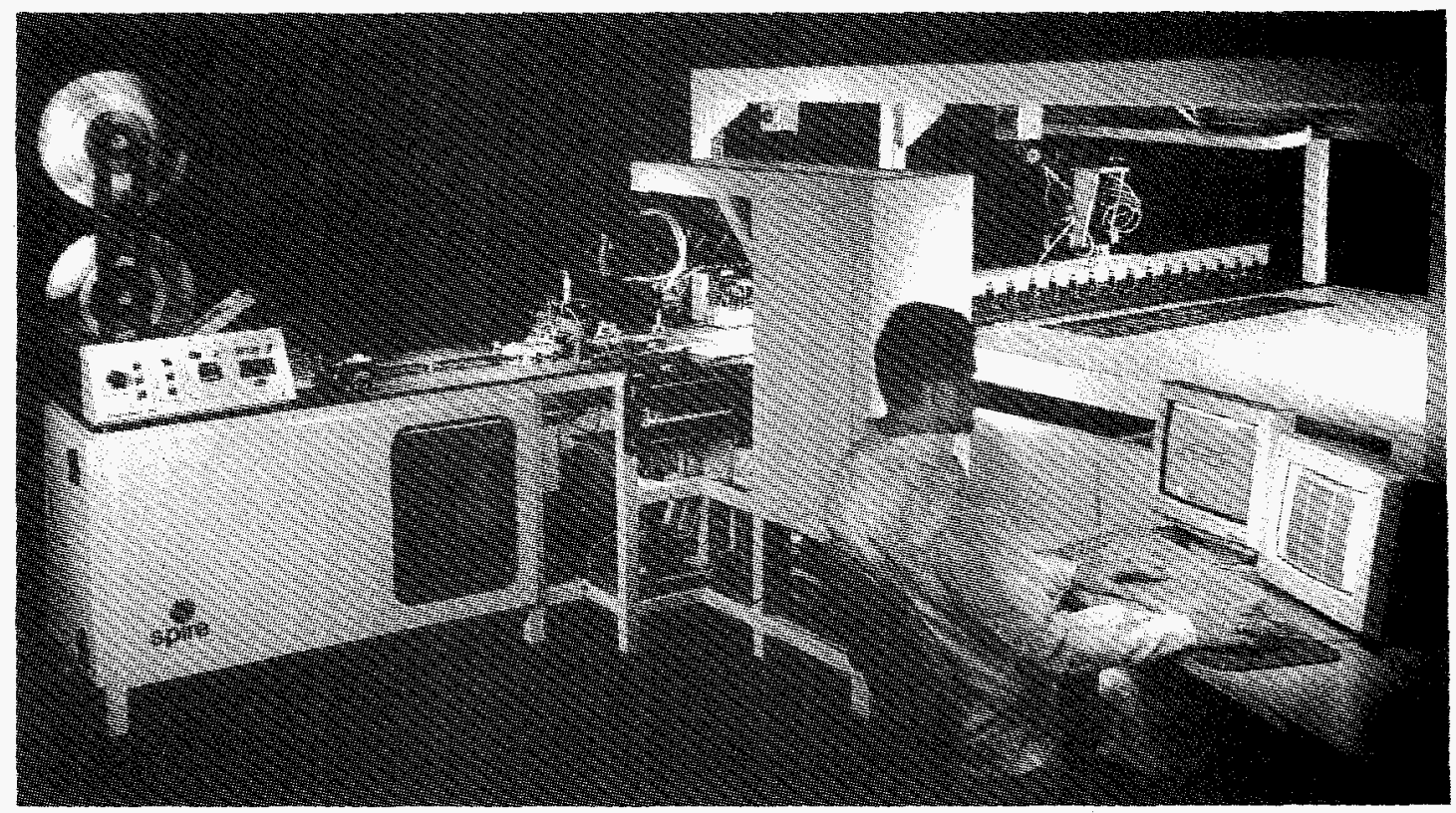

Figure 1 Photograph of the SPI-ASSEMBLER 5000 automated solar cell interconnecting system.

Table 1 Environmental test results for modules fabricated with the SPI-ASSEMBLER 5000 and the SPI-LAMINATOR 350. (Data courtesy of J. Hummel and T. Jester, Siemens Solar.)

\begin{tabular}{|c|c|c|c|c|c|}
\hline \multicolumn{6}{|c|}{ Thermal Cycling Test: T-200 } \\
\hline Module & $\begin{array}{c}\text { Initial Power } \\
P_{\max }(W)\end{array}$ & & $\begin{array}{c}\text { Post T-200 Pov } \\
\mathrm{P}_{\max }(\mathrm{W})\end{array}$ & & $\begin{array}{c}\Delta \mathrm{P}_{\max } \\
(\%)\end{array}$ \\
\hline $\begin{array}{l}1 \\
2 \\
4\end{array}$ & $\begin{array}{l}55.1 \\
54.9 \\
54.8\end{array}$ & & $\begin{array}{l}54.3 \\
54.1 \\
54.4\end{array}$ & & $\begin{array}{l}-1.5 \% \\
-1.5 \% \\
-0.7 \%\end{array}$ \\
\hline \multicolumn{6}{|c|}{ Thermal \& Humidity-Freeze Cycling Test: T-50, HF-10, HF-20 } \\
\hline Module & $\begin{array}{c}\text { Initial Power } \\
P_{\max }(W)\end{array}$ & $\begin{array}{l}\text { Post T-50 } \\
P_{\max }(\mathrm{W})\end{array}$ & $\begin{array}{l}\text { Post HF-10 } \\
P_{\max } \text { (W) }\end{array}$ & $\begin{array}{c}\text { Post HF-20 } \\
P_{\max }(\mathrm{W})\end{array}$ & $\begin{array}{l}\Delta \mathrm{P}_{\max } \\
(\%)\end{array}$ \\
\hline $\begin{array}{l}3 \\
5 \\
9\end{array}$ & $\begin{array}{l}55.2 \\
54.3 \\
55.1\end{array}$ & $\begin{array}{l}55.4 \\
55.4 \\
55.2\end{array}$ & $\begin{array}{l}52.9 \\
53.9 \\
53.7\end{array}$ & $\begin{array}{l}53.5 \\
53.4 \\
53.0\end{array}$ & $\begin{array}{l}-3.1 \% \\
-3.4 \% \\
-3.8 \%\end{array}$ \\
\hline \multicolumn{6}{|c|}{ Damp Heat Soak Test: DH-1000 } \\
\hline Module & $\begin{array}{c}\text { Initial Power } \\
P_{\max }(W)\end{array}$ & & $\begin{array}{l}\text { Post DH-1000 P } \\
\mathrm{P}_{\max }(\mathrm{W})\end{array}$ & & $\frac{\Delta \mathrm{P}_{\max }}{(\%)}$ \\
\hline $\begin{array}{c}7 \\
8 \\
10\end{array}$ & $\begin{array}{l}55.2 \\
55.0 \\
54.6\end{array}$ & & $\begin{array}{l}53.9 \\
54.1 \\
53.8\end{array}$ & & $\begin{array}{l}-2.4 \% \\
-1.6 \% \\
-1.5 \%\end{array}$ \\
\hline
\end{tabular}


Title: $\quad$ Advanced Development of PV Encapsulants

Organization: Springborn Materials Science Corp.

Enfield, Connecticut

Contributors: W.H. Holley, principal investigator, J. P. Galica, administrative manager; S.C. Agro, L. Thoma, R.S. Yorgensen; M. Ezrin and P. Klemchuk, Institute of Materials Science, University of Connecticut

\section{Objectives}

Despite the widespread acceptance of ethylene vinyl acetate (EVA) for flat plate photovoltaic (PV) module encapsulation, we have, over the years, heard a number of reports concerning the yellowing or browning of the EVA encapsulant. The very sporadic nature of these claims--discoloration has been experienced in PV modules of all ages and in a number of different, albeit warmer, geographic regions--caused us to question whether the EVA itself might actually be at fault. An extensive literature search and field survey by the authors revealed that such yellowing has not been limited to the modules of any one manufacturer, nor to the EVA encapsulant sheet of any single supplier.

Thus, starting with the one clue that the degree of discoloration does appear to loosely correlate with the degree of average daily direct normal solar radiation, and a module's approximate maximum operating temperature, the purpose of our current work is to isolate the variable(s) of EVA browning by conducting controlled accelerated aging studies in the laboratory. All in-house produced test results are further being compared to results from field-aged modules that have exhibited browning.

\section{Technical Approach}

To this end, we began by studying field-aged EVA encapsulated modules (both mirror enhanced and non-mirror enhanced arrays) from an affected site in Carrisa Plains, Califormia. These samples were autopsied and analyzed for vinyl acetate content, unsaturation, and concentration of additive levels by Thermogravimetric Analysis (TGA), Fourier Transform Infrared Analysis (FTIR), Gas Chromatography/Mass Spectrometry (GC/MS), and Gas Chromatography/Flame Ionization Detection (GC/FID).

Comparative laminates measuring $2.70 \times 2.75$ inches were then assembled in-house using either "fast-cure" 15295 EVA or "standard-cure" A9918P EVA, and one of four different low iron glasses. The majority of these laminates were glass/glass so as to facilitate visual, colormetric, and spectrographic measurements, and all were prepared by vacuum lamination on laboratory-scale equipment using the commercial time/temperature/vacuum schedule recommended for "standardcure" EVA with Lupersol 101. Accelerated aging was conducted at 0.55 watts per square centimeter at $340 \mathrm{~nm}$--roughly the equivalent of two suns, and a 100 degrees $C$ black panel temperature--in an Atlas Ci35A Xenon Arc Weather-Ometer fitted with a quartz/borosilicate filtering system on its xenon arc lamp. The nominal lower end UV cut-off was $285 \mathrm{~nm}$, and no water spray was used. Complete exposure lasted for a minimum of 17 weeks, or until significant degradation/discoloration had occurred.

These laminates were also evaluated periodically for yellowing by colorimeter per ASTM D 1925, and for percent light transmission (\%T) in the range from 250 to $900 \mathrm{~nm}$. Destructive testing methods concentrated on additive concentration analysis and vinyl acetate content analysis. 
As for the specific glass superstrates selected, we tested AFG's Solite (samples from the mid-1980s), PPG's Starphire, AFG's Solatex II (containing less than 4 percent cerium-oxide by weight), and PPG's Airphire (also containing cerium-oxide). Low iron glass with cerium-oxide was chosen for its ability to screen out much of the UV-B transmission, or the 285 to $330 \mathrm{~nm}$ wavelength region suspected of being responsible for EVA discoloration/degradation.

Additional testing included vinyl acetate content analysis of virgin Elvax 3185 EVA pellets and fully formulated "standard-cure" EVA encapsulant, both before and after field aging, and FTIR analysis of both browned and non-browned areas of the fielded Carrisa Plains modules. Examination of the virgin and aged EVA samples was by complete thermolysis of the acetate group using TGA.

\section{Results}

\section{"Fast-Cure" EVA Reduces Discoloration}

Our examination of the Carrisa Plains samples found that all modules which had experienced EVA browning used "standard-cure" EVA. This finding was further corroborated by an extensive survey of flat plate PV modules which had experienced browning. Conversely, there were no reported cases of EVA browning with "fast-cure" 15295 EVA. It therefore became apparent that the EVA crosslinker may play a part in EVA browning. "Standard-cure" EVA contains Lupersol 101 peroxide as the crosslinker while "fast-cure" 15295 EVA is crosslinked with Lupersol TBEC peroxide.

These same results occurred in our laboratory accelerated UV-aging studies. Measurable amounts of yellowing were evident in "standard-cure" EVA samples after one or two weeks exposure, with visual discoloration observed after four to six weeks. After 20 weeks in the Weather-Ometer, samples laminated with "standard-cure" EVA had a color index of 48 compared to only 21 for laminates made with "fast-cure" 15295 (see Fig. 1).

Further analytical studies have since confirmed that the Lupersol 101 peroxide contained in the "standard-cure" A9918P EVA grade is a major contributor to photothermally induced color development.

\section{Glass With Cerium-Oxide Reduces Discoloration}

Laminates with "standard-cure" A9918P EVA which used either Solatex II or Airphire glass as the superstrate also showed greatly reduced discoloration. After 20 weeks in the Weather-Ometer, these cerium-oxide containing samples exhibited a color index of only 10-11, compared to Solite glass control samples which had a color index of 48. Yellowness of the control samples was also similar to that of the fielded modules without mirror enhancement (see Fig. 2).

We note here that the amount of available energy sacrificed due to the selection of cerium-oxide containing glass for PV laminates is only in the 300 to $350 \mathrm{~nm}$ region--an area of the spectrum where solar cells, at best, operate at only 50 percent of their normal efficiency. Figure 3 demonstrates this minimal loss, using the spectral irradiance of terrestrial sunlight as a reference (see Fig. 3 ).

\section{"Fast-Cure" EVA With Cerium-Oxide Shows No Discoloration}

After 21 weeks in the Weather-Ometer, laminates prepared with "fast-cure" 15295P EVA and either an Airphire or Solatex II superstrate had color indices of only 4.4 and 3.3--a yellowness which is not discernable to the human eye. In contrast, control samples with the Solite superstrate had a color index of 24.0 (see Fig. 4). 


\section{Aged EVA Shows No Loss of Vinyl Acetate Content}

In all cases, vinyl acetate content of the virgin and fully formulated "standard-cure" resins were equivalent and within experimental error standards. Also, similar tests performed on browned EVA samples from the mirror enhanced modules of Carrisa Plains showed no evidence of photothermolysis of the vinyl acetate groups (see Table 1).

\section{Non-Colored EVA in Fielded Modules Shows Oxidation}

The browned EVA samples from Carrisa Plains showed no significant double-bond absorbances in areas of the spectrum where one would expect them. This functionality would have been prevalent if the browning was due to conjugated unsaturation in the EVA backbone. A similar FTIR analysis of the non-browned areas of these same fielded modules-namely those areas between cells and above cracks--showed strong absorbance at $3550 \mathrm{~cm}^{-1}$. This finding is ascribed to hydroxyl groups resulting from oxidation of the encapsulant (see Fig. 5). Further testing of the browned EVA samples at $3550 \mathrm{~cm}^{-1}$ showed much weaker double-bond absorbance in samples taken from areas over the cells.

Thus, oxidation appears to have occurred only in areas where the EVA had access to atmospheric oxygen.

\section{Conclusions}

It appears that the browning of EVA in fully EVA encapsulated flat plate PV modules results not from a chemical breakdown of the EVA polymer but rather from the interaction of additives in the presence of temperature and UV, with a combination of Lupersol 101 peroxide and other additives proving particularly damaging. Thus, we conclude that the use of EVA as a base polymer for flat plate solar cells is a sound choice, and find EVA a very strong candidate for the development of an encapsulant with improved outdoor exposure stability.

Additionally, glass superstrates containing cerium-oxide are effective in minimizing EVA encapsulant discoloration as they screen a majority of the received UV-B radiation. We therefore suggest the use of an alternate crosslinking agent, such as Lupersol TBEC, in combination with a cerium-oxide-containing superstrate for the virtual elimination of the problem of EVA discoloration over a PV module's normal life span.

\section{References}

1. Agro, S.C., Galica, J.P., Holley, W.H., and Yorgensen, R.S., "Case Histories of EVA Encapsulant Discoloration in Fielded Modules," AIP Conference Proceedings 306, 12th NREL Photovoltaic Program Review, Denver, Colorado, October 1993, American Institute of Physics Press, 1994, pp. 586-596.

2. Holley, W.H., Agro, S.C., Galica, J.P., Thoma, L.A., Yorgensen, R.S., Ezrin, M., Klemchuk, P., and Lavigne, G., "Investigation into the Causes of Browning in EVA Encapsulated Flat Plate PV Modules," First World Conference on Photovoltaic Energy Conversion, Waikoloa, Hawaii, December 5-9, 1994.

3. Holley, W.H., Agro, S.C., Galica, J.P., Thoma, L.A., Yorgensen, R.S., and Thomas, H., "Effects of Glass Superstrates on Browning of Accelerated U.V. Aged EVA Encapsulants," NREL Photovoltaic Performance and Reliability Workshop, Lakewood, Colorado, September 21-23, 1994. 
4. Holley, W.H., Agro, S.C., and Thoma, L.A., "Improved Non-Discoloring Encapsulant for Flat Plate PV," NREL Photovoltaic Performance and Reliability Workshop, Golden, Colorado, September 8-10, 1993, Report NREL/CP-410-6033, pp. 387-393.

5. Holley, W.H., Agro, S.C., Galica, J.P., Thoma, L.A., White, R.A., and Yorgensen, R.S., "Advanced Development of PV Encapsulants," National Renewable Energy Laboratory, Subcontract ZAG-3-11219-02-105661, Annual Subcontract Report for 30 December 1992-31 March 1994.

Figur 3 and Table

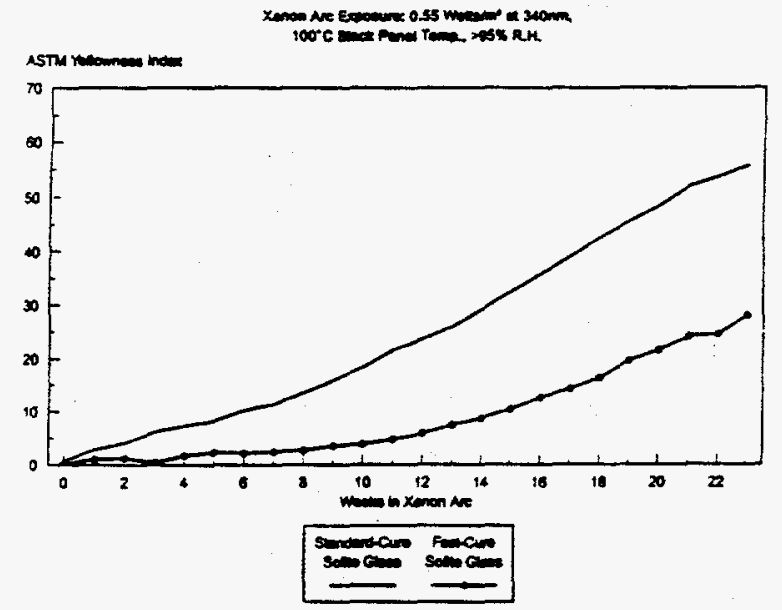

Figure 1. Accolerated UV Aging of "Standard-Cure" Versus "Fast-Cure" EVA Encapsulants

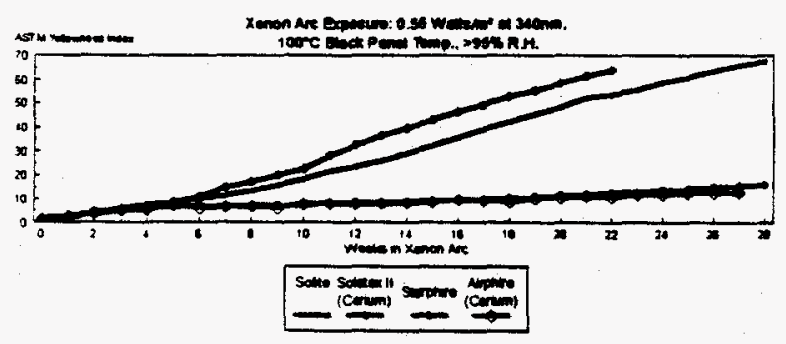

Figure 2. Elfects of Glass Composition on Accelertited UV Aging of "Standard-Curs" A0018P EVA

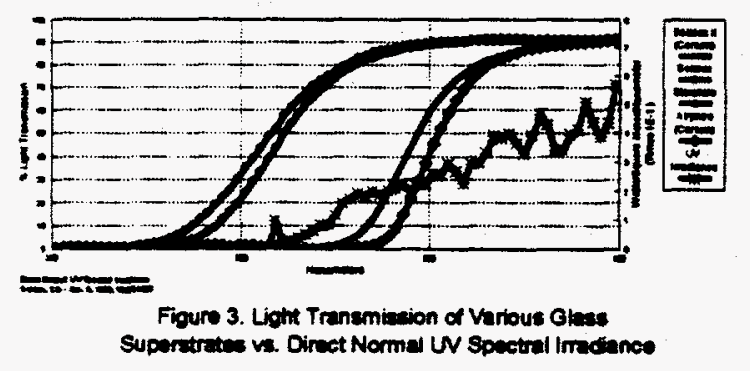

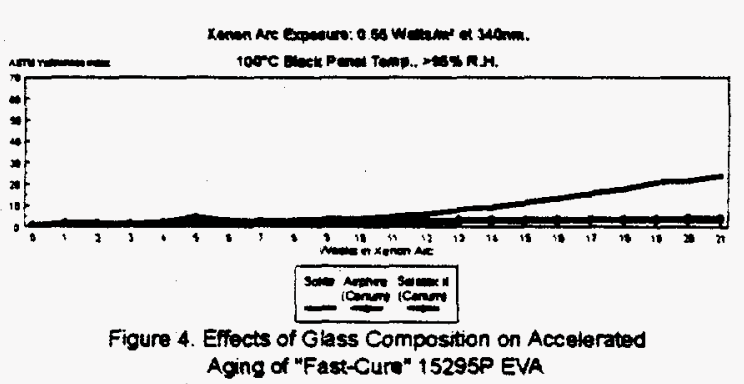

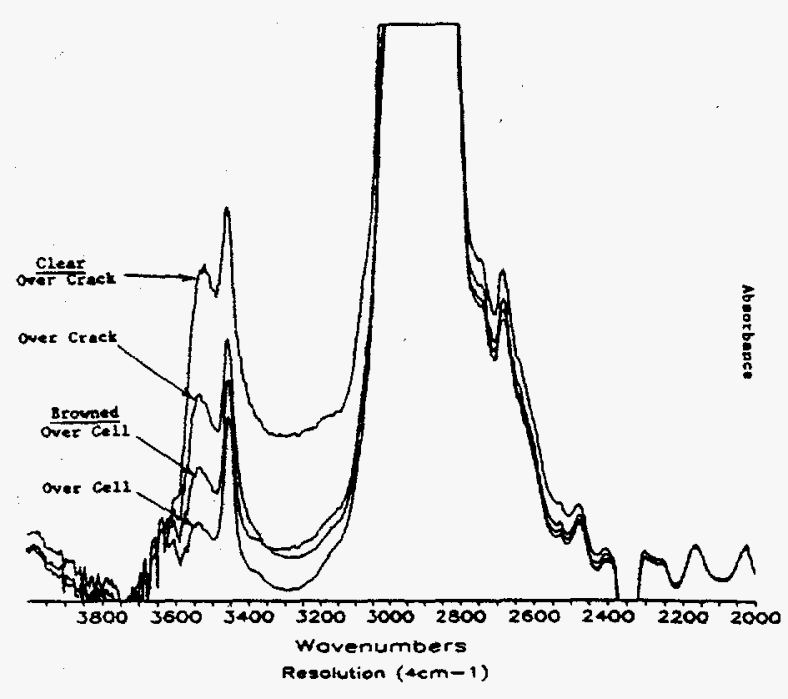

Figure 5. Infrered Spectroscopy of Browned Versus Clear EVA

Table 1. \% Viny Acotats Content by TGA

\begin{tabular}{|c|c|c|c|}
\hline Senpie id. & Serniple Dencriplen & color & $\begin{array}{l}\text { \%VA } \\
\text { comerind for }\end{array}$ \\
\hline Eve 160 & 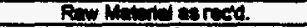 & None & 320 \\
\hline AFTCP & Thar stive & $\operatorname{lom}$ & 33.2 \\
\hline 2000 & ord Ln cl Lun & $\operatorname{Nan}$ & 34.0 \\
\hline Pend $\mathrm{k}$ & 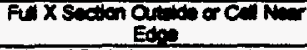 & Nom & 34.5 \\
\hline$\frac{\text { Pen }}{\text { Pen }}$ & 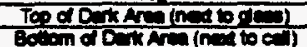 & $\frac{8001}{d x}$ & $\frac{346}{36.6}$ \\
\hline Poners & $\cos x \times \operatorname{sen} 1$ & orr & 3 \\
\hline Peneis & $0+x \times \operatorname{sen}$ & 8 & 339 \\
\hline Pendis & Too ofDin $\times$ sacion & Eving & 335 \\
\hline
\end{tabular}




\author{
Organization: Utility Power Group \\ Los Angeles, California \\ Contributors: $\quad$ G. Duran, Principal Investigator; \\ K. Mackamul, D. Metcalf
}

\title{
Introduction
}

This effort is part of the PVMaT Project, Phase 2A, which addresses problems that are process-specific and are generally unique to a technology. Utility Power Group (UPG) and Advanced Photovoltaic systems, Inc. (APS) are engaged in a unique collaborative effort which integrates the strengths of both organizations, and its implementation will help to ensure that the U.S. PV industry enhances its world leadership role in the commercial development and manufacture of PV systems. The synergistic relationship that exists between UPG and APS is possible due to the similarities of the PV products produced by the two organizations. Both UPG and APS utilize glass superstrates as the structural base for their PV modules, both have chosen amorphous silicon as the semiconductor material in the PV cell, and both have demonstrated the manufacturability and reliability of their PV products in an electric utility setting as demonstrated by the Photovoltaics for utility Scale Applications (PVUSA) project. Many of the innovations developed by UPG for its PVUSA Emerging Module Technology (EMT) array were subsequently incorporated by APS into their PVUSA Utility scale (US) system. The innovations in manufacturing technology of UPG coupled with the large capacity manufacturing facility of APS serve to form the basis for this collaborative PVMaT effort.

\section{Objectives}

The objectives of this effort over its (3) three year duration are to significantly advance the PV manufacturing technologies; reduce module production costs; increase average module performance; and increase PV module production capacity.

\section{Approach}

UPG has concentrated its efforts on research in the area of encapsulation with consideration given to approaches that do not require a second glass plate, and on automation of electrical termination. 
Utility Power Group has evaluated the advanced substitute materials and processes for thin-film module encapsulation identified in the first year of this effort. All materials were evaluated in terms of the level of environmental protection provided to the thin-film module, manufacturing cost reduction, and PV system related factors such as the effect on module weight and module mounting within a panel or sub-array. The advanced encapsulation material is composed of silicone structural adhesive with the addition of glass beads for enhanced mechanical strength. UPG has designed and debugged an automated encapsulation station utilizing the advanced pricessing method and material identified earlier.

UPG has applied the advanced encapsulation material to prototype modules utilizing tempered substrate glass for qualification (NREL/TR-213-3624) testing. In addition, UPG has investigated the use of alternative materials as PV module superstrates which will not require heat tempering.

The goal of this task was to obtain a cost reduction of $50 \%$ in the UPG encapsulation process. UPG has significantly improved upon that goal by demonstrating an encapsulation system which is $72 \%$ lower in cost than the previous glass/EVA/glass system.

\section{POWERGLASS Module Termination}

UPG has optimized the materials and processes utilized in the electrical termination of the POWERGLASS modules. All terminal components have been designed for use with automated insertion and assembly equipment. The terminal design has been analyzed in terms of manufacturing cost reduction. UPG has designed and debugged an automated termination station for the application of the advanced termination systems designed in the first year of this effort.

The goal of this task was to yield an optimized termination system for the POWERGLASS module capable of demonstrating a cost reduction of $70 \%$ in the UPG termination process. UPG has improved upon this goal through the realization of an $81 \%$ reduction in termination cost.

\section{REFERENCES}

1. Annual Subcontract Report, 1 May 1992 - 30 April 1993, NREL Subcontract No. ZM-2-11040-06 (1994).

2. Semiannual Subcontract Report, 1 May 1993 - 31 October 1993 , NREL Subcontract No. ZM-2-11040-06 (1994).

3. G. Duran, K. Mackamul, D. Metcalf, "Progress in Thin-Film Module Encapsulation Design and Reliability," presented at NREL Photovoltaic Performance and Reliability Workshop, Denver, CO, September 1994. 


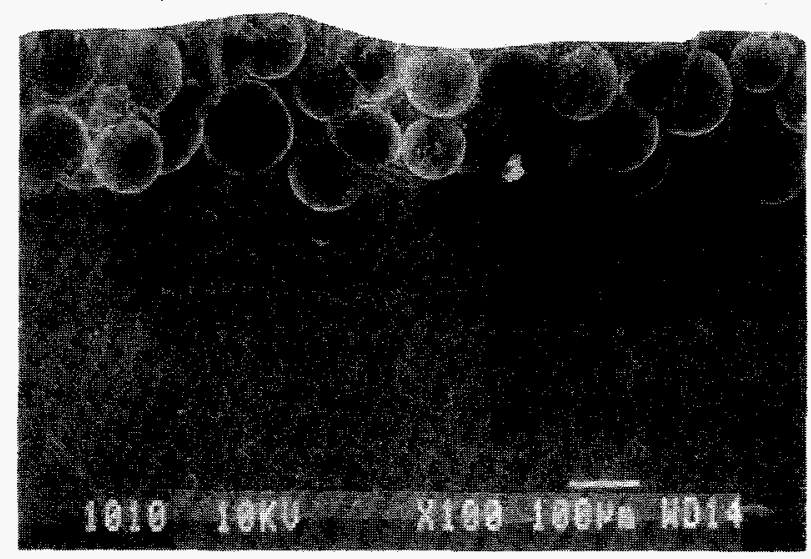

Figure 1. SEM photo illustrating glass beads embedded in the silicone adhesive.
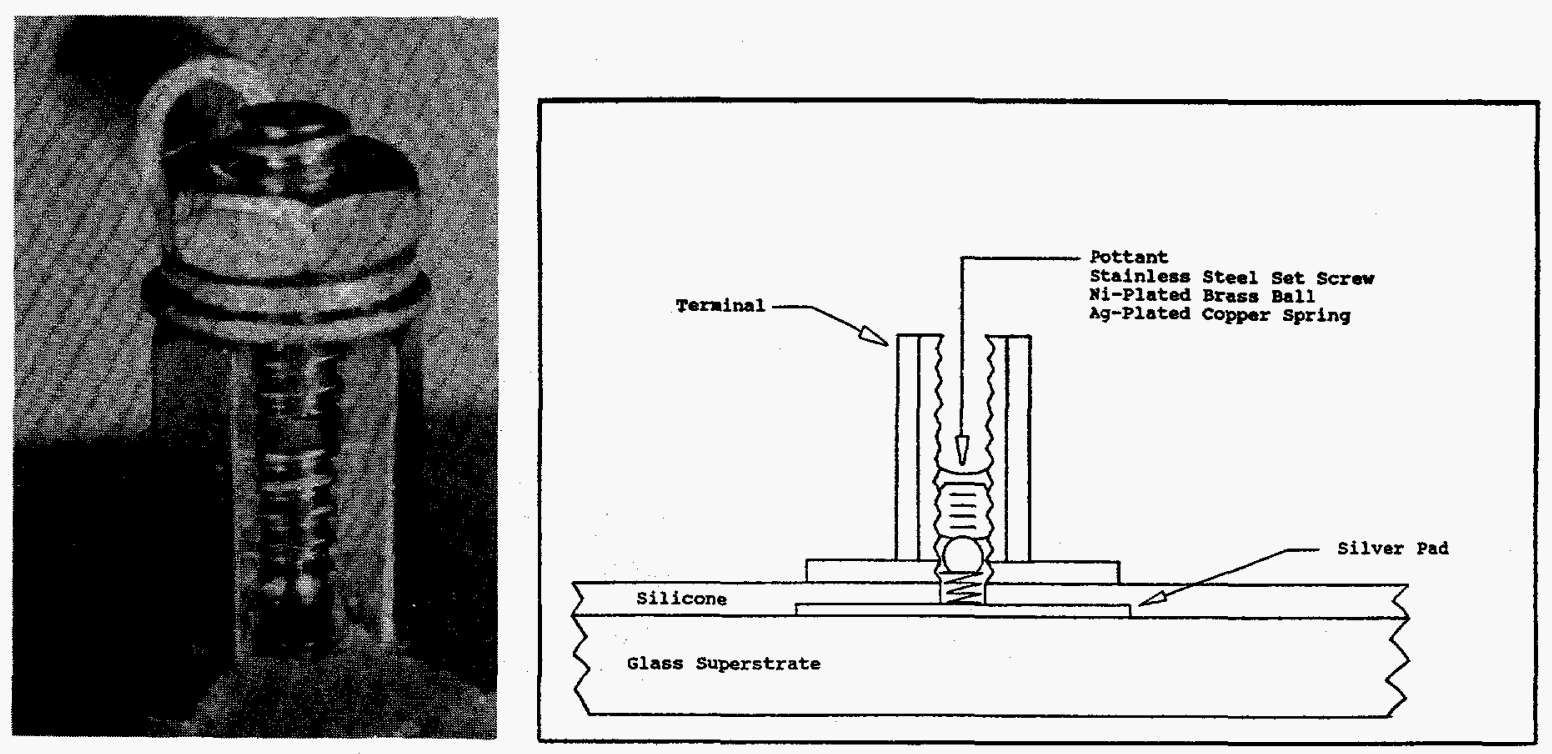

Figure 2. Advanced terminal system. 


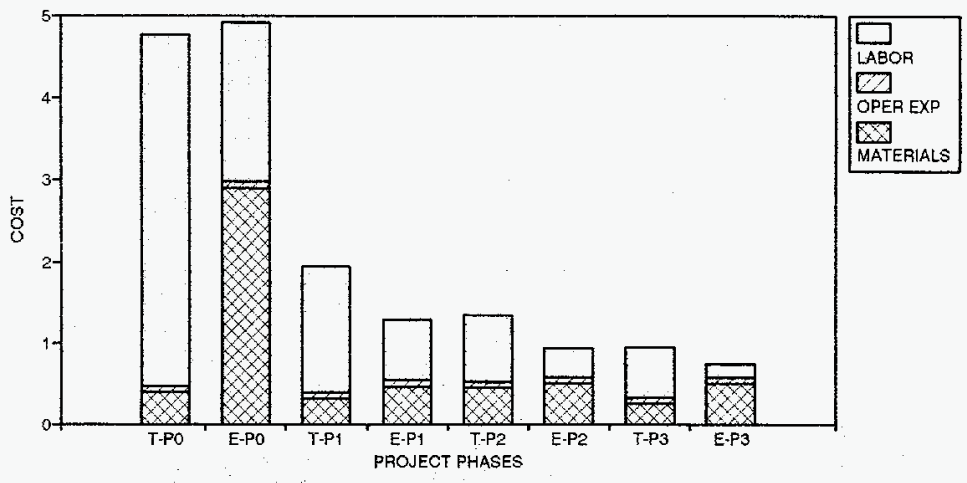

Figure 3. Termination and encapsulation cost reductions broken down by process (Termination or Encapsulation); Phases zero (P0), $1(\mathrm{P} 1), 2(\mathrm{P} 2)$, and 3 (P3); and cost component (naterials, operating expenses, and direct labor). Phase zero represents the Pre-PVMaT period.

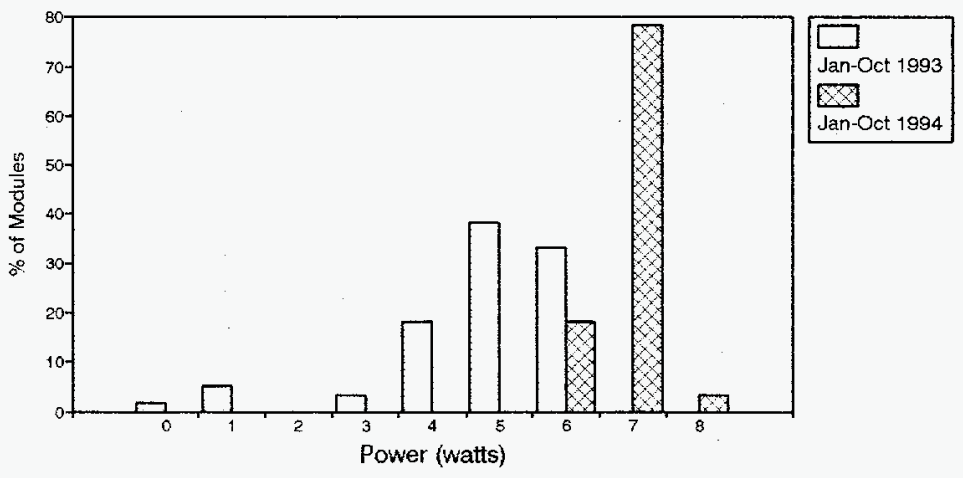

Figure 4. Electrical distribution improvement is a result of both an increase in the unit area conversion efficiency and the tightening of the yield in the manufacturing process.

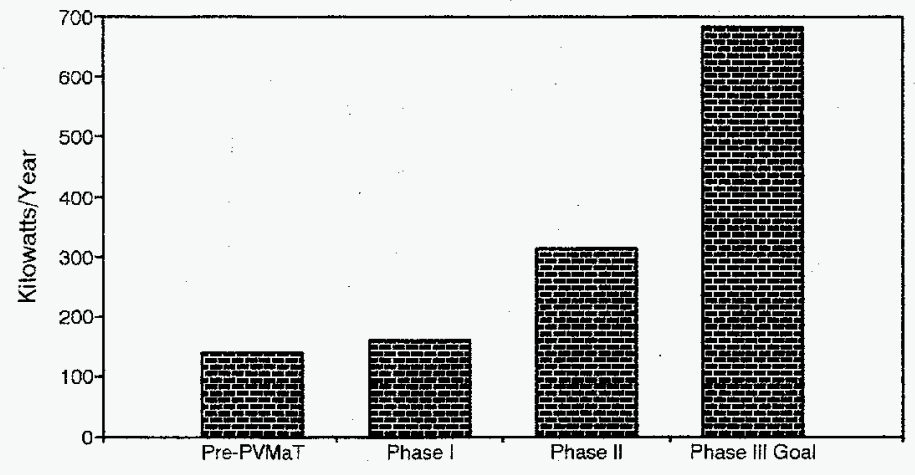

Figure 5. With the increase in throughput, and the module performance enhancement, the annual capacity of UPG'S manufacturing line will increase from $140 \mathrm{~kW}$ for the Pre-PVMaT process to 680kW for the new POWERGLASS process developed through the PVMaT Project. 


\title{
5.0 PHOTOVOLTAIC MODULE AND SYSTEM PERFORMANCE \\ AND ENGINEERING PROJECT
}

\author{
Richard DeBlasio, Manager
}

The objective of this project is to conduct module and systems development through state-of-theart PV module and system research, engineering, testing, evaluation, and analysis tasks. This includes providing technical results, solutions to technical issues, technical assistance, and coordination for developing PV standards, codes, and criteria for test and evaluation procedures. The objective further includes maintaining and enhancing supporting facilities and capabilities that are consistent with the DOE National Photovoltaic Program, complementary to other DOE PV projects, and that will ensure project capabilities and facilities are available resources for cooperative research and use by the U.S. PV R\&D community.

\section{Technical Approach}

The project primarily includes NREL in-house activities and a small number of subcontracts. The project is organized under the primary areas shown in Table 5-1. Each group supports PV standards and codes activities.

\begin{tabular}{|l|l|l|}
\hline \multicolumn{3}{|c|}{ Table 5-1. Photovoltaic Module and System Performance and Engineering } \\
Project Contacts (R. DeBlasio, Manager, 303-275-3786)
\end{tabular}


Photovoltaic Efficiency Measurements-Standard Reporting Conditions. The performance of PV devices of all sizes and technologies is evaluated by providing secondary calibrations of PV cells, efficiency measurements with respect to standard reporting conditions, efficiency verification of subcontract deliverables, and current-voltage (I-V) measurements under varying temperature, spectral irradiance, and total irradiance. Capabilities also support the entire PV community by providing information on PV measurement equipment and systems appropriate for the end user, I-V measurement procedures, and measurement uncertainty analysis, and also being committed to obtaining the lowest possible uncertainty in the measurement of the standardized PV performance of single- and multi-junction cells and modules. Included in the uncertainty analysis are the determination of potential artifacts in the I-V results because of equipment or procedures and the realistic estimates of the elemental error sources.

Photovoltaic Module Materials and Encapsulation Research focuses on polymeric pottant materials, building on past successes such as identifying the causes of failure or change in ethylene vinyl acetate that limit the service life or reduce the performance of PV modules. Ongoing focus is directed at developing and demonstrating new or improved pottant materials, documenting degradation and failure mechanisms that can be used to guide research to identify improved materials, and understanding PV-specific compatibility and durability intrinsic problems at material interfaces in cells/modules. The technical approach consists of conducting experimental work, interpreting the results, establishing test methods, and seeking solutions in concert with industry. Developing and implementing subcontracts furthers this work.

Photovoltaic Module Qualification Testing and Test Method Development. This approach seeks to investigate, understand, and improve PV module long-term performance and reliability, including developing test methods and procedures and performing module exploratory/qualification tests on all commercially available PV modules and on R\&D prototypes and contract deliverables, with special emphasis on thin-film PV technologies. The team is working to establish a correlation between indoor (artificial, accelerated) test results and outdoor (natural, real-time) exposure as related to reliability/stability/weathering issues. In addition, theoretical and experimental studies are undertaken on PV module lifetime prediction from accelerated testing. Further, the work with the NREL failure analysis group is aimed at developing methods and techniques to perform failure analysis on PV modules, establishing means of determining the mechanisms of failure, and obtaining possible solutions to these module performance and reliability problems.

Photovoltaic Component Diagnostics and Failure Analysis is concerned with conducting and documenting module and system reliability performance. Data are obtained from operating systems, demonstration or prototype units, qualification and exploratory tests, research results, dedicated studies, and the literature. Dedicated activities are pursued to uncover and reproduce identified failure mechanisms, validate proposed hypotheses, explore areas that are believed to be of high priority for long-term PV reliability, recommend new tests for screening or qualification, and develop better understanding for improved reliability and design. The cause of PV module failure is determined through nondestructive and destructive evaluation procedures. From these evaluations, more basic materials determinations can be confirmed and probable causes and mechanisms proposed. System-level concerns are addressed, with emphasis extending outward from the module interface. 
Photovoltaic Module and System Technology Validation encompasses activities for monitoring and evaluating state-of-the-art and emerging PV technologies on the module and system level. Technology development and validation are also supported by analyzing the performance and reliability of PV modules and systems in support of the DOE National Photovoltaic Program. The approach includes maintaining a data base on performance and reliability, performing thinfilm module stability testing outdoors with comparison to indoor light-soak experiments, and researching and developing performance evaluation methodologies for modules (for example, seasonal performance, spectral measurements, and techniques for evaluating modules on instantaneous power output and energy production). System performance activities also include supporting the PV for Utility-Scale Applications (PVUSA) project, Niagara Mohawk Power Company, the U.S.-Brazil electrification projects (for example, providing a technical review committee member and additional technical support), PVMaT and PV:BONUS projects, and managing the Amorphous Silicon Utility/Industry PV Power project that develops and performs qualification testing on a-Si modules and ultimately deploys and monitors systems in the field at various locations throughout the country.

Photovoltaics Solar Radiation Research directly supports characterizing, measuring, testing, designing, and understanding the performance of PV cells, submodules, modules, and systems. These activities are pursued by providing scientific and engineering understanding of incident solar and artificial irradiance, and by developing instruments and measurement methods. The approach focuses on providing integrated technical support (for example, developing and providing broadband and spectral radiometric measurements and instrument calibrations, characterization, and use to assist PV performance measurements). The approach includes operating reference radiometric sensors, calibrating solar simulators, and developing and operating a reference irradiance and meteorological station at NREL.

Photovoltaics Standards and Codes focuses on coordinating the consensus development of PV standards, codes, and criteria for test and evaluation procedures. For example, NREL holds an annual PV Standards and Codes Forum. The coordination includes participating and performing major leadership roles in the IEEE Standards Coordinating Committee 21 and in the ASTM Subcommittee E44.09, conducting round-robin cell and module test method verification, developing criteria for module certification and test-facility accreditation through the PV community with balanced industry representation and subcontract support, revising the National Electrical Code Article 690, developing PV energy rating standards, and working in the international arena (for example, through the NREL-managed Solar Energy Industries Association [SEIA] subcontract for International Electrotechnic Committee TC-82 secretariat and U.S. Technical Advisory Group coordination of international PV standards development). 


\section{PV Module and System Performance \& Engineering In-House Research}



Title:

Organization:

Contributors:
PV Efficiency Measurements - Standard Reporting Conditions

\author{
NREL PV Module and Systems Performance and \\ Engineering Project
}

K.A. Emery team leader; S. Rummel, Y. Caiyem

H. Field, D. Dunlavy

\section{Objectives}

The performance of photovoltaic devices of all sizes and technologies are evaluated by this activity. This team supports the entire photovoltaic community by providing: secondary calibrations of photovoltaic cells, efficiency measurements with respect to a given set of standard reporting conditions, efficiency verification of contract deliverables, current versus voltage (I-V) measurements under varying temperature, spectral irradiance and total irradiance. Support is provided for in-house programs in device fabrication, module stability, module reliability, PV systems, and alternative rating methods by performing baseline testing, specialized measurements and other assistance when required. This activity also supports the entire PV community by providing information on: PV measurement equipment and systems that are appropriate for the end user, I-V measurement procedures, and uncertainty analysis. Included in the uncertainty analysis are the determination of potential artifacts in the I-V results because of equipment or procedures, and realistic estimates of the elemental error sources. This activity is committed to obtaining the lowest possible uncertainty in the measurement of the standardized PV performance of single- and multijunction cell and modules.

\section{Technical Approach}

The photovoltaic current versus voltage characteristics are measured with respect to standard terrestrial reporting conditions $\left(25^{\circ} \mathrm{C}\right.$ temperature, $1000 \mathrm{Wm}^{-2}$ total irradiance and ASTM E892 global reference spectrum). The intensity of the Spectrolab X-25 solar simulator ( $30 \mathrm{~cm}$ square beam) is adjusted until the measured short-circuit current of a reference cell is equal to it's calibration value corrected for spectral mismatch. The current versus voltage characteristics are then measured using 4-terminal Kelvin connections to the PV device with a custom data acquisition system designed to give a random error of less than $\pm 0.1 \%$ and a non random error of less than $\pm 1 \%$. The I-V system has a voltage range of $\pm 50 \mathrm{~V}(0.1 \mu \mathrm{V}$ resolution) and $\pm 8 \mathrm{~A}$ to $\pm 1 \mathrm{pA}$. These procedures have been shown to be valid for any given tabular reference spectrum including AM0 and the ASTM direct normal reference spectrum. Because of the wide current and voltage range the system is also used for concentrator and dark I-V measurements. For two-terminal multi-junction devices the spectrum of the Spectrolab model X25 solar simulator is adjusted, using a special filter plate developed at NREL, until each junction is producing the correct photo-current. In the limit as the number of junctions becomes 1 the multi-junction and single-junction measurement procedures are identical. The I-V system is also used for examining the effects of maximum power versus illumination time, Voc vs. time, and Isc vs. time on the PV performance. This system is also used to examine hysteresis related artifacts.

Two spectral response measurement systems have been developed The filter spectral response system uses periodic $(440 \mathrm{~Hz})$ monochromatic light directed through one of 5110 $\mathrm{nm}$ bandwidth interference filters covering the spectral range from 290 to $1900 \mathrm{~nm}$. The system is capable of providing steady-state light bias levels up to $2 \mathrm{~A}$ and voltage bias levels 
from $\pm 1 \mathrm{mV}$ to $\pm 40 \mathrm{~V}$. The intensity of the bias light is normally adjusted to give the shortcircuit current under standard reporting conditions. The custom operational amplifier based data acquisition system allows for a wide range of gains $\left(1\right.$ to $\left.10^{6}\right)$. The intense light from the $1000 \mathrm{~W}$ lamp illuminating the $5 \mathrm{~cm}$ diameter filters allows for small cells to large modules to be illuminated with adequate signal to noise. The uncertainty in the relative spectral response as a function of wavelength is less than $\pm 5 \%$. The data can be made absolute by forcing the integrated quantum efficiency to agree with the measured spectral response. The absolute spectral response can also be measured directly with this system giving an uncertainty of $\pm 10 \%$. The limiting factor in the uncertainty is the $\pm 5-10 \%$ spatial nonuniformity of the monochromatic beam. The second grating monochrometer based system has a wavelength range from $300-10,000 \mathrm{~nm}$ with a $1 \mathrm{~nm}$ wavelength resolution and $\sim 5 \mathrm{~nm}$ bandwidth. The grating system was designed for accurate absolute spectral response measurements by illuminating a small $1 \mathrm{~mm}$ by $3 \mathrm{~mm}$ rectangular area and measuring the power of the entire beam. The system uses all reflective optics so chromatic aberrations and beam wander with wavelength are not present. The grating system uses an operational amplifier for the current-to-voltage conversion allowing a $\pm 15 \mathrm{~V}$ voltage bias range with $0 \pm 1$ $\mathrm{mV}$ being the normal configuration. A lock-in amplifier is used to detect the ac signal. The system is capable of broadband or filtered light bias for multi-junction or nonlinear devices. Both systems rely on accurate pyroelectric detectors for the measurement of the light power.

The efficiency versus concentration measurements are measured with an unfiltered $1000 \mathrm{~W}$ $\mathrm{Xe}$-arc light source that is focused to a small area. and the one-sun data acquisition system described above. Using the cell's Isc measured at one sun (ASTM E891-87 direct normal reference spectrum at a total 1-sun irradiance of $1000 \mathrm{Wm}^{-2}$ ) and assuming linearity in the current with total irrariance, the I-V characteristics are measured. Using a technique developed at NREL the temperature of the space charge region temperature can be accurately set to a given value even though large temperature gradients $\left(>10^{\circ} \mathrm{C}\right)$ may exist between the plate temperature and junction temperature. The procedure involves setting the sample temperature to the reference temperature e.g. $25^{\circ} \mathrm{C}$ without illumination (no heat load). Using a high speed shutter and voltmeter the open-circuit voltage is sampled (1000 readings / sec). The highest measured Voc is then taken to be the Voc under concentration. The thermoelectrically controlled vacuum plate is then cooled until this Voc is reached. This same procedure is used in the Spectrolab X25 system for samples on thermally insulating substrates where temperature gradients of $5-10^{\circ} \mathrm{C}$ are typical.

All the efficiency measurements for the group are traceable to calibrated reference cells. The primary calibration procedure involves measuring the short-circuit current, total irradiance, and spectral irradiance at the same time outdoors with the same field of view. The total irradiance is measured with a primary absolute cavity radiometer with a $5.00^{\circ}$ field of view, and the spectral irradiance is measured with a LICOR LI-1800 spectroradiometer with a $5.00^{\circ}$ field of view. The uncorrected average calibration value $\langle\mathrm{CVu}\rangle$ is calculated for the 30 Isc and Etot readings taken during the 30 seconds required to measure $\operatorname{Es}(\lambda)$. The atmospheric constraint for a valid calibration value is that the range (maximum to minimum) over the 30 second time period in Isc be less than $0.25 \%$, in Etot be less than $0.07 \%$, and in $\langle\mathrm{CVu}\rangle$ be less than $0.1 \%$. Once a valid $\langle\mathrm{CVu}\rangle$ is obtained the short-circuit current is corrected for temperature to $25^{\circ} \mathrm{C}$ and the spectrally corrected calibration value $\mathrm{CV}$ is computed. Since the measurement of $\operatorname{Es}(\lambda)$ does not encompass the limits of the reference spectrum, the measured spectrum is extended using a computer model developed by the group to encompass the range of the reference spectrum $(300-4000 \mathrm{~nm})$. The calibration 
value $\mathrm{CV}$ is computed at least 20 times for at least 3 separate days giving a single primary calibration value. This procedure has been shown to have a total uncertainty of less than $\pm 1 \%$ by rigorous uncertainty analysis, intercomparison with primary AMO standards and other intercomparisons.

The I-V characteristics of modules are routinely evaluated using the Spire $240 \mathrm{~A}$ solar Simulator. This system has a $0-100 \mathrm{~V}$ and $0-20 \mathrm{~A}$ range for a 61 by $122 \mathrm{~cm}$ area. The Spectrolab Large Area Pulsed Solar Simulator (LAPSS) produces a one-sun beam of light ( $2 \mathrm{~m}$ by $2 \mathrm{~m}$ area and could illuminate a $4 \mathrm{~m}$, by $4 \mathrm{~m}$ area). The custom $\mathrm{I}-\mathrm{V}$ system of the LAPSS has 3 modes of operation: capacitive sweep from Isc towards Voc, current at a fixed power supply voltage, and bi-directional sweeping over a user defined or automatically determined voltage range. The system is designed to handle $\pm 200 \mathrm{~V}$ maximum ( $\mu \mathrm{V}$ minimum), $\pm 30 \mathrm{~A}$ maximum $(0.01 \mathrm{~mA}$ minimum) with 10 voltage and current ranges to ensure accuracy. A commercial data acquisition system has been purchased that will greatly enhance the systems availability and reliability and is expected to arrive in mid FY95. The concentrator lamp housing allows measurement to 2000 suns.

During FY94 standardized Outdoor module I-V measurements were performed on a variety of one-sun and concentrator modules using one of two fixed-tilt platforms or a 2-axis Martin Marietta Tracker. For flat-plate modules the total irradiance was measured with an Eppley pyranometer and a crystal-Si reference cell in a module package mounted in the plane-ofarray. A normal incidence pyrheliometer calibrated against NREL's cavity radiometers is used to measure the direct normal irradiance for concentrator modules. The module temperature is measured with a platinum RTD temperature sensor attached to the back surface of the module. The spectral irradiance is measured during the I-V measurement using a LICOR LI-1800 spectroradiometer with a Teflon dome and temperature controlled detector. Meteorological parameters at the time of measurement including direct to diffuse ratio, wind speed, wind direction, barometric pressure, air temperature and relative humidity are stored with the data. The outdoor I-V measurement system is capable of handling devices with a voltage and current range of $\pm 100 \mathrm{~V}$ and $\pm 40 \mathrm{~A}$ maximum to $\pm 0.01 \mathrm{mV}$ and $\pm 1 \mu \mathrm{A}$ minimum. A broad band spectroradiometer $(300-2000 \mathrm{~nm})$ that will be dedicated to this system is being evaluated and will be installed in early FY95.

\section{Results}

During FY94 the team calibrated 1253 cells (I-V under standard reporting conditions), and performed over 1745 module measurements on the SPIRE 240A solar simulator (1009 module measurements) and outdoors under clear sky prevailing conditions (total irradiance > $950 \mathrm{~W} / \mathrm{m}^{2}$ ). These measurements were on representative cells and modules of all PV technologies being investigated at NREL, in the US and worldwide. Approximately 40 papers acknowledged the support of this group during FY94. This group has assisted a variety of module manufactures with their QA/QC programs by providing telephone consultations, and calibration tractability through $\mathrm{I}-\mathrm{V}$ and spectral response measurements on their cells and modules. The move of the cell measurement activities from building 16 to SERF was completed with a minimum of disruption to customers (delays of less than 2 weeks). All of the laboratory software has been removed from the HP1000 and is currently operational on a networked Macintosh based computer system using Labview with some specialized code written in Pascal.

\section{Publications}


1. L.L. Kazmerski and K.A. Emery, "Photovoltaic Technologies: Evaluation and Prospects," 4th Arab International Solar Energy Conference, Nov. 20-25, 1993, Amman Jordan.

2. M.A. Green and K. Emery, "Short Communication: Solar Cell Efficiency Tables (version 3), Progress in Photovoltaics Research and Applications, January 1994, Vol. 2, pp. $27-$ 34, 1994.

3. R.J. Matson, K.A. Emery, I.L. Eisgruber, and L.L. Kazmerski, "The Large-Scale Laser Scanner: Milli-Characterization of Photovoltaic Drvices and Modules," Proc. 12th European PV Solar Energy Conf., April 11-15, 19,4.

4. R.K. Ahrenkiel, B.M. Keyes, D.L. Levi, K. Emery, T.L. Chu, and S.S. Chu, Appl. Phys. Lett. vol. 64, pp. 2879-2881, 1994.

5. K. Emery, "Photovoltaic Efficiency Measurements," June 1994, MRS Bulletin, pp. 64-66.

6. K. Emery, "Performance Characterization of Thermophotovoltaic Cells," Proc. The First NREL Conference on Thermophotovoltaic Generation of Electricity, Copper Mountain, CO, July 24-28, 1994, AIP Conference proceedings 321.

7. M.A. Green and K. Emery, "Short Communication: Solar Cell Efficiency Tables (version 4), Progress in Photovoltaics Research and Applications, July 1994, Vol. 2, pp. 231 234, 1994.

8. K. Emery, "Measurement Artifacts," proc. Photovoltaic Performance and Reliability Workshop, Lakewood, CO, September 21-23, 1994.

9. H. Field and K. Emery, "Measurements of CdTe and CIS Solar Cells and Modules Exhibiting Depending on Premeasurement Conditions," proc. Photovoltaic Performance and Reliability Workshop, Lakewood, CO, September 21-23, 1994.

10. K. Emery and H. Field, "Artificial Enhancements and Reductions in the PV Efficiency," proc. 1st World Conference on Photovoltaic Energy Conversion, Waikoloa, HW December $5-9,1994$.

11. T. Strand, L. Mrig, R. Hansen, and K. Emery, "Technical Evaluation of a Dual-Junction Same-Band-Gap Amorphous Silicon Photovoltaic System at NREL," proc. 1st World Conference on Photovoltaic Energy Conversion, Waikoloa, HW, December 5 -9, 1994.

12. B. Kroposki, K. Emery, D. Myers, and L. Mrig, "A Comparison of Photovoltaic Module Performance Evaluation Methodologies for Energy Ratings," proc. 1st World Conference on Photovoltaic Energy Conversion, Waikoloa, HW, December 5 -9, 1994.

13. S. Kurtz, K. Emery, and J. Olson, "Methods for Analysis of Two-Junction, Two-Terminal Photovoltaic Devices," proc. 1st World Conference on Photovoltaic Energy Conversion, Waikoloa, HW, December 5 -9, 1994.

14. M. Wanlass, J. Ward, K. Emery, T. Coutts, "GaxIn1-xAs Thermophotovoltaic Converters," proc. 1st World Conference on Photovoltaic Energy Conversion, Waikoloa, HW, December 5 -9, 1994.

15. R. Venkatasbramanian, M. Timmons, J. Hutchby, H. Field, and K. Emery, "GaAs and Al0.2 Ga0.8As Solar cells with an Indirect-Bandgap Al0.8Ga0.2As Emitter; Heterojunction Cells - A Novel Approach to Higher performance?," proc. 1st World Conference on Photovoltaic Energy Conversion, Waikoloa, HW, December 5 -9, 1994.

16. I. Eisgruber, R. Matson, J. Sites, K. Emery, "Interpretation of Laser Scans from ThinFilm Polycrystalline Photovoltaic Modules," proc. 1st World Conference on Photovoltaic Energy Conversion, Waikoloa, HW, December 5 -9, 1994.

17. M.W. Wanlass, J.S. Ward, K.A. Emery, and T.J. Coutts, "Improved, Large-Area, TwoTerminal InP/Ga0.47In0.53As Tandem Solar Cells," proc. 1st World Conference on Photovoltaic Energy Conversion, Waikoloa, HW, December 5 -9; 1994. 
Title:

Organization:

Contributors:
PV Module Materials and Encapsulation Research

PV Module and System Performance and Engineering Project NREL PV Program

A.W. Czanderna, Leader; F. J. Pern, S. H. Glick, J. D. Webb

\section{Objectives:}

The goals of the task are to develop and demonstrate innovative flat plat PV module encapsulation materials that will provide optimum module performance and protection from the environment for a 30-year operational life in the field. The long term objectives are (1) to identify, understand, and then mitigate the causes of changes in module materials that alter crucial materials properties, reduce the performance, and/or limit the service life of the module; and (2) to develop new or improved materials that offer greater promise for a module life expectancy of 30 years.

The objectives for the task during FY 1994 and FY 1995 are to (a) identify, test and screen new or improved polymeric materials and formulations that offer great promise than ethylene vinyl acetate (EVA) copolymer for a module life expectancy of 30 years and meet low cost goals, (b) use accelerated and real-time life testing to narrow the potential candidate materials for larger scale evaluation, (c) develop a data analysis method (procedure) to correlate the results from various spectrophotometric measurements (FA, UV-vis, and color index) for ALT-degraded polymers, (d) identify and test various superstrate materials (polymers and glass) for improving the EVA photostability, and (e) test commercially available adhesive films for simplifying the PV module encapsulation process.

\section{Technical Approach:}

The five objectives are being addressed primarily for contemporary thin film and crystalline silicon flat plat PV module technologies. The technical approach consists of conducting experimental work, interpreting the results, establishing test methods, and seeking solutions to module reliability and materials durability problems. The background and problems associated with EVA degradation in PV modules have been summarized.1,2 Summaries of FY 1993 progress are available. 1,3

The generic module materials durability studies are being performed primarily on polymeric pottant materials using the recent results obtained for degraded ethylene vinyl acetate (EVA). Efforts to modify the present formulation of EVA with the optimimum concentratations of Cyasorb UV 531, Tinuvin 770, and Naugard P and with improved UV and thermal stabilizers are being continued and the modified materials are being ranked from accelerated life testing (ALT) against the existing formulations (A9918, 15295, and 15295P), all of which use Cyasorb UV 531. The modified formulations that rank the best versus A9918 and/or 15295 and equivalents will be prepared in sufficient quantity for more quantitative ALT and deployment for real time testing in minimodules. In addition, samples are being obtained for EVA, commercial variations of EVA, DuPont Butacite and Vamac, ethylene methyl acrylate (EMA), modified polymethylmethacrylate (PMMA), and other candidate pottants, and will be studied as separate materials, materials in contact with other module 
components, and in complete mini-modules. The materials are being subjected to both real time testing using field deployment and ALT using DSET Suntest units, Oriel solar simulators, and other existing light sources for test-size specimens. Designed experiments are being used to separate mechanisms thought to include UV, T, and metal ion catalyzed effects.

The degradation will be and is being monitored from characterization measurements made before, during, and after testing, and these emphasize UV-vis, fluorescence analysis, spectrocolorimetry (yellowness index), HPLC, and gel content. Additional cheracterization that is made as deemed appropriate include microgravimetric, FT-IR, GPC, XPS, TGA, conductivity, SIMS, and LCR measurements. As a lower priority than for the new or modified materials studies, the same characterization measurements will be made on field degraded EVA secured from and in collaboration with ASE Americas, Siemens Solar Industries, Solarex, USSC, SWTDI, Ben Gurion University in Israel, and other industrial partners requesting assistance. Non-destructive measurements of degradation that can be used in the field will be sought. Degradation mechanisms will be identified to assist in selecting new or modified materials options. The mechanisms will eventually be necessary for projecting module service lifetime.

Efforts are being continued to identify an appropriate low-cost cover glass or polymer (e.g., modified Tefzel and/or PMMA) that will serve as an effective UV screen for EVA. UV-vis measurements will be made for the candidate materials, which will be laminated with EVA and added to our ALT matrix of experiments. The potential will be assessed for using selfassembled monolayers in surface modification schemes to inhibit degradative reactions, particularly, at metalization/polymer interfaces. Efforts also will be continued to conduct microscopic failure analyses on defective PV USA modules.

\section{Results: Degradation of EVA in Crystalline Silicon Modules}

Modified EVA and Potential EVA Replacements: We have identified the critical concentration ratios among the UV absorber (Cyasorb UV 531), UV light stabilizer (Tinuvin 770), and thermal anti-oxidant (Naugard P) in the EVA formulation that showed, when tested in the form of thin films, an improvement factor of $\sim 4$ for stabilizing the UV absorber (Cyasorb UV 531) against photodecomposition. ${ }^{4}$ We used 3-D and 2-D contour graphical analysis to determine the optimal concentration ratios. The optimized modified EVA formulation is currently being tested further. We have also identified several combinations of stabilizers other than Tinuvin 770 and/or Naugard P that showed when tested in the form of thin films, an improvement factor ranging from 4.2 to 14.5 for stabilizing Cyasorb UV 531 against photodecomposition. The new EVA formulations are currently being tested as thin films and laminated thick films in combination with a modified curing condition.

Degradation Mechanisms: Summaries of the known degradation mechanisms are available. 2,5,6 We have determined the significance of the curing agent (Lupersol TBEC vs. Lupersol 101) used in the EVA formulation by showing that the fast-curing Lupersol TBEC produces considerably less UV-excitable chromophores, which in turn results in a much slower Cyasorb photodepletion and EVA discoloration. As a result of our extensive studies of the faster discoloration of the EVA A9918 and 15295 formulations, we recommended that the PV industry will benefit by using the fast-cure, EVA 15295 formulation in preference to A9918.7 Although the 15295 EVA discolors and degrades 8 by the same mechanism as 
A9918 $\mathrm{EVA}^{6}{ }^{6}$ it does so at a slower rate, as our studies have shown during this past year. ${ }^{7}$ We have also reproduced for the first time in accelerated exposure tests a discoloration pattern of laminated EVA that is nearly identical to that observed for the EVA on fieldweathered PV modules.

Polymers to Replace EVA: Efforts and studies were initiated for identifying new polymeric materials for replacing EVA. The new materials are polyacrylic or methacrylic polymers. Testing was initiated to identify reliable formulations with various stabilizers including the identification and use of suitable cross-linking agent(s) for optimal curing of these polymers. We also monitored a related subcontract by $\mathrm{C}$. Bowman that is summarized at the end of this Project Summary. We initiated studies to investigate (1) the activation spectrum of the EVA copolymer, (2) the stabilizing-discoloring effects of individual and mixtures of the stabilizers used in the EVA, and (3) the discoloring effects of various metal ions on EVA. All studies are presently on-going.

Data Analysis: We have established a method (procedure) for handling the large volume of data generated from transmission, color index, and fluorescence measurements. We demonstrated that the discoloration of laminated EVA A9918 follows a sigmoidal pattern regardless of the light intensity in the accelerated exposure test. Correlation studies of this data analysis method with solar cell efficiency losses are planned for FY1995 to establish preliminary information that will be necessary in any model for service lifetime prediction.

Efficiency Losses: We have continued or initiated several projects to monitor the efficiency losses from yellowed EVA in modules or minimodules. Details are available in reports from SWDTI ${ }^{9}$ and NREL. 1,7

Equipment: Additional laboratory equipment was purchased, installed, and used; new procedures for polymer thin film preparation, testing, and analysis were developed; and some existing equipment was repaired. We installed a HunterLab Ultra Scan Spectrocolorimeter that has been used extensively in various studies on EVA discoloration. We also initiated and completed the purchase of a custom-made, vacuum-bag laminator for minimodules that was delivered in October, 1994, by AstroPower, Inc. The laminator expands our capabilities and activities for testing/optimizing encapsulants, superstrates, lamination/curing conditions, and novel encapsulation methods. Finally, we prepared specifications and ordered a custommade Weatherometer that will permit IV measurements to be made on minimodules during the exposure of samples to $3 x$ xenon, UV, $\mathrm{T}$ to $100^{\circ} \mathrm{C}$, and R.H. to $95 \%$. We also prepared specifications and requests for quotes to assemble and reduce to practice a degradation monitor that is based on our prior fluorescence analysis work. 11

Industrial Interactions: We have interacted extensively with over 30 personnel from the PV and chemical industries to exchange information and/or secure samples of various polymer encapsulant candidates and glass superstrate materials for improving EVA photostability.

Other Activities: An invention disclosure was filed on June 7, 1994, as a very satisfying result of testing a commercial adhesive tape for use in encapsulating solar cells. We contributed parts for a chapter entitled, Polymers for Solar Energy Devices, ${ }^{12}$ and filed a patent application related to stabilizing interfaces using organized molecular assemblies. ${ }^{13} \mathrm{We}$ also assisted in editing a book on polymer/inorganic interfaces. 14 


\section{References}

1. A. W. Czanderna, Current Status of EVA Degradation in Si Modules and Interface Stability in CdTe/CdS Modules, in R. Noufi and H. Ullal, eds., 12th NREL Photovoltaics Program Review, AIP Conference Proceedings, Vol. 306, Am. Inst. Physics, Woodbury, NY, 1994, pp. 147-155, NREL/TP-412-5997, October, 1993.

2. A.W. Czanderna, Conference Record of Photovoltaic Performance and Reliability Workshop, NREL/CP-410-6033, L. Mrig, ed., (National Renewable Energy Laboratory, Golden, CO, October, 1993) pp. 311-357.

3 F.J. Pern, Proc. 23rd PVSC, Louisville, KY, IEEE, NY, 1993, pp. 1113-18, NREL/TP412-5495; F.J. Pern and S. Glick, NREL/TP-412-5996, October, 1993.

4. J. Pern, in L. Mrig, ed., Proceedings of a PV Module Reliability Workshop. NREL/CP410-6033, September 8-10, 1993, pp. 358-374.

5. F.J. Pern and A.W. Czanderna, in AIP Conference Proceedings, R. Noufi, ed., 268, Photovoltaic Advanced Research and Development Project, Amer. Inst. Physics, NY, 1992, pp. 445-451.

6. A.W. Czanderna and F.J. Pern, draft document for publication in 1995.

7. F.J. Pern, Conference Record of a Photovoltaic Performance and Reliability Workshop, L. Mrig, ed., (National Renewable Energy Laboratory, Golden, CO, October, 1994) pp. 329-347.

8. D. Faiman, ibid, pp. 289-314.

9. A. Rosenthal and S. Durand, SWTDI; a summary of this subcontracted activity is given at the end of this Project Summary.

10. F.J. Pern, Conference Record of Photovoltaic Performance and Reliability Workshop, SERI/CP-411-5184, L. Mrig, ed., (National Renewable Energy Laboratory, Golden, CO, September, 1992) pp. 327-344.

11. F.J. Pern, Polymer Deg. Stability, 41, 125 (1993); F.J. Pern and S.H. Glick, op. cit. ref.1, pp. 573-585, NREL/TP-412-5996, October, 1993.

12. G. Jorgensen, J. Pern, S. Kelley, A.W. Czanderna, and P. Schissel, in R. Arshady, ed., Functional Polymers for Emerging Technologies. American Chemical Society, Washington, DC, 1995, In Press. (NREL/TP-454-6097, November, 1993).

13. Patent Application: Molecular Assemblies as Protective Barriers and Adhesion Promoter Interlayer, D.E. King, A.W. Czanderna, and C. Kennedy, 6/10/94.

14. Polymer/Inorganic Interfaces, R.L. Opila, F.J. Boerio, and A.W. Czanderna, eds., Symposium Proceedings, Vol. 304, Materials Research Society, Pittsburgh, PA, 1993. 
Organization:

Contributors:

\author{
PV Module and System Performance and Engineering Project \\ NREL PV Program
}

\author{
Joseph Burdick, Team Leader \\ Jim Pruett, Master Technician \\ Elvira Beck, Research Associate
}

\section{Objectives}

The research team objectives are to design, develop and perform tests on photovoltaic modules and interact with the PV industry (manufacturers, test facilities, end-users, etc.) with the intent of helping to improve PV modules in terms of both long-term performance and reliability; to perform exploratory qualification and reliability tests on PV modules in order to evaluate long-term module reliability as well as to validate ASTM and IEEE test methods, procedures, and standards; to establish a correlation between indoor (artificial, accelerated) test results and outdoor (natural, real-time) exposure as related to reliability/ stability/weathering issues; to help identify module failure mechanisms and work to obtain solutions to reliability problems; and to develop methods to help predict PV module service lifetime in the field.

\section{Technical Approach}

Our research team's approach is to investigate, understand, and improve photovoltaic module longterm performance and reliability by developing test methods and procedures and by performing module exploratory/qualification tests on all commercially-available PV modules, as well as on R\&D prototypes and contract deliverables, with special emphasis on thin-film photovoltaic technologies. We are working to establish a correlation between indoor (artificial, accelerated) test results and outdoor (natural, real-time) exposure as related to reliability/stability/weathering issues. In addition, we undertake theoretical and experimental studies on PV module lifetime prediction from accelerated testing. Further, our work with the failure analysis group is an attempt to develop methods and techniques to perform failure analysis on PV modules, establishing means of determining the mechanisms of failure, and then obtaining possible solutions to these module performance and/or reliability problems. Finally, we interact with the PV community through written and verbal communications, reports and publications, to exchange ideas and results with the goal of improving the long-term performance and reliability of photovoltaic modules.

\section{Results}

\section{Module Qualification Testing:}

MQT Sequence Testing: A test plan was developed for testing commercially-available modules and R\&D prototypes from various manufacturers by running them through the complete MQT sequence. The first batch of modules was begun in April, 1994; the second batch will be run when Batch \#1 is completed in early 1995. The test plan involves modules of several different PV technologies from the following companies: Batch \#1 -a-Si (APS and Solarex), a-Si /a-Si (UPG and USSC), CIS (Siemens Solar), mono x-Si (Siemens Solar), multi x-Si (Solarex), Spheral-Si (Texas Instruments); Batch \#2 - a-Si/a-Si/a-Si:Ge (ECD), CdTe (Golden Photon and Solar Cells, Inc.), Silicon-Film (AstroPower). The modules were received, inventoried, and baselined and then the following tests have been completed thus far: Initial Tests (Ground Continuity, Dry and Wet Hi-Pot, Wet Insulation-Resistance), Sequence A (200 Thermal Cycles, Surface-Cut Susceptability), Sequence C (Damp Heat, Dry Hi-Pot), and Sequence D (Outdoor Exposure). The remaining tests will be completed in the near future. The key MQT results to date were published in a technical paper presented at the 1st World Conference on Photovoltaic Energy Conversion in December, 1994. One of the important results thus far was that some modules (both thin-film and crystalline silicon) which had passed the wet megger test subsequently failed the wet hi-pot test. This was significant because the IEEE committee reviewing the module qualification document has since reversed its position and voted to reinstate the wet hi-pot test into the MQT sequence. 
Electrical Integrity Testing: A new cage was constructed at the Joyce Street Facility Module Testing Laboratory to house the dry and wet hi-pot, ground-continuity and wet insulation-resistance tests. The cage was completed, these tests were set up to the appropriate safety standards, and readiness verifications were performed for ES\&H personnel. Now completed, these electrical integrity tests have been performed on all the modules going through Batch \#1, listed above.

Thermal Cycling, Humidity-Freeze Cycling, Damp Heat Testing: The old BMA environmental test chamber had earlier been modified to allow the monitoring of the temperature of each module under test, as well as to electrically identify if and when an open-circuit or short-to-ground failure occurs during thermal or humidityfreeze cycling, or during the damp heat test. This chamber is primarily used for the damp heat test (1000 hours at $85^{\circ} \mathrm{C}, 85 \%$ R.H.). The new BMA environmental chamber was received and installed in late 1993, and is now fully operational. This apparatus has a large internal capacity, allowing us to test many more modules simultaneously, as well as larger modules. This chamber has been successfully used to run the 200 thermalcycle test $\left(-40^{\circ} \mathrm{C}\right.$ to $\left.+90^{\circ} \mathrm{C}\right)$, and has performed very well. We are in the process of modifying this chamber to also enable the in-situ monitoring of module temperature, open-circuits, and ground-faults, as described above.

Hail Impact Test System (H.I.T.S.): This apparatus has been used to test several products, prototypes, and modules, including: Golden Photon, Utility Power Group and Mobil Solar. The tests helped these manufacturers redesign and improve their PV modules.

Mechanical Flex Tester (MFT): This machine has been set up, tested, and is now fully operational. This particular unit applies a cyclic pressure load $\left(35 \mathrm{lbs} / \mathrm{ft}^{2}\right)$ alternately to each face of a flat-plate module in order to simulate wind and other dynamic mechanical loads that a module would experience in field-installed applications. Once the final instrumen-tation has been installed -- flat pressure transducers to measure the pressure on the front and back of the module during testing - the apparatus will be used in the MQT sequence.

UV/Thermal Test Apparatus: This apparatus has been designed and constructed and will be installed in the new BMA environmental chamber. This apparatus consists of PV module test racks, as well as UV/fluorescent lamps and ballasts. We will be testing the proposed IEEE UV/thermal test over the next few months with both UV-A and UV-B lamps to investigate the validity of this test procedure.

Static Load Test Apparatus: This apparatus was designed and construction has now been completed. This unit will allow static mechanical loads $\left(50 \mathrm{lbs} / \mathrm{ft}^{2}\right)$ to be applied to each face of a PV module to simulate snow or other such static mechanical loads which a module might experience in the field.

New Qualification Test Method Development: New module qualification tests and methods are being developed. The following tests have been designed and set up thus far: (1) thermal and humidity-freeze cycling, (2) hail impact test, (3) mechanical flex test (dynamic), (4) scratch or surface-cut test, (5) groundcontinuity test, (6) dry and wet hi-pot tests, (7) wet insulation-resistance (megger) test, (8) damp heat test, (9) saltwater immersion and corrosion test, and (10) static load test. Additional tests presently under development are: (1) non-intrusive hot-spot endurance test, (2) UV/thermal test using fluorescent lamps, (3) accelerated weathering (UV/solar radiation/thermal /moisture) test using filtered-xenon lamps in an environmental test chamber, (4) mechanical twist/termination robustness test, and (5) bypass diode thermal test.

Photostability Studies:

Tenney Environmental Chamber/Vortek Argon-arc Lamp: This chamber/light-source exposure system has undergone several improvements over the past year, including: (1) the improvement of the lamp's spectral irradiance in the near infrared region of the spectrum $(\sim 700-1300 \mathrm{~nm})$ with the placement of a large-area heatabsorbing glass filter in front of the arc/reflector assembly to absorb a portion of this infrared energy, thereby shaping the spectral content of the lamp's output to be closer to that of the sun; (2) the construction of a hinge/turnbuckle arrangement to allow for the adjustment of the lamp's reflector assembly (top, bottom and side mirrors) to improve the irradiance uniformity at the test plane (from $\pm 20 \%$ to $\pm 5 \%$, with the reduction of the usable test area to $\left.5^{\prime} \times 3^{\prime}\right) ;(3)$ the addition of fans and blowers to increase internal air circulation to promote more uniform module temperatures during testing, as well as the construction of an enclosure around the lamp itself to exhaust the excess heat out of the test chamber; and (4) further improvements in the test structure and wiring of the test area to enable electrical and thermal monitoring of each of the PV modules under test. 
Light-Soak Testing: Light-Soak Test \#3 was completed in the 1994. It involved the light-soaking of multijunction a-Si PV modules from four different manufacturers (APS, Solarex, USSC and ECD). They were exposed to one-sun illumination at $50^{\circ} \mathrm{C}$, loaded at Pmax. Some modules were placed outdoors for further testing. Light-Soak Test \#4 will begin in early 1995, at lower module temperatures during exposure, to try to better simulate the photostability that the modules exhibit outdoors. In addition, modules for the a-Si Utility/Industry PV Power Project are also being light-soaked $\left(600 \mathrm{hrs}\right.$. at $\left.50^{\circ} \mathrm{C}\right)$.

Indoor/Outdoor Photostability Correlation Study: The outdoor portion of this testing is ongoing as part of our long-term outdoor module testing program. The correlation study will involve a comparison of the outdoor photostability of commercial a-Si PV modules with various types of indoor testing performed on identical modules, such as: (1) continuous illumination and constant temperature, and (2) cycled illumination and temperature. The improvement in the spectrum of the Vortek argon-arc lamp as well as exposure under more realistic temperatures will hopefully help to enhance the correlation between indoor and outdoor results of this testing.

\section{Accelerated Weathering:}

New Accelerated-Weathering System (AWS): Vendors have responded to a request-for-quotes (RFQ) which was sent out in 1994. This procurement is for an accelerated-weathering system consisting of three parts: (A) an indoor large-area test chamber that will provide accelerated weathering -- UV/Vis/IR radiation, thermal and humidity cycling -- on a group of PV modules, (B) an outdoor solar-tracking concentrator apparatus for accelerated outdoor weathering of PV modules, and (C) a multiplexing module I-V curve tracer to obtain I-V characteristics of module under test. This area of testing will help us to observe the synergistic effect of various outdoor weather conditions (solar radiation, temperature and humidity) on PV modules in an accelerated environment. This, in turn, will help us to correlate the indoor, accelerated test results with outdoor, real-time exposure testing. The procurement should be completed in early 1995.

\section{Module Performance Testing:}

Multi-Reference-Cell Package: A multiple reference-cell package in a module enclosure has been designed to enable us to instantaneously and simultaneously measure various portions of the incident spectrum during outdoor or indoor testing. Several packages will be fabricated: one of these will be used with PV systems installed at the outdoor test site, two will be used with the Standardized Outdoor Measurement System (SOMS), one will be used with the outdoor experimental test rack and module energy ratings study, one will be used with the Reference Irradiance/Meteorology System (RIMS), and two will be used for indoor module testing.

Irradiance/Temperature Coefficients and PERMM Study: An apparatus called the SolarClimatic 1600 was procured from Atlas Electric Devices and delivered and installed at NREL in October, 1994. It holds up to a $2^{\prime} \times 5^{\prime} \mathrm{PV}$ module, with the ability to vary the irradiance from $200-1100 \mathrm{~W} / \mathrm{m}^{2}$ and the temperature from $-10^{\circ}$ $\mathrm{C}$ to $+90^{\circ} \mathrm{C}$. This allows us to measure the I-V characteristics of various PV modules at different illuminations and temperatures, thereby providing us with the irradiance and temperature coefficients of voltage, current, fill factor, power, and efficiency. We performed an extensive study of five different PV technologies -- dual- and triple-junction a-Si, CIS, mono x-Si and multi x-Si -- at six irradiances $(200,400,600$, $\left.800,1000,1100 \mathrm{~W} / \mathrm{m}^{2}\right)$ and six temperatures $\left(0,15,25,40,60,80^{\circ} \mathrm{C}\right)$. We obtained I-V curves for each of these modules at each of these irradiances and temperatures. With these results, we wrote a computer program to acquire and convert existing weather data (solar irradiation, ambient temperature, and wind speed) for eight sites (Boston, Boulder, Detroit, Miami, Phoenix, San Francisco, Seattle, and St. Louis) and five different years $(1961,1968,1975,1982,1990)$. We then interpolated our I-V vs irradiance and temperature data to obtain the power, energy and efficiency for each module for each site and for each year. This work -called the PERMM (PV Energy Rating Method for Modules) study - was submitted to the Technical Review Committee evaluating new energy rating methods in San Ramon, CA, in November, 1994.

\section{Eacilities:}

FTLB Laboratory \#156: This laboratory contains the Tenney, Vortek light source, old BMA, and new BMA environmental test chambers, described above. Maintaining and improving the equipment, running tests, updating the Safe Operating Procedures (SOPs), etc., are all part of the ongoing, daily functions of our research team. 
Joyce Street Facility (JSF): The Module Testing Laboratory at the JSF contains the Hail Impact Test System (H.I.T.S.), the Mechanical Flex Tester (MFT), the safety cage housing the hi-pot, ground-continuity, insulationresistance, and other electrical-integrity tests, and the SolarClimatic 1600, described above. Maintaining and improving the equipment, running tests, updating the Safe Operating Procedures (SOPs), etc., are all part of the ongoing, daily functions of our research team.

Outdoor Test Facility (OTF): The design for the OTF was completed, and the blueprints for the lower-level laboratories were drawn, reviewed, and reworked several times over the past year. Utility and physical requireme. " $s$, as well as equipment locations, were also specified. Construction on the building finally began in 1994. Muve-in and fit-up is expected to occur in the Spring of 1995.

Outdoor PV Test Site: The RD-1200 Multi-Tracer (Multiplexing PV Module I-V Curve Tracer) was delivered in the Summer, 1993. This apparatus was tested and calibrated and was installed at the outdoor PV test site (FETA - Field Evaluation Test Area) in September, and was connected to PV modules from different manufacturers and various technologies, including: mono $x-\mathrm{Si}$, multi $\mathrm{x}-\mathrm{Si}, \mathrm{CIS}, \mathrm{CdTe}$, single-, dual-and triple-junction a-Si. The computer-controlled apparatus -- which includes a software package for calibration, control, monitoring, storing and plotting the acquired data -- can electrically load and measure up to a total of 15 modules simultaneously, and has been acquiring in-situ I-V curves of the various PV modules every 30 minutes during daylight hours for most of 1994. Vmax, Imax, POA irradiance, and back-of-module temperature are acquired every 5 sec and averaged hourly. In early 1994, the outdoor experimental test rack on which the modules are mounted was moved to its present location on the roof of one of the trailers at the outdoor test site. The Multi-Tracer and computer were moved inside that trailer. The data aquired is being used for multiple purposes, including: PV energy rating studies, indoor/outdoor photostability testing, AOCS studies, etc.

\section{Belevant Conferences:}

NREL Standards and Codes Forum: Golden, CO, June, 1994.

Photovoltaic Performance and Reliability Workshop: Lakewood, CO, September, 1994.

PV Energy Rating Methods Technical Review Committee Meeting: San Ramon, CA, Nov. 1994.

ASTM Standards Meeting: Phoenix, AZ, November, 1994.

1st World Conference on Photovoltaic Energy Conversion: Waikoloa, Hawaii, December, 1994.

\section{Photovoltaic Standards:}

ASTM: The ASTM Standard entitled, "Test Method for Measurement of the Electrical Performance and Spectral Response of Multi-junction Photovoltaic Cells and Modules", is presently being written. An early unfinished draft was presented to the Standards and Codes Forum at NREL in June, 1994, and a later draft was presented to the ASTM standards subcommittee in Phoenix in November, 1994.

IEEE : The IEEE Document entitled, "Recommended Practice for Qualification of Photovoltaic Modules", which outlines the test sequence and procedures for module qualification testing, is presently being reviewed and amended. The final draft will be submitted to the PV community for review and comments in early 1995.

\section{Subcontract Monitoring:}

Spire PVMaT Contract: Technical Monitoring Team member.

PV Laboratory Accreditation / Module Certification: Technical Review Team member.

\section{References / Publications}

1. "Improvements in Indoor Light-Soak Test Equipment", Photovoltaic Performance and Reliability Workshop, Lakewood, CO, September, 1994.

2. "Qualification Testing of Thin-Film and Crystalline Photovoltaic Modules", 1st World Conference on Photovoltaic Energy Conversion, Waikoloa, Hawaii, December, 1994. 
Title: Photovoltaic Module and System Technology Validation

Organization: Photovoltaic Module and System Performance and Engineering Project
NREL PV Program

Contributors: L. Mrig, Team Leader; B. Stafford, T. Strand, B. Kroposki, R. Hansen

\section{Objectives}

The objective of this research activity is to monitor and evaluate state-of-the-art and emerging photovoltaic technologies on the module and system level. This team also assists in the development and validation of PV technologies by analyzing the performance and reliability of PV modules and systems in support of the DOE National Photovoltaic Program. The results of this activity are then disseminated to the PV community in the form of publications, test reports and presentations. In addition, this team interacts with PV manufacturers and other test facilities to exchange information, results, and experience. Finally, the team aids in the development of national and international standards and codes for use by the PV community.

\section{Technical Approach}

Module Testing for Performance, Reliability, and Stability

The team maintains a database on the performance and reliability of flat-plate PV module technologies. The team continues to perform module stability testing on all thin-film PV technologies. To address the issue of module stability, long-term outdoor experiments are conducted. This entails placing the module outdoors under a fixed resistive load (at its maximum power point). Then, periodically the test modules are measured for performance under outdoor (prevailing) and controlled indoor conditions (STC). Amorphous silicon module stability is also characterized indoors under controlled light-soak experiments. Modules are exposed to an equivalent of 1 sun for up to 2000 hours. Module performance is measured periodically during the test. The stability performance data are then shared with individual manufacturers to aid in product evaluation and development.

\section{Module Performance Evaluation Methodology Development}

The team is researching and developing performance evaluation methodologies for modules. These studies include seasonal performance, spectral measurements, and techniques for evaluating modules on instantaneous power output and energy production. For module evaluation, I-V curve data is taken periodically during the day. This data is used in conjunction with radiometric and meteorological data to develop models that predict power output. Once models for power output are developed, energy over specific periods of time can then be predicted. This team has established a technical review committee made up of representatives from PV manufactures, PV users, and government labs to resolve the issues associated with energy ratings and performance evaluation methodology development.

\section{System Performance and Testing}

The team monitors and evaluates the performance of emerging PV technology modules and small systems (approximately $1 \mathrm{kWp}$ each) at NREL's PV Outdoor Test Site. Photovoltaic systems are designed and deployed at this test site to conduct in-situ technical evaluations of the PV array, system performance, and reliability. This activity focuses on emerging PV module technology evaluation/validation, flat-plate module and system development, and failure analysis. This testing is being conducted at NREL to assist the PV manufacturers and system integrators in evaluating array/system performance and identifying technical issues, if any, by fielding these small PV systems in actual operating environments. The systems 
are monitored extensively to help identify trends in various operating parameters and to provide detailed array/system evaluations. This research furnishes data to the PV community on system performance and reliability. This information provides confidence in sizing and fielding PV systems employing these emerging technologies as they become more mature and commercially available.

The technical approach of this activity centers around an in-depth test plan specifically developed for each PV technology/system under test. Each system's performance is monitored by an autonomous data acquisition system (DAS). Each DAS is interrogated nightly by the "Systems Data Base Manager" at the PV Outdoor Test Facility. The systems data base is comprehensive and used for correlating meteorological data, radiometric data, solar spectrum, and individual module and array/system performance. Techniques used in this research activity center around an in-depth analysis of the data collected in the systems data base and special testing conducted on each system periodically (i.e. power quality, IR camera (hot spot), wet resistance tests (megger), and array/module I-V traces). The PV modules are obtained commercially or through subcontracts with PV manufacturing companies or through no-cost agreements as joint NREL/PV manufacturer cooperative research agreements. The PV array and system performance data collected forms the basis of technical publications and reports. A major aspect of this cooperative array/system research activity at NREL is the technical exchange of results obtained from these evaluations with manufacturers and industry.

\section{Amorphous Silicon Utility/Industry Power Project}

This team assisted in the development and qualification of utility-scale amorphous silicon modules and helped to improve the reliability of utility-scale amorphous silicon modules and grid-connected systems. Several subcontracts to photovoltaic manufacturers have been awarded, and this task managed these subcontracts.

\section{Standards and Codes}

The team has members on IEEE SCC21, NEC Article 690 TRC, IEC TC/82, and USTAG to help in the development of national and international standards and codes, through consensus, for the PV industry. The team was involved with generating a comprehensive module qualification document, based on the NREL IQT, which incorporates crystalline silicon technology modules as well. Also the team is leading the effort to revise NEC Article 690.

\section{Support for Internal Proiects}

The team provided support for PVMaT, PVBONUS, Polycrystalline Thin Film, and PVUSA. The team also provided support for several small system designs as well as conducting field testing of PV systems.

\section{Results}

\section{Module Stability and Performance}

There are several important conclusions regarding thin film PV technology. Current production singleand dual-junction a-Si modules degrade $25 \%$ to $30 \%$ from their initial efficiency when deployed outdoors. Most of this initial degradation is due to the light-induced degradation effect normally found in a-Si. The a-Si modules also show an approximate $10 \%$ variation in performance which correlates with season in Golden, Colorado. This may be due to temperature changes, spectral influences, or a combination of both. Current prototype triple-junction modules show two important improvements in module design. First, some modules showed a higher stabilized efficiency $(\sim 6.5 \%)$ compared to commercially available singleor dual-junction modules. Second, others showed less initial light-induced degradation (less than 10\%) in the first 3 months of outdoor exposure. Copper Indium Diselenide modules show long-term stability for several sets of modules. The newer vintage modules also have higher efficiencies $(\sim 9 \%)$. Preliminary temperature coefficients for CIS modules were calculated. These numbers were in the range of $0.6 \%$ and 
$0.7 \%$ change in normalized power per ${ }^{\circ} \mathrm{C}$ for several modules. Cadmium Telluride modules show stability over approximately 1 year of outdoor exposure. Some of these modules also show relatively high efficiencies $(-7.5 \%)$. More results on module performance and reliability can be found in the references $[1,2,4,5]$.

Module Performance Evaluation Methodology Development

A study was conducted on the accuracy of different energy rating techniques, the results can be found in a paper presented at the 24th IEEE [7].

\section{System Evaluation Results}

In-situ testing of advanced thin-film PV systems has been in progress since December 1992. Several systems have been deployed and are currently being monitored. The effects of weather, temperature, and solar spectrum on system/array performance are being studied. Work currently in-progress include in-depth studies of performance versus temperature and spectrum, system energy ratings, and determination of temperature coefficients and system losses. Photovoltaic systems under test at NREL include advanced thin-film PV technologies of amorphous silicon (a-Si), CuInSe ${ }_{2}$ (CIS), and CdTe/CdS. Two identical utility-interconnect PV systems (X-Si) have been installed on the roof of the Solar Energy Research Facility (SERF). All systems were deployed to conduct in-situ technical evaluations of the PV array, system performance, and reliability. A paper detailing each systems current performance/status was presented at the 7th Photovoltaic Performance and Reliability Workshop, Sept. 21-23, 1994, Lakewood, $\mathrm{CO}[3]$.

A paper detailing the performance of a $1.8-\mathrm{kW}_{\mathrm{ac}}$ a-Si dual-junction same band-gap utility-interconnect $\mathrm{PV}$ system under evaluation at NREL's PV test site was presented at the First World Conference on Photovoltaic Energy Conversion, Waikoloa, Hawaii, December, 1994 [6]. Losses and changes in array performance were identified and quantified. System losses (dc only) were found to be less than $10 \mathrm{~W}$ for all data points. Losses due to soiling were measured at approximately $3 \%$. The loss due to module mismatch was calculated to be approximately $3.6 \%$. The change in performance due to temperature, from winter to summer, was found to range between $-3 \%$ and $+4 \%$. Changes in performance due to spectrum from summer to winter were found to be between $1.6 \%$ and $8.6 \%$. After correcting for these losses and changes, the measured (prevailing) array peak power was brought within approximately $11 \%$ of the STC rating for winter operation and $3 \%$ for summer operation. The array peak power was found to degrade by $7.5 \%$ from summer to winter of 1993 and rebound (anneal) by $7.7 \%$ from the winter of 1993 to the summer of 1994 . The lowest uncorrected array peak power, normalized to $1000 \mathrm{~W} / \mathrm{m}^{2}$ for the winter of 1993 , was $77.7 \%$ of the $2244 \mathrm{~W}$ array peak power rating. The highest uncorrected array peak power, normalized to $1000 \mathrm{~W} / \mathrm{m}^{2}$ for the summer of 1994 , was $86.2 \%$ of the $2244 \mathrm{~W}$ array peak power rating.

Test reports specific to the various PV systems under test have been compiled and delivered to the respective manufacturers. These reports delivered include the following:

1. Field Evaluation, 2.5kW Solar Electric Pump Irrigation System Test Report PV46-0103.01, November 16, 1993

2. USSC Integrated/Direct Mount PV Roofing Module System Performance Test Report PV46-0103001, March 8, 1994

3. Siemens Solar CIS System Interim Test Report PV46-0103-00, May 26, 1994

4. 1.8kWac United Solar System Corporation (USSC) Open Circuit Voltage Test Report PV46-010300, April 22, 1994

5. Golden Photon Preliminary Field Test Report PV46-0103.00, August 5, 1994

6. SCI CdTe PV System Performance Status Report SCI-SR56-0103-0, November 23, 1994 


\section{Conclusions and Future Plans}

This team plans to continue in its role as engineering analysis and evaluation for the module and system testing conducted at NREL. In addition, several goals for FY95 are listed below:

1. Technical Report on all emerging technology systems at NREL, August 1995

2. Status Report on the Brazilian Lighting Systems, August 1995

3. Technical Report on the stability of emerging technology modules under test at the PV Outdoor Test Site, August 1995

4. Technical Report on Energy Ratings Methodology, September 1995

5. Organize and Lead a Photovoltaic Performance and Reliability Workshop, September 1995

6. Design and deploy the PV Module High Voltage Test Bed at the PV Outdoor Test Site, September 1995

7. Deploy three PV systems employing emerging technology modules (APS, ASE Americas, Solarex a-Si, Astro Power, Golden Photon, TI) at the PV Outdoor Test Site

8. Relocate to and aid in the fit-up of the Outdoor Test Facility

\section{References}

1. Photovoltaic Module Outdoor, Module Qualification, and System Testing at NREL, L. Mrig, B. Kroposki, Y. Caiyem, S. Rummel, R. Hansen, and T. Strand, 1994 International Solar Energy Conference, San Francisco, CA, March 1994

2. Amorphous Silicon Lightsoak Criterion Development, L. Mrig, 7th Photovoltaic Performance and Reliability Workshop, Sept. 21-23, 1994, Lakewood, CO

3. Technical Evaluation of Photovoltaic Systems at NREL, T. Strand, L. Mrig, and R. Hansen, 7th Photovoltaic Performance and Reliability Workshop, Sept. 21-23, 1994, Lakewood, CO

4. Thin Film Module Stability Testing at NREL, B. Kroposki and L. Mrig, 7th Photovoltaic Performance and Reliability Workshop, Sept. 21-23, 1994, Lakewood, CO

5. Outdoor Performance Stability and Controlled Lightsoak Testing of Multijunction Amorphous Silicon PV Modules at NREL, L. Mrig , J. Burdick, W. Luft, and B. Kroposki, First World Conference on Photovoltaic Energy Conversion, Waikoloa, Hawaii, December, 1994

6. Technical Evaluation of a Dual-Junction Same-Band-Gap Amorphous Silicon Photovoltaic System, T. Strand, L. Mrig, R. Hansen, and K. Emery, First World Conference on Photovoltaic Energy Conversion, Waikoloa, Hawaii, December, 1994

7. A Comparison of Photovoltaic Module Performance Evaluation Methodologies for Energy Ratings, B. Kroposki, L. Mrig, and K. Emery, First World Conference on Photovoltaic Energy Conversion, Waikoloa, Hawaii, December, 1994 


\title{
Organization: NREL PV Module and Systems Performance and Engineering Project
}

\author{
Contributors: T. S. Basso, System Lead; and T. J. McMahon, Module Lead
}

\section{Objectives}

The objectives are to conduct and document module and system reliability and failure activities. The purposes are to provide technical results, recommendations or solutions to technical issues, technical assistance, and, technical inputs. These are intended for the development of PV standards, codes, criteria for test and evaluation procedures, and, design input. This activity aims to establish, develop, enhance, and provide support facilities, equipment, and approaches that are complementary to, or extensions of, other PV means.

\section{Technical Approach}

Module and system level concerns will be addressed through working relationships with the industry, users and other colleagues. Results are generally considered confidential, usually only disclosing generic data in the open literture.

Module and system reliability performance data is obtained from operating systems, demonstration or prototype units, qualification and exploratory tests, research results, dedicated studies, and, from the literature. This is used for establishing a comprehensive database for individual module and array/system component performance with emphasis on reliability and operational characteristics. Analyses will be performed, including the correlation of real time exposure testing, accelerated testing, and deployed system results. Dedicated tasks are pursued to uncover and reproduce identified failure mechanisms, to validate proposed hypotheses, to explore areas that are believed to be of high priority for long term PV reliability, to recommend new tests for screening or qualification, and to develop better understanding for improved reliability and design.

The cause of PV module failure will be determined through nondestructive and destructive evaluation procedures. Nondestructive testing (NDT) includes measuring individual cells of modules using methods such as the newly devised two-terminal shunt resistance measurement technique, the chopped light cell output determination, and, laser scanning. After NDT, module de-encapsulsation may be done, or, samples cored or cut from the module, to allow further evaluation of the suspect areas. This allows further characterization such as chemical analyses, microscopy, spectroscopy, etc. From these evalutions, more basic materials determinations can be confirmed and probable causes and mechanisms can be proposed.

System level concerns are addressed with emphasis extending outward from the module interface. We will build upon ongoing and past activities, since minimal resources are available for new thrusts.

\section{Results}

We have developed a non-destructive test method that allows us to know if the shunt resistance $\left(R_{\text {sh }}\right)$ of individual cells is affecting the output of a two-terminal, series-connected PV module [Reference 1]. The method directly, and non-intrusively measures $R_{\mathrm{sh}}$ of the individual cells. Being a phase-sensitive, lock-in technique, $R_{s h}$ is measured over a wide range, from a fraction of an ohm to thousands of ohms. The twoterminal $R_{s h}$ values compare well to the individually measured values as seen for a special test module that allowed contact to each individual cell. Further, the two-terminal measurements are more accurate for cells that have lower shunt resistance, i.e., the problem cells (Figures $1 \mathrm{a}$ and $1 \mathrm{~b}$ ). 


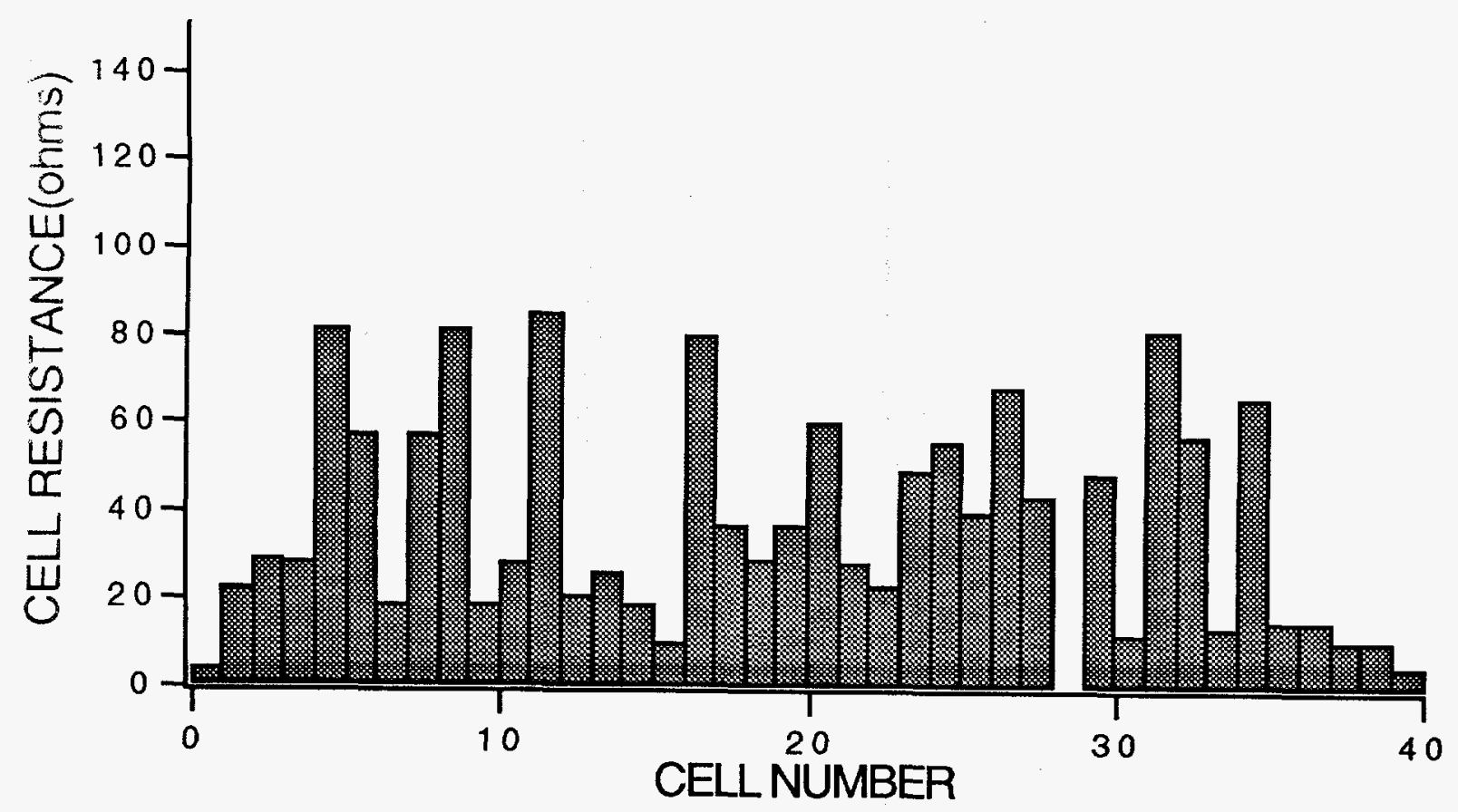

Figure 1a. Individual cell shunt resistances measured by the two-terminal technique.

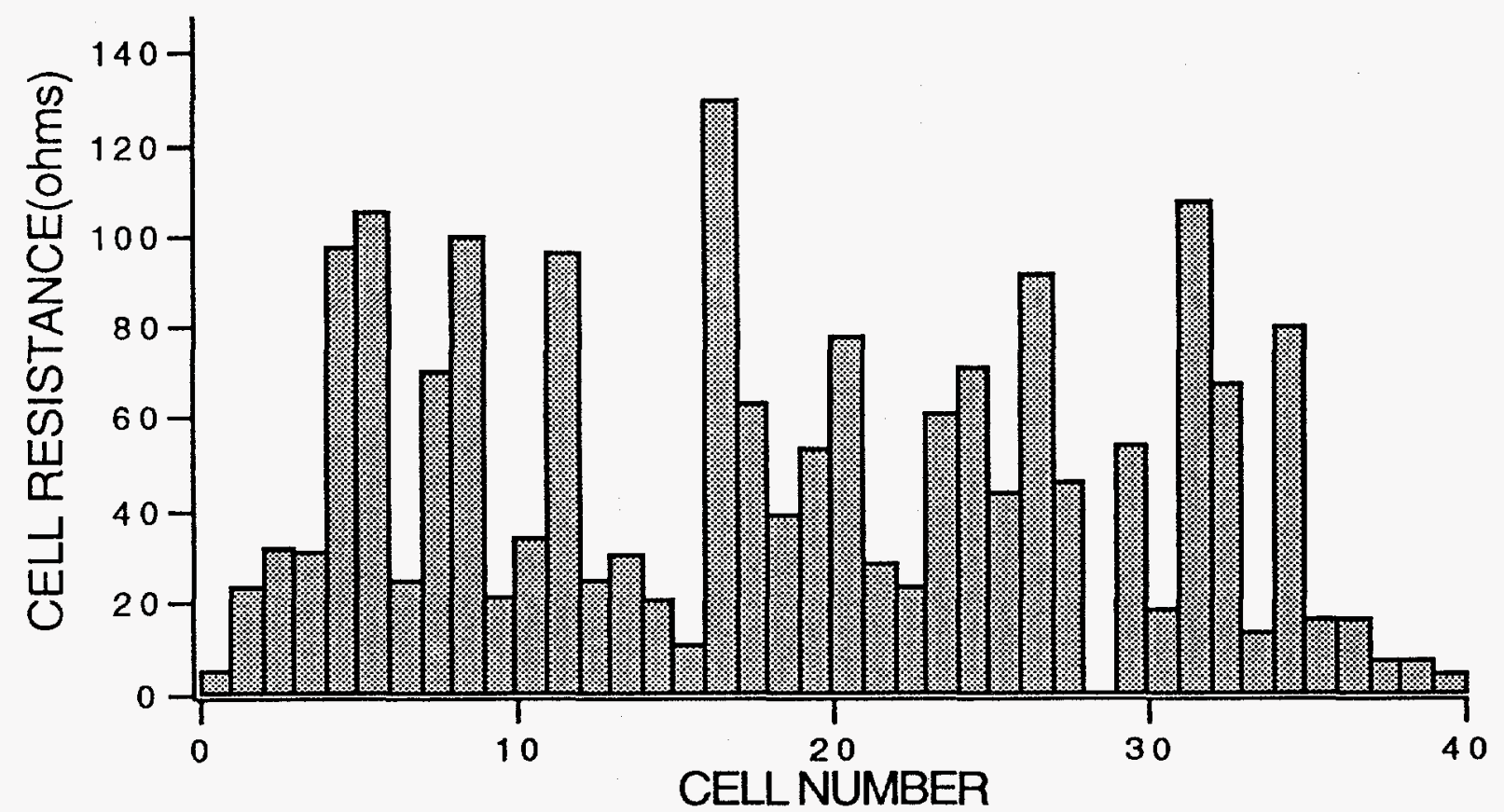

Figure 1b. Individual cell shunt resistances measured by contact of indivdual cells.

A shunt path in a cell carries only so much current. At high light levels, a shunted cell may seem to work at $100 \%$ of its capability. However, as the light intensity is reduced, such as on cloudy days or during daylight hours when the sun is lower in the sky, it becomes evident that the shunt path may be bleeding away some or all of the cell's photocurrent; useful output of the module is reduced. The importance of knowing the individual cell shunt resistances can be seen in the following three application areas: Qualification testing -- cell-by-cell values for $\mathrm{R}_{\mathrm{sh}}$ rather than the gross module output lets us know which cells are degrading module performance; Failure analysis -- non-intrusive cell-by-cell evaluation of a 
failed module is possible, whereas de-encapsualtion usually alters the cell properties making it impossible to pinpoint the exact location of a failure; Module cell performance -- $R_{\mathrm{sh}}$ allows us to determine at what light intensity each cell will stop contributing to the module output.

We have applied the two-terminal method to amorphous $\mathrm{Si}(\mathrm{a}-\mathrm{Si})$, silicon, and copper indium diselenide based modules. Some modules have as few as eight cells in series, but usually 28 to 68 cells. We measured modules from the Photovoltaics for Utility Scale Applications Project Utility Scale system deployed by the Advanced Photovoltaic Systems Corporation (APS); the Amorphous Silicon Utility/Industry Power Project Maspeth system deployed by the Integrated Power Corporation using United Solar Systems Corporation (USSC) modules; the NREL technology validation system deployed by Siemens Solar Systems, Inc.; pre-production modules from Astropower Corporation; prototype modules from Texas Instruments; and other modules undergoing light-soaking/qualification testing at NREL, e.g., APS, Energy Conversion Devices, Kanaka Corporation, Solarex Corporation, and USSC.

We investigated thin film modules from three systems. The modules were brought to our attention after visual anomalies or defects had developed. The two-terminal $\mathbf{R}_{\mathrm{sh}}$ method determined that not all the areas exhibiting the defects had unusually low shunt resistance, but we did not have any baseline measurements prior to deployment. Visual study, literature references, and discussions with two of the manufacturers led us to conclude the defects in their modules were primarily due to stresses incurred during manufacture. These two manufacturers informed us that they have resolved this issue and they do not observe any such visual defects in subsequently fabricated modules. For one of those two manufacturers, there were module regions void of the cell material. For those regions we further hypothesized that the loss of cell material may have been caused by "hot spots" [Reference 2] - localized regions reaching a high temperature due to high current densities. The third manufacturer's modules defects were primarily near the bottom edgeseal. We conjectured that water may have penetrated the edge.

The analyses of the electrical response of modules is being pursued, currently focusing on using PSpice ${ }^{R}$ a circuit simulation and analysis software program. PSpice ${ }^{R}$ is a member of the SPICE (simulation program with integrated circuit emphasis) "family" of circuit simulators. PSpice ${ }^{\mathrm{R}}$ interprets how to run the circuit simulation based on the circuit component descriptions and the commands entered. Preliminary PV module circuit simulations have been run. Results are favorable that PSpice $^{\mathrm{R}}$ is a valuable tool for helping to understand the d.c. and pulsed response of modules. Understanding the equivalent circuitry will help in diagnosing failed modules, analysing "what if" situations, and making simulated test measurements. The electrical analyses of module performance will be expanded to include integrated system components using PSpice ${ }^{\mathrm{R}}$ and other methods as proves appropriate.

A previously initiated, and ongoing collaboration with Solarex Corporation to more fundamentally understand metastable shunt problems is nearly concluded. We (T. McMahon and M. Bennett) believe that metastable shunts, and possibly other shunts, depend on the back contact $\mathrm{ZnO}$ processing [Reference 3]. Specifically, the stepwise increases in reverse bias current are due to a selective etch on the peaks of the a-Si stack that are a result of the underlying $\mathrm{SnO}$. Possibly, a native $\mathrm{SiO}_{\mathrm{x}}$ layer is etched away on those peaks allowing shunt paths to form by metal diffusion from or through the $\mathrm{ZnO}$. This is plausible as we observed that S. Hegedus' devices shunted metastably and badly, but with no steps; those devices have no native $\mathrm{SiO}_{\mathrm{x}}$ between the $\mathrm{ZnO}$ and the a-Si. The switching metastability in a-Si cells is due to something (diffusing) from the ( $\mathrm{Al}) \mathrm{ZnO}$ and the discrete, stepwise quality is due to shunt paths formed on peaks of the textured transparent conducting oxide. Shunt path on- and off-states are observed to have memory; some sort of defect relaxation process must stabilize the conduction paths with biasing, e.g., a trapped charge becomes stabilized on a defect through a relaxation in the lattice surrounding that defect. 


\section{Conclusions}

We have developed a two-terminal, non-destructive, module cell-shunt-resistance measurement technique useful for qualification testing, failure analysis, and module cell performance characterization. This technique will continue to be used at NREL and is readily available for others to implement.

For two PV systems brought to our attention, the thin film module visual defects that we investigated are believed to be due to stresses incurred during manufacture. For one of those, there were module regions void of the cell material. We hypothesized that was the loss of cell material caused by "hot spots." We are planning to reproduce this defect in $s$ laboratory. For modules from a third thin film system that we investigated, we conjectured the visual defect in the modules was water entering through the edgeseal.

The more fundamental work on a-Si has led to the conclusion that metastable shunts, and possibly other shunts, depend on the back contact $\mathrm{ZnO}$ processing. However, the switching metastability in a-Si cells is due to something (diffusing) from the ( $\mathrm{Al}) \mathrm{ZnO}$ and the discrete, stepwise quality is due to shunt paths formed on peaks of the textured transparent conducting oxide; the memory behavior of shunt path on- and off-states are believed due to some sort of defect relaxation process that stabilizes the conduction paths with biasing.

New activities for module and integrated system component reliability include the development of system engineering standardized performance criteria to improve system component compatibility, sizing, and integration. This will be accomplished through in-house research. System and component level failure modes will be tested for reliability in the field through the design and construction of a high voltage system/array test structure outdoors to study performance characteristics (e.g. module/component/array leakage current, etc.) and allow for real-time exposure testing of modules and components under normal and elevated voltage levels to accelerate and identify potential failures. This will provide a test bed to evaluate the requirement established for compatibility of materials and components, identify failures and failure mechanisms, establish field data required in setting qualification test (acceleration) parameters. Results of this activity will provide new or revised design criteria for advanced modules and system components as well as the technical basis for developing new or revised qualification tests needed in assuring field service requirements. As funding may allow, through subcontract research support, we will address system engineering standardized performance to advance the module and component integration and compatibility requirements for reliable design and performance in the field with emphasis on safety, technical assistance, and meeting the Underwriters Laboratories, Inc. and National Electrical Code requirements.

\section{References}

1. McMahon, T. J.; Basso, T. S. (1994). Two Terminal Diagnostic for Cells in Series Connected Modules. Proceedings of the NREL Photovoltaic Performance and Reliability Workshop, September 1994, Lakewood, Colorado.

2. Gonzalez, C. C. and Jetter, E., Hot Spot Durability Testing of Amorphous Silicon Cells and Modules, Proceedings of the 18th IEEE Photovoltaic Specialist Conference, October 1985.

3. McMahon, T. J. and Bennett, M. S. (1994). Metastable Shunt Paths In a-Si Solar Cells, prepared for the Proceedings of the First World Conference on Photovoltaic Energy Conversion, December 1994. 
Title: Photovoltaic Solar Radiometric Measurements and Evaluation

Organization: NREL PV Module and Systems Performance and Engineering Project

Contributors: D.R. Myers, Team Leader; T.W. Cannon, Senior Engineer; D. Trudell, Master Technician

\section{Objectives}

The Photovoltaic (PV) Solar Radiation Measurements and Evaluation (PV-SRME) team provides scientific understanding of incident natural and artificial radiation, supporting characterization and performance testing of PV cells, modules, and systems. Radiometric instrumentation systems, measurement techniques, and calibrations are applied to reduce uncertainty in PV device and system performance testing and evaluation. The team addresses broadband [ 300 nanometers $(\mathrm{nm})$ to $2400 \mathrm{~nm}$ ] and spectral (wavelength dependent) radiometric measurements required to characterize artificial and natural radiation sources for PV reference standards, prototype, and commercially available PV devices tested within the PV Module and System Performance and Engineering Project and other NREL PV Program participants. The team exchanges technical information with industry and NREL PV engineers and researchers concerning radiometric measurements, instrumentation, analysis, and PV solar radiation resource data. In co-operation with the NREL Metrology Laboratory, we provide radiometric calibrations in support of other DOE program activities at NREL.

\section{Technical Approach}

Radiometric calibrations are performed in the Optical Metrology Laboratory, shared with NREL's Metrology Laboratory. Spectral and total irradiance reference standard sources, purchased directly from the National Institute of Standards and Technology (NIST), and quality electronic equipment support improved radiometer calibration and measurement techniques.

Spectral and broadband optical radiometric instrumentation is calibrated, maintained, operated, and loaned out to researchers to satisfy measurement needs of NREL internal and external PV program participants. NREL engineers and subcontractors obtain technical consultation on radiometric measurement problems and data analysis or interpretation critical to PV performance calibrations, testing, and evaluation. This includes the NREL PV Reference Meteorological and Irradiance System (RMIS), Atmospheric Optical Calibration System (AOCS), which monitor radiometric and atmospheric parameters with respect to standard reporting conditions during outdoor PV testing, and five spectroradiometric systems for measuring natural and artificial optical radiation sources. $[1,2,3]$

Team personnel meet NREL PV related optical radiometric measurement needs by maintaining optical metrology expertise, instrumentation and capabilities. We also contribute to the field, participating in organizations such as American Society for Testing Materials (ASTM), Institute of Electrical and Electronic Engineers (IEEE), the Council for Optical Radiation Measurements (CORM); the Society of Photo-Optical and Instrumentation Engineers (SPIE), and the NIST Radiometric Physics Division. T. Cannon is a member of the CORM board of directors. 


\section{Results}

Team accomplishments in Fiscal Year 94 include:

NREL Pulse Analysis Spectroradiometer System (PASS) measurements of spectral distribution of several SPIRE 240A pulsed solar simulator, providing spectral mismatch for emerging technology PV devices tested under this source.

Spectral content $(250 \mathrm{~nm}$ to $2400 \mathrm{~nm})$ measurements of:

-PV Cell and Module Efficiency Measurements task X-25 Solar simulator

-PV Module Exploratory Qualification Testing and Test Method Development task Vortek (light soaking) and Atlas Solar Climatic simulators

-Spectral distribution of various Ultraviolet sources used in the PV Modules Materials and Component Durability Task. Figure 1 shows the spectral distribution of the various sources measured.

-Spatial variation of spectral distributions at the focus of a concentrating collector. Figure 2 shows the changes in spectral distribution across the focal plane of a Fresnel Lens $40 \mathrm{X}$ concentrator (highest curve on axis, each succeeding smaller curve at locations off axis)

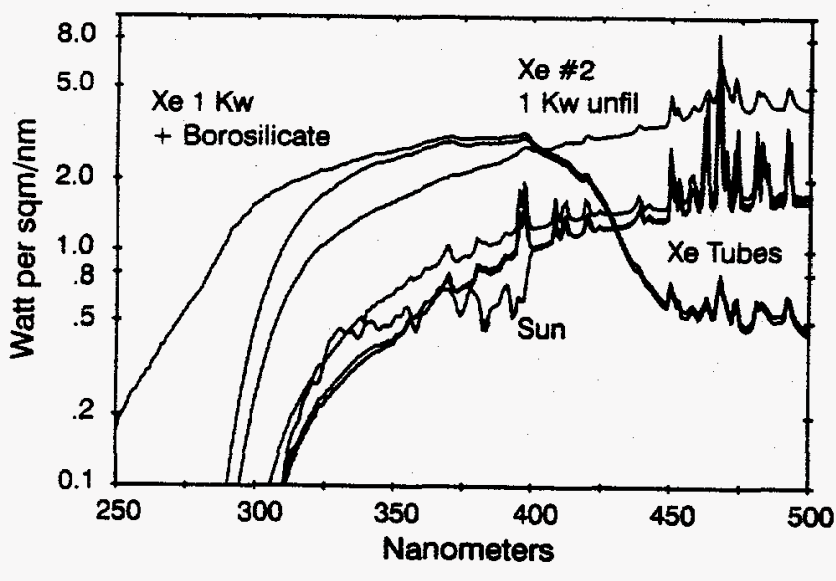

Figure 1: UV Source Spectral Data

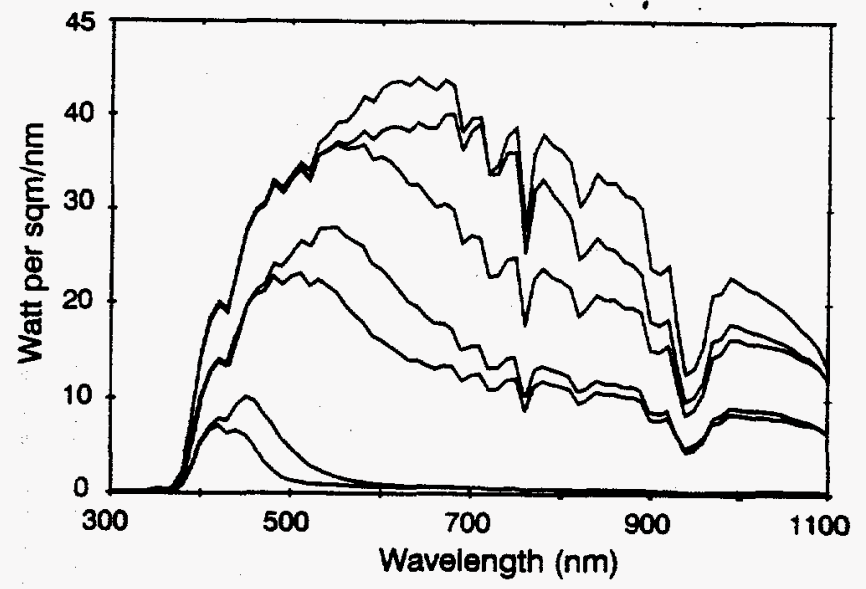

Figure 2: Spatial Spectral Variation Across Concentrator Focal Plane.

The team contributed technical evaluation of draft standards for PV accelerated weathering, qualification, and performance evaluation testing, including draft radiometric requirements for UV exposure testing. at the 1994 Standards and Codes Forum, May 1994, at Golden CO.

A spectroradiometer system $(280 \mathrm{~nm}$ to $3000 \mathrm{~nm})$ for all weather operation at the Field Experiment Test Site PV Outdoor Standardized Outdoor Measurement System (SOMS) test area. 
Real time spectral model and PV spectral mismatch correction factor calculations were implemented in the AOCS at the NREL test site. Figure 3 is a copy of the real-time display of computed spectra and spectral mismatch factors derived from AOCS parameters.

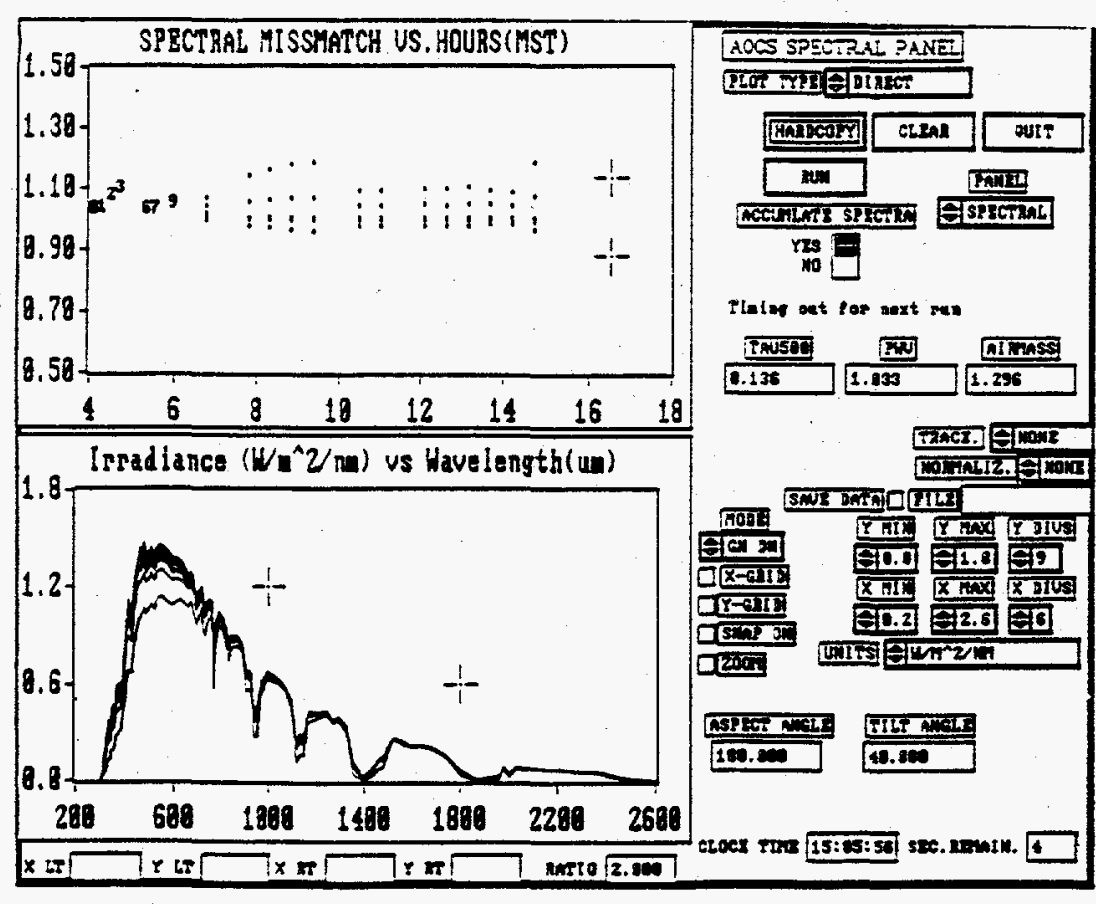

Figure 3. Real Time Display of Computed Spectral Data and Mismatch Factors
The task collected third site-year of solar resource data for the Public Service Company of Colorado at the Fort St. Vrain Generating Plant, Platteville, $\mathrm{CO}$.

A partial list of PV-SRME interactions and technical information transfer in FY 93 include the following:

Industry:

ENRON Emerging Technologies, Inc. Endecon, Inc.

Atlas Corporation

Vortek, Inc.

Optronics Laboratories

Texas Instruments, Inc.

United Solar Systems Corporation

Oriel Instruments

Yankee Environmental Systems, Inc. Geophysical Environmental Research

University, State, National Labs: State University of New York, Albany; Arizona State University;NIST; World Meteorological Organization, Geneva, Switzerland; Smithsonian Environmental Research Center, Solar Radiation Laboratory, Rockville, Md.

Professional Organizations:American Society for Testing of Materials; Institute of Electrical \& Electronic Engineers; Council for Optical Radiation Measurements; Society for Photo-optical Instrumentation Engineers; American Solar Energy Society

\section{Conclusions}

PV test engineers conducting system performance and stability monitoring activities at the NREL PV outdoor test site compare plane of array (POA) radiometric data with high quality data from the RMIS. The PV-SRME team operate, maintain, and calibrate the components of the RMIS, and archive 1 minute time resolution data for further analysis and correlation studies. Calibrations of plane-of-array sensors are co-ordinated by the team, providing traceability and known uncertainty for POA measurements.

Technical contributions to draft standards for PV performance, qualification, and durability testing were made at the 1994 Standards and Codes Forum, and contributed data and analysis for two 
papers [5,6] at the First World Conference on Photovoltaic Energy Conversion at Waikoloa, Hawaii. Unique measurement instrumentation and techniques permit the examination of spectral effects on multijunction and amorphous silicon PV technologies. The team maintains an on-call measurement capability for existing and future solar simulators and radiometric sources.

Outdoor PV performance data can be correlated with AOCS and RMIS data. Integration of real time spectral error correction routines to quantify spectral mismatch factors as a function of AOCS parameters will improve the database for evaluating performance charac astics as a function of climatic variables. Future work includes integration of AOCS param. rs into the Reference Meteorological and Irradiance Station data distribution to PV performance test engineers, continued radiometric calibrations and characterization of radiometers, and radiometric data quality assurance and analysis to meet PV research and engineering needs.

Team members presented papers at the following meetings, workshops and conferences:

DOE Office of Program Assessment Office of Energy Research Program Review, August 10, 1994, Denver Co.

Critical Issues in Air Ultraviolet Metrology, May 26-27, 1994, National Institute of Standards and Technology, Gaithersburg, MD. [4]

PV Performance and Reliability Workshop, Sep 21-23, 1994, Golden, CO. [3]

\section{References}

1. Cannon, T., R. Hulstrom, "Atmospheric Optical Calibration System for Outdoor Testing of Photovoltaic Devices" Metrologia, 30, No. 3, 1993.

2. Cannon, T.W., R.L. Hulstrom, D.T. Trudell. "New Instrumentation for Measuring Spectral Effects During Outdoor and Indoor PV Device Testing", Conference Record of the Twenty Third IEEE Photovoltaic Specialists Conference-1993, p. 1176.

3. Cannon, T.W., "Advances in Radiometric Instrumentation for PV Performance Evaluation", PV Reliability and Performance Workshop, Golden CO. Sep 21-23, 1994.

4. Myers, D.R., "The Uncertainty Challenge in Terrestrial Ultraviolet Measurements", Proceedings of Critical Issues in Air Ultraviolet Metrology Workshop, National Institute of Standards and Technology, May 26-27 1994, Gaithersburg MD. To be Published in Metrologia, Spring, 1995.

5. B. Kroposki, L. Mrig, D.R. Myers, K. Emery, "A Comparison of Photovoltaic Module Performance Evaluation Methodologies for Energy Ratings." First World Conference on Photovoltaic Energy Conversion, December 5-6, Waikoloa, Hawaii, 1994

6. Strand, T., K. Emery, R. Hansen, L. Mrig, "Technical Evaluation of a Dual Junction Same Band Gap Amorphous Silicon Photovoltaic System at NREL." Ibid. 
Title:

PV Standards and Codes

Organization: NREL PV Module and System Performance and Engineering Project

Contributors: C.R. Osterwald

\section{Objectives:}

The objective of this work was to provide coordination for the development of PV standards, codes, criteria for test and evaluation procedures that are crnsistent with the DOE National Photovoltaics Five-Year Plan. Included in this objective is the development of criteria for a possible third-party module certification / laboratory accreditation program.

\section{Technical Approach:}

Technical approaches used for these objectives included participation with the IEEE Standard Coordination Committee 21, and chairing ASTM subcommittee E44.09, titled "Photovoltaic Electric Power Conversion." The module certification (emphasizing qualification testing) and test facility accreditation criteria development effort was initiated through subcontract support to Arizona State University. Other efforts included coordination of the PEP'93

Intercomparison of reference cell calibrations and cell performance measurements, and coordination of the ASTM cell and module measurement round-robin. To facilitate these activities, a second "PV Standards and Codes Forum" was organized to hold individual and joint meetings at the same location and establish coordination of efforts and flow of information between committees on standards issues.

\section{Results:}

The PEP'93 Intercomparison coordination continued with the circulation of the sample sets. Two separate sample sets are being circulated. The first is composed entirely of $2 \times 2 \mathrm{~cm}$ single-crystal Si reference cells that each participant will calibrate with their best calibration method. The results of these calibrations will be analyzed and used to establish the World PV Scale (WPVS) of primary reference cells. The second sample set consists of devices from newer PV technologies that have unique measurement problems, such as narrow bandgap cells, large-area cells, and series-connected tandem cells. Twelve laboratories from throughout the world are participating in the intercomparison., with each participant assigned two months to complete measurements for each sample set, and the sample circulation is scheduled to be complete on June 30,1995 . Seven of the twelve laboratories have completed measurements of the sample sets.

PV standards activities within ASTM during FY1994 dealt with several issues of importance to the PV industry. At the November 1993 meeting of ASTM subcommittee E44.09, work was begun on two new draft standards titled "Test Method for Mechanical Integrity of Photovoltaic Modules" and "Test Method Electrical Performance and Spectral Response of Multi-junction Photovoltaic Devices." The multi-junction test method will document pit-falls and problems of measurements of these relatively new devices (both cells and modules), and the mechanical integrity test method will build on existing tests that were originally developed by JPL as part of the LSA project. Two existing reference cell calibration standards, E 1039 and E 1125, were revised and began the ASTM balloting process in 1994. Two 
important new standards were approved in January 1994. E 1596 provides three methods of testing modules for solar UV weathering, and E 1597 is a saltwater immersion test developed by the U.S. Coast Guard.

A subcontract, titled "PV Module Certification and Laboratory Accreditation Criteria Development," was awarded to Arizona State University (ASU). The major deliverable for this 1 year subcontract was to develop and document criteria and recommendations that specify guidelines to be used to certify a PV module for performance and reliability, along with the specific equipment, facilities, quality assurance procedures, and technical expertise an accredited laboratory needs in order to perform PV module certification. Development of the criteria and the documentation took place through a consensus committee, formed by ASU, of representatives of PV manufacturers, end users, standards and codes organizations, and testing laboratories. 30 organizations committed to work on the project. On March 16, 1994, ASU held the first meeting of the PV industry criteria development committee. A short tutorial on laboratory accreditation and product certification was presented, and possible strategies for implementation of the accreditation/certification program were outlined. Following the tutorial, issues that came from committee members during circulation by mail of the first two sections of the criteria document were presented and answered. These sections define assessment of laboratories for accreditation and a PV module certification and labeling program. The committee then discussed the final section of the document that details the testing, facilities, and expertise needed for module certification. A major topic of this discussion was the issue of certifying module performance, including whether this should be done through a certification program, and possible ways performance measurements could be made part of the existing module labeling already done by manufacturers in different ways.

The 1994 PV Standards \& Codes Forum was held on June 27 through June 30. Nearly 40 representatives from manufacturers, test laboratories, end-users, and the National Laboratories attended meetings of IEEE Standards Coordinating Committee 21, the U.S. Technical Advisory Group to IEC Technical Committee 82, ASTM subcommittee E44.09, and the Criteria Development Committee for the module certification and laboratory accreditation effort. A report on the efforts to propose changes to the National Electric Code Article 690 for the 1996 review cycle was also presented during the Forum. Three draft standards were reviewed during the ASTM E44.09 meeting, including the module wet insulation integrity test, and the two new drafts for module mechanical integrity testing and multijunction device performance testing. IEEE SCC21 held simultaneous working group meetings for document development, including: PV module qualification sequence, PV system battery sizing, PV system safety, and PV system field acceptance testing. Arizona State University conducted the Criteria Development Committee meeting. The goal of this NREL-sponsored program is to develop a document that details the performance and qualification testing needed for certification of PV modules, and the facilities, expertise, and quality assurance a laboratory needs in order to be accredited for certification testing. Another tutorial on the structure of certification bodies was presented. A discussion followed about how a certified module should be performance-tested and how this information should be placed on the module with the certification mark. It was felt that modules submitted to the third-party laboratory for certification should be tested by both the laboratory and the manufacturer's production test facilities. If the results agree, and the modules pass the subsequent qualification testing, the 
manufacturer would be allowed to apply the certification mark along with the manufacturer's existing labels stating the module performance.

\section{Conclusions:}

The two FY 1994 milestones for this subtask were both met. The first was to organize and lead the PV Standards and Codes Coordination Forum that will include consensus standards and codes organizations (meeting, 6/94). Arizona State University prepared and sent to NREL a final draft of the certification/accreditation criteria development report in October 1994, fulfilling the second deliverable.

\section{References:}

1. "Test methods for solar radiation weathering of photovoltaic modules," ASTM standard E 1596, ASTM, Philadelphia, PA, USA

2. "Test method for saltwater pressure immersion and temperature testing of photovoltaic modules for marine environments," ASTM standard E 1597, ASTM, Philadelphia, PA, USA 


\section{PV Module and System Performance \& Engineering Subcontracts}



Organization: Department of Chemical Engineering, University of Colorado, Boulder, CO

Contributors: $\quad$ C. N. Bowman, principal investigator

\section{Objectives}

The goals of this subcontracted work are (i) to conduct a methodical evaluation of potential PV module encapsulants to replace EVA, and (ii) to formulate, prepare, and deliver for test modified Elvax 150 (the principal ingredient in EVA) with different UV absorber-stabilizer combinations and other appropriate ingredients for enhancing the stability, processibility, polymer initiation, and other needs as determined by its intended use in PV modules, and (iii) to identify potential replacements for EVA and critically evaluate them.

\section{Results: Identify Potential Replacements for EVA and Critically Evaluate Them}

Ethylene-vinyl acetate (EVA) copolymers currently used for photovoltaic (PV) cells have exhibited evidence of long term degradation that is detrimental to the efficiency of the PV modules. It is thought that this degradation is catalyzed by ultraviolet (UV) light as well as thermal energy and is also enhanced by the presence of acetic acid and the metal at the surface of the polymer. The first part of this work, objective (i) as proposed, focused on identifying the mechanisms by which EVA copolymers degrade, determining the possible success of stabilizers and UV absorbers, and providing a preliminary evaluation of alternative materials and has been summarized [1]. For objective (ii), samples of Hostavin N20 and N30 that were identified last year [1] were made and delivered to NREL for their use. An alternative to using the commercially available Hostavin is to synthesize an ethylene copolymer with hindered amine light stabilizer (HALS), e.g., $-\left(\mathrm{CH}_{2} \mathrm{CH}_{2}\right)_{n}-\left(\mathrm{CH}_{2}-\mathrm{CHX}\right)-$ in which $\mathrm{X}$ is $\mathrm{C}-\mathrm{CO}-\mathrm{CH}_{2}-\left(\mathrm{CH}_{2}\right)_{8}-$ HALS. This copolymer should provide resistance to depletion because it is in the main chain, and would eliminate solubility limiting problems of the HALS. Several pottant formulations that could serve as alternatives to the current EVA copolymer systems have been identified and preliminary testing of them was conducted. The formulations were selected with the primary objective of increased stability in the presence of thermal and radiative energy. Two approaches were taken to determine the widest range of options: first, to modify the stabilizer and processing conditions for the current EVA formulation and secondly, to select entirely new polymers and processes. With respect to the first option, it is suggested that oxygen be removed from the lamination process, lamination processes should be developed at lower temperatures, hindered amine stabilizers be used, such as a combination of Hostavin N20 and $\mathrm{N} 30$, and a new hindered amine functionalized EVA be synthesized to provide the best longterm stability without sacrificing transparency.

For the second option, two distinct approaches could be taken. First, polymers could be selected that would continue to use the current lamination process. Secondly, liquid lacquers could be developed that would be photopolymerized into a coating in situ. For the first approach, it is highly recommended that ethylene-methyl acrylate (EMA) copolymers be used to replace EVA copolymers as pottant materials. EMA copolymers have nearly identical physical properties when compared to EVA copolymers; however, when they degrade, no labile acid is produced as is the case with the production of acetic acid from EVA copolymers. 
A number of materials were considered as potential replacements for EVA in which the objectives were better stability and being more suitable as a pottant for PV modules. The starting point was the JPL final report [2], but additional polymers were considered. Because the cost of using a pottant involves initial material costs, processing costs, and service lifetime (durability), ethlene-methyl acrylate (EMA) copolymers, polymerizable butyl acrylate (BA), and polyurethane (PU) were critically reviewed and the JPL rationale used for deemphasizing them [2] is questioned. Materials not identified by JPL are PEG-DA, which offer possible improvements versus the crosslinked BA systems, and agar, a cellulosic material that may or may not be simple to work with secause of insufficient characterization of its behavior and properties. The properties of EVt. EMA, BA, PU, PEG-DA, and agar are compared in Table 1 with the specification made in the JPL report [2]. These in situ, photopolymerizable resins offer a number of advantages, especially, because of using a reduced amount of material and significantly lower processing costs would likely result. Each of these resins would provide improved stability when compared with EVA systems. Preliminary testing of the PEG-DA systems showed no moisture problems, complete transparency, and a high double bond conversion. The continued presence of the photoinitiator also acts, to a limited extent, as a UV absorber by absorbing a high percentage of the UV light in a very thin sample. Small quantities of PEG-DA samples were made available and delivered to NREL.

Cellulosic materials were also explored as a possible laminatable material. Unfortunately, our preliminary experiments show a significant amount of clouding and hazing develops when these samples are exposed to moisture. The reduced transparency of these materials is not acceptable. Silicon rubbers (SRs) which have the most outstanding set of properties as pottant materials, are also the most expensive. Silicone rubbers may be poured, cured at room temperature, are extremely transparent, very flexible, have low $\mathrm{Tg}$, and excellent resistance to weathering, oxidative, and thermal degradation. With regard to degradation, these materials are the best of the polymers currently available.

A brief summary of the analysis of possible replacements for EVA requires setting guidelines for choosing which material(s) to pursue. It is likely that the PEG-DA films will prove to be significant improvements over the current materials but at a somewhat higher cost. For those deemphasized by JPL [2], several of their alternatives are likely to be improvements over the current EVA formulations. Many of these would cost additional money, and some would require development of new techniques to handle chemicals with strong odors.

No material is a perfect pottant. EVA has degradation problems, SRs are prohibitively expensive, BA systems have a strong odor, and PU, agar, and PEG-DA have some cost disadvantages. However, improvements can easily be made. For one, a switch to EMA, or at least further characterization, is necessary. It has nearly all of the desirable features of EVA and will not produce a labile acid that will attack and corrode the metal connections or yield degradation products that autocatalyze the EVA degradation. Other avenues should also be pursued once priorities have been set on which properties/costs are most important.

\section{References:}

1. C. N. Bowman, in NREL/TP-410-6285, August, 1994, pp. 310-11.

2. E. Cuddihy, C. Coulbert, A. Gupta, and R. Liang, Flat-Plate Solar Array Project Final Report, Volume VIII: Module Encapsulation, DOE/JPL-1012-125, October, 1986. 
Table 1. Characteristics and Specifications of EVA and Five Polymers that are Potential Replacements for EVA

\begin{tabular}{|c|c|c|c|c|c|c|c|}
\hline Characteristics & Specifications [2] & $\overline{\text { EVA }}$ & $\overline{\text { EMA }}$ & $\overline{\mathrm{BA}}$ & $\overline{\mathrm{PU}}$ & $\begin{array}{l}\text { PEG- } \\
\text { DA }\end{array}$ & Agar \\
\hline $\begin{array}{l}\text { Glass transition } \\
\text { temperature, } \mathrm{Tg}\end{array}$ & $<-40^{\circ} \mathrm{C}$ & $-43^{\circ} \mathrm{C}$ & Unk & $\begin{array}{l}\text { Unk } \\
>20^{\circ} \mathrm{C}\end{array}$ & $-23^{\circ} \mathrm{C}$ & \begin{tabular}{|l|}
$-40^{\circ} \mathrm{C}-$ \\
$-20^{\circ} \mathrm{C}$ \\
\end{tabular} & $\begin{array}{l}\text { low- } \\
\text { Unk }\end{array}$ \\
\hline $\begin{array}{l}\text { Total hemispherical } \\
\text { light trans ssion } \\
\text { through a sil thick } \\
\text { film integra d over the } \\
\text { wavelength range from } \\
0.4 \mu \mathrm{m} \text { to } 1.1 \mu \mathrm{m}\end{array}$ & $>90 \%$ of incident & $90.5 \%$ & $90.5 \%$ & $89 \%$ & $89 \%$ & $>90 \%$ & $>90 \%$ \\
\hline Hydrolysis & $\begin{array}{l}\text { None at } 80^{\circ} \mathrm{C}, 100 \% \\
\mathrm{RH}\end{array}$ & $\sim 0$ & $\sim 0$ & $\sim 0$ & $\sim 0$ & $\sim 0$ & Unk \\
\hline $\begin{array}{l}\text { Resistance to thermal } \\
\text { oxidation }\end{array}$ & Stable up to $85^{\circ} \mathrm{C}$ & $\bar{S}$ & $\mathrm{~S}$ & $\bar{S}$ & $\bar{S}$ & $S$ & $\bar{S}$ \\
\hline Mechanical creep & None at $90^{\circ} \mathrm{C}$ & $S$ & $S$ & $\bar{S}$ & $\bar{S}$ & $S$ & $S$ \\
\hline $\begin{array}{l}\text { Tensile Modulus as } \\
\text { measured by initial } \\
\text { slope of stress-strain } \\
\text { curve }\end{array}$ & $<3000 \mathrm{lb} / \mathrm{in}^{2}$ at $25^{\circ} \mathrm{C}$ & $850 \mathrm{psi}$ & $\begin{array}{l}1620 \\
\text { psi }\end{array}$ & 293 psi & $160 \mathrm{psi}$ & variable & variable \\
\hline Fabrication temperature & $\begin{array}{l}<170^{\circ} \mathrm{C} \text { for either } \\
\text { lamination or liquid } \\
\text { pottant systems }\end{array}$ & $150^{\circ} \mathrm{C}$ & $150^{\circ} \mathrm{C}$ & $60^{\circ} \mathrm{C}$ & $90^{\circ} \mathrm{C}$ & $25^{\circ} \mathrm{C}$ & $25^{\circ} \mathrm{C}$ \\
\hline $\begin{array}{l}\text { Fabrication pressure for } \\
\text { lamination pottants }\end{array}$ & $\leq 1 \mathrm{~atm}$ & $\bar{S}$ & $\bar{S}$ & $\bar{S}$ & $\bar{S}$ & $\bar{S}$ & 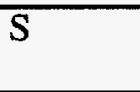 \\
\hline Chemical inertness & $\begin{array}{l}\text { No reaction with } \\
\text { embedded copper } \\
\text { coupons at } 90^{\circ} \mathrm{C}\end{array}$ & $\bar{U}$ & $\bar{S}$ & $\bar{S}$ & $\bar{S}$ & $\bar{S}$ & $\bar{S}$ \\
\hline $\begin{array}{l}\text { UV absorption } \\
\text { degradation }\end{array}$ & $\begin{array}{l}\text { None at wavelength } \\
>0.35 \mu \mathrm{m}\end{array}$ & $\mathrm{U}$ & S? & S? & S? & $\bar{S}$ & Unk \\
\hline Hazing or clouding & $\begin{array}{l}\text { None at } 80^{\circ} \mathrm{C}, 100 \% \\
\text { RH }\end{array}$ & $S$ & $\bar{S}$ & $\bar{S}$ & $\bar{S}$ & $\mathrm{~S}$ & Unk \\
\hline $\begin{array}{l}\text { Minimum thickness on } \\
\text { either side of solar cells } \\
\text { in fabricated modules } \\
\end{array}$ & 6 mils & $S$ & $\bar{S}$ & $\overline{\mathbf{S}}$ & $\bar{S}$ & $\bar{S}$ & $\bar{S}$ \\
\hline $\begin{array}{l}\begin{array}{l}\text { Odor, human hazards } \\
\text { (toxicity) }\end{array} \\
\end{array}$ & None & $\bar{S}$ & $\bar{S}$ & $\overline{\mathrm{U}}$ & $\bar{S}$ & $\bar{U}$ & $\bar{S}$ \\
\hline Dielectric strength & $\begin{array}{l}\text { Sufficient to } \\
\text { electrically isolate } \\
\text { the cells and } \\
\text { interconnects }\end{array}$ & $\mathrm{S}$ & 3.1 & $\bar{S}$ & $S$ & $S$ & $\bar{S}$ \\
\hline Process compatibility & $\begin{array}{l}\text { Compatible with } \\
\text { automated cell } \\
\text { handling and } \\
\text { encapsulation } \\
\text { equipment }\end{array}$ & $\bar{S}$ & $\bar{S}$ & $\begin{array}{l}\text { Mold } \\
\text { Needed }\end{array}$ & $\begin{array}{l}\text { Mold } \\
\text { Needed }\end{array}$ & \begin{tabular}{|l|} 
Mold \\
Needed
\end{tabular} & $\bar{S}$ \\
\hline $\operatorname{Cost}^{+}$ & as low as possible & $\$ 0.6 / 1 b$ & $\$ 0.6 / 1 b$ & $\$ 0.4 / \mathrm{lb}$ & $\$ 2.2 / \mathrm{lb}$ & $\sim \$ 5 / \mathrm{lb}^{*}$ & $\sim \$ 2 / 1 b$ \\
\hline
\end{tabular}

$S$ is Satisfactory, $U$ is Unsatisfactory, and Unk is Unknown. +Cost is in terms of 1982 dollars. Also, the production cost for the liquid polymerizable systems is only $70 \%$ of the cost of the lamination process. *The cost of PEG-DA systems is difficult to estimate as no large scale production involves these materials. 
Title:

Organization:

Contributors:

\section{PV Module Degradation--EVA Pore Volume}

Polymer Science Dept., Penn State Univ., University Park, PA

D. L. Allara, principal investigator

\section{Objective:}

The objectives of this effort are (i) to conduct a methodical evaluation of the feasibility of using spectroscopic ellipsometry (SE) as a non-destructive means of detecting the pore or void volume changes in EVA as a result of increased curing of the polymer, and (ii) to use data from objective (i) to evaluate the potential of using SE for correlating the extent of degradation in field-degraded samples with the expected increase in gel content from prior NREL studies of clear, light yellow, yellow, yellow brown, and brown EVA.

\section{Technical Approach:}

As background, the long term degradation of modules fabricated with ethylene vinyl acetate (EVA) copolymer has become a concern to the DOE PV Program and to PV manufacturers. Evidence indicates that the EVA degradation is related to the formulation of EVA as well as the EVA copolymer itself. A need exists for measuring the extent of degradation of EVA for field deployed modules as well as for laboratory test specimens. One measure of the degradation is the gel content of the EVA, and how this changes with time. The increase in gel content will of necessity cause a change in the pore volume in the polymer from cross linking. The latter alters the physical properties of the polymer that may cause the EVA to lose important components of their physical properties.

EVA is being prepared with varying gel content as specimens that will be suitable for making state of the art SE measurements of the pore or void volume. The gel content is being varied from $50 \%$ to at least $100 \%$ and determined by an extraction procedure. When a suitable correlation is established for the EVA thickness that is optimal for SE measurements, the thickness will be varied to approach commercial reality and to determine the feasibility of SE measurements for determining pore sizes in EVA of increasing thicknesses.

SE will then be used to establish the feasibility of obtaining a correlation between the pore size and distribution in EVA prepared by NREL staff. The EVA, which will be degraded to various stages of yellowing from criteria already known to NREL staff, will be prepared on substrates and/or in the configuration required by the subcontractor to provide optimum results from the SE measurements. Then samples will be prepared at several thicknesses that approach reality to establish how well the SE measurements still correlate with the extent of degradation. Finally, SE measurements will be used as with the laboratory degraded specimens and for the same purpose except the samples supplied by NREL will be taken from field-degraded PV Modules.

\section{Results and Discussion:}

The work has been delayed because of the untimely departure of a key support person for the subcontractor. Some of the initial work is promising as outlined in the second paragraph in the technical approach. The subcontractor is expected to complete the work in 1995 under a no-cost extension of the effort. 


\section{Title: $\quad$ Long Term Effects on Roof-Mounted Photovoltaic Modules}

Organization: Southwest Technology Development Institute (SWTDI)

Contributors: $\quad$ S. Durand and A. Rosenthal

\section{Objectives}

The objectives of this effort were (i) to conduct a methodical monitoring of roof-mounted, PV module performance degradation, and (ii) to document module operating temperatures as a function of roof-mount design.

\section{Technical Approach}

The long term degradation of modules fabricated with EVA is a concern to the DOE PV Program and to PV manufacturers. The rate of EVA degradation is related to module operating temperature, which raises concerns about roof-mounted modules since they operate hotter than ground- or rack-mounted modules.

In coordination with NREL staff, SWTDI selected 12 modules of a specific design from each of 4 manufacturers that have been dedicated to a long term evaluation effort. Modules with a significant exposure history (installed prior to 1985) were selected. Both crystalline and thin film modules were chosen for evaluation.

All of the modules available for this project had already been mounted on the rooftops of prototype buildings at the SWTDI. Selected modules on each prototype building were rewired so that each module is electrically terminated at a terminal strip to facilitate taking individual module IV curves. Each of these modules has a thermocouple bonded to its back surface and terminated at the terminal strip. For the modules selected, SWTDI records were reviewed to retrieve available data on the "as delivered" module efficiencies. Manufacturers were also contacted to determine if records of individual module IV curves are available and if they would be able to retrieve IV curve data for the selected modules. When individual module efficiencies could not be obtained, measured array efficiencies were used to estimate individual module efficiencies.

For the selected modules, SWTDI measured the baseline individual module efficiencies at the contract initiation, and obtained IV curves (light only) at a measured module temperature. SWTDI developed a detailed measurement protocol that was reviewed and approved by NREL prior to beginning the measurements. SWTDI then made the measurements according to the approved protocol at 3-month intervals for two and one quarter years. SWTDI provided NREL with a copy of the data as well as with a summary report after data collection. Following baseline efficiency measurements, one module of each type was packaged and shipped to NREL for destructive analysis. The remaining modules remained mounted at SWTDI for continued, real-time, exposure testing in the Las Cruces, NM solar environment.

For objective (ii), SWTDI prepared a summary report documenting all module mounting designs installed at SWTDI along with an evaluation of the module operating temperatures. The summary report included a commentary on the various mounting methods that discusses 
the design tradeoffs such as ease of installation, ease of maintenance, water tightness, and the design impact on module operating temperature. Results of a 1985 SWRES study of module temperature vs. module spacing above the roof were also included. This summary report was delivered to NREL in 1992, about three months after the contract was initiated. For objective (i), the final report was delivered to NREL in May, 1994.

The criteria for selecting the four arrays/module types used in this study are as follows. All candidate modules had to have been encapsulated with EVA (ethylene vinyl scetate) as per JPL Block V specifications. Preference was given to the arrays and mc iles that had achieved the longest cumulative exposure to sunlight and for which early performance documentation was available.

The four arrays/module types selected for the study are (a) Solarex model MIT\#1002967 polycrystalline silicon modules installed in July 1981, (b) Mobil Solar model Ra-180 single crystal silicon modules installed in October 1983, (c) Solarex model SA-20 amorphous silicon modules installed in May 1988, and (d) Sovonics model P-101 amorphous silicon modules installed in January 1989. All performance reports presented mean ratings (normalized to conditions of $1000 \mathrm{~W} / \mathrm{m}^{2}$ and $45^{\circ} \mathrm{C}$ module temperature) for the modules in each of the four arrays. Normalization was achieved using coefficients derived according to the ASTM standard E1036-85. That method utilizes IV data obtained over a range of temperatures. The coefficients for each module type are presented for each module in the final report to the technical monitor. The mean module rating is presented in Table 1 for each array under test since the project was begun in 1992.

\section{Table 1. Normalized Mean Module Ratings In Watts (W)}

$\begin{array}{lrrrrrrr} & (7 / 92) & (1 / 93) & (4 / 93) & (7 / 93) & (12 / 93) & (4 / 94) & \text { Loss } \\ \text { Array } & (\mathrm{W}) & (\mathrm{W}) & \frac{(\mathrm{W})}{(\mathrm{W})} & \frac{(\mathrm{W})}{(\mathrm{W})} & \frac{\%}{47.62} & 3.6 \% \\ \text { Solarex MIT } & 49.05 & 49.60 & 47.87 & 48.19 & 49.96 & 47.62 & 1.4 \% \\ \text { Mobil Ra-180 } & 129.45 & 128.45 & 129.40 & 131.10 & 128.53 & 127.63 & 11.81 \\ \text { Solarex SA-20 } & 12.21 & 11.31 & 11.91 & 12.5 & 10.75 & 11.81 & -\ldots \\ \text { Sovonics P-101 } & 10.07 * * & 7.50 & 7.61 & 8.72 & 7.5 & 7.61 & \ldots\end{array}$

*See text for a $10 \%$ decline in one year for one of the eight modules.

**Inaccurate first rating reflects improper normalization.

After two years of study, some of the modules demonstrate an unambiguous decline in performance, as can be seen by the loss in percent over the 2-year period. For example, the Solarex MIT modules exhibit a collective loss of $3.6 \%$ per module. However, a detailed analysis on a module by module basis for the ten Solarex modules deployed in 1981 show declines of $9.9,6.8,5.6,4.0,3.1,1.6,1.4,1.4,1.4$, and $0.4 \%$. While the average of all ten modules is $3.6 \%$ or $1.8 \%$ per year, three of the modules exhibited declines in excess of $2.5 \% / y e a r$. All of the modules have a yellow-brown color to the EVA. The widening variances in the module power output can be a source of mismatch losses in large arrays, but the only possible mismatch losses in the present study is for differences in the output of each individual cell in series with each other. The results of the study plus additional data will be presented by A. Rosenthal at the PV Module Performance and Reliability (PVMR) Workshop in September, 1995. Additional comments on each of the four types of modules are now given. 
The Solarex MIT modules have had constant solar exposure since 1981. The modules show EVA yellowing around the centers of the polycrystalline cells. Initial estimates of the module normalization coefficients were revised over time as a database of module IV curves was acquired under a variety of operating conditions. Derivation of the normalization coefficients requires IV data collected over a range of operating temperatures. In the field, this range of operating temperatures is only attainable over a period of months. Initially, 12 modules were placed under test. At present, 10 modules are still under test with two having been removed in accord with the test plan. For the 10 modules still under test, the mean module rating has dropped about $2 \mathrm{~W}$ in the course of two years of tec ng. While this is within the limits of measurement error and can not be considered indicative of performance degradation, there are individual modules whose rating decline is significant and measurable. One module has undergone performance degradation of over $9 \%$ in the two years of study. Its normalized rating has dropped from $48 \mathrm{~W}$ in July 1992 to about $43 \mathrm{~W}$ in April 1994. At the same time, the fill factor for this module has dropped from $61.5 \%$ to $56.9 \%$. Other modules show virtually no performance degradation over the same period as given in the preceding paragraph.

The Mobil Ra-180 array was installed in October, 1983. The modules show considerable EVA browning around the centers of the crystalline cells. As with other arrays in the study, the initial estimates of the module normalization coefficients were revised over time as a database of module IV curves was acquired under a variety of operating conditions. Derivation of the normalization coefficients requires IV data collected over a range of operating temperatures. In the field, this range of temperatures is only attainable over a period of months. Initially, 12 modules were placed under test. At present, 10 modules are still under test with two having been removed in accord with the test plan. For the 10 Mobil modules still under test, the mean module rating has dropped only about $2 \mathrm{~W}$ in the course of two years of testing. This is within the limits of measurement error and can not be considered indicative of performance degradation. However, unlike the Solarex MIT array, none of the modules in the Mobil array have degraded significantly more than the others. All ten modules have undergone a decline of between $1.5 \%$ and $2 \%$ in their normalized ratings while fill factors have remained practically constant.

The Solarex SA-20 array was installed in May 1988. These single junction amorphous silicon modules show no evidence of EVA browning. As with the other arrays in the study, initial estimates of the module normalization coefficients were revised over time as a database of module IV curves was acquired under a variety of operating conditions. Derivation of the normalization coefficients requires IV data collected over a range of operating temperatures. In the field, this range of operating temperatures is only attainable over a period of months. Initially, 12 modules were placed under test. At present, 8 modules are still under test. Two modules have been removed from the array in accord with the test plan. Two other modules have failed. The mean module rating in January, 1993 was $11.31 \mathrm{~W}$. For April 1994, the mean is $11.81 \mathrm{~W}$. However, the assessment of performance of amorphous silicon modules is complex. The apparent performance of the modules changes with the season and the normalization equations are not well defined. For all but one of the modules under test, the range of seasonal performance has remained constant. That is, the rating achieved in the summer months is regained each year. The single exception is module 9 , which has undergone performance degradation of approximately $10 \%$ in the two years of study. Its normalized summer rating has dropped from $11.5 \mathrm{~W}$ in July, 1992 to about $10.4 \mathrm{~W}$ in July, 1993. Comparison of its winter ratings shows a similar decline: $10.1 \mathrm{~W}$ in November, 1992 and $8.9 \mathrm{~W}$ in December, 1993. At the same time, the fill factor for this module has dropped from $45 \%$ to $42 \%$. For comparison, other modules show no performance degradation 
over the same period. By comparing summer and winter data, both their normalized rating and fill factor have remained stable over the two years of study.

The Sovonics P-101 array was installed in January, 1989. These single junction amorphous silicon modules show no evidence of EVA browning. As with other arrays in the study, initial estimates of the module normalization coefficients were revised over time as a database of module IV curves was acquired under a variety of operating conditions. Derivation of the normalization coefficients requires IV data collected over a range of operating temperatures. In the field, this range of operating temperatures is only attainable over a period of months. Initially, 12 modules were placed under test. At present, 10 modules are still under test. Two modules have been removed from the array in accord with the test plan. Unlike the other three arrays under test, the modules of the P-101 array have not degraded measurably either collectively or individually. Comparing like-season data, the summer and winter ratings for each module in the array have remained constant for all the modules under test throughout the two years of study.

\section{Future}

The SWTDI subcontract expired in April, 1994; a new subcontract has been placed to cover an additional 15 months of monitoring beginning in November, 1994. The work reported by SWTDI and NREL analysis of the data demonstrate that monitoring individual modules is essential to identify which ones are undergoing accelerated degradation. The modules that degrade the most can be more gainfully subjected to post mortem analyses to learn more about the causes of the degradation.

The only other systematic monitoring of performance losses of individual modules in an array was presented at the PVMR Workshop by D. Faiman of Ben Gurion U. (BGU), Israel in September, 1994. Both the SWDTI and BGU studies would be better if I-V data had been gathered on each individual module at the time of deployment and periodically thereafter, and if dark IV data were also collected during the present and ongoing monitoring. The latter has been specified in the SWTDI contract extension. 
Title: NREL Module Energy Rating Methodology Project

Organization: Endecon Engineering, San Ramon, CA

Contributors: $\quad$ C. Whitaker, Principal Investigator;; J. Newmiller; W. Howard; L. Mrig, TRC Chairman; B. Kroposki; D. Myers; K. Emery; J. Anderson. Also industry representatives that comprise the Technical Review Committee.

\section{Objective}

o Develop a simple measure of PV performance that will provide a realistic estimate of how one or more modules will perform in a user's specific application.

\section{Approach}

o Develop and validate a PV performance model of sufficient accuracy

o Define weather data profiles that will describe the range of operating conditions in the U.S. and emphasize performance differences between various module types

o Develop a strawman approach to a module energy rating

o Convene an industry-representative Technical Review Committee (TRC) to provide feedback and guidance on the strawman and subsequent documents

\section{Results}

The contract for this project was initiated in May and a kickoff meeting was held at NREL on May 12. A research approach document was developed in June. A list of potential TRC participants - representing manufacturers, users, National Labs, and academia - was compiled and a participation request letter was issued in August. Response to this letter was quite high. An overview of the Module Energy Rating Project was presented at the NREL PV performance and Reliability Workshop in September.

The first TRC meeting was held in November where the strawman approach was presented and decisions on many items were made. The TRC in general agreed with the approach presented by the MER team. The following list summarizes the group's conclusions:

o Target audience is primarily the end user (consumer, system integrator, OEM, distributor, utility engineer).

o Rating will provide stabilized single-module energy values in Watt-hours.

o Energy values will be provided for both peak power and fixed voltage operation.

o Flat plate modules will be modeled with fixed latitude-tilt orientation. Will attempt to develop multipliers for tracking orientations.

o Concentrator modules will be modeled with two-axis tracking orientation.

o Energy values will be based on (approximately) four extreme condition days and one average day.

o Input data will include irradiance (global and direct), ambient temperature, wind speed, location and date (for sun position), and spectrum.

Permission was obtained from PG\&E to use data from their outdoor test facility (the PV test Facility, PTF) in San Ramon, CA and from the PVUSA project. Review of potential PV 
performance models was initiated and several candidate models were obtained. Three models from the PTF were selected for further analysis and testing. These modules included single crystal, poly crystalline, and amorphous silicon, and there are $20 \mathrm{~kW}$ arrays at PVUSA of identical or similar design. The three modules were sent to NREL in October for flash testing. Results of these flash tests were used to generate model coefficients. Based on these models and weather data from San Ramon, performance under a range of weather conditions was estimated. Similar data were generated for several NREL modules. These results were presented at the TRC meeting.

\section{Future Plans}

The project will continue to evaluate available performance models and methods of generating modeling coefficients. In addition to irradiance and ambient temperature, spectral, thermal (i.e. annealing) and solar incidence angle effects will be investigated. Module characterization tests will be redone at NREL using different equipment in an attempt to simplify the procedure and obtain more consistent results.

U.S. weather data will be reviewed with NREL climatologists to obtain appropriate reference days. A second (and possibly third) TRC meeting will be held to present the latest results. Results will be generated for the resulting models and weather data.

Once the rating method is finalized, it will be offered to IEEE Standards Coordinating Committee 21 as a basis for a new IEEE standard. 
Title:

Organization: Sunset Technology Co., Highlands ranch, Colorado.

Contributors: $\quad$ A.J. Anderson, Principal Investigator. In addition, R. DeBlasio, L. Mrig, and B. Kroposki of NREL contributed guidance, technical advice, and testing assistance for this effort.

\section{Objectives}

o To develop new equations for translating photovoltaic (PV) performance values from one temperature and irradiance condition to another in a simple, accurate manner.

o To participate in the development of a new energy rating system for PV modules which will provide the user with day-long energy gathering values for a variety of environmental conditions. o To participate in all PV standards activities both nationally and internationally. These include the IEEE, ASTM, UL, USTAG and IEC standards organizations, and the ASU Certification and Accreditation Committee.

\section{Technical Approach}

o The development of the new translation equations was based on a combined analytical and experimental approach. The equations were initially developed based on logic, and were verified and modified by comparison of the analytical results with actual experimental results.

o The energy rating task consisted of two elements. The first was a supportive effort of the Endecon energy rating contract. The second was to develop an independent approach based on testing modules in the laboratory using a simple temperature-irradiance matrix, and then using that data to evaluate the modules energy gathering ability for 5 hypothetical days of operation (4 extreme days plus one moderate day).

o The standards technical approach consisted of attending all of the standard meetings in the U.S. and around the world which were related to PV modules. A very active role was pursued by the actual writing of new standards, and by participating in the leadership of the standards organizations.

\section{Results}

o New translation equations were developed for translating all of the key PV performance parameters over a very wide range of temperature and irradiance conditions. These equations are more universal and more accurate than current equations, and are simpler to use. The equations were verified by comparison to actual test data from a limited number of amorphous silicon, single-crystal silicon, and polycrystalline silicon modules.

o Two meetings were attended on the Energy Rating task with NREL, Endecon, and industry representatives. In addition, an independent energy rating approach was developed with 4 main sub-parts identified by the acronyms UMP, REF, WIFE and COP: 
UMP - Universal Map of Performance: This is a matrix of irradiance and module temperatures for which module performance would be measured.

REF - Reference Environmental Functions: This is a group of five reference sets of data of irradiance and ambient temperature versus time of day. Four of these "reference days" represent extremes of weather in the United States, and the fifth represents a nominal (moderate) type of day.

WIFE - Wattage Influence Factor Effects: This segment consists of modifiers which take into account module temperature factors such as wind speed, ambient temperature, irradiance and incidence angle; atmospheric spectral irradiance effects such as air mass, water vapor, and turbidity; spectral effects; and the effect of incident angle losses such as surface reflections.

COP - Coefficients of Performance: These present the results of the evaluation of the UMP module data versus the five REF "reference day" irradiance and temperature characteristics, and then modified by the corrective WIFE factors. These are presented as watt-hour values for maximum power applications, and as amp-hour values for battery charging applications.

o All of the IEEE/SCC21, ASTM E44.09, USTAG, and IEC TC82 meetings on PV modules were attended in 1994. In addition, all of the ASU meetings on Certification and Accreditation were attended. Participation on the NEC and IEEE PAR 1374 reviews were performed via mail, FAX, and phone. In addition to the meeting attendance, standards are also being prepared for mechanical load testing, marine environmental qualification, linearity determination of PV devices, world-wide qualification with multiple degrees of difficulty, and for more accurate definition of solar simulator performance. Standards leadership roles include the IEC TC 82 Secretariat, and the IEEE/SCC21 Secretary duties.

\section{Future Plans}

All of the work described will continue into 1995.

o The translation equation work will continue if the optional Task 1.1 is authorized by NREL. The translation equations will be checked against the newer technologies such as CIS and CdTe, and against modules exhibiting performance flaws. Additionally, some equation modifications will be evaluated to further improve the accuracy.

o The energy rating work will be documented with a final report on the UMP/REF/WIFE/COP concept. The collaborative work with Endecon will continue, and a final concept merging the best aspects of each effort should lead to the preparation of an IEEE standard. A parallel effort is being worked on in the IEC TC 82/WG 2 committee.

o The standards work will continue, but a greater effort will be made to contribute to the U.S. IEEE SCC21 effort. 
Title:

\section{Organization:}

Contributors:

\section{Support for the National Electrical Code}

\author{
Solar Energy Industries Association
}

D. H. Meakin, Program Manager; L. Mrig, R. DeBlasio, W. Bower, J. Wiles, H. Meyer, R. Wills, SEIA's NEC Technical Review Committee.

\section{Objectives}

The primary objective of this activity is to support the PV industries representation on the NEC Code Making Panel 3 (CMP3) Task Group for NEC Article 690 and to facilitate the industries input to the Task Group through the Solar Energy Industries Association's NEC-Technical Review Committee (NEC-TRC). The primary charge of the Task Group (TG) is addressing action items assigned to it by the chair of CMP3 concerning proposed amendments to NEC Article 690 for the current code cycle. Its secondary objective is to identify code issues relevant to the industry and to work with industry to address those issues with respect to possible effects on the content of the NEC.

\section{Technical Approach}

Each member of the Task Group was charged with gathering information on a particular issue and reporting back to the committee on their findings. Sources included experts both inside and outside the industry. This was done to insure that each issue was thoroughly addressed from several points of view. Exploration of a given issue by several committee members was also encouraged and on several occasions occurred.

To facilitate industry input, the TG and the NEC-TRC met jointly. These joint meetings helped to insure that the committee's findings and recommendations represented consensus opinion as much as possible. Members of the task group also presented their findings and discussed the issues under consideration at several important industry workshops and standards meetings.

The Task Group met in person approximately every three months over the course of the year to review the work done by its members and to consider inputs and comments from the NEC-TRC members as well as other concerned individuals.

\section{Results}

The committee was able to make recommendations on the majority of the action items it received from CMP\#3. These resolved action items included the following issues.

1. Addition of a section to Article 690 addressing requirements for PV systems over 600 volts nominal.

2. Inclusion of a warning regarding the phenomenon of electroendosmosis.

3. Clarification of language in several sections of Article 690 .

The committee was able to identify several issues that need to be addressed during the 1999 code cycle. This code cycle begins in September of 1995. Task Group is currently preparing a 
preliminary outline for its activities at this time.

The feedback generated from the presentations given by task group members at various industry meetings and to standard groups provided valuable input in guiding the group in its activities.

\section{Conclusions}

The Photovoltaic industry and the Task Group have been able to effectively address important issues and resolve them satisfactorily. However, because the NEC is a continuing activity so must the industries efforts be.

To keep the NEC current with PV technology SEIA and the industry must continue to work with the TG to address Article 690. There must also be a concerted effort to educate the industry as to the various activities regarding the NEC. 
Title:

Organization: $\quad$ Solar Energy Industries Association (SEIA), Washington, D.C.; Institute of Electrical and Electronics Engineers, Inc.;IEEE, NY, NY; American National Standards Institute, ANSI, New York, NY.

Contributors: A. J. Anderson, Secretariat IEC/TC82; S. Chalmers, Assistant Secretariat IEC/TC82; R. DeBlasio; L. Mrig; R. D'Aiello; J. Wohlgemuth; D. Feder; C. Whitaker; D. Meakin. In addition, all experts that comprise IEEE Standards Coordinating Committee (SCC) 21, Photovoltaics; United States Technical Advisory Group (USTAG) to IEC/TC82, responsible for United States input into the international PV arena; ASTM Committee E44.09, PV Electric Power Systems, members serving as a team to assist in the development of the PV standards.

\section{Objective}

The objective is the transfer of the technology of photovoltaic modules, balance of systems, components, and services from the US standards arena to the international standards arena. This effort seeks to transfer the collective experience, consensus opinions and standards of the US photovoltaics industry to the development of international photovoltaic standards.

\section{Technical Approach}

Technical transfer is achieved by drafting national and international standards that are valid for use by both industrialized and emerging third world nations. Such objective of these standards are to reduce product cost, increase product performance and open larger world markets to the photovoltaic and related industries.

The objective is being pursued by sponsoring the secretariat of the International Electrotechnical Commission (IEC) Technical Committee 82, Solar Photovoltaic Energy Systems. In support of the secretariat activities, this effort also supports communications with the US industry through the IEEE/SCC21, ASTM committee E44.09, and the USTAG to IEC/TC82.

\section{Results}

The following international standards have been published.

1. IEC 904-5, determination of the Equivalent Cell Temperature (ECT) of Photovoltaic (PV) Devices by the Open-Circuit Method.

2. IEC 904-6, Requirements for Reference Solar Modules 
Standards on the following subjects are currently in various stages of development.

1. Characteristics of the Utility Interface for Photovoltaic (PV) Systems.

2. Thin-Film Terrestrial Photovoltaic Modules - Design Qualification and Test.

3. Equipment and Safety Specifications for Direct Coupled PV Pumping Systems.

4. Safety Test Procedures for Utility Grid Connected 1 . sotovoltaic Inverters.

5. Reference Solar Day.

6. Photovoltaic System Performance Monitoring - Guidelines for Measurement, Data Exchange and Analysis.

7. Procedures for Measuring Efficiency of Power Conditioners Used in Photovoltaic Systems.

8. Safety Testing Requirements for PV Modules.

9. On-Site Measurements of Crystalline Silicon Photovoltaic (PV) Array I-V Characteristics.

10. General Description of Photovoltaic (PV) Power Generating Systems.

11. Guidance for Spectral Measurement of Photovoltaic (PV) Devices.

12. Solar Simulator Performance Requirements.

13. Computation of Spectral Mismatch Error Introduced in the Testing of a Photovoltaic Device.

14. Susceptibility of a Module to Accidental Impact Damage.

15. Rating of Direct Coupled Photovoltaic Pumping Systems.

16. Salt Mist Corrosion Testing of Photovoltaic Modules. 
Title: PV Utility/Industry Engineering Projects

\author{
Organizations: Advanced Photovoltaic Systems \\ Energy Conversion Devices \\ Energy Photovoltaics \\ Integrated Power Corporation \\ Solarex \\ Ut lity Power Group
}
Contributors, Ron Matlin, Advanced Photovoltaic Systems Masat Izu, Energy Conversion Devices
Alan Delahoy, Energy Photovoltaics
Dave Panico, Integrated Power Corporation
Jim Morris, Solarex
Gilbert Duran, Utility Power Group

\title{
Objective
}

The objectives of the project are 1) to assist in the development and qualification of utility-scale amorphous silicon photovoltaic modules, 2) to improve the reliability of utility-scale amorphous silicon modules and utility grid-connected systems, 3) to demonstrate potential markets for amorphous silicon photovoltaic modules and systems, and 4) to form utility/manufacturer/user teams to promote photovoltaics within the utility sector.

\section{Technical Approach}

Amorphous silicon photovoltaic technology has a significant potential for reducing the cost of photovoltaics from today's costs. Before that potential is realized, newer modules must be developed and validated in systems. A Request for Proposal (RF-1-11061) was issued in 1991 and all awards were completed in 1993. Awards were made to Advanced Photovoltaic Systems, Energy Conversion Devices, Energy Photovoltaics, Integrated Power Corporation, Solarex, and Utility Power Group. Module development coupled with system integration reinforces the need to consider the system as a whole. The subcontracts awarded for this project consist of Phase 1 for module development and qualification and Phase 2 for system design, deployment, and monitoring. In some subcontracts the system design is part of Phase 1.

For Phase 1 module performance goals are based on stabilized performance not initial performance. Amorphous silicon photovoltaic modules exhibit an initial 15-30\% decrease in power within the first two months of system deployment. After this initial power decrease the performance appears to stabilize. The stabilized module power output is defined as the power output measured under standard test conditions after the module has been exposed to $1000 \mathrm{~W} / \mathrm{m}^{2}$ of nominally white light at a module temperature of $50^{\circ} \mathrm{C}$ and under a fixed resistive load. The stabilized power output is a better indicator of future performance 
than the initial power output. Also, modules developed for Phase 1 must pass NREL's "Interim Qualification Tests and Procedures for Terrestrial Photovoltaic Thin-Film Flat-Plate Modules" (SERI/TR-213-3624). Design reviews of the proposed systems will take advantage of the expertise at NREI, Sandia National Laboratory, and the PVUSA Project.

For Phase 2 the modules will be deployed in grid-connected systems ranging from $3.4 \mathrm{kWp}(\mathrm{ac})$ to $50 \mathrm{kWp}(\mathrm{ac})$. The performance of the system will be determined after 60 days of operation. Performance will be monitored by a data acquisition system supplied by the PVUSA Project. NREL will monitor the performance and the data will be shared with the photovoltaic manufacturer, the utility, and PVUSA. Because of DOE funding limitations, only one system is expected to be constructed.

\section{Energy Conversion Devices}

Subcontract AI-2-11061-1 was awarded to Energy Conversion Devices, Troy, Michigan. The objectives are to develop triple-junction amorphous silicon photovoltaic modules with a $7 \%$ average stabilized total-area efficiency, certify that these modules pass NREL's Interim Qualification Tests and Procedures for Terrestrial Photovoltaic Thin-Film Flat-Plate Modules, and deploy these modules in a $10 \mathrm{kWp}(\mathrm{ac})$ system. During Phase 1, ECD will develop triple-junction amorphous silicon photovoltaic modules with dimensions of approximately $34 \mathrm{~cm}$ by $126 \mathrm{~cm}$ and certify that these modules have passed NREL's Interim Qualification Tests and Procedures for Terrestrial Photovoltaic Thin-Film Flat-Plate Modules. In FY 1994, NREL light-soaked 4 triple-junction modules. After 600 hours of light-soaking, the total-area average efficiency of the 4 modules is $6.8 \%$ measured under a Spire solar simulator. ECD is in the process of certifying the modules according to NREL's Interim Qualification Tests and Procedures for Terrestrial Photovoltaic Thin-Film Flat-Plate Modules.

\section{Solarex}

Subcontract AI-3-11061-2 was awarded to Solarex Thin Film Division. The objectives are to develop triple-junction amorphous silicon photovoltaic modules with a $7 \%$ average stabilized total-area efficiency, certify that these modules pass NREL's Interim Qualification Tests and Procedures for Terrestrial Photovoltaic Thin-Film Flat-Plate Modules, and deploy these modules in three systems totalling $10.2 \mathrm{kWp}(\mathrm{ac})$. Phase 1 is negotiated to develop multi-junction amorphous silicon modules with dimensions of approximately $30 \mathrm{~cm}$ by $90 \mathrm{~cm}$ and certify that these modules have passed NREL's Interim Qualification Tests and Procedures for Terrestrial Photovoltaic Thin-Film Flat-Plate Modules. Solarex has sent 4 modules with an average initial total-area efficiency of 8.4\%. The modules use a tandem device structure of a-Si and a-SiGe cells. These modules are being light-soaked for 600 hours. Solarex is in the process of certifying the modules according to NREL's Interim Qualification Tests and Procedures for Terrestrial 
Photovoltaic Thin-Film Flat-Plate Modules.

\section{Integrated Power Corporation}

Subcontract AI-2-11061-3 was awarded to Integrated Power Corporation, Rockville, Maryland. During Phase 1, IPC delivered 4 modules (UPM-880) from United Solar Systems Corporation that NREL light soaked for 600 hours at $50^{\circ} \mathrm{C}$ under nominally white light of $1000 \mathrm{~W} / \mathrm{m} 2$. Measured under standard conditions, the average power output of the four light-soaked modules was $21.5 \mathrm{~W}$, which was within measurement error of the gocl of $22 \mathrm{~W}$ per module. USSC certified that these modules passed NREL's Interim Qualification Tests and Procedures for Terrestrial Photovoltaic Thin-Film Flat-Plate Modules. IPC installed the system on the roof of a New York Transit Authority warehouse in Maspeth, New York. The system uses a unique ballast-weighted configuration using precast concrete curbs that avoids the need to penetrate the roofing membrane. The PV system became operational on June 18, 1993. After 60 days of operation, the system was rated at $17 \mathrm{~kW}(\mathrm{ac})$ which is greater than the contractual requirement of $15 \mathrm{~kW}(\mathrm{ac})$. Data are routinely collected on system performance. A paper on Maspeth PV System: One Year Later was presented in 1994 [1].

\section{Energy Photovoltaics}

Subcontract AI-3-11061-4 was awarded to Energy Photovoltaics, Princeton, New Jersey. The objectives are to develop amorphous silicon photovoltaic modules with a $6.2 \%$ average stabilized total-area efficiency, certify that these modules pass NREL's Interim Qualification Tests and Procedures for Terrestrial Photovoltaic Thin-Film Flat-Plate Modules, and deploy these modules in three $10 \mathrm{kWp}(\mathrm{ac})$ systems. During Phase 1, EPV will develop amorphous silicon modules with dimensions of approximately $30 \mathrm{~cm}$ by $90 \mathrm{~cm}$, certify that these modules have passed NREL's Interim Qualification Tests and Procedures for Terrestrial Photovoltaic Thin-Film Flat-Plate Modules, and design three $10 \mathrm{kWp}(\mathrm{ac})$ system using these modules. To date, EPV has not sent any amorphous silicon modules.

\section{Utility Power Group}

Subcontract AI-3-11061-5 was awarded to Utility Power Group, Chatsworth, California. The objectives are to develop amorphous silicon photovoltaic modules with a $6.3 \%$ average stabilized total-area efficiency, certify that these modules pass NREL's Interim Qualification Tests and Procedures for Terrestrial Photovoltaic Thin-Film Flat-Plate Modules, and deploy these modules in a $20 \mathrm{kWp}$ (ac) system. During Phase 1, UPG will develop amorphous silicon modules with dimensions of approximately $33 \mathrm{~cm}$ by $37 \mathrm{~cm}$ and certify that these modules have passed NREL's Interim Qualification Tests and Procedures for Terrestrial Photovoltaic Thin-Film Flat-Plate Modules: UPG has sent two intermediate module deliverables with an average initial total-area efficiency of $6.3 \%$. 


\section{Advanced Photovoltaic Systems}

Subcontract AI-3-11061-6 was awarded to Advanced Photovoltaic Systems, Princeton, New Jersey. The objectives are to develop amorphous silicon photovoltaic modules with an average stabilized power output of $63.5 \mathrm{~W}$, certify that these modules pass NREL's Interim Qualification Tests and Procedures for Terrestrial Photovoltaic Thin-Film Flat-Plate Modules, and deploy these modules in a $50 \mathrm{kWp}(\mathrm{ac})$ system. Phase 1 was renegotiated to develop 63.5 $W$ amorphous silicon modules with a total area of approximately 1.2 $\mathrm{m}^{2}$ and submit a preliminary design for a $50 \mathrm{kWp}(\mathrm{ac})$ system using these modules. During Phase 1 APS sent 4 modules with an average initial power output of $75 \mathrm{~W}$. There will be no Phase 2 photovoltaic system deployed.

\section{References}

1. Byron Stafford, "Maspeth PV System: One Year Later," Conference Proceedings of the Photovoltaic Performance and Reliability Workshop, Lakewood, Colorado, September 21-23, 1994, NREL/CP-411-7414. 


\title{
6.0 PV APPLICATIONS AND MARKET DEVELOPMENT
}

\author{
Roger Taylor, Manager
}

During FY 1994 the PV Applications and Market Development project continued to provide an NREL focus for supporting the increasingly important analytical, applications, and market development efforts of the DOE National Photovoltaics Program and the $r$ ice of Solar Energy Conversion (OSEC). The overall objective of this project is to conduc: coordinated set of market conditioning and project development activities (through NREL in-house research, NREL subcontracted research, and technical support to DOE GO-contracted efforts) that lead toward sustainable domestic and international markets for PV systems.

The project is divided into seven interrelated areas of activity, each led by an experienced NREL staff member, as shown in Table 6-1. In many instances, the specific activities implemented within the project cross the activity and personnel boundaries implied below. This crossfertilization is encouraged by PV Program and NREL line management, and in every case it leads to better activities and products. The delineations listed in Table 6-1 are largely for administrative convenience and to identify a lead contact.

The accomplishments and ongoing efforts in each activity area are briefly discussed in the following sections.

\section{NREL Project Management}

Overall management of the PV Applications and Market Development project includes providing full-time support to OSEC and the DOE PV Program.

\section{Domestic Markets and Utilities}

PV-DSM Analysis. NREL continues to lead the growing U.S. and international interest in PV analysis as a demand-side management (DSM) option. Coordination and management of two key subcontracts, along with many meetings and interactions with the Utility PhotoVoltaics Group (UPVG) and others, has established NREL leadership in this exciting new area. Coupled with the product development activities under PV:BONUS, PV as a DSM option is moving closer to reality.

Center for Energy and Environmental Policy Research at the University of Delaware is conducting a project entitled,"Evaluation of DSM Opportunities for Photovoltaics." The objectives of this ongoing project are to:

1. Evaluate existing residential, commercial, and industrial DSM incentive programs with regard to incentive levels and utility avoided costs

2. Analyze the interactions between incentive programs, avoided costs, and regulatory influences 
3. Develop a spreadsheet evaluation model to describe the economic costs and benefits to electric utilities and their customers

4. Develop and promote the role PV can play in residential and commercial DSM programs.

\begin{tabular}{|c|c|c|}
\hline Project Activity & Contact Name & Telephone \\
\hline $\begin{array}{l}\text { NREL Project Management } \\
\text { DOE-HQ staff support }\end{array}$ & $\begin{array}{l}\text { Roger Taylor } \\
\text { Dave Mooney }\end{array}$ & $\begin{array}{l}(303) 384-6432 \\
(202) 586-1725\end{array}$ \\
\hline $\begin{array}{l}\text { Domestic Markets and Utilities } \\
\text { PV-DSM Analysis } \\
\text { Utility support (UPVG, PV4U, SCE, etc.) }\end{array}$ & $\begin{array}{l}\text { William Wallace } \\
\text { Christy Herig }\end{array}$ & $\begin{array}{l}\text { (303) 384-6476 } \\
\text { (303) 384-6546 }\end{array}$ \\
\hline $\begin{array}{l}\text { PV-Buildings } \\
\text { PV:BONUS } \\
\text { EPA Data Analysis }\end{array}$ & $\begin{array}{l}\text { Roger Taylor } \\
\text { Cecile Leboeuf }\end{array}$ & $\begin{array}{l}303) 384-6432 \\
(303) 384-6466\end{array}$ \\
\hline Sunrayce 95 & $\begin{array}{l}\text { Byron Stafford } \\
\text { Jeff Shapiro }\end{array}$ & $\begin{array}{l}(303) 384-6426 \\
(303) 384-6735\end{array}$ \\
\hline $\begin{array}{l}\text { PV Education, Assistance and Training } \\
\text { Solar } 2000 \text { Quarterly } \\
\text { NREL PV Technology Transfer }\end{array}$ & $\begin{array}{l}\text { John Thornton } \\
\text { Jack Stone } \\
\text { Robert McConnell }\end{array}$ & $\begin{array}{l}(303) 384-6469 \\
(303) 384-6470 \\
(303) 384-6419\end{array}$ \\
\hline $\begin{array}{l}\text { International Markets } \\
\text { Brazil } \\
\text { China } \\
\text { India } \\
\text { South Africa } \\
\text { Pakistan } \\
\text { Bulgaria } \\
\text { Russia } \\
\text { Egypt } \\
\text { Indonesia } \\
\text { Village Power Hybrid Team }\end{array}$ & $\begin{array}{l}\text { Roger Taylor } \\
\text { Cecile Leboeuf } \\
\text { William Wallace } \\
\text { Jack Stone } \\
\text { Robert McConnell } \\
\text { John Thornton } \\
\text { Dave Mooney } \\
\text { Ken Touryan } \\
\text { Larry Kazmerski } \\
\text { Roger Taylor } \\
\text { Cecile Leboeuf }\end{array}$ & 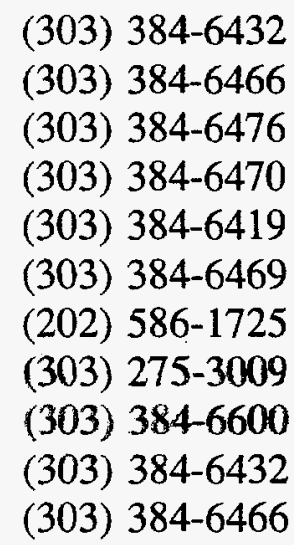 \\
\hline
\end{tabular}


The first part of this work was completed in 1993. During 1994, the effort was expanded to focus on developing the computer-based economic analysis tool (item 3) that captures utility and customer benefits of PV-DSM. This tool is being validated during 1995 with several participating utilities and will be made available to UPVG and others upon completion.

Atmospheric Sciences Research Center, State University of New York at Albany continued a project entitled,"Solar Resource/Utility Load Matching Assessment." The objectives of this study are to:

1. Investigate the viability of using insolation data measured by geostationary satellites

2. Infer the load-matching capability of PV systems in selected electric utilities across the United States.

By comparing PV load-matching capability estimated from the satellite data with that obtained from ground measurements, satellite data can be used to provide a good indication of PV effective load- carrying capability. During 1993, 20 participating utilities received a preliminary evaluation of PV's potential for contributing to meeting their system load requirements and are now better informed about how to pursue an interest in PV development. As with the DSM evaluation, the first part of this work was completed in early 1993. During 1994 additional utilities were matched with their solar resource data-an activity being conducted in cooperation with UPVG. In 1995 the effort is being expanded to include similar analysis of solar-buildings correlations using data from the EPA project sites, and discussions are under way regarding possible expansion to utilities in other countries.

DOE-GO Utility Support (UPVG, PV4U, SCE, etc). The PV Applications and Market Development group provides technical support to GO activities related to commercializing PV in the utility industry. During 1994, primary efforts went into critically reviewing and evaluating the UPVG/ TEAM-UP/PV-COMPACT proposals. In addition, activities being initiated by Southern California Edison and Georgia Tech (for the 1996 Olympics) are supported by the group. An NREL staff addition during the first part of FY 1995 will considerably enhance this support to DOE-contracted programs.

\section{Photovoltaic Buildings}

During 1993 a major multi-year three-phase procurement was initiated that is directed at supporting the development of cost-effective PV products and product-supply/product-user relationships that provide a sustainable market for PV in the buildings sector. Titled PV:BONUS (Building Opportunities in the U.S. for PV), the 5-year, \$25 million (DOE), government/industry cost-shared initiative is being contractually administered by DOE-GO as a financial assistance (cooperative agreement) program. The PV:BONUS program was fully under way during 1994, with Phase 1 team-building activities completed and Phase 2 product development efforts now under way.

Five cooperative agreements presently constitute the PV:BONUS Program. 
Energy Conversion Devices (EDC) (along with subtier organizations USSC, Arizona Public Service, Bechtel, Detroit Edison, MERRA, Michigan PSC, Minoru Yamasaki Associates, NAHB Research Center, Oakland CC, and Solar Design Associates) is developing two product lines: a standing seam metal roofing system and a shingle-like roofing system based on the ECD/USSC a-Si/stainless-steel PV technology.

Delmarva Power \& Light (DP\&L) (along with subtier organizations University of Delaware, Asc asion Technologies, and AC Battery Corporation) is conducting economic evaluations and proc act testing for a dispatchable PV system that DP\&L may market to commercial electric utility customers.

Solar Design Associates (along with subtier organizations Solarex and Kawaneer, among others) is developing a high-power (250-300-W), totally self-contained ac PV module that will generate $120-\mathrm{V}, 60-\mathrm{Hz}$ power. Such a module will be individually power tracked and can be paralleled together and connected directly into a building's electric service entrance or other electrical interface.

Fully Independent Residential Solar Technology, Inc. (along with subtier organizations Bradley Builders and Avis America, among others) is developing integrated PV energy systems for the modular home industry. These systems and homes will be marketed as stand-alone remote homes and as grid-connected units.

Advanced Photovoltaic Systems (APS) (and several subtier organizations) is now under contract to develop large-area PV window wall technology using the APS a-Si-on-glass modules.

In addition to the direct support of GO for the PV:BONUS procurement, NREL has contracted with the American Institute of Architecture (AIA)/Association of Collegiate Schools of Architecture, Joint Council on Architectural Research to conduct a project entitled, "Design for Photovoltaics: Curriculum Development for North American Architecture School Faculty." The objective of the project is to begin to institutionalize PV-buildings concepts by developing guidance for architectural school faculty in North America on how to incorporate PV information into the design curriculum. The materials developed over the course of this project are directly targeted at the 3000 members and 32,000 students active in North American schools of architecture in any given year. In 1994 the resource package was distributed to 50 key North American schools of architecture. The curriculum package consists of:

- The BOOKSHELF, a collection of five books, twelve reports and pamphlets, one magazine, two software programs, and two manufacturers' videos

- The SLIDE SET, consisting of nearly 500 slides of PV basics and applications

- The DESIGN GUIDELINES, which give students a quick way of approximately sizing PV arrays, assessing annual and daily electricity generation along with use profiles for various building types 
- $\quad$ The QUESTIONS that are used to generate class discussion during solar energy seminars. During 1995 and 1996, the course materials will be repackaged and refined to make up a self-study course that will be available through AIA as part of its new continuing education requirement for AIA member architects.

\section{Sunrayce 95}

Sunrayce is a DOE-sponsored biennial university design competition for solar-powered electric vehicles. It is dedicated to the educational development of university science and engineering students with its themes of education, energy, and the environment.

Following on the successful Sunrayce 93, the PV Applications and Market Development project has been assigned by DOE to manage the race again for 1995-Sunrayce 95 . The competitive procurement for participating schools and the necessary internal management structuring for the 1995 race were completed in 1994. The project is expected to result in a successful race from Indianapolis to Denver in June 1995.

\section{Photovoltaics Education, Assistance, and Training}

The technical assistance, education, and training activity continues and enhances specific actions directed at accelerating the commercialization of currently cost-effective PV products. It also supports utilities and other organizations that investigate the possible purchase of a PV system to meet the electrical needs of a particular site, and develops and provides educational materials for student training.

The stated goal of this subtask is to accelerate the acceptance and use of PV via technical assistance, facilitation, education, and the leveraged use of DOE funds. Specific activities engaged in during 1994 included:

- $\quad$ Facilitating contacts between potential users of PV and industry suppliers

- Conducting workshops for federal and state facility managers on how to specify, purchase, design, install, and maintain PV systems

- $\quad$ Providing information on PV system applications for public utility commission hearings

- Characterizing new market sectors; documenting case studies of cost-effective PV applications

- Performing special analyses in support of the National Photovoltaic Program.

Twenty to 30 inquiries, ranging from requests for literature and reports to actual support of projects, were received and dealt with each month. Funding was provided for the SOLAR 95 and SOLTECH 95 conferences. Testimony was provided for public utility commission hearings in Arizona, Colorado, Nevada, and Oregon. In at least two of these cases, the testimony was instrumental in getting renewable-oriented public policies adopted. 
Most subtask activities were domestically oriented, but training was also provided to support the U.S. DOE Country Studies Program. Under this program, other countries are being encouraged to develop renewables and other clean technologies to mitigate greenhouse gases. Engineers and planners are brought to the U.S. to receive training over a 2-week period. NREL staff provided a 1-day introductory workshop on all types of renewable energy systems.

\section{International Market Development}

Brazilian Rural Electrification Pilot Project. DOE/OSEC, through NREL, is undertaking a cooperative joint pilot project with eight states and the Centro de Pesquisas de Energia Eletrica (CEPEL) in the Federal Republic of Brazil. The objective is to establish and assess the efficiency, operability, and reliability of solar-energy-based rural electrification. During 1994, the Phase 1 activity in the states of Ceara and Pernambuco was completed, and major efforts were devoted to a competitive procurement that will result in a Phase 2 expanded program-including two $50-\mathrm{kW}$ hybrid power systems-being implemented in six additional states in 1995.

China. During 1994, NREL devoted considerable effort to helping DOE establish project opportunities in China. NREL organized two official visits for the DOE in January/February and in September/October 1994. These visits involved the participation of high-level and broad technical personnel from the United States, meeting with all key agencies in Beijing involved in renewable energy technology and policy development in China. During these visits, trips were also made to the provinces of Inner Mongolia, Gansu, and Hainan to explore specific renewable opportunities involving PV, wind, biomass, and solar thermal technologies.

During Secretary of Energy Hazel O'Leary's visit, three NREL staff members served on her advance team helping to organize meeting events in Beijing and Shanghai. The groundwork laid in these visits led directly to the signing of the Energy Efficiency and Renewable Energy Protocol by the Secretary of Energy with the Chinese State Science and Technology Commission in February 1995.

NREL will be involved in implementing renewable energy projects under this protocol agreement. In addition, during 1994, NREL signed a subcontract with the Chinese Academy of Sciences to initiate a renewable energy study in Inner Mongolia as a cooperative project. This project will also involve the participation of the Center for Energy and Environmental Policy at the University of Delaware.

India. During 1994, NREL devoted considerable effort to helping DOE establish project opportunities in India. For 4 weeks in January and February, NREL representatives visited some 30 companies in India who had expressed interest in diversifying their businesses into renewable energy. They prepared an extensive trip report that was distributed widely to the PV industry in the United States. Based on expressed interest, a number of firms were invited to join the trade mission organized by Secretary O'Leary in July. Several U.S. PV manufacturers signed joint-venture agreements with Indian companies to market and produce products in India. 
NREL began discussions with the Solar Energy Centre and the Tata Energy Research Institute to establish formal cooperative agreements. Both interactions were planned to result in signed memoranda of understanding between the institutions early in FY 1995.

NREL approached the Ministry of Non-Conventional Energy Sources (MNES) in India to jointly fund with DOE/NREL a sustainable renewable energy rural electrification project in several states in India, similar to the model followed in Brazil. The project was accepted by Minister Krishna Kumar of MNES, with implementation scheduled to begin in FY 1995. This 50-50 cost-shared project is expected to result in expanded business opportunities for U.S. industry in the growing Indian renewable energy market.

Russia. At the request of DOE-OTFA, project staff participated in a scoping trip to Moscow to investigate possible collaborative project opportunities between DOE/NREL and Russian scientists and engineers. Project funds are being provided by OTFA to investigate hybrid power system opportunities at three Russian sites during 1995 and to facilitate further collaboration and demonstrations with the InterSolarCenter in Moscow.

Egypt. In October 1994, NREL participated in and facilitated a significant solar and wind energy conference in Cairo, Egypt. NREL, Cairo's Ain-Shams University, the United Nations Educational Scientific and Cultural Organization, Alafandi Est., the Egyptian government's New and Renewable Energy Authority (NREA), the World Renewable Energy Network, and the International Energy Foundation sponsored the International Conference on Solar Electricity: Photovoltaics and Wind. The conference was held in Cairo, Egypt, from October 3-6, 1994. About 180 people attended, and there were more than 70 oral and poster presentations. More than 20 exhibitors, primarily companies from the United States and the Middle East, showed their products and services. Egypt's Minister of Electricity and Energy, M. Abaza, gave a presentation at the opening session of the Conference, followed by a $1 \frac{1}{2}$-hour visit with the conference exhibitors. Minister Abaza's presentation was highlighted in the Cairo newspapers.

During the conference, Minister Abaza and M. S. Zannoun, Director of NREA, met with DOE and NREL representatives, who emphasized the potential role of PV and wind technologies in Egypt's extensive rural electrification and reclamation programs. They accepted Minister Abaza's directive to provide a report, prepared jointly with NREA, documenting the cost effectiveness of PV in remote rural applications. The preparation of the report by NREL and NREA led to the identification of three projects for potential co-funding by Egypt and USAID in the areas of rural electrification of farms, desalination, and PV education. In a survey conducted after the conference, many of the exhibitors said the conference had led to sales and sales opportunities and they would be interested in attending future conferences.

Indonesia. Project staff members continue to support the IPC/Westinghouse efforts to commercialize village hybrid power systems in Indonesia. Numerous discussions were held during 1994, and financial support for installing and testing a prototype unit at NREL's National Wind Technology Center (NWTC) in 1995 is being implemented. 
Village Power Hybrid Team. As part of the growth in village power opportunities, which largely focuses on diesel fuel displacement, an NREL Director's Development Fund award was provided to help establish a sustainable village power focus and test capability at the NWTC. The PV Applications and Market Development project has participated in this effort, which closely aligns with several ongoing (Brazil) and future (India, China, Indonesia) hybrid project opportunities. 
Title:

Organization:

Contributors:
Design for Photovoltaics: Curriculum Development for North American Architecture School Faculty

\author{
AIA/ACSA Council on Architectural Research \\ Washington, DC
}

D. Evans, Principal Investigator; J. Reynolds, University of Oregon, Te nical Director; J. Cook, Arizona State University; R. Diamond, Ha vard University; D. Procos, Technical University of Nova Scotia; S. Strong, Solar Design Associates

\section{Objectives}

The purpose of the project was to undertake a sustained program of information development and dissemination focused on teaching photovoltaics-in-buildings in North American schools of architecture.

The focus of the work effort was a comprehensive "resource package" targeted at architecture school faculty and students. The package was developed by a team of technical advisors with expertise in both research and teaching of energy issues and consisted of the following elements:

- a basic primer on photovoltaics and their use in buildings, including background materials and teaching aids;

- guidelines on "how to teach" the information included in the primer, targeted at two different settings:

- in a lecture course on energy/environmental systems,

- as part of a studio design course;

- a series of case studies on successful use of photovoltaics in buildings;

- information on successful teaching of photovoltaics in architecture school curriculum.

In addition to the resource materials developed under the project, a feedback mechanism was also established whereby successful applications of the information in schools of architecture could be reported to the Research Council and made available to all other ACSA schools on a regular basis as part of an updated resource package. 


\section{Approach}

The project undertook the following interrelated series of tasks.

\section{A. Select Project Team}

In January 1993 the Research Council issued a call for qualifications to select the project team to develop the curriculum package. The call went to over 2500 faculty at the 118 member schools of the ACSA.

Responses to the call were reviewed in mid-March and the final team was selected:

John Reynolds, Team Leader and Principal Investigator

Jeffrey Cook, Team Member

Jeffrey Diamond, Team Member

Dimitri Procos, Team Member.

Steven Strong was then added to the team as a special technical advisor.

\section{B. Planning Meeting}

On April 16 and 17, 1993 the full project team met at NREL headquarters for a planning meeting to kick-off the project. At the meeting the team discussed the scope and content of the resource package and developed a basic workplan for the remainder of the project.

\section{Draft Resource Package}

Over the course of the summer Professor Reynolds and his assistants, with input from other project team members, worked to develop the resource package. Portions of the package were circulated to the team for comment in mid-August. Additional items were included in the final draft package, which was forwarded to each team member in midSeptember 1993 for pilot testing. The final package included 16 publications, 2 computer programs, 2 video tapes, one magazine article and close to 500 slides.

\section{Pilot Test - Resource Package Update}

Over the course of the Fall 1993 semester the project team pilot tested the resource package at their schools. The package was used in a variety of lecture and studio settings and each team member provided written comments to Professor Reynolds at the conclusion of the semester.

On March 25, 1994 the project team reconvened at NREL headquarters to review the results of the pilot test and to map out necessary changes to the resource package. At the conclusion of the meeting it was agreed that the final package would contain the following: 
1. Binder: Part \#1: Teaching results from Fall 1993, Book Summaries, Design Guidelines, Questions \& Answers;

Part \#2: $\quad 310$ Slides, annotations, and supporting information;

2. PV Cell in photo holder

3. Bookshelf: Photovoltaics in Architecture, Othar Humm and Peter Toggweiler, Birkhäuser Verlag, 1993

Photovoltaics in the Built Environment: Architectural Ideas

Competition, The Netherlands Agency for Energy and the

Environment, 1994

Building Integrated Photovoltaics, Kiss Cathcart Anders Architects, January 1993

Photovoltaic Fundamentals, Gary Cook, Lynn Billman, and Rick Adcock, SERI/TP, September 1991

Solar Industry Journal, Solar Energy Industries Association (Volume 5, Issue 1)

The Solar Electric House, Steven J. Strong, William G. Scheller, 1987, 1991

Evaluation of Roof Integrated PV Module Designs and Systems, Florida Solar Energy Center, July, 1992

Solar Radiation Data Manual for Flat-Plate and Concentrating Collectors, NREL, April, 1994

Stand-Alone Photovoltaic Systems: A Handbook, Sandia National Laboratories, November, 1991

E. Disseminate Resource Package

In mid-April the Research Council issued a call for qualifications to 2800 faculty at ACSA member schools. The call requested qualifications from faculty who would commit to using the resource package in their Fall 1994 classes. Responses were received in May and faculty at 41 schools were selected to receive the package. Complete packages were then distributed to all selected faculty.

F. Coordinate IEA/NAEE ideas competition

Concurrent with the preceding tasks, and as a result of a modification to the original subcontract, the Research Council undertook to act as the United States coordinator for an international ideas competition on Photovoltaics in Buildings. The work effort entailed developing and disseminating a general call for entries to architecture practitioners, faculty and students. The Council also coordinated submission of projects. The calls went out in mid-September 1993 and the jury was held in Amsterdam in 
January 1993. The results were noteworthy in that three of the four schools represented on the project team submitted entries and were awarded citations.

The task resulted in a publication which was included in the final version of the resource package.

\section{Importance and Impact}

As a result of this project information on Photovoltaics in Buildings will be incorporated into curricula at 41 schools of architecture nationwide. Assuming a studio or lecture course can contain anywhere from 20 to 60 students, the number of students affected by the project will range from 820 to 2460 . These students will eventually become the gatekeepers to specifying new technologies such as PV for buildings. It is therefore very efficient to expose them to the topic now and to prepare them to become "early adopters" as practitioners in the field.

As part of its commitment to the project, the Research Council intends to continue to make the resource package available to ACSA member schools on a ongoing basis. It is anticipated that the package will be updated each year to include comments from faculty who have used it in the preceding year. Announcements concerning the package's availability will be made annually, in time for interested faculty to obtain information for use in Fall or Spring. In this way the project will sustain itself well beyond the initial subcontract period of performance.

RC36/nrelarpt.1-5 
Title:

Organization:

Contributors:
Joint U.S./Brazilian Rural Electrification Pilot Project

Centro de Pesquisas de Energia Eletrica (CEPEL) Brazil

Roger Taylor, Cecile Leboeuf, Mauricio Moszkowicz

\section{Joint U.S./Brazilian Rural Electrification Pilot Project}

The U.S. Department of Energy, through the National Renewable Energ aboratory (NREL) is implementing a joint technology research and demonstration effort with thie Centro de Pesquisas de Energia Elétrica (CEPEL) in Brazil, the Brazilian equivalent of the Electric Power Research Institute. In this program, DOE is cost-sharing renewable energy pilot projects with Brazilian state-owned utilities. The pilot projects demonstrate PV systems in a variety of applications. DOE commits a maximum of $50 \%$ of project costs, with the states supplying the remainder. The DOE contribution takes the form of U.S. PV modules and system components that cannot be found in Brazil, such as controllers and inverters. The approach of this joint program has been to:

1) establish technical, institutional, and economic confidence in using renewable energy (PV and wind) systems to meet the needs of the citizens of rural Brazil,

2) establish ongoing institutional, individual, and business partnerships with Brazil that are necessary to implement sustainable programs and commitments that benefit both Brazil and the U.S., and

3) lay the groundwork for large-scale rural electrification through the use of renewable energy systems based on U.S. technology.

To date, over $2000 \mathrm{PV}$ systems have been installed under this program in five Brazilian states. One of these states, Bahia, has announced that $5 \%$ of its new generation will come from renewables.

And, hardware for two $50-\mathbf{k W}$ hybrid village power systems will arrive this spring in Rio de Janeiro for installation in remote villages of Amazonia. There, PV and wind are replacing consumption of diesel fuel, which is expensive to transport, unreliable in quality, and environmentally damaging. The situation is particularly acute in Amazonia when river access to some remote villages for fuel delivery is often impossible for several months of the year.

The program has succeeded because it developed key institutional relationships between U.S. and Brazil to enhance the movement of U.S. hardware into this foreign country, and established the necessary in-country training and manpower. In essence, DOE has opened the Brazil utility door for U.S. PV companies.

The project has yielded a number of benefits:

- enhanced sales, profits, and eirtployment opportunities for U.S. renewable energy manufacturers

- offered cost-competitive alternatives to diesel, kerosene, and grid-extension

- enabled the rural population to receive electric power for the improvement of economic and social conditions 
Title:

Organization:

Contributors:
Solar Resource Utility Load Matching Assessment

Atmospheric Sciences Research Center, The University at Albany, Albany, New York

Richard Perez, principal investigator, and Robert Seals

\section{OBJECTIVES}

The project was initiated in August 1991, its initial objective was to estimate the load matching capability of photovoltaics (PV) for a sample of US utilities. This application required time coincident hourly electric load and local PV output data obtained via simulation from satellite remote sensing. Hence a secondary, but fundamental, objective of this work was to demonstrate the accuracy of satellite remote sensing for the considered application.

In March 1993, the project's objective was updated to include a larger sample of US utilities as well as "sub-utility" loads (i.e., substations, large customers, etc.). The rationale was to confirm and refine the relationships observed between load shapes and the effective capacity of PV. Secondary objectives were set to investigate the climatological representativeness of the test data used (1987 and 1988), and to identify the logistical requirements of effectively complementing ground based irradiance measurement with satellite data for providing an operational solar resource capability suited for localized PV-load investigations.

The current and future objectives of this projects are:

(1) to continue increasing the experimental load sample, providing both a service to the considered utilities and adding experimental data to validate the load shape-PV capacity relationships; in particular small customerspecific and more recent (1993-94) loads will be added to the analysis;

(2) to investigate whether PV's effective capacity may be inferred based on daily irradiance data, with the rationale of possibly expanding PV-utility studies where no hourly data are available (e.g., internationally)

(3) To map PV's effective capacity in the United States, based on load shape information from the majority of US utilities, making use of the relationship observed between PV capacity and load shape.

\section{RESULTS AND DISCUSSION OF ACTIVITIES COMPLETED IN FY 94}

\section{Recap on completed work}

The following results have been documented during initial phases of this study:

- Satellite-based site/time hourly solar radiation data have been shown to be effective for addressing PV-utility interaction issues at arbitrary locations in the US. This work was documented in $[1,2,3$ and 4].

- The effective capacity of PV, as quantified using several statistical and deterministic benchmarks was found to be substantially greater than the resource's capacity factor for a large number of US utilities. Regions of high PV capacity were not found to always coincide with the regions traditionally identified for solar energy development opportunities. On the other hand, a strongly defined relationship between PV's effective capacity and simple load parameters was identified. This work has been documented in [3,5 and 6].

- The experimental solar radiation data set used for this investigation (1987 and 1988) was found to be climatologically satisfactory for the stated objective. This work was documented in [7 and 8]. 


\section{Update on current investigations}

\section{PV's effective Capacity}

A total of 82 load-years have been analyzed, including 37 utilities and 9 "subutilities. The sub-utilities include substation and large end-use loads (hospitals, airports and administrative buildings, currently limited to the New York metropolitan area). As can be seen in Fig. 1 the considered service areas are very diverse (ranging from a point location to several $100,000 \mathrm{~km}^{2}$ ), and geographically distributed in most climatic regions of the US.

The studied loads span several orders of magnitude and their distribution is presented in Fig. 2 .

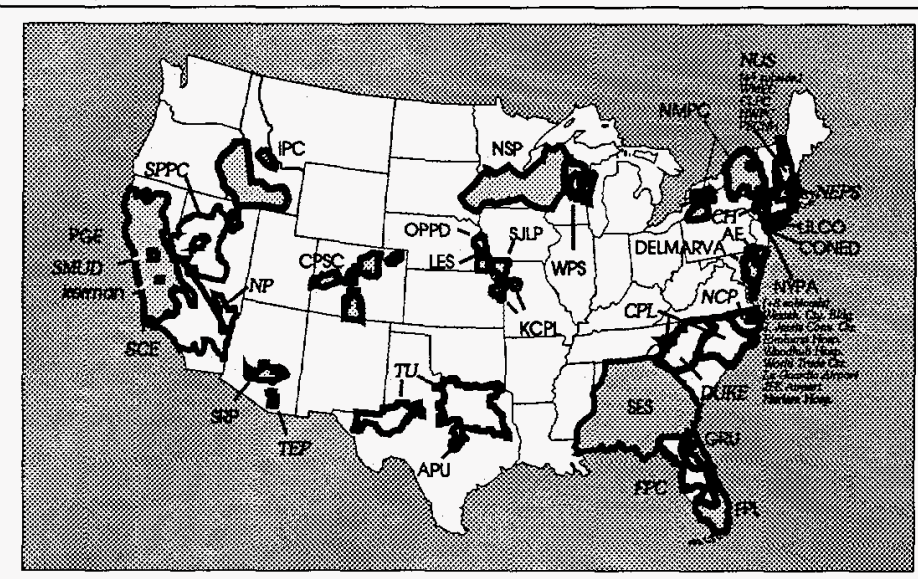

Figure 1: Geographical distribution and service area of investigated loads

In each case, PV capacity is quantified using four benchmarks including the Effective Load Carrying Capability (ELCC), the Minimum Buffer Energy Storage (MBES), the Mean Output during the Highest Loads (MOHL) and the Normalized Energy Worth (NEW). Each benchmark was described in detail in [3]. For this update, our attention is focused on the ELCC benchmark. The ELCC of each load studied is illustrated in Fig. 3 for 2-axis tracking systems at $2 \%$ PV penetration. It is remarkable that in the great majority of cases the ELCC of PV far exceeds its capacity factor. Following a request from several groups involved in the valuation of the PV resource, in

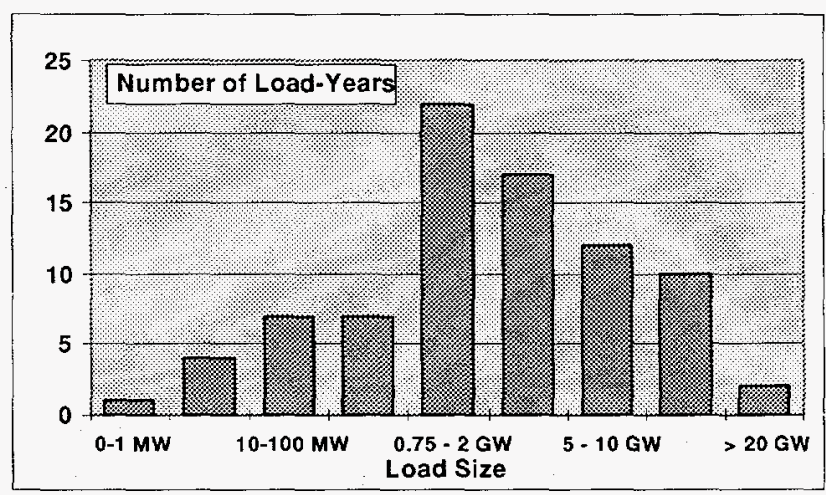

Figure 2: Size distribution of investigated loads particular PV4U [9], we also produced load matching benchmarks corresponding to the prevailing summer rates (nominally May to September). The summer ELCC of PV (2-axis, 2\% penetration) for each of the 82 load years is plotted in Fig. 4, where the trend apparent in Fig. 3 is considerably reinforced. This should have noteworthy implications for winter peaking utilities which had exhibited weak year around effective PV capacities.

The trend observed between key load shape parameters and the overall ELCC of PV has been refined. This trend is presented in Fig. 5, where observed ELCC for each of the 82 load years studied is plotted against a composite load shape parameter. This parameter is primarily the summer-to-winter peak load ratio (SWPR) previously introduced by the authors $[3,5]$, with minor adjustments to account for the time of the summer peak, the extent of the summer

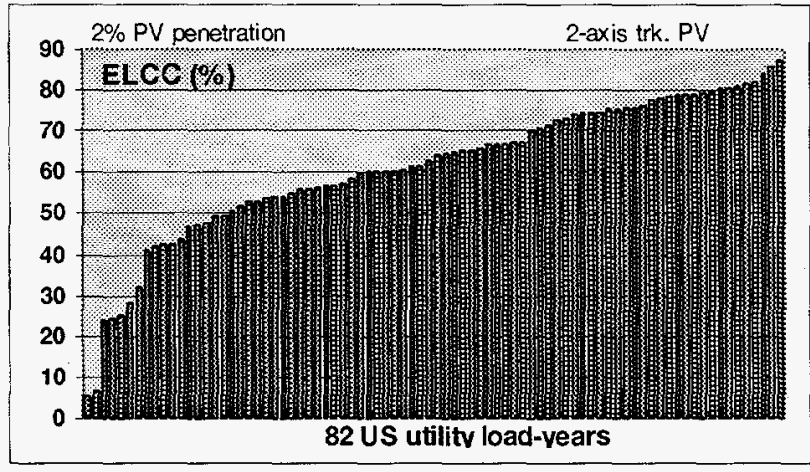

Figure 3: Effective Load Carrying Capability of PV

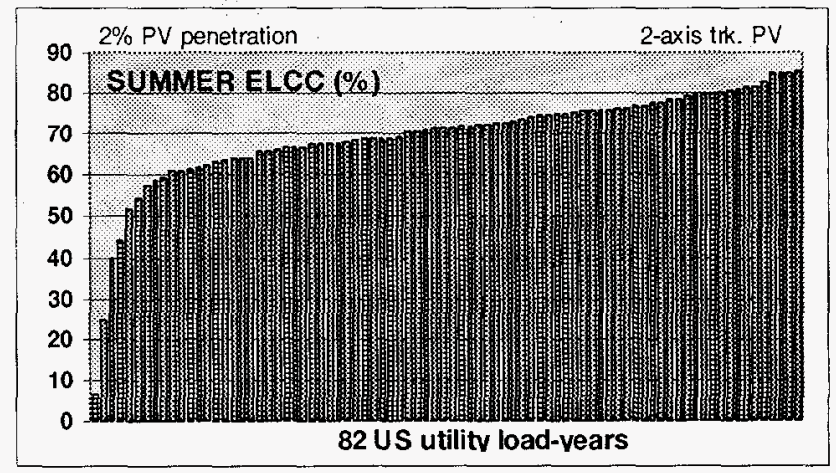

Figure 4: Summer PV Effective Load Carrying Capability 
evening peak shoulder, the summer valley-to-peak excursion, the size of the off-season peak load, the broadness of the winter peak, and the size of the considered load. It should be noted that Fig. 5 includes points derived form the 1987-1988 satellite and load data as well as points derived from ground measurements and load data for other time periods.

The observed relationship can be used as a tool to estimate PV's effective capacity where no site/time specific solar resource data exist. An direct application of this relationship is used for the mapping exercise discussed below.

\section{Estimating load matching from daily irradiance}

The technical objective of this exercise is to evaluate the accuracy of a daily time-site specific

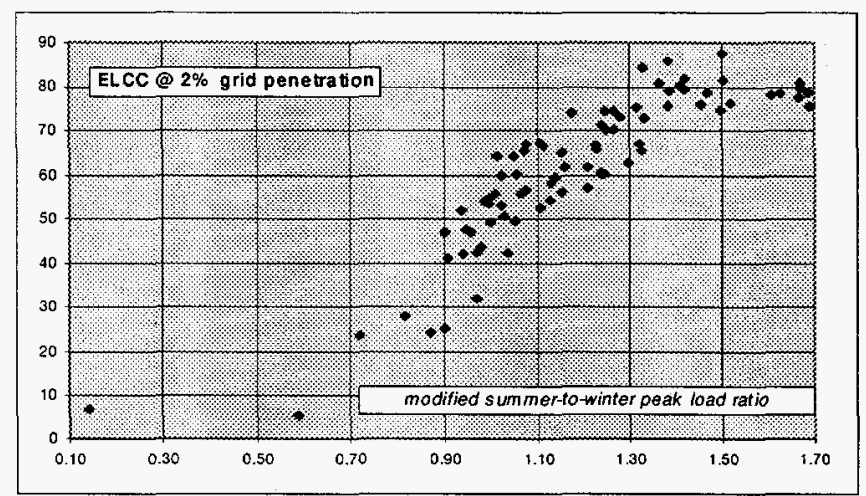

Figure 5: PV's ELCC vs. load shape characteristics solar radiation resource, as compared to an hourly resource to estimate the effective capacity of PV. If accuracy is found to be acceptable, daily solar resource data, that are historically more commonly available than hourly data (e.g., in [10]), could be used, thereby extending our potential domain of investigation.

Methods: our approach consists, for each of the 82 load-years mentioned above, to start from the corresponding hourly global and direct irradiance data, and for each day, summing that data and generating a new set of hourly data with constant daily clearness index. The new set of data, that is based solely the daily information input, is then used in conjunction with hourly load data to calculate new load matching benchmarks that may be compared to original benchmarks.

Preliminary results: These are illustrated for the ELCC and MBES benchmarks for two-axis tracking PV configuration and $2 \% \mathrm{PV}$ penetration. The agreement between daily-derived and hourly-derived ELCC is remarkable (Fig. 6), with the largest bias occurring in the middle range. A simple correction may be applied and correct most of this bias. For the MBES, the agreement is not as strong (Fig. 7) but nevertheless quite remarkable. Because of the deterministic nature of this benchmark, this is more sensitive to hourly irregularities in the solar resource. It is interesting to note that the largest discrepancies are found for late-peaking loads in areas subject to afternoon thunderstorm activity. The absence of behavior difference between ground data points and satellite derived data points in this exercise should be brought to the credit of the satellite resource. Also remarkable is the absence of behavior difference small site-specific loads and large extended utility loads.

As preliminary as these results are, it is apparent that a daily solar resource could be used to estimate PV-utility match when an hourly resource is not available.

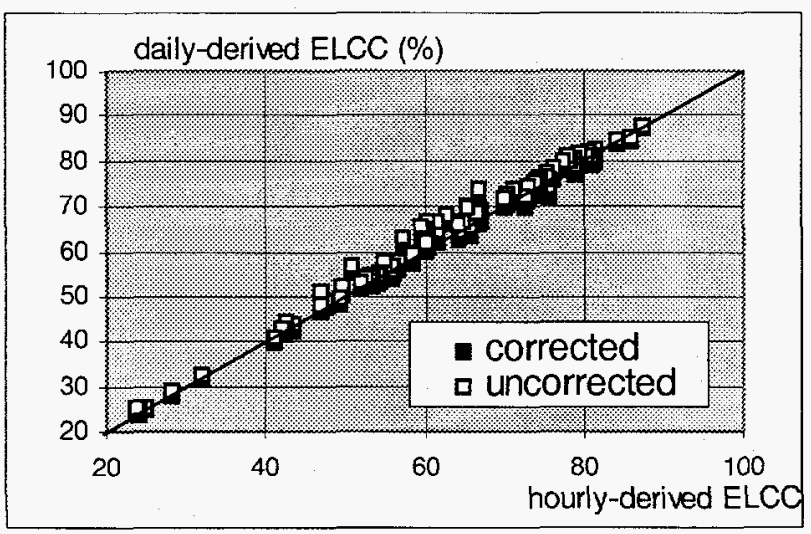

Figure 6: Daily vs. hourly-derived ELCC

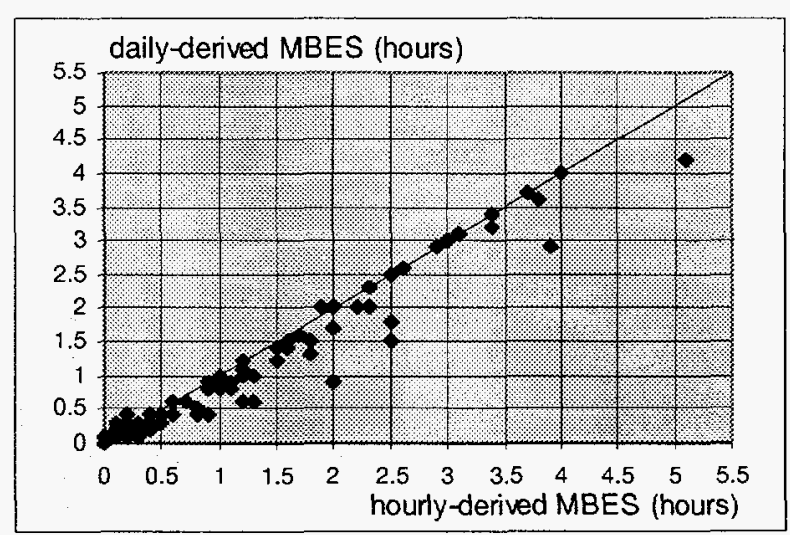

Figure 7: Daily vs. hourly-derived MBES 
We obtained preliminary load shape information for the great majority of US utilities for the Year 1993 [12], consisting of summer and winter peak load data. Although the relationship in Fig. 5 includes several load shape parameters, the summer and winter peaks do explain most of the load-ELCC relationship and are deemed to be sufficient for this initial mapping exercise. Utility service territories will be gridded on a $2^{\circ}$ latitudeby $-2^{\circ}$ longitude map. The effective capacity for each grid cell will be estimated from a weighted average of (or portions of) utilities composing each cell. A preliminary version of this map is given in Figure 8, with its resolution currently limited to State boundaries. Nevertheless, it is already quite apparent that the geographical distribution of PV's capacity is considerably

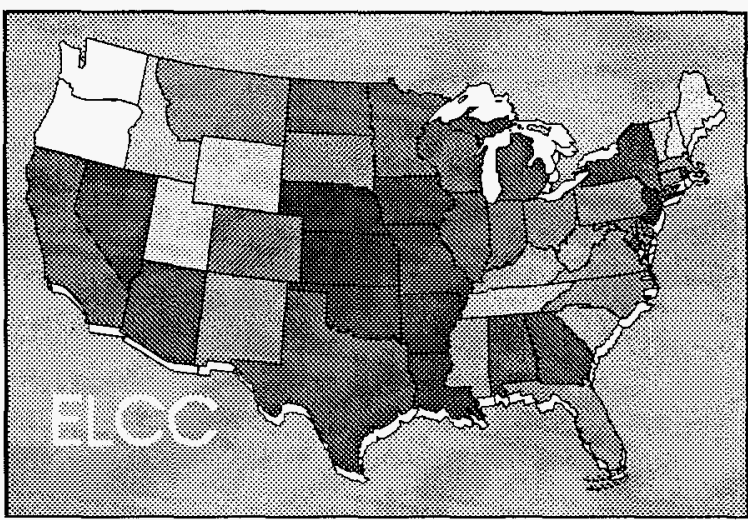

Figure 8: Preliminary mapping of PV ELCC, limited to State-boundarv resolution different from that of the standard solar energy resource, confirming the partial evidence based on the 82 experimental load data sets discussed above. The high capacities observed in the mid-Atlantic seaboard and in the Central US are most remarkable.

Acknowledgment: This effort is supported by NREL (contract \# XR-1111-681), with William Wallace and Roger Taylor as program managers.

\section{REFERENCE}

1. Perez, R. Seals, R. Stewart, A. Zelenka and V. Estrada-Cajigal (1994): Using Satellite-Derived Insolation Data for the SitelTime Specific Simulation of Solar Energy Systems Solar Energy Vol. 53, 6; 7 pp.

2. Perez, R., R. Seals, R. Stewart, A. Zelenka and V. Estrada-Cajigal, (1993): Using Satellite-derived Insolation Data to Evaluate the Utility Load Matching Capability of Photovoltaics. Proc., ISES World Congress, Budapest, Hungary.

3. Perez, R. Seals, R. Stewart, (1994): Solar Resource -- Utility Load-Matching Assessment. NREL Report. No. TP-411-6292, 48 pp.

4. Beyer, D. Heinemann, R. Perez, C. Reise and R. Seals, (1994): Satellite-Derived Radiation Data for PV System Studies -- an Appraisal. Proc. 12th European Photovoltaic Solar Energy Conference, Amsterdam, The Netheriands

5. Perez, R., R. Seals and R. Stewart, (1993): Assessing the Load Matching Capability of Photovoltaics for US Utilities Based Upon Satellite-Derived Insolation Data, IEEE Transactions, pp. 1146-1149 (23d. PV Specialists, Louisville, KY).

6. Perez, R. Seals and R. Stewart, (1994): Matching Utility Peak Loads with Photovoltaics. Proc. RENEW-94, NESEA's Annual Meeting, 6 pp.

7. Perez, R., R. Seals and R. Stewart, (1993): Long Term Variability of Solar Radiation in the US -- A Preliminary Utilization of the NREL National Solar Radiation Data Base, intermediate contract report.

8. Perez, M. Rymes and D. Renné, (1994): Assessment of Photovoltaic Load Matching Potential -- Impact of Long term Insolation Variations. Proc. ASES Annual Meeting, San Jose, CA, 6 pp.

9. Whitlock, C. H. et al., (1993): WCRP Surface Radiation Budget Shortwave Data Product Description Version 1.1. NASA TM 107747, NASA Langley, Hampton, VA

10. e.g., see Weissman, J., (1994); PV Market Development -- Collaborative Action at State Level. Proc. RENEW94, NESEA's Annual Meeting, 6 pp.

11. Bergman, M. (1994): Personal_Communication, UPVG, Washington, DC, USA 


\title{
Title: $\quad$ Evaluation of DSM Incentive Opportunities for Photovoltaics
}

\author{
Organization: Center for Energy and Environmental Policy (CEEP) \\ University of Delaware \\ Newark, Delaware 19716 \\ TEL: (302) 831-8405 FAX: (302) 831-3098
}

Contributors: John Byrne, principal investigator; Young-Doo Wang and Abigail Jahiel, investigators; Willett Kempton, research support; Steven Letendre, Chandrasekhar Govindarajalu, Maithili Iyer and Christine Egan, graduate students

\section{Objectives}

The development of intermediate markets for photovoltaics (PV) is vital for near-term growth of the PV industry. One key utility market sector for PV receiving increasing attention for intermediate applications is the use of PV in the building sector as a demand-side management (DSM) tool to shave peak loads. The Center for Energy and Environmental Policy (CEEP) at the University of Delaware has been studying the possibilities for utilizing PV as a DSM option. The objectives of this research during FY 1994 were:

- To convert models for PV-DSM value analysis into spreadsheet analysis tools that can be used by utility companies, regulatory and consumer advocate staff, and others in performing resource evaluations.

- To extend the analysis of the value to utilities and their customers of PV as a DSM option, incorporating complementary PV-DSM benefits derived from emergency power applications.

- To organize and conduct a PV-DSM workshop for utilities, PV manufacturers and researchers in order to assemble and transfer available information concerning the use of PV as a DSM application.

Tasks and Research Results

\section{Task 1: $\quad$ Spreadsheet Applications-Case Studies}

CEEP, in cooperation with NREL staff, identified five utilities in 1994 to participate in case studies of the use of the PV-DSM Spreadsheet Analysis Tool created by CEEP for the purpose of evaluating the economics of PV-DSM in these utilities' service territories. The five utilities under investigation are diverse in terms of their geographic location, size, and rate structure. The participating utilities are Niagara Mohawk Power Corporation (NMPC), Austin Electric (AUSTIN), Sacramento Municipal Utility District (SMUD), Delmarva Power \& Light (DP\&L), and an East-Coast Urban Utility (ECUU). ${ }^{3}$

The performance of a $10 \mathrm{~kW}$ PV-DSM system with a 25 year life was assessed for use in the service territories of each of the case utilities. Both non-dispatchable and dispatchable system designs ${ }^{2}$ were

${ }^{1}$ This investor-owned utility has requested that its name not be used in published papers and reports.

${ }^{2}$ Non-dispatchable PV systems have limited or no storage, providing mainly energy conservation ( $\mathrm{kWh}$ ) value. Dispatchable PV systems incorporate storage sized to allow utilities and customers to credit units with reliable peak-shaving capacity $(\mathrm{kW})$, as well as energy conservation value. 
analyzed. Utility-owned and customer-owned systems were analyzed, along with an innovative utilitycustomer partnership, our proposed "Green Investment Fund" (GIF). Under the GIF, customers and utilities contribute to a fund for the purpose of purchasing a PV-DSM system, much in the same manner that "green pricing" joins customers and utilities in a common effort to make advance purchase of technologies that are currently not cost-effective to the individual parties. Utility contributions are in the form of a rebate for access to dispatchable PV systems for the purpose of shaving utility load peaks. Customers agree to pay for grid electricity at existing service levels and dedicate potential bill savings to the acquisition of PV systems.

Our analysis showed that dispatchable PV had higher customer and utility value than non-dispatchable systems in all five service territories. For this report, therefore, we have focused on the economic performance of dispatchable PV systems. Table 1 compares the benefit-cost ratios of dispatchable systems for three investment options: customer-owned; utility-owned; and GIF. GIF calculations assumed that the customer contributes 80 percent of the potential bill savings and 100 percent of the tax savings available from the operation of the system. The utility was assumed to pay a rebate to the Fund equivalent to the avoided capacity costs (at zero capacity factor) of a gas-fired combustion turbine based on the credited capacity of the PV system. In the five utility service territories, the economics of dispatchable PV-DSM are more favorable for customer-owned than utility-owned systems (due largely to the tax treatment of capital investments afforded to customers). The GIF was the most economic vehicle for developing dispatchable PV-DSM.

Table 1. PV-DSM Investment Options: Benefit/Cost Ratio Comparisons

(Dispatchable System)

\begin{tabular}{lccccc}
\hline & NMPC & DP\&L & ECUU & SMUD & AUSTIN \\
\hline $\begin{array}{l}\text { Utility } \\
\text { Owned }\end{array}$ & 0.41 & 0.66 & 0.52 & N/A & N/A \\
$\begin{array}{l}\text { Customer } \\
\begin{array}{l}\text { Owned } \\
\text { Green } \\
\text { Investment } \\
\text { Fund }\end{array}\end{array}$ & 0.69 & 0.76 & 0.98 & 0.73 & 0.75 \\
\hline
\end{tabular}

Note: * Winter-peaking; all others are summer-peaking.

N/A: These are municipal utilities and, therefore, the methods for estimating avoided costs and carrying charges are different from those employed by investor-owned utilities. Currently, methods are being refined to allow for meaningful comparisons. For this reason, a GIF scenario could not be run.

Source: Center for Energy and Environmental Policy, University of Delaware

\section{Task 2: $\quad$ Extended Development of PV-DSM Value Analysis}

In addition to PV's DSM value, CEEP extended the concept of PV-DSM to incorporate the complementary benefits of emergency power options. This takes advantage of further value-added benefits generated by the operational capabilities of dispatchable PV systems located at customer sites. The battery storage component of a PV-DSM system can additionally provide customers with on-site power for critical functions in the event of grid power failure. Most commercial buildings now purchase some form of emergency power, typically, emergency lighting. Since PV-DSM can potentially obviate or reduce the need for that purchase, it can increase the value of PV-DSM systems to building customers. 
Two emergency power options which are widely used in the commercial sector were evaluated: emergency lighting and uninterruptible power supplies (UPS) for computer operations. Commercial buildings are required by code to provide lighting for safe occupant exit in the case of power loss, primarily in halls and stairways. The BOCA code requires that this lighting be able to operate for at least one hour. Three vendors were contacted for emergency lighting evaluations (Brite-Lite, Lighting Design Center and United Electric Supply). Computer UPS can supply either a few minutes of power, intended to allow a rational shutdown of computer operations, or power for much longer period of time, to allow continuous use of computers throughout a power outage. Dispatchable PV-DSM would probably be able to serve only short-term UPS needs (vendors contacted for UPS evaluation were American Power Conversion and Controlled Power Company).

Figure 1 compares different emergency systems meeting divergent needs on comparable energy storage costs per $\mathrm{kW}$. The figure indicates that current markets place a premium value on in-building emergency power, far exceeding values common in utility calculations. The largest cost is for battery-based emergency lighting systems, at $\$ 2,500-\$ 6,000$ per $\mathrm{kW}$. Much cheaper generator technology is available (as indicated in the figure for diesel gen-sets) but is valuable only for larger scale needs and when only short interruptions are permissible. The more expensive battery-based systems are required for small units (such as modular emergency lighting) and in applications requiring fast switchover time. According to the manufacturer of the AC Battery module, a PV-DSM unit could be modified to serve the functions of emergency power at little additional cost.

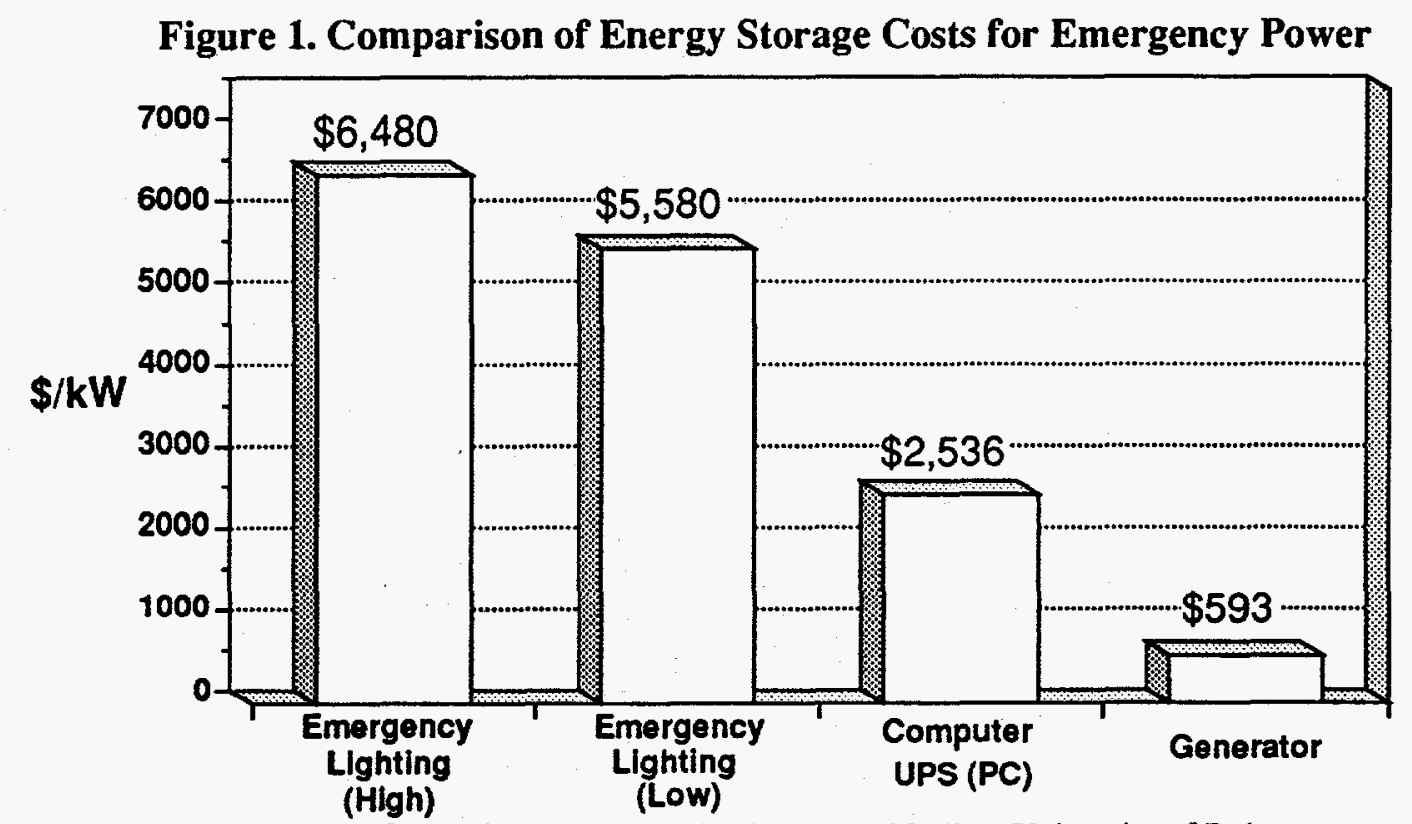

Source: Center for Energy and Environmental Policy, University of Delaware

Task 3:

\section{PV-DSM Workshop}

CEEP organized a PV-DSM Workshop with the objective of assembling the best available information on PV-DSM utility applications and transferring that information throughout the utility sector and to PV manufacturers. The workshop was closely coordinated with NREL and complemented the PV:BONUS workshop, also held at the University of Delaware. The workshop concept was co-initiated by, and received enthusiastic support from, the PV:BONUS program managers. The overall result of the workshop increased synergy between the PV-DSM and PV:BONUS programs combining PV-DSM and PV building integration approaches to identify near-term applications. 
The workshop was held on October 19-20, 1994 at the University of Delaware, and the contents were published in a proceedings titled Forging PV-Buildings Bridges: A Joint NREL/DOE, PV-DSM and $P V: B O N U S$ Workshop. The PV-DSM workshop included information on: i) the economics of PV-DSM, ii) the technical potential and performance of PV-DSM systems, iii) regulatory and other policy issues related to the commercialization of PV-DSM systems, iv) the status and use of the PV-DSM Spreadsheet Analysis Tool, v) a discussion of case study results from Task 1, and vi) methodological and conceptual issues in the assessment of PV-DSM.

\section{References}

1. J. Byrne, S. Hegedus and Y-D. Wang. 1994. "Photovoltaics as a Demand-Side management Technology: an Analysis of Peak-shaving and Direct Load Control Options." Progress in Photovoltaics: Research and Applications. Vol. 2, 235-248.

2. J. Byrne, S. Letendre, R. Nigro and Y-D. Wang. 1994. "PV-DSM As a Green Investment Strategy." Proceedings of the Fifth National Conference on Integrated Resource Planning. May 15-18, Kalispell, Montana. National Association of Regulatory Utility Commissioners. Washington, DC.

3. J. Byrne, S. Letendre, R. Nigro, W. Wallace and Y-D. Wang. 1994. "Photovoltaics in HighValue, Utility Markets: A Demand-Side Management Strategy." Proceedings of the American Solar Energy Society Solar '94 Conference. June 25-30, San Jose, CA.

4. J. Byrne, C. Govindarajalu, W. Kempton and Y-D. Wang. 1994. Emergency Power As an Additional Value From Dispatchable PV Peak Shaving. Prepared for the National Renewable Energy Laboratory. July. Center for Energy and Environmental Policy, University of Delaware.

5. J. Byrne, Y-D. Wang, R. Nigro and S. Letendre. 1994. "Photovoltaics in a Demand-Side Management Role." Proceedings of the ACEEE 1994 Summer Study on Energy Efficiency in Buildings. August. American Council for Energy-Efficient Economy. Washington, DC.

6. J. Byrne, S. Letendre, R. Nigro, C. Govindarajalu, W. Wallace and Y-D. Wang. 1994. "Photovoltaics For Demand-Side Management Utility Markets: A Utility/Customer Partnership Approach." Proceedings of the First World Conference on Photovoltaic Energy Conversion. December 4-9, Waikoloa, Hawaii.

7. J. Byrne, Y-D. Wang, S. Letendre, C. Govindarajalu, R. Nigro and W. Bottenberg. 1994. "Deployment of a Dispatchable Photovoltaic System: Technical and Economic Results." Proceedings of the First World Conference on Photovoltaic Energy Conversion. December 4-9, Waikoloa, Hawaii.

8. J. Byrne, A.M. Barnett, et al. 1994. "Dispatchable Commercial Building Photovoltaic DemandSide Management System Tests." Proceedings of the 12th European Photovoltaic Specialists Conference. 
Title:

Organization:

Contributors:

\section{Rooftop PV System}

Energy Conversion Devices, Inc.

Troy, Michigan 48084

Energy Conversion Devices, Inc.:

M. Izu, Principal Investigator; H.C. Ovshinsky, K. Whelan, L. Fatalski, T. Ellison and S.R. Ovshinsky

United Solar Systems Corp.:

T. Glatfelter, K. Younan, K. Hoffman,

A. Banerjee, J. Yang, and S. Guha

\section{Objectives}

The principal goals of this PV:Bonus project are to 1) develop and demonstrate architecturally integrated rooftop PV systems; 2) verify technical and economic performance; 3) develop a market plan; and 4) lay the foundation for high volume commercialization. It is expected that in large volume production the installed cost of the proposed system will be approximately $\$ 2.60$ per peak watt. Successful implementation of the program will result in cost-effective, aesthetically pleasing, easy-to-install rooftop photovoltaic (PV) systems which will greatly accelerate acceptance and penetration of photovoltaics in the enormous building and utility markets.

\section{Technology}

During the past fifteen years, Energy Conversion Devices, Inc. (ECD) has made important progress in the development of materials, device designs and manufacturing processes required for the continued advancement of PV technology. Among these accomplishments ECD has pioneered, and continues to develop, two key proprietary technologies with significant potential for achieving the cost goals necessary for widespread growth of the photovoltaic market: (1) a low-cost, roll-to-roll continuous substrate thin-film solar cell manufacturing process; and (2) a high-efficiency, multiple-junction, spectrum-splitting thin-film amorphous-silicon alloy device structure.

ECD and United Solar Systems Corp. (United Solar) are now developing, lightweight, flexible photovoltaic modules that can replace conventional roofing materials and be economically and aesthetically integrated into residential and commercial buildings. The modules will be fabricated from high-efficiency multi-junction a-Si alloy solar cells developed by ECD and United Solar. These cells are produced on thin, flexible, stainless steel substrates and encapsulated with polymer materials.

\section{Scope and Method of Approach}

Two types of products, $1 \mathrm{ft}$ by $10 \mathrm{ft}$ overlapping PV shingles and $1.3 \mathrm{ft}$ by $20 \mathrm{ft}$ PV roof panels, are being developed by United Solar and ECD, respectively[1-3]. United Solar's shingle type design uses a roof mounting procedure similar to those used with conventional asphalt shingles, 
while ECD's PV panel uses mounting procedures conforming to metal roof systems. Both types of rooftop PV modules are lightweight and flexible. Thus, they can be installed on roof sheathings, replacing ordinary shingles or metal roofing panels, on a standard wood roof construction. The product design features and installation methods have been refined through discussions and reviews with building industry experts including the NAHB Research Center, Solar Design Associates, and Minoru Yamasaki Associates.

United Solar's roofing module is designed to emulate the conventional asphalt shingle in form, structure, function, and installation. The module's geometry (form) is represented in Fig. 1. Several PV sub-panels will be integrated into this shingle module by laminating the elements together. The materials used in the module are illustrated in Fig. 2. Environmental protection is provided by ethylene vinyl acetate (EVA) and Tefzel. The sub-panels consist of multi-layer thinfilm solar cells deposited onto thin sheets of stainless steel. The shingle also includes interconnecting wires and current bypass diodes (not shown). Electrical inter-module connections will be made below the sheaves of the roof. There will be one roof penetration for each module via the use of a sealed insert. The result will be a flexible PV module that bears a close resemblance to an asphalt shingle. The PV shingles will protect the roof in the same manner as standard shingles by providing water run off. A clear separation of tasks among the building trades is envisioned for the installation of these modules. The roofer would tack down the shingles with customary roofing tacks. An electrician would subsequently complete the electrical connections in the attic spaces beneath the roof.

ECD's PV modules are installed water tight and vertically, using existing standing seam or low profile continuous sealing batten designs. These features can be seen in Fig. 3 which shows drawings of a demonstration home proposed to be built by NAHB Research Center. Wiring will be along the roof peak in a trough that also functions as a roof vent, eliminating the need for any holes in the roof. The long continuous watertight vertical panel design eliminates horizontal seams as a source of roof leaks.

Members of the project team include NAHB Research Center, Detroit Edison, Arizona Public Service Company (APS), Solar Design Associates, Minoru Yamasaki Associates and others. The project team will verify technical and economic performance of the products and develop a marketing/business plan.

The NAHB Research Center has performed a preliminary market acceptance survey within the building industry and has identified several key issues that are important for acceptance by the building industry of this new technology.

\section{Major Accomplishments}

1) Module Development

ECD has completed design and construction of its large-area laminator ( $2.5 \mathrm{ft}$ by $22 \mathrm{ft})$. ECD has begun fabrication of prototype modules. 
ECD has optimized and refined $1.3 \mathrm{ft}$ by $20 \mathrm{ft}$ metal roof-type PV module design by utilizing standard metal roofing materials and installation techniques.

United Solar has designed and constructed a $2 \mathrm{ft}$ by $10 \mathrm{ft}$ laminator and is fabricating its prototype $1 \mathrm{ft}$ by $10 \mathrm{ft}$ shingle-type modules.

United Solar has completed the design and installation method of its $1 \mathrm{ft}$ by $10 \mathrm{ft}$ shingle-type modules.

United Solar has constructed a demonstration roof structure with prototype modules for outdoor testing and architectural evaluation.

\section{2) Demonstration Program}

The detailed engineering and architectural specifications for remote PV installation on Beaver Island, Michigan have been defined and the components have been purchased.

Detailed drawings are being developed for the proposed NAHB Research Center demonstration site and a commercial building in Arizona, and a home at the site of the next Olympics in Atlanta, Georgia.

\section{3) Business Development}

APS has completed a preliminary market assessment and identified a potential economically attainable national market of low-cost rooftop PV modules for about 1.7 Giga Watts without DSM incentives and about 4.2 Giga Watts with DSM incentives.

The NAHB Research Center has performed a preliminary market acceptance survey within the building industry and has identified several key issues that are important for acceptance by the building industry of this new technology.

\section{References}

1. "Lightweight Flexible Rooftop PV Module", M. Izu, H.C. Ovshinsky, K. Whelan, L. Fatalski, S.R. Ovshinsky, T. Glatfelter, K. Younan, K. Hoffman, A. Banerjee, J. Yang, and S. Guha, First World Conference on Photovoltaic Energy Conversion, December 1994.

2. M. Izu, H.C. Ovshinsky, K. Whelan, L. Fatalski, S.R. Ovshinsky, T. Glatfelter, K. Younan, K. Hoffman, A. Banerjee, and S. Guha. Presentation at: Forging PVBuildings Bridges, a Joint NREL/DOE PV-DSM/PV:Bonus Workshop, "Roofing Systems Development", Presented on October 19, 1994.

3. Presentation at: The International Energy Agency Workshop on Building-Integrated PV, "Building-Integrated PV Activities at ECD and United Solar", M. Izu, H.C. Ovshinsky, K. Whelan, L. Fatalski, S.R. Ovshinsky, T. Glatfelter, K. Younan, K. Hoffman, A. Banerjee, J. Yang, S. Guha and R. Blieden, Presented on September 16, 1994. 


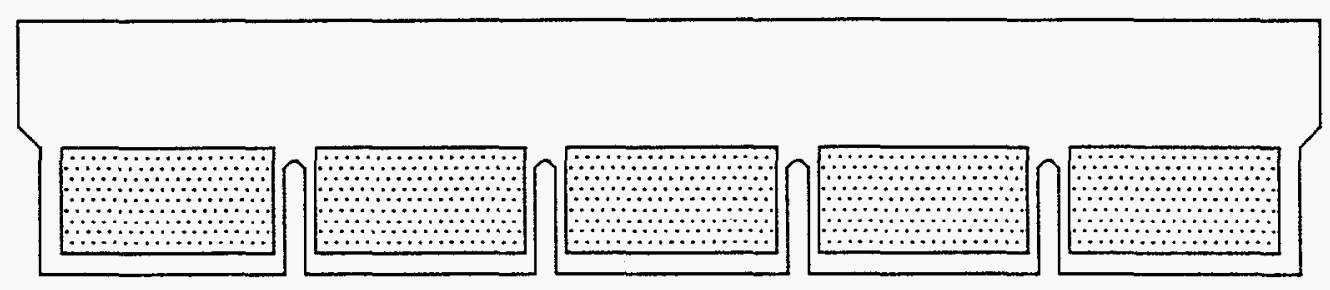

Figure 1 Schematic of shingle module footprint

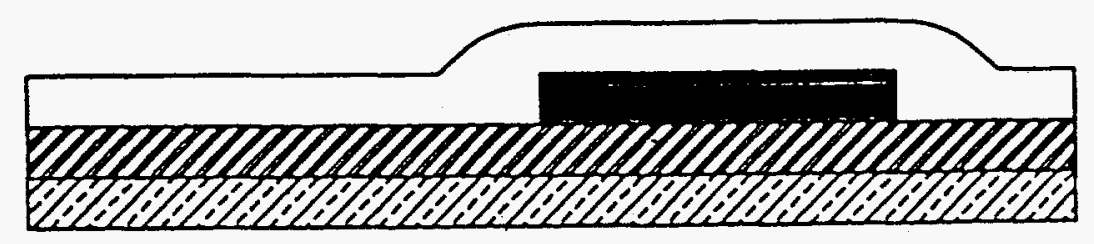

Tefzel/EVA Composite PV Sub-Module EVA

Nylon/EVA Composite

Figure 2 Shingle module materials stack-up

NAHB TOWNHOUSE (ARCHITECTURAL DRAWING)

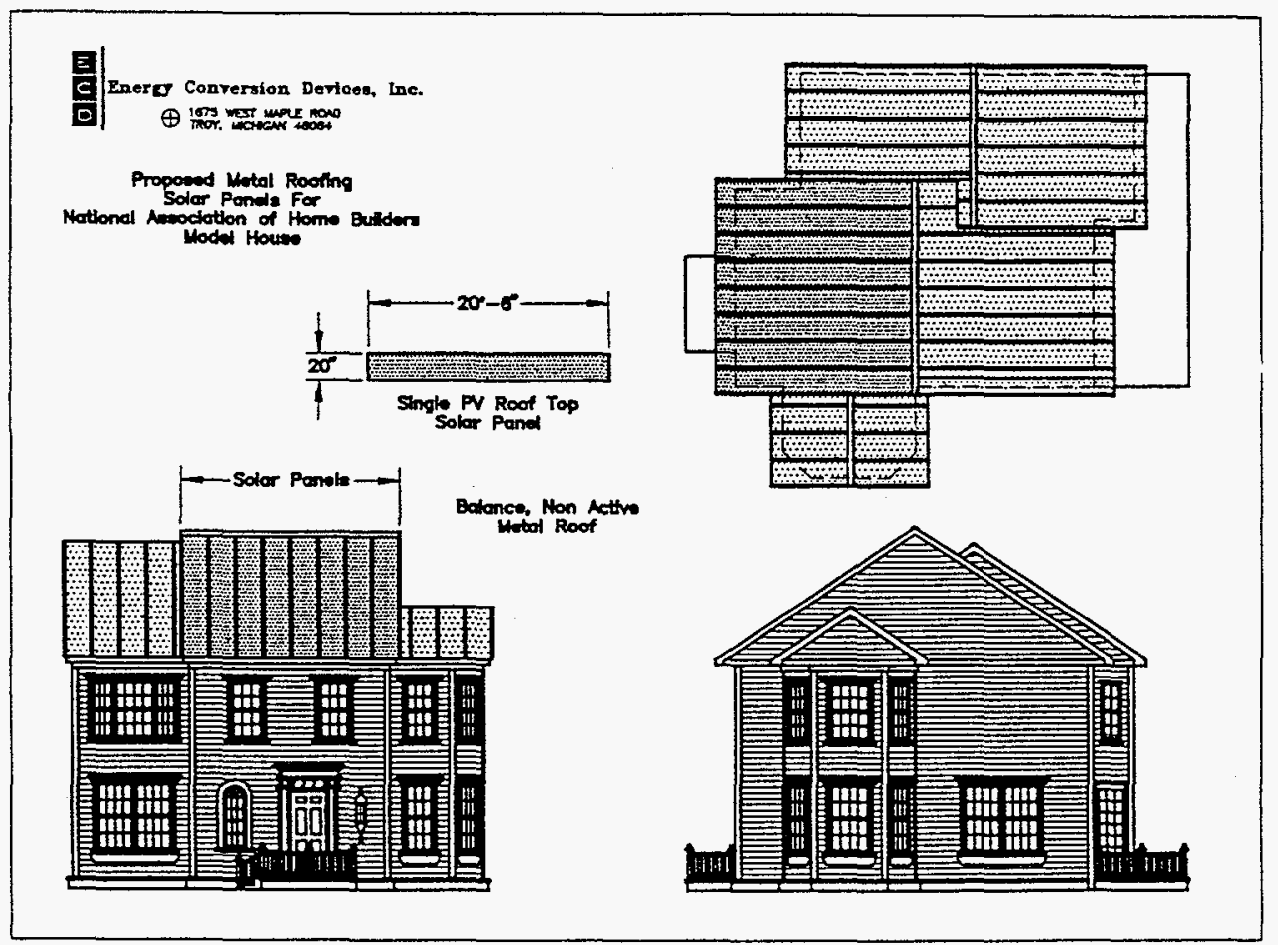

Figure 3 Demonstraton home proposed by NAHB Research Center 
Title:

PV Program Support to Electric Utilities

Organization: NEOS Corporation, Lakewood, Colorado

Contributors: $\quad$ K. Stokes, program manager; C. Lane, circuit rider; and P. Saito

\section{Introduction}

Within the last few years, nearly 50 rural electric cooperatives (RECs) in the western United States have begun to investigate the use of photovoltaic (PV) power as both a cost-cutting and new service option. Presently, approximately $15 \mathrm{RECs}$ in this region have implemented pilot programs to provide PV services to their consumers. These small utilities are recognizing that PV power can significantly reduce the cost of service to small outlying or difficult-to-serve loads (e.g., lighting systems, livestock water pumping, and cathodic protection) without compromising service reliability or customer satisfaction.

Despite these benefits, initiating any new program within a small utility with limited resources is difficult. Generally, utility member service personnel are committed to other full-time responsibilities and taking on new tasks requires careful examination. An additional obstacle to program implementation in the case of a new technology such as PV is the lack of local vendors to provide equipment and support. Furthermore, it is difficult to find impartial technical experts.

\section{Objectives}

As a small utility's ability to provide or find competent PV service support may be limited, the goal of this program is to create an energy service organization to provide PV support services to interested utilities. To obtain this goal, the following three program objectives were identified and implemented:

1. A PV service technician was employed and equipped with transportation, tools, and hardware to provide services that fulfilled the combined needs of the member utilities. Many of these services are provided on site at each utility location by the PV "service center on wheels."

2. A comprehensive list of necessary support services was compiled.

3. During the two-year pilot program phase, the PV support service was being funded primarily by outside sources with a minimal annual contribution from each member utility.

\section{Approach}

The majority of the funding for the pilot program has been provided through contributions from interested federal, state, and private organizations. The "seed" funding for the pilot program is provided by member utilities and indicates a level of interest and commitment to the program by the participating utilities. Outside funding is provided by Sandia National Laboratories, National 
Renewable Energy Laboratory, Electric Power Research Institute, Colorado Office of Energy Conservation and Western Area Power Administration.

The PV support service is oriented for use by both utilities that are ready to offer PV as a service alternative to their customers, and those that have not yet made that decision. The support service can help a utility determine if there is a need for a PV service within their customer base. It also provides information and assistance to utility consumers with small loads who cannot afford a new line extension or high service charges for an existing service. Support services also help utilities find cost-effective applications within their own operations.

The implementation of the pilot phase of the program required NEOS to carry out several key functions. These functions included:

- Screening and hiring a PV service technician;

- Training the technician in the PV services required by the utilities;

- Providing instructional, secretarial, and on-site support of the technician's activities;

- Tailoring the PV support services and activities to the needs of each member utility;

- Administering the pilot program funding obtained from outside sources and membership fees;

- Providing office space and telephone, fax, copier and computer services to technician; and

- Leasing and insuring an appropriate vehicle for the technician and equipping it with all the necessary tools and equipment.

\section{Results}

The 17 utility members include 14 RECs and 3 municipalities. The following charts detail the service activities of the PV Circuit Rider for the first year of the project. Figure 1 shows the percent of time the PV Circuit Rider spent on each activity.

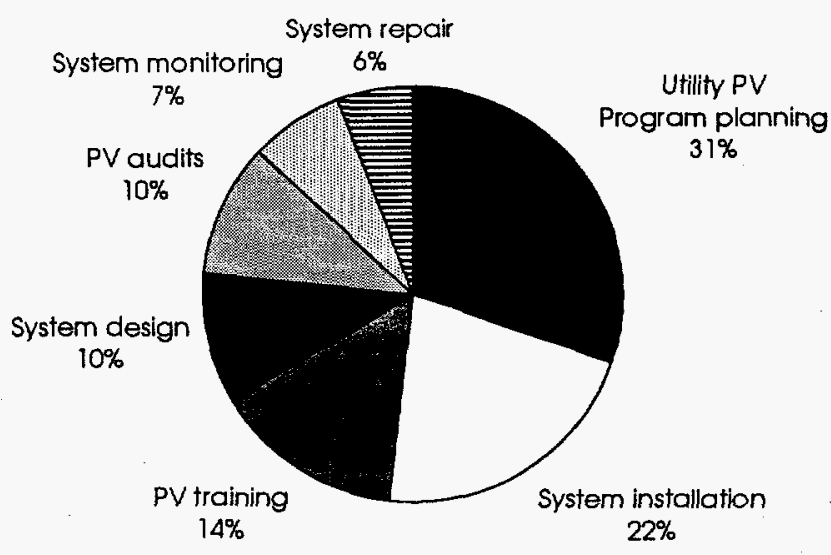

Figure 1-Activities Performed for PV Circuit Rider Members 
Figure 2 shows the PV applications that were serviced by the PV Circuit Rider. As shown, the most prevalent application was PV water pumping.

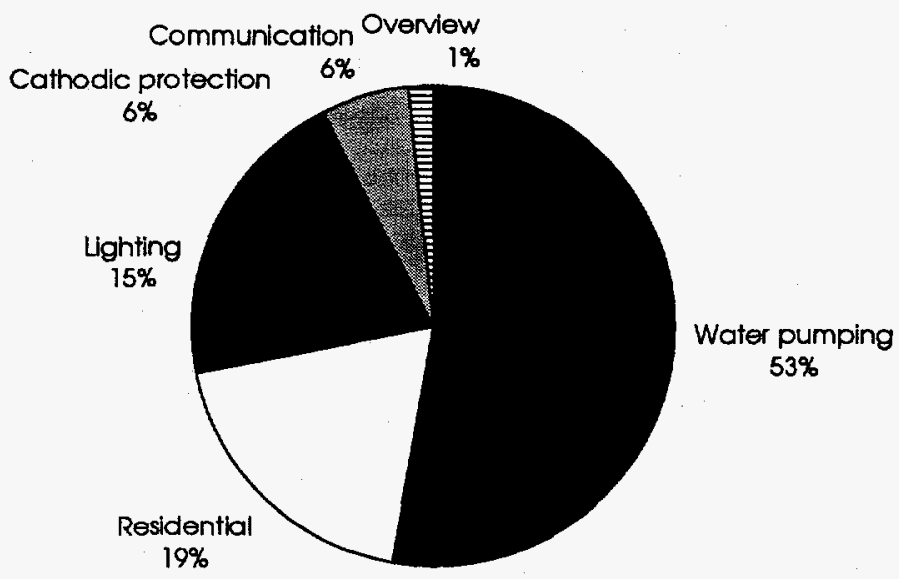

\section{Figure 2-Applications Serviced for PV Circuit Rider Members}

The PVCircuit Rider News newsletter was developed to aid in information transfer to utilities and facilitate exchange between utilities interested in the PV Circuit Rider Program. Many utilities and organizations outside the PV Circuit Rider Program have requested the newletter. The current mailing list exceeds 90 people.

The following has been learned from the first year of the PV Circuit Rider Program.

- Utilities are pleased with the program. In a recent survey 80 percent of the PV Circuit Rider members were either pleased or very pleased with the program. (Twenty percent did not respond.)

- The benefits of the program, as stated by the member utilities, include:

- Technical support for installations and procurement;

- Audit procedures and methods to easily determine cost-effective applications;

- Education for both utility employees and their customers;

- Information on the latest PV products; and

- Publication of the monthly newsletter, PV Circuit Rider News.

- Local PV vendors are needed and wanted, but when they do exist, utilities do not always trust their expertise. The PV Circuit Rider members need more information on the capabilities of existing PV vendors. The utilities also stated that they want the pump dealers to maintain and repair PV pumps.

- PV Program and activity implementation barriers do exist within utilities: 
- Traditional responsibilities of utility personnel tend to have a high priority leaving little time for PV.

- Some board of directors are not always supportive of PV services and usually tend to support the status quo, such as liberal line extension policies.

- Some customers are skeptical of the unfamiliar PV services, especially for residential applications.

\section{Conclusion/Future Plans}

There has been significant interest in expanding the geographic area served and the scope of services offered by the PV Circuit Rider program. To address these interests, a non-profit utility organization, the Photovoltaic Services Network (PSN), has been formed. The PSN will continue circuit rider support, as needed, and will also provide support for PV product specifications development, PV system testing, and PV system bulk purchasing agreements.

In order to meet all these needs, the next goal will be to create a sustainable organization which does not require non-utility funding. However, it will be necessary to increase the contribution by the utility members above the current level of approximately $10 \%$ of the total project cost.

\section{References}

1. PV Circuit Rider News, June ‘93 - May '95.

2. PV Circuit Rider Program Quartley Reports from June '93 though April '95.

3. "PV Circuit Rider Program: PV Program Support for Electric Utilities", C. Lane, P. Plate, ASES ‘94, San Jose 


\subsection{LIST OF ACTIVE SUBCONTRACTS}

\begin{tabular}{|c|c|c|c|c|}
\hline \multicolumn{5}{|c|}{ Subcontracts for FY 1994} \\
\hline $\begin{array}{l}\text { Contractor, Principal } \\
\text { Investigator, Address }\end{array}$ & $\begin{array}{l}\text { Work Title } \\
\text { (Research Activity) }\end{array}$ & $\begin{array}{l}\text { Contract } \\
\text { Number }\end{array}$ & $\begin{array}{l}\text { FY } 1994 \\
\text { Funding } \\
\text { (\$K) }\end{array}$ & $\begin{array}{l}\text { Start' } \\
\text { End } \\
\text { Dates }\end{array}$ \\
\hline \multicolumn{5}{|c|}{ CRYSTALLINE SILICON AND ADVANCED DEVICES PROJECT } \\
\hline $\begin{array}{l}\text { Califomia Institute of } \\
\text { Technology } \\
\text { H. Atwater } \\
\text { Pasadena, CA } 91125\end{array}$ & $\begin{array}{l}\text { Highly Crystalline } \\
\text { Si Films }\end{array}$ & XAL-4-13357-01 & 75.0 & $\begin{array}{l}7 / 94 \\
7 / 97\end{array}$ \\
\hline $\begin{array}{l}\text { Georgia Institute of Tech } \\
\text { A. Rohatgi } \\
\text { Atlanta, GA } 30332\end{array}$ & $\begin{array}{l}\text { Post-Growth } \\
\text { Quality } \\
\text { Enhancement }\end{array}$ & $\mathrm{XD}-2-11004-2$ & 130.0 & $\begin{array}{l}4 / 92 \\
6 / 95\end{array}$ \\
\hline $\begin{array}{l}\text { Duke University } \\
\text { T. Tan } \\
\text { Dept. of Mech. Engin. } \\
\text { Durham, NC } 27706\end{array}$ & $\begin{array}{l}\text { Influence of Self- } \\
\text { Interstitials Injected } \\
\text { by Phosphorus } \\
\text { Diffusion on Defect } \\
\text { Structures and } \\
\text { Electronic } \\
\text { Properties in } \\
\text { Crystalline Si }\end{array}$ & XD-2-11004-1 & 145.0 & $\begin{array}{l}3 / 92 \\
6 / 95\end{array}$ \\
\hline $\begin{array}{l}\text { Johns Hopkins University } \\
\text { Dr. Searson } \\
\text { Baltimore, MD } 21218\end{array}$ & $\begin{array}{l}\text { Solar Electric } \\
\text { Conversion at Dye- } \\
\text { Sensitized } \\
\text { Nanostructured } \\
\text { Electrodes }\end{array}$ & XAD-3-12114-4 & 50.0 & $\begin{array}{l}7 / 93 \\
7 / 96\end{array}$ \\
\hline $\begin{array}{l}\text { Massachusetts Institute of } \\
\text { Technology } \\
\text { L. Kimerling } \\
\text { Cambridge, MA } 02139\end{array}$ & $\begin{array}{l}\text { Role of Point } \\
\text { Defects and } \\
\text { Impurities in } \\
\text { Processing and } \\
\text { Performance of } \\
\text { Crystalline Si Solar } \\
\text { Cells }\end{array}$ & $\mathrm{XD}-2-11004-4$ & 125.0 & $\begin{array}{l}7 / 92 \\
7 / 95\end{array}$ \\
\hline $\begin{array}{l}\text { North Carolina State } \\
\text { University } \\
\text { S. Bedair } \\
\text { Raleigh, NC } 27695\end{array}$ & $\begin{array}{l}\text { High Efficiency } \\
\text { Cascade Solar Cells }\end{array}$ & XAD-2-1104-5 & 99.3 & $\begin{array}{l}12 / 93 \\
16 / 95\end{array}$ \\
\hline
\end{tabular}




\begin{tabular}{|c|c|c|c|c|}
\hline \multicolumn{5}{|c|}{ Subcontracts for FY 1994} \\
\hline $\begin{array}{l}\text { Contractor, Principal } \\
\text { Investigator, Address }\end{array}$ & $\begin{array}{l}\text { Work Title } \\
\text { (Research Activity) }\end{array}$ & $\begin{array}{l}\text { Contract } \\
\text { Number }\end{array}$ & $\begin{array}{l}\text { FY } 1994 \\
\text { Funding } \\
(\$ K)\end{array}$ & $\begin{array}{l}\text { Start/ } \\
\text { End } \\
\text { Dates }\end{array}$ \\
\hline $\begin{array}{l}\text { North Carolina State } \\
\text { University } \\
\text { G. Rozgonyi } \\
\text { Raleigh, NC } 27695 \\
\end{array}$ & $\begin{array}{l}\text { Electrical and } \\
\text { Structural } \\
\text { Characterization of } \\
\text { PV Si Substrates }\end{array}$ & XD-2-11004-6 & 130.0 & $\begin{array}{l}5 / 92 \\
6 / 95\end{array}$ \\
\hline $\begin{array}{l}\text { Princeton University } \\
\text { S. Forrest } \\
\text { Princeton, NJ } 08544\end{array}$ & $\begin{array}{l}\text { Crystalline Organic } \\
\text { Multiple Quantum- } \\
\text { Well Solar Cells } \\
\end{array}$ & XAI-3-11670-3 & 50.0 & $\begin{array}{l}8 / 93 \\
9 / 95\end{array}$ \\
\hline $\begin{array}{l}\text { Research Triangle } \\
\text { Institute } \\
\text { R. Vankatasubramanian } \\
\text { RTI, NC } 27709\end{array}$ & $\begin{array}{l}\text { High Efficiency } \\
\text { GaAs Solar Cells }\end{array}$ & YAL-4-13357-03 & 250.0 & $\begin{array}{l}8 / 94 \\
8 / 97\end{array}$ \\
\hline $\begin{array}{l}\text { Spire Corporation } \\
\text { Vernon/Maruska } \\
\text { Bedford, MA } 01730\end{array}$ & $\begin{array}{l}\text { High Performance } \\
\text { (Porous) Si Solar } \\
\text { Cells }\end{array}$ & XAI-3-11670-2 & 0.0 & $\begin{array}{l}10 / 93 \\
9 / 95\end{array}$ \\
\hline $\begin{array}{l}\text { SUNY at Buffalo } \\
\text { W. Anderson } \\
\text { Amherst, NY } 14228\end{array}$ & $\begin{array}{l}\text { Thin Film Si by } \\
\text { Constitutional } \\
\text { Supercooling }\end{array}$ & XR-1-1168-1 & 0.0 & $\begin{array}{l}5 / 93 \\
6 / 95\end{array}$ \\
\hline $\begin{array}{l}\text { Texas Tech University } \\
\text { Stefan Eistreicher } \\
\text { Lubbock, TX } 79409\end{array}$ & $\begin{array}{l}\text { Theoretical Analysis } \\
\text { of Hydrogen- } \\
\text { Vacancy Complex } \\
\text { Formation and } \\
\text { Diffusion in } \mathrm{Si}\end{array}$ & XAI-4-14195-1 & 25.0 & $\begin{array}{l}2 / 93 \\
6 / 95\end{array}$ \\
\hline $\begin{array}{l}\text { Univ. of Calif. Berkeley } \\
\text { E. Weber } \\
\text { Los Angeles, CA } 90089\end{array}$ & $\begin{array}{l}\text { Identification and } \\
\text { Control of Lifetime } \\
\text { Reducing Defects }\end{array}$ & XD-2-11004-3 & 160.0 & $\begin{array}{l}7 / 92 \\
7 / 95\end{array}$ \\
\hline $\begin{array}{l}\text { Univ. of Califomia, LA } \\
\text { E. Yablonovitch } \\
\text { Los Angeles, CA } 90024\end{array}$ & $\begin{array}{l}\text { Optical } \\
\text { Confinement in the } \\
\text { Wave Limit }\end{array}$ & XAL-4-13357-02 & 166.0 & $\begin{array}{l}7 / 94 \\
7 / 97\end{array}$ \\
\hline $\begin{array}{l}\text { University of Florida at } \\
\text { Gainsville } \\
\text { T. Anderson } \\
\text { Gainesville, FL } 32611\end{array}$ & $\begin{array}{l}\text { New Plasma Source } \\
\text { of Hydrides }\end{array}$ & XCG-4-14194-01 & 54.0 & $\begin{array}{l}6 / 94 \\
6 / 97\end{array}$ \\
\hline $\begin{array}{l}\text { Univ. of South Florida } \\
\text { L. Jastrzebski } \\
\text { Tampa, FL } 33620\end{array}$ & $\begin{array}{l}\text { Optimization of } \\
\text { Gettering Processes } \\
\text { for PV Si }\end{array}$ & XD-2-11004-5 & 130.0 & $\begin{array}{l}2 / 92 \\
6 / 95\end{array}$ \\
\hline
\end{tabular}




\begin{tabular}{|c|c|c|c|c|}
\hline \multicolumn{5}{|c|}{ Subcontracts for FY 1994} \\
\hline $\begin{array}{l}\text { Contractor, Principal } \\
\text { Investigator, Address }\end{array}$ & $\begin{array}{l}\text { Work Title } \\
\text { (Research Activity) }\end{array}$ & $\begin{array}{l}\text { Contract } \\
\text { Number }\end{array}$ & $\begin{array}{l}\text { FY } 1994 \\
\text { Funding } \\
\text { (\$K) }\end{array}$ & $\begin{array}{l}\text { Start/ } \\
\text { End } \\
\text { Dates }\end{array}$ \\
\hline $\begin{array}{l}\text { University of Illinois } \\
\text { A. Rockett } \\
\text { Champaign, IL } 61820\end{array}$ & $\begin{array}{l}\text { Effects of Crystal } \\
\text { Defects on CuInSe }\end{array}$ & $X A D-3-12114-1$ & 85.0 & $\begin{array}{l}5 / 93 \\
6 / 96\end{array}$ \\
\hline $\begin{array}{l}\text { University of Utah } \\
\text { C. Taylor } \\
\text { Salt Lake City, UT } 84112\end{array}$ & $\begin{array}{l}\text { Electronic Processes } \\
\text { in Thin-Film PV } \\
\text { Materials }\end{array}$ & XAD-3-12114-2 & 188.0 & $\begin{array}{l}3 / 93 \\
6 / 96\end{array}$ \\
\hline $\begin{array}{l}\text { Univ. of South Florida } \\
\text { D. Morel } \\
\text { Tampa, FL } 33620\end{array}$ & $\begin{array}{l}\text { Heterojunctions in } \\
\text { Thin-Film Solar } \\
\text { Cells }\end{array}$ & $\mathrm{XAD}-3-12114-3$ & 130.0 & $\begin{array}{l}4 / 93 \\
5 / 96\end{array}$ \\
\hline \multicolumn{5}{|c|}{ THIN FILM PV TECHNOLOGIES PROJECT FY 1994} \\
\hline $\begin{array}{l}\text { Advanced Photovoltaic } \\
\text { Systems } \\
\text { R. Maitland } \\
\text { Princeton, NJ } 08542\end{array}$ & $\begin{array}{l}\text { Stable Hi-Efficiency } \\
\text { a-Si Multijunction } \\
\text { Modules }\end{array}$ & ZM-1-19033-2 & 500.0 & $\begin{array}{l}12 / 91 \\
9 / 94\end{array}$ \\
\hline $\begin{array}{l}\text { AstroPower, Inc. } \\
\text { J. Rand } \\
\text { Newark, DE } 19711\end{array}$ & $\begin{array}{l}\text { Monolithically } \\
\text { Integrated Si-Film } \\
\text { PV Modules }\end{array}$ & ZR-1-11064-1 & 369.0 & $\begin{array}{l}5 / 91 \\
6 / 95\end{array}$ \\
\hline $\begin{array}{l}\text { Colorado State Univ. } \\
\text { J. Sites } \\
\text { Fort Collins, CO } 80523\end{array}$ & $\begin{array}{l}\text { Role of } \\
\text { Polycrystallinity in } \\
\text { CdTe and CuInSe }{ }_{2} \\
\text { PV }\end{array}$ & $X A X-4-14000-1$ & 92.0 & $\begin{array}{l}4 / 90 \\
12 / 96\end{array}$ \\
\hline $\begin{array}{l}\text { Colorado School of Mines } \\
\text { J. Trefny } \\
\text { Golden, CO } 80401\end{array}$ & $\begin{array}{l}\text { CdTe Solar Cells } \\
\text { Fabricated by } \\
\text { Electro-deposition }\end{array}$ & $X G-2-11036-4$ & 174.5 & $\begin{array}{l}3 / 92 \\
12 / 96\end{array}$ \\
\hline $\begin{array}{l}\text { Colorado School of Mines } \\
\text { D. Williamson } \\
\text { Golden, CO } 80401\end{array}$ & $\begin{array}{l}\text { Small-Angle X-Ray } \\
\text { Scattering/Micro- } \\
\text { voids a-Si } \\
\text { Semiconductors }\end{array}$ & $X G-1-10063-3$ & 70.0 & $\begin{array}{l}1 / 91 \\
4 / 97\end{array}$ \\
\hline $\begin{array}{l}\text { Energy Conversion } \\
\text { Devices } \\
\text { X. Deng } \\
\text { Troy, MI } 48084\end{array}$ & $\begin{array}{l}\text { A-Si Multijunction } \\
\text { Cells }\end{array}$ & ZAN-4-13318-11 & 135.0 & $\begin{array}{l}7 / 94 \\
7 / 97\end{array}$ \\
\hline $\begin{array}{l}\text { Energy Photovoltaics } \\
\text { A. Delahoy } \\
\text { Lawrenceville, NJ } 08648\end{array}$ & $\begin{array}{l}\text { Ultra-Thin CIS } \\
\text { Devices with Non- } \\
\mathrm{H}_{2} \mathrm{Se}\end{array}$ & $X G-2-12051-1$ & 892.0 & $\begin{array}{l}3 / 92 \\
2 / 95\end{array}$ \\
\hline
\end{tabular}




\begin{tabular}{|c|c|c|c|c|}
\hline \multicolumn{5}{|c|}{ Subcontracts for FY 1994} \\
\hline $\begin{array}{l}\text { Contractor, Principal } \\
\text { Investigator, Address }\end{array}$ & $\begin{array}{l}\text { Work Title } \\
\text { (Research Activity) }\end{array}$ & $\begin{array}{l}\text { Contract } \\
\text { Number }\end{array}$ & $\begin{array}{l}\text { FY } 1994 \\
\text { Funding } \\
(\$ K)\end{array}$ & $\begin{array}{l}\text { Start } \\
\text { End } \\
\text { Dates }\end{array}$ \\
\hline $\begin{array}{l}\text { Energy Photovoltaics } \\
\text { \& Delahoy } \\
\text { L. wrenceville, NJ } 08648\end{array}$ & $\begin{array}{l}\text { A-Si Multijunction } \\
\text { Cells }\end{array}$ & ZAF-5-14142-04 & 135.0 & $\begin{array}{l}1 / 95 \\
3 / 95\end{array}$ \\
\hline $\begin{array}{l}\text { Florida Solar Energy } \\
\text { Center } \\
\text { N. Dhere } \\
\text { Cape Canaveral, FL } \\
32920\end{array}$ & $\begin{array}{l}\text { Polycrystalline } \\
\text { CuInSe }_{2} \text { and CdTe } \\
\text { PV Solar Cells }\end{array}$ & $X G-2-11036-5$ & 77.8 & $\begin{array}{l}4 / 92 \\
4 / 95\end{array}$ \\
\hline $\begin{array}{l}\text { Georgia Institute of } \\
\text { Technology } \\
\text { A. Rohatgi } \\
\text { Atlanta, GA } 30332\end{array}$ & $\begin{array}{l}\text { MOCVD CdTe } \\
\text { Sputtering }\end{array}$ & $X G-2-11036-3$ & 128.0 & $\begin{array}{l}2 / 92 \\
1 / 95\end{array}$ \\
\hline $\begin{array}{l}\text { Golden Photon, Inc. } \\
\text { S. Albright } \\
\text { Golden, CO } 80401\end{array}$ & $\begin{array}{l}\text { High-Efficiency, } \\
\text { Large-Area CdTe } \\
\text { Panels }\end{array}$ & ZN-0-19019-1 & 465.0 & $\begin{array}{l}6 / 90 \\
2 / 95\end{array}$ \\
\hline $\begin{array}{l}\text { Harvard University } \\
\text { R. Gordon } \\
\text { Cambridge, MA } 02138\end{array}$ & $\begin{array}{l}\text { Optimization of } \\
\text { Transparent and } \\
\text { Reflecting Films for } \\
\text { a-Si Solar Cells }\end{array}$ & XAN-4-13318-5 & 120.0 & $\begin{array}{l}5 / 91 \\
5 / 97\end{array}$ \\
\hline $\begin{array}{l}\text { Iowa State University } \\
\text { V. Dalal } \\
\text { Ames, IA } 50011\end{array}$ & $\begin{array}{l}\text { Stability and } \\
\text { Electronic } \\
\text { Properties of a-Si:H } \\
\text { and a-SiGe:H } \\
\text { Alloys }\end{array}$ & XAN-4-13318-8 & 125.0 & $\begin{array}{l}3 / 91 \\
5 / 97\end{array}$ \\
\hline $\begin{array}{l}\text { International Solar } \\
\text { Electric Technology } \\
\text { V. Kapur } \\
\text { Inglewood, CA } 90301\end{array}$ & $\begin{array}{l}\text { Novel Two-Stage } \\
\text { Selenization } \\
\text { Methods for } \\
\text { Fabrication of CIS } \\
\text { Cells \& Sub- } \\
\text { Modules }\end{array}$ & YI-2-12069-1 & 545.0 & $\begin{array}{l}3 / 92 \\
2 / 94\end{array}$ \\
\hline $\begin{array}{l}\text { National Institute of } \\
\text { Standards and Technology } \\
\text { A. Gallagher } \\
\text { Boulder, CO } 80303\end{array}$ & $\begin{array}{l}\text { Growth } \\
\text { Mechanisms and } \\
\text { Characterization of } \\
\text { a-Si:H Alloy Films }\end{array}$ & DAD-4-14084-1 & 150.0 & $\begin{array}{l}1 / 91 \\
1 / 95\end{array}$ \\
\hline
\end{tabular}




\begin{tabular}{|c|c|c|c|c|}
\hline \multicolumn{5}{|c|}{ Subcontracts for FY 1994} \\
\hline $\begin{array}{l}\text { Contractor, Principal } \\
\text { Investigator, Address }\end{array}$ & $\begin{array}{l}\text { Work Title } \\
\text { (Research Activity) }\end{array}$ & $\begin{array}{l}\text { Contract } \\
\text { Number }\end{array}$ & $\begin{array}{l}\text { FY } 1994 \\
\text { Funding } \\
\text { (\$K) }\end{array}$ & $\begin{array}{l}\text { Start } \\
\text { End } \\
\text { Dates }\end{array}$ \\
\hline $\begin{array}{l}\text { North Carolina State } \\
\text { University } \\
\text { G. Lucovsky } \\
\text { Raleigh, NC } 27695\end{array}$ & $\begin{array}{l}\text { Electrical and } \\
\text { Optical Properties } \\
\text { of Micro-crystalline } \\
\text { Si and a-Si,N:H } \\
\text { Alloys }\end{array}$ & $X G-10063-2$ & 33.0 & $\begin{array}{l}2 / 91 \\
2 / 94\end{array}$ \\
\hline $\begin{array}{l}\text { Pennsylvania State } \\
\text { University } \\
\text { R. Collins } \\
\text { Univ. Park, PA } 16801\end{array}$ & $\begin{array}{l}\text { In-Situ } \\
\text { Characterization of } \\
\text { Growth and } \\
\text { Interfaces In a-Si:H } \\
\text { Devices }\end{array}$ & $X G-1-10063-10$ & 120.0 & $\begin{array}{l}4 / 91 \\
4 / 94\end{array}$ \\
\hline $\begin{array}{l}\text { Purdue University } \\
\text { J. Gray } \\
1021 \text { Hovde Hall, Rm } \\
301 \\
\text { West Lafayette, IN } 47907 \\
\end{array}$ & $\begin{array}{l}\text { Development and } \\
\text { Application of a } \\
\text { Computer Model } \\
\text { for CdTe and } \\
\mathrm{CuInSe}_{2}\end{array}$ & $X G-2-11036-2$ & 72.0 & $\begin{array}{l}3 / 92 \\
2 / 95\end{array}$ \\
\hline $\begin{array}{l}\text { Siemens Solar Industries } \\
\text { K. Mitchell } \\
\text { Camarillo, CA } 93011\end{array}$ & $\begin{array}{l}\text { High Efficiency } \\
\text { Large-Area CIS } \\
\text { Modules }\end{array}$ & $\mathrm{ZN}-1-19019-5$ & 700.0 & $\begin{array}{l}5 / 91 \\
4 / 95\end{array}$ \\
\hline $\begin{array}{l}\text { Solar Cells Inc. } \\
\text { T. Zhou } \\
\text { Toledo, OH } 43615\end{array}$ & $\begin{array}{l}\text { Fabrication of } \\
\text { Stable Large-Area } \\
\text { Thin-Film CdTe PV } \\
\text { Modules }\end{array}$ & ZR-1-11059-1 & 604.0 & $\begin{array}{l}5 / 91 \\
11 / 96\end{array}$ \\
\hline $\begin{array}{l}\text { Solarex } \\
\text { R. Arya } \\
\text { Newtown, PA } 18940\end{array}$ & $\begin{array}{l}\text { Research on Poly- } \\
\text { crystalline Thin } \\
\text { Film Submodules } \\
\text { Based on CuInSe }_{2} \\
\text { Materials }\end{array}$ & ZAN-4-13318-1 & 604.0 & $\begin{array}{l}5 / 90 \\
9 / 97\end{array}$ \\
\hline $\begin{array}{l}\text { Solarex } \\
\text { R. Arya } \\
\text { Newtown, PA } 18940\end{array}$ & $\begin{array}{l}\text { A-Si Multijunction } \\
\text { Cells }\end{array}$ & ZAN-4-13318-01 & 175.0 & $\begin{array}{l}9 / 94 \\
9 / 97\end{array}$ \\
\hline $\begin{array}{l}\text { Syracuse University } \\
\text { E. Schiff } \\
\text { Syracuse, NY } 13244\end{array}$ & $\begin{array}{l}\text { Research on Defects } \\
\text { and Transport in a- } \\
\text { Si Based } \\
\text { Semiconductors }\end{array}$ & XG-1-10063-7 & 90.0 & $\begin{array}{l}2 / 91 \\
6 / 95\end{array}$ \\
\hline $\begin{array}{l}\text { Univ. of Califomia, LA } \\
\text { R. Braunstein } \\
\text { Los Angeles, CA } 90024\end{array}$ & $\begin{array}{l}\text { A-Si Photocharge } \\
\text { Transfer }\end{array}$ & $\begin{array}{l}\text { XAN-4-13318- } \\
10\end{array}$ & 30.0 & $\begin{array}{l}5 / 94 \\
4 / 97\end{array}$ \\
\hline
\end{tabular}




\begin{tabular}{|c|c|c|c|c|}
\hline \multicolumn{5}{|c|}{ Subcontracts for FY 1994} \\
\hline $\begin{array}{l}\text { Contractor, Principal } \\
\text { Investigator, Address }\end{array}$ & $\begin{array}{l}\text { Work Title } \\
\text { (Research Activity) }\end{array}$ & $\begin{array}{l}\text { Contract } \\
\text { Number }\end{array}$ & $\begin{array}{l}\text { FY } 1994 \\
\text { Funding } \\
\text { (\$K) }\end{array}$ & $\begin{array}{l}\text { Start/ } \\
\text { End } \\
\text { Dates }\end{array}$ \\
\hline $\begin{array}{l}\text { University of Colorado } \\
\text { A. Hermann } \\
\text { Boulder, CO } 80309\end{array}$ & $\begin{array}{l}\text { Novel Thin Film } \\
\text { CuInSe }_{2} \text { Fabrication }\end{array}$ & $X C-0-10012-1$ & 28.0 & $\begin{array}{l}3 / 90 \\
1 / 95\end{array}$ \\
\hline $\begin{array}{l}\text { University of Delaware- } \\
\text { IEC } \\
\text { S. Hegedus } \\
\text { Newark, DE } 19716\end{array}$ & $\begin{array}{l}\text { Stable High- } \\
\text { Efficiency a-Si- } \\
\text { Based Solar Cells } \\
\text { with Low Hydrogen } \\
\text { Content }\end{array}$ & $X G-1-10063-4$ & -0 & $\begin{array}{l}3 / 91 \\
4 / 94\end{array}$ \\
\hline $\begin{array}{l}\text { University of Delaware- } \\
\text { IEC } \\
\text { R. Birkmire } \\
\text { Newark, DE } 19716\end{array}$ & $\begin{array}{l}\text { Thin Film Materials } \\
\text { and Devices }\end{array}$ & XAV-3-13170-1 & 1342.0 & $\begin{array}{l}11 / 92 \\
1 / 97\end{array}$ \\
\hline $\begin{array}{l}\text { University of Illinois } \\
\text { J. Abelson/N. Maley } \\
\text { Champaign, IL } 61820\end{array}$ & $\begin{array}{l}\text { Research on Si- } \\
\text { Carbon Alloys and } \\
\text { Interfaces }\end{array}$ & $X G-1-10063-6$ & 25.0 & $\begin{array}{l}2 / 91 \\
4 / 95\end{array}$ \\
\hline $\begin{array}{l}\text { Univ. of North Carolina } \\
\text { H. Daxing } \\
\text { Chapel Hill, NC } 27599\end{array}$ & $\begin{array}{l}\text { Recombination and } \\
\text { Metastability in a-Si } \\
\text { and } \mathrm{Si} \mathrm{Ge} \mathrm{Alloys}\end{array}$ & $\mathrm{XG}-1-10063-5$ & 50.0 & $\begin{array}{l}2 / 91 \\
1 / 94\end{array}$ \\
\hline $\begin{array}{l}\text { University of Oregon } \\
\text { J. Cohen } \\
\text { Eugene, OR } 97403\end{array}$ & $\begin{array}{l}\text { Origins of } \\
\text { Metastable Effects } \\
\text { in a-Si:H and Deep } \\
\text { Defect } \\
\text { Characterization in } \\
\text { a-Si, Ge:H Alloys }\end{array}$ & $X G-1-10063-1$ & 120.0 & $\begin{array}{l}2 / 91 \\
4 / 94\end{array}$ \\
\hline $\begin{array}{l}\text { Univ. of South Florida } \\
\text { D. Morel } \\
\text { Tampa, FL } 33620\end{array}$ & $\begin{array}{l}\text { Advanced } \\
\text { Processing } \\
\text { Technology for } \\
\text { High Efficiency } \\
\text { Thin Film CIS and } \\
\text { CdTe }\end{array}$ & $X G-11036-1$ & 200.00 & $\begin{array}{l}5 / 92 \\
2 / 95\end{array}$ \\
\hline $\begin{array}{l}\text { University of Toledo } \\
\text { A. Campaan } \\
\text { Toledo, OH } 43606\end{array}$ & $\begin{array}{l}\text { Thin Film CdTe PV } \\
\text { Cells }\end{array}$ & $\mathrm{ZN}-1-19019-3$ & 125.0 & $\begin{array}{l}7 / 90 \\
1 / 94\end{array}$ \\
\hline $\begin{array}{l}\text { United Solar Systems } \\
\text { Corp. } \\
\text { S. Guha } \\
\text { Troy, MI } 48084\end{array}$ & $\begin{array}{l}\text { High-Efficiency } \\
\text { Multi-Gap Multi- } \\
\text { Junction a-Si-Based } \\
\text { Submodules }\end{array}$ & $\mathrm{ZM}-1-19033-2$ & 738.0 & $\begin{array}{l}1 / 91 \\
4 / 94\end{array}$ \\
\hline
\end{tabular}




\begin{tabular}{|c|c|c|c|c|}
\hline \multicolumn{5}{|c|}{ Subcontracts for FY 1994} \\
\hline $\begin{array}{l}\text { Contractor, Principal } \\
\text { Investigator, Address }\end{array}$ & $\begin{array}{l}\text { Work Title } \\
\text { (Research Activity) }\end{array}$ & $\begin{array}{l}\text { Contract } \\
\text { Number }\end{array}$ & $\begin{array}{l}\text { FY } 1994 \\
\text { Funding } \\
(\$ K)\end{array}$ & $\begin{array}{l}\text { Start/ } \\
\text { End } \\
\text { Dates }\end{array}$ \\
\hline $\begin{array}{l}\text { United Solar Systems } \\
\text { Corp. } \\
\text { S. Guha } \\
\text { Troy, MI } 48084\end{array}$ & A-Si Research & ZAN-4-13318-02 & 175.0 & $\begin{array}{l}7 / 94 \\
7 / 97\end{array}$ \\
\hline $\begin{array}{l}\text { Washington State Univ. } \\
\text { L. Olsen } \\
\text { Pulman, WA } 99164\end{array}$ & $\begin{array}{l}\text { CuInSe }{ }_{2} \text { Solar Cells } \\
\text { Based on ZnSe } \\
\text { Windows }\end{array}$ & $X G-2-11036-6$ & 90.0 & $\begin{array}{l}2 / 92 \\
2 / 95\end{array}$ \\
\hline $\begin{array}{l}\text { Xerox Corporation } \\
\text { R. Street } \\
\text { Palo Alto, CA } 94304\end{array}$ & $\begin{array}{l}\text { Stability, Electronic } \\
\text { and Structural } \\
\text { Properties of a-Si } \\
\text { Silicon Alloys }\end{array}$ & $X G-1-10063-9$ & 208.0 & $\begin{array}{l}6 / 91 \\
5 / 95\end{array}$ \\
\hline \multicolumn{5}{|c|}{ PHOTOVOLTAIC MANUFACTURING TECHNOLOGY (PVMaT) PROJECT FY 1994} \\
\hline $\begin{array}{l}\text { AstroPower, Inc. } \\
\text { S. Collins } \\
\text { Newark, DE } 19711\end{array}$ & $\begin{array}{l}\text { Silicon-Film } \\
\text { PVMaT }\end{array}$ & ZM-2-11040-5 & 1511.0 & $\begin{array}{l}1 / 92 \\
2 / 95\end{array}$ \\
\hline $\begin{array}{l}\text { Energy Conversion } \\
\text { Devices } \\
\text { M. Izu } \\
\text { Troy, MI } 48084 \\
\end{array}$ & $\begin{array}{l}\text { Continuous Roll-to- } \\
\text { Roll a-Si PVMaT }\end{array}$ & $\mathrm{ZM}-1-11040-7$ & 1809.0 & $\begin{array}{l}4 / 92 \\
7 / 95\end{array}$ \\
\hline $\begin{array}{l}\text { ENTECH, Inc. } \\
\text { M. O'Neill } \\
\text { Dallas-Ft. Worth Airport } \\
\text { TX } 74261\end{array}$ & $\begin{array}{l}\text { PVMaT } \\
\text { Improvements for } \\
\text { ENTECH's } \\
\text { Concentrator } \\
\text { Module }\end{array}$ & ZM-2-11040-4 & 600.0 & $\begin{array}{l}2 / 92 \\
5 / 95\end{array}$ \\
\hline $\begin{array}{l}\text { Mobil Solar Energy Corp. } \\
\text { J. Kalejs } \\
\text { Billerica, MA 01821-3980 }\end{array}$ & $\begin{array}{l}\text { Thin Edge-Defined } \\
\text { Film-Fed Growth } \\
\text { (EFG) Octagons }\end{array}$ & ZM-1-11040-3 & 599.8 & $\begin{array}{l}4 / 92 \\
1 / 94\end{array}$ \\
\hline $\begin{array}{l}\text { Siemens Solar Industries } \\
\text { T. Jester } \\
\text { Camarillo, CA } 93011\end{array}$ & $\begin{array}{l}\text { Photovoltaic } \mathrm{Cz}-\mathrm{Si} \\
\text { Manufacturing } \\
\text { Technology } \\
\text { Improvements }\end{array}$ & ZM-1-11040-1 & 1695.0 & $\begin{array}{l}3 / 92 \\
5 / 95\end{array}$ \\
\hline $\begin{array}{l}\text { Solarex Corp. } \\
\text { R. Oswald } \\
\text { Newtown, PA } 18940\end{array}$ & $\begin{array}{l}\text { Large-Area Triple } \\
\text { Junction a-Si Alloy } \\
\text { Production Scale- } \\
\text { Up Project }\end{array}$ & ZM-2-11040-2 & 1659.0 & $\begin{array}{l}3 / 92 \\
6 / 95\end{array}$ \\
\hline
\end{tabular}




\begin{tabular}{|c|c|c|c|c|}
\hline \multicolumn{5}{|c|}{ Subcontracts for FY 1994} \\
\hline $\begin{array}{l}\text { Contractor, Principal } \\
\text { Investigator, Address }\end{array}$ & $\begin{array}{l}\text { Work Title } \\
\text { (Research Activity) }\end{array}$ & $\begin{array}{l}\text { Contract } \\
\text { Number }\end{array}$ & $\begin{array}{l}\text { FY } 1994 \\
\text { Funding } \\
\text { (\$K) }\end{array}$ & $\begin{array}{l}\text { Start/ } \\
\text { End } \\
\text { Dates }\end{array}$ \\
\hline $\begin{array}{l}\text { Spire Corporation } \\
\text { M. Nowlan } \\
\text { Bedford, MA } 01730\end{array}$ & $\begin{array}{l}\text { Automated Solar } \\
\text { Cell Assembly } \\
\text { Teamed Process } \\
\text { Research }\end{array}$ & ZAG-3-11219-01 & 533.0 & $\begin{array}{l}1 / 93 \\
1 / 95\end{array}$ \\
\hline $\begin{array}{l}\text { Springbom Laboratories } \\
\text { W. Holley } \\
\text { Enfield, CT } 06082\end{array}$ & $\begin{array}{l}\text { Teamed R\&D on } \\
\text { Discoloration and } \\
\text { Degradation of } \\
\text { EVA }\end{array}$ & ZAG-3-11219-02 & $-0-$ & $\begin{array}{l}12 / 92 \\
6 / 96\end{array}$ \\
\hline $\begin{array}{l}\text { Utility Power Group } \\
\text { G. Duran } \\
\text { Chatsworth, CA } 91311 \\
\text { and } \\
\text { Advanced Photovoltaics } \\
\text { Systems } \\
\text { H. Volltrauer } \\
\text { Princeton, NJ } 08542\end{array}$ & $\begin{array}{l}\text { A-Si PV } \\
\text { Manufacturing } \\
\text { Technology }\end{array}$ & $\begin{array}{l}\text { ZM-1-11040-6 } \\
\text { Lower Tier } \\
\text { Subcontract }\end{array}$ & 1420.0 & $\begin{array}{l}4 / 92 \\
7 / 95\end{array}$ \\
\hline $\begin{array}{l}\text { Golden Photon, Inc. } \\
\text { S. Johnson } \\
\text { Golden, CO } 80401\end{array}$ & $\begin{array}{l}\text { PVMaT, Phase 2B } \\
\text { Process-Specific } \\
\text { Issues }\end{array}$ & ZAI-4-11294-03 & 1798.5 & $\begin{array}{l}1 / 94 \\
1 / 97\end{array}$ \\
\hline $\begin{array}{l}\text { Texas Instruments } \\
\text { J. Skelly } \\
\text { Dallas, TX } 75243\end{array}$ & $\begin{array}{l}\text { PVMaT, Phase 2B- } \\
\text { Solar Technology }\end{array}$ & ZAI-4-11294-4 & 2000.0 & $\begin{array}{l}1 / 94 \\
1 / 95\end{array}$ \\
\hline $\begin{array}{l}\text { Solar Cells, Inc. } \\
\text { D. Sandwisch } \\
\text { Toledo, OH } 43607\end{array}$ & $\begin{array}{l}\text { High Throughput } \\
\text { Manufacturing of } \\
\text { Thin Film CdTe PV } \\
\text { Modules }\end{array}$ & ZAI-4-11294-02 & 1189.4 & $\begin{array}{l}11 / 93 \\
11 / 96\end{array}$ \\
\hline $\begin{array}{l}\text { Solarex Corp. } \\
\text { R. Oswald } \\
\text { Newtown, PA } 18940\end{array}$ & $\begin{array}{l}\text { Large-Area Triple } \\
\text { Junction a-Si Alloy } \\
\text { Scale-Up Project }\end{array}$ & $\mathrm{ZM}-2-11040-2$ & 1658.7 & $\begin{array}{l}3 / 92 \\
6 / 95\end{array}$ \\
\hline
\end{tabular}




\begin{tabular}{|c|c|c|c|c|}
\hline \multicolumn{5}{|c|}{ Subcontracts for FY 1994} \\
\hline $\begin{array}{l}\text { Contractor, Principal } \\
\text { Investigator, Address }\end{array}$ & $\begin{array}{l}\text { Work Title } \\
\text { (Research Activity) }\end{array}$ & $\begin{array}{l}\text { Contract } \\
\text { Number }\end{array}$ & $\begin{array}{l}\text { FY } 1994 \\
\text { Funding } \\
\text { (\$K) }\end{array}$ & $\begin{array}{l}\text { Start/ } \\
\text { End } \\
\text { Dates }\end{array}$ \\
\hline $\begin{array}{l}\text { Solarex Corp. } \\
\text { J. Wohlgemuth } \\
\text { Frederick, MD }\end{array}$ & $\begin{array}{l}\text { PVMaT Phase 2B, } \\
\text { Process-Specific } \\
\text { Issues }\end{array}$ & ZAI-4-11294-01 & 1134.3 & $\begin{array}{l}12 / 93 \\
12 / 96\end{array}$ \\
\hline \multicolumn{5}{|c|}{ PV MODULE AND SYSTEM PERFORMANCE AND ENGINEERING PROJECT FY 1994} \\
\hline $\begin{array}{l}\text { Arizona State University } \\
\text { R. Hammond } \\
\text { Tempe, AZ }\end{array}$ & $\begin{array}{l}\text { Laboratory } \\
\text { Accreditation } \\
\text { Module } \\
\text { Certification } \\
\text { Criteria }\end{array}$ & AAF-4-13301-01 & 100.0 & $\begin{array}{l}12 / 94 \\
12 / 95\end{array}$ \\
\hline $\begin{array}{l}\text { Endecon } \\
\text { C. Witiker } \\
\text { San Ramon, CA }\end{array}$ & PV Energy Rating & AA-4-14192-01 & 50.0 & $\begin{array}{l}4 / 94 \\
4 / 95\end{array}$ \\
\hline $\begin{array}{l}\text { SEIA } \\
\text { D. Mekin } \\
\text { Washington, D.C. } 20001\end{array}$ & $\begin{array}{l}\text { National Electric } \\
\text { Code (NEC } \\
\text { Support) }\end{array}$ & HI2-12006-27 & 36.0 & $\begin{array}{l}6 / 94 \\
6 / 95\end{array}$ \\
\hline $\begin{array}{l}\text { SEIA } \\
\text { D. Mekin } \\
\text { Washington, D.C. } 20001\end{array}$ & $\begin{array}{l}\text { Management and } \\
\text { Administration of } \\
\text { IEC/PV/TEC- } 83 \\
\text { Secretariat and } \\
\text { International } \\
\text { Standards } \\
\text { Development }\end{array}$ & HI-2-12006-9 & 55.0 & $\begin{array}{l}6 / 94 \\
6 / 95\end{array}$ \\
\hline $\begin{array}{l}\text { Sunset Technology } \\
\text { J. Anderson } \\
\text { Denver, CO }\end{array}$ & $\begin{array}{l}\text { PV Performance } \\
\text { Modeling }\end{array}$ & TAD-4-14166-01 & 25.0 & $\begin{array}{l}4 / 94 \\
4 / 95\end{array}$ \\
\hline \multicolumn{5}{|c|}{ PV APPLICATIONS AND MARKETS DEVELOPMENT PROJECT FY 1994} \\
\hline $\begin{array}{l}\text { AIA/ACSA Research } \\
\text { Council } \\
\text { Washington, D.C. } 20006\end{array}$ & $\begin{array}{l}\text { PV/Buildings } \\
\text { Curriculum } \\
\text { Development for } \\
\text { North American } \\
\text { Architecture School } \\
\text { Faculty }\end{array}$ & AD-3-13008-01 & 85.0 & $\begin{array}{l}12 / 92 \\
9 / 94\end{array}$ \\
\hline
\end{tabular}




\begin{tabular}{|c|c|c|c|c|}
\hline \multicolumn{5}{|c|}{ Subcontracts for FY 1994} \\
\hline $\begin{array}{l}\text { Contractor, Principal } \\
\text { Investigator, Address }\end{array}$ & $\begin{array}{l}\text { Work Title } \\
\text { (Research Activity) }\end{array}$ & $\begin{array}{l}\text { Contract } \\
\text { Number }\end{array}$ & $\begin{array}{l}\text { FY 1994 } \\
\text { Funding } \\
(\$ K)\end{array}$ & $\begin{array}{l}\text { Start } \\
\text { End } \\
\text { Dates }\end{array}$ \\
\hline $\begin{array}{l}\text { Centro de Pesquisas de } \\
\text { Energia Eletrica (CEPEL) } \\
\text { A. Mosse } \\
\text { Rio de Janeiro } \\
\text { Brazil } 20001-970\end{array}$ & $\begin{array}{l}\text { Brazilian Rural } \\
\text { Electrification Pilot } \\
\text { Project }\end{array}$ & $\begin{array}{l}\text { AAD-3-13179- } \\
01\end{array}$ & 121.2 & $\begin{array}{l}12 / 92 \\
12 / 96\end{array}$ \\
\hline $\begin{array}{l}\text { Neos Corporation } \\
\text { C. Lane } \\
\text { Lakewood, CO } 80215\end{array}$ & $\begin{array}{l}\text { PV Circuit Rider, } \\
\text { Phase II }\end{array}$ & $\begin{array}{l}\text { AAX-4-14410- } \\
01\end{array}$ & 30.0 & $\begin{array}{l}6 / 93 \\
3 / 95\end{array}$ \\
\hline $\begin{array}{l}\text { Solar Design Associates } \\
\text { S. Strong } \\
\text { Harvard, MA } 01451\end{array}$ & $\begin{array}{l}\text { International PV } \\
\text { Buildings Review }\end{array}$ & $\begin{array}{l}\text { AAX-3-13463- } \\
01\end{array}$ & 50.0 & $\begin{array}{l}9 / 93 \\
3 / 94\end{array}$ \\
\hline $\begin{array}{l}\text { SUNY at Albany } \\
\text { R. Perez } \\
\text { Albany, NY } 12201\end{array}$ & $\begin{array}{l}\text { Solar Resource } \\
\text { Utility Load- } \\
\text { Matching } \\
\text { Assessment }\end{array}$ & XR-1-11168-1 & 100.0 & $\begin{array}{l}9 / 91 \\
12 / 94\end{array}$ \\
\hline $\begin{array}{l}\text { University of Delaware } \\
\text { J. Byme } \\
\text { Newark, DE } 19616\end{array}$ & $\begin{array}{l}\text { Evaluation of DSM } \\
\text { Incentive } \\
\text { Opportunities for } \\
\text { PV }\end{array}$ & XR-2-11248-1 & 100.0 & $\begin{array}{l}10 / 91 \\
6 / 95\end{array}$ \\
\hline $\begin{array}{l}\text { Competitive RFP } \\
\text { (TBD) }\end{array}$ & $\begin{array}{l}\text { Brazil Phase } 2 \\
\text { Rural Electrification }\end{array}$ & AAD-5-13504 & 1155.0 & $\begin{array}{l}9 / 94 \\
9 / 95\end{array}$ \\
\hline $\begin{array}{l}\text { Miscellaneous Support } \\
\text { Subcontractors }\end{array}$ & $\begin{array}{l}\text { Sunrayce } 95 \text { and } \\
\text { Miscellaneous } \\
\text { Regulatory and } \\
\text { Education }\end{array}$ & --- & 200.00 & $\begin{array}{l}1 / 94 \\
1 / 95\end{array}$ \\
\hline
\end{tabular}




\subsection{BIBLIOGRAPHY}

\section{October 1993-September 1994}

Ahrenkiel, R.K., Keyes, B.M., Levi, D.L., Emery, K., Chu, T.L., and Chu, S.S. (1994). "Spatial Uniformity of Minority-Carrier Lifetime in Polycrystalline CdTe Solar Cells." Applied Physics Letters (64:21), pp. 2879-2881.

Albright, S.P. and Johnson, S.X. (1994). Module Process Optimization and Device Efficiency Improvement for Stable, Low-Cost, Large-Area, Cadmium Telluride-Based Photovoltaic Module Production: Final Subcontract Report, 1 July 1990-30 April 1994. SERI/TP-413-6633. Work performed by Golden Photon, Inc., Golden, CO. Available NTIS: Order No. DE94011817.

Alonso, R.G., Mascarenhas, A., Horner, G.S., Bertness, K.A., Kurtz, S.R., and Olson, J.M. (1993). "Spontaneous Ordering in GaInP: A Polarized-Piezomodulated-Reflectivity Study." Physical Review. B, Condensed Matter (48:16), pp. 11833-11837.

Arent, D.J., Alonso, R., Horner, G., Bode, M., Olson, J.M., Yin, X., DeLong, M.C., SpringThorpe, A.J., and Majeed, A. (1994). "Ordered and Randomly Disordered AlAs/GaAs Short-Period Superlattices." Journal of Vacuum Science and Technology. B, Microelectronics and Nanometer Structures (12:2), pp. 1009-1012.

Arent, D.J., Alonso, R.G., Horner, G.S., Kibbler, A.E., Olson, J.M., Yin, X., DeLong, M.C., SpringThorpe, A.J., Majeed, A., Mowbray, D.J., and Skolnick, M.S. (1994). "Optical Properties and Electroluminescence of Ordered and Disordered AlAs/GaAs Superlattices." Physics and Applications of Defects in Advanced Semiconductors: Proceedings of Materials Research Society Symposium, 29 November-1 December 1993, Boston, MA, Materials Research Society Symposium Proceedings, Volume 325, Manasreh, M.O., et al., eds. Pittsburgh, PA: Materials Research Society, pp. 499-505.

Arent, D.J., Alonso, R.G., Horner, G.S., Levi, D., Bode, M., Mascarenhas, A., Olson, J.M., Yin, X., DeLong, M.C., SpringThorpe, A.J., Majeed, A., Mowbray, D.J., and Skolnick, M.S. (1994). "Optical Properties of Ordered and Randomly Disordered AlAs/GaAs Short-Period Superlattices." - Physical Review. B, Condensed Matter (49:16), pp. 11173-11184.

Arya, R.R., Bennett, M., Chen, L., Fieselmann, B., Li, Y., Maley, N., Newton, J., Podlesny, R., and Yang, L. (1994). Research on Stable, High-Efficiency, Amorphous Silicon Multijunction Modules: Final Subcontract Report, I May 1990-30 June 1993. SERI/TP-411-6841. Work performed by Solarex Thin Film Division, Newtown, PA. Available NTIS: Order No. DE94011826.

Banerjee, A., Yang, J., Hoffman, K., and Guha, S. (1994). "Characteristics of Hydrogenated Amorphous Silicon Alloy Solar Cells on a Lambertian Back Reflector." Applied Physics Letters, (65:4), p. 472. 
Banerjee, A., Yang, J., Glatfelter, T., Hoffman, K., and Guha, S. (1994). "An Experimental Study of P-Layers in 'Tunnel' Junctions for High Efficiency Amorphous Silicon Alloy Multijunction Solar Cells and Modules."

Basol, B. M., Kapur, V. K., Halani, A., and Leidholm, C. (1993). Novel Two-Stage Selenization Methods for Fabrication of Thin-Film CIS Cells and Submodules: Annual Subcontract Report, 25 March 1992-28 February 1993. SERI/TP-413-5844. Work performed by International Solar Electric Technology, Inglewood, CA. Available NTIS: Order No. DE94000220.

Basso, T.S. (1994). "Institute of Electrical and Electronic Engineers Standards Coordination Committee 21 (IEEE SCC21) Photovoltaic Systems Overview." Proceedings of the NREL Photovoltaic Performance and Reliability Workshop, September, Lakewood, CO.

Basso, T.S. (1994). "Photovoltaics: Technology Providing Global Energy Solutions." $A$ Collection of Technical Papers, Part 4: Proceedings of the 29th Intersociety Energy Conversion Engineering Conference, 7-11 August 1994, Monterey, CA. Washington, D.C.: American Institute of Aeronautics and Astronautics, pp. 1784-1789.

Benson, D.K., Potter, T.F., and Tracy, C.E. (1994). Design of a Variable-Conductance Vacuum Insulation. SERI/TP-452-5814. Presented at the SAE 1994 Annual Meeting, 28 February-3 March 1994, Detroit, MI. Available NTIS: Order No. DE94000270.

Bertness, K.A., Friedman, D.J., Kibbler, A.E., Kramer, C., Kurtz, S.R., and Olson, J.M. (1994). "High Efficiency GaInP/GaAs Tandem Solar Cells." NREL Photovoltaic Program Review: Proceedings of the 12th Review Meeting, 13-15 October 1993, Denver, CO. AIP Conference Proceedings No. 306, R. Noufi and H.S. Ullal, eds. New York: American Institute of Physics, pp. $100-111$.

Bertness, K.A., Kramer, C., Olson, J.M, and Moreland, J. (1994). "In-Situ Observation of Surface Morphology of InP Grown on Singular and Vicinal (001) Substrates." Journal of Electronic Materials (23:2), pp. 195-200.

Bertness, K.A., Kurtz, S.R., Friedman, D.J., Kibbler, A.E., Kramer, C., and Olson, J.M. (1994). "29.5\%-Efficient GaInP/GaAs Tandem Solar Cells." Applied Physics Letters (65:8), pp. 989-991.

Bertness, K.A., Kurtz, S.R., Friedman, D. J., Kibbler, A.E., Kramer, C., and Olson, J.M. (1994). "High Efficiency GaInP/GaAs Tandem Solar Cells." A Collection of Technical Papers, Part 1: Proceedings of the 29th Intersociety Energy Conversion Engineering Conference, 7-11 August 1994, Monterey, CA. Washington, D.C.: American Institute of Aeronautics and Astronautics, pp. 251-256.

Bhattacharya, R.N. and Blaugher, R.D. (1994). "Processing of Ag Doped Thick-Film Electrodeposited Thallium-Oxide Superconductors." Physica. C, Superconductivity (225), pp. 269-274. 
Bhattacharya, R.N. and Blaugher, R.D. (1994). "Preparation of High-Temperature Superconductor Oxide Powders by Electrodeposition." Physica C (229), pp. 244-250.

Bhattacharya, R.N., Duda, A., Ginley, D.S., DeLuca, J.A., Ren, Z.F., Wang, C.A., and Wang, J.H. (1994). "Superconducting Thallium Oxide Films via an Electro-Deposited Precursor." Physica C (229), pp. 145-151.

Birkmire, R.W., Phillips, J.E., Buchanan, W.A., Hegedus, S.S., McCandless, B.E., Shafarman, W.N., and Yokimcus, T.A. (1994). Processing and Modeling Issues for Thin-Film Solar Cell Devices: Annual Report, I6 January 1993-15 January. 1994. SERI/TP-451-7056. Work performed by Institute of Energy Conversion, University of Delaware, Newark, DE. Available NTIS: Order No. DE94011895.

Bode, M.H., Tuttle, J., and Al-Jassim, M.M. (1994). "TEM Investigations on Ordered Phases

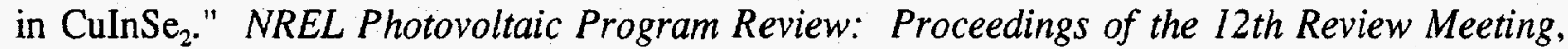
13-15 October 1993, Denver, CO, AIP Conference Proceedings No. 306. New York: American Institute of Physics, pp. 421-428.

Bottenberg, W.R., Hall, R.B., Jackson, E.L., Lampo, S., Mulligan, W.P., and Barnett, A.M. (1994). Silicon-Film ${ }^{T M}$ Photovoltaic Manufacturing Technology: Annual Subcontract Report, 15 January 1992-15 November 1992. SERI/TP-411-5815. Work performed by AstroPower, Inc., Newark, DE. Available NTIS: Order No. DE94000202.

Bottenberg, W.R. (1994). Silicon-Film ${ }^{T M}$ Photovoltaic Manufacturing Technology: Semiannual Subcontract Report, 15 November 1992-15 May 1993. SERI/TP-411-6110. Work performed by AstroPower, Inc., Newark, DE. 'Available NTIS: Order No. DE94000258.

Branz, H.M., Asher, S.E., Nelson, B.P., and Kemp, M. (1994). "Hydrogen Diffusion Mechanism in Amorphous Silicon from Deuterium Tracer Studies." Proceedings of the 15th International Conference on Amorphous Semiconductors: Science and Technology, Cambridge, England, pp. 269-272.

Branz, H.M. and Iwaniczko, E. (1993). "Evidence for the Hydrogen-Glass Model of Metastability Annealing in Phosphorus-Doped Amorphous Silicon." Physical Review B, Condensed Matter (48:23), pp. 17114-17120.

Burdick, J. and Pruett, J. (1994). "Overview of Photovoltaic Module Reliability Testing at NREL." NREL Photovoltaic Program Review: Proceedings of the 12th Review Meeting, 13-15 October 1993, Denver, CO. AIP Conference Proceedings No. 306, R. Noufi and H. S. Ullal, eds. New York: American Institute of Physics, pp. 156-163.

Carr, K., Carlson, N., Sopori, B.L., Marshall, C., Allen, L., Marshall, T., and Murphy, R. (1994). "SDMS-A Tool for Characterizing Commercial PV Si Materials and Cells." Role of Point Defects/ Defect Complexes in Silicon Device Processing: Book of Abstracts from the Fourth Workshop, 27-29 June 1994, Beaver Creek, CO, NREL/CP-413-7033. p. 16. Available NTIS: Order No. DE94011828. 
Chen, W.S., Stewart, J.M., Mickelsen, R.A., Devaney, W.E., and Stanbery, B.J. (1993). Research on Polycrystalline Thin-Film CuInGaSe ${ }_{2}$ Solar Cells: Annual Subcontract Report, 3 May 1991-21 May 1993. SERI/TP-413-5835. Work performed by Boeing Defense \& Space Group, Seattle, WA. Available NTIS: Order No. DE94000203.

Cohen, J.D. (1994). Microscopic Origins of Metastable Effects in a-Si:H and Deep Defect Characterization in a-Si,Ge:H Alloys: Final Subcontract Report, I February 1991-31 January 1994. SERITP-451-7163. Work performed by Department of Physics and Material Science, University of Oregon, Eugene, OR. Available NTIS: Order No. DE94011887.

Cohen, J.D., Zhong, F., Unold, T., and Hautala, J. (1994). "Electronic Properties of Amorphous Silicon and Amorphous Silicon-Germanium Alloys." NREL Photovoltaic Advanced Research and Development Review: Proceedings of the 12th Review Meeting, 13-15 October 1993, Denver, CO. AIP Conference Proceedings No. 306, R. Noufi and H.S. Ullal, eds. New York: American Institute of Physics, p. 452.

Collins, R.W., Wronski, C.R., An, I., Lu, Y., and Nguyen, H.V. (1994). In-Situ Characterization of Growth and Interfaces in a-Si:H Devices: Final Subcontract Report, 1 May 1991-3I May 1994. SERI/TP-451-7045. Work performed by Materials Research Laboratory, Pennsylvania State University, University Park, PA. Available NTIS: Order No. DE94011847.

Collins, R.W., Wronski, C.R., An, I., Lu, Y., and Nguyen, H.V. (1994). In-Situ Characterization of Growth and Interfaces in a-Si:H Devices: Annual Subcontract Report, 1 May 1992-30 April 1993. SERI/TP-451-6135. Work performed by Pennsylvania State University, University Park, PA. Available NTIS: Order No. DE94000291.

Collins, S.R. and Hall, R.B. (1994). Silicon-Film ${ }^{T M}$ Photovoltaic Manufacturing Technology: Annual Subcontract Report, 15 November 1992-15 October 1993. SERI/TP-411-6857. Work performed by AstroPower, Inc., Newark, DE. Available NTIS: Order No. DE94011832.

Compaan, A.D. and Bohn, R.G. (1993). Thin-Film Cadmium Telluride Photovoltaic Cells: Annual Subcontract Report, I November 1991-31 October 1992. SERI/TP-451-5813. Work performed by Department of Physics and Astronomy, University of Toledo, Toledo, $\mathrm{OH}$. Available NTIS: Order No. DE94000201.

Compaan, A.D. and Bohn, R.G. (1994). Thin-Film Cadmium Telluride Photovoltaic Cells: Final Subcontract Report, 1 November 1992-1 January 1994. SERI/TP-451-7162. Work performed by Department of Physics and Astronomy, University of Toledo, Toledo, OH. Available NTIS: Order No. DE94011886.

Contreras, M.A., Gabor, A.M., Tennant, A.L., Asher, S., Tuttle, J., and Noufi, R. (1994). "16.4\% Total-Area Conversion Efficiency Thin-Film Polycrystalline $\mathrm{MgF}_{2} / \mathrm{ZnO} / \mathrm{CdS} / \mathrm{Cu}(\mathrm{In}, \mathrm{Ga}) \mathrm{Se}_{2} / \mathrm{Mo}$ Solar Cell." Progress in Photovoltaics: Research and Applications (2), pp. 287-292. 
Contreras, M.A., Gabor, A.M., Tennant, A.L., Asher, S., Tuttle, J., and Noufi, R. (1994). $" 16.4 \%$ Total-Area Conversion Efficiency Thin-Film Polycrystalline $\mathrm{MgF}_{2} / \mathrm{ZnO} / \mathrm{CdS} / \mathrm{Cu}(\mathrm{In}, \mathrm{Ga}) \mathrm{Se}_{2} / \mathrm{Mo}$ Solar Cell." Progress in Photovoltaics, (2:4); p. 287.

Contreras, M.A., Tuttle, J., Yu, D., Qi, Y., Swartzlander, A., Tennant, A., and Noufi, R. (1993). "Graded Band-Gap $\mathrm{Cu}(\mathrm{In}, \mathrm{Ga}) \mathrm{Se}_{2}$ Thin-Film Solar Cell Absorber with Enhanced Open-Circuit Voltage." Applied Physics Letters (63:13).

Cudzinovic, M., Deng, X., Marshall, C., and Sopori, B. (1994). "Solar Cell Contact Formation by Optical Processing." Role of Point Defects/Defect Complexes in Silicon Device Processing: Book of Abstracts from the Fourth Workshop, 27-29 June 1994, Beaver Creek, CO, NREL/CP-413-7033. p. 23. Available NTIS: Order No.94011828.

Czanderna, A.W. (1993). "EVA Degradation Mechanisms: A Review of What Is and Is Not Known." Proceedings of a PV Module Reliability Workshop, L. Mrig, ed. NREL/CP-410-6033, pp. 311-357.

Czanderna, A.W. (1994). "Current Status of EVA Degradation in Si Modules and Interface Stability in CdTe/CdS Modules." NREL Photovoltaic Advanced Research and Development Review: Proceedings of the 12th Review Meeting, 13-15 October 1993, Denver, CO. AIP Conference Proceedings No. 306, R. Noufi and H.S. Ullal, eds. New York: American Institute of Physics, pp. 147-155.

Dalal, V.L., Baldwin, G., Ping, E.X., Leonard, M., and Bhan, M. (1994). "Fabrication of More Stable Materials and Devices in Silicon." NREL Photovoltaic Advanced Research and Development Review: Proceedings of the 12th Review Meeting, 13-15 October 1993, Denver, CO. AIP Conference Proceedings No. 306, R. Noufi and H.S. Ullal, eds. New York: American Institute of Physics, p. 460.

Delahoy, A.E., Britt, J., Faras, F., and Kiss, Z. (1994). Non- $\mathrm{H}_{2} \mathrm{Se}$, Ultra-Thin CuInSe Devices: $^{2}$ Annual Subcontract Report, 10 November 1992-9 November 1993. SERI/TP-413-7165. Work performed by Energy Photovoltaics, Inc., Princeton, NJ. Available NTIS: Order No. DE94011894.

Deng, X. and Sopori, B. (1994). "Experimental Results in Support of Hydrogen Diffusion via a H-V Mechanism." Role of Point Defects/Defect Complexes in Silicon Device Processing: Book of Abstracts from the Fourth Workshop, 27-29 June 1994, Beaver Creek, CO, NREL/CP-413-7033, p. 38.

Dhere, N.G. (1994). Polycrystalline CuInSe $e_{2}$ and CdTe Solar Cells: Annual Subcontract Report, 15 April 1992-14 April 1993. SERI/TP-451-6637. Work performed by Florida Solar Energy Center, Cape Canaveral, FL. Available NTIS: Order No. DE94011875. 
Dhere, R.G., Ramanathan, K., Keyes, B.M., and Moutinho, H.R. (1994). "Growth and Properties of CdS/CdTe Heterojunctions on Soda Lime Glass Substrates." NREL Photovoltaic Advanced Research and Development Review: Proceedings of the 12th Review Meeting, 13-15 October 1993, Denver, CO. AIP Conference Proceedings No. 306, R. Noufi and H.S. Ullal, eds. New York: American Institute of Physics, pp. 335-344.

Duran, G., Mackamul, K., Metcalf, D., Koniares, A., Skinner, D., and Volltrauer, H. (1994). Amorphous Silicon Photovoltaic Manufacturing Technology-Phase 2A: Annual Subcontract Report, l May 1992-30 April 1993. SERI/TP-411-6263. Work performed by Utility Power Group, Chatsworth, CA and Advanced Photovoltaic Systems, Inc., Princeton, NJ. Available NTIS: Order No. DE94000294.

Duran, G., Mackamul, K., Metcalf, D., and Volltrauer, H. (1994). Amorphous Silicon Photovaltaic Manufacturing Technology-Phase 2A; Semiannual Subcontract Report, 1 May 1993-31 October 1993. SERI/TP-411-6470. Work performed by Utility Power Group, Chatsworth, CA and Advanced Photovoltaic Systems, Inc., Princeton, NJ. Available NTIS: Order No. DE94006895.

Emery, K. (1994). "Performance Characterization of Thermophotovoltaic Cells." Proceedings of the First NREL Conference on Thermophotovoltaic Generation of Electricity, 24-27 July 1994, Copper Mountain, CO, NREL/CP-413-6186, p. 165. Available NTIS: Order No. DE94011831.

Fitzgerald, M. (1994). PV Working with Industry: National Renewable Energy Laboratory Quarterly Technical Report, June. SERI/TP-410-7049.

Friedman, D.J., Kurtz, S.R., Bertness, K.A., Kibbler, A.E., Kramer, C., Emery, K., Field, H., and Olson, J.M. (1994). "GaInP/GaAs Tandem Concentrator Cells." NREL Photovoltaic Advanced Research and Development Review: Proceedings of the 12th Review Meeting, 13-15 October 1993, Denver, CO. AIP Conference Proceedings No. 306, R. Noufi and H.S. Ullal, eds. New York: American Institute of Physics, pp. 521-528.

Friedman, D.J., Horner, G.S., Kurtz, S.R., Bertness, K.A., Olson, J.M., and Moreland, J. (1994). "Effect of Faceting on the Band Gap of Ordered GaInP." Applied Physics Letters (65:7), pp. $878-880$.

Gabor, A.M. and Hermann, A.M. (1994). Novel Thin-Film CuInSe ${ }_{2}$ Fabrication: Annual Subcontract Report, 1 May 1992-31 October 1993. SERI/TP-451-7055. Work performed by the University of Colorado, Department of Physics, Boulder, CO. Available NTIS: Order No. DE94011874.

Gabor, A.M., Hermann, A.M., Tuttle, J.R., Contreras, M.A., Albin, D.S., Tennant, A.L., and Noufi, R. (1993). "High Efficiency Polycrystalline Cu(In,Ga)Se ${ }_{2}$-Based Solar Cells." NREL Photovoltaic Advanced Research and Development Review: Proceedings of the 12th Review Meeting, 13-15 October 1993, Denver, CO. AIP Conference Proceedings No. 306, R. Noufi and H.S. Ullal, eds. New York: American Institute of Physics, pp. 59-66. 
Gabor, A.M., Tuttle, J.R., Albin, D.S., Contreras, M.A., Noufi, R., and Hermann, A.M. (1994). "High Efficiency CuIn $\mathrm{Ga}_{1-\mathrm{x}} \mathrm{Se}_{2}$ Solar Cells Made from $\left(\mathrm{In}_{\mathrm{x}}, \mathrm{Ga}_{1-\mathrm{x}}\right)_{2} \mathrm{Se}_{3}$ Precursor Films." Applied Physics Letters (65:2), pp. 198-200. Work performed by the National Renewable Energy Laboratory, Golden, $\mathrm{CO}$, and the University of Colorado, Boulder, $\mathrm{CO}$.

Gabor, A.M., Tuttle, J.R., Albin, D.S., Tennant, A.L., Contreras, M.A., Noufi, R., and Hermann, A.M. (1994): "High Efficiency Polycrystalline $\mathrm{Cu}(\mathrm{In}, \mathrm{Ga}) \mathrm{Se}_{2}$-Based Solar Cells." 'NREL Photovoltaic Advanced Research and Development Review: Proceedings of the 12th Review Meeting, 13-15 October 1993, Denver, CO. AIP Conference Proceedings No. 306, R. Noufi and H.S. Ullal, eds. New York: American Institute of Physics, pp. 59-66. Work performed by the National Renewable Energy Laboratory, Golden, CO, and the University of Colorado, Boulder, $\mathrm{CO}$.

Gabor, A.M., Tuttle, J.R., Albin, D.S., Contreras, M.A., and Noufi, R. (1994). "High Efficiency $\mathrm{CuIn} \mathrm{Ga}_{1-\mathrm{x}} \mathrm{Se}_{2}$ Solar Cells Made from $\left(\mathrm{In}_{\mathrm{x}}, \mathrm{Ga}_{1-\mathrm{x}}\right)_{2} \mathrm{Se}_{3}$ Precursor Films." Applied Physics Letters (65:2), pp. 198-200.

Gabor, A.M., Tuttle, J.R., Albin, D.S., Matson, R., Franz, A., Niles, D.W., Contreras, M.A., Hermann, A.M., and Noufi, R. (1994). "A Microstructural Comparison of $\mathrm{Cu}(\mathrm{In}, \mathrm{Ga}) \mathrm{Se}_{2} \mathrm{Thin}$ Films Grown from $\mathrm{Cu}_{\mathrm{x}} \mathrm{Se}$ and (In, $\left.\mathrm{Ga}\right)_{2} \mathrm{Se}_{3}$ Precursors." Materials Research Society Symposium Proceedings (343).

Gabor, A.M., Tuttle, J.R., Contreras, M.A., Albin, D.S., Franz, A., Niles, D.W., and Noufi, R. (1994). "High-Efficiency Thin-Film Cu(In,Ga)Se 2 Solar Cells: Achievement of a Confirmed World-Record 16.4\% Total-Area Efficiency." Proceedings of the 12th European Photovoltaic Solar Energy Conference, Amsterdam, The Netherlands, p. 939.

Gale, R.P. (1994). High-Efficiency, Thin-Film Solar Cells: Annual Subcontract Report, 1 July 1991-30 June 1992. SERI/TP-451-5746. Work performed by Kopin Corporation, Taunton, MA. Available NTIS: Order No. DE94000250.

Gallagher, A., Tanenbaum, D., Laracuente, A., and Kalra, P. (July 1994). Growth Mechanisms and Characterization of Hydrogenated Amorphous-Silicon-Alloy Films: Final Subcontract Report, 15 February 1991-14 April 1994. SERI/TP-411-6864. Work performed by the National Institute of Standards and Technology, Boulder, CO. Available NTIS: Order No. DE94011842.

Gessert, T.A. and Coutts, T.J. (1994). "Development and Analysis of Cu-Doped ZnTe for Use as a Back Contact Interface for CdS/CdTe Solar Cells." NREL Photovoltaic Advanced Research and Development Review: Proceedings of the 12th Review Meeting, 13-15 October 1993, Denver, Colorado. AIP Conference Proceedings No. 306, R. Noufi and H.S. Ullal, eds. New York: American Institute of Physics; pp. 345-353.

Gheorghita, L., Cocivera, M., and Nelson, A.J. (1994). "Formation of a Silver-Rich Layer on Electrodeposited CdTe." Journal of the Electrochemical Society (141:2), p. 529. 
Ghosh, M., Kampas, F., and Xi, J. (1994). Research on Stable, High-Efficiency Amorphous-Silicon Multijunction Modules: Annual Subcontract Report, 1 November 1992-31 March 1994. SERI/TP-411-6854. Work performed by Advanced Photovoltaic Systems, Princeton, NJ. Available NTIS: Order No. DE94011839.

Gordon, R.G., Hu, J., Lacks, D., Musher, J., Thornton, J., and Liang, H. (1994). Optimization of Transparent and Reflerting Electrodes for Amorphous-Silicon Solar Cells: Final Subcontract Report, I May 1991-3. April 1994. SERITP-411-6856. Work performed by Harvard University, Department of Chemistry, Cambridge, MA. Available NTIS: Order No. DE94011841.

Gray, J.L., Schwartz, R.J., and Lee, Y.J. (1994). Development of a Computer Model for Polycrystalline Thin-Film CuInSe ${ }_{2}$ and CdTe Solar Cells: Annual Subcontract Report, 1. March 1992-28 February 1993. SERI/TP-451-6398. Work performed by Purdue University, School of Electrical Engineering, West Lafayette, IN. Available NTIS: Order No. DE94006853.

Gregg, B.A. and Kim, Y.I. (1994). "Redox Polymer Contacts to Molecular Semiconductor Films: Toward Kinetic Control of Interfacial Exciton Dissociation and Electron-Transfer Processes." Journal of Physical Chemistry (98:9), pp. 2412-2417.

Guha, S. (1994). Research on Stable, High-Efficiency Amorphous Silicon Multijunction Modules: Semiannual Subcontract Report, I March 1993-30 November 1993. SERI/TP-411-6396. Work performed by United Solar Systems Corporation, Troy, MI. Available NTIS: Order No. DE94000298.

Guha, S., Xu, X., and Yang, J. (1994). "Saturation Behavior and Thermal Annealing Recovery of Light-Induced Degradation in a-SiGe:H Alloy Solar Cells." Proceedings: 1994 MRS Spring Meeting.

Guha, S., Yang, J., Banerjee, A., Glatfelter, T., Hoffman, K., Ovshinsky, S.R., Izu, M., Ovshinsky, H.C., and Deng, X. (1994). "Amorphous Silicon Alloy Photovoltaic Technology from R\&D to Production." Proceedings: 1994 MRS Spring Meeting.

Guha, S., Yang, J., Banerjee, A., Glatfelter, T., Hoffman, K., and Xu, X. (1994). "High Efficiency Multijunction Amorphous Silicon Alloy-Based Solar Cells and Modules." NREL Photovoltaic Advanced Research and Development Review: Proceedings of the 12th Review Meeting, 13-15 October 1993, Denver, Colorado. AIP Conference Proceedings No. 306, R. Noufi and H.S. Ullal, eds. New York: American Institute of Physics, p. 127.

Gunes, M., Wronski, C.R., and McMahon, T.J. (1994). "Charged Defect States in Intrinsic Hydrogenated Amorphous Si Films." Journal of Applied Physics (76), p. 2260.

Herdt, G.C. and Czanderna, A.W. (1993). "Penetration of Vacuum Deposited Silver into Octadecanethiol Self-Assembled Monolayers Studied with ISS." Surface Science Letters, 297, L109. NREL/TP-4121-5842. 
Herdt, G.C. and Czanderna, A.W. (1994). "Metal Overlayers on Self-Organized Molecular Assemblies. IV. Ion Scattering Spectroscopy of the Ag/CH3 Interface." Journal of Vacuum Science and Technology A, Vacuum, Surfaces, and Films (12:4), pp. 2410-2414.

Holley, W.H. (1993). Advanced Development of PV Encapsulants: Semiannual Subcontract Report, I January 1993-30 June 1993. SERI/TP-411-5800. Work performed by Springborn Laboratories, Inc., Enfield, CT. Available NTIS: Order No. DE94000219.

Horner, G.S., Mascarenhas, A., Alonso, R.G., Froyen, S., Bertness, K.A., and Olson, J. M. (1994). "Photoluminescence and Excitation-Photoluminescence Study of Spontaneous Ordering in GaInP ${ }_{2}$ " Physical Review. B, Condensed Matter (49:3), pp. 1727-1731.

Iwaniczko, E., Nelson, B.P., Molenbroek, E.C., Schropp, R.I., Crandall, R.S., and Mahan, A.H. (1994). "Solar Cells Incorporating Hot-Wire Deposited Amorphous Silicon." NREL Photovoltaic Advanced Research and Development Review: Proceedings of the 12th Review Meeting, 13-15 October 1993, Denver, CO. AIP Conference Proceedings No. 306, R. Noufi and H.S. Ullal, eds. New York: American Institute of Physics, p. 458.

Izu, M. (1993). Continuous Roll-to-Roll a-Si Photovoltaic Manufacturing Technology: Annual Subcontract Report, I April 1992-31 March 1993. SERI/TP-411-6003. Work performed by Energy Conversion Devices, Inc., Troy, MI. Available NTIS: Order No. DE94000260.

Izu, M. (1994). Continuous Roll-to-Roll Amorphous Silicon Photovoltaic Manufacturing Technology: Semiannual Subcontract Report, 1 April 1993-30 September 1993. SERI/TP-411-6624. Work performed by Energy Conversion Devices, Inc., Troy, MI. Available NTIS: Order No. DE94011816.

Izu, M., Ovshinsky, S.R., Deng, X., Krisko, A., Ovshinsky, H.B., Narasimhan, K.L., and Young, R. (1994). "Continuous Roll-to-Roll Amorphous Silicon Photovoltaic Manufacturing Technology." American Institute of Physics, p. 198.

Jackson, W.B., Johnson, N., Nickel, N., Nebel, C., Hack, M., Santos, P., Schumm, G., Street, R.A., Thompson, R., Tsai, C.C., and Walker, J. (1994). Research on the Stability, Electronic Properties, and Structure of a-Si:H and Its Alloys: Annual Subcontract Report, I June 1992-31 May 1993. SERI/TP-451-6192. Work performed by Xerox Palo Alto Research Center, Palo Alto, CA. Available NTIS: Order No. DE94000292.

Jester, T. (1994). PV Cz Silicon Manufacturing Technology Improvements: Semiannual Subcontract Report, 1 April 1993-30 September 1993. SERI/TP-411-6634. Work performed by Siemens Solar Industries, Camarillo, CA. Available NTIS: Order No. DE94011818.

Jester, T. (1994). PV Cz Silicon Manufacturing Technology Improvements: Annual Subcontract Report, 1 April 1992-31 March 1993. SERI/TP-411-6010. Work performed by Siemens Solar Industries, Camarillo, CA. Available NTIS: Order No. DE9400248. 
Jorgensen, G., Pern, J., Kelley, S., Czanderna, A. W., Schissel, P. (1995). "Polymers for Solar Energy Devices." Functional Polymers for Emerging Technologies, R. Arshady, ed., American Chemical Society, Washington, D.C. NREL/TP-454-6097. In press.

Jung, D.R. and Czanderna, A.W. (1994). "Metal Overlayers on Organic Functional Groups of Self-Organized Molecular Assemblies. III. X-Ray Photoelectron Spectroscopy of $\mathrm{Cr} / \mathrm{CN}$ on 12-Mercaptododecanenitrile and of $\mathrm{Cr} / \mathrm{CH}_{3}$ on Octadecanethiol at Sample Temperatures from 173 to 373 K." Journal of Vacuum Science and Techrology A, Vacuum, Surfaces, and Films (12:4), pp. 2402-2409.

Jung, D.R. and Czanderna, A.W. (1994). "Chemical and Physical Interactions at Metal/SelfAssembled Organic Monolayer Interfaces." Critical Reviews in Solid State and Materials Sciences (19:1), pp. 1-54.

Kalejs, J.P. (1994). Mobil Solar Energy Corporation Thin EFG Octagons: Final Subcontract Report, I April 1992-3I January 1994. SERI/TP-411-6770. Work performed by Mobil Solar Energy Corporation, Billerica, MA. Available NTIS: Order No. DE94011829.

Kalejs, J.P. (1994). Thin EFG Octagons: Semiannual Subcontract Report, 1 April 1993-30 September 1993. SERL/TP-411-6399. Work performed by Mobil Solar Energy Corporation, Billerica, MA. Available NTIS: Order No. DE94006855.

Kalejs, J.P. (1994). Thin EFG Octagons: Annual Subcontract Report, I April 1992-3I March 1993. SERI/TP-411-6046. Work performed by Mobil Solar Energy Corporation, Billerica, MA. Available NTIS: Order No. DE94000257.

Keyes, B., Dhere, R., and Ramanathan, K. (1994). "Time-Resolved Photoluminescence of Polycrystalline CdTe Grown by Close-Spaced Sublimation." NREL Photovoltaic Advanced Research and Development Review: Proceedings of the 12th Review Meeting, 13-15 October 1993, Denver, CO. AIP Conference Proceedings No. 306, R. Noufi and H.S. Ullal, eds. New York: American Institute of Physics, pp. 364-369.

Khattak, C.P., Schmid, F., Sopori, B.L., and Schubert, W.K. (1994). "Characterization of HEM Multicrystalline Silicon." Role of Point Defects and Defect Complexes in Silicon Device Processing: Summary Report and Papers of the Second Workshop, 24-26 August 1992, Breckenridge, CO. Golden, CO: National Renewable Energy Laboratory, pp. 33-39. Available NTIS: Order No. DE94011876.

King, D.E. and Czanderna, A.W. (1994). "Oxidation Behavior of Thin Copper Films on a Mercaptoundecanoic Acid Organized Molecular Assembly.". Langmuir (10:5), pp. 1630-1631.

Knapp, K.E. and Gay, R.R. (1994). Research on High-Efficiency, Large-Area, CuInSe ${ }_{2}$-Based Thin-Film Modules: Annual Subcontract Report, I May 1992-15 August 1993. SERI/ TP-413-6216. Work performed by Siemens Solar Industries, Camarillo, CA. Available NTIS: Order No. DE94000259. 
Kocha, S.S., Turner, J.A., and Nozik, A.J. (1994). "Study of the Schottky Barrier and Determination of the Energetic Positions of Band Edges at the N- and P-Type Gallium Indium Phosphide Electrode/Electrolyte Interface." Journal of Electroanalytical Chemistry (367), pp. $27-30$.

Kurtz, S.R., Olson, J.M., Arent, D.J., Bode, M.H., and Bertness, K.A. (1994). "Low-Band-Gap $\mathrm{Ga}_{0.5} \mathrm{In}_{0.5} \mathrm{P}$ Grown on (511)B GaAs Substrates." Journal of Applied Physics (75:10), pp. $5110-5113$.

Kurtz, S.R., Arent, D.J., Bertness, K.A., Olson, J.M. (1994). "The Effect of Phosphine Pressure on the Band Gap of $\mathrm{Ga}_{0.5} \mathrm{In}_{0.5} \mathrm{P}$." Compound Semiconductor Epitaxy, Materials Research Society Conference Proceedings, No. 340, San Francisco, CA.

Kurtz, S.R., Olson, J.M., Friedman, D.J., Kibbler, A.E., and Asher, S. (1994). "Ordering and Disordering of Doped $\mathrm{Ga}_{0.5} \mathrm{In}_{0.5} \mathrm{P} . "$ Journal of Electronic Materials (23:5), pp. 431-435.

Lu, Z.W., Zunger, A., and Fox, A.G. (1994). "First-Principles Simulated Annealing Study of Phase Transition and Short Range Order in Transition Metal and Semiconductor Alloys." Physical Review B (50), pp. 6642-6661. (OER/BES)

Lu, Z.W. and Zunger, A. (1994). "Unequal Wavevectors in Short vs Long Range Ordering in Intermetallic Compounds." Physical Review B (50), pp. 6626-6636. (OER/BES)

Luft, W., Branz, H.M., Dalal, V.L., Hegedus, S.S., and Schiff, E.A. (1994). "Recent Progress in Amorphous Silicon PV Technology." NREL Photovoltaic Advanced Research and Development Review: Proceedings of the 12th Review Meeting, 13-15 October 1993, Denver, CO. AIP Conference Proceedings No. 306, R. Noufi and H.S. Ullal, eds. New York: American Institute of Physics, pp. 31-45.

Luft, W. (1994). "NREL/Industry Interaction: Amorphous Silicon Alloy Research Team Formation." NREL Photovoltaic Advanced Research and Development Review: Proceedings of the I2th Review Meeting, 13-15 October 1993, Denver, CO. AIP Conference Proceedings No. 306, R. Noufi and H.S. Ullal, eds. New York: American Institute of Physics, pp. 46-50.

Luo, J.S., Olson, J.M., Bertness, K.A., Raikh, M.E., and Tsiper, E.V. (1994). "Investigation of Spontaneous Ordering in GaInP Using Reflectance Difference Spectroscopy." Journal of Vacuum Science and Technology. B, Microelectronics Processing and Phenomena (12:4), pp. 2552-2557.

Mader, K.A. and Zunger, A. (1994). "Effects of Atomic Clustering on the Optical Properties of III-V Alloys." Applied Physics Letters (64:21), pp. 2882-2884. (OER/BES)

Mao, D., Kim, K., and Frank, A.J. (1994). "Open-Circuit Photovoltage and Charge Recombination at Semiconductor/Liquid Interfaces." Journal of the Electrochemical Society (141:5), pp. 1231-1236. 
Marshall, T. and Sopori, B. (1994). "Light Trapping in Thin-Film Cells." Role of Point Defects/ Defect Complexes in Silicon Device Processing: Book of Abstracts from the Fourth Workshop, 27-29 June 1994, Beaver Creek, CO. NREL/CP-413-7033, p. 45. Available NTIS: Order No. DE94011828.

McMahon, T.J. and Xiao, Y. (1993). "Electron Spin Resonance Study of the Dangling Bond in Amorphous Si and Porous Si." Applied Physics Letters (63), p. 1657.

McMahon, T. and Basso, T.S. (1994). "Two Terminal Diagnostic for Ceils in Series-Connected Modules." Proceedings of the NREL Photovoltaic Performance and Reliability Workshop, September 1994, Lakewood, CO.

McNutt, P. (1994). U.S. Manufacturers of Commercially Available Stand-Alone Photovoltaic Lighting Systems. SERI/TP-411-6406. Available NTIS: Order No. DE94006917.

Morel, D. L. and Ferekides, C.S. (1994). Advanced Processing Technology for High-Efficiency, Thin-Film CuInSe $e_{2}$ and CaTe Solar Cells: Annual Subcontract Report, 1 March 1993-28 February 1994. SERI/TP-451-6638. Work performed by the University of South Florida, Department of Electrical Engineering, Tampa, FL. Available NTIS: Order No. DE94011838.

Moskowitz, P.D., Fthenakis, V.M., Crandall, R.S., and Nelson, B.P. (1994). "Analysis of the Risks Associated with Hazardous Production Materials." Solid State Technology (121).

Mrig, L., Caiyem, Y., Rummel, S., Hansen, R., Kroposki, B., and Strand, T. (1994). "Photovoltaic Module and System Performance Testing at NREL." NREL Photovoltaic Advanced Research and Development Review: Proceedings of the 12th Review Meeting, 13-15 October 1993, Denver, CO. AIP Conference Proceedings No. 306, R. Noufi and H.S. Ullal, eds. New York: American Institute of Physics, pp. 164-169.

Mrig, L., Caiyem, Y., Rummel, S., Hansen, R., Kroposki, B., and Strand, T. (1994). "Photovoltaic Module Outdoor, Module Qualification, and System Testing at NREL." Solar Engineering 1994: Proceedings of the ASME/JSME/JSES International Solar Energy Conference, 27-30 March 1994, San Francisco, CA. New York: American Society of Mechanical Engineers, pp. $437-444$.

Myers, D.R., Cannon, T.W, and Trudell, D. (1994) "Technical Overview of Photovoltaic Solar Radiation Research at NREL." NREL Photovoltaic Advanced Research and Development Review: Proceedings of the 12th Review Meeting, 13-15 October 1993, Denver, CO. AIP Conference Proceedings No. 306, R. Noufi and H.S. Ullal, eds. New York: American Institute of Physics, pp. 137-146.

National Renewable Energy Laboratory. (1993). PVMaT: A Government/Industry Partnership for U.S. Competitiveness; History and Current Status, October 1993. SERI/MK-411-5850. Available NTIS: Order No. DE94000205. 
National Renewable Energy Laboratory. (1994). Sunrayce 93: The Hottest New Sport on Campus. SERI/TP-411-6767. Available NTIS: Order No. DE94006927.

National Renewable Energy Laboratory. (1994). Role of Point Defects/Defect Complexes in Silicon Device Processing: Book of Abstracts from the Fourth Workshop, 27-29 June 1994, Beaver Creek, CO. SERI/CP-413-7033. Available NTIS: Order No. DE94011828.

Naziripour, A., Hermann, A.M., Swartzlander, A.B., Nelson, A. J., and Dong, C. (')93). "The Influence of Processing Condition on the Morphology and Electronic Properties C: Tl-Ba-Ca$\mathrm{Cu}-\mathrm{O}$ (TBCCO) Superconducting Thin Films." Proceedings of the Beijing International Conference on High $T_{c}$ Superconductivity, Beijing, China. Philadelphia: World Scientific Press, p. 735 .

Nelson, A.J. (1994). "Investigation on the Effect of Isoelectronic Substitution in $\mathrm{ZnS}_{1-\mathrm{x}} \mathrm{Se}_{\mathrm{x}}$ Alloys." Presented at the National AVS Symposium, Orlando, FL, November 1993. Journal of Vacuum Science Technology (A12:4), p. 1090.

Nelson, A.J. (1994). "X-Ray Photoemission Spectroscopy." Microanalysis of Solids, B.G. Yacobi, D.B. Holt, and L.L. Kazmerski, eds. New York: Plenum Publishing, pp. 247-267.

Nelson, A.J., Bode, M.H., Horner, G.S., Sinha, K., and Moreland, J. (1994). "Epitaxial Growth and Characterization of the Ordered Vacancy Compound $\mathrm{CuIn}_{3} \mathrm{Se}_{5}$ on $\mathrm{GaAs}$ (100) Fabricated by Molecular Beam Epitaxy." Compound Semiconductor Epitaxy, Materials Research Society Conference Proceedings No. 340, San Francisco, CA, p. 599.

Nelson, A.J., Frigo, S.P., and Rosenberg, R.A. (1994). "Chemistry and Electronic Structure of the $\mathrm{H}_{2}$ Plasma Passivated Surface of CdTe." Journal of Applied Physics (75:3), pp. 1632-1637.

Nelson, A.J., Hasoon, F., and Levi, D. (1994). "Processing and Characterization of Large-Grain Thin-Film CdTe." Journal of Vacuum Science Technology (A12:5), p. 2803.

Nelson, A.J., Horner, G.S., Sinha, K., and Bode, M.H. (1994). "Ordered Vacancy Compound $\mathrm{CuIn}_{3} \mathrm{Se}_{5}$ on $\mathrm{GaAs}(100)$ : Epitaxial Growth and Characterization." Applied Physics Letters (64:26), pp. $3600-3601$.

Nelson, A.J., Niles, D.W., Schwerdtfeger, C.R., Wei, S.-H., Zunger, Z., and Hochst, H. (1994). "Prediction and Observation of II-VI/CuInSe $\mathrm{e}_{2}$ Heterojunction Band Offsets." Proceedings of the Fifth International Conference on Electron Spectroscopy, Kiev, Ukraine. Journal of Electron Spectroscopy and Related Phenomena (64/65), p. 185.

Nelson, A.J., Niles, D.W., Schwerdtfeger, C.R., Wei, S.H., Zunger, A., and Hochst, H. (1994). "Prediction and Observation of the II-VI/CuInSe, Heterojunction Band Offsets." Journal of Electron Spectroscopy and Related Phenomena (68), pp. 185-193. Work performed by the National Renewable Energy Laboratory, Golden, CO, and Synchrotron Radiation Center, University of Wisconsin-Madison, Stoughton, WI. 
Nelson, A.J., Swartzlander, A.B., Tuttle, J.R., Noufi, R., Patel, R., and Hochst, H. (1993). "Photoemission Investigation of the Electronic Structure at Polycrystalline CuInSe $\mathrm{T}_{2}$ Thin-Film Interfaces." Journal of Applied Physics (74:9), pp. 5757-5760.

Nelson, A.J., Tuttle, J.R., Noufi, R., Rioux, D., Patel, R., and Hochst, H. (1993). "Photoemission Investigation of the Electronic Structure at the Polycrystalline CuInSe $\mathrm{T}_{2}$ Thin-Film Interfaces." Journal of Applied Physics (74:9), p. 5757.

Nelson, B.P., Iwaniczko, E., Schropp, R.I., Mahan, A.H., Molenbroek, E.C., Salamon, S., and Crandall, R.S. (1994). "Solar Cells Incorporating Hot-Wire Deposited Amorphous Silicon." Proceedings of the 12th European PV Solar Energy Conference, Amsterdam, The Netherlands, p. 1.

Northrup, J.E. and Froyen, S. (1993). "Energetics of GaAs (100)-(2x4) and GaAs (100)-(4x2) Reconstructions." Physical Review Letters (71:14), pp. 2276-2279. (Xerox/NREL)

Nowlan, M.J., Hogan, S.J., Darkazalli, G., Breen, W.F., Murach, J.M., and Sutherland, S.F. (1994). Automated Solar Cell Assembly Teamed Process Research: Semiannual Subcontract Report, 7 January 1993-30 June 1993. SERI/TP-411-6264. Work performed by Spire Corporation, Bedford, MA. Available NTIS: Order No. DE94000295.

Nowlan, M.J., Hogan, S.J., Darkazalli, G., Breen, W.F., Murach, J.M., Sutherland, S.F., and Patterson, J.S. (1994). Automated Solar Cell Assembly Team Process Research: Annual Subcontract Report, l January 1993-31 December 1993. SERI/TP-411-6769. Work performed by Spire Corporation, Bedford, MA. Available NTIS: Order No. DE94011819.

Olsen, L.C. (1994). Investigation of Polycrystalline Thin-Film CuInSe ${ }_{2}$ Solar Cells Based on ZnSe Windows: Annual Subcontract Report, 15 February 1992-14 February 1993. SERI/TP-451-6469. Work performed by Electronic Materials Laboratory, Washington State University at Tri-Cities, Richland, WA. Available NTIS: Order No. DE94006936.

Oswald, R. and O'Dowd, J. (1994). Large-Area Triple-Junction a-Si Alloy Production Scale Up: Annual Subcontract Report, 17 March 1992-18 March 1993. SERI/TP-411-6047. Work performed by Solarex Thin Film Division, Newtown, PA. Available NTIS: Order No. DE94000249.

Oswald, R., O’Dowd, J., Ashenfelter, W., Chen, L.F., Hainsworth, M.C., Jansen, K., Johnson, B., Kloss, T., Morris, J., Raquet, J., Stabinsky, R., Twesme, E., Weiss, P., Willing, F., and Yang, L. (1994). Large-Area Triple-Junction a-Si Alloy Production Scale-Up: Semiannual Subcontract Report, 17 March 1993-18 September 1993. SERI/TP-411-6397. Work performed by Solarex Thin Film Division, Newtown, PA. Available NTIS: Order No. DE94006856.

Padilla, S., ed. (1993). Annual Report, Materials Science Branch, FY 1992. SERITP-451-5733. Available NTIS: Order No. DE94000204. 
Paranthaman, M., Foldeaki, M., Balzer, D., Ledbetter, H., Nelson, A. J., and Hermann, A. M. (1994). "Enhanced Flux Pinning via Chemical Substitution in Bulk Superconducting Tl-2212." Superconductor Science Technology 7, p. 227.

Perez, R., Seals, R., and Stewart, R. (1994). Solar Resource-Utility Load-Matching Assessment: Interim Subcontract Report, 20 September 1991-19 December 1993. SERI/TP-411-6292. Work performed by Atmospheric Sciences Research Center, State University of New York, Albany, NY. Available NTIS: Order No. DE94000297.

Perez, R. and Wallace, W. (1993). Solar Resource-Utility Load Matching Assessment, NREL Photovoltaic Project Summary. SERI/TP-411-5836. Work performed by Atmospheric Sciences Research Center, State University of New York, Albany, NY. Available NTIS: Order No. DE94000232.

Pern, F.J. and Glick, S.H. (1994). "Fluorescence Analysis as a Diagnostic Tool for Polymer Encapsulation Processing and Degradation." NREL Photovoltaic Advanced Research and Development Review: Proceedings of the 12th Review Meeting, 13-15 October 1993, Denver, CO. AIP Conference Proceedings No. 306, R. Noufi and H.S. Ullal, eds. New York: American Institute of Physics, pp. 573-585. NREL/TP-412-5996.

Pern, F.J. (1993). "Modification of EVA Formulation for Improved Stability." Proceedings of a PV Module Reliability Workshop, L. Mrig, ed., pp. 358-374. NREL/CP-410-6033.

Picorel, R., Chumanov, G., Cotton, T.M., Montoya, G., Toon, S., and Seibert, M. (1994). "Surface-Enhanced Resonance Raman Scattering Spectroscopy of Photosystem II Pigment-Protein Complexes." Journal of Physical Chemistry (98:23), pp. 6017-6022.

Picorel, R., Williamson, D.L., Yruela, I., and Seibert, M. (1994). "State of Iron in the OxygenEvolving Core Complex of the Cyanobacterium Phormidium laminosum: Mossbauer Spectroscopy." Biochimica et Biophysica Acta (1184:2/3), pp. 171-177.

Pohle, L., ed. (1994). NREL Photovoltaic Program FY 1993 Bibliography. SERITP-411-6270. Available NTIS: Order No. DE94000272.

Pohle, L., ed. (1993). Photovoltaic Advanced Research and Development Project: Technical Summary Report, September 1993. SERI/MP-410-5993.

Rajh, T., Micic, O.I., Nozik, A.J. (1993). "Synthesis and Characterization of Surface-Modified Colloidal CdTe Quantum Dots." Journal of Physical Chemistry (97:46), pp. 11999-12003.

Rand, J.A., Cotter, J.E., Ingram, A.E., Ruffins, T.R., Thomas, C.J., Hall, R.B., and Barnett, A.M. (1994). Development of Large-Area Monolithically Integrated Silicon-Film ${ }^{T M}$ Photovoltaic Modules: Annual Subcontract Report, 1 January 1993-31 December 1993. SERI/TP-413-6853. Work performed by AstroPower, Inc., Newark, DE. Available NTIS: Order No. DE94011830. 
Rockett, A., Yang, L.C., Kenshole, G., Banda, E., and Feen, A. (1994). Alternative Fabrication Techniques for High-Efficiency CuInSe $e_{2}$ and CuInSe $e_{2}$-Alloy Films and Cells: Final Subcontract Report, I March 1990-31 August 1992. SERI/TP-451-6862. Work performed by the Department of Materials Science and Engineering, University of Illinois, Urbana, IL. Available NTIS: Order No. DE94011846.

Rohatgi, A., Chou, H.C., Kamra, S., and Bhat, A. (1994). Development of High-Efficiency, Thin-Film CdTe Solar Cells: Annual Subcontract Report, 1 January 1993-31 December 1993. SERI/TP-451-7065. Work performed by the School of Electrical and Computer Engineering, Georgia Institute of Technology, Atlanta, GA. Available NTIS: Order No. DE94011885.

Rosenwaks, Y., Hanna, M.C., Levi, D.H., Szmyd, D.M., Ahrenkiel R.K., and Nozik, A. (1993). "Hot-Carrier Cooling in GaAs: Quantum Wells Versus Bulk." Physical Review. B, Condensed Matter (48:19), pp. 14675-14678.

Rosenwaks, Y., Thacker, B.R., Nozik, A.J., Shapira, Y., Huppert, and D. (1993). "Recombination Dynamics at InP/Liquid Interfaces." Journal of Physical Chemistry (97:40), pp. 10421-10429.

Schiff, E.A., Antoniadis, H., Gu, Q., Lee, J.K., Wang, Q., and Zafar, S. (1994). Research on Defects and Transport in Amorphous-Silicon-Based Semiconductors: Final Subcontract Report, 20 February 1991-19 April 1994. SERI/TP-451-7164. Work performed by the Department of Physics, Syracuse University, Syracuse, NY. Available NTIS: Order No. DE94011888.

Schiff, E.A., Branz, H.M., Han, D., Melcher, D.C., and Silver, M. (1994). "Photocarrier Drift and Recombination in a-Si:H: The Vital Importance of Defect Relaxation." Proceedings of the 15th International Conference on Amorphous Semiconductors: Science and Technology, Cambridge, England, pp. 331-334.

Shinar, J., Jia, H., Shinar, R., Chen, Y., and Williamson, D. (1994). "Microvoid, Si, H, and Al Dynamics in a-Si: $\mathrm{H} / \mathrm{Al}_{2} \mathrm{O}_{3} / \mathrm{Al}$ Structures: A Small-Angle X-Ray Scattering and InfraredAbsorption Study." Physical Review B (50:11), p. 7358.

Silver, M., Han, D.X., Wang, K.D., and Kemp, M. (1994). Recombination and Metastability in Amorphous Silicon and Silicon-Germanium Alloys: Final Subcontract Report, I February 1991-31 January 1994. SERI/TP-451-6491. Work performed by the University of North Carolina, Chapel Hill, NC. Available NTIS: Order No. DE94011837.

Silver, M. (1993). Recombination and Metastability in Amorphous Silicon and Silicon Germanium Alloys: Annual Subcontract Report, 1 February 1992-31 January 1993. SERI/TP-451-5834. Work performed by the University of North Carolina, Chapel Hill, NC. Available NTIS: Order No. DE94000237.

Sinha, K., Mascarenhas, A., Alonso, R.G., Horner, G.S., Bertness, K.A., Kurtz, S.R., and Olson, J.M. (1994). "Optical Investigation of the Influence of Substrate Orientation on Spontaneous Ordering in $\mathrm{Ga}_{0.5} \mathrm{In}_{0.5} \mathrm{P}$ Alloy." Solid State Communications (89:10), pp. 843-847. 
Sinha, K., Mascarenhas, A., Horner, G.S., Alonso, R.G., Bertness, K.A., and Olson, J.M. (1993). "Resonance Raman Study of Spontaneous Ordering in GaInP ${ }_{2}$ " Physical Review. B, Condensed Matter (48:23), pp. 17591-17594.

Sites, J.R. (1994). Role of Polycrystallinity in CdTe and CuInSe 2 Photovoltaic Cells: Final Subcontract Report, l April 1990-30 November 1993. SERI/TP-451-7047. Work performed by Colorado State University, Fort Collins, CO. Available NTIS: Order No. DE94011849.

Sites, J.R. (1994). Role of Polycrystallinity in CdTe and CuInSe, Photovoltaic Cells: Annual Subcontract Report, 1 April 1992-31 March 1993. SERI/TP-451-6215. Work performed by Colorado State University, Fort Collins, CO. Available NTIS: Order No. DE94000293.

Sopori, B.L. (1994). "Defect Engineering Concepts for Low-Cost, High Efficiency Solar Cell Process Design." Role of Point Defects and Defect Complexes in Silicon Device Processing: Summary Report and Papers of the Second Workshop, 24-26 August 1992, Breckenridge, CO. National Renewable Energy Laboratory, pp. 11-14. Available NTIS: Order No. DE94011876.

Sopori, B.L. (1994). "Towards 18\% Commercial Si Solar Cells: Research Issues and Approach." Role of Point Defects/Defect Complexes in Silicon Device Processing: Book of Abstracts from the Fourth Workshop, 27-29 June 1994, Beaver Creek, CO. NREL/CP-413-7033, pp. 1-2. Available NTIS: Order No. DE94011828.

Sopori, B.L. and Tan, T.Y., eds. (1994). Role of Point Defects and Defect Complexes in Silicon Device Processing: Summary Report and Papers of the Second Workshop, 24-26 August 1992, Breckenridge, CO. SERI/TP-413-7064. Available NTIS: Order No. DE94011876.

Sopori, B.L. and Tan, T.Y., eds. (1994). Role of Point Defects and Defect Complexes in Silicon Device Processing: Summary Report and Papers of the Third Workshop, 16-18 August 1993, Vail, CO. SERI/TP-413-7061. Available NTIS: Order No. DE94011873.

Sopori, B.L. (1994). "Characterization of Commercial PV Silicon Substrates." NREL Photovoltaic Advanced Research and Development Review: Proceedings of the 12th Review Meeting, 13-15 October 1993, Denver, CO. AIP Conference Proceedings No. 306, R. Noufi and H.S. Ullal, eds. New York: American Institute of Physics, Pp. 3-11.

Stafford, B. (1994). "Design Considerations and Performance of Maspeth a-Si PV System." NREL Photovoltaic Advanced Research and Development Review: Proceedings of the 12th Review Meeting, 13-15 October 1993, Denver, CO. AIP Conference Proceedings No. 306, R. Noufi and H.S. Ullal, eds. New York: American Institute of Physics, pp. 180-187.

Stafford, B. (1994). "Zero Emission Vehicles." Presented at the 45th Annual Earthmoving Industry Conference, April 12-13, 1994, Peoria, IL. SAE Technical Paper Series \#941108.

Stanbery, B.J. (1994). New Plasma Source of Hydrides for Epitaxial Growth: Final Subcontract Report, 15 April 1991-3 September 1993. SERI/TP-451-6400. Work performed by Boeing Defense and Space Group, Seattle, WA. Available NTIS: Order No. DE94006918. 
Strand, T. and McNutt, P. (1994). "Installation and Preliminary Evaluation of Six Photovoltaic Stand-Alone Area Lighting Systems." NREL Photovoltaic Advanced Research and Development Review: Proceedings of the 12th Review Meeting, 13-15 October 1993, Denver, CO. AIP Conference Proceedings No. 306, R. Noufi and H.S. Ullal, eds. New York: American Institute of Physics, pp. 565-572.

Surek, T. (1993). State of the Art of Thin-Film Photovoltaics. SERITP-410-6049. Presented at the K.W. Boer Solar Energy Medal of Merit Award Ceremony, 16 February 1993, Newark, DE. Available NTIS: Order No. DE94000224.

Tan, T., Jastrzebski, L., and Sopori, B. (1994). Fourth Workshop on the Role of Point Defects/ Defect Complexes in Silicon Device Processing: Conference Summary Report. SERI/TP-413-7050. Available NTIS: Order No. DE94011850.

Taoka, S., Jursinic, P.A., and Seibert, M. (1993). "Slow Oxygen Release on the First Two Flashes in Chemically Stressed Photosystem II Membrane Fragments Results from Hydrogen Peroxide Oxidation." Photosynthesis Research (38:3), pp. 425-431.

Thornton, J.P. (1994). "Economics of Photovoltaics Versus Line Extensions: Selected Case Studies." Solar '94: Proceedings of the 1994 American Solar Energy Society Annual Conference, 25-30 June 1994, San Jose, CA. American Solar Energy Society, pp. 102-105.

Trefny, J.U., Furtak, T.E., Williamson, D.L., and Kim, D. (1994). Polycrystalline Thin Film Cadmium Telluride Solar Cells Fabricated by Electrodeposition: Annual Subcontract Report, 20 March 1993-19 March 1994. SERI/TP-451-7046. Work performed by the Department of Physics, Colorado School of Mines, Golden, CO. Available NTIS: Order No. DE94011848.

Tuttle, J.R., Albin, D.S., Tennant, A.L., Gabor, A.M., Contreras, M., and Noufi, R. (1994). "Characterization of Variable-Band-Gap Thin-Film $\mathrm{Cu}(\mathrm{In}, \mathrm{Ga}) \mathrm{Se}_{2}:$ A Simple Model for the Interdiffusion of In and Ga in Alloy Structures." Solar Energy Materials and Solar Cells (35); Amsterdam, The Netherlands: Elsevier Press, pp. 193-201.

U.S. Department of Energy. (1994). Biomass Power Industry: Assessment of Key Players and Approaches for DOE and Industry Interaction (January). DOE/CH10093-257. Prepared by the National Renewable Energy Laboratory, Golden, CO. Available NTIS: Order No. DE94000238.

Ullal, H.S., Zweibel, K., and von Roedern, B.G. (1994). "Thin-Film CdTe and CuInSe Photovoltaic Technologies." Proceedings: ISES Solar World Congress: Harmony with Nature, Volume 3: Photovoltaics, M. Palfy, ed. Budapest, Hungary: Hungarian Energy Society, p. 187.

Vanacek, M., Crandall, R. S., and Mahan, H. (1994). "On the Microscopic Origin of the Higher Stability of Amorphous Silicon Prepared by the Hot-Wire Technique." Proceedings of the 12th European PV Solar Energy Conference, Amsterdam, The Netherlands, p. 2.

Wang, L.W. and Zunger, A. (1994). "Large Scale Electronic Structure Calculations Using the Lanczos Method." Computational Materials Science (2), pp. 326-340. (OER/BES) 
Wang, L.W. (1994). "Calculating the Density of States and Optical-Absorption Spectra of Large Quantum Systems by the Plane-Wave Moments Method." Physical Review. B, Condensed Matter (49:15), pp. 10154-10158. (OER/BES)

Wang, L.W. and Zunger, A. (1994). "Dielectric Constants of Silicon Quantum Dots." Physical Review Letters (73), pp. 1039-1042. (OER/BES)

Wang, L. and Zunger, A. (1994). "Electronic Structure Pseudopotential Calculations of Large (approx. 1000 Atoms) Si Quantum Dots." Journal of Physical Chemistry (98:8), pp. 2158-2165. (OER/BES)

Wang, L.W. and Zunger, A. (1994). "Solving Schrodinger's Equation Around a Desired Energy: Application to Silicon Quantum Dots." Journal of Chemical Physics (100:3), pp. 2394-2397. (OER/BES)

Ward, J.S., Wanlass, M.W., Wu, X., and Coutts, T.J. (1994). "High Performance Contacts to GaInAs TPV Converters." Proceedings of the First NREL Conference on Thermophotovoltaic Generation of Electricity, 24-27 July 1994, Copper Mountain, CO. NREL/CP-413-6186, p. 147. Available NTIS: Order No. DE94011831.

Weaver, P.F, and Maness, P.C., inventors. (1993). Photoconversion of Gasified Organic Materials into Biologically-Degradable Plastics, U.S. Patent No. 5,250,427. Assignee: Midwest Research Institute, Kansas City, MO.

Webb, J.D., Dunlavy, D.J., Ciszek, T., Ahrenkiel, R.K., Wanlass, M.W., Noufi, R., and Vernon, S.M. (1993). "Room-Temperature Measurement of Photoluminescence Spectra of Semiconductors Using an FT-Raman Spectrophotometer." Applied Spectroscopy (47:11), pp. $1814-1819$.

Wei, S.H. and Zunger, A. (1994). "Strain Effects on the Spectra of Spontaneously Ordered $\mathrm{Ga}_{\mathrm{x}} \mathrm{In}_{\mathrm{l}-\mathrm{x}} \mathrm{P} . "$ Applied Physics Letters (64:6), pp. 757-759.

Wei, S.H. and Zunger, A. (1993). "Band Offsets at the CdS/CuInSe ${ }_{2}$ Heterojunction." Applied Physics Letters (63:18), pp. 2549-2551.

Wei, S.H. and Zunger, A. (1994). "Optical Anisotropy and Spin Polarization in Ordered GaInP." Applied Physics Letters (64:13), pp. 1676-1678.

Wei, S. and Zunger, A. (1994). "Optical Properties of Zinc-Blende Semiconductor Alloys: Effects of Epitaxial Strain and Atomic Ordering." Physical Review. B, Condensed Matter (49:20), pp. 14337-14351.

Williamson, D.L., Jones, S.J., and Chen, Y. (1994). Small-Angle X-Ray Scattering Studies of Microvoids in Amorphous-Silicon-Based Semiconductors: Annual Subcontract Report, I February 1992-31 January 1993. SERI/TP-451-6395. Work performed by the Department of Physics, Colorado School of Mines, Golden, CO. Available NTIS: Order No. DE94006931. 
Williamson, D.L., Chen, Y., and Jones, S.J. (1994). "Microstructure of a-Si:H, a-SiGe:H, and a-SiC:H Solar Cell Materials." NREL Photovoltaic Advanced Research and Development Review: Proceedings of the 12th Review Meeting, 13-15 October 1993, Denver, CO. AIP Conference Proceedings No. 306, R. Noufi and H.S. Ullal, eds. New York: American Institute of Physics, p. 442.

Williamson, D.L., Jones, S.J., and Chen, Y. (1994). Small-Angle X-Ray Scattering Studies of Microvoids in Amorp: as-Silicon-Based Semiconductors: Final Subcontract Report, 1 February 1991-31 January 199;-. SERI/TP-411-6855. Work performed by the Department of Physics, Colorado School of Mines, Golden, CO. Available NTIS: Order No. DE94011840.

Witt, C.E., Mitchell, R.L., Thomas, H., and Herwig, L.O. (1994). "Photovoltaic Manufacturing Technology Project (PVMaT) After Three Years." A Collection of Technical Papers, Part 4: Proceedings of the 29th Intersociety Energy Conversion Engineering Conference, 7-11 August, Monterey, CA. American Institute of Aeronautics and Astronautics, pp. 1688-1691.

Witt, C.E., Mitchell, R.L., Thomas, H., and Herwig, L. (1994). PVMaT Project After Three Years. SERI/TP-411-6639. Prepared for the 29th Intersociety Energy Conversion Engineering Conference, 7-11 August 1994, Monterey, CA. Available NTIS: Order No. DE94011877.

Witt, C.E., Mitchell, R.L., Mooney, G.D., Herwig, L.O., Hasti, D., and Sellers, R. (1993). Progress in Phases 2 and 3 of the Photovoltaic Manufacturing Technology Project (PVMaT). SERI/ TP-411-5501. Presented at the 23rd IEEE Photovoltaic Specialists Conference, 9-14 May 1993, Louisville, KY. Available NTIS: Order No. DE94000206.

Wolverton, C.M., Zunger, A., and Lu, Z.W. (1994). "Long- Versus Short-Range Order in $\mathrm{Ni}_{3} \mathrm{~V}$ and $\mathrm{Pd}_{3} \mathrm{~V}$ Alloys." Physical Review. B, Condensed Matter (49:22), pp. 16058-16061. Work performed by the National Renewable Energy Laboratory, Golden, CO, and the Department of Physics, University of California, Davis, CA.

Xiao, Y., McMahon, T.J., Pankove, J.I., and Tsuo, Y.S. (1994). "Existence of a $\mathrm{P}_{\mathrm{bl}}$-Like Defect Center in Porous Silicon." Journal of Applied Physics (76), p. 2260.

Yang, S.-Y., Yang, Y., Abelson, J.R., An, I., Lu, Y., Wronski, C.R., and Collins, R.W. (1994). "Hydrogen Effects on the TCO/a-Si,C:H Interface: Progress by the NREL Wide Band Gap Team." NREL Photovoltaic Advanced Research and Development Review: Proceedings of the 12th Review Meeting, 13-15 October 1993, Denver, CO. AIP Conference Proceedings No. 306, R. Noufi and H.S. Ullal, eds. New York: American Institute of Physics, p. 431.

Yeh, C.Y., Zhang, S.B., and Zunger, A. (1993). "Identity of the Light Emitting States in Porous Silicon Wires." Applied Physics Letters (63), p. 3455.

Yeh, C.Y., Zhang, S.B., and Zunger, A. (1994). "Pressure Dependence of the Band Gaps in Si Quantum Wires." Applied Physics Letters (64:26), pp. 3545-3547. (OER/BES) 
Yeh, C.Y., Wei, S.H., and Zunger, A. (1994). "Relationships Between the Band Gaps of the Zinc-Blende and Wurtzite Modifications of Semiconductors." Physical Review. B, Condensed Matter (50:4), pp. 2715-2718. (OER/BES)

Yeh, C.Y., Zhang, S.B., Froyen, S., and Zunger, A. (1994). "Confinement Effects in Supported vs Isolated Quantum Structures: A Study of Si(100) Film." Superlattices and Microstructures (14), pp. 141-148.

Yeh, C.Y., Zhang, S.B., and Zunger, A. (1993). "Identity of the Light-Emitting States in Porous Silicon Wires." Applied Physics Letters (63:25), pp. 3455-3457.

Zhang, S.B. and Zunger, A. (1993). "Prediction of Unusual Electronic Properties of Si Quantum Films." Applied Physics Letters (63), pp. 1399-1401.

Zhang, J.G., Benson, D.K., Tracy, C.E., and Deb, S.K. (October 1993). "Influence of Microstructure on the Electrochromic Properties of $\mathrm{Li}_{x} \mathrm{WO}_{3}$ Films: Part II Limiting Mechanisms in Coloring and Bleaching Processes." Journal of Materials Research (8:10), pp. 2657-2667.

Zhang, S.B., Yeh, C.Y., and Zunger, A. (1993). "Electronic Structure of Semiconductor Quantum Films." Physical Review. B, Condensed Matter (48:15), pp. 11204-11219.

Zhang, J.-G., Tracy, C.E., Benson, D.K., and Deb, S.K. (1993). "Influence of Microstructure on the Electrochromic Properties of $\mathrm{Li}_{x} \mathrm{WO}_{3}$ Films: Part I. Ion Diffusion and Electrochromic Properties." Journal of Materials Research (8:10), pp. 2649-2656.

Zunger, A. (1994). "First Principles Statistical Mechanics of Semiconductor Alloys and Intermetallic Compounds." Statics and Dynamics of Alloy Phase Transformations. New York: Plenum Press, pp. 361-419.

Zunger, A. (1993). "Prediction of New Semiconductor and Transition Metal Structures and Their Properties." Japan Journal of Applied Physics Supplement (32:3), pp. 14-21.

Zunger, A. and Mahajan, S. (1994). "Atomic Ordering and Phase Separation in Epitaxial III-V Alloys." Handbook of Semiconductors, Vol. 3, second edition. Amsterdam, The Netherlands: Elsevier Press, pp. 1399-1514.

Zweibel, K. (1994). "Thin Film PV Partnership Program (1995-1999)." Proceedings of Solar '94, The 1994 American Solar Energy Society Annual Conference, 25-30 June 1994, San Jose, CA. Boulder, CO: American Solar Energy Society, pp. 15-23.

Zweibel, K., Ullal, H.S., von Roedern, B.G., Noufi, R., Coutts, T.J., and Al-Jassim, M.M. (1994). "Perspectives and Opportunities in Polycrystalline Thin-Film Photovoltaic Technologies." Proceedings: Conference Record of the 23rd IEEE Photovoltaic Specialists Conference, 10-14 May 1993, Louisville, KY, p. 379. 
Public reponing ourden for this collection of information is estimated to average 1 hour per response, including ihe time tor reviewing instructions, searching existing zata

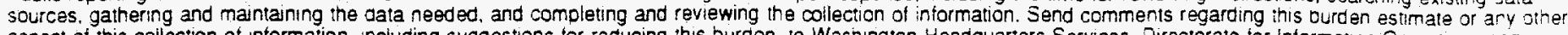
ascect of this coilection of Information. including suggestions for reducing this burden. to Wasnington Headquarters Services, Directorate ior information Ooerations ano Reports, 1215 Jefferson. Davis Highway, Sute 1204, Arlington. VA 22202-4302, and to the Office of Management and Budget Paperwork Feduction Projeci 10704-9: a8. Washington, DC 20503.

\begin{tabular}{|l|l|l} 
1. AGENCY USE ONLY (Leave blank) & $\begin{array}{c}\text { 2. REPORT DATE } \\
\text { June } 1995\end{array}$ & $\begin{array}{l}\text { 3. REPORT TYPE AND DATES COVERED } \\
\text { Annual Report }\end{array}$
\end{tabular}

4. TITLE AND SUBTITLE
NREL Photovoltaic Program FY 1994 Annual Report

5. FUNDING NUMBERS

C: N/A

6. AUTHORIS

TA: PV510101

K.A. Summers, Coordinator

7. PERFORMING ORGANIZATION NAME(S) AND ADDRESS(ES)

9. SPONSORING/MONITORING AGENCY NAME(S) AND ADDRESS(ES)

National Renewable Energy Laboratory

1617 Cole Blvd.

Golden, CO $80401-3393$

10. SPONSORING/MONITORING AGENCY REPORT NUMBER

PERFORMING ORGANIZATION REPORT NUMBER

TP-410-7993

DE95009244

\section{SUPPLEMENTARY NOTES}

NREL Technical Monitor: N/A

12a. DISTRIBUTION/AVAILABILITY STATEMENT

UC -1250

13. ABSTRACT (Maximum 200 words)

This report summarizes the in-house and subcontracted research and development (R\&D) activities under the National Renewable Energy Laboratory (NREL) Photovoltaics (PV) Program from October 1, 1993, through September 30 , 1994 (fiscal year [FY] 1994). The NREL PV Program is part of the U.S. Department of Energy's (DOE) National Photovoltaics Program, as described in the DOE Photovoltaics Program Plan; FY 1991-FY1995. The mission of the DOE National Photovoltaic Program is to: "Work in partnership with U.S. industry to develop and deploy photovoltaic technology for generating economically competitive electric power, making photovoltaics an important contributor to the nation's and the world's energy use and environmental improvement. The two primary goals of the national program are to (1) maintain the U.S. PV industry's world leadership in research and technology development, and (2) heip the U.S. industry remain a major, profitable force in the worid market. The NREL PV Program provides leadership and support to the national program toward achieving its mission and goals.

14. SUBUECT TERMS

photovoltaics ; solar cells

15. NUMBER OF PAGES 439

16. PRICE CODE

17. SECURITY CLASSIFICATION OF REPORT Unclassified 18. SECURITY CLASSIFICATION
OF THIS PAGE Unclassified
19. SECURITY CLASSIFICATION OF ABSTRACT Unclassified
20. LIMITATION OF ABSTRACT

UL 